NASA Technical Memorandum 87798

\title{
Molecular Clouds in the Carina Arm
}

David Andrew Grabelsky

NASA Goddard Institute for Space Studies

New York, New York

\section{N/Sก}

National Aeronautics and Space Administration

Scientific and Technical Information Branch 


\section{TABLE OF CONTENTS}

ACKNOWLEDGEMENTS

I. INTRODUCTION

A. The Carina Arm: A Brief Review 5

1. Optical Studies 5

2. Radio Continuum 8

3. Radio Recombination Line Studies 9

4. HIStudies 10

5. Molecular Line Observations 11

B. Present Work 13

11. INSTRUMENTATION AND OBSERVING TECHNIQUES 15

A. instrumentation $\quad 15$

1. Antenna 16

2. Mount and Drive System 17

3. Receiver 19

4. Spectrometer 20

5. Computer System 21

B. Calibration and Observing Techniques 24

III. OBSERVATIONS 30

PREZZDiNe PAGE BLAMK NOT FILMED 
IV. LARGE-SCALE PROPERTIES OF MOLECULAR GAS IN THE CARINA ARM

A. Kinematics and Distribution of $\mathrm{CO}$ in the Carina Arm

1. The I, V Diagram

2. The Spatial Maps

42

3. The Distribution About the Galactic Plane

B. Comparison with $\mathrm{HI}$

1. The i, Diagrams

50

2. The Spatial Maps

54

3. The z-Distribution

55

C. Summary

57

V. THE CARINA ARM MOLECULAR CLOUDS

59

A. Identification of the Clouds 61 Distances 63

Masses 64 
B. The $\eta$ Carinae Molecular Cloud 65

1. Identification of the Cloud Complex 65 Mass 68

2. The $\eta$ Carinae Nebula and the Molecular Cloud 69

Stellar Winds 74

Rocket Effect 77

3. Star Formation Efficiency in the $\eta$ Carinae Molecular Cloud $\quad 79$

4. Summary 86

C. The Carina Arm in the Galaxy 87

D. Notes on Individual Clouds 90

$\begin{array}{ll}\text { VI. SUMMARY } & 108\end{array}$

$\begin{array}{ll}\text { REFERENCES } & 112\end{array}$

APPENDIX A: TELESCOPE POINTING 120

A. Determination of Pointing Parameters 121

B. Pointing Accuracy 122

1. Shaft Encoders 122

2. Starpointing 122

3. Sunpointing 123 
APPENDIX B: ADDITIONAL I,V AND b,V MAPS

1. IV at each $b$; full resolution

2. $b, v$ at each /; full resolution

251

3. I, $\mathrm{V}$ at each $\mathrm{b} ; 0.5^{\bullet}$ Superbeam

312

4. $b, v$ at each $1 ; 0.5^{\bullet}$ superbeam

333 


\section{ACKNOWLEDGEMENTS}

Working with Patrick Thaddeus, my adviser, has been one of the highlights of my graduate studies at Columbia University. His passion for science coupled with his gift for recasting complex concepts in intuitive terms made studying under him stimulating and rewarding. It is a pleasure to thank him for his support, guidance, and encouragement during the years 1 spent on the Southern Millimeter-Wave Telescope Project.

Richard Cohen, my co-adviser, deserves thanks for numerous reasons, but two in particular. First, he is largely responsible for the success of the Southern Millimeter-Wave Telescope. Without Richard's scientific and technical know-how and direction, the Southern Telescope would still be a pile of nuts, bolts, waveguides, and transistors in New York. Second, with his special brand of intellectual challenge, he did much to help me steer clear of sloppy thinking. I learned a great deal working with Richard, and I am pleased to express my appreciation to him for sharing his talents.

Throughout all phases of my work on this thesis, from helping to build and test the telescope in New York to analysis and interpretation of the data after I returned from Chile, Tom Dame provided valuable advice and assistance, as well as encouragement and friendship. For this -- and for his good company on several long runs in Central Park -- I am grateful.

I would like to thank many other people, in both New York, at the Goddard Institute for Space Studies and Columbia University, and in Chile, for helping make this thesis possible. Sam Palmer and Dennis Mumma helped construct the telescope and taught me how to trouble-shoot its electronics. Dorn Peterson had an integral part in the receiver effort. Leo Bronfman assembled the receiver and kept it running, and, as my fellow graduate 
student on the project, was an important partner and companion in all aspects of the work. Joe Montani and Mustafa Koprucu helped bulld the telescope and, with the aid of Jorge May, Hector Alvarez, and Monica Rubio of the University of Chlle, kept it running smoothly. Pat Osmer, as director of CTIO, along with Victor Blanco, John Graham and the rest of the observatory staff put many of the observatory's facilities at the disposal of the Columbia Millimeter-Wave Project and provided a harmonious working environment for the Columbia group. I am also very appreciative of Victor and Betty Blanco for their warm hospltality during my stay in Chile.

Thanks also go to the (past and present) faculty of the Astronomy Department at Columbla University, Bruce Elmegreen in particular, for their interest and support, and to my fellow graduate students for their friendship. At the Goddard Institute for Space Studies, Richard Stothers provided several informative discussions regarding clusters and the use of the intial mass function. Allison Smith alded in the reduction of the data; and Ellin Sarot helped iron out some kinks in the manuscript.

I am indebted to my entire family -- my parents, my brothers and sisters and their children, and my in-laws -- for their perpetual love, support, and encouragement throughout the many years that led to this thesis. Finally, my deepest affection and gratitude go to my son Jonah for preventing me from growing old over the last two years, and to my wife Becky for her tolerance, understanding, and unparalleled love during the entire course of this work. 


\section{INTRODUCTION}

A spiral arm in Carina was first proposed by Bok in 1937, just fourteen years after spiral nebulae were conclusively shown to be distant, extragalactic stellar systems. Having noted stellar concentrations elongated in the directions of Carina and Cygnus, Bok (1937) concluded his monograph The Distribution of Stars in Space:

The observer in the tropics should not find it difficult to accept as a working model for our Milky Way one with a distant centre in Sagittarius and in which a spiral arm passes from Carina through the Sun toward Cygnus.

Since then, the Carina arm has been firmly established in all spiral tracers as one of the best-defined major spiral arm segments in the Galaxy. Following the pioneering studies of local spiral structure in the Northern Milky Way by Morgan, Sharpless, and Osterbrock (1952) and Morgan, Whitford, and Code (1953), complementary studies were undertaken in the Southern galactic plane. It soon became evident that the Carina region was host not only to Bok's "elongated" grouping of stars, but also to a rich and varied collection of Population I objects which appeared to be spread out over considerable distances. Whether the observed relative paucity of optical spiral tracers preceding (in galactic longitude) the Carina concentration was due to overlying extinction or represented a true boundary to the bright feature was not certain. By comparing the optical data and radio continuum observations in the region, Sher (1965) argued that the edge was real and showed that the 0 and $B$ stars, Cepheids, young clusters, and emission nebulae appeared to trace a spiral arm aligned nearly parallel to the line of sight in Carina. Bok, who from the beginning had held 
that a major spiral feature existed in Carina, delivered additional strong support for Sher's interpretation. From a comparison study of Population I in Carina which made use of a substantial amount of new data, including the OB star survey of Graham and Lyngå (1965) and the (then unpublished) radio recombination line survey of Wilson, Bok et al.(1970) provided what has become the canonical picture of the Carina arm: the arm opens outward starting from a point $\sim 2 \mathrm{kpc}$ from the Sun near $l=295^{\circ}$; it is viewed tangentially at $l \approx 283^{\circ}$, where it crosses the solar circle, then extends to $\sim 10 \mathrm{kpc}$ from the Sun as it bends back toward higher longitudes. The evidence that led to this picture of the arm is reviewed below. How the Carina arm connected with other spiral arms on the other side fo the galactic center (i.e., in the first quadrant) was (and remains) a matter of some debate and it is a question which will be addressed in this thesis.

Part of the problem of connecting the Carina arm with the rest of the Galaxy comes from the difficulty in interpreting the neutral hydrogen observations in terms of large-scale galactic structure. The early $21-\mathrm{cm}$ pictures of spiral structure differed from the optically-determined local picture, the neutral hydrogen suggesting nearly circular arms while the optical tracers indicated high-pitched arms. If the young stars formed from the $\mathrm{HI}$, shouldn't both trace the same arms? Although part of this discrepancy could be traced to the different types of distances used -optical versus kinematic -- no direct observational link existed between the massive stars and the neutral hydrogen from which presumably they formed.

With the advent of millimeter-wave astronomy in the late 1960's, it soon became apparent that molecular gas -- $\mathrm{H}_{2}$ in particular -- was at least as important a constituent of the interstellar medium in the interior 
regions of the Galaxy as $\mathrm{HI}$, and the $\mathrm{J}=1 \rightarrow 0$ rotational transition of $\mathrm{CO}$ emerged as the best tracer of the abundant $\mathrm{H}_{2}$ molecule. The placental material of massive stars was discovered: giant molecular clouds. As the birth sites of $O$ and early $B$ stars and their accompanying $H \|$ regions (see e.g., Blitz 1978), giant molecular clouds might at last provide a physical link between the distant but obscured radio arms and the local optical arms. The first $\mathrm{CO}$ surveys of the inner Galaxy showed that $\mathrm{H}_{2}$ was concentrated in a broad "ring" about the galactic center, but they did not reveal any apparent large-scale spiral structure (Burton et al. 1975; Scoville and Solomon 1975). However, these early surveys were severely undersampled, and subsequent, well-sampled surveys in the first galactic quadrant of fer a more complete view of CO spiral structure (Cohen et al. 1980; Dame 1983; Sanders et al. 1985).

Observed in CO, giant molecular clouds now appear to be superior to neutral hydrogen as a tracer of large-scale spiral structure. Like the $\mathrm{HI}$, they are easily observed throughout the galactic disk and, in the first and second galactic quadrants, delineate the same arms (Cohen et al. 1980). But unlike the $H$ l, the $C O$ appears to exhibit high arm-interarm contrast (Dame 1983). It does not necessarily follow, however, that molecular clouds are more concentrated in the arms than $\mathrm{HI}$, since streaming motions of only a few kilometers per second can mimic spiral arms in the data (Liszt and Burton 1981). Using the Columbia CO survey of the first quadrant, Dame (1983) argued that molecular clouds are indeed more confined to the arms by demonstrating that the $\mathrm{CO}$ and $\mathrm{H} I$ share the same large-scale kinematics, and thereby ruling out differences in streaming motions as the cause of the apparently higher arm-interarm contrast seen in $\mathrm{CO}$ than in $\mathrm{H} \mathrm{I}$. 
The degree to which molecular clouds are themselves confined to the arms, at least in the region of the "molecular ring," is still controversial. Recently, for example, Solomon et al.(1985) argued that two distinct populations of molecular clouds, represented by $\sim 2000$ warm and cold "cores" identifled between $/=20^{\circ}$ and $50^{\circ}$, exist in the Galaxy. They claim that the warm cores appear to reside in the spiral arms, while the cold cores, outnumbering the warm 3 to 1 , show a kinematic distribution consistent with no concentration to the arms. Alternatively, Dame et al. (1985), taking partial inventory of the most massive molecular clouds in the first quadrant and locating them in the Galaxy by a variety of techniques, found that spiral arms are well traced by the largest clouds. The mass spectrum of molecular clouds in the Galaxy being dominated by the largest clouds (see e.g., Dame 1983; Sanders et al. 1985), the question of conf inement of molecular clouds to the arms is better addressed considering clouds by mass rather than by number. In this thesis the approach of Dame et al. is used to show that in the Carina arm, as in the iirst quadrant, the largest molecular clouds are good spiral-arm tracers, and that just outside the Carina arm, the total mass of molecular gas is relatively small.

A significant characteristic of the Carina region, recognized since the earllest studies of the Carina arm, is its high optical transparency. Some stars and optical $H \| I$ regions can be seen to distances greater than $10 \mathrm{kpc}$. Several radio $H I I$ regions have distances spanning the same range, many of them the radio counterparts of the optical $H$ II regions. The Carina arm provides a rare example in the Galaxy of a spiral arm which is delineated over many kiloparsecs in all the classic radio and optical tracers, and so provides an opportunity to compare the classic optical spiral-arm tracers 
and their parent molecular clouds over the same large distance. Based on the first well-sampled co survey in the Carina arm, this thesis offers an initial contribution to such a comparison. To place the observations presented here in the context of the known structure of the Carina arm, the following brief review of previous observations of that region is given.

A. The Carina Arm: A Brief Review

\section{Optical Studies}

Studies of OB stars in Carina have been made by several authors, among them Bok and van Wijk (1952), Graham and Lyngå (1965), and Feinstein (1969), whose main results are worth discussing briefly.

Based on the observations of Bok and van Wijk, Bok (1956) pointed out the large concentration of $O B$ stars in Carina from distances of $\sim 1.5$ to $4 \mathrm{kpc}$, and, expanding on his 1937 study of the region, suggested we were viewing a spiral arm oriented roughly along the line of sight. Bok also noted that throughout the region the optical extinction was generally low and that there was an apparent edge to the stellar distribution at $/ \approx 283^{\circ}$. Feinstein obtained UBV photometry for 135 OB stars with known spectral types in the region and found they extended at least to $6 \mathrm{kpc}$ but no closer than $1.6 \mathrm{kpc}$. Using the objective prism survey of Graham and Lyngå of 436 $O B$ stars, Graham (1970) determined that the stars span distances of 2 to $10 \mathrm{kpc}$ between $/=282^{\circ}$ and $292^{\circ}$. He found the distribution to have a sharp boundary that became more distant with longitude. Arguing that this apparent boundary represented a true edge in the $O B$ star distribution, Graham proposed that we were seeing the outer edge of the Carina arm and 
located the tangent direction at $/ \approx 285^{\circ}$. In addition, he pointed out that the stars were found primarily at negative latitudes, with a $z$-displacement that increased with distance.

A statistical study of southern OB stars by Lynga (1970) led to the identification of eight $O B$ concentrations between $I=284^{\circ}$ and $330^{\circ}$, five of them in the Carina region. Some are galactic clusters, while others are OB associations within $\sim 3.5 \mathrm{kpc}$ (Humphreys 1972;1978). Young clusters in the Carina region show a wide range of distances, and their distribution traces a spiral arm in good agreement with that traced by the individual OB stars (Vogt and Moffat 1975b and references therein).

The list of stellar spiral tracers in Carina also includes young Cephelds, observed to $10 \mathrm{kpc}$ (Fernie 1968; Tammann 1970), and supergiants of all spectral types (Humphreys 1970). Based on the Schmldt rotation curve, Humphreys (1970) found evidence among the supergiants for noncircular motions which varled systematically within the longitude range $280^{\circ}$ to $300^{\circ}$. Since the supergiants fit the same spatial distribution as the other tracers, Humphreys interpreted the negative velocity residuals (i.e., with galactic rotation in the fourth quadrant) between $l=280^{\circ}$ and $292^{\circ}$ and the positive residuals (against galactic rotation) between $I=292^{\circ}$ and $300^{\circ}$ as streaming motions along the outer and inner edges of the arm.

Three properties of the stellar distributions that led investigators to suggest a lengthwise spiral arm in Carina were the richness of the stellar content (particularly the $\mathrm{OB}$ stars), the great distances present in the narrow longitude range, and the rather abrupt appearance of the concentration, beginning at $/ \approx 283^{\circ}$. These same properties were evident in the Ho surveys of Hoifleit (1953), Gum (1955), and Rodgers, Campbell, and Whiteoak (1960, hereaf ter RCW), although distances to each emission 
region were not initially available. Particularly noticable in the RCW atlas (Rodgers, Campbell, Whiteoak, Bally, and Hunt 1960) is the relatively low $\mathrm{H \alpha}$ intensity preceeding the large array of emission regions which sets in near the $\mathrm{OB}$ star edge. The entire region between $/=283^{\circ}$ and $297^{\circ}$ is rich in $\mathrm{H} \alpha$, as a glance at the RCW atlas demonstrates. Further evidence that the edge near $283^{\circ}$ marked the tangent direction of the Carina arm was provided by the radio continuum observations, as described below. Some of the brighter nebulae in the Carina region include RCW 53 ( $\eta$ Carinae Nebula), RCW 54, RCW 57 (NGC 3576 and NGC 3603), and RCW 62 (IC 2944). Interferometric studies in Ho by Georgelin and Georgelin (1970a; 1970b) ylelded kinematic distances to enough $\mathrm{H} I \mathrm{Il}$ regions to trace the Carina arm to $8 \mathrm{kpC}$ near $I=290^{\circ}$. A similar investigation by Bigay et al. (1972) showed the $\mathrm{H} I \mathrm{I}$ regions near $l=282^{\circ}$ to lie between 2.5 and $5 \mathrm{kPC}$, straddling the solar circle in that direction. Many of the Horegions included in early catalogs were subsequently detected in radio recombination lines, and the wide range of observed velocities implied that the optical regions were spread out over considerable distances.

The large number of optical objects seen to such great distances in the narrow longitude range of the Carina arm implies generally low optical extinction. Indeed, this well-known characteristic of the Carina region motivated some of the optical studies. With data available on Cepheids, Sher (1965) indicated that visual absorption was less than one magnitude per kiloparsec out to $\sim 5 \mathrm{kpc}$. Bok et al. (1970) estimated 0.5 magnitudes per kiloparsec between $/=282^{\circ}$ and $305^{\circ}$ to comparable distances, and more recent studies of the large-scale distribution of interstellar extinction (Sundman 1979; Loden and Sundman 1980; Neckel and Klare 1980) also noted the high transparency of the Carina region. 
2. Radio Continuum

Mills (1959) suggested that steps in the radio continuum along the galactic plane were due to lines of sight along tangent directions to spiral arms, noting one such a step (among others) at $/ \approx 281^{\circ}$ in the $85.5 \mathrm{MHz}$ survey of Hill et al.(1958). Sher (1965) emphasized the coincidence of the trough in the $1.4 \mathrm{GHz}$ continuum map of Mathewson, Healy and Rome (1962a, hereafter MHR) with the low Ho intensity just anead of the bright edge of the Carina concentration in the RCW atlas. Sher reasoned that the optically dark region truly lacked Population I material since optical absorption cannot be responsible for a radio continuum dip. The coincidence of the sudden jump in the continuum at $I \approx 283^{\circ}$ with the onset of the optical Population I concentration then led to the conclusion that this longitude marked the outer edge of the Carina arm.

In the MHR survey, which had a spatial resolution of $50^{\circ}$, fourteen discrete sources were Identified between $I=280^{\circ}$ and $300^{\circ}$; between $270^{\circ}$ and $280^{\circ}$ only two sources were found. Based on their spectral indices (determined by comparison with the $85.5 \mathrm{MHz}$ survey of Hill et al) eight of the fourteen sources were classified as thermal and two as nonthermal (Mathewson, Healy and Rome 1962b). Surveys with higher spatial resolution were carried out at $2650 \mathrm{MHz}$ (Thomas and Day 1969), at $5000 \mathrm{MHz}$ (Goss and Shaver 1970), and at $408 \mathrm{MHz}$ (Shaver and Goss 1970a), all aimed at observing previously identified sources. The spatial resolution of the $2650 \mathrm{MHz}$ survey was $8.2^{\prime}$; the other two surveys had resolutions of $4^{\circ}$ and $3^{\circ}$. At $2650 \mathrm{MHz}, 40$ sources were found between $/=289^{\circ}$ and $300^{\circ}$, six of them identified (some tentatively) with MHR, Hill et al., or RCW sources. At 
$408 \mathrm{MHz}, 37$ sources between $l=280^{\circ}$ and $300^{\circ}$ were found (versus 29 at $5000 \mathrm{MHz}$ ); of these, 28 were observed at both frequencles. Where determinations could be made, 15 sources were classifled as thermal and eight nonthermal (Shaver and Goss 1970b). More recently, Haynes et al. (1978) carried out a high-sensitivity survey at $5 \mathrm{GHz}$ with a $4^{\prime}$ beam, finding 104 sources between $I=280^{\circ}$ and $300^{\circ}$ (Haynes et al. 1979). A prominent characteristic of the continuum maps of all these surveys is the clustering of strong sources between $I=282^{\circ}$ and $292^{\circ}$.

In addition to these continuum surveys, high resolution studies of individual sources in the arm have also been made, including 50" resolution observations of NGC 3576 and NGC 3603 (Retallack and Goss 1980) and the $\eta$ Carina Nebula (Retallack 1983). These measurements will be considered in Chapter V, where the molecular clouds associated with these objects are discussed.

3. Radio Recombination Line Studles

The most comprehensive study of radio recombination lines in the Southern Milky Way was by Wilson et al.(1970), in H109 $\alpha$ Between $I=282^{\circ}$ and $300^{\circ} 24$ sources were found, nine of them belonging to the Carina Nebula. Less extensive observations have been made in the following lines: $H 126 \alpha$ and $H 127 \alpha$ (MCGee and Gardner 1968), He 109 $\alpha$ and C109 $\alpha$

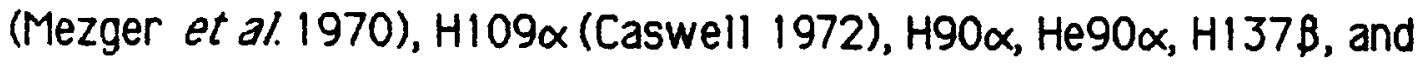

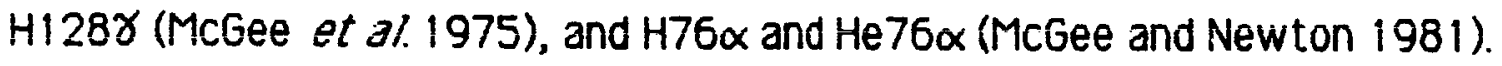
None of these found any additional sources in the Carina arm. The significant result of all these studies (Wilson et al, in particular) is that within $l=280^{\circ}$ and $300^{\circ}$ the wide range of velocities observed implies a 
wide range of kinematic distances. As already noted, the radio $\mathrm{H} / \mathrm{l}$ regions paint a picture of the Carina arm in complete accord with the optical picture.

The $\eta$ Carinae Nebula has been the subject of a number of recombination line investigations; these will be mentioned in Chapter $V$ in a detalled discussion of the giant molecular cloud associated with this very bright $\mathrm{H} I \mathrm{l}$ region.

\section{HI Studies}

An arm-like concentration of $\mathrm{H} I$ in Carina was evident in the earliest 21 -cm surveys of the Southern Milky Way (see e.g., Kerr et al. 1957 and Oort et al. 1958). Taken together, Northern and Southern surveys suggested the $\mathrm{HI}$ in Carina passed through the Sun toward Cygnus (although one might interpret the early $21-\mathrm{cm}$ plane-of-the-Galaxy maps as having all the "arms" passing through the Sun). Bok (1959) quickly recognized the good agreement of the neutral hydrogen results with the distribution of OB stars, HII regions, and Cepheids, and again argued for a Carina-Cygnus arm. A general property of the $\mathrm{HI}$ in the Southern Galaxy to emerge from the first $21-\mathrm{cm}$ studies was an apparent warping of the $\mathrm{H} I$ plane to negative latitudes, which increased with distance from the galactic center. (Graham [1970] found a similar trend in the OB stars and pointed out the agreement with the $H$ I results.) In later attempts to deduce the overall spiral structure of the Galaxy from $21-\mathrm{cm}$ observations, considerable disagreement has arisen on how to connect the Carina with the Northern spiral arms. For example, Kerr (1970) maintained that the arms were nearly circular and that Carina connected with Cygnus through the Sun -- the 
picture Bok favored. Weaver (1970), on the other hand, proposed higherpitched arms, Joining Carina and Sagittarius in a single arm. (A review of the arguments for both alternatives can be found in Simonson [1970]). Recently, Henderson et al.(1982) indicated that the Carina arm extends to longitudes below $280^{\circ}$ beyond the solar circle, which, if true, makes the Carina-Sagittarius connection unlikely.

Aside from the question of how to match the north and south, all $21-\mathrm{cm}$ studies confirm three properities of the $\mathrm{H} /$ distribution in the Southern Galaxy: the downward warping of the galactic $\mathrm{H}$ I plane, the widening of the $\mathrm{H} /$ layer with galactocentric distance, and the persistence of the Carina arm to distances of $\sim 20 \mathrm{kpc}$. The Carina arm is unquestionably a major spiral feature. In Chapter IV the $21-\mathrm{cm}$ picture of the Carina arm is examined in more detail and the question of its place in the Galaxy will be considered in light of a comparison of the $\mathrm{HI}$ and the $\mathrm{CO}$.

5. Molecular Line Observations

Molecular absorption-line surveys of the Southern Galaxy were carried out in the $1667 \mathrm{MHz}$ line of $\mathrm{OH}$ (Caswell and Robinson 1974) and in the $4830 \mathrm{MHz}$ line of $\mathrm{H}_{2} \mathrm{CO}$ (Whiteoak and Gardner 1970; 1974). The observations, taken toward known continuum sources, detected lines over a wide range of velocities in Carina, which, like the recombination line measurements, imply a wide range of distances. The same conclusion can be drawn from the 1662 and $1665 \mathrm{MHz} \mathrm{OH}$ emission-line observations of Manchester et al. (1970). These studies provided the first indications that molecular material fits the same pattern as the other spiral tracers in the Carina arm. 
Other molecules detected in the arm include $\mathrm{NH}_{3}, \mathrm{H}_{2} \mathrm{O}, \mathrm{HCN}, \mathrm{HCO}, \mathrm{HCO}$, and $\mathrm{CO}$ (Whiteoak 1983 and references therein). Nine $\mathrm{H}_{2} \mathrm{O}$ masers, all associated with known $H$ II regions in the Carina arm, have been discovered between $I=284^{\circ}$ and $300^{\circ}$ (Scalise and Schall 1977; Kaufmann et al. 1977; Scalise and Braz 1980; Batchelor et al. 1980; Braz and Scalise 1982).

The first CO survey in the Southern Galaxy was made in the $J=1 \rightarrow 0$ line by Gillispie et al.(1977). Five detections were made between $I=284^{\circ}$ and $300^{\circ}$ in the direction of well-known $\mathrm{H} / 1$ regions, including the Carina Nebula, NGC 3576, and NGC 3603. Subsequently, the Carina nebula was observed in the $2 \rightarrow 1$ transition of CO by de Graauw et al.(1981), who mapped a small portion of the region. Further observations in the $2 \rightarrow 1$ line toward individual sources were also made by White and Phillips (1983). In the $J=1 \rightarrow 0$ CO survey at $b=0^{\circ}$ made with the $4 \mathrm{~m}$ telescope at CSIRO (McCutcheon et al. 1982; Robinson et al. 1983) the distant portions of the Carina arm were barely detected, because this part of the arm lies almost entirely below the plane (see Chapter IV); the tangent region, lying beyond the low-longitude boundary $\left(294^{\circ}\right)$ of this survey, was completely missed. A $J=2 \rightarrow 1$ survey by Israel et al.(1984) covered $I=270^{\circ}$ to $355^{\circ}$ at $b=0^{\circ}$, but again, owing to the survey's sparse sampling, restricted latitude coverage, and low sensitivity, the Carina arm went largely undetected. 
B. Present Work

This thesis reports the results of the first well-sampled, large-scale $\operatorname{CO}(J=1 \rightarrow 0)$ survey of molecular clouds in the Carina arm. The survey was made with the Columbia $1.2 \mathrm{~m}$ millimeter-wave telescope at Cerro Tololo, Chile. Prior to carrying out the observations presented here, I participated in the construction and testing of the instrument in New York City and its subsequent installation at Cerro Tololo. Being a close copy of the Columbia millimeter-wave telescope in New York City, the Chile telescope not only incorporated a proven design, but also offered the added benefit that new Southern CO surveys and previously completed Northern CO surveys (made with the New York telescope) could be joined together with almost none of the calibration problems that have traditionally plagued the North-South matching of galactic surveys made with different instruments. Since the largest molecular clouds in the first and second quadrants have proven to be excellent spiral tracers (Dame et al. 1985), one would hope that a survey of these objects in the Carina arm would provide an answer to the question of the Carina arm's connection with the Northern arms as seen in Co. It does. The first results of this survey of the Carina arm were reported by Conen et al. (1985a). In that paper, we showed that the arm is traced exceptionally well over $25 \mathrm{kpc}$ by the molecular clouds and that the Carina arm apparently joins the Sagittarius arm, as defined by molecular clouds identified in the Columbia CO survey of the first quadrant (Dame et al. 1985). This thesis presents the observations and analysis that led to these conclusions.

In the Chapter II the telescope and observing techniques are described, and in Chapter III the observations of the Carina region are presented in various forms and some of the reduction techniques are described. 
Chapter IV focuses on the large-scale characteristics of the arm, including a discussion of the z-distribution of the molecular layer and a comparison of the $\mathrm{CO}$ and the $\mathrm{HI}$. In Chapter $\mathrm{V}$ the individual giant molecular clouds are identified and cataloged, with special attention given to the cloud associated with the $\eta$ Carinae Nebula; the clouds are used to trace the arm over $25 \mathrm{kpc}$ and compared with clouds found in the first and second quadrants to determine how the Carina arm connects with the rest of the Galaxy. Chapter VI presents a summary of the results. 


\section{INSTRUMENTATION AND OBSERVING TECHNIQUES}

The observations described here were carried out at the Cerro Tololo Inter-American Observatory (CTIO) in Chile with the Columbia millimeterwave telescope (hereafter the Southern Millimeter-Wave Telescope, or just the Southern Telescope), a close copy of the Columbia millimeter-wave telescope in New York City (the Northern MIllimeter-Wave Telescope, or the Northern Telescope). Prior to installation at CTIO, the telescope was thoroughly tested in New York City on the roof of the Goddard Institute for Space Studies (GISS). In 1982 November it was dismantled and shipped to Cerro Tololo where it arrived near the end of 1982 December; by the beginning of 1983 January the Southern Millimeter-Wave Telescope was in full operation. The first part of this chapter presents a description of the instrument, and the second part a discussion of the calibration and observing techniques.

A. Instrumentation

The telescope consists of five principal components: 1) the antenna; 2) the mount and drive system; 3) the receiver; 4) the spectrometer; and $5)$ the computer system. Each of these is briefly described. My primary responsibllity having been the mount and drive and the computer system, these sections will be emphasized. Results of the pointing tests are presented in Appendix A; a more detailed description of the antenna and the recelver can be found in Bronfman (1985). See the theses of $R$. Conen (1977) and G. Chin (1977) and Cohen et al. (1985b) for details regarding the 
pointing and computer system of the Northern Millimeter-Wave Telescope that are relevant to the Southern Millimeter-Wave Telescope.

\section{Antenna}

The antenna is a Cassegrain system consisting of a 1.2 meter parabolic primary reflector $(f / D=0.375)$ and a $17.8 \mathrm{~cm}$ hyperbolic secondary reflector. The effective focal ratio of the primary plus the secondary is 3.79 . The primary was manufactured by Philco Ford with a measured Rris surface accuracy of 36 microns, but was subsequently resurfaced to an RMS accuracy of $\sim 6$ microns as a prototype for a balloon-borne telescope for continuum observations in the far infrared. Deformation of the primary under gravitational stress contributes at most an additional 6 microns (Chin 1977), giving a total surface accuracy of 12 microns, corresponding to $\sim \lambda / 200$ at $2.6 \mathrm{~mm}$, about four times the usual criterion for a diffraction limited system.

To check that the primary, secondary, and horn were all properly aligned and that the antenna was diffraction limited as claimed, the antenna pattern was measured following the general procedure described by Conen (1977). With a $115 \mathrm{GHz}$ transmitter located atop Cerro Morado, a mountain a few kilometers from Cerro Tololo, the antenna pattern was mapped by scanning across the transmitter in azimuth and elevation. The full width at the half-power points (FWHM) of the beam was found to be $8.8^{\circ}$, and comparison of the measured pattern and the pattern calculated from scalar diffraction theory showed good agreement (Bronfman 1985), assuring the system was diffraction limited. 


\section{Mount and Drive System}

Bullt by the machine shop of the Columbia University Phyics Department, the altitude-azimuth mount of the telescope (Fig. $11-1$ ) is almost identical to the New York telescope's mount, except for a slightly larger housing for the azimuth section (allowing easy access for maintenance). Each axis is equipped with a direct-drive torque motor, a tachometer generator, and a 16-bit optical shaft encoder. The computer reads the position and velocity of each axis 100 times per second and issues torque commands to the motors based on the commanded position and an approximate solution to the telescope's equation of motion. The telescope is made to track by converting source right ascension and declination to altitude and azimuth five times per second. Data taken when pointing errors exceed about one arcminute are automatically discarded. The telescope's light weight allowed it to change position by as much a $10^{\circ}$ in about one second, so position-switching (used for the observations presented in this thesis) can be carried out quickly and efficiently. The entire telescope system is housed under a 16-foot dome in a small building at the summit of Cerro Tololo. A 5-foot wide slit in the dome for observing is covered with a low-loss ( $0.15 \mathrm{~dB}$ ) plastic fabric called Grifolin that mechanically protects the telescope from the wind and allows a nearly constant temperature to be maintained inside. The azimuth tracking of the dome is controlled by the computer.

A stringent check of the telescope pointing is achieved with a small optical telescope coaligned with the radio axis of the primary. Starting with approximate pointing parameters (see Appendix $A$ ), the radio center of 
the Sun is located automatically by determining the symmetry points of azimuth and elevation scans across its limb. The optical and radio axes are then collimated to within $\sim 0.25^{\circ}$ by tracking the Sun's radio center and mechanically adjusting the optical telescope until the Sun's optical image is centered in the reticle. Finally, the positions of about 40 stars, well distributed across the sky, are checked with the optical telescope, and an analysis of the pointing errors yields improved pointing parameters.

From this "starpointing" procedure, which included no corrections for possible nonperpendicularity of the altitude and azimuth axes or the altitude and radio axes, peak and RMS pointing errors of $0.5^{\prime}$ and $0.2^{\circ}$ were found. Inclusion of nonperpendicularity corrections produced negligible improvements in the pointing accuracy so such corrections were omitted. Subsequent periodic starpointings showed a gradual deterioration of the pointing accuracy due to a slow drift of the azimuth axis with respect to the vertical. When the RMS error exceeded about one arcminute, new pointing parameters were determined and the accuracy restored.

As a daily check on the pointing, the radio position of the Sun was monitored by scanning across its limb, as described above. Using the pointing parameters from the starpointing procedure, an offset of about one arcminute was found between the expected and observed positions of the Sun. The source of this discrepancy was never completely determined, but being less than $1 / 8$ of a beam it did not pose a serious problem.

At the beginning of each observing session, a spectrum was obtained at the peak position of Orion A. The good day-to-day agreement of these spectra (see Section $B$ below) provided a further check on the pointing (as well as a check on other components of the system). 
Details of the pointing model can be found in the thesis of R. Cohen (1977); Its application to the Southern Millimeter-Wave Telescope and a summary of the results are given here in the Appendix $A$.

\section{Receiver}

A double sideband receiver, based on a very stable liquid nitrogen cooled $(77 \mathrm{~K})$ Schottky barrier diode mixer, was used to detect the incoming (RF) signal. In the first stage, the RF signal collected at a scalar feed horn and the local osclllator (LO) signal introduced by means of a resonant ring injection cavity are mixed to produce a first intermediate frequency (IF) signal of $1390 \mathrm{MHz}$. The LO signal is the frequency-doubled output of a 57 $\mathrm{GHz}$ klystron that is phase-locked to a computer-controlled frequency synthesizer. By setting the sythesizer frequency, the LO is tuned to correct for the Doppler shift of the CO line due to the earth's motion with respect to the local standard of rest. The first IF signal passes through an impedancematching transformer and is amplified by a low-noise (22 K) FET amplifier with $30 \mathrm{~dB}$ of gain. Except for the feed horn, all the components in the first stage are maintained at $77 \mathrm{~K}$ by liquid nitrogen in a vacuum dewar. In the second stage, the first If signal is further amplified, then down-converted to a second intermediate frequency centered $150 \mathrm{MHz}$, and, after amplification, the second IF signal is sent to the spectrometer. The single sideband noise temperature measured at the feed horn is $\sim 385 \mathrm{~K}$.

For further detalls on the recelver, see the thesis of L. Bronfman (1985). 


\section{Spectrometer}

The filter bank spectrometer (or "backend"), built at GISS from the NRAO design (Mauzy 1974), has 256 channels, each with spectral resolution of $0.5 \mathrm{MHz}\left(1.3 \mathrm{~km} \mathrm{~s}^{-1}\right.$ at $\left.115 \mathrm{GHz}\right)$, for a total bandwidth of $330 \mathrm{~km} \mathrm{~s}^{-1}$. The $150 \mathrm{MHz}$ signal from the receiver enters the IF section of the backend where it is divided into sixteen $8 \mathrm{MHz}$ wide bands, each centered on $8 \mathrm{MHz}$. Each of these signals is routed to one of sixteen identical filter boards; on each filterboard, sixteen contiguous $0.5 \mathrm{MHz}$ filters followed by square-law detectors yield the power spectrum of the input signal to the board. In the next stage, 256 integrators on sixteen identical integrator boards integrate the output of each detector for 48 milliseconds. A 5-millisecond hold period follows during which each integrator output is read, digitized, and sent to the computer where it is added to the sum of all previous cycles for that channel. The integrators are then reset in preparation for the next integration cycle.

During the course of post-observation data reduction it was discovered that a weak ( $1 \sigma$ ) false signal persisted in three channels, even in absence of any real emission. The problem could be traced to a 5-10\% deviation from square-law behavior of the detectors in these channels. At a given source position, the final spectrum is the difference between a spectrum taken on-source and a spectrum taken off-source (with the baseline determined from a straight-line fit; see Section II.B below); it can be shown that, in the difference spectrum, the deviations from square-law behavior lead to residual signal levels which are $3-6 \%$ above the level in the remaining good (baseline) channels. These residuals account for the 
measured false signals. In the few data processing procedures where this problem became noticable, e.g., the summing together of several spectra channel-by-channel (an operation that led to the discovery of the bad channels), the bad channels were replaced with an interpolation using their neighboring, good channels. Aside from these cases, the bad channels produced no effect greater than the noise, so the problem was generally ignorable. (The bad channels have since been corrected.)

\section{Computer System}

The computer system, built around a Data General Nova $4 / X$ minicomputer with $128 \mathrm{~K}$ bytes of memory, controls the telescope pointing, data acquisition, and synthesizer frequency setting. On-line data processing and a moderate amount of programming may be done by the observer while the computer carries out the primary telescope-control tasks.

Figure 1I-2 shows a block diagram of the overall system. A crystal oscillator generates an interrupt every 0.01 sidereal second; all timekeeping tasks are performed and then the telescope pointing programs are executed. The computer interface with the telescope drive electronics receives telescope (and dome) position and velocity information and sends torque commands, effecting the pointing (see Section 2 above). A microcomputer-controlled reference generator (not shown in Fig. 11-2) issues the "Integrate-hold-reset" commands to the spectrometer's integrators. The "hold" command causes a Nova interrupt, initiating the readout of the integrators via the Nova/spectrometer interface. The Nova is interfaced with an IEEE-488 General Purpose Interface Bus (GPIB) for 
communication with the frequency synthesizer (and other IEEE-488 programmable devices).

Data storage facilities consist of a CDC $9427 \mathrm{H}$ moving head disk drive, with a 5 megabyte fixed disk and a 5 megabyte removable disk cartridge, and Cypher F 100 dual density (800/1600 bits per inch) tape drive. After each observation is completed, the data are transferred from a buffer in the computer to the disk cartridge, along with an information header (position, synthesizer frequency, date, etc.). Each cartridge can hold about 2000 scans. A variety of telescope system and Data General system (RDOS) programs are stored on the fixed disk. Data are transferred daily and weekly to tapes both for backup and for transport from the telescope to New York, where full-scale processing is carried out.

Operator interaction with the system is through a Hewlett-Packard 2623A Graphics Terminal. In addition to commanding the source coordinates, the observer may display and manipulate both completed and in-progress observations. Hard coples of graphics data may be obtained from a bulit-in printer in the graphics terminal, and text may be output to a dot matrix printer used to maintain an automatic log of each observation. A TV monitor, updated every second, displays the status of the system.

A second Nova $4 / X$ computer is avallable both as a backup and for program development and data analysis without interruption to the telescope operations. A spare disk drive, tape drive, graphics terminal, and a graphics printer are included.

The telescope operating system consists of a collection of elementary ("low-level") routines written in Data General assembler, and a collection of complex ("high-level"), interactive programs written in a language, similar to FORTH, called the Dictionary. The low-level routines, always complied in 
the computer memory, carry out the telescope-control tasks and provide the rudiments of the Dictionary in the form of approximately 270 permanent "words" (subroutines) that can be commanded from the keyboard or from other programs. The permanent words make the low-level telescope-control routines accessible to the operator, provide a library of mathematical functions, and allow the programmer to extend the Dictionary by "defining" new words in terms of permanent and previously defined words. The extended Dictionary code resides on the fixed disk, although during normal operation part of it is compiled in the computer memory. Other programs are complled only at execution time and are subsequently discarded from memory. The complete operating system, including the low-level routines and about 1000 permanent and defined Dictionary words, occuples about $90 \%$ of the available memory. With the remaining $10 \%$ users may create programs for special purposes.

Some of the hardware differences between the Northern and Southern Millimeter-Wave Telescopes required modifications to the operating system, the most complicated of these software changes being the Implementation of frequency-sw/tching in the Southern Telescope. In the Northern Telescope, frequency-switching was achieved by alternating between two oscillators (with distinct frequencies) in the LO circuitry of the receiver, the alternation being synchronized with the start of each data acquisition cycle of the backend. The computer kept track of the switching phase, but had no control over which oscillator was active. In the Southern Telescope, a new scheme for irequency-switching was used in which the LO circuit had only one oscillator in place of these two, and the computer set the switching phase and frequency of the LO signal through the programmable frequency synthesizer. To effect this scheme, I developed 
assembly-level and high-level routines that controlled the synthesizer. The interrupt-servicing program was modified to allow a frequency-command routine to be placed in a queue (with other routines) upon completion of the drive interrupt routine (similarly queued-up routines calulate and command torques, recompute telescope pointing coordinates, and process backend data; the order in which they are executed depends on their preassigned priority). The final program gave the observer the flexibility to choose the frequency difference and the switching rate.

Various other minor differences between the Northern and Southern Telescopes required a number of less major modifications to the operating system. For example, a larger calibration chopper wheel on the Southern Telescope required more time to go from rest to its uniform rotation rate than did the chopper on the Northern Telescope, and a time delay had to be added in the program that processed calibration data from the backend.

\section{B. Calibration and Observing Techniques}

Weather conditions at Cerro Tololo are extremely favorable for yearround observations at millimeter wavelengths and only few a days of operation were lost because of rain or snow between 1983 January and August. (Year-round, round-the-clock observing posed a challenge to keeping the telescope system maintenance-free, but very little observing time was lost to technical problems.) The approximately 7000 spectra on which this thesis is based were collected in less than one year.

The receiver was calibrated using the standard chopper wheel technique discussed by Penzias and Burrus (1973), with refinements suggested by Davis and Vanden Bout (1973) and Kutner (1978). In the 
original method the measured difference between a room-temperature blackbody and the sky produces a calibration signal that, assuming the ambient and atmospheric temperatures to be equal, depends on atmospheric attenuation in the same way as does the measured spectral line signal. The ratio of the measured line signal to the calibration signal then yields a line temperature that is independent of the atmospheric attenuation. The calibration signal is obtained by pointing at the sky while rotating a twobladed chopper wheel across the feed horn and subtracting the sky signal (clear horn) from the absorber signal (blocked horn).

Two ref inements, suggested by Davis and Vanden Bout (1978), were made to the original procedure. The first is a correction applicable to double sideband receivers. If the atmospheric opacity is different in the two sidebands, the total calibration signal will be the sum of two terms with differing amounts of attenuation, while the observed spectral line will be affected only by the attenuation in one of the sidebands. In this case, the ratio of line signal to total calibration signal will give the wrong result. (Another potential source of error is unequal gains in the two sidebands, but because laboratory measurements of the receiver on the Northern Millimeter-Wave Telescope indicated very nearly equal gains in each sideband [Cohen et al., 1985b], and since the present recelver and the Northern Telescope's receiver have similar electronics in the signal path, it was assumed that the gains in the two sidebands of the present receiver were equal.) The second refinement takes into account the difference between the atmospheric and the amblent temperatures, the atmospheric temperature being generally lower. The effect of this temperature difference on the original method is to increase the calibration signal, leading to a derived line temperature below its true value. Both corrections 
are elevation dependent and require knowledge of the atmospheric opacity as a function of frequency.

In a third refinement, the primary sources of atmospheric attenuation in the millimeter region, molecular oxygen and water vapor, are treated in a two-layer atmospheric model, following Kutner (1978). The upper, oxygen layer is well mixed throughout the atmosphere and has a large scale height; its temperature and opacity, stable over long time scales, are taken to be constant in the model. The water vapor layer is close to the ground, and its temperature and opacity can change significantly in a matter of hours or minutes. The opacity and effective temperature of the water are determined by antenna tipping -- measuring the sky brightness as function of elevation -- and fitting the data with the two-layer model.

With all three refinements to the original method, the final calibration signal for a given source is a function of: 1) the measured calibration voltage; 2 ) the source elevation; 3 ) the ratio of gains in the two sidebands, assumed for the present receiver to be unity; 4) the $\mathrm{O}_{2}$ layer temperature and opacity in both sidebands ("s" for signal and " $i$ " for image), calculated to be: $T_{O S}=255 \mathrm{~K}, T_{O i}=254 \mathrm{~K}, \tau_{O S}=0.21$, and $\left.\tau_{O i}=0.10 ; 5\right)$ the $\mathrm{H}_{2} \mathrm{O}$ layer temperature and opacity, determined from an antenna tipping; and 6) $\eta_{f}$, the forward beam filling factor for the source, equal to the main beam efficlency, $\eta$, for a source that just fllls the main beam. Using the same chopper wheel method for the Northern Millimeter-Wave Telescope, conen et al.(1985b) estimate the calibration is accurate to within 15\%. In daily observations with the Southern Millimeter-Wave Telescope of the peak position in orion $A$, the peak temperature varied typically by $3 \%$ with a maximum deviation of $12 \%$; typical variation of the integrated temperature was $3 \%$ with a $15 \%$ maximum deviation. 
All line intensities reported in this thesis are antenna temperatures corrected for atmospheric attenuation and main beam efficiency, and are equal to the radiation temperature, $T_{R}{ }^{*}$, for a source that just fills the main beam. The main beam efficiency, $\eta$, having not been measured directly, was inferred by scaling the data for a number of standard sources to the results from the Northern Millimeter-Wave Telescope for the same sources. The antenna temperatures (corrected for atmospheric attenuation), $T_{A}{ }^{*}$, of the sources measured at the Southern Telescope were found to be about $17 \%$ higher than those measured at the Northern Telescope, a result primarily reflecting the slightly different optics (and efficiencies) of the two antennas, and a small error in the calculated oxygen opacities (and thus the calibrated antenna temperatures) at the Northern Telescope (Dame, private communication). Since $T_{R}^{*}=T_{A}^{*} / \eta$ (for a source filling the main beam), and the calculated main beam efficiency of the Northern Telescopeis 0.81 (Cohen et al. 1985b), then the requirement that $T_{R}{ }^{*}$ (Southern) $=T_{R}{ }^{*}$ (Northern) implies that $\eta=0.95$ for the Southern Telescope. This efficiency for the Southern telescope, implied by the scaling, could be about $10 \%$ too high due to the oxygen opacity error just noted.

The temperature scale of the Northern Millimeter-Wave Telescope compared with that of the Bell Labs $7 \mathrm{~m}$ telescope is within $17 \%$ for individual molecular clouds and within $10 \%$ for total galactic plane emission; comparison with the NRAO $11 \mathrm{~m}$ telescope gives $8 \%$ for individual clouds and $26 \%$ for total galactic plane emission (Cohen et al. 1985b). The two types of comparisons, using either individual molecular clouds or galactic plane emission, give different results because of the different efficlencles with which these sources couple with the beam. 
The Southern Milky Way passes high overhead at Cerro Tololo, and the observations reported here were all made at elevations between $45^{\circ}$ and $70^{\circ}$, and usually above $55^{\circ}$. At the start of every observation a 5 second calibration scan, at the same elevation as the source, was made in order to determine a calibration signal for that observation. Antenna tippings were done at the beginning of each observing session (about every eight hours). The optical depth of the water vapor per air mass seldom exceeded 0.12 , and during the dry winter months was often half this value.

Position-switching was used to remove instrumental contributions to the spectrum, and ylelded very flat baselines (Fig. $|1|-1$ ). In this standard method of observing, a comparison spectrum of a position free of CO emission (off) is subtracted from the spectrum of the source (on). For a given observation, the telescope alternates, every 15 seconds, between the ow and the off, the computer separately recording the spectrum at each position. The final spectrum, the difference of the two, is formed at the end of the observation. Since the sky contribution to the total power is elevation dependent, it is important that the ows and offs be at nearly equal elevations. For this purpose, I developed a program that displayed graphically, for a given ow at any time, all offs within some radius (usually $1^{\circ}$ ) of the on. The choice to observe the on (at a specific time) was then based on finding an off within some acceptable elevation range. Using this program, several hours of observations could be scheduled in a few minutes. The results were on-off elevation differences of typically $0.2^{\circ}$, and extremely flat baselines. Reference positions, checked by positionswitching against one another, were verified to be emission-free to about $0.07 \mathrm{~K}$, or half the tyical RMS noise of the survey. Table $11-1$ lists the reference positions used in this survey. 
The receiver was tuned so that $0 \mathrm{~km} \mathrm{~s}^{-1}$ (with respect to the local standard of rest) corresponded to the center of the spectrometer. All positions were observed typically for five minutes, yielding an RMS noise per channel of about $0.14 \mathrm{~K}$. To determine the baseline of each spectrum, a straight line was subtracted from it; the straight line was the leastsquares fit to the emission-free ends (usually the first and last 40 channels) of the spectrum. Any spectrum that, after this procedure, exhibited baseline curvature greater than 1 to 2 times the RMS was rejected and the point re-observed. 


\section{OBSERVATIONS}

The observations presented in this chapter comprise two large-scale CO surveys in the fourth galactic quadrant which cover all the emission arising in the Carina arm from $/=270^{\circ}$ to $300^{\circ}$, and provide most of the evidence for conclusions drawn in this thesis. Where the Carina arm extends beyond $I=300^{\circ}$, the high longitude limit of these surveys, observations from a third, adjoining survey, carried out concurrently with the same telescope by L. Bronfman, will be used in the discussion.

Figure $111-1$ shows the region of the sky covered by the two surveys. Both extended from $I=270^{\circ}$ to $300^{\circ}$ and covered $330 \mathrm{~km} \mathrm{~s}^{-1}$ (centered at $V_{L S R}=0$ ) with a spectral resolution of $1.3 \mathrm{~km} \mathrm{~s}^{-1}$, but differed in latitude coverage and spatial resolution. The main or "full resolution" survey, indicated by the region filled with small circles in the figure, had a spatial resolution of $8.8^{\circ}$ (one beam) and a sampling interval of $0.125^{\circ}$, or $7.5^{\circ}$ in latitude and longitude. Although the nominal latitude range was within $b= \pm 1^{\circ}$, all Carina arm sources (longitudes greater than $\sim 280^{\circ}$ ) were followed in latitude until the line antenna temperature dropped below $\sim 0.5$ $K$. The irregularly-shaped latitude boundary in the figure reflects the general pattern of those latitude extensions. For longitudes less than about $280^{\circ}$, the emission is mostly local in origin (see Chapter IV), and not from the Carina arm, so the full resolution survey includes only $|b| \leq 1^{\circ}$ for is $280^{\circ}$. The total area of the full resolution survey, about $85 \mathrm{deg}^{2}$, was covered by $\sim 5500$ observations.

The second, companion survey had a spatial resolution of $0.5^{\circ}$ and covered a latitude range of $|\mathrm{b}| s 5^{\circ}$ with a sampling interval of $0.5^{\circ}$ in 
latitude and longitude. Nearly 16 times the solid angle of the main beam, the half-degree "beam" is obtained by stepping the telescope's position through a $4 \times 4$ array during data acquisition. The resultant rapid, on-line spatial smoothing permits mapping a large area in a relatively short time. This survey, dubbed the "Superbeam" survey (after the computer program, developed by $T$. Dame, that controls the telescope in this observing mode), was carried out for two reasons: 1) to provide a quick overview of the emission in the Vela-Carina-Centaurus region of the galactic plane and 2) to help determine appropriate latitude limits for the full resolution survey. The Superbeam survey includes 1281 spectra covering 300 deg$^{2}$.

Typical spectra from the full resolution survey are shown in Figure III-2. The RMS noise per channel is about $0.14 \mathrm{~K}$; only first-order (straight line) fits were used in determining the baselines of all the spectra. The flat baselines and high signal-to-noise evident in this sample are characteristic of the quality of the spectra in both surveys.

Recorded as a "cube" $T\left(/_{i}, b_{j}, V_{k}\right)$, data in this and subsequent chapters will usually be displayed by projecting the integral of temperature over one of the independent variables onto the plane of the other two, the integration limits depending on the emission characteristics being discussed. As mentioned in Chapter II, three bad channels were discovered in the spectrometer after the survey had been completed. The defect, no more than $1 \sigma(0.14 \mathrm{~K})$ in any individual spectrum, is noticable only when like channels in several spectra are summed together ( $e . g$, in integrated $I, V$ and $b, \vee$ maps), the result being a false signal at the velocity of the bad channels (at least 9 spectra must be summed for the false signal to exceed the $3 \sigma$ level of the sum). (The problem can be Ignored in spatlal maps where different channels within individual spectra are summed, or in $I V$ maps at 
single values of $b$, and $b, v$ maps at single values of 1.) Maps displaying bad channeis (which appear as narrow lines at constant velocity) have been repaired by replacing the bad-channel sums with the interpolated value of the neighboring, good-channel sums.

The total molecular cloud emission from the Superbeam survey, integrated from -50 to $+50 \mathrm{~km} \mathrm{~s}^{-1}$, is shown in the plane of the sky in Figure $111-3$. Containing no velocity information, this map, analogous to a continuum map, is useful for showing overall planar distribution of the emission without regard for its distribution along the line of sight. For example, between $I=270^{\circ}$ and $280^{\circ}$ the emission appears diffuse and has a large latitude extent, while for longitudes above $280^{\circ}$ the emission occurs largely in well defined molecular clouds which are concentrated near the galactic plane. It will be shown later that the wide-latitude emission is mostly local and that the narrow layer is the much more distant Carina arm.

The similar spatial map in Figure III-4 is a hybrid of the Superbeam data from Figure $111-3$ and the data from the full resolution survey, also integrated from -50 to $+50 \mathrm{~km} \mathrm{~s}^{-1}$. This figure lllustrates that the latitude coverage of the full resolution survey is sufficient to cover all the Carina arm clouds: outside the dotted line, where the Superbeam data is shown, we see wide-latitude emission at / less than $280^{\circ}$, but very little else. It is also evident from the figure that the molecular clouds seen in the Superbeam map show considerable structure at full resolution. The region between $I=280^{\circ}$ and $285^{\circ}$, in particular, is quite complex. From $I=285^{\circ}$ to $295^{\circ}$ strong sources immersed in the complex low-level background appear to be clustered on scales $\sim 1^{\circ}$, suggesting that the smaller beam is resolving big molecular clouds. 
To emphasize the most intense features, spatial maps were also produced in which all spectral channels below about $3 \sigma(0.5 \mathrm{~K})$ were "clipped" (i.e., set to zero) before integration; the resultant maps (integrated from -100 to $+100 \mathrm{~km} \mathrm{~s}^{-1}$ ), are shown in Figures $111-5$ (Superbeam) and $111-6$ (full resolution combined with Superbeam). Even with the velocity-blending in these maps, having removed most of the weak background by clipping, a few large clouds can now be recognized. For example, most of the emission between $I=285^{\circ}$ and $288^{\circ}$ is part of a large cloud complex associated with the $\eta$ Carinae Nebula. The emission between $I=289^{\circ}$ and $290.5^{\circ}$ in the plane is a cloud assoclated with the $H$ II region RCW 54a, and most of the emission between $I=291^{\circ}$ and $296^{\circ}$ comes from a cloud complex assoclated with the H II regions NGC 3576, RCW 60, RCW 61, and RCW 62. More will be said about these and other giant molecular clouds in the Carina arm in Chapter V.

The kinematics of molecular gas in the galactic disk is best studied by displaying the data in the longitude-velocity plane, as in Figure $111-7$. Covering most of the fourth quadrant, the,$v$ map in this figure was produced by integrating the full resolution data over all latitudes where observations have been made; we emphasize that the data have not been clipped in this map. Although the integration ilmits are not uniform in l, they should include essentlally all nonlocal (Carina arm) emission at longitudes between $280^{\circ}$ and $300^{\circ}$, as the total emission spatial map (Fig. $\mid 11-4$ ) Indicates. For / > 300 only full resolution data were available, but the Carina arm (seen in this part of the iv diagram as the lane of positive velocity emission) is covered completely within the latitude limits of the survey in this part of the fourth quadrant. The I,V map in Figure $111-7$ will generally be referred to as the "I, map" or the "I,V diagram." 
The dominant feature of the I, $\mathrm{V}$ map is the open loop of emission formed by a negative velocity segment from $l \approx 280^{\circ}$ to $300^{\circ}$ and a positive velocity segment at $/ 2280^{\circ}$ joined by the intense emission centered at $v \approx 0 \mathrm{~km} \mathrm{~s}^{-1}$ near $/=280^{\circ}$. This loop is the Carina arm, its near side being traced by the negative velocity emission, its far side by the positive velocity emission, and its tangent marked by the strong emission connecting the two. A more detailed discussion is deferred until Chapter IV. (The strong emission in the upper left of this map originates in the inner Galaxy and will be largely ignored here; see Bronfman [1985] for a full discussion.)

Figure $111-8$ shows the corresponding Superbeam $I, V$ map made by integrating from $b=-5^{\circ}$ to $+5^{\circ}$. The weakness in the superbeam map of the lane of positive velocity emission seen in the full resolution map, and the greater intensity in the superbeam map of the emission below $/ \approx 280^{\circ}$, are readily understood. Being closely confined to the galactic plane, the positive velocity emission is diluted by the large beam and wide latitude coverage of the Superbeam survey and falls below the 30 level (roughly the first contour) of the superbeam $/ v$ map. At $/ s 280^{\circ}$ much of the emission that spills out of the latitude boundaries of the full resolution survey is covered in the Superbeam survey (see Fig. $111-4$ ), so more emission is included in the Superbeam $I, V$ map below $/ \approx 280^{\circ}$ than in the full resolution iv map. Bearing in mind these differences, both maps are fully consistent.

An I,V map made by smoothing the full resolution $I V$ map in longitude to $0.5^{\circ}$ (Fig. III-9) more closely resembles the Superbeam I, $\mathrm{V}$ map, the main difference between the two being the difference in the strength of the local material below $I=280^{\circ}$. Between $/=280^{\circ}$ and $300^{\circ}$, the Superbeam map contains about $30 \%$ more integrated emission (above the $3 \sigma$ level) than the 
full resolution map, again indicating that most of the emission at $/ 2280^{\circ}$ is accounted for in the full resolution I, $v$ map.

A set of 54 full resolution I, $V$ maps at each sampled latitude in the survey $\left(b=+2.5^{\circ}\right.$ to $\left.-4.125^{\circ}\right)$ is shown in Figure $B-1$ (placed in an appendix due to their bulk). For $|b|>1^{\prime}$, where the latitude coverage varies, dotted lines mark the separation between observed and unobserved sections of longitude (except for $1<280^{\circ}$, there little or no emission is expected in the unobserved portions of each map, as we have just seen). The positive velocity emission in these maps is strongest between $b=0^{\circ}$ and $-1^{\circ}$, while strong negative velocity emission can be seen over a wider latitude range, roughly from $b=1.5^{\circ}$ to $-2.5^{\circ}$. The differing latitude extents of the positive and negative velocity emission simply reflect the different distances to the far and near sides of the Carina arm. Emission from the tangent region of the arm $\left(/ \approx 280^{\circ}, v \approx 0 \mathrm{~km} \mathrm{~s}^{-1}\right)$, evident between $b=0.625^{\circ}$ and $-2.75^{\circ}$, is, as in the far side, strongest at negative latitudes. The loop that traces the Carina arm in the integrated iv map is apparent in these maps primarily in the narrow latitude range between $D=-0.25^{\circ}$ and $-0.875^{\circ}$. It is quite clear from these maps that the Carina arm could be overlooked in a co survey restricted to $b=0^{\circ}$.

There being no other Co survey of the Carina arm with comparable coverage, it seems approprlate to include here (also in Appendix B), in addition to the maps to be used in the next two chapters, a larger set of maps for general use. Figure B-2 shows 241 full resolution b,v maps at each longltude observed in the full resolution survey; Figure B-3 shows 21 Superbeam $I, V$ maps at each latitude observed in the Superbeam survey; and Figure B-4 shows 61 Superbeam b, $v$ maps at each longitude observed in the Superbeam survey. The maps are presented here without further discussion. 


\section{LARGE SCALE PROPERTIES OF MOLECULAR GAS IN THE CARINA ARM}

Between longitudes $280^{\circ}$ and $300^{\circ}$, the Carina arm has been well established in a variety of optical and radio spiral tracers. CO, being an excellent spiral tracer in the first and second quadrants (Dame 1983), may be expected to join the list of tracers in the Carina arm. As we will see below, the Carina arm is indeed traced exceptionally well by molecular gas, and is actually the best example to date of a CO spiral arm in the Galaxy. As the birth sites of massive stars and their accompanying $H / I$ regions, giant molecular clouds provide an important physical link between the optical and the classic $21-\mathrm{cm}$ arms. In the Carina arm in particular, where the optical tracers can be seen to very great distances ( $10 \mathrm{kpc}$ ), the good agreement between the optical and molecular pictures of the arm underscores the importance of $\mathrm{CO}$ in the study of large-scale galactic structure in regions of the Galaxy where the arms are optically obscured.

The optical transparency of the Carina region of the galactic plane ends with increasing $/$ near $/=298^{\circ}$, but the molecular arm continues much further: at least to $I=329^{\circ}$, as will be shown. This chapter focuses on the large scale characteristics of the arm. First, the Carina arm's appearance in the I,V diagram is discussed. Using the I, diagram to locate the near side, tangent region, and far side of the arm in velocity space, these three segments of the arm next are viewed as they appear in the plane of the sky. Then, for the emission from beyond the solar circle, the distribution of molecular gas about the galactic plane is examined, and the surface density of molecular hydrogen as a function of galactocentric radius determined. Finally, the co results are compared with the large-scale distribution of neutral atomic hydrogen observed at $21-\mathrm{cm}$. 
A. Kinematics and Distribution of Co in the Carina Arm

\section{The I, D Dlagram}

In the fourth galactic quadrant, materlal within the solar circle $\left(R_{0}=10 \mathrm{kpc}\right)$ is generally overtaking the sun in its galactic orbit, so radial velocities in the local standard of rest are negative. Similarly, material beyond the solar circle is falling behind, so radial velocities are positive. From previous optical and radio studies (see Chapter 1 ), the tangent point of the Carina arm is known to be near the solar circle at $/ \approx 281^{\circ}$. Within the solar circle, the arm approaches a point about $2 \mathrm{kpc}$ from the sun near $I \approx 296^{\circ}$, and beyond the solar circle it moves further from the galactic center (and from the Sun) with increasing longitude. The expected signature of the Carina arm in the $I, V$ diagram, then, is a loop with the near side at negative velocities between $/ \approx 281^{\circ}$ and $296^{\circ}$, the far side at positive velocites at longitudes greater than $\sim 281^{\circ}$, and the connecting tangent region near zero velocity at $/ \approx 281^{\circ}$. This is exactly what is seen in the integrated IV map (Fig. $111-7$ ). Because velocity crowding and noncircular motions influence the appearance of the IV map (e.g., Burton 1971), a closer look at the Carina "loop" is worthwhile. For the following discussion, a schematic representation of the main features in the,$V$ diagram is displayed in Figure IV-I $\mathrm{b}$ beside the integrated I, $\mathrm{V}$ diagram to the same scale (Fig. IV-la).

The correspondence of the near side of the arm with the high velocity ridge between $I=280^{\circ}$ and $300^{\circ}$ ralses the question of whether velocity crowding, rather than a true density enhancement, could be responsible for 
the enhanced emission in this part of the I, diagram. While there are undoubtedly some chance line of sight coincidences of distinct molecular clouds near the terminal velocity, the sharp gap in emission between $l=289^{\circ}$ and $291^{\circ}$ (and to a lesser extent between $l=296^{\circ}$ and $297^{\circ}$ ) along the high velocity ridge indicates that velocity blending of many small, randomly distributed clouds is not likely to be the origin of this ridge. This may be seen by considering the following two-dimensional picture.

The velocity extent of the emission gap at $/ \approx 290^{\circ}--$ through the high velocity ridge and all the way to zero velocity -- corresponds to a path length of about $7 \mathrm{kpC}$ through the galactic plane; the implied region of the galactic plane that is iree of molecular clouds is from the sun to the solar circle between $l=289^{\circ}$ and $291^{\circ}$. The area lies between 9 and $10 \mathrm{kpc}$ from the galactic center and covers about $8 \times 10^{5} \mathrm{pc}^{2}$. Let $\Sigma$ be this total cloudfree area, and let $S_{M}$ be the number surface density $\left(\mathrm{pc}^{-2}\right)$ of all molecular clouds in the mass range $M_{1} \leq M \leq M_{2}$ in the galactic plane between $R \approx 9$ and $10 \mathrm{kpc}$. Then, if the clouds in this mass range are distributed in the plane with a Poisson distribution of mean $S_{M}$, the probability, $P_{0}$, that no clouds with masses between $M_{1}$ and $M_{2}$ occupy $\Sigma$ is:

$$
P_{0}=\exp \left(-s_{M} \Sigma\right)
$$

For a given mass range, $M_{1} \leq M \leq M_{2}$, with mean mass $\langle M\rangle$, we can write $s_{M} \approx f_{M} \sigma /\langle M\rangle$, where $\sigma$ is the mass surface density of molecular gas, and $f_{M}$ is the fraction of $\sigma$ contained in clouds with masses between $M_{1}$ and $M_{2}$. From the axisymmetric distribution derived by Dame (1983) and relation $(I V-1)$ introduced later in this chapter, we have $\sigma \approx 3 \mathrm{M}_{\odot} D C^{-2}$ between $\mathrm{R} \approx 9$ and $10 \mathrm{kpc}$. Using the molecular-cloud mass spectrum of Dame 
(1983), $f_{M}$ and hence $S_{M}$ have been estimated for six half-decade wide logarithmic mass intervals with centers ranging from $\log \left(M / M_{\odot}\right)=4.0$ to 6.5; the smallest mass corresponds roughly to the sensitivity limit of the full resolution survey for clouds at $7 \mathrm{kpc}$. The computed values of $P_{0}$ for the respective mass intervals (least to most massive) are found to be: 0.002 , $0.031,0.147,0.320,0.549$, and 0.702 . Evidently, if velocity crowding of small clouds were the origin of the high velocity ridge in this part of the $I, V$ diagram, a gap such as the one observed at $I=290^{\circ}$ would be a very unlikely occurence. As larger and larger clouds are considered, however, the existence of the gap becomes less unlikely, suggesting that the high velocity ridge emission comes from large clouds. The gap at $/ \approx 290^{\circ}$ in the high velocity ridge is just the line of sight between very large clouds that happen to lie near the terminal velocity.

Large clouds also are subject to velocity crowding, but they are relatively few in number, and, out of four identified near the terminal velocity between $I=280^{\circ}$ and $300^{\circ}$, two can be placed in the near side of the Carina arm on the basis of associations with well-known optical objects that trace the arm (see Chapter $V$ ). It seems reasonable, therefore, to attribute the high velocity ridge emission largely to the near side of the Carina arm.

Between $1 \approx 280^{\circ}$ and $284^{\circ}$, near zero velocity the tangent region of the arm is marked by intense $\mathrm{CO}$ emission. A long path length through the arm lies in a narrow range of longitudes near the tangent, accounting for the observed brightness of the emission and rapid increase in the velocity width as $/$ increases from $280^{\circ}$ to $284^{\circ}$. Some local emission may be mixed in as well, but the coincidence of the bright $\mathrm{CO}$ edge with the classic Carina tangent suggests that the local contribution is slight. The relatively weak 
intensity just before $I=280^{\circ}$ corresponds to an interarm region outward in galactocentric radius from the Carina arm tangent.

The abrupt onset of the tangent emission as our line of sight sweeps toward $I=280^{\circ}$ from below is illustrated especially well in Figure IV-2, which shows the emission from the full resolution survey integrated over latitude and velocity as a function of longitude. Plotting the total intensity of a spiral tracer versus longitude is a classic method for locating the tangent directions of spiral arms. In such an intensity-longitude or $" I(/)$ graph, the tangent directions appear as upward steps in the intensity, and the ratio of brightness in the tangent direction to that in the preceding interarm direction indicates the arm-interarm contrast of the spiral tracer (Dame 1983). In the first quadrant, the CO I( /) graph shows a typical tangent-interarm brightness ratio of about 2 to 1 (Dame 1983), and in the fourth quadrant (Fig. IV-2) similar ratios are seen in the tangent directions of the Centaurus and Norma arms, at $/ \approx 310^{\circ}$ and $l \approx 330^{\circ}$. For the Carina tangent at $1=280^{\circ}$, however, the ratio is much higher: about to 13 to 1 . (The nearby step down from $/ \approx 270^{\circ}$ to $272^{\circ}$ results mostly from local emission.) There are three possible explanations for such a high ratio. First, the latitude coverage of the full resolution survey fans out at about the same longitude as the step in the $I(/)$ graph, suggesting that the strength of the step might be an artifact of the sampling. This possibility was carefully considered by comparing $I(/)$ graphs produced from the Superbeam survey to the full resolution $1(/)$ graph smoothed in longitude to $0.5^{\circ}$. The smoothed full resolution $1(/)$ graph was found to be completely consistent with the Superbeam $I(/)$ graphs, and therefore, since the sampling of the superbeam survey is uniform in $/$ and $b$, the irregular sampling pattern of the full resolution survey fails to explain the high 
tangent-interam brightness ratio. Second, since co emission from the tangent region of the Carina arm is kinematically indistinguishable from local, low velocity CO emission, the strength of the step in the $I(/)$ graph could be due in part to contributions from local emission. However, as noted above, such contributions cannot be substantial, as evidenced by the observation of distant, optically visible $\mathrm{H}$ II regions at the Carina arm tangent (Bigay et al. 1972), and the absence of Population I material ahead of (at lower longitudes than) the tangent coupled with the step in the radio continuum intensity observed in the direction of the Carina tangent. Third, the high tangent-interarm brightnesss ratio is a true indication of the physical arm-interarm contrast in the Carina arm. This explanation is by far the most plausible of the three, and the conclusion is unavoidable: there is 13 times more molecular gas in the Carina arm than in the interarm region ahead of the tangent. This finding provides significant support for the claim that molecular clouds in the Galaxy are confined largely to the spiral arms (Cohen et al. 1980; Dame 1983), and demonstrates emphatically the importance of $C O$ as a spiral tracer.

Above $I=280^{\circ}$, at positive velocities, the tangent region extends continuously to the far side of the Carina arm. Because the far side lies beyond the solar circle, there is no kinematic distance ambiguity in the co emission. A fairly sharp low velocity edge for longitudes above $\sim 284^{\circ}$ marks the inner side of the arm, and the relative emptiness of the region between this edge and zero velocity is very similar to the interarm gap observed between the locai arm and the Perseus arm in the second quadrant (Cohen et al. 1980). As is the case with the near side, individual molecular clouds identified in the far side between $/=280^{\circ}$ and $300^{\circ}$ coincide with previously known Carina arm tracers, including some very distant optical 
H $1 /$ regions. Beyond $I=300^{\circ}$, the arm extends to the top of the $I, V$ map: the small object at $/ \approx 329^{\circ}, v \approx 30 \mathrm{~km} \mathrm{~s}^{-1}$ is not noise, but a distant, $6 \times 10^{5} M_{\odot}$ molecular cloud in the Carina arm; an even more distant cloud observed at $I=335^{\circ}$ extends the arm even farther (Chapter $V$ ).

Running down the center of the l, diagram, near zero velocity, is a lane of emission mostly local in origin, although a small amount of weak, distant emission may be present here as well. The local emission is recognizable by the narrow lines and, in the case of the narrow-velocity component of the feature seen between $1=293^{\circ}$ and $295^{\circ}$, by its large latitude displacement (see the $I, V$ maps from $b=-2^{*}$ to $-3^{\circ}$ in Fig. $B-1$ ). The sparseness of the emission that appears between the near and far sides in the l,V diagram is also evident in the plane-of-the-sky map of the tangent region (Fig. IV-3b) discussed below.

Except for the local emission near zero velocity, the region between the near and far sides of the arm in the I,V dlagram is remarkably free of emission. This gap corresponds to the interarm region interior to the Carina arm. With the exception of the Perseus arm (Cohen et al. 1980), no other CO spiral arm, when viewed in IV space, exhibits such a sharp intensity drop inside its inner edge; it is the relative emptiness of the region inward of the arm that makes the Carina loop stand out in the I,V diagram.

\section{The Spatial Maps}

To view the near side, tangent region, and far side of the Carina arm on the plane of the sky, the emission was integrated over the approximate velocity widths of these features as they appear in the integrated IV diagram. The velocity boundarles are indicated in the key to the I, dlagram 
(Fig. IV -1 b) as the dotted lines at constant velocity, and the resultant spatial maps are shown in Figures IV-3a to IV-30. For / $300^{\circ}$, the spatial maps combine the full resolution and Superbeam data; from $/=300^{\circ}$ to $330^{\circ}$, only full resolution data are avallable. In the following discussion the spatial maps are referred to according to the segment of the arm displayed, i.e., the "near side map" (Fig. IV-3a), the "tangent region map" (Fig. IV-3b; this map contains some local material as well -- see below), and the "far side maps" (Figs. IV-3C and IV-3d).

For $/>284^{\circ}$, the near and far stdes of the arm are well contained within the adopted velocity ranges of the spatial maps. Below $284^{\circ}$, where the near and far sides of the arm merge continuously into the tangent, the velocity boundaries at $-9 \mathrm{~km} \mathrm{~s}^{-1}$, the inner edge of the near side, and at $7 \mathrm{~km} \mathrm{~s}^{-1}$, the inner edge of the far side, sandwich the tangent region somewhat arbitrarlly. Even so, these limits evidently enclose most of the tangent emission, as a comparison of the tangent region map to the total emission spatial map (Fig $|1|-6$ ) shows. Almost all the peaks between $I=280^{\circ}$ and $285^{\circ}$ in the total emission map appear in the tangent region map. (Notable exceptions are the peaks at $/=283^{\circ}, \mathrm{b}=1.5^{\circ} ; /=284.25^{\circ}$, $b=0.75^{\circ}$; and $l=284.5^{\circ}, b=0.5^{\circ}$, the first two being seen in the near side map, the third in the far side map.) The abrupt onset of the tangent emission, prominent in the total emission map, is no less evident in the tangent region map; and although the total emission map is more intense than the tangent region map between $I=280^{\circ}$ and $285^{\circ}$, the complicated appearance of the emission is similar in each. This is because the large path length sampled by the small velocity extent of the tangent region map accounts for most of the tangent region emission. 
Two other characteristics of the emission in the tangent region map should be noted. First, the wide latitude emission between $/=270^{\circ}$ and $275^{\circ}$ is completely accounted for within the low velocities of the tangent region map (compare the near and far side and tangent region maps), supporting the eariler assertion that this wide latitude emission is local. Second, the emission near zero velocity between the near and far sides of the arm in the $I, V$ diagram is weak when viewed in the plane of the sky, especially compared with the near side and far side emission between $l=285^{\circ}$ and $300^{\circ}$. This clearly supports the conclusion that the interarm region interior to the Carina arm is comparatively free of molecular clouds.

The spatial map of the near side (Fig. IV-3a) is characterized by welldefined molecular clouds or cloud complexes between $I=284^{\circ}$ and $296^{\circ}$. For example, the cluster of strong peaks between $I=285^{\circ}$ and $288^{\circ}$ near the galactic plane is a single cloud complex associated with the $\eta$ Carinae Nebula, the brightest optical feature in the Carina arm. The emission between $l=292^{\circ}$ and $295^{\circ}$ comprises a large cloud complex associated with a group of $H \| 11$ regions, Including RCW 62 and NGC 3576, also optically prominent Carina arm objects. Between $I=280^{\circ}$ and $284^{\circ}$ below the galactic equator, where the near side merges with the tangent region (compare Figs. IV-3a and IV-3b), and for $/>297^{\circ}$, where our line of sight moves toward the inner Galaxy, the emission has a more confused appearance, making identification of individual clouds less certain. The total latitude extent of the near side is 3 to 4 degrees, corresponding to a total thickness of about 160 to 210 pc, assuming an average distance of 3 $\mathrm{kpc}$ to this part of the arm.

In the far side of the arm (Figs. IV-3c and IV-3d), most of the emission is concentrated below the galactic equator. As the longitude increases, the 
latitude width of the layer shrinks, reflecting the increasing distance to the far side with longitude, in agreement with the kinematic picture of the far side.

\section{The Distribution About the Galactic Plane}

Because no kinematic distance ambiguity exists for emission beyond the solar circle, a unique distance from the galactic plane, $z$, may be assigned for the emission at each observed $l, b$ and $v$ for $R>R_{0}$, and the distribution of molecular material about the galactic plane in the outer Galaxy can be determined directly. In this section the dependence on galactocentric distance of the average midplane, layer thickness, and surface and volume density of molecular hydrogen beyond the solar circle is derived, ignoring for the moment the dependence of these properties on galactocentric azimuth.

A qualitative comparison between the observed radial distribution and the assumed axial symmetry can be gleaned from Figure IV-4. In the figure, the galactocentric rings of constant radius are shown transformed to I, space (using a flat rotation curve, $V(R)=250 \mathrm{~km} \mathrm{~s}^{-1}$ ) and superimposed on an outline of the $I, v$ data. The opening outward of the Carina arm with increasing longltude is quite apparent, particularly between $/ \approx 280^{\circ}$ and $310^{\circ}$ Because this nonaxisymmetric structure is ignored below, and individual clouds in the arm have velocity widths that "spread them out" over a few klloparsecs in kinematic radius, the arm will be largely washed out in the following treatment. But even so, we will see that by considering a restricted range of longltudes the arm and interarm regions are easily distinguishable. 
To convert the observed co luminosity at each postition to density, the integrated emission, $\int T^{*}{ }_{R} d v$, denoted $W(C O)$, is assumed to be proportional to the column density of molecular hydrogen, $\mathrm{N}\left(\mathrm{H}_{2}\right)$. Even though the ${ }^{12} \mathrm{CO}$ line is optically thick, $W(C O)$ has been shown empirically to be a good tracer of molecular cloud mass in the Galaxy (Lebrun et al. 1983; Bloemen et al. 1985). We adopt here the $W(\mathrm{CO})-\mathrm{N}\left(\mathrm{H}_{2}\right)$ relation of Bloemen et al:

$$
\mathrm{N}\left(\mathrm{H}_{2}\right) / \mathrm{W}(\mathrm{CO})=2.8 \times 10^{20} \mathrm{~cm}^{-2}\left(\mathrm{~K} \mathrm{~km} \mathrm{~s}^{-1}\right)^{-1}
$$

Their result was derived using gamma rays as a tracer of the total gas column density and the $21-\mathrm{cm}$ line as a tracer of the atomic hydrogen column density.

Writing expression (IV-1) in the differential form,

$$
d N\left(H_{2}\right)=2.8 \times 10^{20} T_{R}^{*} d V\left(\mathrm{~cm}^{-2}\right)
$$

It follows that the density at any point $(R, Z)$ can be written as

$$
\rho(R, z)=6.17 T_{R^{*}}^{*}(l, b, v) \times|d r / d v|^{-1} M_{\odot} D C^{-3},
$$

where $T_{R}$ is the $C O$ line temperature; $R$ and $z$ (for $R>R_{0}$ ) are uniquely determined by $l, b$, and $v$ (in $\mathrm{km} \mathrm{s}^{-1}$ ), $r$ is the kinematic distance to the point in parsecs, and the mean molecular weight per $\mathrm{H}_{2}$ molecule has been taken to be $2.76 \mathrm{~m}_{H}$ (Allen 1973). The factor $|\mathrm{dr} / \mathrm{dv}|^{-1}$ can be calculated from the rotation curve using the expression for the observed radial velocity due to differential galactic rotation, 


$$
V_{L S R}=R_{0}\left[\omega(R)-\omega\left(R_{0}\right)\right] \sin (/) \cos (b),
$$

where $\omega(R)$ is the angular velocity at radius $R$. As a reasonable first approximation, and to facilitate comparison of the CO results with the $\mathrm{H} \mathrm{I}$ results of Henderson et al. (1982), a flat rotation curve was used: $V(R)=250 \mathrm{~km} \mathrm{~s}^{-1}$. The projected surface density, $\sigma(R)$, is just $\int \rho(R, z) d z$, where the integral runs over all $z$. In this analysis, the computed densities at each point, $(\not, b, V)$, between $I=280^{\circ}$ and $335^{\circ}$, weighted according to the respective volume elements, were summed in bins in $R$ and $z$, yielding a sampled mass per bin. Dividing the total mass sampled by the total volume sampled in a given bin then gave the average density for that bin.

The results for $p(R, z)$ are plotted as a function of $z$ at fixed $R$, starting at $10.5 \mathrm{kpc}$, in Figures IV-5a-f; the data are shown by the solid line. At each radius, a Gaussian with the form

$$
p(z)=p_{0} \exp \left[-\left(z-z_{0}\right)^{2} \ln (2) /\left(z_{1 / 2}\right)^{2}\right]
$$

was fitted to the data, and the parameters determined: $p_{0}$, the peak density of the Gaussian; $z_{0}$, the $z$-displacement of the peak; and $z_{1 / 2}$, the halfthickness at half the peak density. The fits, drawn with a dotted line in the figures, are evidently fairly good representations of the actual distribution at each radius. Table IV-I gives the parameters from the fits, the $\mathrm{H}_{2}$ surface density based on the fit, and, for comparison, the surface density measured directly from the area under the solid (data) curves in the figures. The Co midplane, layer thickness, and surface density are plotted versus radius in Figures $\mathrm{IV}-12 \mathrm{a}-\mathrm{c}$. 
The mean of the distribution dips below the plane for $R_{0}<R<12.5$ $\mathrm{kpc}$, reaching a maximum displacement of $z=-166 \mathrm{pc}$ at $12.5 \mathrm{kpc}$. The half-thickness of the layer grows with increasing radius. By $13 \mathrm{kpc}$, there is very little molecular hydrogen, and the measurements, as shown, are highly uncertain. In general, the surface density of $\mathrm{H}_{2}$ beyond the solar circle is known to be smaller than in the inner Galaxy (e.g. Sanders et al. 1984) and drops of $f$ with increasing radius beyond $R_{0}$. Between $/=280^{\circ}$ and 335", however, a peak is seen in $\sigma\left(\mathrm{H}_{2}\right)$ at $R=11.5 \mathrm{kpC}$. This peak is probably caused by the three or four large molecular clouds between $I=290^{\circ}$ and $320^{\circ}$ that straddle the $11.5 \mathrm{kpc}$ ring in Figure IV-4. With the results of the axisymmetric model for the inner Galaxy used by Dame (1983), the $\mathrm{H}_{2}$ surface density at the $11.5 \mathrm{kpc}$ peak is found to be only a factor of $\sim 4$ below the value at the peak of the "molecular ring" in the first quadrant. Taking the peak at $11.5 \mathrm{kpc}$ to be representative of the surface density in the Carina arm suggests that a single spiral arm can account for a significant fraction of the $\mathrm{H}_{2}$ surface density observed in the interior region of the Galaxy.

If attention is restricted to longitudes $290^{\circ}$ to $310^{\circ}$ and just the two rings at $10.5 \mathrm{kpC}$ and $11.5 \mathrm{kpc}$ are considered (refer to Fig. IV-4), we can get some idea of the arm-interarm contrast in terms of surface density. In this case, we find that $\sigma\left(\mathrm{H}_{2}\right)=1.1 \mathrm{M}_{\odot} D c^{-2}$ at $10.5 \mathrm{kPC}$, and $4.9 \mathrm{M}_{\odot} \mathrm{pc}^{-2}$ at 11.5 $\mathrm{kPC}$, giving an arm-interarm surface density contrast of $\sim 4.5$ to 1 . Because the preceding analysis tends to wash out nonaxisymmetric structure (i.e., spiral arms), the actual contrast is likely to be higher -- recall that a 13 to 1 contrast is seen in the $1(/)$ graph (Section A. 1 above). To a distant extragalactic millimeter-wave astronomer with a face-on view of our Galaxy, the Carina arm must be a prominent feature. 
Finally, the total mass of $\mathrm{H}_{2}$ in the sector of the Galaxy considered $\left(/=280^{\circ}\right.$ to $335^{\circ}, R=10.25 \mathrm{kpc}$ to $13.25 \mathrm{kpc}$ ) may be obtained by multiplying the $\mathrm{H}_{2}$ surface density of each ring segment by the area of the segment and summing over the segments. The result using the surface densities from the fits is $1.0 \times 10^{8} \mathrm{M}_{\odot}$, whereas using the measured surface densities (the numerical integrals of the solid curves in Figs. IV-5a-f) gives $1.1 \times 10^{8} \mathrm{M}_{\odot}$. These numbers are about 1.7 to 1.9 times the value obtained in the next chapter by considering the total mass in a catalog of Carina arm molecular clouds. This factor of $\sim 2$ difference can be largely reconciled by evaluating the completeness of the molecular cloud catalog within the projected area of the galactic plane considered in the present section. If account is taken of: 1) molecular clouds too faint to be included in the catalog, and 2) a few molecular clouds interior to the Carina arm that contribute to the surface density derived above but are not included in the catalog of Carina arm clouds, about half of the "missing mass" is recovered. The mass in clouds in category (2) may total as much as $107 \mathrm{M}_{\odot}$ (Bronfman, private communication). Another $\sim 107 \mathrm{M}_{\odot}$ is estimated to reside in clouds below the mass threshold for inclusion in the catalog. This estimate was obtained by using the molecular cloud mass spectrum of Dame (1983), the threshold apparent $\mathrm{CO}$ luminosity for clouds in the catalog, and an average distance to each of the galactocentric ring segements to calculate roughly the fraction of the surface density (and hence of the total mass) in each ring segment that falls below the threshold. With corrections (1) and (2) to the total mass in the cataloged Carina arm clouds, then, the total mass found from cloud counting agrees to within $\sim 25 \%$ of the total derived from the axisymmetric averaging of the $\mathrm{CO}$ emission. 


\section{B. Comparison with $\mathrm{HI}$}

In this section the large scale distribution of $\mathrm{H}_{2}$ and of $\mathrm{H} \mathrm{I}$ are compared. Simllar comparisons in the first quadrant have shown a good correlation between the $\mathrm{CO}$ and $\mathrm{HI}$, although the $\mathrm{CO}$ appears to be more conf ined to the spiral arms (Dame 1983). The HI data used to produce the maps discussed below are from a neutral hydrogen survey of the Southern Milky Way made with the Parkes $18 \mathrm{~m}$ telescope. A magnetic tape containing the survey was kindly provided by Dr. Frank Kerr.

\section{The IV Diagrams}

Figure $\mid V-6$ is the $\mathrm{HI}, \mathrm{V}$ map integrated within $|\mathrm{b}| \leq 2^{\circ}$, covering the same region of $I, V$ space as the CO I,V map in Figure $111-7$. As in the first quadrant (e.g., Burton and Gordon 1978; Dame 1983; Sanders et al 1984), the $\mathrm{CO}$ and $\mathrm{HI}$ terminal velocity curves are quite simllar. For example, between $I=318^{\circ}$ and $315^{\circ}$, the slope in $/$ of the high velocity ridge becomes almost vertical in both $\mathrm{CO}$ and $\mathrm{H}$ I; at $I=313^{\circ}$, a slight positive velocity indentation flanked on elther side (in $/$ ) by two negative velocity bumps is seen in both the $\mathrm{CO}$ and $\mathrm{H} I$ high velocity ridges. These and other easily recognizable coincidences of the $\mathrm{CO}$ and $\mathrm{H}$ I terminal velocity curves suggest that the two species share the same large-scale kinematics.

More quantitative evidence is provided by comparing the emissionweighted mean velocities of $\mathrm{CO}$ and $\mathrm{H} I$ along their respective high velocity ridges. To make this comparison we define the mean velocity of the high velocity ridge of species $X(\mathrm{CO}$ or $H 1)$ at longitude $/,\left\langle V(/)_{H \cup R}\right\rangle$, to be 


$$
\left\langle v(/)_{H V R^{2} x}=\int v_{x}(/) T_{X}(/, v) d v / \int T_{X}(/, v) d v\right.
$$

Where the integrals extend over $\pm 25 \mathrm{~km} \mathrm{~s}^{-1}$ of the assumed rotation curve at the given longitude, and before integrating, the CO data is smoothed in $/$ to the resolution of the Parkes survey. The difference between these mean velocities, $\Delta\left\langle v_{H V R}(/)\right\rangle$, where

$$
\Delta\left\langle V(/)_{H V R}\right\rangle\left\langle V(/)_{H V R}\right\rangle_{C O}-\left\langle V(/)_{H V R}\right\rangle_{H I}
$$

should be approximately independent of the rotation curve used. Figure IV-7 shows these velocity residuals plotted against longitude; to demonstrate the relative insensitivity to the rotation curve, the residuals were calculated using both the Burton and Gordon (1978) rotation curve (solid line for $\Delta V\left(/ /_{H V R}\right)$ and a simple straight line in the,$V$ plane from $v=-85 \mathrm{~km}^{-1}, \quad l=330^{\circ}$, to $0 \mathrm{~km} \mathrm{~s}^{-1}, 270^{\circ}$ (dotted line for $\Delta\left\langle V(/)_{H V R}\right)$ ). A large discrepancy between the $\mathrm{CO}$ and $\mathrm{H} /$ terminal velocities appears near $I=290^{\circ}$, the longitude of the gap in the co high velocity ridge, but because there is no CO emission in this region, the weighting function in equation $(I V-4)$ is just noise and $\left\langle V(/ /)_{H V R}{ }^{\prime}\right.$ co is not well defined. Aside from this direction, however, the magnitude of $\Delta\left\langle V(/ /)_{H V R}\right\rangle$ is generally less than $5 \mathrm{~km} \mathrm{~s}^{-1}$, quantifying both the agreement of the $\mathrm{CO}$ and $\mathrm{H} /$ terminal velocities and the similarity of the large-scale kinematics of the two species. (The possibility of differences between the small-scale kinematics of the two species is not precluded by the foregoing computation.) 
In the outer Galaxy $(v>0)$, the $\mathrm{H}$ I is more widespread in velocity than the $\mathrm{CO}$, but by far the strongest $\mathrm{H} I$ feature is the Carina arm. A striking resemblence between the $\mathrm{CO}$ and $\mathrm{HI}$ pictures of the outer Carina arm can be seen by comparing the $\mathrm{CO} I, V$ diagram to an $\mathrm{H} I$ IV diagram produced by setting all spectral channels below $50 \mathrm{~K}$ to zero before integrating in latitude. Such a map, shown in Figure IV-8, emphasizes the strongest $\mathrm{H} \mathrm{I}$ features. Nearly every $\mathrm{HI}$ peak in the outer Carina arm has a corresponding peak or complex of peaks in the CO map, each of these CO peaks being a giant molecular cloud (see Chapter $v$ ). Note especially the features at: $I \approx 303^{\circ}$, $v \approx 32 \mathrm{~km} \mathrm{~s}^{-1} ; / \approx 311^{\circ}, v \approx 35 \mathrm{~km} \mathrm{~s}^{-1}$; and $/ \approx 329^{\circ}, v \approx 30 \mathrm{~km} \mathrm{~s}^{-1}$.

The shapes of these objects, as can be seen, are generally similar in both $c 0$ and $\mathrm{H} \mathrm{I}$. The dominant features in neutral hydrogen, then, appear to be closely associated with giant molecular clouds.

The tangent region is a strong $\mathrm{H} I$ feature, but from the $\mathrm{HI}$ data alone it is not obvious that the far side of the arm is continuous with the tangent feature. This uncertainty of the far side-tangent connection is partly due to the apparent continuity of far side at $1>280^{\circ}$ with the $\mathrm{H}$ I at about the same velocity at $l<280^{\circ}$ (e.g., the concentration at $/=273^{\circ}$, $v=35 \mathrm{~km} \mathrm{~s}^{-1}$ in Fig. IV-6). Recently, Henderson et al. (1982) examined $H I$ in the outer Galaxy by considering emission only at kinematicallydetermined galactocentric radil greater than $11 \mathrm{kpc}$. Intentionally omitting the inner Galaxy from their analysis to avoid the problem of kinematic distance ambiguity, they excluded the tangent region, and interpreted the far side as continuing to $/ \approx 265^{\circ}$ (as inferred from their map of $\mathrm{H} \mathrm{I}$ projected surface density in galactic plane). There are two properties of the $H$ I I,V map (especially the clipped,$V \mathrm{~V}$ map) that do suggest that the far side of the arm does not continue to longltudes less than $280^{\circ}$. First, 
between $v=15$ and $45 \mathrm{~km} \mathrm{~s}^{-1}$, there is a sudden rise of the $\mathrm{H}$ I contours in the direction of increasing $/$ between $/=279^{\circ}$ and $280^{\circ}$ which is uncharacteristic of the more gentle slopes of the contours between the $\mathrm{H}$ concentrations at $/>280^{\circ}$ in the far side. Second, a deep trough in the emission centered at $/ \approx 277^{\circ}, v \approx 35 \mathrm{~km} \mathrm{~s}^{-1}$ that separates the far side at $/>280^{\circ}$ from the feature at $I=273^{\circ}, v=35 \mathrm{~km} \mathrm{~s}^{-1}$ is unlike the shallower depressions between the other far side concentrations. The best evidence against the interpretation that the far side of the Carina arm extends to longitudes less than $280^{\circ}$, however, comes from the CO $\mathrm{lN}$ diagram: the strength of the tangent and the clear continuity of the near and far sides with the tangent. Without the $\mathrm{CO}$ data, extension of the $\mathrm{H} I$ counterpart of the far side to $/<280^{\circ}$ cannot be convincingly ruled out.

An interesting feature in the near side of the arm noted earlier is the $2^{\circ}$ wide gap in the CO high velocity ridge centered near $/=290^{\circ}$. This gap, the existence of which argues against a widespread distribution of small molecular clouds throughout the Carina region, can reasonably be attributed to the line of sight between two large molecular clouds. $\ln \mathrm{H} \mid, \mathrm{a}$ corresponding feature near $I=290^{\circ}$ appears as a shift of the high velocity ridge toward more positive velocities and a spreading out in / of the contours. This detour of the $\mathrm{H} I$ high velocity ridge was interpreted by Humphreys and Kerr (1974) as evidence for a large-scale shock at the inner edge of the Carina arm, but if the claim that we are just looking between molecular clouds at $I=290^{\circ}$ is correct, and if $\mathrm{CO}$ and $\mathrm{H} I$ concentrations are related, as appears to be the case in the far side of the arm, then this notch in the $\mathrm{H} I$ high velocity ridge near $I=290^{\circ}$ may just reflect a true lack of neutral hydrogen near the terminal velocity at this longitude. Nonetheless, it is possible that the absence of $\mathrm{CO}$ and (perhaps) $\mathrm{HI}$ in this 
direction is the result of an energetic event or a large-scale disturbance having cleared out the gas. This region clearly deserves further study. Aside from such indirect evidence for correlation of $\mathrm{HI}$ and $\mathrm{CO}$ in the near side, the general strength of the $\mathrm{H} I$ high velocity ridge makes it difficult to trace the near side of the Carina arm in the $\mathrm{H} \mathrm{I} \mathrm{I,V} \mathrm{map,} \mathrm{an} \mathrm{aspect} \mathrm{of} \mathrm{the} \mathrm{HI}$ emission that, perhaps more than any other, masks the Carina "loop" in the H I l, v map.

2. The Spatial Maps

The H I spatial map of the total emission between -100 and $100 \mathrm{~km} \mathrm{~s}^{-1}$ displayed in Figure IV-9 is qualitatively similar to the CO total emission map (Fig. $|1|-6$ ). The tangent region is recognizable, as is the general latitude-shape of the layer at $/>280^{\circ}$, although most of the individual peaks are washed out. The more diffuse appearance of the $\mathrm{H} I$ relative to the CO is only partly due to the lower spatial resolution of the $21-\mathrm{cm}$ observations, since even when smoothed to the $48^{\prime}$ resolution of the Parkes survey, the co retains more definition than is evident in the $\mathrm{HI}$.

H I spatial maps of the near side, tangent region, and far side of the arm made with the same velocity windows used for the CO spatial maps are shown in Figures IV-10a-d. The tangent region map (Fig. IV-10b) shows the $H$ ! counterpart to the $C O$ tangent, although the $H I$ tangent does not exhibit as abrupt an outer edge at $I \approx 280^{\circ}$ as does the co tangent, and the interarm region at $/>285^{\circ}$ is not as empty in $\mathrm{H}$ I as in CO. In the near side, where the high velolcity ridge dominates the $H I I, V$ diagram, an apparent $H$ I concentration can be seen in the spatial map (Fig. IV-10a) between $/ \approx 280^{\circ}$ and $288^{\circ}$; the sudden shift of the $H$ I high velocity ridge 
toward positive velocities near $I=290^{\circ}$ noted above is marked by a relative trough in the spatial map between $I=288^{\circ}$ and $290^{\circ}$, supporting the interpretation that the notch in the high velocity ridge represents a true lack of material. In the far side spatial maps (Figs. IV-10c,d), although the latitude extent of the $\mathrm{HI}$ is everywhere greater than the $\mathrm{CO}$, the projected thickness of the $\mathrm{H} I$ layer generally decreases from $I=280^{\circ}$ to $330^{\circ}$, and, as with the $\mathrm{CO}$ and $\mathrm{H} \mathrm{I} \mathrm{IV} \mathrm{maps,} \mathrm{the} \mathrm{spatial} \mathrm{maps} \mathrm{of} \mathrm{the} \mathrm{far} \mathrm{side} \mathrm{show} \mathrm{a}$ close correlation between $\mathrm{HI}$ concentrations and the $\mathrm{CO}$ peaks. A strong $\mathrm{HI}$ feature in the far side map at $/ \approx 273^{\circ}, \mathrm{b} \approx-1.5^{\circ}$ could be interpreted as an extension of the far side of the Carina arm to $/<280^{\circ}$. The depression of the contours separating this feature from the emission that begins abruptly at $/ \approx 280^{\circ}$ is, however, deeper than the troughs between any of the $\mathrm{H}$ I concentrations farther along the far side of the arm ( $/>280^{\circ}$ ), suggesting (as did the $I, V$ map) that the far side of the Carina arm does not connect with any feature below $1 \approx 280^{\circ}$ in the outer Galaxy.

\section{The z-Distribution}

The distribution of $\mathrm{HI}$ about the galactic plane in the outer Galaxy has recently been reinvestigated by Henderson et al.(1982). In the Southern Milky Way, their results, in agreement with earlier findings, show an increasing displacement of the $\mathrm{HI}$ midplane below the $b=0^{\circ}$ plane with increasing distance from the galactic center, reaching an extreme value of $z=-850 \mathrm{DC}$ at $R \approx 17 \mathrm{kPC}$ and a galactocentric azimuth of $260^{\circ}$ (where $0^{\circ}$ coincides with $I=0^{\circ}$ and the angle increases counterclockwise). The width of the layer increases outward as well, attaining a half-thickness (def ined as the width about the midplane within which the projected surface density 
equals one-half the total projected surface density) of about $2 \mathrm{kpc}$ near the outer edge of the Galaxy.

Most of the results of Henderson et al. are presented in the form of contour maps in the galactic plane, making a direct comparison with the $C O$ in the overlapping region discussed in Section A.3 above somewhat difficult. For this reason, the same procedure used to obtain the results for the $\mathrm{H}_{2}$ was applied to the Parkes $\mathrm{H} I$ data. The results are shown in Figures IVlla-f (solid line). It is not certain to what extent the high- $z$ wings in the $H$ I density profiles are real: the large velocity dispersion of the $H$ causes some local high latitude material to appear kinematically at $R>10 \mathrm{kpC}$ and ficticiously high $z$ (note especially Fig. IV-11a), but the wings persist to $R>13 \mathrm{kpc}$; stray radiation entering the antenna's sidelobes also might be partly responsible for the observed broad wings. A simple Gaussian shape in the outer Galaxy is not necessarily expected, since, for example, in an analysis of $H I$ in the inner Galaxy, Lockman (1984) found the layer's vertical shape between $R=4$ and $8 \mathrm{kpc}$ is fitted best by the sum of two Gaussians plus an exponential. On the assumption that the deviation in the shape of the $\mathrm{H}$ I layer from a Gaussian in the outer Galaxy is real, the half-thickness and the surface density from the fits (dotted line in figures) have not been used. Instead, the half-width at half-maximum was measured directly from the plots of the data (solid line), and the surface density was taken from the numerical integral (in z) under the same curves. The symmetry of the curves made it possible to measure the average midplane directly off the plots as well. Considering the differences between this analysis and that of Henderson et al., there is good general agreement of the derived quantities; in particular, the surface density as a 
function of galactocentric radius derived here is generally within about $20 \%$ of that found by Henderson et al

Comparisons of the $\mathrm{CO}$ and $\mathrm{HI} \mathrm{z}$-distribution parameters are shown in Figures IV-12a-c. (Because of the high velocity dispersion of the $H I$, the results at $R=10.5 \mathrm{kpc}$ were omitted from this comparison.) The $\mathrm{HI}$ midplane is within about $25 \mathrm{pc}$ of the CO midplane out to $12.5 \mathrm{kpc}$, beyond which very little $\mathrm{CO}$ emission is seen. The $\mathrm{HI}$ layer thickness is about twice that of the $\mathrm{CO}$ at all radil. Similarly, the $\mathrm{H}$ I surface density is much greater than the $\mathrm{H}_{2}$ surface density in the outer Galaxy, although, owing to the presence of the Carina arm, the ratio of $\mathrm{H}_{2}$ surface density to $\mathrm{HI}$ surface density is about twice as high as that derived by Sanders et al.(1984) in the first and second quadrants. The absence of a counterpart in $\mathrm{H} I$ to the peak in the $\mathrm{H}_{2}$ surface density at $11.5 \mathrm{kpC}$ again indicates the higher velocity dispersion of the $\mathrm{HI}$.

\section{Summary}

The Carina arm has the characteristic signature of a spiral arm in the CO I,V diagram. Integrating the CO emission over velocity and latitude reveals a 13 to $1 \mathrm{arm}$-interarm contrast between the tangent direction and longitudes preceding the tangent. The continuity of the near side, tangent region, and far side, and the relative absence of molecular clouds in the interarm region are seen more clearly than in any other $\mathrm{CO}$ spiral arm in the Gaiaxy. in the plane of the sky, the angular width of the layer, as it should, diminishes with distance along the arm. The principal findings of an axisymmetric analysis of the out-of-plane distribution of $\mathrm{CO}$ in the outer Galaxy are: an increasing displacement of the $\mathrm{H}_{2}$ midplane below the $b=0^{\circ}$ 
plane with galactocentric radius; an increase in the width of the layer with galactocentric radius; and a lower limit of $\sim 4.5$ to 1 for the ratio of surface densities in the arm to the interarm region interior to the arm.

A comparison with $\mathrm{H}$ I using the Parkes $18 \mathrm{~m} 21$-cm survey of the Southern Milky Way shows a close correspondence of the far side and tangent of the arm, although the connection of these two features in the IV dlagram is not as apparent in $\mathrm{H}$ I as in $\mathrm{CO}$. The near side of the arm is more difficult to identify in $\mathrm{H} \mathrm{l,} \mathrm{making} \mathrm{the} \mathrm{Carina} \mathrm{arm} \mathrm{loop} \mathrm{less} \mathrm{apparent} \mathrm{in} \mathrm{the}$ $\mathrm{H} I$ than in the CO I, map. In the region of the plane where the $21-\mathrm{cm}$ observations overlap with the CO survey, the well-known warping of the $\mathrm{H} I$ layer follows closely a similar trend found here in the molecular layer; the flaring of the $H$ I layer with galactocentric radius was also found to have a molecular counterpart, although the $H \mid$ layer is about twice as thick as the molecular layer. 


\section{THE CARINA ARM MOLECULAR CLOUDS}

To place the Carina arm in the Galaxy, we now turn to a discussion of the individual clouds that trace the arm in the $/, V$ and $/, b$ maps presented earlier, focusing on the largest ones: those with linear dimensions of 50 to $100 \mathrm{DC}$ and masses greater than $105 M_{0}$. The significance of these objects as galactic spiral tracers, although suspected almost since the discovery of giant molecular clouds, has become clear only in the last five or six years, and owes its recognition largely to extensive, well-sample co surveys made with the Columbia Northern Millimeter-Wave Telescope. As the study of molecular clouds associated with such nearby star-forming regions as Orion began to provide evidence for the existence of large cloud complexes ( $e . g$., Tucker et al. 1973; Kutner et al. 1977), the prospect was raised that giant molecular clouds, as represented by the local examples, are commonplace throughout the Galaxy. Being closely associated with Population I material, giant molecular clouds were obvious candidates for spiral arm tracers. A mass of $10^{6} M_{\odot}$ found for the giant cloud complex associated with $M 17$ (Elmegreen and Lada 1976) further suggested that giant molecular clouds are among the most massive objects in the Galaxy. Although there was evidence from early galactic CO surveys that perhaps as much as $50 \%$ of the total molecular cloud mass in the Galaxy resided in a relatively few clouds more massive than $106 M_{\odot}$ (e.g., Solomon et al. 1979), the small beamsizes and resultant severe undersampling of these early surveys all but precluded the systematic study of such massive clouds as distinct objects, and hence of their galactic distribution. By 1980, CO surveys undertaken with the Northern Millimeter-Wave Telescope had covered enough of the first and second quadrants with sufficient sampling to reveal a string of molecular 
clouds that trace the Perseus arm, as well as to identify the molecular counterparts of the classic $21-\mathrm{cm}$ arms (Cohen et al 1980). With the completion of these surveys it became possible to systematically search the inner Galaxy Co emission for distinct giant cloud complexes and to study their distribution on a galactic scale. Using the Columbia CO survey of the first quadrant, Dame (1983; also see Dame et al. 1985) identifled 25 molecular cloud complexes more massive than $5 \times 10^{5} \mathrm{M}_{\odot}$, including 13 more massive than $10^{6} M_{\odot}$. These objects were found to be excellent spiral arm tracers, with even those clouds at very great distances standing out in the data as discrete features.

The approach taken by Dame is a familiar one in astronomy: to assume that the propertles of a locally-observed class of objects can be used in the global study of these objects. We adopt the same approach in this chapter, using the propertles of locally-observed glant molecular clouds to aid in the identification of the Carina arm clouds. Prototypes of giant clouds, such as those associated with $W 44$ and NGC 7538, have been discussed elsewhere (see e.g., Dame 1983 and Dame et al. 1985), and further discussion here of local examples is omitted. It is shown below that the Carina arm clouds not only delineate the arm over nearly $25 \mathrm{kpC}$-- not a surprising result considering the prominence of the loop in the l,V diagram -- but when viewed in the plane of the Galaxy together with the largest clouds in the first and second quadrants they suggest how the Carina arm connects with the arms in the first galactic quadrant.

First, the general procedure for ldentifying and locating the clouds is described, then, as an example, the large cloud associated with the $\eta$ Carinae Nebula is examined in detall. Next, from a catalog of 43 molecular clouds identified between $I=270^{\circ}$ and $336^{\circ}$, a subset of those 
generally more massive than $10^{5} \mathrm{M}_{\odot}$ is used to trace the Carina arm in the plane of the Galaxy, and the implications for large-scale galactic structure are discussed. Finally, a brief description is given of each cloud in the catalog between $/=270^{\circ}$ and $300^{\circ}$.

\section{A. Identification of the Clouds}

The Carina arm loop in the I, $\mathrm{V}$ diagram consists of about 35 strong, fairly isolated emission features that resemble the largest clouds in the first and second quadrants, with velocity widths of $\sim 10 \mathrm{~km} \mathrm{~s}^{-1}$ and linear sizes (from their angular extents and estimated distances) of $\sim 100 \mathrm{pC}$. Based on these discrete features picked out from the l, diagram, a preliminary list of cloud identifications was compiled. In the far side of the arm most of the clouds are not connected even at the lowest contour level; usually each such isolated feature was considered a separate cloud. In the near side of the arm collections of peaks, as seen for example between $I=291^{\circ}$ and $296^{\circ}$, were tentatively considered as cloud complexes, pending identification of associated optical objects with known distances. Cloud identifications in the tangent region were least certain and still remain subject to revision. Clouds interior to the loop, except for clearly local ones, were included in the preliminary list as well.

Cloud identifications were also made using the full resolution spatial maps in Figures IV-3a-d and a set of spatial maps smoothed to $0.25^{\circ}$ (Figs. $v-(a-d)$. The smoothed maps provided higher signai-to-noise, and highlighted large clouds in the near side. Again, emission features were considered clouds or cloud complexes if they resembled in spatial appearance giant clouds in the first and second quadrants. In general, the 
same clouds and cloud complexes were identified in both the $/ \mathrm{V}$ diagram and the spatial maps, supporting the initial identifications. In a few cases the spatial maps showed that separate clouds at the same longitude had been combined in the I, $\mathrm{V}$ map as a result of integration across the plane. For example, at $l \approx 283^{\circ}$ the two peaks at $b=+1^{\circ}$ and $b=-1.5^{\circ}$ in the near side of the arm form a single peak at $/ \approx 283^{\circ}, v=-15 \mathrm{~km} \mathrm{~s}^{-1}$ in the $\mathrm{I}, \mathrm{V}$ map. Comparing Figures $V-1 a$ and $b$ indicates that the feature at $b=-1.5^{\circ}$ is continuous with the tangent region emission, whereas the feature at $b=+1^{\bullet}$ shows no continuation into the tangent region map; these two features were then considered to be distinct.

The frequent association of giant molecular clouds with $\mathrm{H} / 1$ regions, young stars and $O B$ associations, and other Population I materlal provided an important criterion for refinement of preliminary cloud identifications, particularly in the near side and the tangent region. The association of these young objects with molecular clouds was determined on the basis of close spatial and velocity overlap. Occasionally, clear evidence of interaction between the molecular cloud and an adjacent $H \quad 1 /$ region provided an additional argument for association. in some cases, such as the $\eta$ Carinae molecular cloud (discussed below), an apparent grouping of $\mathrm{CO}$ peaks in the I,V and spatial maps could be identified as a single cloud complex based on association with various optical objects known to be at the same distance. At the same time, these optical objects which have only their similar distances in common, could be shown to be physically related through association with a parent cloud.

The final list of identified clouds is given in Table $V-2$ and their running numbers are Indicated near their locations on the spatial maps in Figures $v-1 a-d$. 
Distances:

Distances to the clouds were usually determined either kinematically or, when possible, by association with optical objects of known distance, preference being given always to optical distances. It was possible to assign optical distances to two of the four clouds identified in the near side; the other two were placed kinematically on the subcentral locus. About half the clouds in the tangent region of the arm could be given optical distances; for those given far kinematic distances, the near-far ambiguity was resolved by one of two ines of evidence: 1) poreground absorption of an associated $H$ II region where the absorption line velocity is more extreme than the $H$ II region velocity, indicating that the $H \|$ region (and the cloud) is on the far side of the subcentral point; or 2) the implled radius of the cloud at the far distance was clearly iavored over that at the near distance by the power law relation between cloud radius and cloud velocity width derived by Dame et al. (see "Masses" below for the definitions of cloud radius and velocity width). In the far side of the arm where there is no kinematic distance ambiguity, all but two clouds (placed optically) were given kinematic distances. A few clouds near zero velocity at longitudes above the tangent region were placed according to the radius-iine width relation, the proximity to the $b=0^{\circ}$ plane, or by the probable association with local dust clouds. 
Masses:

The mass of each cloud, $M_{\text {co, }}$ was determined from its $\mathrm{CO}$ luminosity, using expression $(I V-1)$ to convert from integrated $\mathrm{CO}$ intensity to $\mathrm{H}_{2}$ column density, and a mean molecular weight per $\mathrm{H}_{2}$ molecule of $2.76 \mathrm{~m}_{\mathrm{H}}$ (Allen 1973). Denoting the total Integrated emission over the face of the cloud in units of $\mathrm{K} \mathrm{km} \mathrm{s}^{-1}$ as $\mathrm{I}_{\mathrm{co}}$, and the heliocentric distance to the cloud In klloparsecs as $r$, the total mass of a molecular cloud (including helium) can be expressed as:

$$
M_{C O}=1.9 \times 10^{3} I_{C O} r^{2} M_{\odot}
$$

$I_{c o}$ is obtained by integrating the emission over the full velocity width of the cloud as deduced from the I,V dlagram, and over the face of the cloud as defined in the spatial maps.

For comparison, a virial mass, $M_{\text {vir }}$, for each cloud was computed by assuming the cloud to be in virlal equilibrium, supported against gravity by internal motions. In the case of a uniform density sphere with radius $R$, total mass $M$, and 3-dimensional (Isotropic) velocity dispersion $\sigma$, the virial theorem can be written as:

$$
M \sigma^{2}-3 / 5 G M 2 / R=0,
$$

assuming magnetic pressure and tidal disruption are negligible. Since $M$ is the mass of a cloud in virial eqilibrium, we write $M=M_{\text {vir }}$; for the cloud

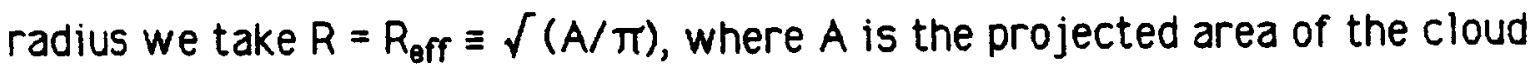
(in $p c^{2}$ ). The isotropic velocity dispersion, $\sigma$, and the observed velocity 
width of the cloud (in $\mathrm{km} \mathrm{s}^{-1}$ ), $\Delta \mathrm{v}$, as measured from the FWHM of a Gaussian fit to the sum of all spectra covering the cloud, are related by:

$$
\sigma=[3 /(2 \ln 2)]^{1 / 2} \Delta v / 2
$$

Solving equation $(V-2)$ for $M_{\text {vir }}$ and substituting more convenient units then gives:

$$
M_{\text {vir }}=210 R_{\text {eff }} \Delta v^{2} \text {, }
$$

where $M_{v i r}$ is in solar masses, $R_{\text {eff }}$ is in parsecs, and $\Delta v$ is in $\mathrm{km} \mathrm{s}^{-1}$. If the observed velocity width includes contributions due to expansion or contraction of the cloud, the expression for $\sigma$ gives an erroneously high velocity dispersion, and $M_{\text {vir }}$ will then be an overestimate of the actual mass. Under the assumptions above, the virial mass is thus an upper limit to the cloud's true mass.

B. The $\eta$ Carinae Molecular Cloud

1. Identification of the Cloud Complex

The general procedure used for identifying molecular clouds in the survey is illustrated by considering the giant molecular cloud associated with the $\eta$ Carinae Nebula, one of the brightest giant $H \|$ ll regions in the Galaxy, and certainly one of the most interesting objects in the Carina arm. In Figure $V-1 a$, a string of five clouds is seen lying approximately on a line from $/=285^{\circ}, b=0.5^{\circ}$ to $/=288^{\circ}, b=-1^{\circ}$. Similar in spatial 
appear ance to, for example, a $106 \mathrm{M}_{\odot}$ complex in the Scutum. arm designated $[22,53]$ in Dame et al, this grouping of clouds can also be seen in the I, map (Fig. $111-7$ ) bewteen $/=285^{\circ}$ and $288^{\circ}$ in the near side. (The cloud at $I=288^{\circ}, b=1.5^{\circ}$ in Figure $V-1$ a blends with the rest of the group in the I, map.) To determine if it is indeed part of a single cloud complex, known optical objects in this region with distances on the near side of the arm were identified, and evidence for the association of these objects with the molecular emission was sought.

Figure $V$-2 shows a blow-up of the near side of the arm (full resolution from Fig. IV-3a) overlaid on a mosaic of the ESO J plates of this region. The $\eta$ Carinae Nebula, also known as NGC 3372, is the bright object between $l=287^{\circ}$ and $288^{\circ}$ in the figure. Its position at the edge of the cloud is similar to that of many other $H$ II regions associated with giant molecular clouds, such as Orion (e.g Kutner et al. 1977), W3, W4, and W5 (Lada et al. 1978) and the Rosette nebula (Blitz and Thaddeus 1980). The positional coincidence is strong evidence for an association of the molecular cloud with the nebula. Radio recombination line measurements of the nebula indicate that its mean velocity is $-20 \mathrm{~km} \mathrm{~s}^{-1}$ while the mean velocity of the molecular cloud from its CO emission in this region is $-19 \mathrm{~km} \mathrm{~s}^{-1}$, making the association almost certain. The optical distance to the Carina Nebula being $2.7 \mathrm{kpc}$ (e.g., Walborn 1973), this is taken to be the distance to the molecular cloud. The relationship of the cloud to the nebula is discussed in detail below.

At $I=286.2^{\circ}, b=-0.2^{\circ}$ the cluster NGC 3324 can be seen, its location between two CO peaks and along a CO ridge suggestive of association with this molecular gas. The cluster's age is $\sim 2.2 \times 10^{6}$ years (Claria 1977) and it contains the 06-7 star HD92206 (Goy 1973). The Hoc region G 31, also 
seen in this direction, apparently is ionized by the bright stars in the cluster (Gum 1955). No direct velocity information is avallable for $G 31$, but Humphreys (1972) gives $-21.2 \mathrm{~km} \mathrm{~s}^{-1}$ as the velocity of the cluster based on measurements of stellar velocities (using the notation after Hoffleit (1953), Humphreys refers to $G 31$ as $\mathrm{H} \mathrm{31}$ ). The good agreement in velocity between the cluster and the molecular cloud, coupled with the spatial coincidence, again indicates that the two are associated. Various distances to NGC 3324 and $G 31$ range from 1.9 to $3.28 \mathrm{kPC}$ (Humphreys 1976; Hofflelt 1953; Georgelin 1975; Turner et al. 1980; Clarla 1977; Moffat and Vogt 1975), the average being $2.7 \mathrm{kpC}$, which is the distance to the Carina Nebula. A connection between the Co emission seen toward the Carina Nebula and the emission toward NGC 3324 seems likely, since in each case the optical sources are associated with the coincident molecular gas and both sources are at the same distance.

Another cluster, NGC 3293, is coincident with the $\mathrm{H} \alpha$ region $\mathrm{G} 30$ and sits on a steep ridge of CO emission at $1=285.9^{\circ}, b=0.1^{\circ}$. This cluster is older than NGC 3324 -- about 5-10 $\times 10^{6}$ years according to Turner et al. (1980) and Stothers (1972). The velocity of the cluster is $-25.5 \mathrm{~km} \mathrm{~s}^{-1}$ (Humphreys 1972), in good agreement with the molecular cloud's velocity. Distance estimates again average around 2.7 kpc (e.g.s. Humphreys 1972; Turner et al. 1980), and we conclude that NGC 3293 is also associated with the same cloud as the Carina Nebula and NGC 3324.

Based on its possible association with the cluster IC 2581, again at about $2.7 \mathrm{kPC}$ (Turner 1978 ), the CO cloud at $1=285.2^{\circ}, \mathrm{b}=0.4^{\circ}$ may aiso be part of this same cloud complex. At $/=284.7^{\circ}, D=0.1^{\circ}$, IC 2581 sits near the edge of this cloud; the cluster has an age of about $10^{7}$ years (Stothers 1972). The velocity of the cluster is $-13 \mathrm{~km} \mathrm{~s}^{-1}$ (Humphreys 
1972) whereas the strongest emission from the clump lies between -23 and $-21 \mathrm{~km} \mathrm{~s}^{-1}$ (see Fig. $\mathrm{V}-4 d$ ), so the agreement is not 50 good as in the three cases just considered. Nevertheless, in view of the cluster's distance and proximity to a co cloud at the same velocity as the rest of the cloud complex, it seems likely that IC 2581 is associated with the CO cloud and that the cloud is part of the same complex.

All the emission from $l=284.7^{\circ}$ to $289^{\circ}$ along the plane, then, apparently forms a single giant molecular cloud complex. At a distance of $2.7 \mathrm{kpc}$, its projected length is $\sim 150 \mathrm{pc}$, typical for the largest complexes found in the first and second quadrants. Turner et al. (1980) suggested IC 2581, NGC 3292, NCG 3324, and the clusters within the Carina Nebula $(\operatorname{Tr} 14,15,16$, and $C r 228)$, are all genetically related, based on the similar distances, the sequential orientation nearly along the galactic plane, and the apparent age gradient from the young clusters in the Carina Nebula to the older IC 2581. The large cloud complex we have identified confirms this picture by revealing the common parent material of these clusters.

Mass:

Using expression $(V-1)$, the co mass of the cloud complex was found to be $6.7 \times 10^{5} \mathrm{M}_{\odot}$, somewhat smaller than the largest molecular complexes in the first and second quadrants. With a full velocity width, $\Delta v$, of $9.9 \mathrm{~km} \mathrm{~s}^{-1}$ and an effective radius, $R_{\text {eff }}$, of $66 \mathrm{pC}$, the virial mass of the cloud complex from expression $(V-3)$ is $1.4 \times 10^{6} M_{0}$. 
2. The $\eta$ Carinae Nebula and the Molecular Cloud

We now compare the distribution and kinematics of the molecular gas just in the vicinity of the $\eta$ Carinae Nebula with what is known about this spectacular object from observations at optical, radio, infrared, ultraviolet, and $X$-ray wavelengths. Taken together, these data suggest a simple, preliminary model for the geometry and interaction of the gas and stars in this region. In the following discussion we first establish the spatial relationship and relative motions of the $\mathrm{H} I \mathrm{I}$ region and its constituents, and the molecular cloud, showing that a portion of the cloud is apparently expanding away from the main body. Then giving brief consideration to the possible dynamical relationship between the $\mathrm{H} I 1$ region and the expanding cloud fragment, we show that sources within the $\mathrm{H} I I$ region can supply sufficient mechanical energy to drive the expansion.

The $\eta$ Carinae Nebula covers about $4 \mathrm{deg}^{2}$ centered near $I=287.5^{\circ}$, $b=-0.5^{\circ}$ (Fig. V-2); dark dust lanes are seen to cross the bright central portion of the nebula. In the immediate vicinity of the bright gas the dust lane is vee-shaped, but it extends beyond the $H \|$ II region in the direction of NGC 3324 and NGC 3293. For the purposes of this discussion, the apparently connected dust lanes are divided into four pieces as follows (see the schematic diagram in Fig. $V-3$ ): 1) eastern leg, extending from $I \approx 287.8^{\circ}, b \approx-0.4^{\circ}$ on the eastern side of the bright gas to $1 \approx 287.6^{\circ}$, $b \approx-0.7^{\circ}$ at the vertex of the vee; 2 ) western leg, extending from the vertex of the vee to $/ \approx 287^{\circ}, \mathrm{b} \approx-0.5^{\circ}$, just beyond the bright gas to the east of this dust (the eastern leg and the western leg comprise the vee); 3 ) western extension, the continuation of the western leg to $/ \approx 286^{\circ}$, 
$D \approx-0.3^{\circ}$, the region below NGC 3324 and NGC $3293 ; 4$ ) northern extension, passing between NGC 3324 and NGC 3293.

Near the nebula's bright center is a large collection of early type stars making up the young clusters $\operatorname{Tr} 14$ and $\operatorname{Tr} 16 . \operatorname{Tr} 16\left(/=287.61^{\circ}\right.$, $\left.b=-0.65^{\circ}\right)$ is centered just above the vertex of the vee-shaped dust lane, and $\operatorname{Tr} 14\left(/=287.42^{\circ}, b=-0.58^{\circ}\right)$ sits about $5^{\circ}$ east of the western leg. A far-infrared continuum peak in the western leg is likely due to the heating of the dust by $\operatorname{Tr} 14$ (Harvey et al. 1979). Among the many young stars found in these two clusters are at least 160 stars, inlcuding five classified by Walborn $(1971 ; 1973)$ as $03 \mathrm{~V}$. Another young cluster, $\mathrm{Cr} 228$ $\left(I=287.52^{\circ}, \mathrm{D}=-1.03^{\circ}\right)$, seen toward the bright gas beneath the vee, contains 60 stars (Walborn 1973). All three clusters are at about $2.7 \mathrm{kpC}$ (Turner et al. 1980; Feinstein et al. 1973, 1976; Walborn 1973) and have ages less than $3 \times 10^{6}$ years, perhaps as young as $10^{6}$ years (Feinstein et al. 1976; Turner et al. 1980). Tr $15\left(/=287.40^{\circ}, \mathrm{b}=-0.36^{\circ}\right)$, a cluster north of $\operatorname{Tr} 16$, also at about $2.7 \mathrm{kpc}$, may be slightly more evolved than the others (Feinstein et al. 1980; Walborn 1973). This large concentration of young stars, the coincidence with the bright $\mathrm{H} \| \mathrm{r}$ region, and the identification of an associated molecular cloud provides strong evidence for the true spatial localization of these clusters in a massive star-forming region.

A good correlation is apparent between the obscuring dust and the observed molecular cloud. A small co peak can be seen in Figure $V-2$ at $I=287.8^{\circ}, \mathrm{b}=-0.8^{\circ}$ coincident with, and having the same general shape, as the eastern leg. $A$ bright peak at $I=287.5^{\circ}, b=-0.6^{\circ}$ marks the boundary between the bright gas and the western leg. Some of this intense co emission overlaps with the western leg, indicating a connection between 
the CO and this dust, but a large amount of the molecular gas is spatially coincident with the bright gas to the left of the western leg and must therefore correspond to molecular material behind the nebula. The co emission at $/<287^{\circ}$ mimics the general shape of the western and the northern extensions. Physical contact between the lonized gas and the western leg is apparent from the existence of bright rims along the projected boundary. This boundary is also marked by an enhancement of the diffuse $X$-ray emission that pervades the entire nebula (Seward and Chlebowski 1982). The co peak seen in this direction provides further evidence for the interaction between the ionized gas and dust, and so between the ionized gas and the molecular cloud.

Two radio continuum sources, Car I and Car 11 , are observed in the nebula. Observations at $3.4 \mathrm{~cm}, 6 \mathrm{~cm}, 11 \mathrm{~cm}$, and $21 \mathrm{~cm}$ (Huchtmeier and Day 1975; Gardner et al. 1970; Beard and Kerr 1966) conf irm that both are thermal. Car I peaks at the interface of the bright gas and the western leg, about 3.5' from TR 14. High resolution observations at $1415 \mathrm{MHz}$ show Car I to be elongated parallel to the western leg dust lane, suggesting the source marks an ionization front at the gas-dust boundary (Retallack 1983). Car II peaks about $6^{\circ}$ west of $\operatorname{Tr} 16$, above the vertex of the vee. Radio recombination lines throughout the nebula show a mean velocity of about $-20 \mathrm{~km} \mathrm{~s}^{-1}$. In the immedlate vicinity of Car II, double lines with separations of up to $45 \mathrm{~km} \mathrm{~s}^{-1}$ are seen; the overall distribution of recombination lines suggests the presence of an expanding shell of lonized gas (Huchtmeier and Day 1975; Beard and Kerr 1966; Wilson et al. 1970).

The $\mathrm{OH}$ and $\mathrm{H}_{2} \mathrm{CO}$ absorption line measurements of Gardner et al. (1973) and Dickel et al.(1973) identified the vee-shaped dust lane as the molecular cloud in the $\eta$ Carinae Nebula. Dickel and Wall (1974) demonstrated the 
physical association of the dust (in the vee) with the molecular gas by comparing a map of $A_{v}$ in the nebula (derived from the ratio of the $5 \mathrm{GHz}$ continuum intensity to the $\mathrm{H}_{\alpha}$ intensity) to the $\mathrm{OH}$ optical depth map of Gardner et al. The mean dust velocity of $-24 \mathrm{~km} \mathrm{~s}^{-1}$ from the $\mathrm{OH}$ measurements shows the neutrai material in the vee is blueshifted with respect to the bulk of the ionized gas behind it. A somewhat more extensive molecular cloud was revealed by the $\mathrm{CO}(J=2 \rightarrow 1)$ observations of de Graauw et al.(1981), who found two CO sources, one associated with what we have called the eastern leg, the other corresponding to the western leg plus material behind the ionized nebula, east of the western leg. They proposed that near the line of sight to Car II, the molecular cloud lies below and on the near side of $\operatorname{Tr} 16$ (corresponding to the eastern leg), and in the direction of $\mathrm{Car} 1$, the cloud stretches to the west of $\operatorname{Tr} 14$ and bends around to the far side of the nebula. It was evident to de Graauw et al. that the molecular cloud extended beyond the limits of their map, making some of their conclusions tentative. The CO observations presented here confirm the essential features of their model and place it in the context of the large cloud complex in this region.

The $\mathrm{CO}$ emission as a function of velocity through the cloud from -31 to $-10 \mathrm{~km} \mathrm{~s}^{-1}$ is shown in a set of eight spatial maps in adjacent, $2.6 \mathrm{~km} \mathrm{~s}^{-1}$ (two channels) wide velocity windows (Figs. $v-4 a-h$ ). In conjunction with the optical, $\mathrm{OH}$ absorption, and recombination line observations, these maps demonstrate that radial velocity is a good indicator of position (along the line of sight) in the cloud in the vicinity of the Carina Nebula. The most intense $\mathrm{CO}$ emission lies between -20 and $-18 \mathrm{~km} \mathrm{~s}^{-1}$ (Fig. $\mathrm{V}-4 \mathrm{e}$ ), the same as the mean velocity of the $H \|$ region. In particular, the emission between $I=287^{\circ}$ and $287.5^{\circ}$ being spatially coincident with the bright gas, must lie 
behind the $H$ II region. The CO counterpart of the vee, seen most strongly between -28 and $-23 \mathrm{~km} \mathrm{~s}^{-1}$ (Figs. $V-4 b, c$ ), clearly represents only a small part of the whole cloud complex (accounting for less than $10 \%$ of the total mass, as shown below). This molecular feature or filament, as it will be referred to here, corresponds to the structure identified by Gardner et al. (1973) in $\mathrm{OH}$ absorption. As we approach the mean cloud velocity of $-19 \mathrm{~km} \mathrm{~s}^{-1}$ from $-30 \mathrm{~km} \mathrm{~s}^{-1}$, the portion of the filament corresponding to the eastern leg disappears, while, between -26 and $-20 \mathrm{~km} \mathrm{~s}^{-1}$, the co peak near the western leg shifts from a region of high optical obscuration to a line of sight coincident with the brightest nebulosity. Thus, the filament on the near side of the nebula joins the more massive portion of the cloud on the far side in the region to the west of Car I. The most negative velocities correspond to material on the near side of the cloud, i.e, the obscuring dust lanes, while more positive velocity material lies next to, and behind the nebula. The difference between the mean velocity of the filament and that of the rest of the molecular cloud is $\sim 7 \mathrm{~km} \mathrm{~s}^{-1}$.

A schematic representation of the molecular cloud and its surroundings is shown in Figure $V-5$. The expanding $H \|$ II region sits at the eastern edge of the cloud, with a portion of the cloud seen in projection behind the $\mathrm{H} \mathrm{II}$ region (near $\mathrm{Tr}$ 14). The fllament passes in front of the ionized gas and, west of $\operatorname{Tr} 14$, wraps around the nebula to connect with the main body of the cloud. The far-infrared peak to the west of $\operatorname{Tr} 14$ arises in this region where the filament joins the rest of the cloud. This picture of the molecular cloud cradling the nebula was also deduced by de Graauw et al., but because most of the cloud lies beyond the boundaries of their map, they could not recognize that the filament represents only a small portion of the total cloud. Since our observations show that the mean velocity of the 
entire cloud is the same as the $\mathrm{H} / 1$ region while the filament is blueshifted with respect to the rest of the cloud, it is likely that the fllament is expanding away from the main body of the cloud. The situation resembles that in the Pelican nebula (Bally and Scoville 1980), with an $\mathrm{H} / 1$ region forming inside a molecular cloud and then breaking out into the intercloud medium when the expanding Stomgren sphere ruptures the edge of the cloud. The question arises as to whether the motion of the filament can be due to sources within the nebula. Brief consideration is now given to two possible mechanisms: stellar winds, and the rocket effect. The rough calculations made below place useful constraints on our preliminary cloud model and are not intended to be a rigorous treatment of the dynamics within the cloud.

Stellar Winds:

Observations of optical and ultraviolet interstellar absorption lines in the direction of several stars in the nebula show velocities ranging over $550 \mathrm{~km} \mathrm{~s}^{-1}$ (Walborn 1982; Walborn and Hesser 1982; Laurent et al. 1982), indicating that the stars are injecting a lot of mechanical energy into the surrounding medium. Assuming the absorption lines arise in stellar winds and an age of $\sim 3 \times 10^{6}$ years for the clusters, Walborn (1982) estimated a total energy output of $3 \times 10^{51}$ ergs from the known 0 and $W N-A$ stars. The observation of diffuse $X-r a y$ emission throughout the Carina Nebula can likewise be interpreted as a result of stellar winds with a similar energy input (Seward and Chlebowski 1982). To compare the mechanical energy from stellar winds with the kinetic energy of the expansion, the CO mass of the fllament is determined by integrating its emission over velocity. This integration, from $v=-31$ to $-24 \mathrm{~km} \mathrm{~s}^{-1}$, yields a CO mass of 
$2.2 \times 10^{4} M_{\odot}$, only $3 \%$ of the total co mass found above for the entire complex, so the filament is really only a small part of the whole cloud. Taking the expansion velocity to be $7 \mathrm{~km} \mathrm{~s}^{-1}$ and a mass of $2.2 \times 10^{4} \mathrm{M}_{\odot}$ yields a kinetic energy of $\sim 1049 \mathrm{ergs}$, well within the energy available from stellar winds.

The energy supply being sufficient, a further constraint is the momentum of the filament, $P_{\text {filament }}=3.1 \times 1043 \mathrm{gm} \mathrm{cm} \mathrm{s}^{-1}$. For a single star with a constant mass loss rate, $\mathrm{dM} / \mathrm{dt}$, and a constant wind velocity $v_{w}$, the momentum supplied directly from stellar mass loss to the surrounding medium over a time $\tau$ is simply $P_{\text {in }}=(d M / d t) v_{w} \tau$. Walborn (1982) tabulated the numbers of $O$ and $W N-A$ stars in the Carina Nebula along with the mass loss rates and wind velocities (taken from the literature) for the various spectral types. Following Walborn, we take the age of the clusters to be $3 \times 10^{6}$ years and assume the $\mathrm{WN}-\mathrm{A}$ and luminosity class I stars have existed as supergiants for $10^{5}$ years. The total momentum input from stellar winds is then found to be $P_{\text {in }}=1.9 \times 10^{43} \mathrm{gm} \mathrm{cm} \mathrm{s}^{-1}$, only marginally enough to account for $P_{\text {filament. }}$ The momentum that can actually be transferred directly from the wind to the filament is $P_{\text {in }}$ reduced by the fraction, $f$, of $4 \pi$ steradians subtended at the stars by the filament. From the spatial map (Fig. V-2) the projected area of the filament is approximately $1000 \mathrm{pc}^{2}$; taking this to be its true area and assuming a distance of $20 \mathrm{pc}$ between the stars and the filament (the projected separation between the tip of the eastern leg and the CO peak west of $\operatorname{Tr} 14$ ) then gives $f=0.2$. Therefore the filament could have acquired a momentum of $3.8 \times 10^{42} \mathrm{gm} \mathrm{cm} \mathrm{s}-1$ from stellar winds, about a factor of ten below its observed value.

The effect of a strong wind from a star on the surrounding medium has been investigated by Castor et al. (1975). They show that an expanding 
cavity or bubble forms around the star, bounded by a thin shell of material swept up by the expansion into the ambient medium. Between the shell and the cavity is a layer of not, shocked gas which acts like a piston driving the shell outward. The momentum of the shell can be greater than that supplied directly by the wind. To examine this picture in the context of the $\eta$ Carinae molecular cloud, the ambient medium with density $\rho_{0}$ is taken to be the molecular cloud, with the filament corresponding to the shell. Castor et al. give for the radius of the shell:

$$
R_{s}(t)=0.76\left[(d M / d t) V_{w} 2 /\left(2 p_{0}\right)\right]^{1 / 5} t^{3 / 5}
$$

Differentlating with respect to time, and identifying the velocity of the shell, $v_{s}$, with that of the cloud filament, $v_{c}$, we have:

$$
v_{c}=d R_{s}(t) / d t=2.7 \times 10^{3}\left[(d M / d t) v_{w}^{2} n_{0}^{-1}\right]^{1 / 5} t^{-2 / 5} \mathrm{~km} \mathrm{~s}^{-1},
$$

where $d M / d t$ is in units of $M_{\odot} y r^{-1}, v_{w}$ is in $\mathrm{km} \mathrm{s}^{-1}, t$ is in years, and $n_{0}$ is particle density in $\mathrm{cm}^{-3}$; a mean molecular weight per particle of $2.76 \mathrm{~m}_{H}$ (Allen 1973) has be assumed. As a lower limit on no we take $8 \mathrm{~cm}^{-3}$, the average density in the entire cloud obtained from $M_{\text {co }}$ and $R_{\text {eff }}$ (Section B.1 above). Since the density in regions of the cloud where stars are forming will be higher, an upper limit for $n_{0}$ is taken to be the density in the filament. Assuming the filament is sheet with area $1000 \mathrm{pc}^{2}$ and thickness $5 \mathrm{pc}$, and mass $2.2 \times 10^{4} M_{\odot}$, we have $n_{0} \approx 80 \mathrm{~cm}^{-3}$. The tabulation in Walborn (1982) is used to estimate an average mass-loss rate, 〈dM/dt>, and average wind velocity, $\left\langle v_{w}\right\rangle$, for the young clusters in the Carina Nebula, giving $\langle d M / d t\rangle=1.1 \times 10^{-5} M_{\odot} y r^{-1}$, and $\left\langle v_{w}\right\rangle=2900 \mathrm{~km} \mathrm{~s}^{-1}$. Substituting these 
values into the expression for the velocity of the fllament yields:

$v_{c}=7-11 \mathrm{~km} \mathrm{~s}^{-1}$, in good agreement with the observed velocity of the fllament relative to the main body of the $\eta$ Carinae molecular cloud.

We conclude that, while direct transfer of stellar-wind momentum to the fllament cannot account for the fllament's observed motion, the expansion of a wind-driven bubble in the cloud easily explains the moton of the filament with respect to the main body of the cloud.

Rocket Effect:

As discussed by Bally and Scoville (1980, and references therein), when an $\mathrm{H} I 1$ region bursts through the edge of a molecular cloud from within, the resulting depressurization will tend to increase the flux of ionizing radiation at the remaining boundary between the molecular cloud and the ionization front, but the density of lonized gas between the stars and the cloud will be essentially unchanged. Ionized molecular gas at this boundary will stream away from the cloud, accelerating the cloud like a rocket (e.g., Spitzer 1978). If the cloud has mass $M$, velocity $v_{c}$, and loses mass at a rate $\mathrm{dM} / \mathrm{dt}$, then it accelerates according to

$$
v_{8}(d M / d t)=M\left(d v_{c} / d t\right)
$$

where $v_{e}$, the exhaust velocity of the freshly ionized gas from the cloud, is taken to be the isothermal sound speed in the $H \|$ region, $\sim 10 \mathrm{~km} \mathrm{~s}^{-1}$. Integrating this equation gives

$$
v_{c}=v_{\theta} \ln \left(M_{0} / M\right)+v_{0}
$$


where $M_{0}$ and $v_{0}$ are the initial mass and velocity of the cloud. Assuming $v_{0}=0$, taking $M$ to be the presently observed mass, and estimating $M_{0}$, we can evaluate the rocket effect for the filament by comparing the predicted cloud velocity with the observed velocity.

The initial mass of the cloud can be estimated as follows. The flux of ionization off the back of the filament is $n_{H I I} v_{\mathbf{B}}$, where $n_{H I I}$ is the density of the ionized gas in the $\mathrm{H} \|$ region at the filament. The filament then loses mass at a rate

$$
\mathrm{dM} / \mathrm{dt}=A n_{H I I} V_{\mathrm{e}}
$$

where $A=1000 \mathrm{pc}^{2}$ is the area of the filament. As noted by Bally and Scoville, $n_{H I I}$ is about the same before and after the $H$ II region bursts through the edge of the cloud and, at radius $r$ of the $H \|$ II region, is given by

$$
n_{H I I}=\left[3 Q /\left(4 \pi \alpha^{(2)} \Gamma^{3}\right)\right]^{1 / 2},
$$

where $Q$ is the emission rate of Lyman continuum photons from the Ionizing stars, and $\alpha^{(2)}$ is the recombination coefficient to all levels with $n>1$. For the Carina nebula Smith et al. (1978) give $Q=13 \times 10^{49} \mathrm{~s}^{-1}$ for the continuum sources Car I and Car II. Considering only ionized hydrogen and assuming a temperature for the $\mathrm{H} \| \mathrm{l}$ region of $10^{4} \mathrm{~K}$ then, at the fllament $(r \approx 20 \mathrm{pC}$, as above $), n_{\mathrm{HII}} \approx 21 \mathrm{~cm}^{-3}$, and

$$
\mathrm{dM} / \mathrm{dt} \approx 5.3 \times 10^{-3} \mathrm{M}_{\odot} \mathrm{yr}^{-1}
$$


Assuming this rate to be constant, then in $3 \times 10^{6}$ years the filament has lost $\sim 1.6 \times 10^{4} M_{\odot}$, and its initial mass was therefore $M_{0}=4.7 \times 10^{4} M_{\odot}$. Equation $(\mathrm{V}-5)$ then gives $\mathrm{v}_{\mathrm{c}} \approx 4.1 \mathrm{~km} \mathrm{~s}^{-1}$, which is close to the filament's presently observed velocity of $7 \mathrm{~km} \mathrm{~s}^{-1}$ relative the rest of the molecular cloud. We conclude that the rocket effect is also apparently capable of explaining the observed motion of the filament.

The actual $H$ II region-molecular cloud interaction is undoubtedly more complicated than so far assumed, but the basic picture of the $\mathrm{H}$ II region breaking through the cloud's surface and ejecting neutral material (the filament) outward of fers a plausible explanation for the observed motions of the molecular cloud and the ionized gas. It is interesting to note that for the parameters used above, $r=20 \mathrm{pc}$ and $v_{\mathrm{c}}=7 \mathrm{~km} \mathrm{~s}^{-1}$, the expansion time scale is about $3 \times 10^{6}$ years, in good agreement with the estimated age of the young clusters that power the $H \|$ region. The possibility that the expansion of the filament is driven by a supernova was not considered here because no confirmed SNR exists in the vicinity of the Carina Nebula. Although the small nonthermal radio source 6286.5-0.5 is claimed to be a supernova remnant (Jones 1973; Becker et al. 1976; Elliot 1979), Seward and Chleblowski (1982) pointed out that, at the distance of the Carina Nebula, the source's $X$-ray luminosity is too low for a SNR.

3. Star Formation Efficiency in the $\eta$ Carinae Molecular Cloud

The interaction between the $\eta$ Carinae Nebula and its associated molecular cloud of fers an outstanding example of the destructive effect that massive stars can have on the cold, quiescent gas from which they 
form. By dispersing the molecular cloud, massive stars can disrupt star formation in their immedlate neighborhood, leading to a low overall star formation efficiency in the cloud. In this section the star formation efficiency, SFE $=M_{*} /\left(M_{*}+M_{\text {cloud }}\right)$, is determined for the entire $\eta$ Carinae molecular cloud. The result, SFE $\approx 0.02$, illustrates that the efficiency can indeed be low in large clouds that spawn massive stars, and provides a check on the star formation efficiencies recently determined for inner Galaxy clouds where star formation is generally highly obscured. As defined here, the star formation efficiency depends on the total molecular cloud mass, $M_{\text {eloud, }}$ and the total mass of stars associated with the cloud, $M_{*}$. For $M_{\text {cloud }}$ the $C O$ mass determined in Section B.I above is used. The determination of the total stellar mass is the main focus of the following discussion.

The initial mass function (IMF) of Miller and Scalo (1979),

$$
\xi(\log m)=\zeta \exp \left[-1.09(\log m+1.02)^{2}\right]
$$

where $\zeta$ is a proportionality constant, can be used to estimate $M_{*}$. The main drawback of this approach is that, being derived from fleld stars, the Miller-scalo IMF is least certain at the high-mass end, so its application to the young clusters in the $\eta$ Carinae Nebula may not be appropriate. For example, Garmany et al. (1982) find that the slope of the IMF for early-type 0 stars is less steep than the the Miller-Scalo IMF, with a higher proportion of massive stars in young clusters than in the general field. It is possible too that the IMF varies from cluster to cluster. However, because the IMF method is the only way at present to account for the intrinsically faint, low mass stars which (collectively) contain most of the stellar mass, we adopt 
that approach here, making some attempt to alleviate the uncertainty at the high-mass end.

The unusually large number of main sequence 0 stars observed in the Carina Nebula suggests that a substantial fraction of the existing massive stars can be seen, and their total mass obtained simply by counting them. We assume this to be the case and rely on the IMF only for the less massive stars. Specifically, the observed stars are binned in logarithmic mass intervals every $\Delta \log m=0.1$ (where $m$ is in solar masses) beginning with the bin centered at $\log _{\max }$; the mass, $\log _{\mathrm{e}}$, at which the counts appear to fall of $f$ is taken as the dividing line between those stars $\left(m 2 m_{c}\right)$ which are completely tallied and those $\left(m<m_{c}\right)$ which are not. A discontinuity in the slope of $\mathrm{dN} / \mathrm{d}(\mathrm{logm})$ indicates where the counts become incomplete due to selection effects since, if all the stars present could be seen, then the counts would continue to rise with decreasing mass. The IMF contribution to the total mass is obtained by integrating the IMF between $\log _{\mathrm{c}}$ and $\log _{\min }$, where $m_{\min }$ is taken to be $0.1 M_{\bullet}$, and the proportionality constant in the Miller-Scalo IMF follows from fixing the computed number of stars in $\Delta \operatorname{logm}$ about $\log _{\mathrm{c}}$ to the observed number. The total stellar mass is then the IMF contribution plus the total observed mass for stars with $m>m_{c}$.

Table $V-1$ lists all the known early type main sequence stars in the clusters $\operatorname{Tr} 14, \operatorname{Tr} 16$, and $\mathrm{Cr} 228$. The primary references are Walborn (1973), and Levato and Malaroda (1981; 1982); these sources include all cluster members with known spectral type tabulated elsewhere in the literature. To convert from spectral type to mass, a graph (not shown here) of $\log \left(\mathrm{M} / \mathrm{M}_{\odot}\right)$ versus $\log T_{\text {eff }}$ was constructed using the empirical relation between mass and effective temperature given by Habets and Heintze (1981) for spectral types later than (and including) $08.5 \mathrm{~V}$. The graph was extended 
to $03 \mathrm{~V}$, the assumed most massive type in the Carina Nebula, by connecting the points $\left[\log \mathrm{T}_{\text {eff }}, \log \left(M / M_{0}\right)\right]$ for the $08.5 \mathrm{~V}$ and later types and a single, corresponding point for an $03 \mathrm{~V}$ star with a smooth curve. Conti and Burnichon (1975) give $\log T_{\text {eff }}=4.74$, and $M \approx 120 M_{\odot}$ for an $03 \mathrm{~V}$ star; this mass is highly uncertain, but probably reasonable for the present purposes. Using the effective temperatures for spectral types $08.5 \mathrm{~V}$ and later from Habets and Heintze, and from Conti and Burnichon for earlier types, the masses of the stars in Table $V-1$ were read off the $\log T_{\text {eff }}-\log \left(M / M_{0}\right)$ graph.

The mass spectrum of the stars in Table $V-1$ is shown in Figure $V-6$; the solid line shows the logarithm of the number of stars in each mass bin, while the dotted line shows the (log of the) cumulative number as less and less massive stars are considered. If the five $03 \mathrm{~V}$ stars $\left[\log \left(M / M_{\odot}\right)=2.08\right]$ are excluded for the moment, a gradual (albiet uneven) rise in number with decreasing mass can be seen between $\log \left(M / M_{\odot}\right)=1.85$ and 1.15. With the stellar data available it is not possible to say whether the apparent drop in the counts for $\log \left(M / M_{\odot}\right)<1.15$ represents the expected turnover as intrinsically fainter stars are observed or just a fluctuation simllar to the ones seen at $\log \left(M / M_{\odot}\right)>1.15$. Since member stars that are much less massive than $\log \left(M / M_{\odot}\right)=0.95$ would be difficult to distinguish from field stars, it is likely that the turnover does occur at or near $\log \left(M / M_{\odot}\right) \leq 1.15$. Therefore, we assume that all the existing stars in the Carina Nebula for which $\log \left(M / M_{\odot}\right) \geq 1.15$ (Including now the five $03 \mathrm{~V}$ stars) are accounted for, and take $\log m_{c}=1.15$.

From the IMF, the number of stars with mass between $m_{1}$ and $m_{2}$, $N\left(m_{1}, m_{2}\right)$, is just 


$$
N\left(m_{1}, m_{2}\right)=\int \xi(\log m) d \log m
$$

and the total mass between $m_{1}$ and $m_{2}, M\left(m_{1}, m_{2}\right)$, is

$$
M\left(m_{1}, m_{2}\right)=\int m \xi(\log m) d \log m,
$$

where the integrals extend from $m_{1}$ to $m_{2}$. For the Miller-Scalo IMF these quantities can be written,

$$
N\left(m_{1}, m_{2}\right)=0.85 \zeta \operatorname{erf}[1.04(\log m+1.02)]_{m_{2}}^{m_{1}}
$$

and

$$
M\left(m_{1}, m_{2}\right)=0.27 \zeta \operatorname{erf}[1.04 \operatorname{logm}-0.04]_{m_{2}}^{m_{1}}, \quad(v-10)
$$

where erf is the error function. As mentioned above, $\zeta$ is set by fixing $N\left(m_{1}, m_{2}\right)$ to the number of stars actually observed between $\log m_{1}=\log m_{c}+(\Delta \log m) / 2$ and $\log m_{2}=\log m_{c}-(\Delta \log m) / 2$. In this interval 12 stars are observed, yielding $\zeta=1.9 \times 10^{4}$, and the IMF contribution to the stellar mass, $M_{\mathbb{M F}}$, is then:

$$
M_{\text {IMF }} \equiv M\left(m_{c}, 0.1 M_{\odot}\right)=10^{4} M_{\odot}
$$

The total mass observed with $m>m_{c}, M_{\text {obs, }}$ is $1.610^{3} M_{\odot}$, so the total mass in all stars, $M_{T}=M_{I M F}+M_{\text {obs }}$, is: 


$$
M_{T}=1.2 \times 10^{4} M_{0}
$$

For comparison with the observed numbers of stars, the values of $\log \mathrm{N}$ predicted by the IMF in the mass intervals with $\log <<\log _{c}$ are also shown in Figure $V-6$ (filled circles). In the Carina Nebula the massive stars appear to exhibit a flatter distribution than that implied by the Miller-5calo IMF, supporting the similar findings of Garmany et al. from a more general survey of early type stars.

Taking $M_{\text {cloud }}=6.7 \times 10^{5} M_{\odot}$ (Section B.1, this chapter) and $M_{*}=M_{T}$, the resulting star formation efficiency is:

$$
\text { SFE }=0.018
$$

A relative uncertainty of a factor of 2-3 is estimated for SFE, with approximately equal contributions from $M_{\text {eloud }}$ and $M_{*}$. Improper choice of the cloud boundary and the uncertainty in the $W(\mathrm{CO})-\mathrm{N}\left(\mathrm{H}_{2}\right)$ conversion lead to a relative uncertainty of $\sim 2$ in the cloud mass. The estimate for $M_{*}$ may be influenced by a number of factors. By using the mass of the entire cloud we have made the assumption that the mass present in the clusters which are not part of the Carina Nebula (NGC 3324, NGC 3293, and IC 2581) is accounted for in the preceeding stellar mass estimate. This assumption is reasonable because the stars in these clusters, primarily later types than those in the Carina Nebula, lie in the regime of the IMF calculation. A more accurate mass might be obtained by considering these clusters separately. From an analysis of NGC 3293 Herbst and Miller (1982) derived a mass of $\sim 1500 M_{\odot}$; if approximately the same amount of mass is associated with 
NGC 3324 and IC 2581 , M* could be increased by about $40 \%$. More critical is the assumption that all stars with $m=m_{c}$ are observed, since $M_{*} \sim M_{\text {IMF }}$ and MimF depends linearly on the number of stars in the mass bin centered at $m_{c}$; this number could be off by a factor of 2-3 owing to obscuration by the dust. A similar factor probably applies to the number of stars with $m>m_{c}$, but the total mass of these stars, $M_{\text {obs }}$, is only about $15 \%$ of $M_{*}$. (We note also that the number of ionizing photons available in the Carina Nebula as estimated from radio continuum measurements [Smith et al. 1978] is reasonably consistent with the observed numbers of early-type stars, indicating that the star counts are probably not far off.) Finally, even if $M_{\text {obs }}$ correctly measures the total mass in stars more massive than $m_{c}$, no allowance has been made for 0 stars which are no longer present. However, judging from the effects of the $\eta$ Carinae Nebula on the molecular cloud, it is not likely that the number of such stars is much greater than the present day value of $\mathrm{N}\left(\mathrm{m}>\mathrm{m}_{\mathrm{c}}\right)$ since the molecular cloud remains largely intact in the vicinity of the older clusters, and near the $\eta$ Carinae Nebula the motions of the cloud are consistent with the energetics of the present star-forming epoch.

The star formation efficiency derived here for the $\eta$ Carinae molecular cloud agrees well with the efficiencies for giant molecular clouds in the inner Galaxy recently derived by Myers et al. (1985). To obtain M* for each molecular cloud in their sample, Myers et al. used the measured radio continuum and far infrared fluxes of $\mathrm{H} \|$ regions associated with the clouds to infer the mass, $m_{\max }$, of the most massive star present in each $\mathrm{H}$ II region. The Miller-scalo IMF, normalized to one star of mass $m_{\max }$, was then used to calculate the mass of a single cluster containing just one such star, and mass-luminosity relations from the literature were used to predict the 
total luminosity of the cluster. With the assumption that the total stellar luminosity equals the observed FIR luminosity of each $H$ II region, the total stellar mass associated with each $\mathrm{H} \| \mathrm{I}$ region followed from the number of identical clusters required to produce the observed total luminosity. For a given molecular cloud, $M_{*}$ was then just the sum of the total stellar masses in each $\mathrm{H} / 1$ region associated with the cloud. An important principle of this method is that, for a given $H \| I$ region, the ratio of the observed FIR luminosity to the Lyman $\alpha$ luminosity (inferred from the radio continuum), denoted IRE for infrared excess, is an indicator of the amount of nonionizing stellar luminosity present. If IRE is too small, as turns out to be the case with the $\eta$ Carinae Nebula, the method doesn't yield consistent results. From their sample of about 50 giant molecular clouds, Myers et al. found a median SFE of 0.02. Aside from the use of the Miller-Scalo IMF, the method of Myers et al. is quite different from the one used here for the $\eta$ Carinae molecular cloud, and the good agreement between the star formation efficiencies found here and in the inner Galaxy, then, probably indicates that the results are reasonable.

\section{Summary}

A glant molecular cloud complex was found to be assoclated with the $\eta$ Carinae Nebula, NGC 3324, NGC 3293, and IC 2581. The linear extent of the cloud is $\sim 150 \mathrm{pc}\left(R_{\text {eff }}=66 \mathrm{pc}\right)$ and its total mass is $\sim 7 \times 10^{5} \mathrm{M}_{\odot}$, both values typical of giant molecular clouds found elsewhere in the Galaxy. In the vicinity of the Carina Nebula, the interaction of the young stars, Ionized gas, and the molecular cloud can be understood with a simple model in which the prominent dust lanes crossing the face of the nebula trace a fragment of 
the molecular cloud that is expanding away from the bulk of the cloud. Sufficient energy is available from sources within the nebula to drive the expansion. The main body of the cloud sits beside the $H \|$ II region and extends along the galactic plane in the direction of NGC 3324, 3293, and IC 2581. By summing the mass in the observed early type stars and using the IMF to obtain the mass in the late type stars, a star formation efficiency of 0.018 was derived for the entire molecular cloud. This number agrees well with the star formation efficiencies for giant molecular clouds in the inner Galaxy.

\section{The Carina Arm in the Galaxy}

How does the Carina arm connect with the rest of the Galaxy? This question goes back to Bok, whose original idea was a single spiral arm from Carina through Cygnus. The early hopes that $21 \mathrm{~cm}$ line studies of neutral hydrogen would reveal global spiral structure were never realized because of the ubiquity of $\mathrm{H} \mathrm{1}$, the complications arising from noncircular motions, the difficulties in comparing different surveys, and the different interpretations by workers using the same data. One of the main points of controversy has been the way in which the Carina arm connects with the rest of the Galaxy.

Weaver (1970) presented a model in which the Carina and Sagittarius arms form a single major spiral arm with a pitch angle of about $12.5^{\circ}$. Alternatively, Kerr (1970) favored Bok's model which joins the Carina arm with Cygnus in a low-pitch arm; the low density of Population I beyond the Sun in the directions of Carina and Cygnus suggested a localized region of low density where the arm passes through the solar neighborhood (simonson 
1970). Kerr and Kerr (1970) pointed to a gap from $l=292^{\circ}$ to $305^{\circ}$ in the $11 \mathrm{~cm}$ continuum and $\mathrm{H} 109 \alpha$ fluxes as evidence against a CarinaSagittarius connection, arguing that such an arm would be expected to pass just inward of the Sun in this longitude range. They maintained instead that the only $\mathrm{H} \| \mathrm{I}$ regions seen in this gap were distant and could not represent a connecting stretch between Carina and Sagittarius.

Using a variety of spiral tracers, Humphreys (1976) traced the Carina arm into the first quadrant and, like Weaver, connected it with the Sagittarius arm. Similarly, Georgelin and Georgelin (1976), using optical and radio $H$ II regions as tracers, proposed a Carina-Sagittarius arm. However, their Carina-Sagittarius arm is least well-def ined in the region connecting the two arm segments, and between $/ \approx 30^{\circ}$ and $45^{\circ}$ in the Sagittarius arm there is a $6 \mathrm{kpc}$ gap, which is difficult to reconcile with the claim of large-scale continuity.

The evidence offered by the largest molecular clouds -- those more massive than roughly $105 \mathrm{M}_{\odot}--$ strongly supports the Carina-Sagittarius connection. Figure $V-7 a$ shows all the Carina clouds generally more massive than $105 M_{\odot}$ located in the plane of the Galaxy; also shown are similarly massive clouds identifled in earlier co surveys made with the Columbla millimeter-wave telescope in New York City. The dominant feature is the Carina arm. It is traced over a $23 \mathrm{kpc}$ path length by 37 clouds. Spiral arm segments delineating the Perseus and Sagittarius arms are also readily apparent. From the figure, the Carina and Sagittarius features evidently form a continuous spiral arm. The wedge from $/=300^{\circ}$ to $12^{\circ}$, a region for which the data have not yet been fully analyzed for cloud identifications, represents a path length of $\sim 1 \mathrm{kpC}$ along the arm, only slightly larger than the average intercloud spacing of the Carina clouds of $700 \mathrm{pc}$. The dip in the 
continuum and $\mathrm{H} 109 \times$ fluxes between $/=292^{\circ}$ and $305^{\circ}$ noted by Kerr has no counterpart in reduced $C O$ emission. Rather, the I, $V$ and spatial maps show the near side of the arm continuing into the inner Galaxy emission, where it becomes difficult to trace.

A large gap can also be seen between the inner-most clouds in the Carina arm and the clouds in the direction of Cygnus. But while the unanalyzed wedge between the Carina and Sagittarius arms is bridged optically by young clusters (Vogt and Moffat 1975b), giant stars (Humphreys 1976), and weak H II regions (Gerogelin and Georgelin 1976), as well as a tentatively identified molecular cloud (Bronfman, private communication), no similar link can be found between the near side of the Carina arm and Cygnus. The Carina arm would have to spiral outward from $/=300^{\circ}$ to $\sim 85^{\circ}$ to meet Cygnus, making further unlikely any Carina-Cygnus connection.

The linear mass densities of the Carina and Sagittarius arms are the same, also supporting the hypothesis that the two are connected. The total cloud mass in the Carina arm, $58 \times 10^{6} M_{\odot}$, extends over $23 \mathrm{kpc}$, for a linear mass density of $2.5 \times 10^{6} \mathrm{M}_{\odot} \mathrm{PC}^{-1}$; in the Sagittarius arm, $39 \times 10^{6} \mathrm{M}_{\odot}$ extends over $16 \mathrm{kpc}$, again for $2.5 \times 10^{6} \mathrm{M}_{\odot} \mathrm{DC}^{-1}$. By comparison, in the Perseus arm with $9.8 \times 10^{0} \mathrm{M}_{\odot}$ extending over $8 \mathrm{kpc}$, the linear density is much lower, only $1.3 \times 10^{6} \mathrm{M}_{\odot} \mathrm{pc}^{-1}$.

In a plot of galactocentric angle versus the logarithm of galactocentric radius (Fig. V-7b), in which logaritmic spirals will appear as straight lines, the continuity between the Carina and Sagittarius clouds is even clearer. All of the Carina and Sagittarius clouds are seen to fall close to a $10^{\circ}$ spiral with a tangent at $J=281^{\circ}$ (the best-fit inclination is $\left.9.75^{\circ}\right)$. Figure $V-8$, which shows the clouds in the plane of the Galaxy with a 10 logarithmic spiral superimposed, illustrates the conclusion well: the 
Carina and the Sagittarius arms form a single spiral arm nearly $40 \mathrm{kpc}$ long wrapping at least $2 / 3$ of the way around the Galaxy.

D. Notes on Individual Clouds

Table $V-2$ is a catalog of all the clouds identified between $/=270^{\circ}$ and $300^{\circ}$, as well as all the clouds in the far side of the Carina arm beyond $l=300^{\circ}$ identified in the Co survey of L. Bronfman (1985). The catalog is ordered in increasing longitude and includes, with the coordinates $(l, b, v)$, distances, velocity widths, effective radil, and $\mathrm{CO}$ and virial masses of the clouds.

All the clouds identifled between $/=270^{\circ}$ and $300^{\circ}$ are described briefly in the notes below, which summarize cloud identifications, distance determinations, and identification of associated objects. For a description of the Carina arm clouds beyond $l=300^{\circ}$, see Bronfman (1985).

1) $l=270.9^{\circ}, \mathrm{b}=-0.5^{\circ}, v=52.5 \mathrm{~km} \mathrm{~s}^{-1}$

There is no kinematic distance ambiguity for this cloud, its velocity placing it well beyond the solar circle. The cloud does not appear to have any associated objects. Its longitude, about $10^{\circ}$ ahead of the Carina tangent (at $/=280^{\circ}$ ), and its distance indicate that the cloud does not lie in the Carina arm. It may be related to the $H$ I feature in the outer Galaxy noted in Figures IV-6 and IV-8. 
2) $l=279.9, b=-1.6^{\circ}, v=35.1 \mathrm{~km} \mathrm{~s}^{-1}$

This very weak feature has no associated objects. Its kinematic distance, beyond the solar circle, is unambiguous.

3) $l=281.4, b=-1.1^{\bullet}, v=-5.1 \mathrm{~km} \mathrm{~s}^{-1}$

In the heart of the tangent region of the Carina arm, this feature is probably a blend of more than one cloud. The spatial maps of this region (Figs. IV-1b and $V-1 \mathrm{~b}$ ) and the integrated $/, V$ map (Fig. III-7) exhibit several peaks between $/=280^{\circ}$ and $283^{\circ}$. Spatial maps in adjacent velocity windows (Figs. $V-9 a-j$ ) show a fair degree of continuity in the emission between $I=280^{\circ}$ and $283^{\circ}$ as we progress from about -18 to $8 \mathrm{~km} \mathrm{~s}^{-1}$, although the centroid of the emission shifts from about $J=282.7^{\circ}$, $b=-1.7^{\circ}$ to $/=281.6^{\circ}, b=-0.7^{\circ}$. Because of the confused nature of the emission from $I=280^{\circ}$ to $283^{\circ}$, no attempt was made to subdivide this emission further (with two exceptions noted below), any such division being arbitrary.

This cloud complex was placed at $3.2 \mathrm{kpc}$, based on the optical distance to G282.2-2.0 (Bigay et al 1970), an assoclated $\mathrm{H}$ II region. With an $\mathrm{H \alpha}$ velocity of $-12.2 \mathrm{~km} \mathrm{~s}^{-1}$ (Bigay et an.), G282.2-2.0 sits blueward (in velocity) of a CO peak in this direction.

Three other $H$ II regions between $/=280^{\circ}$ and $283^{\circ}$ may also be associated with various components of this cloud; they are: RCW 45, RCW 46, and RCW 47. RCW $45\left(/=282.2^{\circ}, \mathrm{b}=-0.1^{\circ}\right)$ sits at the edge of the CO peak at $/=282.3^{\circ}, \mathrm{b}=-0.5^{\circ}$; its $\mathrm{H} \times$ velocity, $-9.8 \mathrm{~km} \mathrm{~s}^{-1}$ (Bigay et $a h$, places it to the blue of this peak in velocity. No optical distance is 
available for RCW 45. RCW $47\left(/=283.0^{\circ}, b=-2.7^{\circ}\right)$ is situated at the low latitude edge of the cloud where the emission is relatively weak; its $\mathrm{H} \alpha$ velocity of $-11.6 \mathrm{~km} \mathrm{~s}^{-1}$ (Bigay et al) shows that this region, too, is blueward of the the cloud's velocity. Georgelin (1975) gives a distance of $2.7 \mathrm{kpC}$ for $\mathrm{RCW} 47$. (The $l, \mathrm{~V}$ maps at $\mathrm{b}=-2.0^{\circ}$ and $-2.75^{\circ}$ indicate that the assoclation between the cloud and G282.2-2.0 is probably more certain than the association between the cloud and RCW 47; for this reason the distance to G282.2-2.0 was used as the cloud's distance.)

The other $H$ II region, $\mathrm{RCW} 46\left(1=282.4^{\circ}, \mathrm{b}=-1.3^{\circ}\right)$, has an $\mathrm{H} \alpha$ velocity of $-10.6 \mathrm{~km} \mathrm{~s}^{-1}$ (Georgelin 1975) and sits between two CO peaks, one, at $l=281.7^{\circ}, \mathrm{b}=-1.5^{\circ}$, near the center of the tangent region under discussion, the other at $/=282.75^{\circ}, \mathrm{D}=-1.25^{\circ}$. In the integrated $\mathrm{I}, \mathrm{V}$ map and the $I, V$ maps at each latitude between $b=-1.5^{\circ}$ and $0^{\circ}$ (Fig. B-1), the higher longitude peak appears as a distinct feature and was cataloged as a separate cloud, *6 in the list. It is not clear whether RCW 46 is associated with cloud $* 3$ or $* 6$.

The other exception to the decision not to subdivide the tangent region emission between $I=280^{\circ}$ and $283^{\circ}$ is cloud $* 5$, which blends with $* 3$ in the integrated $I V$ map giving the impression of a continuous feature. The spatial maps of the near side of the arm (Figs. IV-3a and $V-1 a$ ) clearly show * 5 as a distinct cloud at $I=282.9^{\circ}, \mathrm{b}=1.3^{\circ}$, with little spillover into the tangent region.

After excluding the emission from *5 and $* 6$, the remaining emission for $* 3$ yields a co mass of $2.3 \times 10^{6} \mathrm{M}_{\odot}$. Alnough some local emission is probably mixed in, the derived mass is not atypical of other large clouds in the Galaxy. 
4) $l=282.0^{\circ}, b=-0.8^{\circ}, v=17.3 \mathrm{~km} \mathrm{~s}^{-1}$

This cloud is apparently associated with the H109 $\alpha$ recombination line source 282.0-1.2 at a velocity of $22.4 \mathrm{~km} \mathrm{~s}^{-1}$ (Wilson et al 1970). Bigay et al (1970) report an $H \alpha$ velocity of $21.6 \mathrm{~km} \mathrm{~s}^{-1}$ for the same source, but no optical distance determination is available. The cloud was therefore placed at its kinematic distance, which has no ambiguity since the velocity is positive.

5) $l=282.9^{\circ}, \mathrm{b}=1.3^{\circ}, \mathrm{v}=-18.7 \mathrm{~km} \mathrm{~s}^{-1}$

No known $\mathrm{H}$ II regions, continuum sources, $\mathrm{OH}$ or $\mathrm{H}_{2} \mathrm{CO}$ emission or absorption features, or apparent optical obscuration are seen toward this cloud. It has not previously been detected in $\mathrm{CO}$. Its velocity places it at the tangent point.

6) $I=282.9^{\circ}, b=-0.7^{\bullet}, v=-4.5 \mathrm{~km} \mathrm{~s}^{-1}$

This cloud appears as a distinct feature in the $I, V$ map at $I=282.9^{\circ}$, $v=-5 \mathrm{~km} \mathrm{~s}^{-1}$. The emission near this longitude and latitude varies continuously in the spatial maps in adjacent velocity windows (Figs. $V-9 a-j$ ) between -10 and $-2 \mathrm{~km} \mathrm{~s}^{-1}$; the strongest emission appears in the $-5 \mathrm{~km} \mathrm{~s}^{-1}$ map at $/ \approx 283^{\circ}, b \approx-1^{\circ}$. It was therefore considered to be separate from cloud *3. Although possibly associated with RCW 46 at $1.8 \mathrm{kpc}$ (Georgelin 1975), a connection is not completely convincing. The cloud was assigned the far kinematic distance on the basis of the radius- 
line width relation (Dame et al. 1985), which clearly favors the far distance.

7) $l=283.8^{\circ}, b=0.0^{\circ}, v=-5.4 \mathrm{~km} \mathrm{~s}^{-1}$

This cloud is probably associated with RCW 48 and RCW 49, placing it at about 3 to $5 \mathrm{kpc}$. RCW 48 , at $l=283.5^{\circ}, \mathrm{b}=-1.0^{\circ}$, is crescent-shaped, both in the optical and in the radio continuum, indicating that the concave side of the optical crescent is not due to obscuration but to an obstruction -- a molecular cloud -- adjacent to the crescent's inner edge. There is little other evidence, such as a bright co peak, of direct contact between the lonized gas on the concave side of the crescent and the molecular cloud. The mean cloud velocity of $-5.4 \mathrm{~km} \mathrm{~s}^{-1}$ is close to the mean $\mathrm{H} \alpha$ velocity for RCW 48 of $-8.2 \mathrm{~km} \mathrm{~s}^{-1}$ (Bigay et al 1972), and the angular displacement between the nebula and the cloud's edge is small, leading to the conclusion that the two are associated.

RCW 49 consists of an optically bright central portion coincident with a strong continuum source at $1=284.3^{\circ}, \mathrm{b}=-0.3^{\circ}$, and a reglon of more diffuse $\mathrm{H} \alpha$ emission extending roughly half a degree about the center. The center of RCW 49 lies midway between two Co peaks in the cloud, and the mean $\mathrm{H} \alpha$ velocity of $-7.8 \mathrm{~km} \mathrm{~s}^{-1}$ (Bigay et ah) compares well with the mean cloud velocity, so RCW 49 also is believed to be associated with the cloud. The H109 $\alpha$ recombination line velocity of RCW 49 measured only toward the continuum source is $-0.7 \mathrm{~km} \mathrm{~s}^{-1}$ (Wilson et an), a value not too discrepant from the $\mathrm{CO}$ and $\mathrm{H} \alpha$ velocities, considering these are mean velocities with spreads of about $\pm 5 \mathrm{~km} \mathrm{~s}^{-1}$. 
Bigay et al suggest that RCW 48 and RCW 49 form a single $H$ II region complex, citing as evidence both the similar velocities, and at about the same velocity, a third, more diffuse $\mathrm{H} \alpha$ emission region (designated 283.9-0.6) that links the two. RCW 48 is at about $3 \mathrm{kpc}$, based on the the distances to two exciting stars (Georgelin 1975). RCW 49 lies between $3 \mathrm{kpC}$, based on the distances to two exciting stars (Georgelin 1975), and $5 \mathrm{kpc}$, based on the distance to the apparently associated cluster Wd2 (Moffat and Vogt 1975). Here a distance of $4 \mathrm{kpc}$ for the cloud is adopted. Although this distance could be off by a kiloparsec, such an error has little effect on the large scale picture of the Carina arm since this cloud is certainly near the tangent of the arm.

8) $l=284.5^{\circ}, b=-0.2^{\circ}, v=11.6 \mathrm{~km} \mathrm{~s}^{-1}$

This cloud lies beyond the solar circle, so its kinematic distance is unambiguous. The radio continuum source G282.0-1.0 sits near the edge of one of the cloud's two CO peaks; the velocity of the source from its $\mathrm{HIO} \alpha \alpha$ recombination line is $4.5 \mathrm{~km} \mathrm{~s}^{-1}$ (Wilson et al 1970), placing it near the edge of the cloud in velocity space; the radio object is very likely to be associated with the molecular cloud. The weak $H \alpha$ source $H \quad 8$ is also coincident with the radio $H \|$ region, but its association with it, while probable, is not certain; no optical distance is available to $\mathrm{H} 8$.

9) $l=285.3^{\circ}, \mathrm{b}=0.0^{\circ}, \mathrm{v}=0.0 \mathrm{~km} \mathrm{~s}^{-1}$

The velocity of this cloud puts it on the solar circle, elther local or quite distant ( $5.3 \mathrm{kpc}$ for this longitude). The far kinematic distance is 
chosen here not only because this compact cloud lies at $b=0^{\circ}$, but, more important, because $\mathrm{H}$ I absorption of a bright continuum source coincident with the cloud is clearly due to nonlocal, intervening gas (Goss et al, 1972). The small $H \propto$ source $H \quad 18$ is the apparent optical counterpart of the radio H II region.

10) $l=286.4^{\circ}, \mathrm{b}=-0.3^{\circ}, \mathrm{v}=14.3 \mathrm{~km} \mathrm{~s}^{-1}$

This cloud was assigned a kinematic distance that places it beyond the solar circle. It has no associated radio or optical objects.

11) $l=287.5^{\circ}, b=-0.5^{\circ}, v=-19.4 \mathrm{~km} \mathrm{~s}^{-1}$

This is the large cloud complex associated with the $\eta$ Carinae Nebula, NGC 3324, NGC 3293, and IC 2581. Its distance was taken to be $2.7 \mathrm{kPC}$, the distance to the associated objects. A detalled discussion of this cloud is presented in section $B$ of this chapter.

12) $I=288.6^{\circ}, b=1.5^{\circ}, v=-21.9 \mathrm{~km} \mathrm{~s}^{-1}$

The kinematic distance to this source places it at the subcentral point. Although the fllamentary $\mathrm{H} \alpha$ source RCW $54 \mathrm{~b}$ lies at the edge of this cloud, no convincing association can be deduced because no information regarding velocity is available for the $\mathrm{H} \alpha$ source. No continuum, $\mathrm{OH}$ or $\mathrm{H}_{2} \mathrm{CO}$ absorption or emission sources are observed toward this cloud. 
13) $l=289.3^{\circ}, b=-0.6^{\circ}, v=22.4 \mathrm{~km} \mathrm{~s}^{-1}$

This is one of two clouds in the far side of the Carina arm that can be assigned an optical distance. The associated $H$ II region, RCW $54 a$ $\left(I=289.7^{\circ}, \mathrm{b}=-1.3^{\circ}\right)$, is situated near the edge of one of the cloud's two CO peaks. The $\mathrm{H} 109 \alpha$ recombination line velocity (Wilson et al 1970) and the $H_{\alpha}$ velocity (Georgelin and Georgelin 1970b) of RCW $54 a$ are 21.9 and $20.7 \mathrm{~km} \mathrm{~s}^{-1}$, respectively, placing the $H \|$ region slightly to the blue of the associated CO peak in velocity space, and implying that ionized gas is streaming away from the molecular cloud that lies partly behind the $\mathrm{H} \mathrm{II}$ region. That RCW $54 a$ is observed optically is consistent with the cloud forming a backdrop to the bright gas.

Another $H$ II region, G289.1-0.4, also associated with the molecular cloud, is seen directly toward the other CO peak, its $\mathrm{H} 109 \alpha$ recombination line (Wilson et an) coinciding with the co peak in velocity, as well. G289.1-0.4 was first identified as nonthermal (Milne et al 1969), but was subsequently determined by Shaver and Goss (1970) to be thermal.

Two other objects are seen close to the smaller co peak. One is the supernova remnant MSHI 1-6/A at $/=290.1^{\circ}, b=-0.8^{\circ}$, which is extremely bright in the radio continuum (Shaver and Goss 1970) and exhibits a faint optical shell (Kirshner and Winkler 1979, hereafter KW; Elliot and Malin 1979, hereafter EM). Although the close spatial coincidence of MSH11-6/A with the cloud suggests an association between them, the supernova's distance, between 3.2 and $5.8 \mathrm{kpc}(\mathrm{KW}$; EM) argues against such a connection. The distance to MSH 1-6/A, however, may be substantially greater than $5.8 \mathrm{kpC}(\mathrm{KW}$; EM), so an association between the molecular cloud and the SNR cannot be ruled out. The other object, the $H \|$ II region 
G289.9-0.8, has an H109a $\alpha$ velocity of $5 \mathrm{~km} \mathrm{~s}^{-1}$ (Dickel 1973) making its assocation with the cloud unlikely. Given the evidence available, no strong arguments can be made regarding the possible relationships between these two objects and the molecular cloud.

14) $l=290.2^{\circ}, \mathrm{b}=-0.2^{\circ}, \mathrm{v}=-1.4 \mathrm{~km} \mathrm{~s}^{-1}$

The far kinematic distance was chosen for this cloud based on its small angular size, its proximity to the galactic plane, and the radius-line width relation which favors the far distance. No optical obscuration is apparent toward this cloud, and any possible connection with the optical H II region $G 37$ (RCW 54C), near the cloud's edge, is ruled out by the $H \alpha$ velocity of about $-22 \mathrm{~km} \mathrm{~s}^{-1}$ for $G 37$ (Georgelin and Georgelin 1970b).

15) $I=290.6^{\circ}, b=-0.2^{\circ}, v=15.4 \mathrm{~km} \mathrm{~s}^{-1}$

The composite spectrum (the sum of all the spectra over the face of a cloud) of this feature shows two velocity components at about 9 and $18 \mathrm{~km} \mathrm{~s}^{-1}$. From the I, $\mathrm{v}$ and spatial maps, though, the feature would appear to be a single cloud. Individual spectra in the sum exhibit wide, single lines (near the velocity of the feature) of fering little evidence for a second peak other than a mild asymmetry, and so this feature was considered to be a single cloud. The supernova remnant MSH11-62 at $I=291.0^{\circ}, \mathrm{b}=-0.1^{\circ}$, near the edge of the cloud, is at an estimated distance of $9.9 \mathrm{kpc}$ (Milne 1979), in good agreement with the kinematic distance to the molecular cloud of $8.7 \mathrm{kpc}$, although this does not confirm an association between the cloud and the SNR. The kinematic distance is adopted for the cloud. 
Another weak, nonthermal continuum source, G290.4-0.9, of fset slightly from the cloud's edge, is probably extragalactic (Shaver and Goss 1970).

16) $I=291.4^{\circ}, b=-0.2^{\circ}, v=-7.0 \mathrm{~km} \mathrm{~s}^{-1}$

The coincidence of this feature with a dark dust cloud on the ESO J plate in its direction indicates that the CO emission is local. On an opacity scale of 1-6 with 6 corresponding to the apparently darkest, Sandqvist (1977) ranked the dust cloud (number 127 in his Table 1) class 5.

Formaldehyde absorption observed in the direction of the dust cloud has a velocity of $-6.5 \mathrm{~km} \mathrm{~s}^{-1}$ (Goss et al 1980), further supporting the connection between the molecular cloud and the obscuring dust. The virtual absence of stars near the dust cloud's core implies that the dust, and therefore the $c 0$, is quite local.

17) $l=291.6^{\circ}, b=-0.4^{\circ}, v=14.7 \mathrm{~km} \mathrm{~s}^{-1}$

This cloud is associated with the giant $H \|$ region NGC 3603 seen directly toward the CO peak and just at the blue end of the cloud in velocity. The optical distance of $7.2 \mathrm{kpc}$ (van den Bergh 1978) was assigned. This cloud coincides spatially with cloud $* 18$ but has a velocity $14 \mathrm{~km} \mathrm{~s}^{-1}$ higher. A composite spectrum of the region containing clouds $* 17$ and $* 18$ gives the impression that these two may be different velocity components of a single cloud complex, but the virial mass of the two-cloud system is about 10 times the sum of the co masses of each component, so clouds $\approx 17$ and $* 18$ were considered to be two separate clouds. 
18) $I=291.6^{\circ}, b=-0.5^{\circ}, v=28.8 \mathrm{~km} \mathrm{~s}^{-1}$

This cloud was has a kinematic distance which places it beyond the solar circle. It is associated with the $H \quad I$ region $H 58$ at $I=291.9^{\circ}$, $D=-0.7^{\circ}$. H 58 has an $H 109 \alpha$ recombination line velocity of $25.5 \mathrm{~km} \mathrm{~s}^{-1}$ (Wilson et al 1970) and an $\mathrm{H} \alpha$ velocity of $22.3 \mathrm{~km} \mathrm{~s}^{-1}$ (Georgelin and Georgelin 1976). No optical distance is available for $H 58$.

The possibility that this cloud and $* 17$ might form a single complex is discussed in the notes to $\approx 17$, where it is concluded that clouds $* 17$ and - 18 are two separate clouds.

19) $l=292.6^{\circ}, \mathrm{b}=-0.3^{\circ}, \mathrm{v}=4.0 \mathrm{~km} \mathrm{~s}^{-1}$

This weak feature has no counterpart at any other wavelength. It was placed at the far kinematic distance based on the radius-line width relation.

20) $l=293.3^{\circ}, b=-1.4^{\circ}, v=-25.1 \mathrm{~km} \mathrm{~s}^{-1}$

This cloud complex was placed at $2.4 \mathrm{kpc}$ based on its association with the bright $H$ II region RWC 62 (also referred to as IC 2944, after the young cluster containing the ionizing stars), one of four associated $H$ II regions. RCW 62 has an $\mathrm{H} 109 \alpha$ velocity of $-20.9 \mathrm{~km} \mathrm{~s}^{-1}$ (WIlson et al. 1970) and lies at one end of the cloud, near $/=295^{\circ}$. Based on similar distances and comparisons of the $H \alpha$ and radio continuum morphology, Georgelin and Georgelin (1976) considered RCW 62 with RCW 60 and RCW 61 as a single $H \|$ region complex. The average $H \alpha$ velocity of these three is $-27.6 \mathrm{~km} \mathrm{~s}^{-1}$, 
in good agreement with both the radio recombination line and the molecular velocities. The common molecular cloud provides further support for the connection of these three regions. Of the three, the best established distance is to RCW 62 (IC 2944) and, following Georgelin and Georgelin (1976), its distance is adopted here for the molecular cloud. The fourth $\mathrm{H} \mathrm{II}$ region, NGC 3576, has an $\mathrm{H109} \alpha$ velocity of $-23 \mathrm{~km} \mathrm{~s}^{-1}$ (Wilson et al. 1970), and is situated immediately next to a strong peak in the co emission near $l=291.5^{\circ}$, at the other end of the cloud. Various published optical distances to NGC 3576 place it between 2.5 and $3.3 \mathrm{kpC}$ (Georgelin and Georgelin 1970b, 1976; Humphreys 1978), the spread due in part to uncertain identification of the exciting stars (Georgelin, private communication). Within the distance errors and given the apparent connection by a common cloud, NGC 3576 may be considered to be at the same distance as RCW 60, RCW 61, and RCW 62. At $2.4 \mathrm{kpc}$, the cloud has a projected length of about $160 \mathrm{pc}\left(R_{\text {eff }}=77 \mathrm{pC}\right)$ and a mass of $7 \times 10^{5} \mathrm{M}_{\odot}$, both values typical of other large clouds in the Galaxy, so the assumption that the four $\mathrm{H} \| \mathrm{I}$ regions are the offspring a single large cloud is reasonable.

An alternative interpretation is that we are viewing two clouds in projection, one associated with RCW 60, RCW 61, and RCW 62 at $2.4 \mathrm{kpC}$, and the other assoclated with NGC 3576 at $3.3 \mathrm{kPC}$. Such a picture is suggested by: 1) the different morphological appearance of the two possible clouds (see to Fig. $V-1 a$ ), one very bright and compact (NGC 3576), the other weaker and more diffuse (RCW 60, RCW 61, RCW 62); 2) the relatively weak link bewteen the two; and 3 ) a hole in the emission centered approximately between the clouds near $l=292.5^{\circ}, \mathrm{b}=-1.0^{\circ}$. There are no obvious explanations for the emission gap, such as a bright $H \|$ 
region or supernova remnant. (A similar hole centered near $/=294.5^{\circ}$, $b=-2.0^{\circ}$ is partially filled by the ionized gas of RCW 62 , indicating that the molecular gas in it may have been cleared out by the expanding $\mathrm{H}$ II region.) However, even if $3.3 \mathrm{kPC}$ is accepted as the distance to NGC 3576 , a distance error of no greater $15 \%$ to this object and to RCW 62 places the lower limit on their separation of $50 \mathrm{pc}$, well within the typical size for large cloud complexes. In addition, the velocities of the "two" clouds, measured from a composite spectrum of each, are nearly identical:

$-25.1 \mathrm{~km} \mathrm{~s}^{-1}$ versus $-24.9 \mathrm{~km} \mathrm{~s}^{-1}$; and spatial maps of the region in adjacent, narrow velocity windows (Figs. $v-10 a-h$ ) show that the strongest components fade in, peak, and fade out at the same velocities. The "one cloud complex" interpretation is therefore adopted here.

Although in Cohen et al (1985a) we chose the "two cloud" picture ( $12^{\text {th }}$ and $16^{\text {th }}$ entries in Table 1), it should be emphasized that our conclusions about the large-scale structure of the Carina arm, including its connection with the rest of the Galaxy, remain essentially the same, regardless of which interpretation is accepted for this cloud.

21) $l=294.0^{\circ}, b=-0.9^{\circ}, v=32.3 \mathrm{~km} \mathrm{~s}^{-1}$

This cloud was placed at its kinematic distance. It does not appear to have any assoclated objects, except possibly the supernova remnant G293.8+0.6 at $9.6 \mathrm{kpC}$ (Milne 1979). The separation between the cloud and the SNR, assuming the cloud's distance of $10.7 \mathrm{kpC}$, is about $90 \mathrm{pC}$, making any connection between the two questionable. 
22) $I=294.4^{\circ}, b=-0.7^{\circ}, v=-2.7 \mathrm{~km} \mathrm{~s}^{-1}$

This cloud does not appear to have any associated optical or radio objects. Its proximity to the galactic plane and radius-line width relation imply it is at the far kinematic distance.

23) $I=295.1^{\circ}, b=-0.8^{\circ}, v=26.0 \mathrm{~km} \mathrm{~s}^{-1}$

With multiple velocity components, this feature has a ragged appearance in the integrated $l, V$ map, making its classification as a single molecular cloud (or cloud complex) suspect. Four reasons can be suggested, however, to support its being one object. First, with a distinct peak at $l=291.25^{\circ}, b=-0.75^{\circ}$, its spatial appearance resembles that of many of the other clouds in our survey. Second, the individual $I V$ maps at $b=0.625^{\circ}$ and $-0.75^{\circ}$ (Fig. $B-1$ ) show that all the emission near $I=295.25^{\circ}$, from $v \approx 20$ to $\sim 40 \mathrm{~km} \mathrm{~s}^{-1}$, is connected. It seems unlikely that within such a narrow range of longitude and latitude a chance coincidence of clouds would be spread out over these velocities. A more probable explanation for the wide velocity extent is the occurence of a violent event, such as a supernova, in a single cloud. Third, the $\mathrm{H} \| \mathrm{I}$ region G295.2-0.6 is seen almost directly toward the cloud's peak. The $H 109 \alpha$ recombination line velocity of this source is $51.1 \mathrm{~km} \mathrm{~s}^{-1}$ (Wilson et al); on the $I V$ map at $b=-0.625^{\circ}$ this $H \|$ region lies about $15 \mathrm{~km} \mathrm{~s}^{-1}$ beyond the highest velocity CO peak in the cloud. Although such a large velocity discrepancy would usually be considered evidence against an association between a cloud and an $\mathrm{H} / 1$ region, in this case the velocity displacement may be consistent with the cloud's already large velocity width. If the large 
velocity extent of the cloud is indeed related to energetic phenomena, then perhaps disagreement between the $H$ II region's velocity and the cloud's velocity is not surprising. Fourth, at least one other example of such a wide velocity molecular cloud complex exists: the cloud associated with the supernova remnant $W 44$ in the first quadrant. The $W 44$ molecular cloud has been shown to be a single object accounting for the emission over nearly $40 \mathrm{~km} \mathrm{~s}^{-1}$ within a $2^{\circ}$ wide longitude range (Dame 1983 ).

In view of these considerations, this feature is considered to be a single cloud with a kinematic distance of $10.5 \mathrm{kpc}$.

24) $I=297.4^{\circ}, b=-0.5^{\circ}, v=21.5 \mathrm{~km} \mathrm{~s}^{-1}$

It is difficult to establish a clear separation between this cloud and *26. If all the emission between $/=297^{\circ}$ and $299.75^{\circ}$ represented a single cloud complex, its projected length would be unreasonably large: about 500 DC at an assumed (kinematic) distance of $11 \mathrm{kpc}$. From the integrated $/ \mathrm{V}$ and spatial maps of this region, $l=298^{\circ}$ would appear a reasonable dividing line between this cloud and cloud $* 26$. The radio continuum (Shaver and Goss 1970a), which exhibits a cluster of sources between $l=298^{\circ}$ and $299^{\circ}$ then drops off just below $298^{\circ}$, lending support to this subdivision. Evidence for the physical connection of the emission between $I=297^{\circ}$ and $298^{\circ}$ at velocities greater than about $10 \mathrm{~km} \mathrm{~s}^{-1}$ can be found by comparing the spatial map (Fig. IV-3C) and the IV maps (Fig. B-1) between $b=-1.0^{\circ}$ and $0.25^{\circ}$. Two components in the spatial map at $l=297.25^{\circ}, b=-1.0^{\circ}$ and $l=297.375^{\circ}, b=-0.125^{\circ}$ appear as distinct features at $30 \mathrm{~km} \mathrm{~s}^{-1}$ and $17 \mathrm{~km} \mathrm{~s}^{-1}$, respectively, in the $I V$ maps at those latitudes. At intervening latitudes the $l, V$ maps show these features joined by a bridge of wide- 
velocity, low-level emission. The dividing line between this cloud and 26 is taken to be $/=298^{\circ}$.

This cloud is placed according to its kinematic distance. The supernova remnant G296.8-0.2 at $8.7 \mathrm{kpc}$ (Milne 1979) sits near its edge and may well be associated. At the cloud's assumed distance of $11 \mathrm{kpc}$, the projected separation between it and the SNR is about $50 \mathrm{pc}$. No other associated objects are apparent.

25) $l=298.6^{\circ}, b=0.1^{\circ}, v=-34.9 \mathrm{~km} \mathrm{~s}^{-1}$

This feature may be a blend of more than one cloud, but there are no associated objects that ald in the placement or identification of possibly separate components. In the absence of further evidence the kinematic distance is adopted, placing the cloud at the subcentral point. This distance should be regarded as highly uncertain, given the cloud's location along the high velocity ridge. The adopted distance makes this the only major cloud between $I=280^{\circ}$ and $300^{\circ}$ that appears not to fit into the Carina arm. Two obvious explanations are: 1) the cloud represents part of a real feature interior to the Carina arm, perhaps a spur (such a picture of the inner edge of the Carina arm has been discussed by Humphreys (1976)); or 2) the adopted distance to the cloud is too big, and the cloud actually resides in the near side of the Carina arm. With regard to the latter possibility, two other major clouds in the near side of the Carina arm, $* 11$ and $* 20$, have been assigned optical distances quite a bit closer than their implied kinematic distances on the subcentral locus. No attempt has been made here to "correct" for streaming motions when computing kinematic distances, although has Humphreys (1970) reported evidence for such motions in 
supergiants in the Carina arm. If the velocity residuals for the stars are used to adjust the observed velocities of clouds $=11$ and $=20$, their "corrected" kinematic distances agree with their optical distances. A similar adjustment could be applied to cloud $\approx 25$, but seems unwarrented without a more systematic comparison of stellar and molecular cloud velocities.

26) $l=298.8^{\circ}, \mathrm{b}=-0.2^{\circ}, v=24.9 \mathrm{~km} \mathrm{~s}^{-1}$

The three peaks between $/=298^{\circ}$ and $300^{\circ}$ in the far side spatlal map (Figs. IV-3C and $V-1 C$ ) are grouped together and assigned a kinematic distance of $11.9 \mathrm{kpc}$. (The estabilishment of $I=298^{\circ}$ as the dividing line between this cloud and $* 24$ is discussed in the notes to $* 24$.) The classification of all this emission as a single complex was suggested in part by the clustering of distant $H \|$ regions and supernova remnants between $I=298^{\circ}$ and $300^{\circ}$. A feature at $I=299.5^{\circ}, v \approx 27 \mathrm{~km} \mathrm{~s}^{-1}$ in the I, map was included because its kinematic distance is nearly the same as that of the rest of the complex. H109 $\alpha$ recombination line velocities of $30.6 \mathrm{~km} \mathrm{~s}^{-1}$ and $24.2 \mathrm{~km} \mathrm{~s}^{-1}$ for $6298.2-0.3$ and G298.9-0.4 (Wilson et al. 1970), respectively, show that the $H I I$ reglons are closely associated with cloud components at the corresponding spatial and velocity coordinates. 6298.2-0.3 is apparently an extremely bright $H$ II region. Infrared continuum observations of this object yield the highest measured $1-20 \mu$ luminosity for any galactic $H \|$ II region (Frogel and Persson 1974), and from infrared emission line measurements Lacy et al. (1982) estimate that four $05.5 \mathrm{~V}$ stars are required to provide the lonizing radiation for this $\mathrm{H} \mathrm{II}$ region. A third $H 109 \alpha$ source, $6298.8-0.3\left(V=25.0 \mathrm{~km} \mathrm{~s}^{-1}\right)$, is also 
associated with the cloud, although this $H$ II region may be part of G298.9-0.4 (Wilson et al. 1970).

Three supernova remnants are also seen in the direction of this cloud complex: G298.5-0.3, 298.6+0.0, and 299.0+0.2 at distances 16.1, 17.2, and $10.6 \mathrm{kPC}$, respectively (Milne 1979). Assuming distance errors of $30 \%$ to these SNRs, it is possible that all three are related to the cloud; given the spatial coincidence with a cloud that appears to be the site of ongoing, massive star formation, the association of at least one of these SNRs with the molecular cloud seems quite likely.

27) $I=299.4^{\circ}, \mathrm{b}=-0.1^{\circ}, v=-6.0 \mathrm{~km} \mathrm{~s}^{-1}$

The far kinematic distance for this cloud is favored by the radius-line width relation. A closer distance (but not the near kinematic distance) of 2.3 to $3.5 \mathrm{kpC}$ is possible if a small $\mathrm{H} \alpha$ source at $l=299.9^{\circ}, \mathrm{b}=0.4^{\circ}$ is associated with the cloud. Georgelin and Georgelin (1970) give an optical distance of $2.3 \mathrm{kpC}$ and an $\mathrm{H} \alpha$ velocity of about $-10 \mathrm{~km} \mathrm{~s}^{-1}$ for the source, but subsequently (Georgelin 1975) the distance was revised to $3.5 \mathrm{kpc}$ and the source excluded from a list of measured $H \alpha$ velocities (Table $V$ in Georgelin 1975). Since the evidence for an association of the $H_{\alpha}$ source and the molecular cloud is not very compelling, the far kinematic distance is chosen for the cloud. 
The distribution of molecular clouds in the Carina arm was determined by surveying the $J=1 \rightarrow 0$ line of $C O$ in the Southern Milky Way with the Columbia University Millimeter-Wave telescope at Cerro Tololo. Ranging from $I=270^{\circ}$ to $300^{\circ}$, and covering more than $60 \mathrm{deg}^{2}$ about the galactic plane at $0.125^{\circ}$ resolution and $300 \mathrm{deg}^{2}$ at $0.5^{\circ}$ resolution, the survey provides the first large-scale CO observations of this region, one of the most interesting in the Milky Way. Various representations of the data were presented, including latitude-integrated $\$ V$ diagrams, I, $\mathrm{V}$ maps at individual latitudes, velocity-integrated spatial maps, and $b, v$ maps at each observed longitude.

The Carina arm stands out with the loop-like signature of a spiral arm in the $I, V$ diagram. Its abrupt tangent near $I=280^{\circ}$ at zero velocity connects with the near side of the arm, at negative velocities (within the solar circle), and the far side, at positive velocities (beyond the solar circle). Extending toward higher longitudes, the near and far sides become separated by as much as $60 \mathrm{~km} \mathrm{~s}^{-1}$, with only weak or local emission in the velocity gap between them. When the emission is integrated over velocity and latitude a 13 -fold jump in intensity is seen as $/$ crosses $280^{\circ}$ from below, indicating an arm-interarm contrast of at least 13 to 1 . The near side, tangent region, and far side of the arm were viewed in the plane of the sky by considering the emission within the velocity ranges of these kinematically defined features. In the resultant spatial maps, the thinning of the CO layer with kinematic distance, which is an expected projection effect, supported the kinematic interpretation of the data. 
At galactocentric distances beyond the solar circle the $\mathrm{CO}$ and $\mathrm{H} \mathrm{I}$ are well-correlated in both space and velocity, tracing the same large-scale segment of the arm and exhibiting coincident concentrations of emission along its length. Within the solar circle, the $\mathrm{CO}$ and $\mathrm{H}$ I define similar terminal velocity curves, but the near side of the arm is more difficult to discern as a distinct feature in the $\mathrm{H} I$ than in the $\mathrm{CO}$. The relative emptiness of the interarm region in the $C O$, $V$ diagram likewise has only a weak counterpart in the $\mathrm{H}$ I I, V dlagram. In general, the Carina arm loop, an obvious feature in the CO,$V$ diagram, is not outstanding in the corresponding $\mathrm{H} I$ map. The absence of apparent continuity between the near and far sides of the $21-\mathrm{cm}$ arm may partly account for the different interpretations by Kerr (1970) and Weaver (1970) of the Carina arm's connection with the rest of the Galaxy.

The radial dependence in the outer Galaxy of the average co midplane, molecular layer thickness, and $\mathrm{H}_{2}$ surface density were determined by averaging the $\mathrm{H}_{2}$ density at each observed position with $\mathrm{R}>\mathrm{R}_{0}$ over longitude. There being no kinematic distance ambiguity for emission arising at points beyond $R_{0}$, a unique distance, $z$, from the galactic plane could be assigned to all such points. For comparison with the $H I$, a parallel analysis of the recent Parkes $18 \mathrm{~m} 21-\mathrm{cm}$ survey data was carried out. The co midplane bends downward increasingly from the $b=0^{\circ}$ plane with distance from the galactic center, dropping from $z=-48$ to $-167 \mathrm{pc}$ between $R=10.5$ and $12.5 \mathrm{kpC}$. A similar warp has been recognized in $\mathrm{H}$ l ever since the earliest $21-\mathrm{cm}$ surveys of the southern galactic plane, and the treatment here of the Parkes data shows the $\mathrm{H} I$ midplane to be within about $25 \mathrm{pc}$ of the molecular midplane at all radii considered. Again, the half-thickness of the CO layer, expanding from 112 to 182 pc between 10.5 
and $12.5 \mathrm{kpC}$ from the galactic center, mimics the long established flaring or the neutral hydrogen layer, although the scale height of the $\mathrm{H} I$ is about twice as great at that of the $\mathrm{H}_{2}$ over the same range of radii. Three or four very large molecular clouds in the CO survey that lie kinematically between 11 and $12 \mathrm{kpc}$ from the galactic center cause an apparent peak of $\sim 3 \mathrm{M}_{\odot} \mathrm{pc}^{-2}$ in the $\mathrm{H}_{2}$ surface density at $\mathrm{R}=11.5 \mathrm{kpc}$. No similar peak is seen in the neutral hydrogen surface density, a result due in part to the higher velocity dispersion of the H I. Comparison of the molecular surface densities in the arm and interarm regions yields an arm-interarm contrast of about 4.5 to 1 for the Carina arm. This ratio is likely a lower limit since 1) it follows from an analysis that tends to wash out nonaxisymmetric (sp/ral) structure by assigning a distinct distance to every point (at $R>R_{0}$ ) in $(l, b, v)$-space and averaging over longitude, and 2) a ratio of 13 to 1 is indicated by the step at $I=280^{\circ}$ in the total ( $b$ - and $v$ - integrated) CO intensity diagram.

To place the Carina arm in the Galaxy, a catalog of individual molecular clouds that trace the arm in the $I, V$ and spatial maps was compiled, the clouds at longitudes greater than $300^{\circ}$ having been identifled in an adjoining CO survey made with the same telescope. The catalog lists the positions, distances, linear dimensions, and masses of the clouds; the masses were calculated from an assumed proportionality between integrated $\mathrm{CO}$ line intensity and $\mathrm{H}_{2}$ column density. The identification of each cloud within $l=270^{\circ}$ and $300^{\circ}$ is discussed briefly, including its distance determination and possible association with other radio and optical objects. One cloud, that associated with the $\eta$ Carinae Nebula, was studied in some detail. The giant H II region NGC 3372 ( $\eta$ Carinae Nebula) is severely disrupting its immediate surroundings, driving a fragment of the assoclated molecular cloud away from the main body of the cloud. The star formation efficiency 
in the $\eta$ Carinae molecular cloud was found to be $\sim 0.02$, in good agreement with the recent results of Myers et al. (1985) for inner Galaxy molecular clouds.

By locating the cataloged clouds in the plane of the Galaxy, clouds more massive than $105 M_{\odot}$ were shown to trace the Carina arm over a length of nearly $25 \mathrm{kpc}$. From $/ \approx 280^{\circ}$ to $300^{\circ}$, where the Carina arm had previously been traced optically to distances as great as $12 \mathrm{kpc}$, the close agreement between the molecular arm and the optical arm emphasized the importance of giant molecular clouds in the study of spiral structure on the galactic scale. With the largest clouds identified in earlier Columbia Co surveys of the first and second quadrants added to the picture, the Carina and Sagittarius arms appear to form a single $10^{\circ}$-spiral arm $40 \mathrm{kpc}$ long, covering at least $250^{\circ}$ in galactocentric angle. 


\section{REFERENCES}

Allen, C.W., 1973, Astrophysical Quantities, The Anthlone Press, University of London.

Bally, J., and Scovllle, N.Z., 1980, AD. J., 239, 121.

Batchelor, R.A., Caswell, J.L., Goss, W.M., Haynes, R.F., Knowles, S.H., and

Wellington, K.J., 1980, Australian J. of Phys., 33, 139.

Beard, M., and Kerr, F.J., 1966, Australian J. of Phys., 19, 875.

Becker, R.H., Boldt, E.A., Holt, S.S., Pravdo, S.H., Rothschlid, R.E.,

Serlemitsos, P.J., and Swank, J.H, 1976, AD. J. (Letters), 209, L65.

Bigay, J.H., Garnier, R., Georgelin, Y.P., and Georgelin, Y.M., 1972, Astr. AD.,

18,301 .

Blitz, L., 1978, Ph.D dissertation, Columbla University.

Blitz, L. and Thaddeus, P., 1980, AD. J. (Letters), 226, L39.

Bloemen, J.B.G.M., Strong, A.W., Blitz, L., Cohen, R.S., Dame, T.M.,

Grabelsky, D.A., Hermsen, W., Lebrun, F., and Thaddeus, 1985, Astr. Ap., in press.

Bok, B., 1937, The Distribution of Stars in Space, University of Chicago Press.

Bok, B.J., and van Wijk, V., 1952, A. J., 57, 213.

Bok, B.J., 1956, vistas in Astronomy, 2, 1522.

Bok, B.J., 1959, The Observatory, 79, 58.

Bok, B.J., Hine, A.A., and Miller, E.W,1970, in IAU Symposium 38, The Spiral Structure of our Galaxy, ed., W. Becker and G. Contopoulos (Dordrecht: Reidel), p. 246.

Braz, M.A., and Scallse, E. Jr., 1982, Astr. AD., 107, 272.

Bronfman, L., 1985, Ph.D dissertation, Columbia University. 
Burton, W.B., 1971, Astr. AD., 10, 76

Burton, W.B., Gordon, M.A., Bania, T.M., and Lockman, F.J., 1975 ,

AD. $\mathcal{L}, 202,30$.

Burton, W.B., and Gordon, M.A., 1978, Astr. AD., 63, 7.

Castor, J., McCray, R., and Weaver, R., 1975, Ap. J. (Letters), 200, L107.

Caswell, J.L., 1972, Australlan J. or Phys., 25, 443.

Caswell, J.L., and Robinson, B.J., 1974, Australian J. of Phys., 27, 597.

Chin, G., 1977, Ph.D. dissertation, Columbia University.

Claria, J.J., 1977, Astr. AD. Suppl, 27, 145.

Cohen, R.S., 1977, Ph.D. dissertation, Columbla University.

Cohen, R.S., Cong, H., Dame, T.M., and Thaddeus, P., 1980, Ap. J. (Letters), 239, L53.

Conen, R.S, Grabelsky, D.A., May, J., Bronfman, L., Alvarez, H., and Thaddeus, P., 1985a, AD. J. (LetterS), 290, L15.

Cohen, R.S., Dame, T.M., and Thaddeus, P., 1985b, Ap. J. Suppl, in press.

Conti, P.S., and Burnichon, M.-L., 1975, Astr. AD., 38, 467.

Dame, T.M., 1983, Ph.D. dissertation, Columbia University.

Dame, T.M., Cohen, R.S., Elmegreen, B.G., and Thaddeus, P., 1985, AD. J, in press.

Davis, J.H., and Vanden Bout, P., 1973, Ap. Letters, 15, 43.

de Graauw, T., Lindholm, S., Fitton, B., Beckman, J., Israel, F.P.,

Nieuwenhuijzen, H., and Vermue, J., 1981, Astr. AP., 102, 257.

Dickel, J.R., and Milne, D.K., 1972, Australian J. or Phys., 25, 539.

Dickel, J.R., 1973, AD. Letters, 15, 61.

Dickel, H.R., and Wall, J.V., 1974, Astr. AD., 31, 5.

Dickel, H.R., 1974, Astr. AD, 31, 11.

Elliot, K.H., 1979, M.N.R. A. S., 186, 9P. 
Elliot, K.H., and Malin, D.F., 1979, M. N. R. A. S., 186, 45P.

Elmegreen, B.G., and Lada, C.J., 1976, AD. J., 81, 1089.

Feinstein, A., 1969, M.N.R. A. S., 143, 273.

Feinstein, A., Marraco, H.G., and Muzzio, J.C., 1973, Astr. Ap. Suppl., 12, 331.

Feinstein, A., Marraco, H.G., and Forte, J.C., 1976, Astr. Ap. Suppl., 24, 389.

Feinstein, A., FitzGerald, M.P., and Moffat, A.F.J., 1980, A. J., 85, 6.

Fernie, J.D., 1968, A. J., 73, 995.

Frogel, J.A., and Persson, S.E., 1974, AD. J, 192, 351.

Gardner, F.F., Milne, D.K., Mezger, P.G., and Wilson, T.L., 1970, Astr. AD., 7, 349.

Gardner, F.F., Dickel, H.R., and Whiteoak, J.B., 1973, Astr. Ap., 23, 51.

Garmany, C.D., Conti, P.S., and Chiosi, C., 1982, AD. J., 263, 777.

Georgelin, Y., 1975, Ph.D. dissertation, Universite de Provence, Observatoire de Marseille.

Georgelin, Y.M., and Georgelin, Y.P., 1976, Astr. AD., 49, 57.

Georgelin, Y.P., and Georgelin, Y.M., 1970a, Astr. AD., 6, 349.

Georgelin, Y.P., and Georgelin, Y.M., 1970D, Astr. AD., 7, 133.

Glllisple, A.R., Huggins, P.J., Sollner, T.C.L.G, Phillips, T.G., Gardner, F.F., and Knowles, S.H., 1977, Astr. Ap., 60, 221.

Gordon, M.A., and Burton, W.B., 1976, AD. J., 208, 346.

Goss, W.M., and Shaver, P.A., 1970, Australian J. of Phys. Supple., 14,1. Goss, W.M., Radhakrishnan, V., Brooks, J.W., and Murray, J.D., 1972, Ap. J. Suppl., 24, 123.

Goss, W.M., Manchester, R.N., Brooks, J.W., Sinclair, M.W., Manefield, G., and Danziger, I.J., 1980, M. N. R. A. S., 191, 533.

Goy, G., 1973, Astr. Ap. Suppl., 12, 277.

Granam, J.A., and Lyngå, G., 1965, Mem. Mt. Stromlo Obs., No. 18. 
Graham, J.A., 1970, A. J., 75, 703.

Gum, C.5., 1955, Mem. R. A. S., 67, 155.

Habets, G.M.H.J., and Heintze, J.R.W., Astr. Ap. Suppl, 46, 193.

Harvey, P.M., Hoffman, W.F., and Campbell, M.F., 1979, AD. J., 227, 114.

Haynes, R.F., Caswell, J.L., and Simons, L.W.J., 1978, Australian J. or Phys. Supple., 45, 1.

Haynes, R.F., Caswell, J.L., and Simons, L.W.J., 1978, Australian J. of Phys. Supple., 48, 1.

Henderson, A.P., Jackson, P.D., and Kerr, F.J., 1982, AD. J., 263, 116.

Herbst, W., and Miller, D.P., 1982, A. J., 87, 1478.

Hill, E.R., Slee, O.B., and Mills, B.Y., 1958, Australian J. or Phys., 11, 530.

Horfleit, D., 1953, Harvard Ann., 119, 37.

Huchtmeier, W.K., and Day, G.A., 1975, Astr. AD. 41, 153.

Humphreys, R.M., 1970, A. J., 75, 602.

Humphreys, R.M., 1972, Astr. Ap., 20, 29.

Humphreys, R.M., 1976, Pub. A. S. P. 58, 647.

Humprheys, R.M., 1978, Ap. J. Suppl., 38, 309.

Humphreys, R.M., and Kerr, F.J., 1974, AD. J., 194, 301.

Israel, R.P., de Graauw, T., deVries, C.P., Brand, J., van de Stadt, H.,

Habing, H.J., Wouterloot, J.G.A, van Amerongen, J., van der Biezen, J.,

Leene, A., Nagtegaal, I., and Selman, F., 1984, Astr. AD., 134, 396.

Jones, B.B., 1973, Australian J. of Phys., 26, 545.

Kaufmann, P., Zisk, S., Scalise, E. Jr., Schaal, R.E., and Gammon, R.H., 1977,

A. J., 82, 577.

Kerr, F.J., Hindman, J.V., and Carpenter, M.S., 1957, Nature, 180, 677.

Kerr, F.J., 1970, in IAU Symposium 38, The Spiral Structure or our Galaxy,

ed., W. Becker and G. Contopoulos (Dordrecht: Reidel), p. 95. 
Kerr, F.J., and Kerr, M., 1970, Ap. Letters, 6, 175.

Kirshner, R.P., and Winkler, F.P. Jr., 1979, AD. J., 227, 853.

Kutner, M.L., Tucker, K.D., Chin, G., and Thaddeus, P., 1977, AD. J., 215, 521.

Kutner, M.L., 1978, Ap. Letters, 19, 81.

Lacy, J.H., Beck, S.C., and Geballe, T.R., 1982, Ap. J., 255, 510.

Lada, C.J., Elmegreen, B.G., Cong, H-I., and Thaddeus, P., 1978, Ap. J. (Letters), 225, L39.

Laurent, C., Paul, J.A., and Pettini, A., 1982, AD. J., 260, 162.

Lebrun, F., Bennet, K., Bignami, G.F., Bloemen, J.B.G.M., Buccheri, R., Caraveo, P.A., Gottwald, M., Hermsen, W., Kanbach, G., Mayer-Hasselwander, H.A., Montmerle, T., Paus, J.A., Sacco, B., Strong, A.W., Wills, R.D., Dame, T.M., Cohen, R.S., and Thaddeus, P., 1983, Ap. J., 274, 231.

Levato, H., and Malaroda, S., 1981, Pub. A. S. P., 93, 714.

Levato, H., and Malaroda, 5., 1981, Pub. A. 5. P., 94, 807.

Liszt, H.S., and Burton, W.B., 1981, AD. J., 243, 778.

Lockman, F.J., 1984, Ap. J., 283, 90.

Loden, L.O., and Sundman, A., 1980, Astr. AD., 91, 59.

Lyngå, G., 1970, Astr. AD., 8, 41.

Manchester, R.N., Robinson, B.J., and Goss, W.M., 1970, Australian J. of Phys., 23,751 .

Mathewson, D.S., Healy, J.R., and Rome, J.M., 1962, Australian J. of Phys., 15, 354.

Mathewson, D.S., Healy, J.R., and Rome, J.M., 1962, Australian J. of Phys., 15, 369.

Mauzy, B., 1974, NRAO Electronics Division Internal Report No. 146. 
McCutcheon, W.H., Robinson, B.J., Whiteoak, J.B., and Manchester, R.N., 1983, in Kinematics, Dynamics, and Structure or the Milky way, ed. W.L.H. Shuter (Dordrecht: Reidel), p. 165. McGee, R.X., and Gardner, F.F., 1968, Australian J. or Phys, 21, 149. McGee, R.X., Newton, L.M., and Batchelor, R.A., 1975, Australian J or Phys., 28, 185.

McGee, R.X., and Newton, L.M., 1981, M. N. R. A. S., 196, 889.

Mezger, P.G., Wilson, T.L., Garnder, F.F., and Milne, D.K., 1970, Ap. Letters, 6, 35.

Miller, G.E., and Scalo, J.M., 1979, Ap. J, 41, 513.

Mills, B.Y., 1959, in IAU Symposium 9, Paris Symposium on Radio Astronomy, ed. R.N. Bracewell (Stanford University Press), p. 431.

Milne D.K., Wilson, T.L., Gardner, F.F., and Mezger, P.G., 1969, Ap. Letters, 4, 121.

Milne, D.K., 1979, Australian J. of Phys., 32, 83.

Moffat, A.F.J., and Vogt, N., 1975, Astr. Ap. Suppl, 20, 125.

Morgan, W.W., Sharpless, S., and Osterbrock, D.E., 1952, A. J., 57, 3.

Morgan, W.W., Whitford, A.E., and Code, A.D., 1953, AD. J, $118,318$.

Myers, P.C., Dame, T.M., Thaddeus, P., Conen, R.S., Silverberg, R.F., Dwek, E., and Hauser, M.G., 1985, in press.

Neckel, Th., and Klare, G., 1980, Astr. Ap. Suppl, 42, 251.

Oort, J.H., Kerr, F.J., and Westerhout, G., 1958, M.N.R. A. S. 118, 379.

Penzias, A.A., and Burrus, C.A., 1973, Ann. Rev. Astr. AD. 11, 51.

Retallack, D.S., and GoSS, W.M., 1980, M. N. R. A. S., 193, 261.

Retallack, D.S., 1983, M.N. R. A. S., 204, 669. 
Robinson, B.J., Manchester, R.N., Whiteoak, J.B., Sanders, D.B., Scoville, N.Z., Clemens, D.P, McCutcheon, W.H., and Solomon, P.M., 1983, Ap. J. (Letters), 283, L31.

Rodgers, A.W., Campbell, C.T., Whithoak, J.B., Baily, H.H., and Hunt, V.O., 1960, An Atlas or Ho Emission in the Southern Milky Way, Canberra.

Rodgers, A.W., Campbell, C.T., Whithoak, J.B., 1960, M. N. R. A. S. 121, 103. Sanders, D.B., Solomon, P.M., and Scoville, N.Z., 1984, Ap. J., 276, 182.

Sanders, D.B., Scoville, N.Z., and Solomon, P.M., 1985, AD. J., 289, 373.

Sandqvist, Aa., 1977, Astr. AD., 57, 467.

Scalise, E. Jr., and Schaal, R.E., 1977, Astr. AD, 57, 475.

Scalise, E. Jr., and Braz, M.A., 1980, Astr. AD, 85, 149.

Scoville, N.Z., and Solomon, P.M., 1975, Ap. J. (Letters), 199, 535.

Seward, F.D., and Chlebowski, T., 1982, AD. J., 256, 530.

Shaver, P.A., and Goss, W.M., 1970a, Australian J. or Phys, 14, 77.

Shaver, P.A., and Goss, W.M., 1970b, Australian J. of Phys., 14, 133.

Sher, D., 1965, Quart. J.R. A. S, 6, 299.

Simonson, S.C. III, 1970, Astr. AD, 9, 163.

Smith, L.F., Biermann, P., and Mezger, P.G., 1978, Astron. AD., 66, 65.

Solomon, P.M., Sanders, D.B., and Scoville, N.Z., 1979, in IAU Symposium 84,

The Large-scale Characteristics of the Galaxy, ed. W.B. Burton (Dordrecht: Reldel), p.35.

Solomon, P.M., Sanders, D.B., and Rivolo, A.R., 1985, Ap. J. (Letters), 292,

Lig.

Spitzer, L. Jr., 1978, Physical Processes in the Interstellar Medium, John Wiley and Sons, New York.

Stothers, R., 1972, Ap. J., 175, 431.

Sundman, A., 1979, Astr. Ap. Suppl, 35, 327. 
Tammann, G.A., 1970, in IAU Symposium 38, The Spiral Structure of our

Galaxy, ed., W. Becker and G. Contopoulos (Dordrecht: Reldel), p. 236.

Thomas, B., and Day, G.A., 1969, Australian J. of Phys. Supple, 11, 3.

Tucker, K.D, Kutner, M.L., and Thaddeus, P., 1973, AD. J. (Letters), 186, L13

Turner, D.G., 1978, A. J, 83, 1081.

Turner, D.G., Grieve, G.R., Herbst, W., and Harris, W.E., 1980, A. J., 85, 1193.

vanden Bergh, S., 1978, Astr. Ap., 63, 275.

Vogt, N., and Moffat, A.F.J., 1975, Astr. AD, 39, 477.

Walborn, N.R., 1971, Ap. J. (Letters), 167, L31.

Walborn, N.R., 1973, AD. J. 179, 517.

Walborn, N.R., 1982, AD. J. Suppl, 48, 145.

Walborn, N.R., and Hesser, J.E., 1982, AD. J. 252, 156.

Weaver, H., 1970 in IAU Symposium 38, The Spiral Structure of our Galaxy,

ed., W. Becker and G. Contopoulos (Dordrecht: Reidel), p. 126.

White, G.J., and Phillips, J.P., 1983, M. N. R. A. S., 202, 255.

Whiteoak, J.B., 1983, in Surveys of the Southern Galaxy, ed. W.B. Burton and

F.P. Israel (Dordrecht: Reidel), p. 31.

Whiteoak, J.B., and Gardner, F.F., 1970, AD. Letters, 5, 5.

Whiteoak, J.B., and Gardner, F.F., 1974, Astr. AD, 37, 389.

Wilson, T.L., Mezger, P.G., Gardner, F.F., and Milne, D.K., 1970, Astr. AD. 6 , 364. 
APPENDIX A

TELESCOPE POINTING

Telescope tracking was carried out under computer control by continuous transformation of source right ascension and declination to azimuth and elevation, the telescope's pointing being maintained by an alogrithm that utilized position and velocity feedback. The pointing model, described in detail in Cohen (1977), was based on the assumption that, for a perfectly vertical azimuth axis and a mechanically perfect mount, all pointing errors can be corrected by applying constant of fsets in azimuth and elevation only. A tilt of the azimuth axis with respect to the vertical is equivalent to a shift in the true geocentric latitude and longitude of the telescope and can be accounted for by using the apparent latitude and longitude in the transformation from right ascension, declination to azimuth, elevation. Nonperpendicularity of either the altitude and azimuth axes or the altitude and optical axes is easily corrected by including an elevation-dependent term for each in the azimuthal pointing. In order to point the telescope, then, a total of six parameters must be determined: one offset each for the elevation position encoder, the azimuth encoder, the latitude, and the longitude; and two constants describing the degree of mount impreciston (nonperpendicularity terms). The determination of these parameters is described briefly in this appendix, and the pointing accuracy of the telescope is discussed. 


\section{A. Determination of Pointing Parameters}

Because the planets and other small continuum sources are not sufficiently bright at millimeter wavelengths to be readily detected by the $1.2 \mathrm{~m}$ antenna, the pointing parameters were determined with a small optical telescope mounted on the back of the antenna. First, with rough alignment of the optical axes of the radio and optical telescopes, and with approximate values for the latitude and longitude of the mount, the optical telescope was pointed at the sun to obtain approximate azimuth and elevation encoder of fsets. Next, using these offsets, the radio center of the Sun was located to $-0.3^{\prime}$ by finding the symmetry points of radio scans (in azimuth and elevation) across the Sun's limb; more accurate encoder of fsets were derived in this way. Then, with the antenna tracking (under computer control) the radio center of the Sun, the optical telescope was mechanically adjusted until the solar image was centered, to within a fraction of an arc minute, in a circular reticle that has the same apparent diameter as the Sun. This last procedure assured good alignment of the optical axes of the radio and optical telescopes. The two nonperpendicularity parameters were assumed to be small and were taken to be zero in the pointing model (see Cohen 1977 for the procedures for the determination of these parameters); the assumption that these terms could be ignored was later justified by the results of the pointing tests.

Having collimated the radio and optical telescopes, improved parameter values were determined through an optical "starpointing" procedure. With the encoder of fsets derived from the Sun pointing and the approximate latitude and longitude in place, but no corrections for possible nonperpendicularity of the mount axes, the telescope was made to track, 
under computer control, a number of bright stars spread across the sky. Each star was centered in the optical telescsope by using a paddle to of fset the automatic tracking, and the deviation of the true position from that predicted by the pointing model was recorded. The computer then makes a least-squares fit of the model to the data, yielding the new parameter values.

B. Pointing Accuracy

1. Shaft Encoders

Azimuth and elevation are measured with Baldwin 16 bit optical shaft encoders connected to the azimuth and elevation axes by flexible couplers. Each bit corresponds to $1 / 2^{16}$ revolutions or 0.33 arc minutes; the specified precision by Baldwin of these encoders was \pm 1 bit. The angular offset between the absolute zero of each encoder and zero degrees azimuth or elevation was determined from the starpointing procedure.

\section{Starpointing}

A second starpolnting, again without any corrections for nonperpendicularity of the mount axes, was carried out using the encoder offsets and telescope coordinates determined from the previous

starpointing. The peak pointing error from this new test was $0.48^{\circ}$ and the RMS error was $0.19^{\prime}$. These numbers compared with the $8^{\circ} 8^{\circ}$ beamwidth of the antenna indicated that the mount was sufficiently precise to ignore the nonperpendicularity terms in the pointing model. Figure Al shows the sky 
coverage of the 37 stars used in this test, and Figures A2a-d show the errors as a function of azimuth and elevation. A quantization of the points in error results from the discrete step-size ( $n$-half bits) of the offsets commanded from the paddle.

Periodic starpointings were performed to check the pointing. The peak and RMS errors from a third point done about two weeks after the second were $0.64^{\circ}$ and $0.35^{\circ}$, respectively. After about one year of operation a drift of the azimuth axis with respect to the vertical resulted in peak errors of 1.6', and new parameters were installed.

\section{Sunpointing}

A daily check on the pointing was made by measuring the deviation between the observed position of the radio center of the sun (located by the method described above) and the expected position. These tests indicated a systematic elevation error of about 1 ' with respect to the starpointing parameters, the observed position of the Sun almost always being lower (in elevation) than the expected position. The source of the error could not be determined, although a slow drift of the azimuth axis may have been a contributing factor since these errors, as well as smaller azimuth errors, appear to have generally worsened with time (Fig. A-3). For 74 sunpointings, the peak total error $\left[\left(\delta e^{2}+\delta a^{2} \cdot \cos ^{2} e\right)^{1 / 2}\right]$ and $R M S$ of the total errors were $1.7^{\prime}$ and $1.1^{\prime}$, repectively (excluding one pointing at $6^{\circ}$ elevation). 
TABLE $\|-1$

CO-FREE REFERENCE POSITIONS

\begin{tabular}{lr}
\hline & \\
\hline$\left.l()^{\bullet}\right)$ & $b\left({ }^{\bullet}\right)$ \\
\hline 272.00 & -5.00 \\
273.00 & -5.00 \\
274.00 & -4.00 \\
275.00 & -5.63 \\
276.18 & -4.95 \\
278.95 & -5.04 \\
282.24 & 3.71 \\
283.50 & 2.20 \\
284.12 & -3.43 \\
284.50 & 0.88 \\
285.00 & 3.00 \\
288.00 & 5.00 \\
288.47 & -3.09 \\
291.00 & 0.75 \\
291.70 & 3.70 \\
292.30 & 4.00 \\
292.67 & 3.01 \\
292.76 & -1.18 \\
293.00 & 5.00 \\
294.71 & 1.46 \\
295.00 & 5.00 \\
297.00 & 4.00 \\
& \\
\hline &
\end{tabular}


TABLE IV-1

Z-DISTRIBUTION OF $\mathrm{H}_{2}$ IN THE OUTER GALAXY TOWARD THE CARINA ARM

\begin{tabular}{cccccc}
\hline $\begin{array}{c}\text { Radius } \\
(\mathrm{kpc})\end{array}$ & $\begin{array}{c}\left\langle z_{0}\right\rangle \\
(\mathrm{pc})\end{array}$ & $z_{1 / 2}$ & $\begin{array}{c}\rho\left(\left\langle z_{0}\right\rangle\right) \\
\left(M_{\odot} p c^{-3}\right)\end{array}$ & $\sigma_{\text {fit }}$ & $\begin{array}{c}\sigma_{\text {measured }} \\
\left(M_{\odot} p c^{-2}\right)\end{array}$ \\
\hline 10.5 & $-48 \pm 18$ & $112 \pm 21$ & $0.0078 \pm 0.0015$ & $1.88 \pm 0.50$ & $2.18 \pm 0.06$ \\
11.0 & $-73 \pm 16$ & $120 \pm 16$ & $0.0077 \pm 0.0008$ & $1.96 \pm 0.34$ & $2.11 \pm 0.06$ \\
11.5 & $-109 \pm 19$ & $126 \pm 25$ & $0.0104 \pm 0.0017$ & $2.79 \pm 0.71$ & $3.00 \pm 0.04$ \\
12.0 & $-145 \pm 20$ & $141 \pm 26$ & $0.0060 \pm 0.0008$ & $1.78 \pm 0.41$ & $2.02 \pm 0.04$ \\
12.5 & $-167 \pm 25$ & $182 \pm 31$ & $0.0034 \pm 0.0004$ & $1.32 \pm 0.28$ & $1.40 \pm 0.04$ \\
13.0 & $-113 \pm 70$ & $277 \pm 86$ & $0.0017 \pm 0.0014$ & $0.95 \pm 0.38$ & $1.02 \pm 0.04$ \\
\hline
\end{tabular}


TABLE V-1

MASSIVE MAIN SEQUENCE STARS IN THE $\eta$ CARINAE NEBULA

\begin{tabular}{|c|c|c|c|c|}
\hline $\mathrm{HD} / \mathrm{No}$. & Sp. Type & ref & $\log \left(M / M_{\odot}\right)$ & Cluster \\
\hline 93161 & $06.5 V((f))$ & 2 & 1.58 & $\operatorname{Tr} 16$ \\
\hline 93204 & $05 \mathrm{~V}$ & 2 & 1.76 & $\operatorname{Tr} 16$ \\
\hline 93205 & $03 V$ & 2 & 2.08 & $\operatorname{Tr} 16$ \\
\hline 93250 & $03: V((f))$ & 2 & 2.08 & $\operatorname{Tr} 16$ \\
\hline 93343 & o8Vn & 2 & 1.42 & $\operatorname{Tr} 16$ \\
\hline 303308 & $03 V((f))$ & 2 & 2.08 & $\operatorname{Tr} 16$ \\
\hline 303311 & $05 \mathrm{~V}$ & 2 & 1.76 & $\operatorname{Tr} 16$ \\
\hline 1 & $09.5 \mathrm{~V}$ & 2 & 1.25 & $\operatorname{Tr} 16$ \\
\hline 2 & B1.5:V: & 2 & 1.02 & $\operatorname{Tr} 16$ \\
\hline 3 & $09: \mathrm{V}:$ & 2 & 1.41 & $\operatorname{Tr} 16$ \\
\hline 5 & $\mathrm{~B} 2: \mathrm{Vn}$ & 2 & 0.96 & $\operatorname{Tr} 16$ \\
\hline 8 & B1.5Vb & 2 & 1.02 & $\operatorname{Tr} 16$ \\
\hline 9 & $09.5 \mathrm{~V}$ & 2 & 1.25 & $\operatorname{Tr} 16$ \\
\hline 10 & BOVn & 2 & 1.19 & $\operatorname{Tr} 16$ \\
\hline 13 & $\mathrm{~B} 2 \mathrm{Vnn}$ & 2 & 0.96 & $\operatorname{Tr} 16$ \\
\hline 16 & $\mathrm{~B} 2 \mathrm{Vb}$ & 2 & 0.96 & $\operatorname{Tr} 16$ \\
\hline 19 & $09.5 \mathrm{~V}$ & 2 & 1.25 & $\operatorname{Tr} 16$ \\
\hline 20 & $B 1: V$ & 2 & 1.08 & $\operatorname{Tr} 16$ \\
\hline 23 & $07 \mathrm{Vn}$ & 2 & 1.54 & $\operatorname{Tr} 16$ \\
\hline 31 & Bovn & 2 & 1.19 & $\operatorname{Tr} 16$ \\
\hline 34 & 08/09:V: & 2 & 1.36 & $\operatorname{Tr} 16$ \\
\hline 64 & B1.5V:b & 2 & 1.02 & $\operatorname{Tr} 16$ \\
\hline 94 & BIVn & 2 & 1.08 & $\operatorname{Tr} 16$ \\
\hline 100 & $05.5 \mathrm{~V}$ & 2 & 1.69 & $\operatorname{Tr} 16$ \\
\hline 104 & $07: \mathrm{V}:$ & 2 & 1.54 & $\operatorname{Tr} 16$ \\
\hline 110 & $07 \mathrm{~V}$ & 2 & 1.54 & $\operatorname{Tr} 16$ \\
\hline 112 & $04.5 V((f))$ & 2 & 1.84 & $\operatorname{Tr} 16$ \\
\hline 115 & O9V & 2 & 1.41 & $\operatorname{Tr} 16$ \\
\hline 305536 & $08.5 \mathrm{~V}$ & $\overline{1}$ & 1.36 & Cr 228 \\
\hline 305522 & $\mathrm{BO} .5 \mathrm{~V}$ & 1 & 1.12 & $\operatorname{Cr} 228$ \\
\hline 305534 & $80.5 \mathrm{~V}:+\mathrm{BIV}$ & $i$ & 1.12 & $\operatorname{Cr} 228$ \\
\hline 93056 & $\mathrm{~B} I \mathrm{Vb}$ & 1 & 1.08 & $\operatorname{Cr} 228$ \\
\hline 305521 & $\mathrm{~B} 0.5 \mathrm{~V}$ & 1 & 1.12 & $\operatorname{Cr} 228$ \\
\hline 21 & $07.5 \mathrm{Vn}$ & $i$ & 1.46 & $\operatorname{Cr} 228$ \\
\hline 305437 & BO.5V & $i$ & 1.12 & $\operatorname{Cr} 228$ \\
\hline
\end{tabular}


TABLE V-1 (continued)

MASSIVE MAIN SEQUENCE STARS IN THE $\eta$ CARINAE NEBULA

\begin{tabular}{rllll}
\hline & & & & \\
\hline HD/No. & Sp. Type & ref & log $\left(\mathrm{M} / \mathrm{M}_{\odot}\right)$ & Cluster \\
\hline 305438 & O7.5V & 1 & 1.46 & $\mathrm{Cr} 228$ \\
305535 & B2.5V & 1 & 0.92 & $\mathrm{Cr} 228$ \\
93208 & O8.5V & 1 & 1.36 & $\mathrm{Cr} 228$ \\
305543 & BIV+BIV & 1 & 1.08 & $\mathrm{Cr} 228$ \\
30 & Bl.5V & 1 & 1.02 & $\mathrm{Cr} 228$ \\
305516 & Bo.5Vb & 1 & 1.12 & $\mathrm{Cr} 228$ \\
36 & B0.5:V:+B0.5:V: & 1 & 1.12 & $\mathrm{Cr} 228$ \\
37 & B2Vb & 1 & 0.96 & $\mathrm{Cr} 228$ \\
305532 & O5V & 1 & 1.76 & $\mathrm{Cr} 228$ \\
39 & O8V & 1 & 1.42 & $\mathrm{Cr} 228$ \\
43 & B2Vb & 1 & 0.96 & $\mathrm{Cr} 228$ \\
305515 & B1.5Vsn & 1 & 1.02 & $\mathrm{Cr} 228$ \\
305533 & B0.5:Vnn+Shell & 1 & 1.12 & $\mathrm{Cr} 228$ \\
48 & B1.5Vb & 1 & 1.02 & $\mathrm{Cr} 228$ \\
93146 & O6V & 1 & 1.61 & $\mathrm{Cr} 228$ \\
66 & O9.5V & 1 & 1.25 & $\mathrm{Cr} 228$ \\
67 & O9V & 1 & 1.41 & $\mathrm{Cr} 228$ \\
68 & BIV & 1 & 1.08 & $\mathrm{Cr} 228$ \\
305528 & B2V & 1 & 0.96 & $\mathrm{Cr} 228$ \\
81 & BO.5Vb & 1 & 1.12 & $\mathrm{Cr} 228$ \\
305538 & BOVb & 1 & 1.19 & $\mathrm{Cr} 228$ \\
89 & B2V & 1 & 0.96 & $\mathrm{Cr} 228$ \\
93576 & O9Vn & 1 & 1.41 & $\mathrm{Cr} 228$ \\
305539 & O7V & 1 & 1.54 & $\mathrm{Cr} 228$ \\
95 & BOVb & 1 & 1.19 & $\mathrm{Cr} 228$ \\
97 & O5V & 1 & 1.76 & $\mathrm{Cr} 228$ \\
305525 & O6V & 1 & 1.61 & $\mathrm{Cr} 228$ \\
& & & &
\end{tabular}


TABLE V-1 (continued)

MASSIVE MAIN SEQUENCE STARS IN THE $\eta$ CARINAE NEBULA

\begin{tabular}{rlccl}
\hline & & & & \\
\hline HD/No. & Sp. Type & ref & $\log \left(\mathrm{M} / \mathrm{M}_{\odot}\right)$ & Cluster \\
\hline 93027 & $09.5 \mathrm{~V}((\mathrm{f}))$ & 3 & 1.25 & $\operatorname{Cr} 228$ \\
93128 & $03 \mathrm{Tr} 14$ \\
$93129 \mathrm{~B}$ & $03 \mathrm{(ff}))$ & 3 & 2.08 & $\operatorname{Tr} 14$ \\
-582620 & $06.5 \mathrm{~V}(\mathrm{f}))$ & 3 & 1.08 & $\operatorname{Tr} 14$ \\
\hline
\end{tabular}

References for spectral types:

1. Levato and Malaroda ( 1981).

2. Levato and Malaroda ( 1981 ).

3. Welborn (1973). 
TABLE V-2

CARINA ARM MOLECULAR CLOUDS

\begin{tabular}{|c|c|c|c|c|c|c|c|c|c|}
\hline No. & (") & b & $\begin{array}{l}\mathrm{v} \\
(\mathrm{km}\end{array}$ & $\begin{array}{r}\Delta v \\
\left.s^{-1}\right)\end{array}$ & $\begin{array}{l}\text { dist } \\
(\mathrm{kpc})\end{array}$ & $\begin{array}{l}R_{\text {eff }} \\
(p c)\end{array}$ & $M_{\text {Co }}$ & $\begin{array}{l}M_{\text {vir }} \\
\left.T_{\odot}\right)\end{array}$ & Notes \\
\hline 1 & 270.9 & -0.5 & 52 & 7 & 6.8 & 57 & 2.2 & 5.1 & $a, d, g, n$ \\
\hline 2 & 279.9 & -1.6 & 35 & 5 & 7.1 & 37 & 1.0 & 2.1 & $a, d, g$ \\
\hline 3 & 281.4 & -1.1 & -5 & 13 & 3.2 & 101 & 22.5 & 37.5 & $a, d, h$ \\
\hline 4 & 282.0 & -0.8 & 17 & 5 & 6.1 & 88 & 2.7 & 4.6 & $c, e, g$ \\
\hline 5 & 282.9 & 1.3 & -19 & 6 & 2.2 & 26 & .7 & 2.2 & $a, d, g, k$ \\
\hline 6 & 282.9 & -0.7 & -5 & 9 & 3.2 & 31 & 2.8 & 5.6 & $a, d, g, i$ \\
\hline 7 & 283.8 & 0.0 & -5 & 10 & 4.0 & 65 & 6.3 & 13.1 & $b, e, h$ \\
\hline 8 & 284.5 & -0.2 & 12 & 8 & 6.6 & 123 & 20.3 & 14.9 & $b, e, g$ \\
\hline 9 & 285.3 & 0.0 & 0 & 10 & 5.3 & 88 & 4.5 & 17.4 & $b, e, g, j$ \\
\hline 10 & 286.4 & -0.3 & 14 & 8 & 7.5 & 84 & 8.3 & 9.9 & $a, d, g$ \\
\hline 11 & 287.5 & -0.5 & -19 & 10 & 2.7 & 66 & 6.7 & 13.6 & $a, d, h$ \\
\hline 12 & 288.6 & 1.5 & -22 & 7 & 3.2 & 56 & 3.5 & 5.8 & $a, d, g, k$ \\
\hline 13 & 289.3 & -0.6 & 22 & 15 & 7.9 & 132 & 31.2 & 64.9 & $a, d, h$ \\
\hline 14 & 290.2 & -0.2 & -1 & 5 & 6.8 & 55 & 2.7 & 3.2 & $a, d, g, i$ \\
\hline 15 & 290.6 & -0.2 & 15 & 10 & 8.7 & 80 & 4.3 & 15.1 & $c, e, g$ \\
\hline 16 & 291.4 & -0.2 & -7 & 4 & - & -- & -- & -- & $a, d, 0$ \\
\hline 17 & 291.6 & -0.4 & 15 & 10 & 7.2 & 73 & 4.1 & 14.1 & $b, e, n$ \\
\hline 18 & 291.6 & -0.5 & 29 & 6 & 10.3 & 105 & 12.5 & 6.7 & $a, d, g$ \\
\hline 19 & 292.6 & -0.3 & 4 & 4 & 8.1 & 31 & 12.0 & 9.1 & $b, e, g, i$ \\
\hline 20 & 293.3 & -1.4 & -25 & 8 & 2.4 & 77 & 7.1 & 9.2 & $a, d, h$ \\
\hline 21 & 294.0 & -0.9 & 32 & 8 & 11.3 & 102 & 12.2 & 13.1 & $a, d, g$ \\
\hline 22 & 294.4 & -0.7 & -3 & 10 & 8.0 & 109 & 12.2 & 22.0 & $a, d, g, i$ \\
\hline 23 & 295.1 & -0.8 & 26 & 11 & 11.0 & 64 & 4.5 & 16.3 & $a, e, g$ \\
\hline 24 & 297.4 & -0.5 & 22 & 18 & 11.2 & 154 & 32.1 & 102 & $a, d, g$ \\
\hline 25 & 298.6 & 0.1 & -35 & 10 & 4.7 & 139 & 26.5 & 26.3 & $a, d, g, k$ \\
\hline 26 & 298.8 & -0.2 & 25 & 16 & 12.0 & 157 & 30.8 & 83.5 & $a, d, g$ \\
\hline 27 & 299.4 & -0.1 & -6 & 9 & 9.4 & 66 & 4.6 & 12.0 & $b, e, g, i$ \\
\hline 28 & 300.3 & -0.2 & 31 & 9 & 12.9 & 61 & 7.0 & 10.4 & $\mathrm{~g}, \mathrm{l}, \mathrm{m}$ \\
\hline 29 & 300.6 & -0.2 & 10 & 7 & 11.1 & 61 & 4.2 & 6.4 & $g, 1, m$ \\
\hline 30 & 301.8 & 0.0 & 24 & 7 & 12.7 & 44 & 4.2 & 4.5 & $g, 1, m$ \\
\hline 31 & 302.3 & -0.7 & 32 & 11 & 13.6 & 97 & 29.4 & 24.6 & $g, 1, m$ \\
\hline 32 & 303.9 & -0.4 & 29 & 13 & 13.7 & 144 & 46.2 & 51.1 & $g, 1, m$ \\
\hline
\end{tabular}


TABLE V-2 (continued)

CARINA ARM MOLECULAR CLOUDS

\begin{tabular}{|c|c|c|c|c|c|c|c|c|c|}
\hline No. & I & ${ }^{b}$ & $\begin{array}{l}v \\
(\mathrm{~km}\end{array}$ & $\begin{array}{c}\Delta v \\
\left.s^{-1}\right)\end{array}$ & $\begin{array}{l}\text { dist } \\
\text { (kpc) }\end{array}$ & $\begin{array}{l}R_{\text {off }} \\
(p c)\end{array}$ & $M_{C o}$ & $M_{\odot}^{M_{\text {vir }}}$ & Notes \\
\hline 33 & 306.9 & -0.5 & 25 & 7 & 14.2 & 50 & 5.6 & 5.2 & $g, 1, m$ \\
\hline 34 & 308.0 & -0.7 & 32 & 11 & 15.1 & 113 & 21.0 & 28.7 & $\mathrm{~g}, \mathrm{l}, \mathrm{m}$ \\
\hline 35 & 311.3 & -0.3 & 27 & 17 & 15.5 & 210 & 109.2 & 127 & $9,1, m$ \\
\hline 36 & 313.2 & -0.3 & 42 & 10 & 17.6 & 100 & 18.2 & 21 & $\mathrm{~g}, \mathrm{l}, \mathrm{m}$ \\
\hline 37 & 314.0 & -0.1 & 28 & 7 & 16.4 & 59 & 4.2 & 6.1 & $\mathrm{~g}, \mathrm{l}, \mathrm{m}$ \\
\hline 38 & 315.3 & -0.3 & 14 & 10 & 15.4 & 72 & 11.2 & 15.1 & $\mathrm{~g}, \mathrm{l}, \mathrm{m}$ \\
\hline 39 & 318.0 & -0.3 & 30 & 15 & 17.6 & 148 & 2.8 & 69.9 & $\mathrm{~g}, \mathrm{l}, \mathrm{m}$ \\
\hline 40 & 320.5 & -0.5 & 26 & 17 & 17.8 & 155 & 12.6 & 94.1 & $\mathrm{~g}, \mathrm{l}, \mathrm{m}$ \\
\hline 41 & 325.3 & -0.1 & 28 & 12 & 19.3 & 115 & 19.6 & 34.8 & $g, 1, m$ \\
\hline 42 & 328.5 & -0.1 & 30 & 6 & 20.4 & 55 & 5.6 & 4.2 & $9,1, m$ \\
\hline 43 & 335.5 & -0.5 & 27 & 5 & 22.0 & 24 & $>0.6$ & 1.3 & $\mathrm{~g}, 1, \mathrm{~m}$ \\
\hline
\end{tabular}

Notes on Table $Y-2$ :

a) Velocity from Gaussian fit to composite spectrum

b) Velocity from hand-drawn "eyeball" fit to composite spectrum

c) Velocity from liv map

d) FWHM from Goussian fit to composite spectrum

e) FWHM from hand-drawn "eyeball" fit to composite spectrum

f) FWHM from l, $\mathrm{T}$ map

g) Kinematic distance

h) Optical distence

1) Distance ambiguity resolved with radius-line width relation (Dame et al. 1985)

j) Distance ambiguity resolved with foreground absorption

k) Yelocity places cloud at subcentral point

1) Cloud identfication from Bronfman (1985)

m) Cloud parameters from Bronfman (private communication)

n) This cloud is probably not in the Car ine arm -- see "Notes on Individual Clouds" in Chapter $y$

o) Associated with local dust; distance unknown -- see "Notes on Individual Clouds" in Chapter $v$ 


\section{FIGURE CAPTIONS}

\section{Chapter II}

Figure 11-1: Schematic diagram of telescope mount and antenna. The housing shown on the base of the mount actually corresponds to the Northern Millimeter-Wave Telescope, but otherwise the Northern and Southern telescopes are mechanically identical. Individual components are discussed in the text.

Figure II-2: Block diagram of telescope system. Analog and digital signals are drawn in thick and thin lines, respectively. The main components are discussed in the text.

\section{Chapter III}

Figure 111-1: Survey coverage and sampling. Observed full resolution positions are marked by circles of $8^{\prime} 8^{\prime}$ diameter (one beam); spacing is $0.125^{\circ}$. The entire area within the map was observed every $0.5^{\circ}$ in $/$ and $b$ in the $0.5^{-}$Superbeam survey; a few squares indicate the $0.5^{\bullet}$ beam sampling pattern. 
Figure 111-2: Sample spectra from the full resolution survey. All spectra cover about $330 \mathrm{~km} \mathrm{~s}^{-1}$ with 256 channels, each $1.3 \mathrm{~km} \mathrm{~s}^{-1}$ wide. A straight line, from a fit to the first and last 40 channels, was removed from each spectrum. Typical integration times were 5 minutes, yielding RMS noise of about $0.14 \mathrm{~K}$ per channel. The temperature scale on the right is in units of radiation temperature.

Figure 111-3: Spatial map from the Superbeam survey showing all emission between $v=-50$ and $+50 \mathrm{~km} \mathrm{~s}^{-1}$. Contours are at $6,12,18, \ldots \mathrm{K} \mathrm{km} \mathrm{s}^{-1}$.

Figure 111-4: Spatial map combining both the full resolution and Superbeam surveys showing all emission between $v=-50$ and $+50 \mathrm{~km} \mathrm{~s}^{-1}$. The full resolution data are shown within the dotted lines, the Superbeam data beyond the dotted lines. Contours are at $6,12,18, \ldots \mathrm{K} \mathrm{km} \mathrm{s}^{-1}$.

Figure 111-5: 5patial map from clipped Superbeam data showing emission between $v=-100$ and $+100 \mathrm{~km} \mathrm{~s}^{-1}$. All spectral channels below $0.5 \mathrm{~K}(\sim 3 \sigma)$ were set to zero before integrating. Contours are at $3,612,18, \ldots \mathrm{K} \mathrm{km} \mathrm{s}^{-1}$.

Figure III-6: Spatial map combining clipped full resolution and clipped Superbeam data showing emission between $v=-100$ and $+100 \mathrm{~km} \mathrm{~s}^{-1}$. All specatral channels below $0.5 \mathrm{~K}(\sim 3 \sigma)$ were set to zero before integrating. Contours are at $3,6,12,18, \ldots \mathrm{Km} \mathrm{s}^{-1}$.

Figure 1II-7: Full resolution integrated IV diagram. The CO emission has been integrated over all latitudes where data were taken (see Fig. III-1 for outline of survey coverage). Contours are at $0.35,0.7,1.4,2.1, \ldots \mathrm{K} \mathrm{deg}$. 
Figure 111-8: Superbeam integrated $/, V$ diagram. The $\mathrm{CO}$ emission has been integrated over all latitudes where data were taken $\left(b=+5^{\circ}\right.$ to $\left.-5^{\circ}\right)$.

Contours are at $1.0,1.5,2.0,2.5, \ldots \mathrm{K}$ deg.

Figure 111-9: Integrated $I, V$ diagram of full resolution data smoothed in $/$ to $0.5^{\circ}$ for comparison with the Superbeam integrated I, $V$ diagram (Fig. III-6). Contours are at $1.0,1.5,2.0,2.5, \ldots \mathrm{K}$ deg.

\section{Chapter IV}

Figure IV-I: (a) Full resolution integrated I,V map; identical to Figure III-5 but on a smaller scale. (b) Key. Approximate hellocentric distances are marked off along the Carina arm. The dark lines are schematic and not derived from a model. The regions enclosed in dotted lines and labeled $a b$, $c$ and $d$ show the velocity limits used in the spatial maps of the near side, tangent region, and far side of the Carina arm (Figs. IV-3a- $d$ and $V-1 a-d$.

Figure IV-2: CO emission integrated over all covered velocities and latitudes (in units of $\mathrm{K} \mathrm{km} \mathrm{s}^{-1} \mathrm{deg}$ ) as a function of longitude. Tyical RMS noise $(1 \sigma)$ per longitude point is $2.2 \mathrm{~K} \mathrm{~km} \mathrm{~s}^{-1}$ deg. 
Figure IV-3: Spatial maps of CO emission integrated over the indicated velocity ranges. Part a contains the near side of the Carina arm; part $b$ the tangent region; parts $c$ and $d$ the far side. Full resolution data are shown inside the dotted lines in the maps covering $/=270^{\circ}$ to $300^{\circ}(a-c)$; Superbeam data are shown beyond the dotted lines. At /> $300^{\circ}$, only full resolution data are avallable. The contour interval is $5 \mathrm{~K} \mathrm{~km} \mathrm{~s}^{-1}$.

Figure IV-4: Galactocentric rings of radi1 10.5, 11.0, 11.5, 12.0, 12.5, and $13.0 \mathrm{kpC}$ are shown transformed to the I, $\mathrm{V}$ plane using a flat rotation curve, $V(R)=250 \mathrm{~km} \mathrm{~s}^{-1}$. An outline of the data is also shown (shaded region).

Figure IV-5: Volume density of $\mathrm{H}_{2}$ as a function of vertical distance, $\mathrm{z}$, from the galactic plane at fixed galactocentric radii beyond the solar circle. Each point in $z$ represents an average over 20 pc bins in $z$ and 500 pC rings in $R$ (see Section IV.A.3 for procedure). Solid line shows the data; dotted line is the best-fit Gaussian. The radii indicated in parts $a-f$ mark the center of the 500 pc rings.

Figure IV-6: Longitude-velocity map of $\mathrm{H}$ I emission integrated in latitude from $\mathrm{D}=+2^{\circ}$ to $-2^{\circ}$. Data, from the Parkes $18 \mathrm{~m} 21-\mathrm{cm}$ survey of the Southern Milky Way (Henderson et al. 1982), are in units of brightness temperature. Contour interval is $25 \mathrm{~K}$ deg. 
Figure IV-7: Comparison of $\mathrm{CO}$ and $\mathrm{H} /$ high velocity ridges. The difference between the mean $\mathrm{CO}$ high velocity ridge and the mean $\mathrm{H} I$ high velocity ridge is plotted. The mean high velocity ridge at each $/$ is defined as the velocity-weighted emission ( $\mathrm{CO}$ or $\mathrm{H} \mathrm{1}$ ) within $\pm 25 \mathrm{~km} \mathrm{~s}^{-1}$ of the expected rotation-curve velocity at that $/$ The solid line shows the result using the rotation curve of Burton and Gordon (1978), and the dotted line shows the result using a simple straight line drawn in the $I, V$ plane from $v=-85 \mathrm{~km} \mathrm{~s}^{-1}, l=330^{\circ}$, to $v=0 \mathrm{~km} \mathrm{~s}^{-1}, l=270^{\circ}$.

Figure IV-8: Longitude-velocity map of clipped $\mathrm{H}$ l emission integrated in latitude from $b=+2^{\circ}$ to $-2^{\circ}$. All spectral channels with $T_{b}<50 \mathrm{~K}$ were set to zero before integration. Contour interval is $25 \mathrm{~K} \mathrm{deg}$.

Figure IV-9: Spatial map of $\mathrm{H}$ l emission integrated from -100 to $+100 \mathrm{~km} \mathrm{~s}^{-1}$. Contours are at $500,2000,4000,6000, \ldots \mathrm{K} \mathrm{km} \mathrm{s}^{-1}$.

Figure IV-10: Spatial maps of $\mathrm{H}$ I emission in the Carina arm integrated over the indicated velocity ranges. These maps correspond to the co spatial maps of the near side, tangent region, and far side of the Carina arm (Figs. IV-3a-d). Contours are at 100, 200, 400,600,... K km s-1. 
Figure IV-11: Volume density of $H$ I as a function of vertical distance, $z$, from the galactic plane at flxed galactocentric radii beyond the solar circle (analogous to Figs. IV-5a-f). Solid line shows the data; dotted line is best-fit Gaussian. The radil indlcated in parts $a-f$ mark the center of the 500 pc rings. Following Henderson et al. (1982), an optical depth correction was applied in calculating the number density at each l, b, and $\mathrm{v}$ : $n_{H}(\not, b, v)=1.823 \times 10^{18} T_{s} \tau(l, b, v)|d v / d r|$ atoms $\mathrm{cm}^{-2}\left(\mathrm{~km} \mathrm{~s}^{-1}\right)^{-1}$, where $r$ is the (kinematic) distance to the point, $T_{S}(=125 \mathrm{~K})$ is the (assumed uniform) spin temperature, and $\tau(l, b, v)$ is the optical depth; $\tau(l, b, v)=-\ln \left[1-T_{b}(l, b, v) / T_{s}\right]$. Each point in $z$ represents an average over $50 \mathrm{pc}$ bins in $z$ and $500 \mathrm{pc}$ rings in $\mathrm{R}$ (see text for procedure).

Figure IV-12: Parameters of the vertical distributions of $\mathrm{H}_{2}$ (circles) and $\mathrm{H} /$ (diamonds) in the outer Galaxy between $\mathrm{R}=10.5$ and $13.0 \mathrm{kpc}$ : (a) average midplanes, $z_{0} ;(b)$ thicknesses, $z_{1 / 2}$; and (c) surface densities, $\sigma$ (note that the $H$ I surface density is divided by 10 to fit on the same scale as the $\mathrm{H}_{2}$ surface density). For $\mathrm{H}_{2}$ the parameters are taken from Gaussian fits (dotted lines in Figs. IV $-5 a-f$ ) to the data (solid lines in same figures). Error bars on the $\mathrm{H}_{2}$ parameters represent the formal errors from the fit in each ring (of average radius $\mathrm{R}$ ) multiplied by a galactocentric dependent correction factor, $\sqrt{ }(\mathrm{N})$, where $N$ is the number of resolution elements (, 0 , and $v$ ) subtended by a typical cloud (50 pc diameter, $6 \mathrm{~km} \mathrm{~s}^{-1}$ wide) at the average distance to the ring; the correction factor approximately accounts for correlation of the data over the emission volume of discete molecular clouds. Because of the uncertainty of the true form of the $\mathrm{H}$ I layer distribution, no attempt has been made to estimate the errors on the parameters. 


\section{Chapter V}

Figure V-1: Spatial maps of $\mathrm{CO}$ emission integrated over the indicated velocity ranges. Part a contalns the near side of the Carina arm, part $b$ the tangent region, parts $c$ and $d$ the far side. Inside the dotted lines in the maps covering $/=270^{\circ}$ to $300^{\circ}(a-c)$ and at $/>300^{\circ}(d)$, full resolution data are shown smoothed to $0.25^{\circ}$ resolution. Beyond the dotted lines in parts $(a-c)$, where $0.5^{\circ}$ Superbeam data are shown, all spectral channels with $T<0.5 \mathrm{~K}$ were set to zero before integration. The contour interval is $3 \mathrm{~K} \mathrm{~km} \mathrm{~s}^{-1}$. The bold-face numbers on these maps identify the molecular clouds in the survey according to their running numbers in Table V-2. For extended clouds, a pair of lines radiating from the ID number indicates the cloud's extent; where the identification may appear ambiguous, a single line from the ID number points to the cloud. A pair of numbers separated by a comma indicates that two clouds with nearly the same spatial coordinates have been blended together in velocity by the integration. The parenthetical " 3 " in the near side map (part $a$ ) refers to a portion of cloud * 3 that spills over from the tangent region map (part $b$ ).

Figure V-2: Optical mosaic of the $\eta$ Carinae Nebula and its surroundings from ESO $J$ plates of the region. The CO contours overlaid on the mosaic are from a blowup of the region shown in the near side spatial map (Fig. IV-3a); the emission is integrated from -50 to $-9 \mathrm{~km} \mathrm{~s}^{-1}$, and the contour interval is $5 \mathrm{Kkm} \mathrm{s}^{-1}$. 
Figure V-3: Schematic representation of the dust, ionized gas, star clusters, and $\mathrm{CO}$ emission in the $\eta$ Carinae Nebula.

Figure V-4: Varlation of CO emission with velocity through the $\eta$ Carinae molecular cloud. Each spatial map shows the emission integrated over two spectral channels $\left(2.6 \mathrm{~km} \mathrm{~s}^{-1}\right)$. The eight maps $(a-h)$ are contiguous in velocity and cover the emission from -31 to $-10 \mathrm{~km} \mathrm{~s}^{-1}$. The contour interval is $1.4 \mathrm{~K} \mathrm{~km} \mathrm{~s}^{-1}$.

Figure V-5: Schematic lllustration of the giant molecular cloud associated $w i t h$ the $\eta$ Carinae Nebula. From right to left, the star clusters represent IC 2581, NGC 3293, NGC 3324, $\operatorname{Tr} 14, \operatorname{Tr} 15$ (top), $\operatorname{Tr} 16$ (center), and Cr 228 (bottom). The feature labelled "wisp" corresponds to the fllament discussed in the text; the arrow indicates its direction of motion with respect to the main body of the cloud.

Figure $V-6$ : The distribution of massive main sequence stars in the clusters $\operatorname{Tr} 14, \operatorname{Tr} 16$, and $\mathrm{Cr} 228$. All observed early-type stars with spectral classifications have been binned in 0.1 -decade wide logarithmic mass intervals centered at $\log \left(M / M_{\odot}\right)=2.15,2.05,1.95, \ldots 0.95$. (See Section V.2.C for a description of the stellar mass determinations.) The solid line shows the logarithm of the number of stars in each interval, and the dotted line shows the logarithm of the number in each interval plus the sum of all higher-mass intervais. The number of stars predicted in the indicated mass intervals by the Miller-Scalo (1979) IMF is shown by the filled circles show. 
Figure V-7: (a) Face-on view of the Galaxy with the locations of the largest molecular clouds. The cloud shown with a dashed circle has a very inaccurate distance and may actually lie in the Carina arm (see notes to cloud $=25$ in Section V.D.). (b) Same as Figure $V-8$ a replotted in rectangular semilog coordinates. The line follows a $10^{\circ}$ logarithmic spiral with a tangent at $I=281^{\circ}$.

Figure V-8: Same as Figure V-7a with a $10^{\bullet}$ logarithmic spiral with a tangent at $l=281^{\circ}$ drawn in.

Figure V-9: Variation of co emission with velocity through the tangent region of the Carina arm. Each spatial map shows the emission integrated over two spectral channels $\left(2.6 \mathrm{~km} \mathrm{~s}^{-1}\right)$. The ten maps $(a-j)$ are contiguous in velocity, and cover the emission from -18 to $+8 \mathrm{~km} \mathrm{~s}^{-1}$ (see notes to cloud $* 3$ in Section V.D.). The contour interval is $1.4 \mathrm{~K} \mathrm{~km} \mathrm{~s}^{-1}$.

Figure $V-10$ : Varlation of Co emission with velocity through the molecular cloud associated with NGC 3576, RCW 60, RCW 61, and RCW 62 (see notes to cloud $* 20$ in Section V.D.). Each spatial map shows the emission integrated over two spectral channels $\left(2.6 \mathrm{~km} \mathrm{~s}^{-1}\right)$. The eight maps $(a-h)$ are contiguous in velocity, and cover the emission from -31 to $-10 \mathrm{~km} \mathrm{~s}^{-1}$. The contour interval is $1.4 \mathrm{~K} \mathrm{~km} \mathrm{~s}^{-1}$. 


\section{Appendix A}

A-1: Azimuth and elevations of the 37 stars used in the final starpointing test used to check the pointing accuracy of the telescope.

A-2: Pointing errors of the 37 stars from the final starpointing test.

(a) Elevation errors as a function of azimuth; (b) azimuth errors times cosine of the elevation as a function azimuth; elevation errors as a funciton of elevation; and (d) azimuth errors times cosine of the elevation as a function of elevation. The quantization in error results from the discrete step size of the applied pointing offsets (see text).

A-3: Sunpointing errors plotted against day of 1983. The vertical axis shows the total error, $\left.\left[\left.\delta e\right|^{2}+\delta a z^{2} \cos ^{2}(e)\right)\right]^{1 / 2}$.

\section{APPENDIX B}

Figure $B-1$ : Full resolution $I, V$ maps at each latitude between $b=2.5^{\circ}$ and $-4.125^{\circ}\left(\Delta D=0.125^{\circ}\right)$. At $|b|>1^{\circ}$, where longitude coverage was not uniform, pairs of dotted lines mark the longitude intervals sampled; an odd number of dotted lines indicates that a dotted line nearest the top or bottom longitude boundary of the survey is paired with that boundary. Contours are at $0.5,1.0,2.0,3.0, \ldots \mathrm{K}$. 
Figure B-2: Full resolution $b, v$ maps at each longitude in the survey $\left(270^{\circ} \mathrm{s} / \mathrm{s} 300^{\circ}, \Delta /=0.125^{\circ}\right)$. The latitude boundarles of the maps show the maximum range observed in the full resolution survey. In general, pairs of dotted lines mark the latitude intervals sampled; an odd number of dotted lines indicates that a dotted line nearest the top or bottom latitude boundary is paired with that boundary. Contours are at $0.5,1.0,2.0,3.0, \ldots \mathrm{K}$.

Figure B-3: Superbeam $I, V$ maps at each latitude in the Superbeam survey $\left(b=+5^{\circ}\right.$ to $\left.-5^{\circ}, \Delta b=0.5^{\circ}\right)$. Contours are at $0.5,1.0,2.0,3.0, \ldots \mathrm{K}$.

Figure $B-4$ : Superbeam $b, v$ maps at each longitude in the survey $\left(270^{\circ} \mathrm{s} / \mathrm{s} 300^{\circ}, \Delta /=0.5^{\circ}\right)$. Contours are at $0.5,1.0,2.0,3.0, \ldots \mathrm{K}$. 


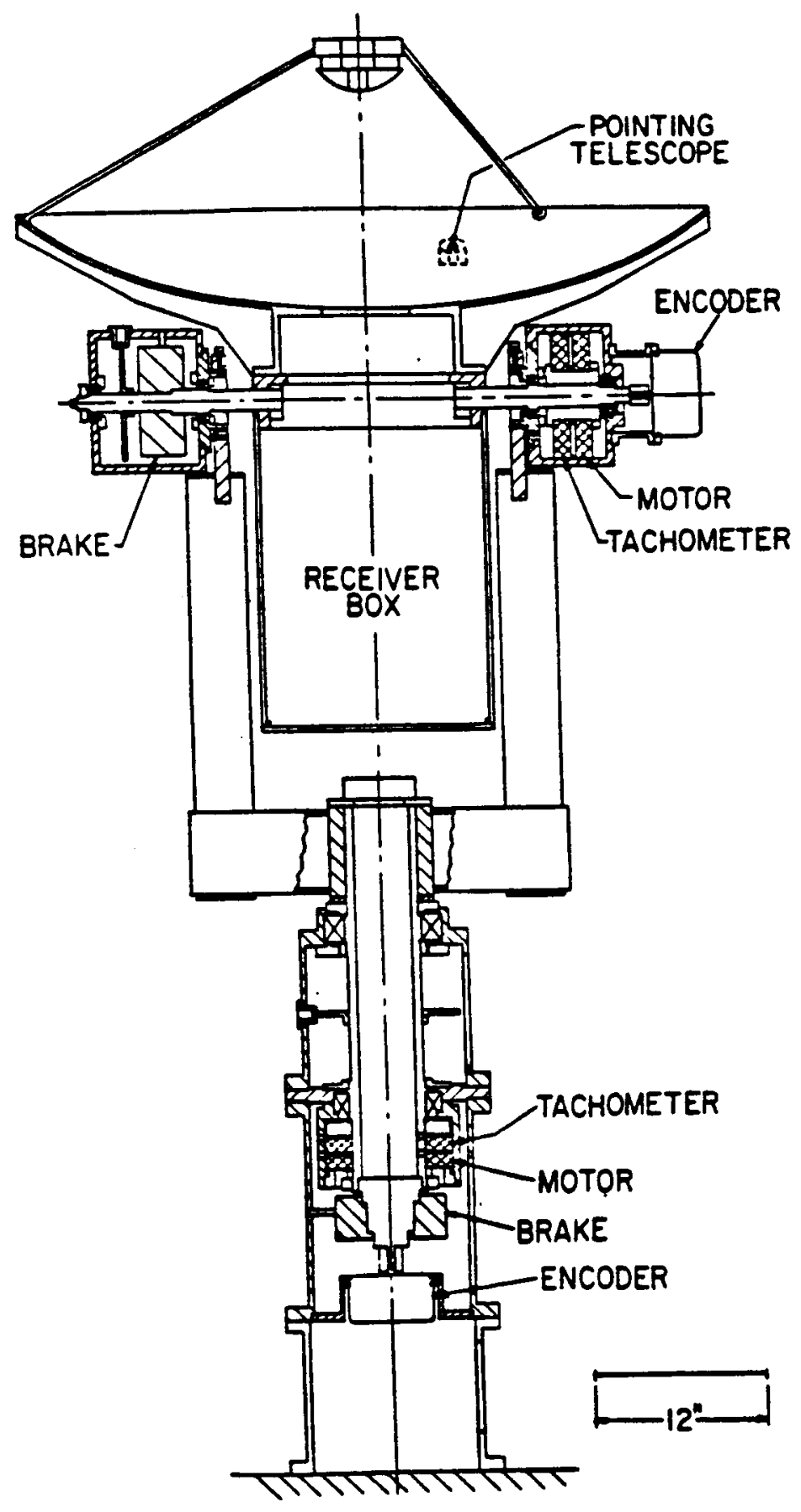

FIGURE $\mid 1-1$ 


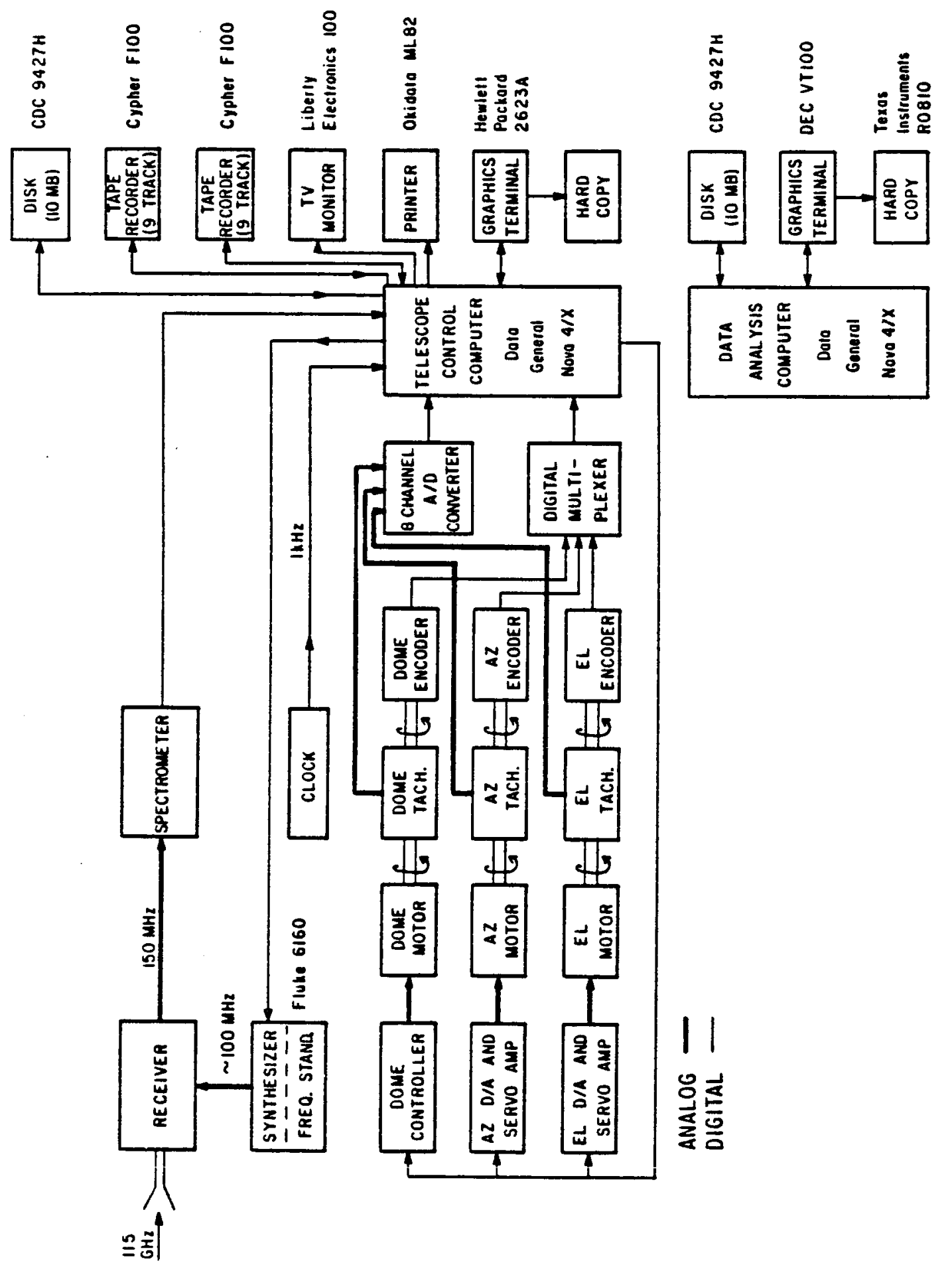

FIGURE $\mid 1-2$ 


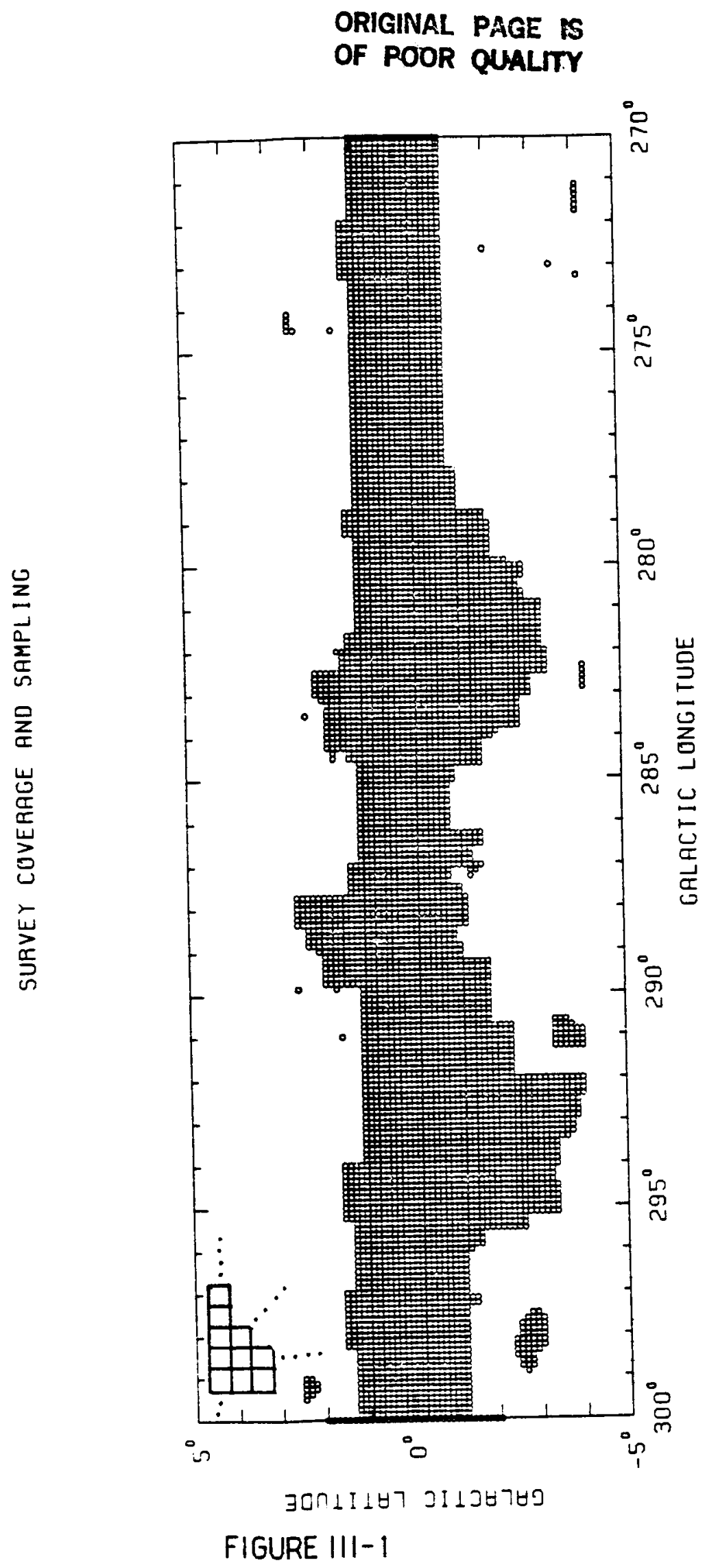




\section{TYPICAL SPECTRA}

$L=286.375$

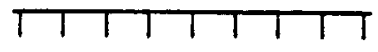

$B=0.375$

0.250

0.125

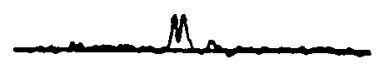

0.0

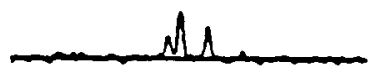

$-0.125$

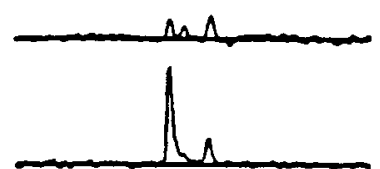

$-0.250$

$-0.375$

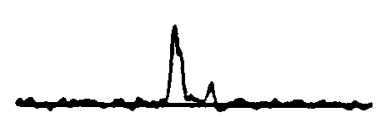

$-0.500$

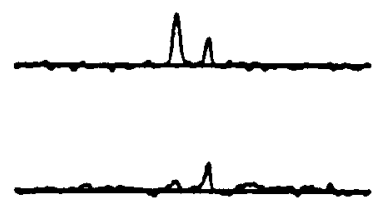

286.250
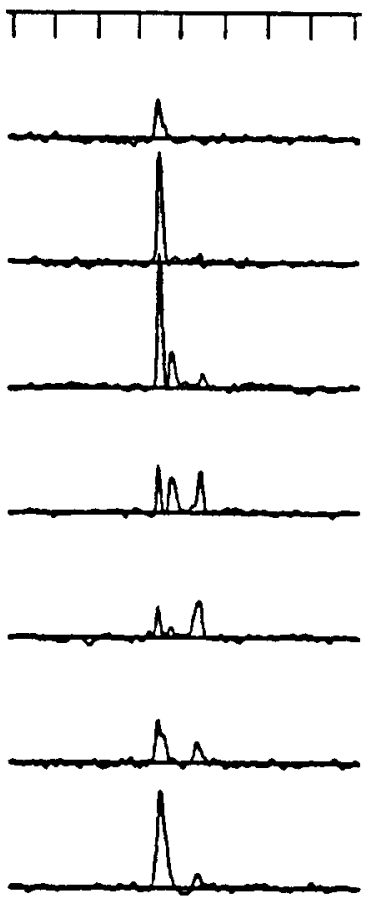

As

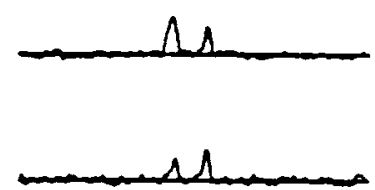

286.125
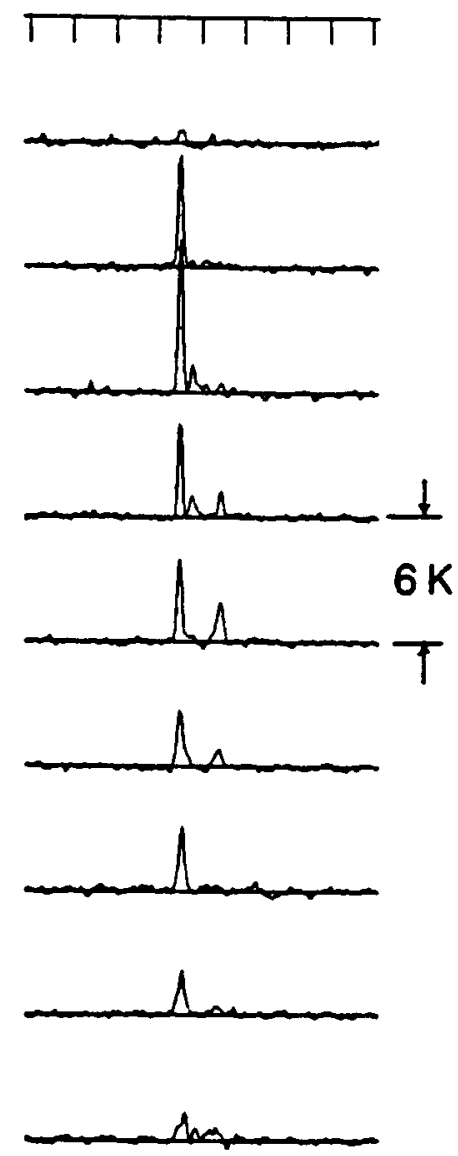

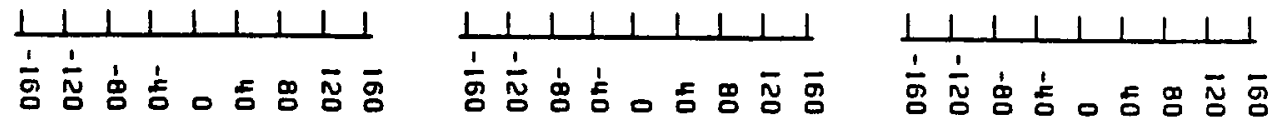
LSR RADIAL VELOCITY ( $\left.\mathrm{km} \mathrm{s} \mathrm{s}^{-1}\right)$

FIGURE III-2 


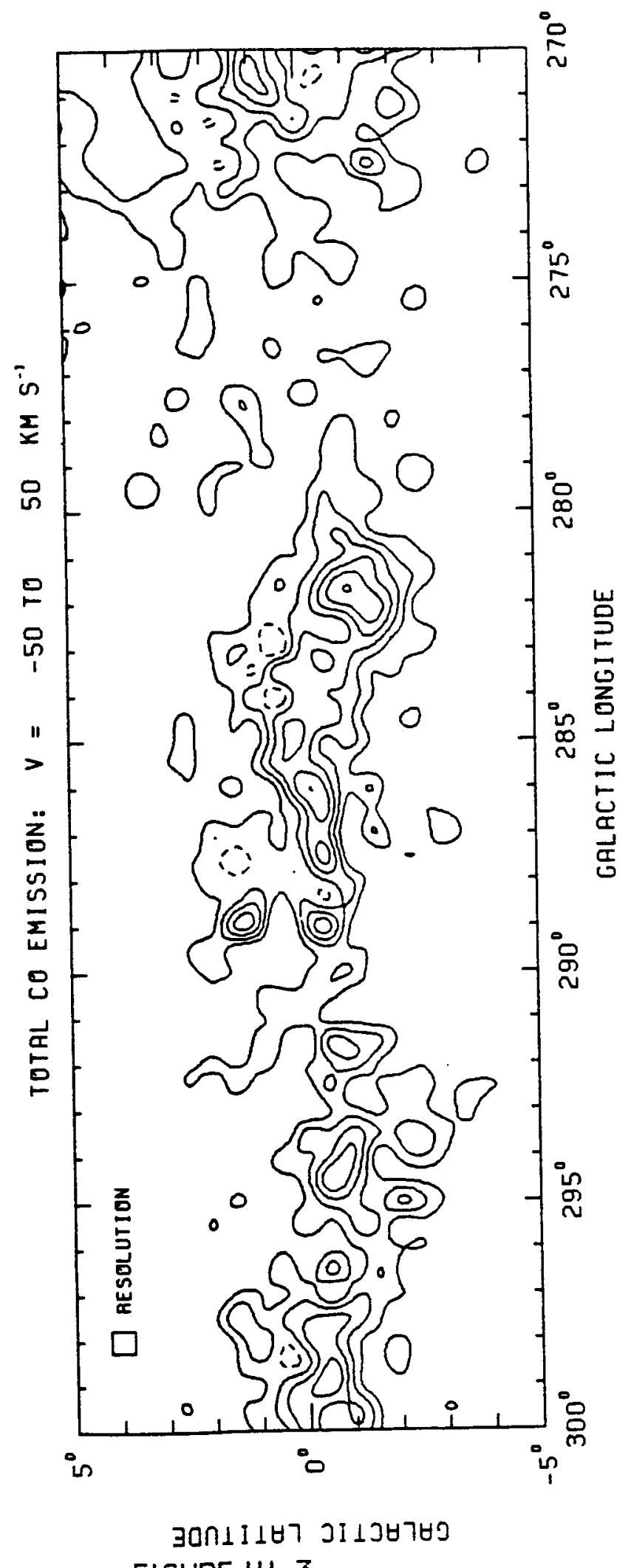

FIGURE $111-3$ 


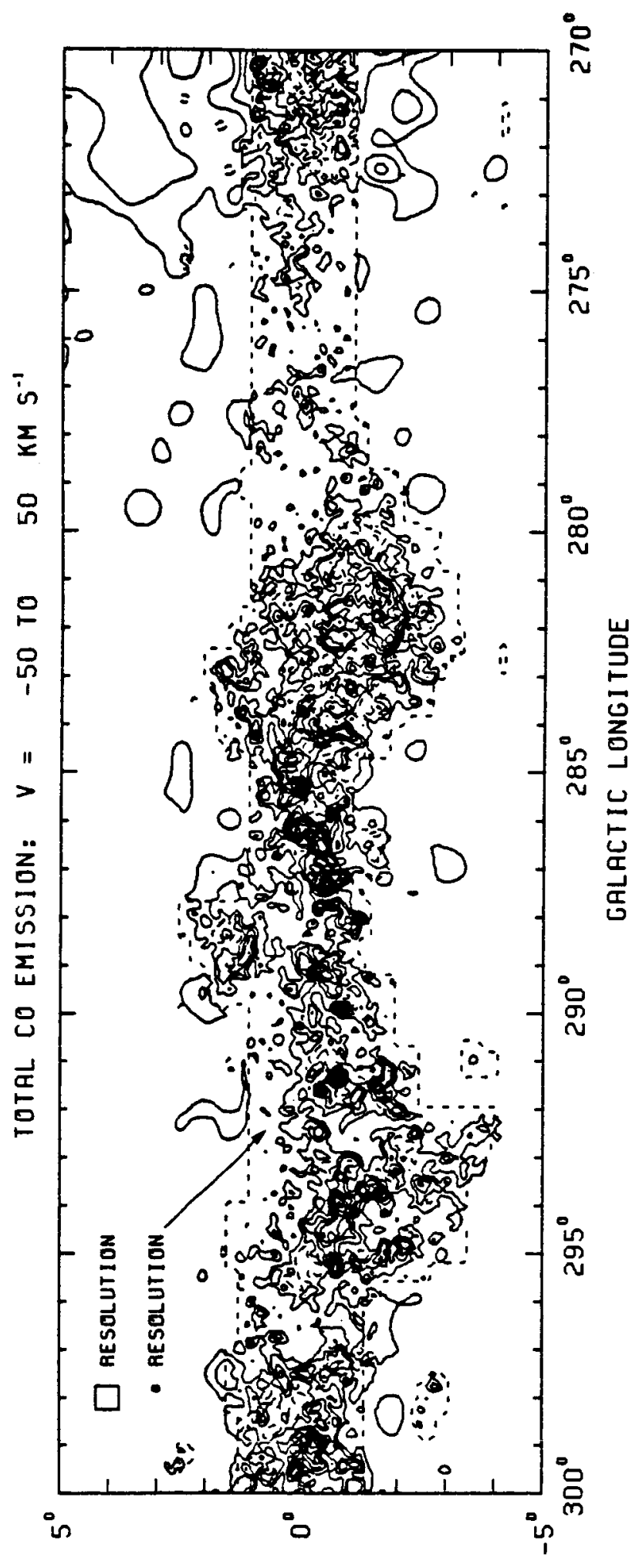

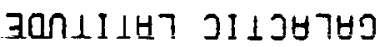

FIGURE III-4 


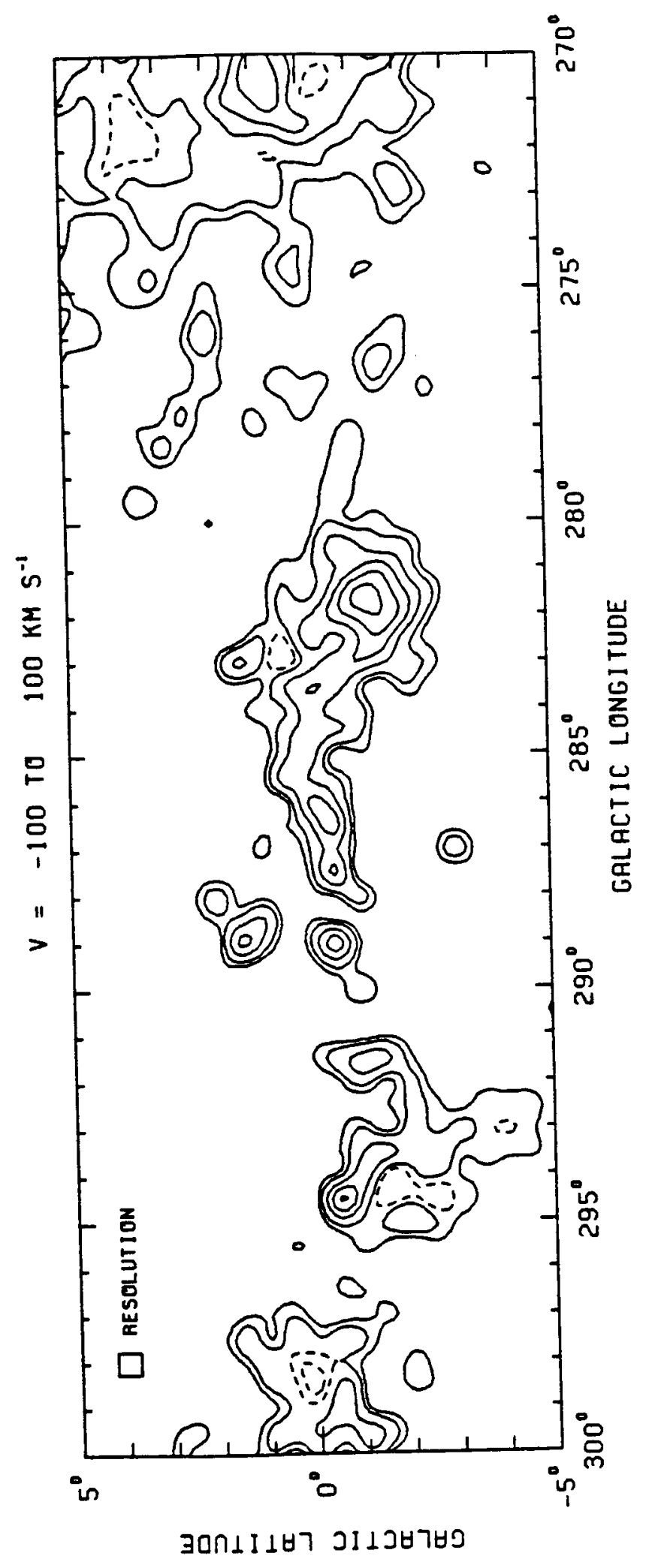

FIGURE 


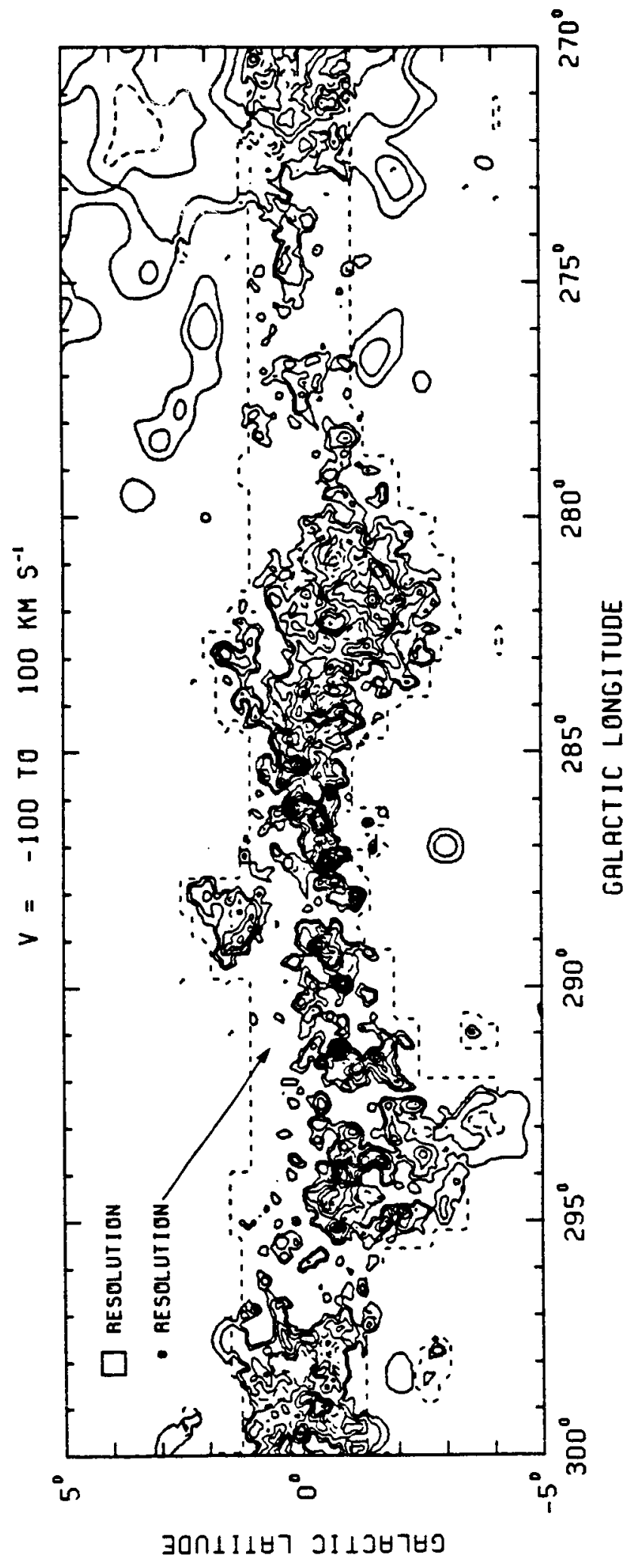

FIGURE III-6 


\section{ORIGINAL PAGE IS \\ OF POOR QUALTT}

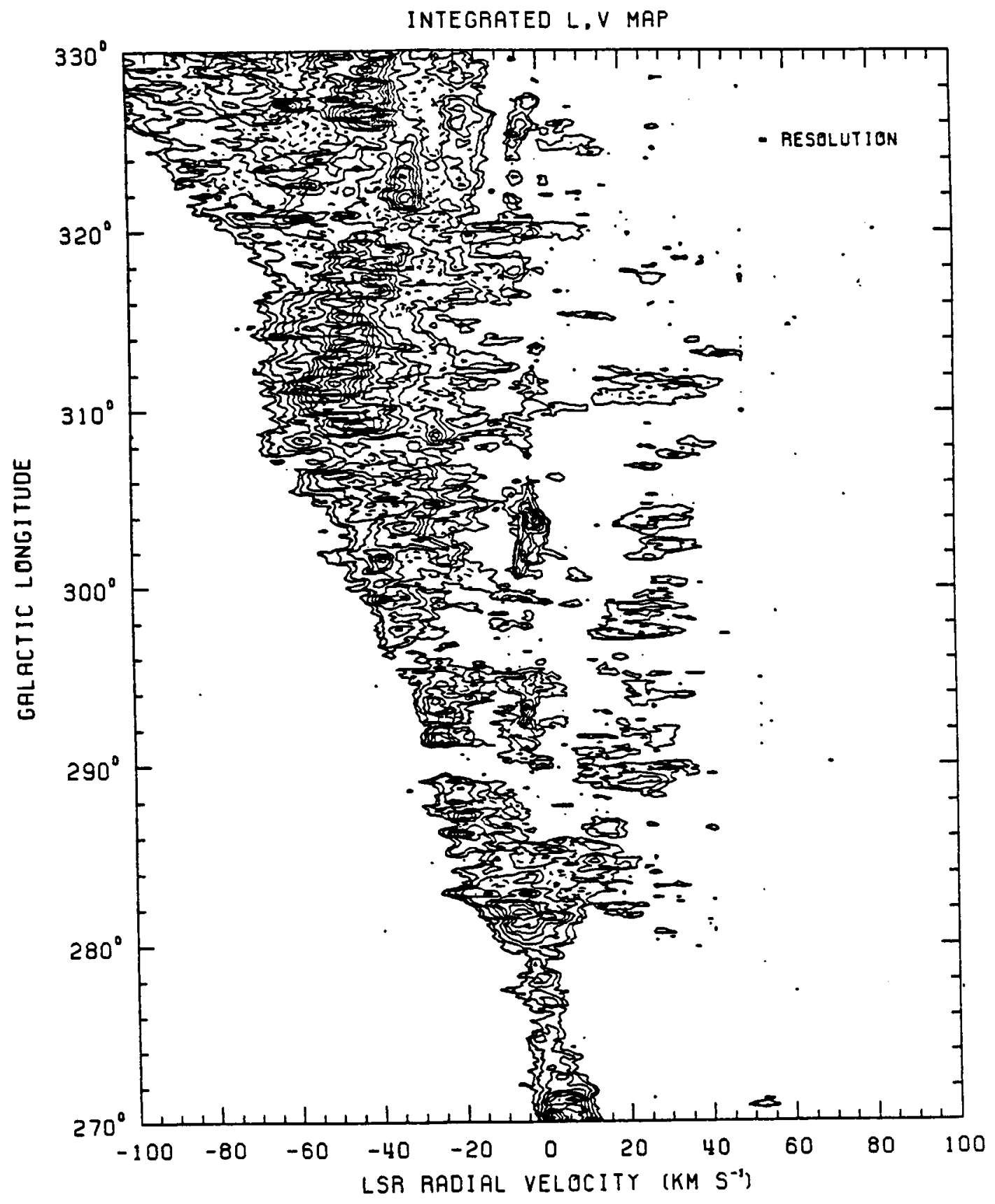

FIGURE $|1|-7$ 


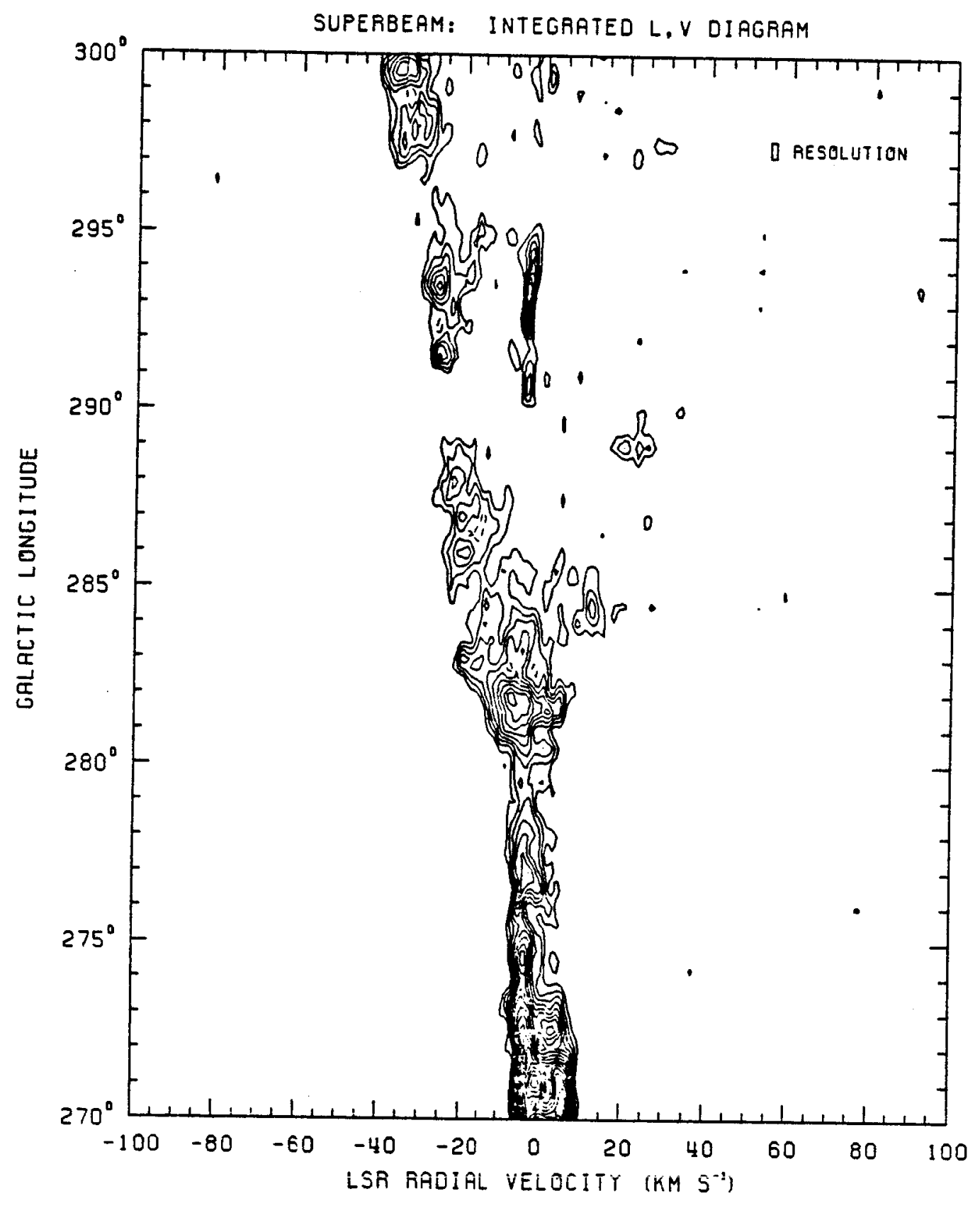

FIGURE III-8 


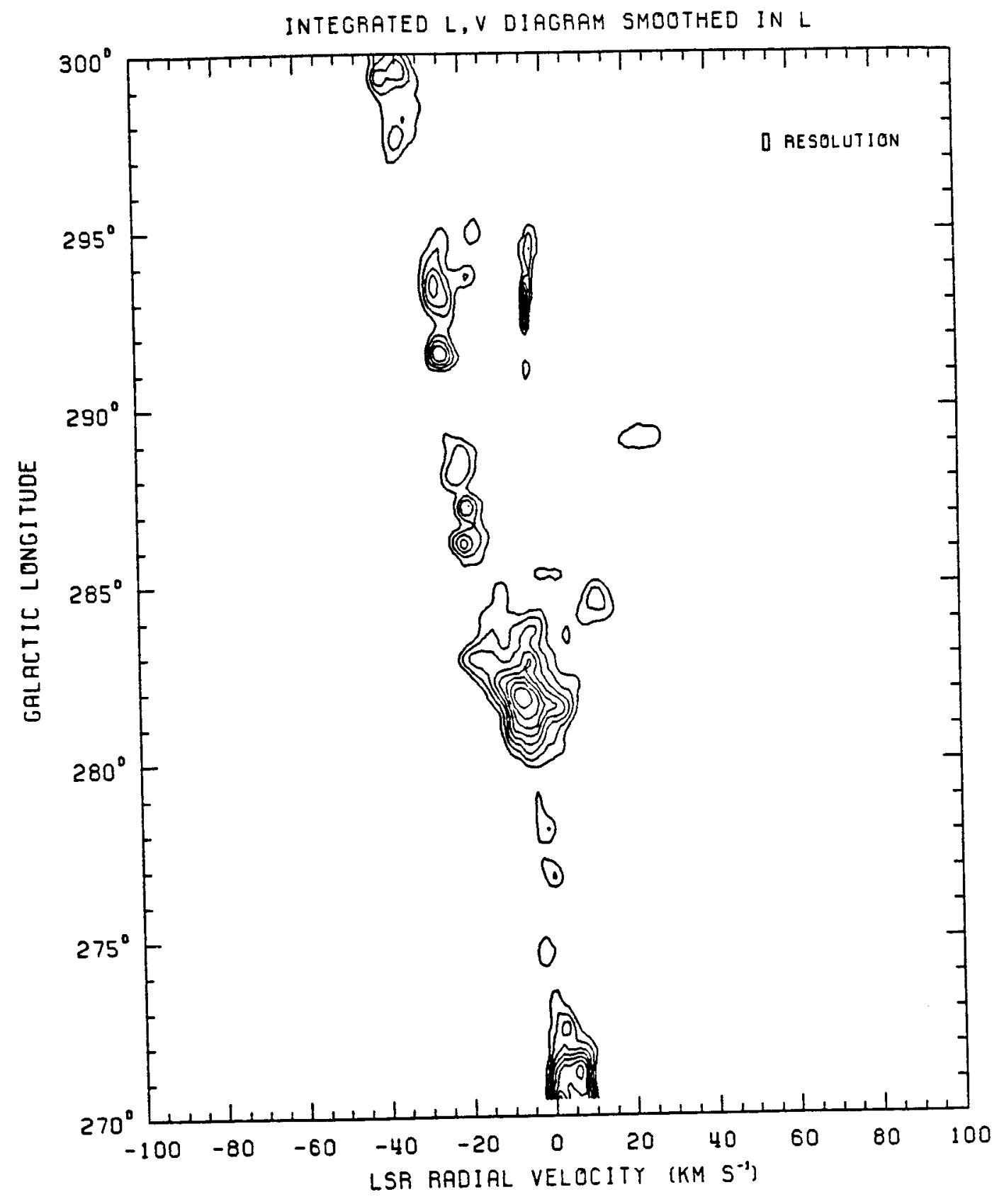

FIGURE $|I|-9$ 


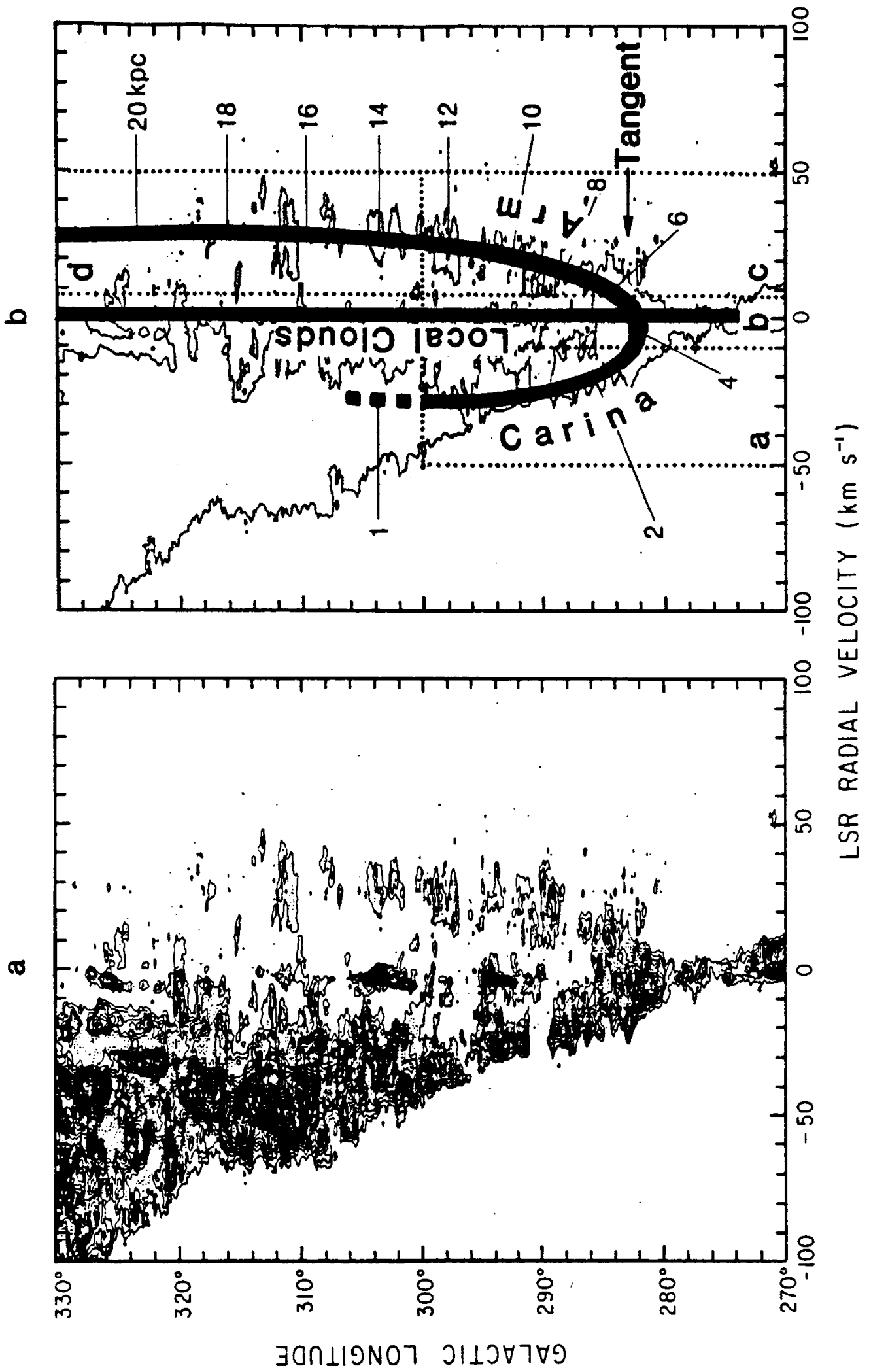

FIGURE IV-1 


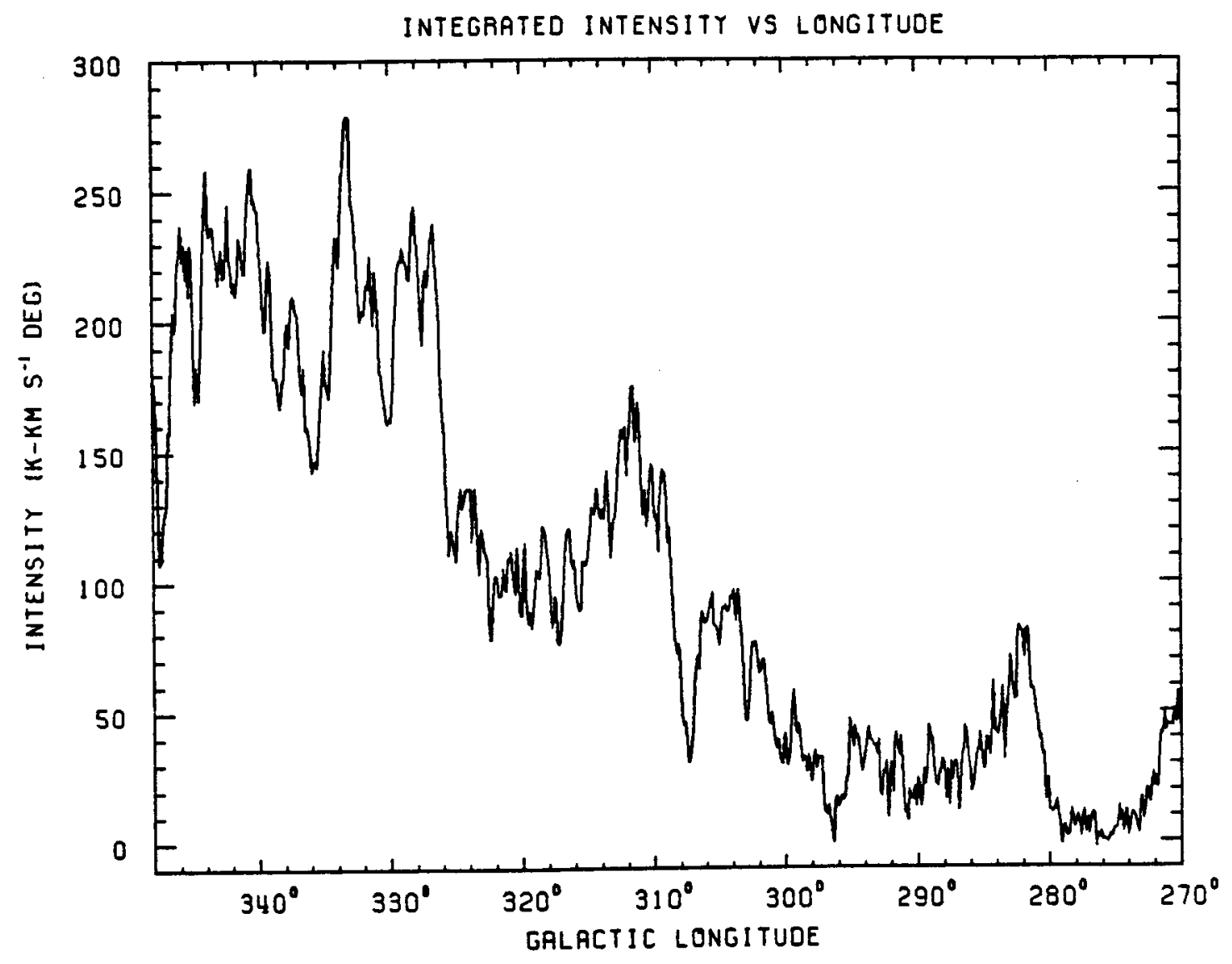

FIGURE IV-2 


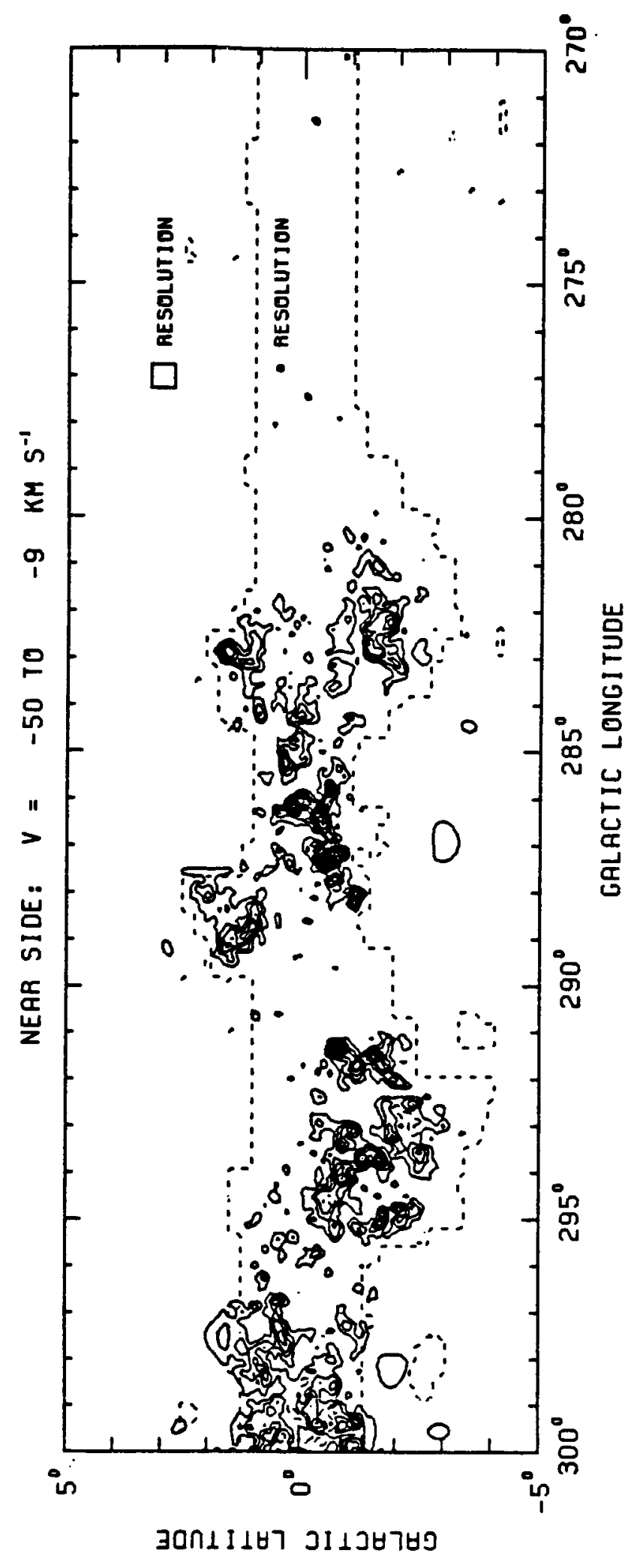

FIGURE IV-sa 


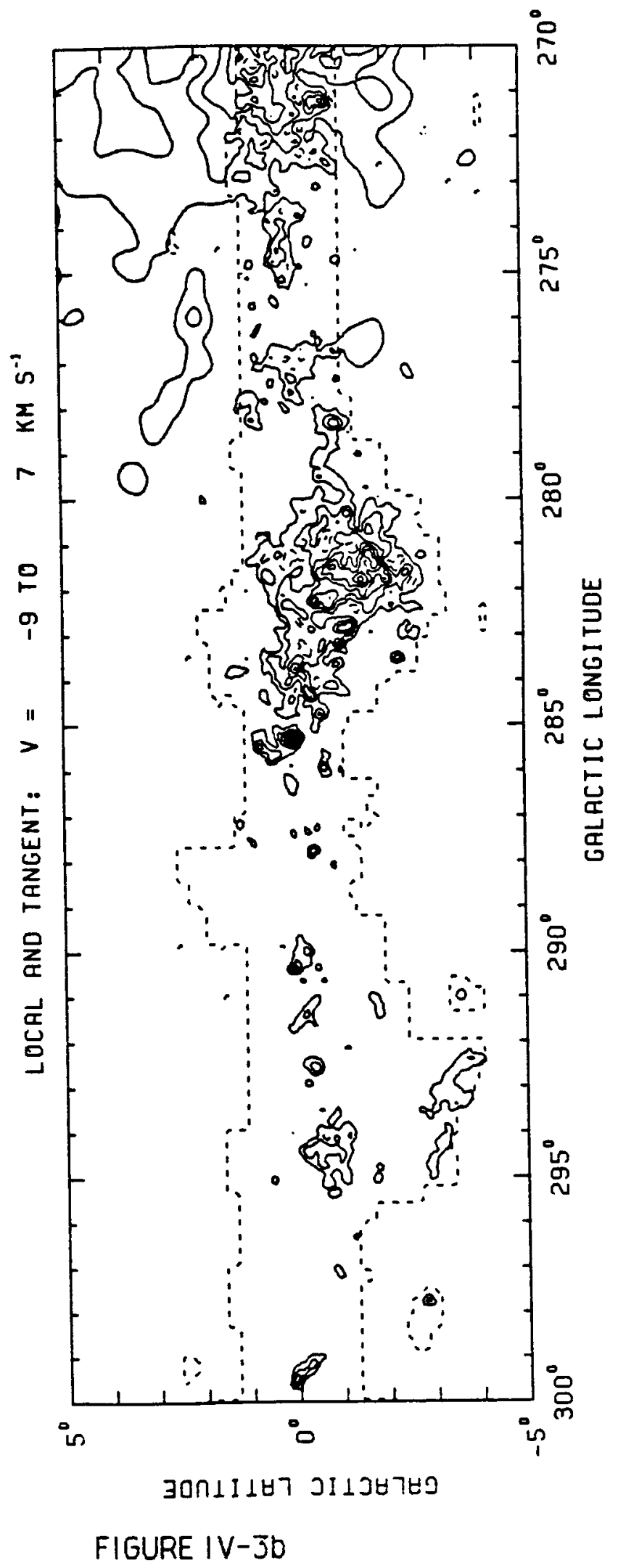

156 
ORIGINAL PAGE IS OF POOR QUALITY

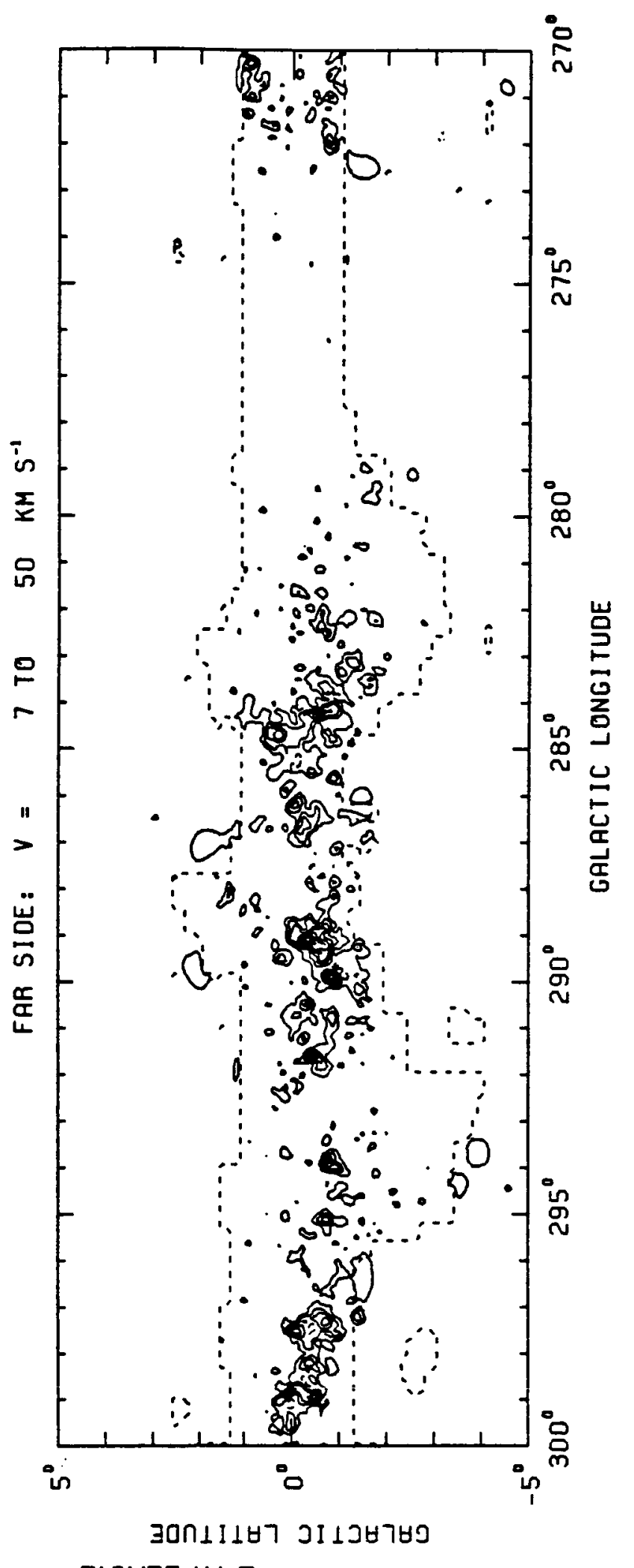

FIGURE IV-JC 


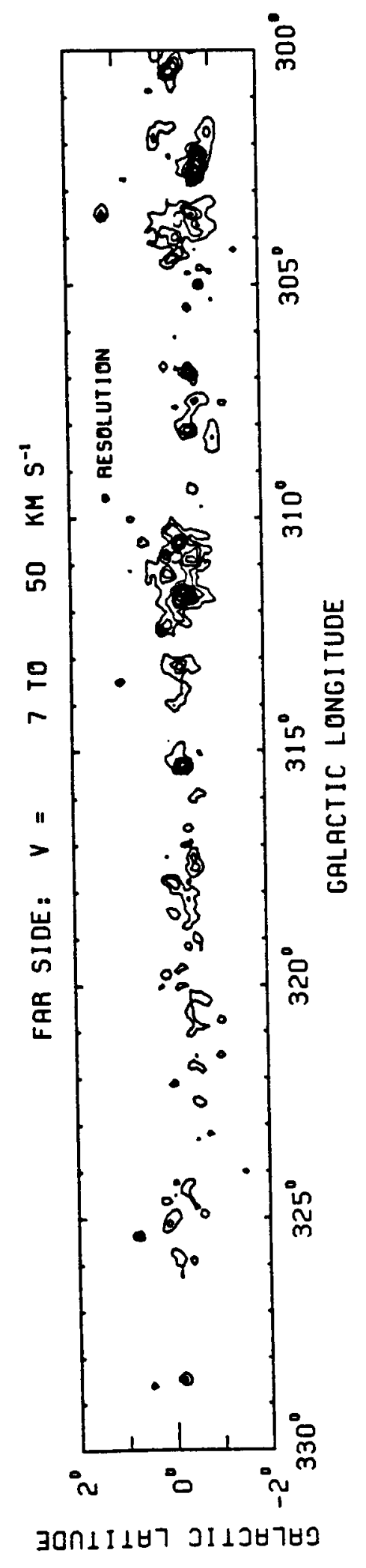

FIGURE IV-3d 


\section{ORIGINAL PAGE IS
OF PCOR GI:ALITY}

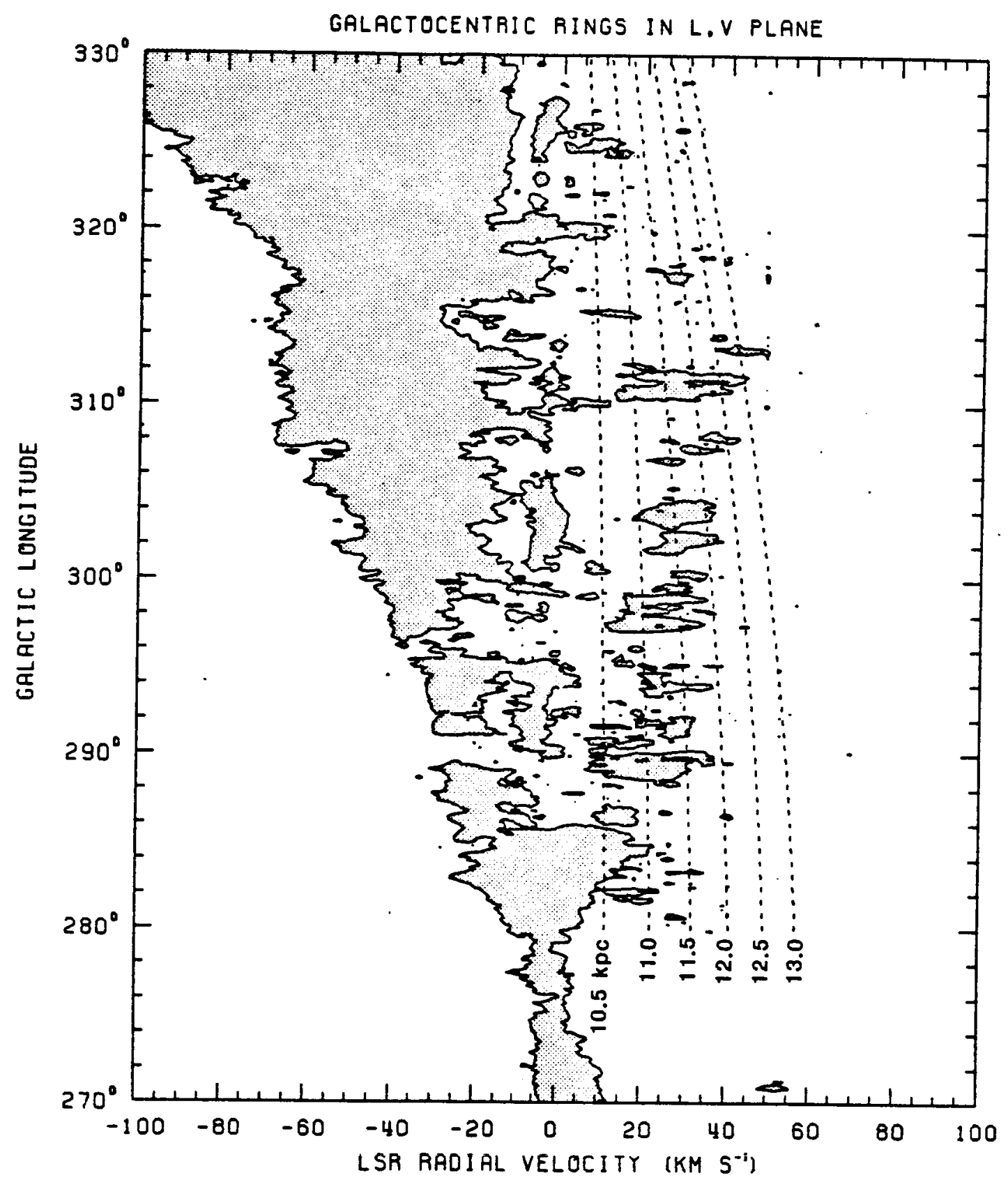

FIGURE IV-4 

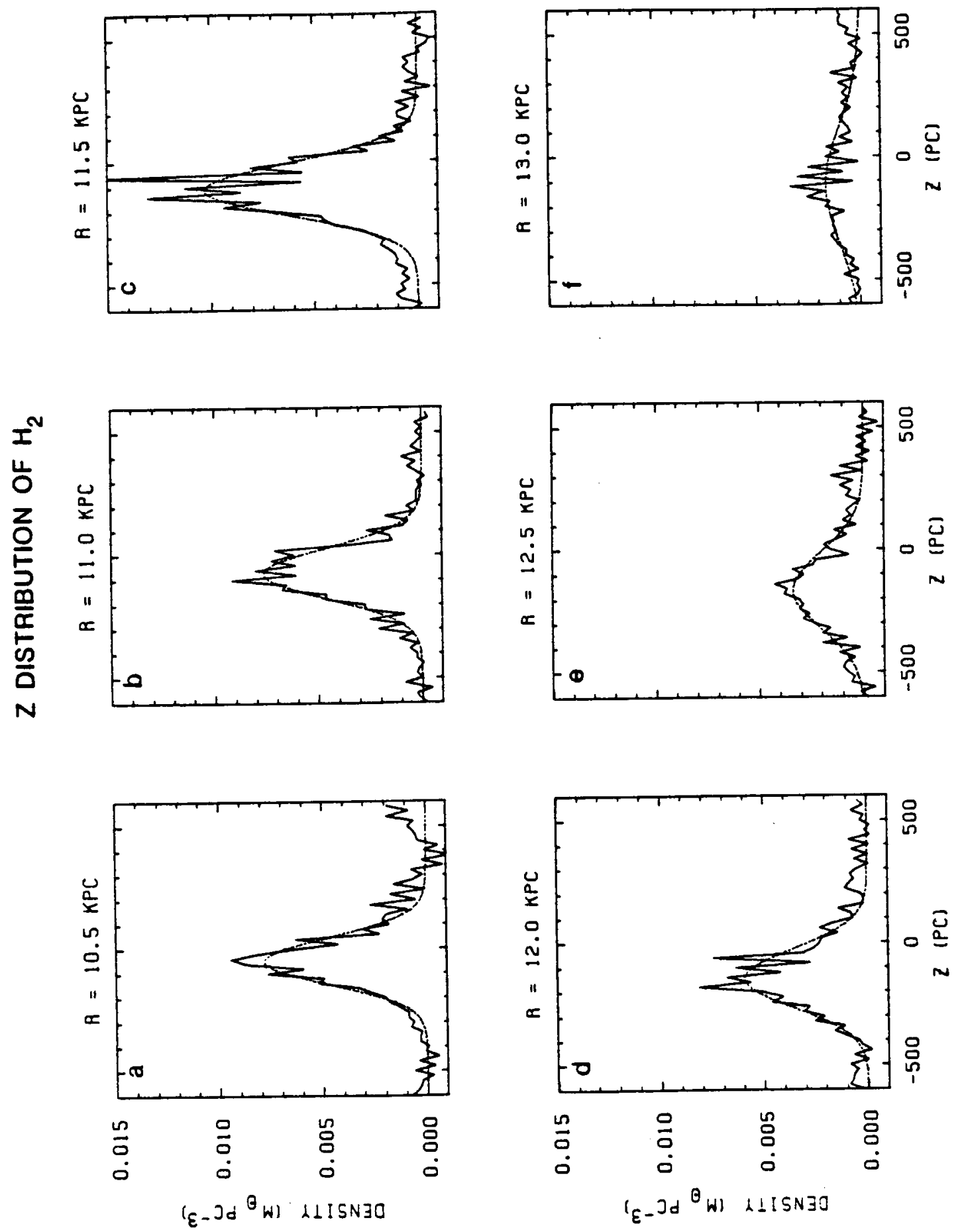

FIGURE IV-5 


\section{ORIGINAL PAGE IS
OF POOR QUALITY}

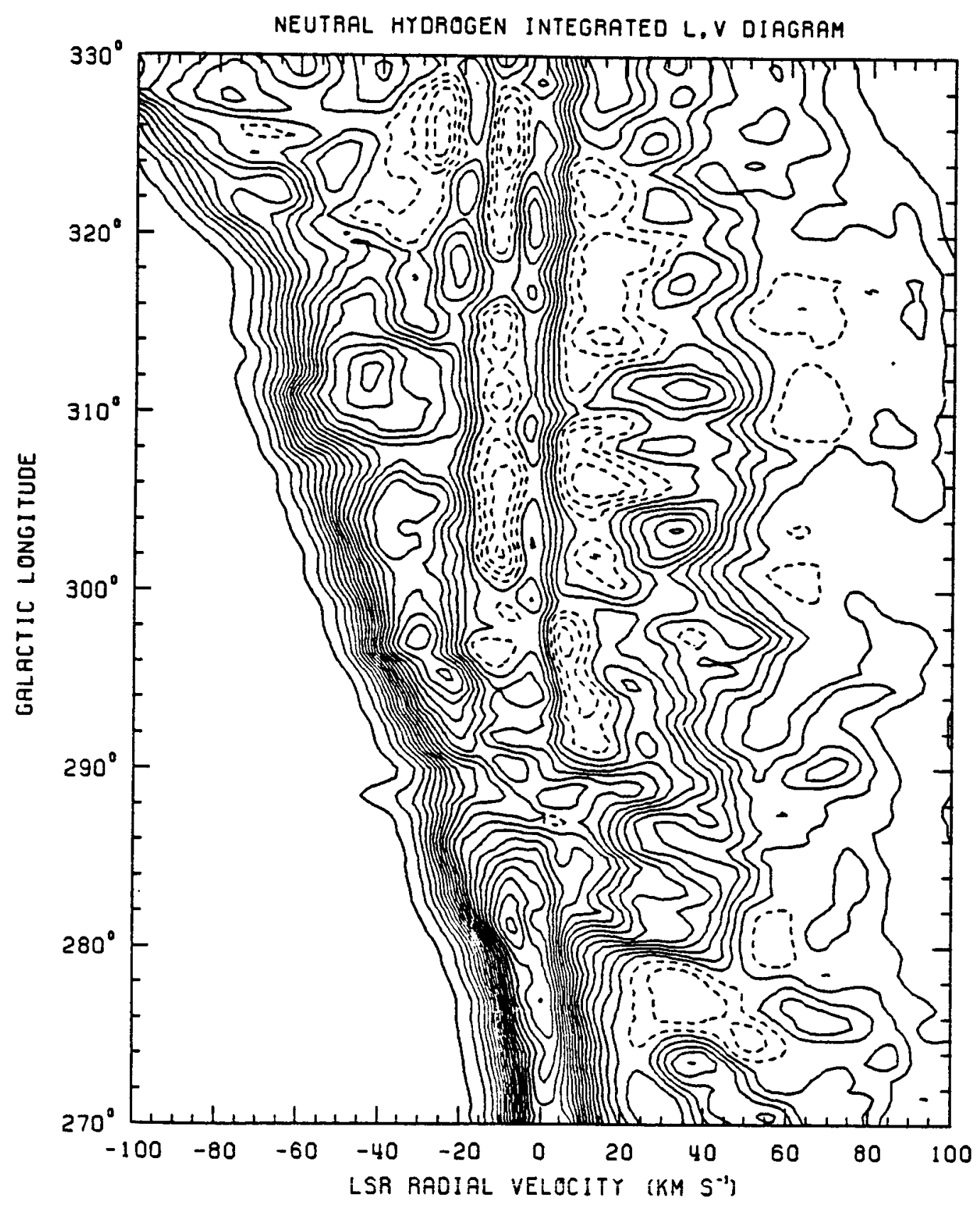

FIGURE IV-6 


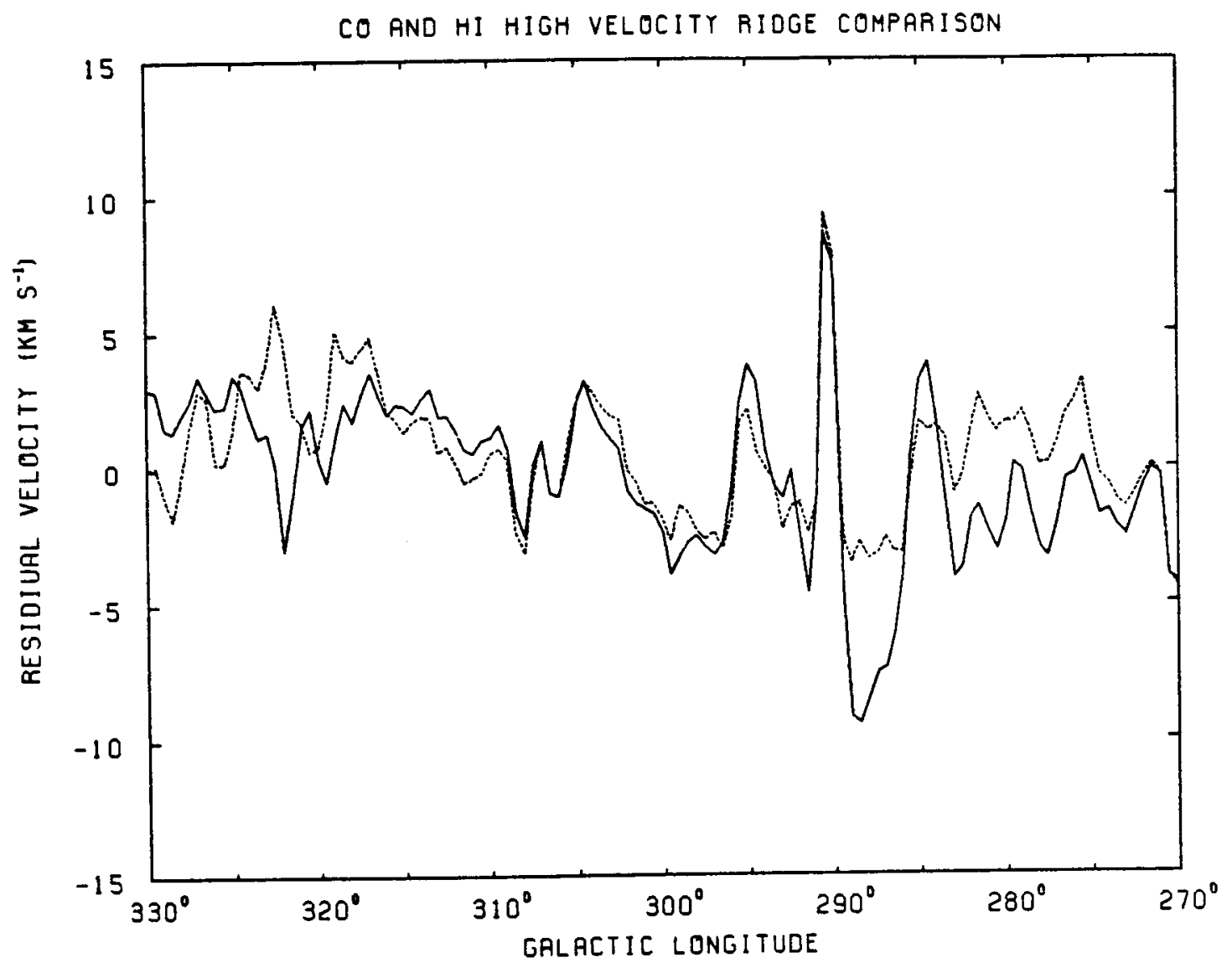

FIGURE IV-7 
ORIGINAL PAOE IS

OF. POOR QUALITY

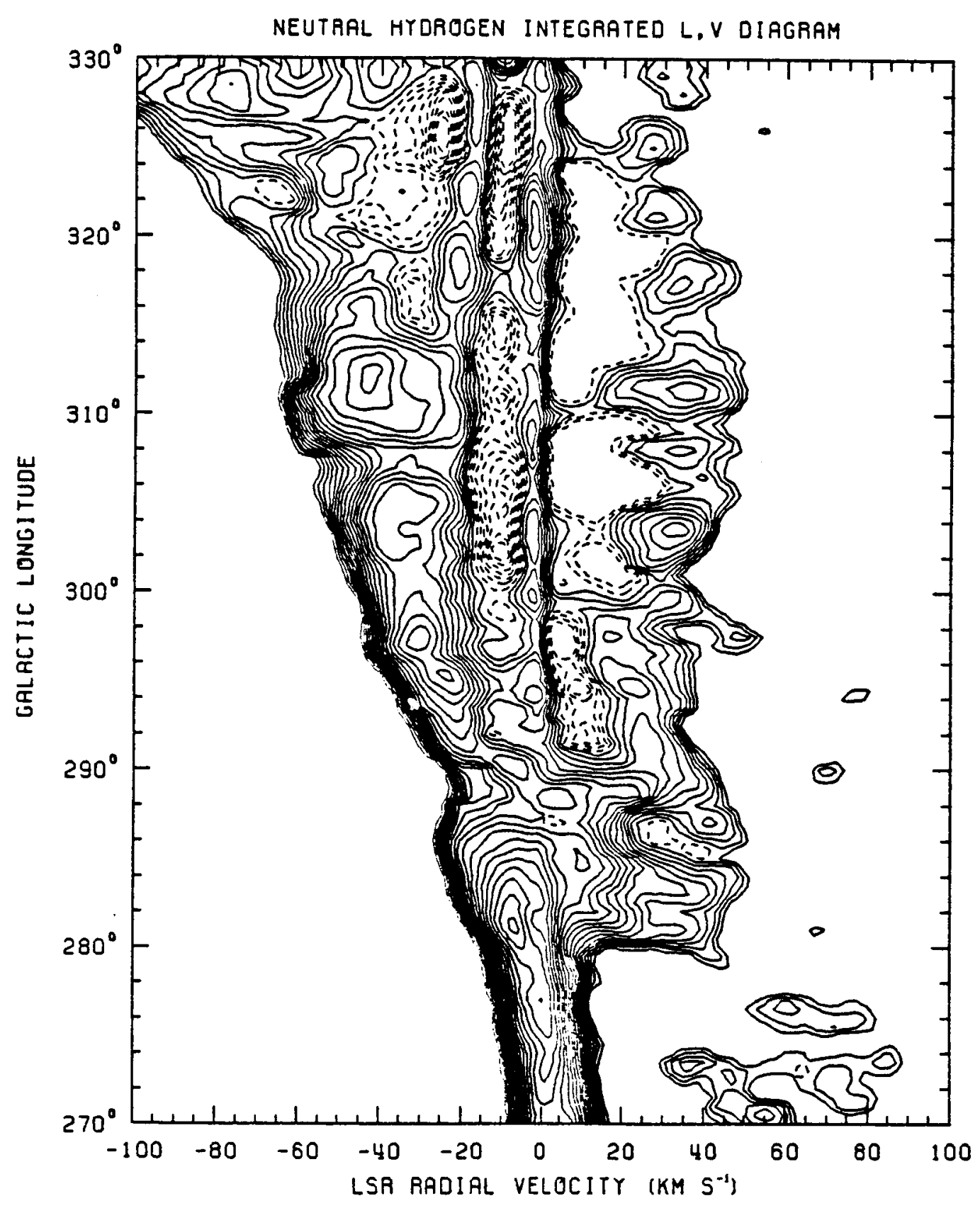

FIGURE IV-8 


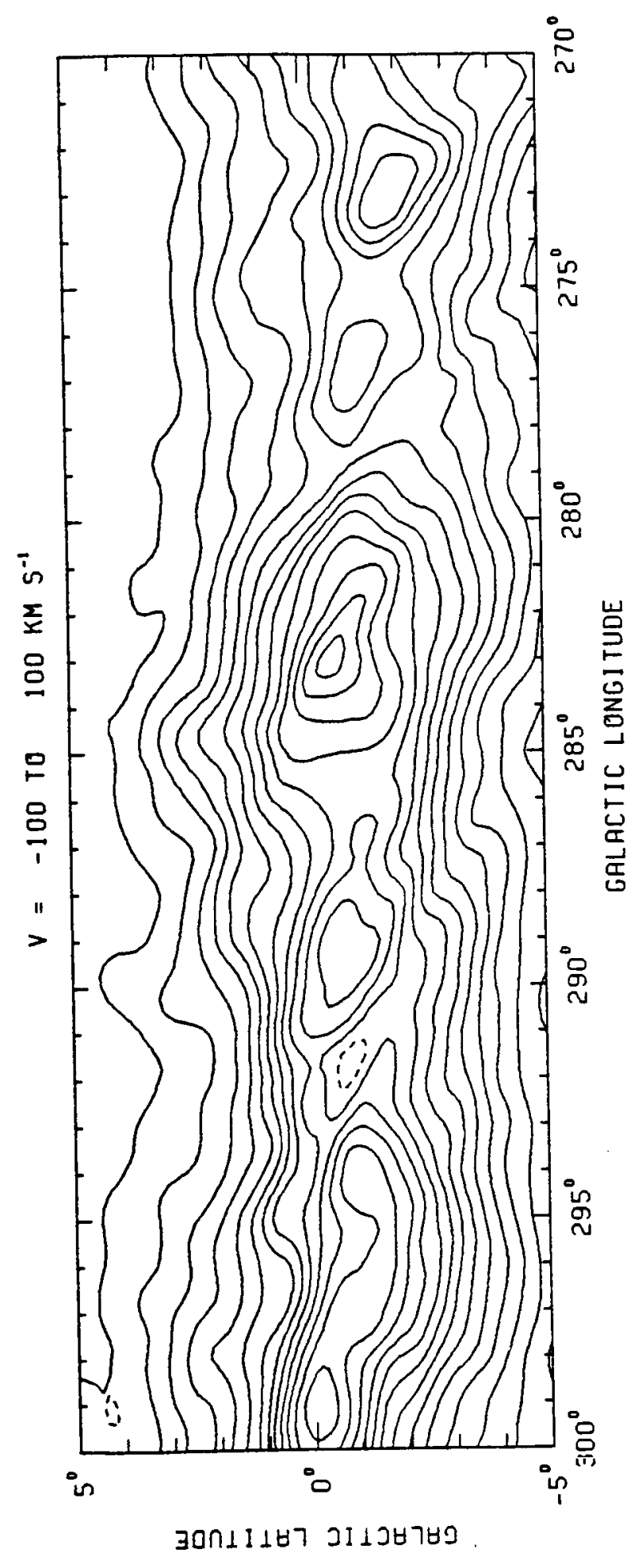

FIGURE IV-9 


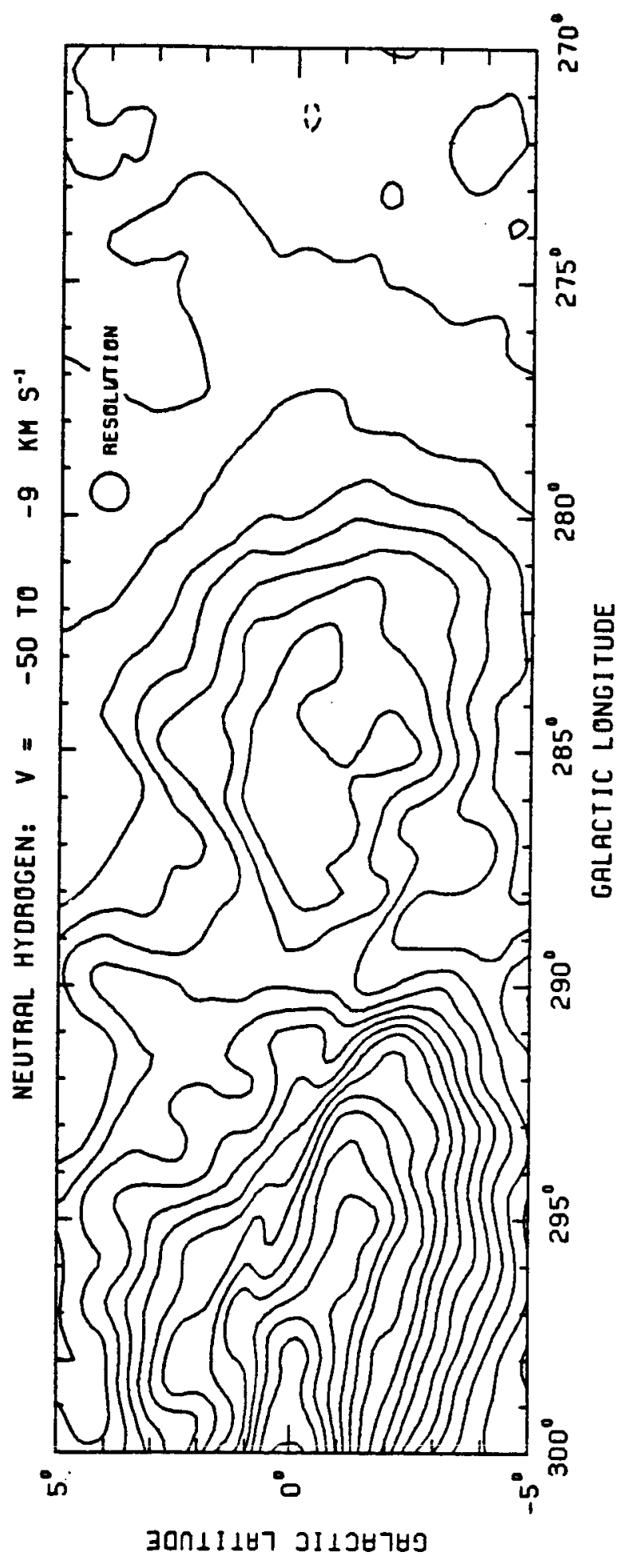

FIGURE IV-10a

165 


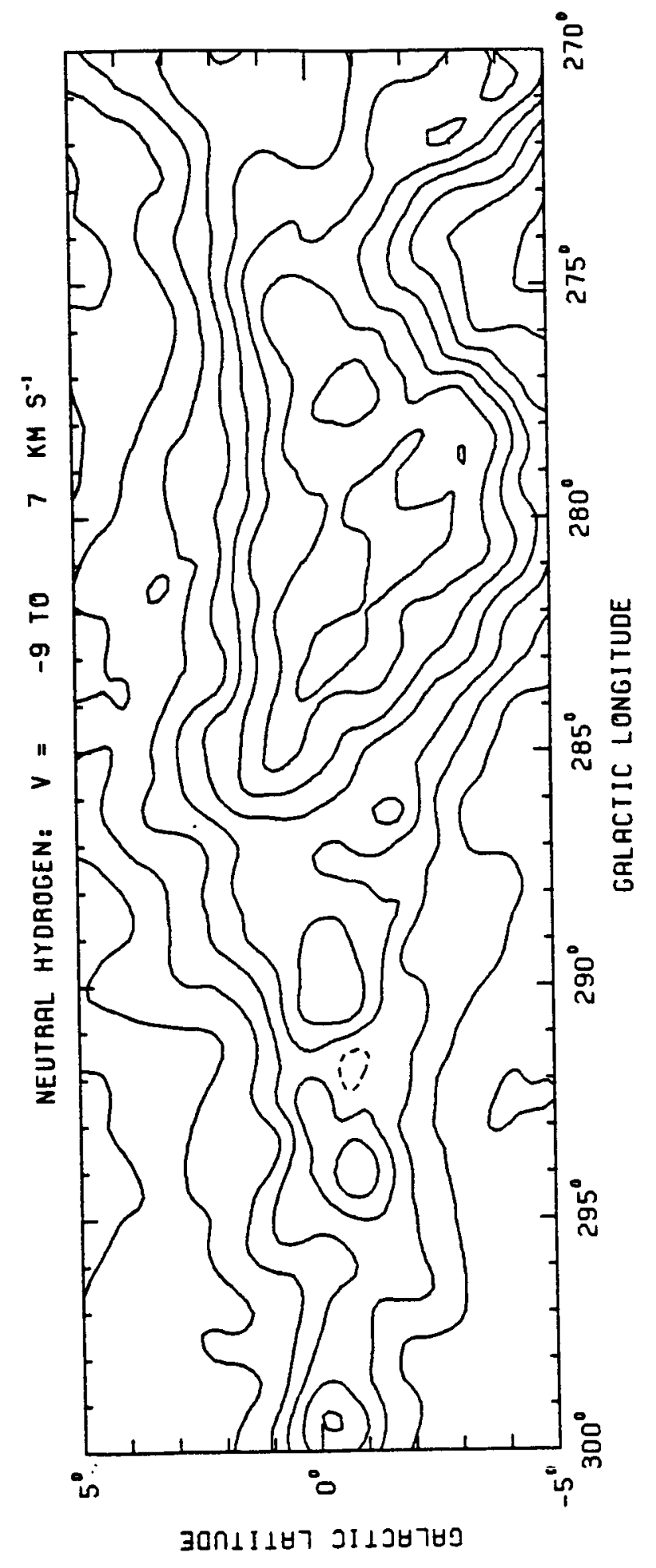

FIGURE IV-10D 


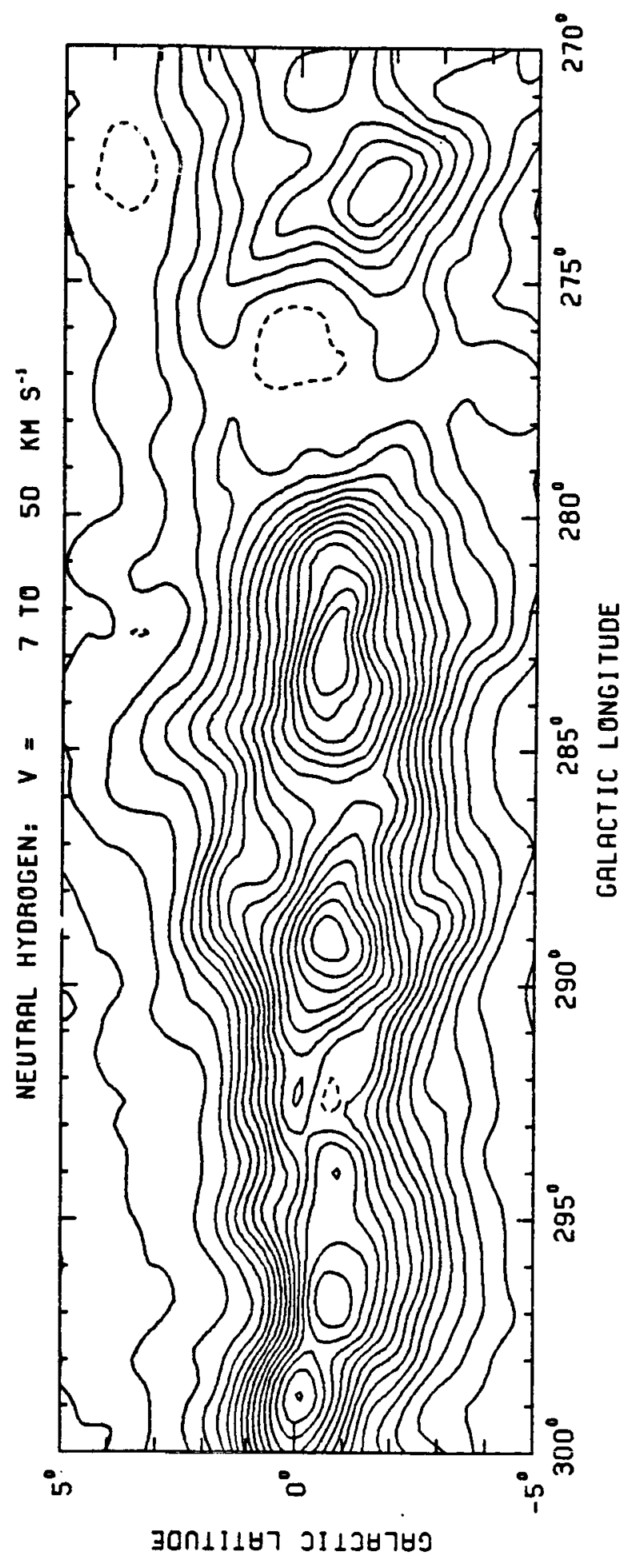

FIGURE IV-10C 


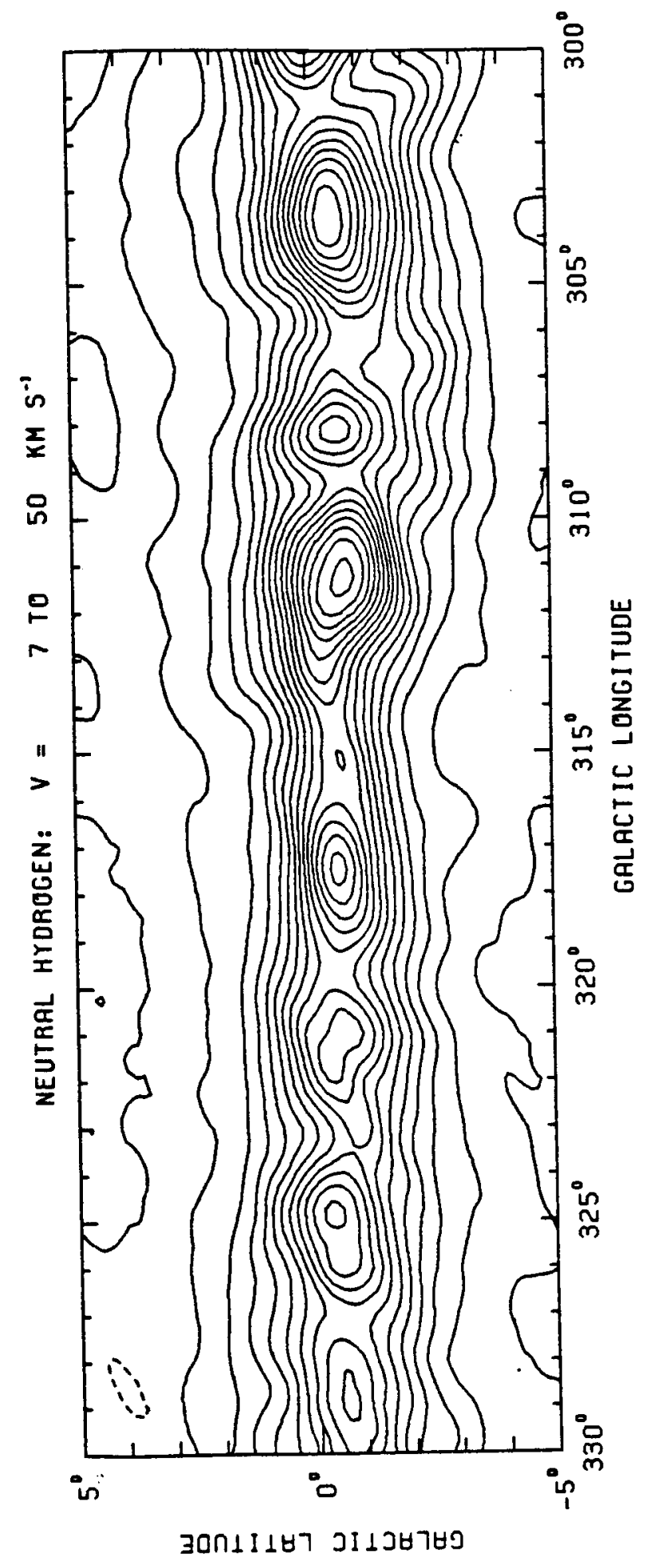

FIGURE IV-IOd 

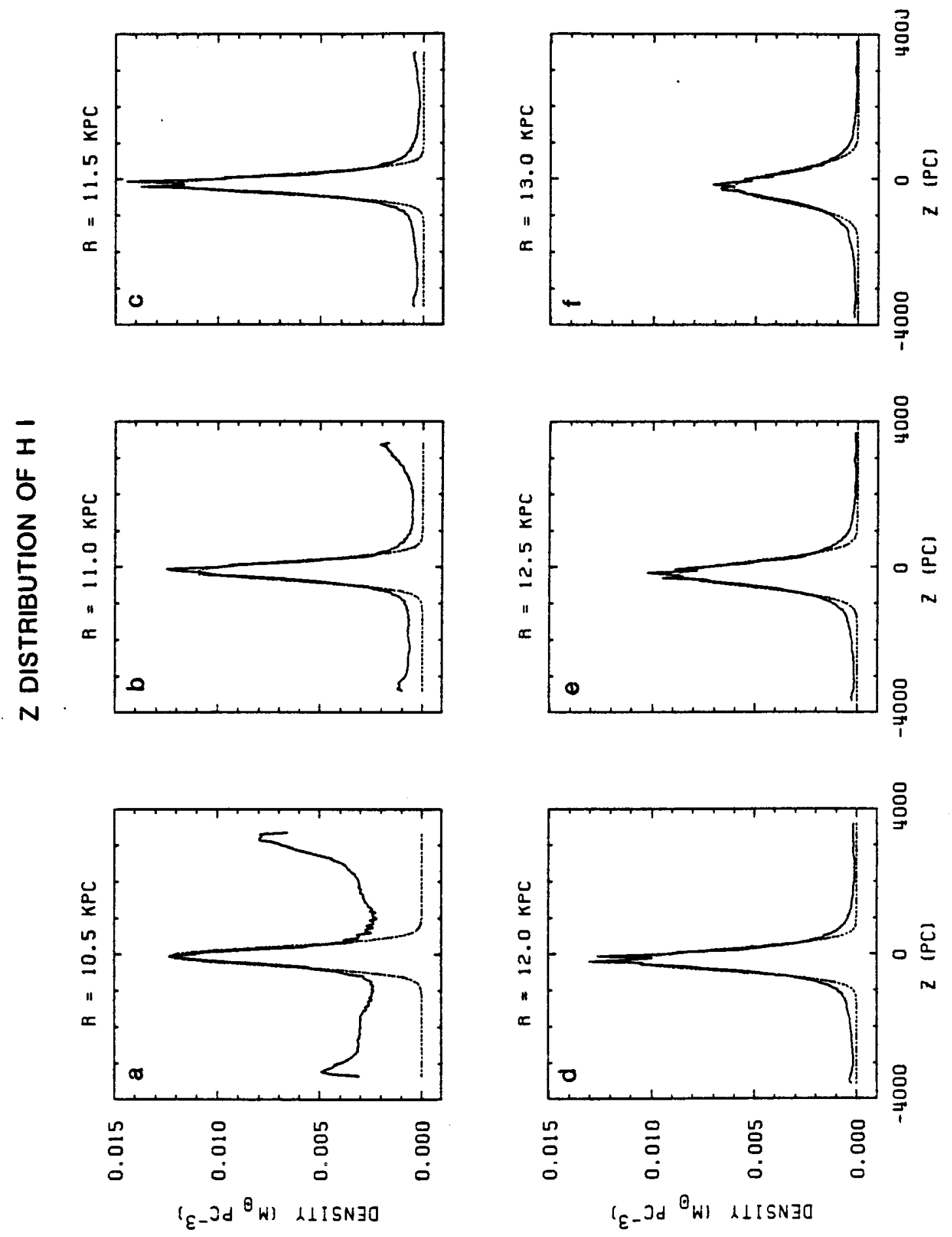

FIGURE IV $-1 \mid$ 

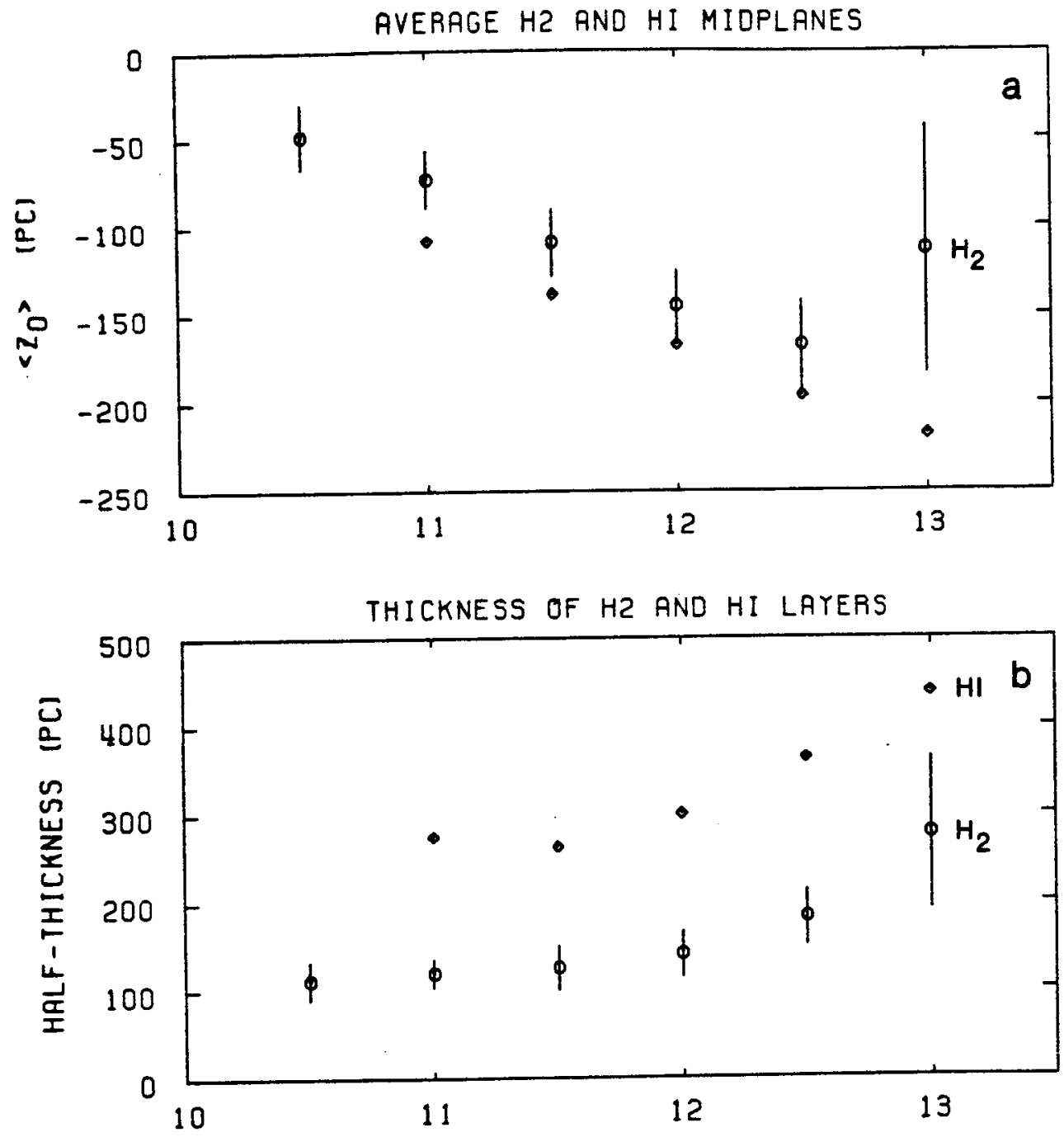

H2 AND HI SURFACE DENSITIES

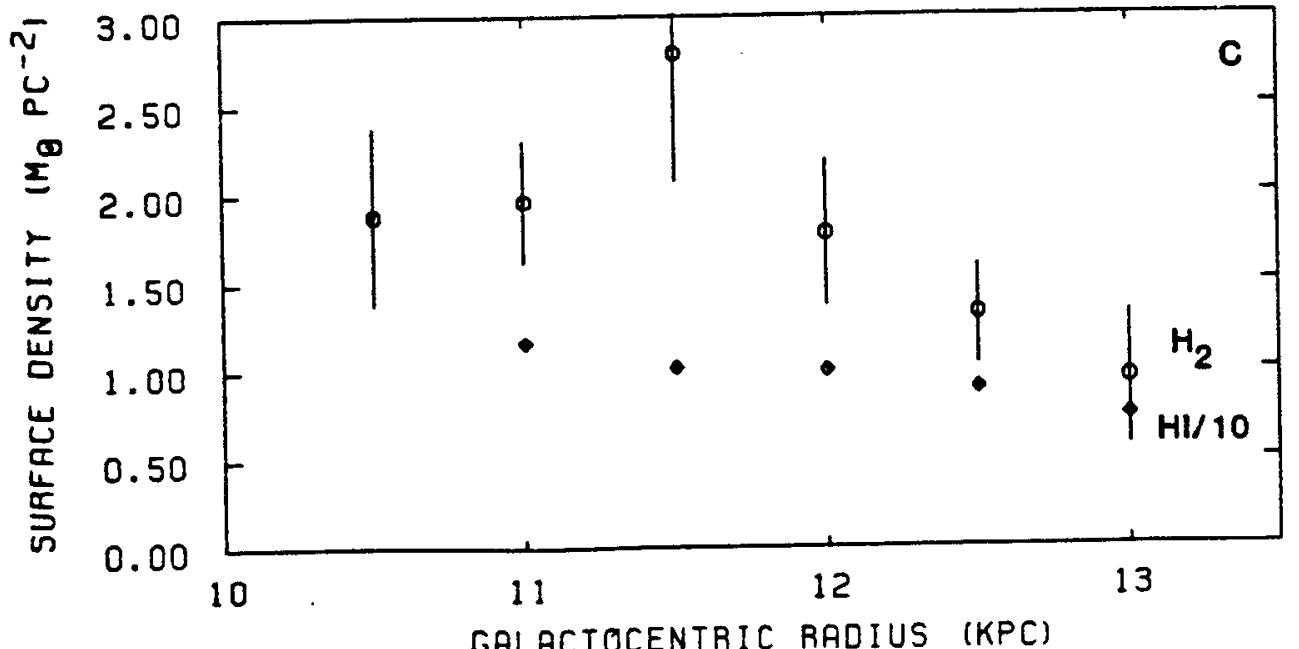

FIGURE IV-12 
ORIGINAL PAGE IS

OF POOR OIALITY

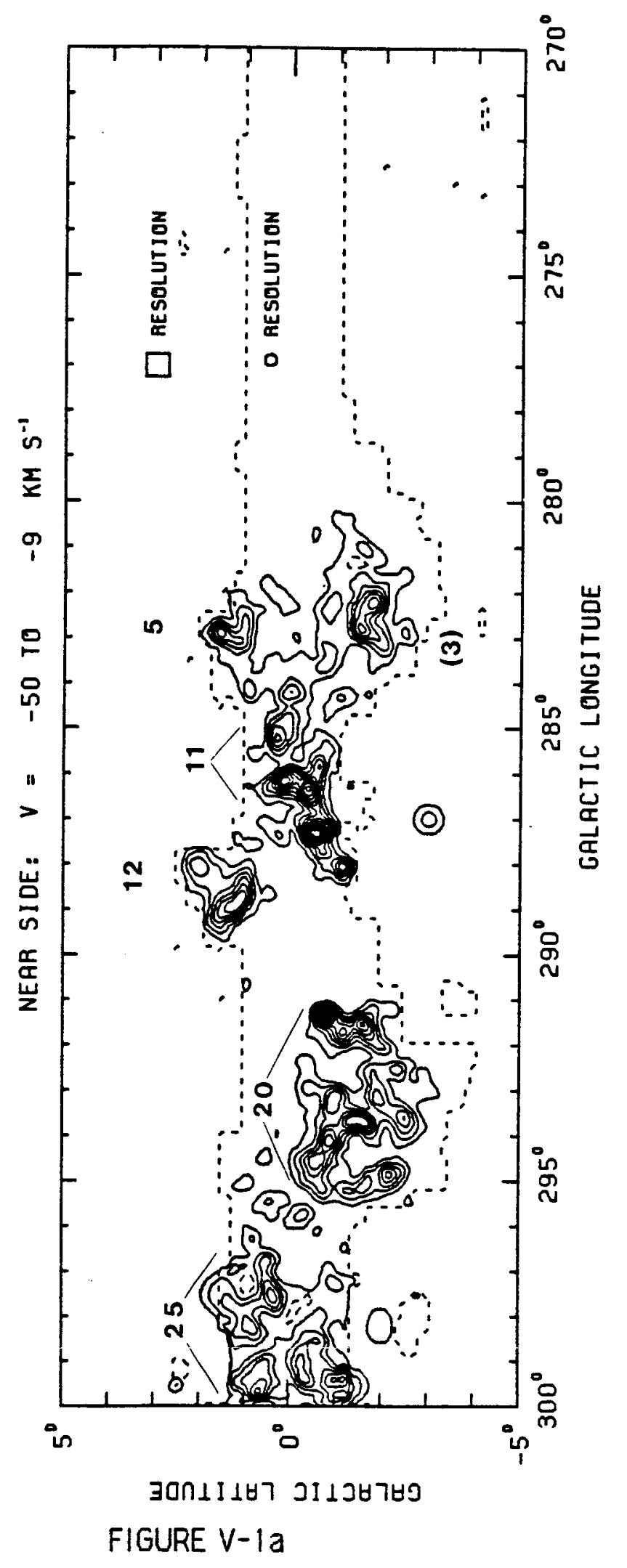




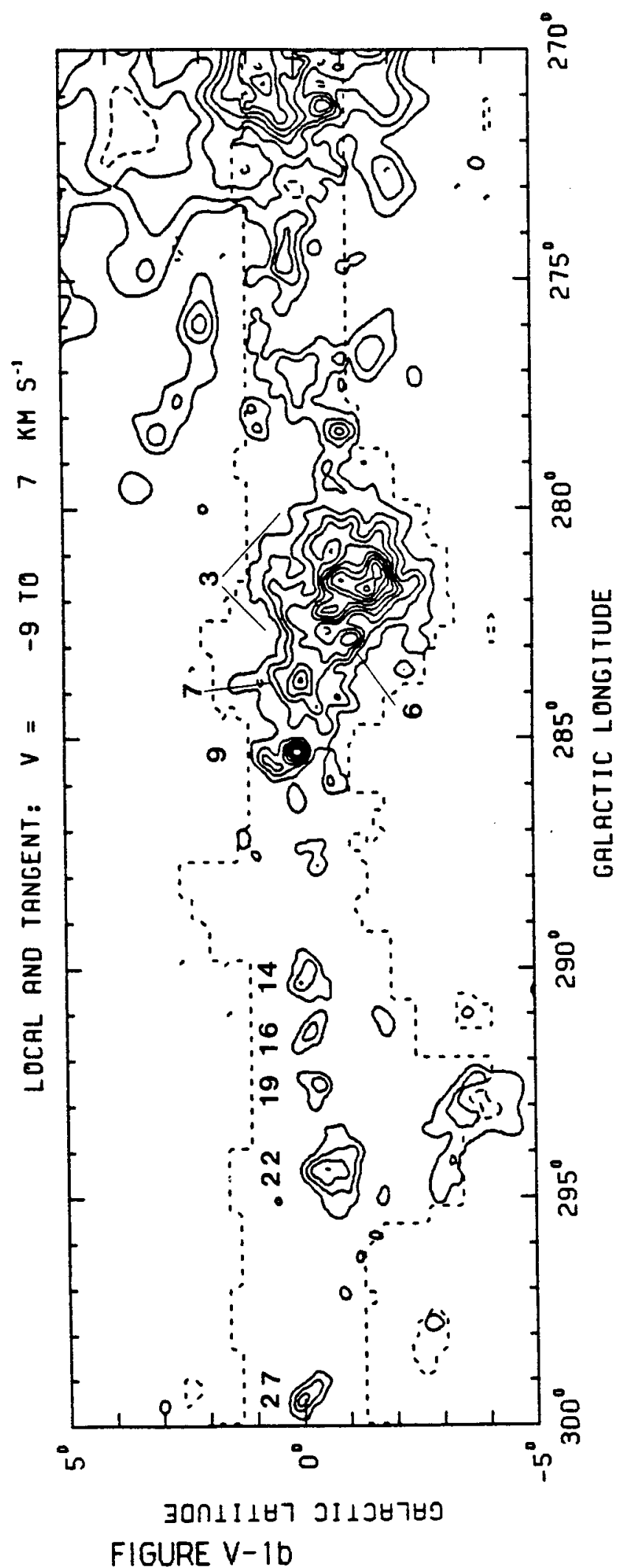




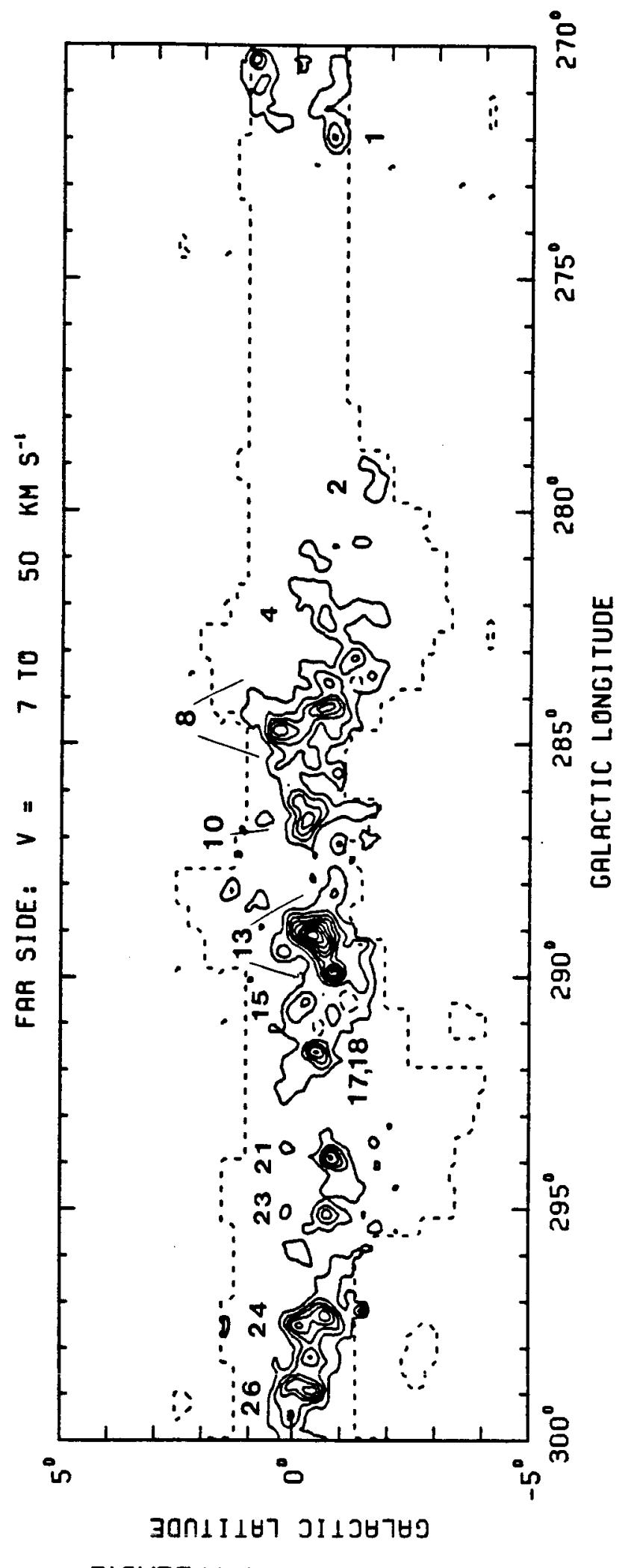

FIGURE $V-1 C$ 


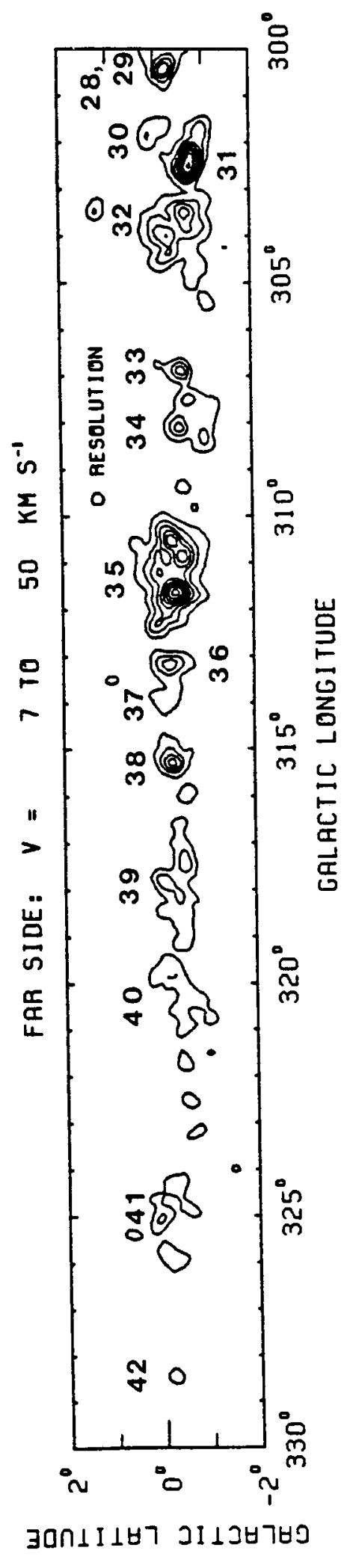

FIGURE $V-1 d$ 


\section{ORIGINAL PAGE OF POOR QUALITY}

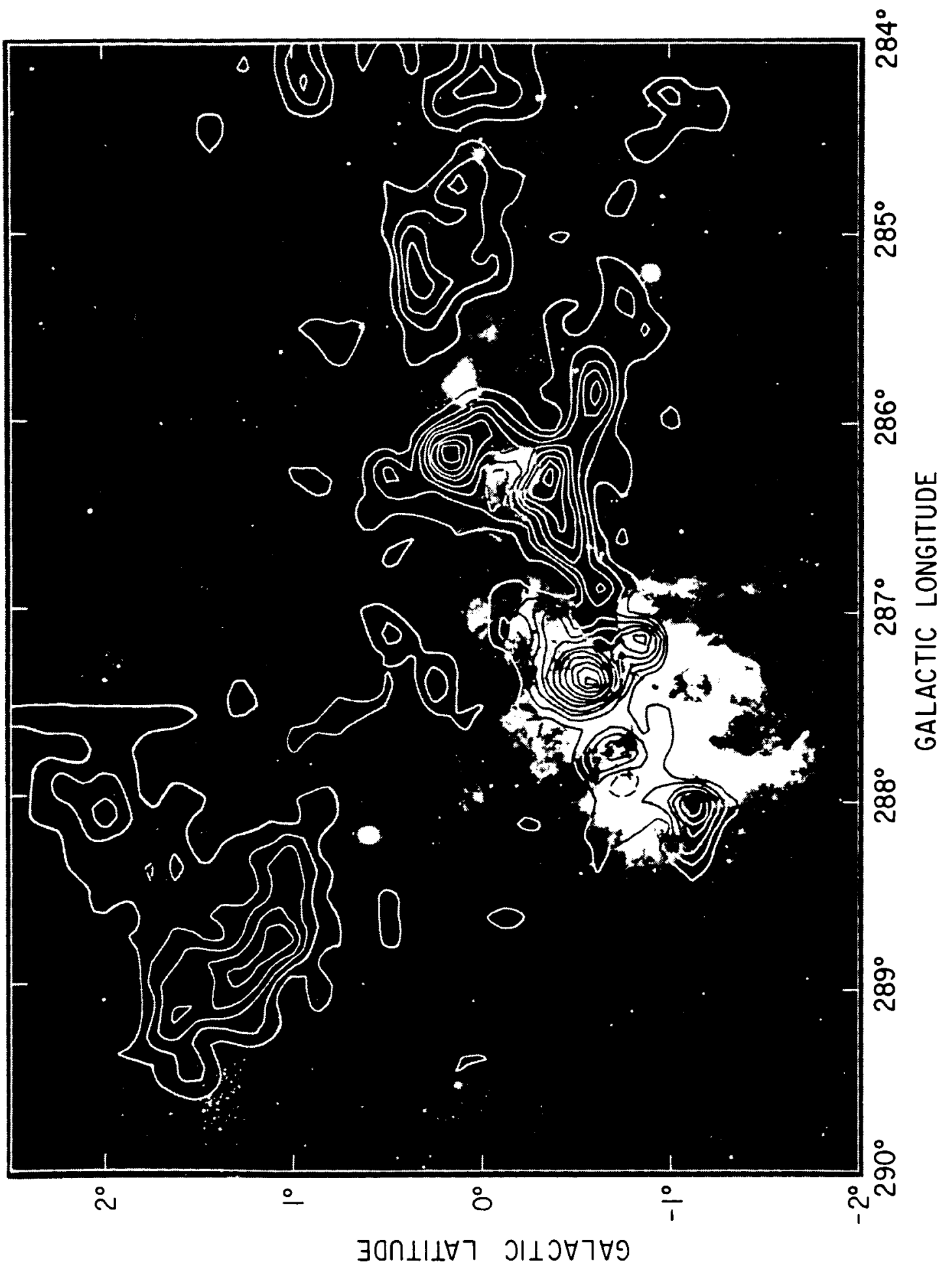

FIGURE $V-2$ 


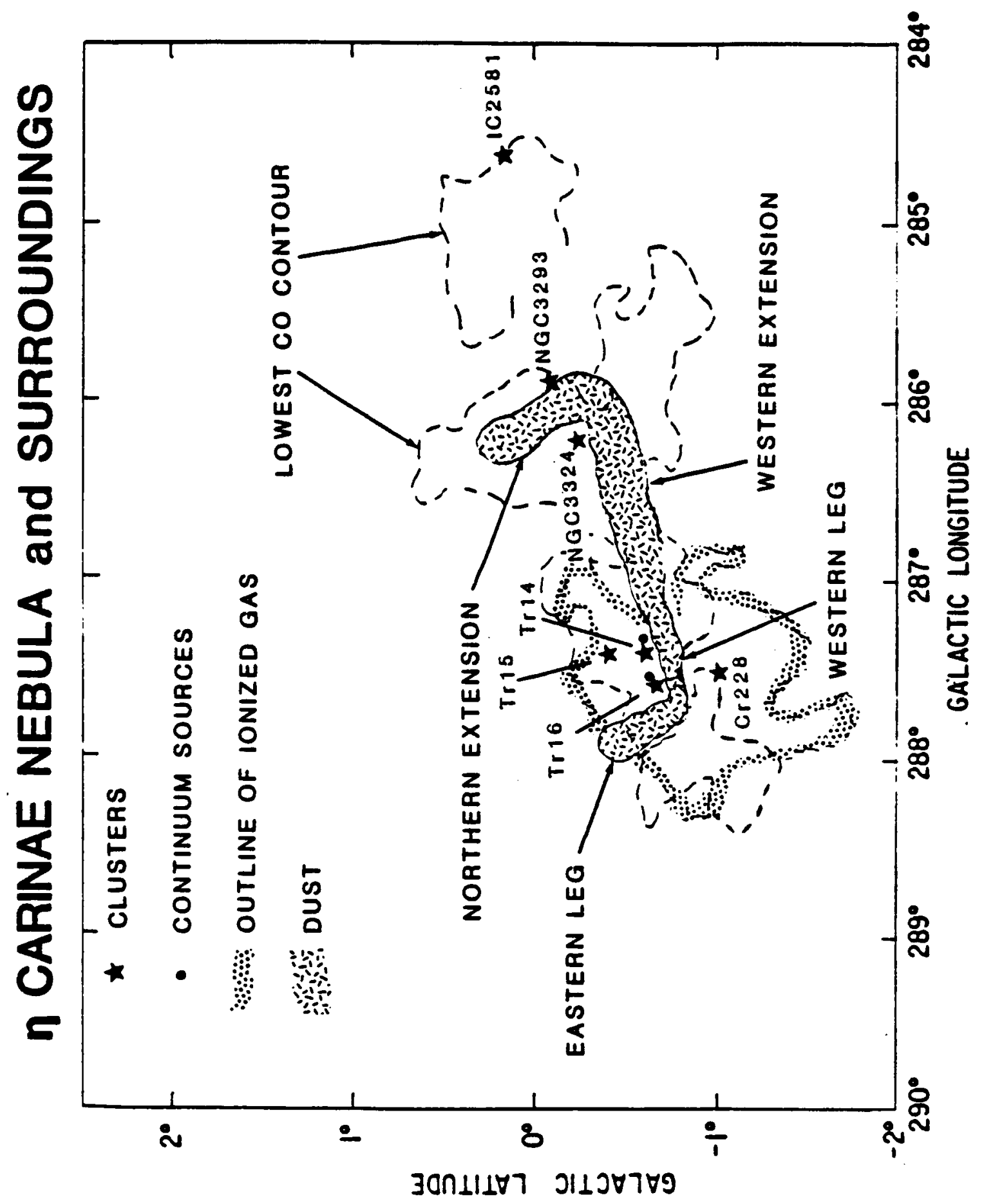

FIGURE V-3 

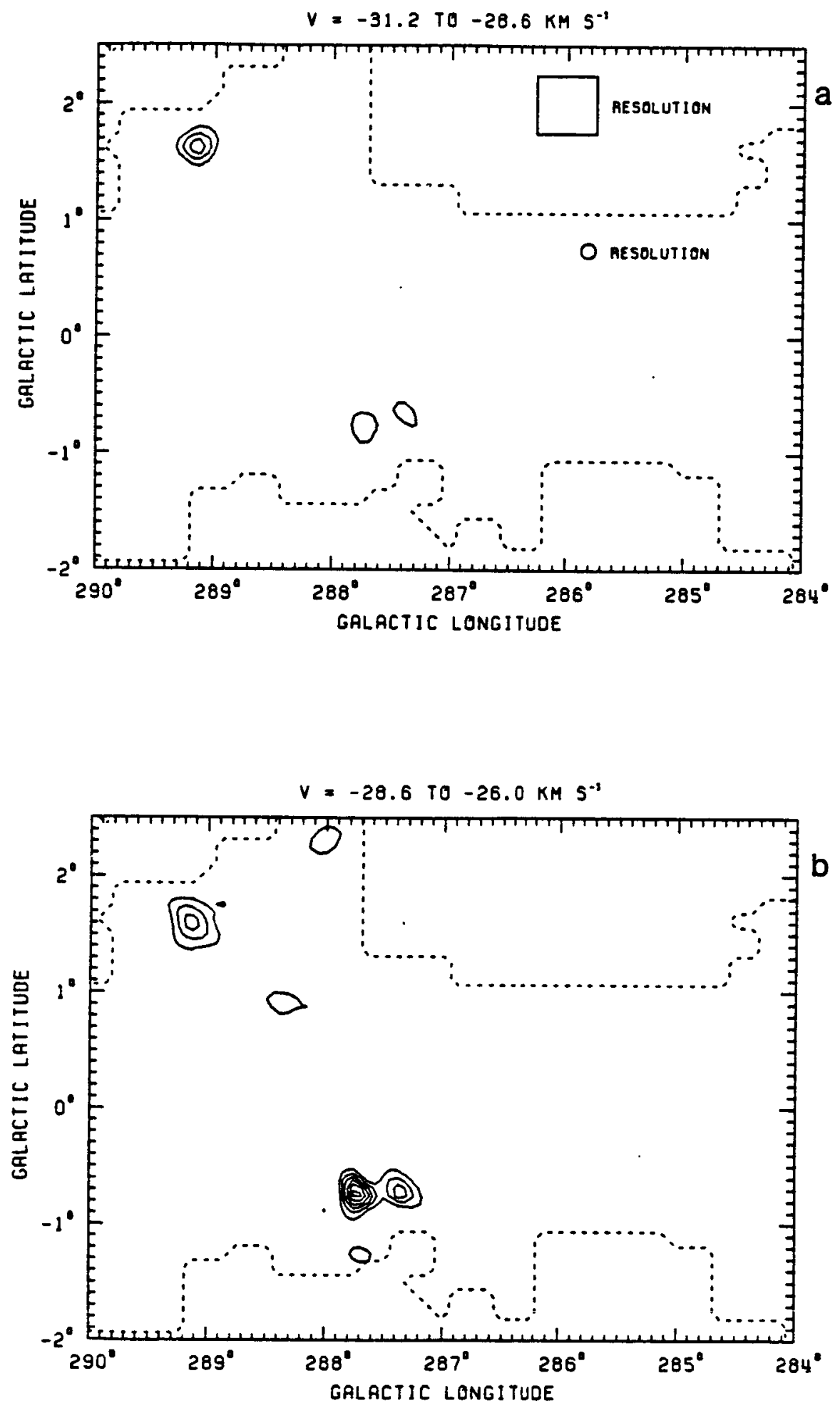

FIGURE V-4 

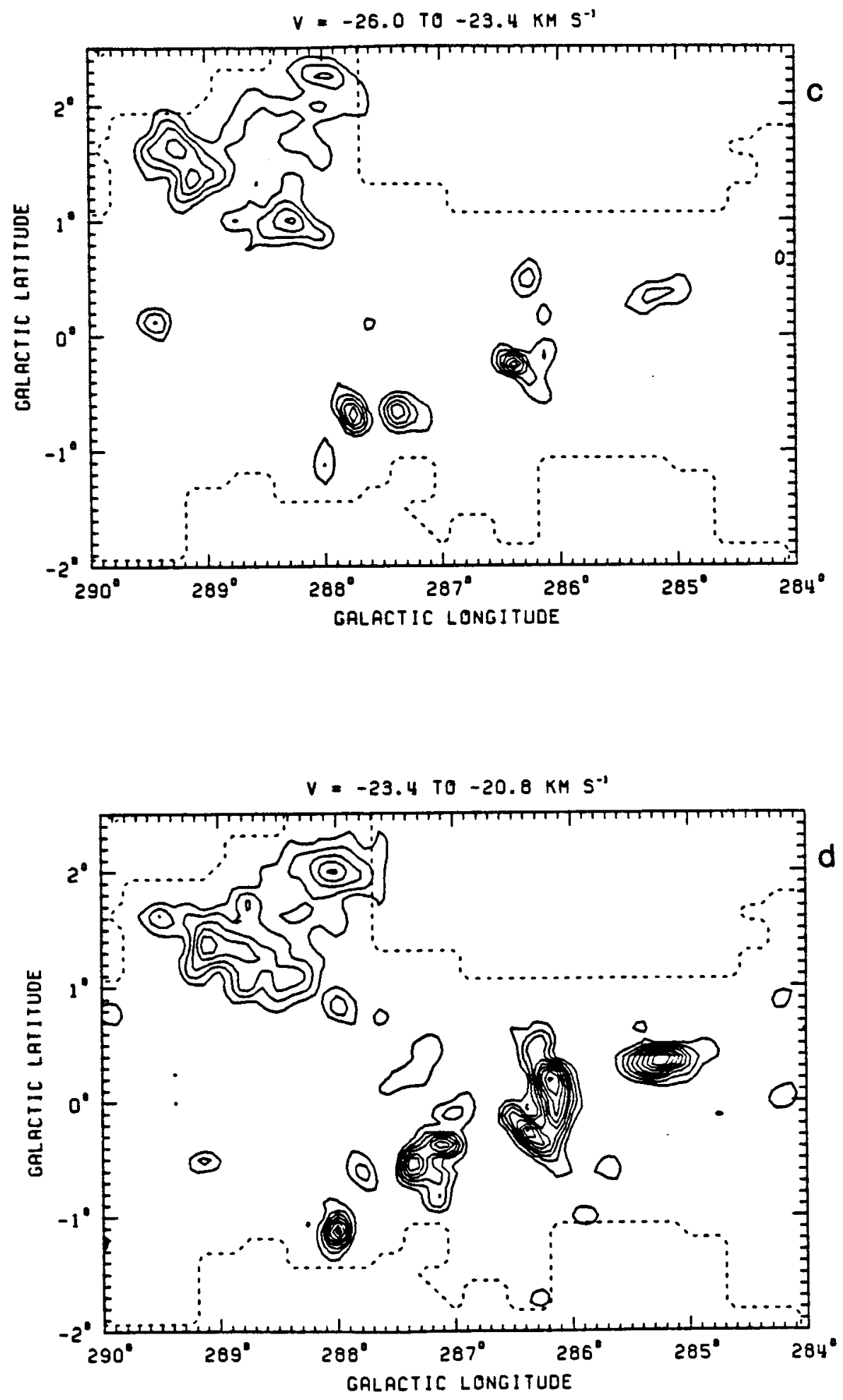

FIGURE V-4 (continued) 


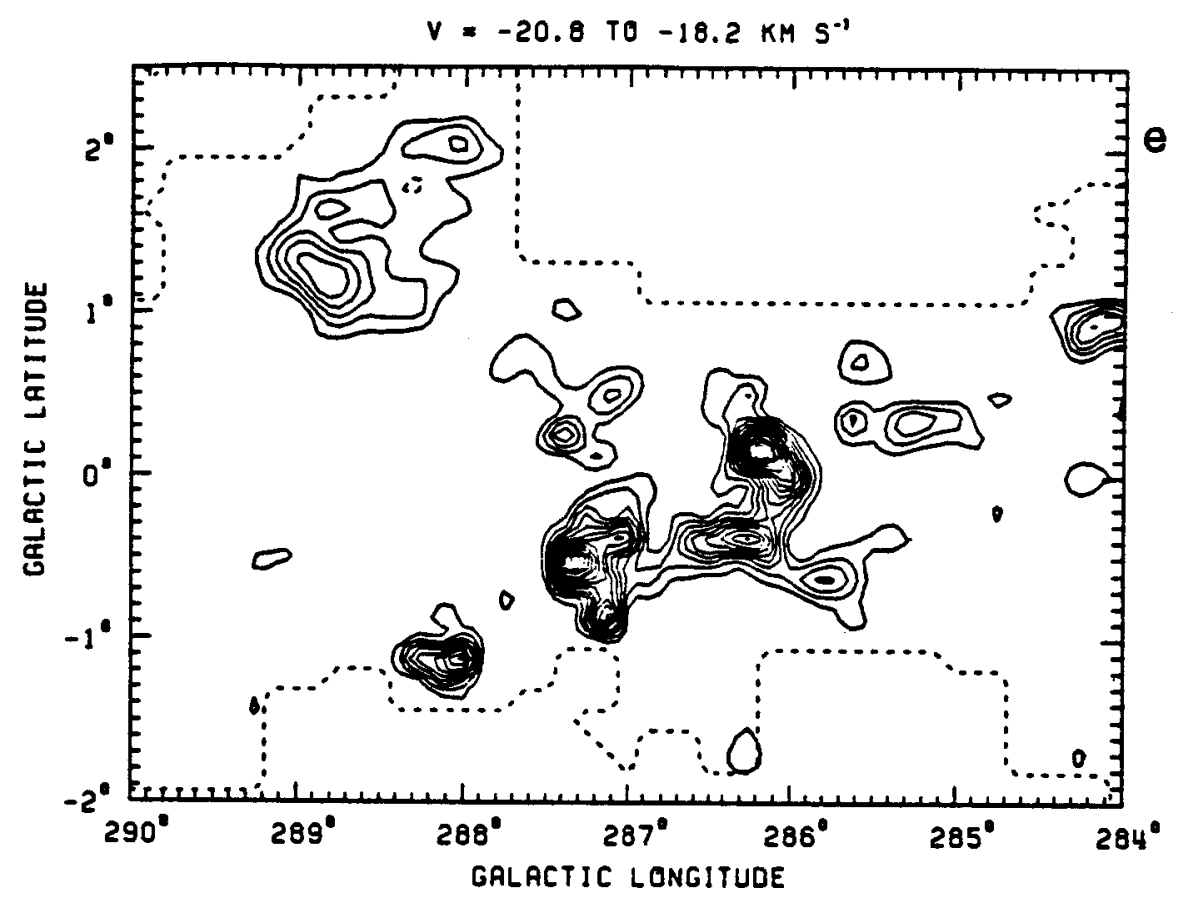

$V=-18.210-15.6 \mathrm{KM} \mathrm{S}-1$

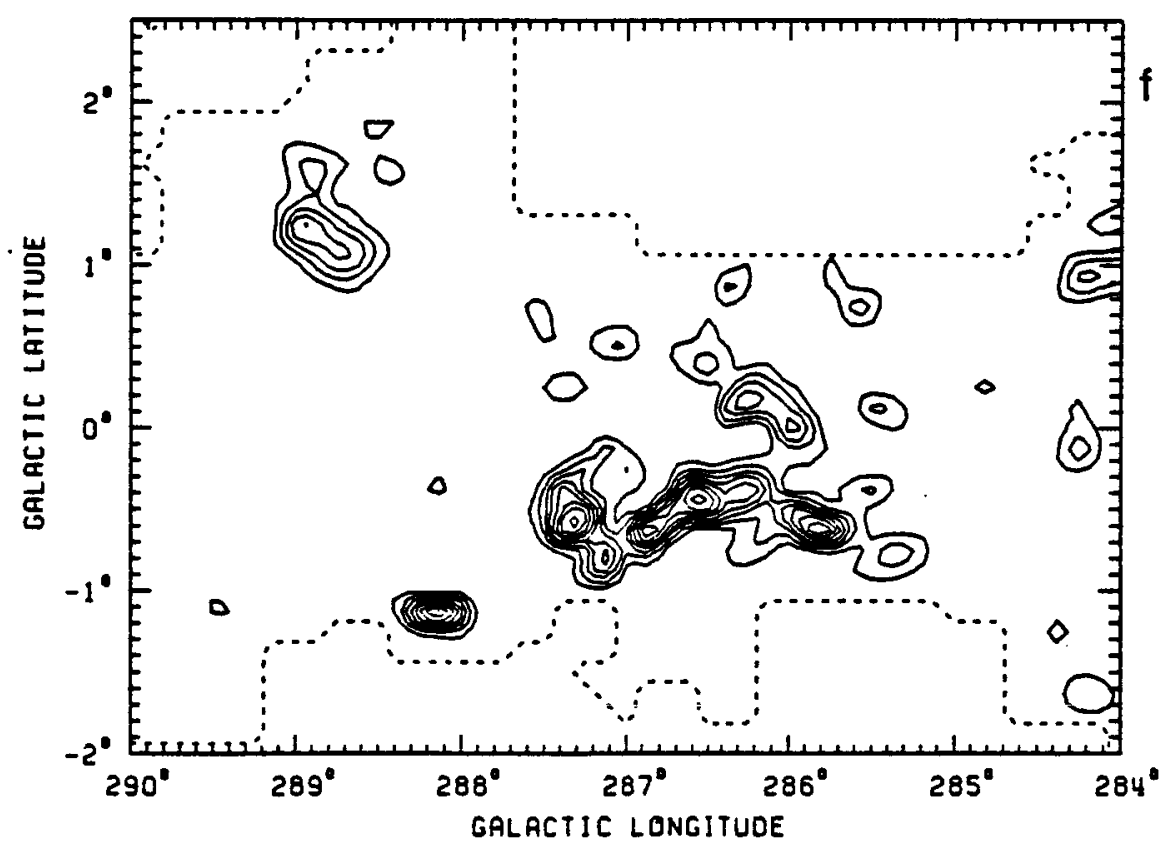

FIGURE V-4 (continued) 

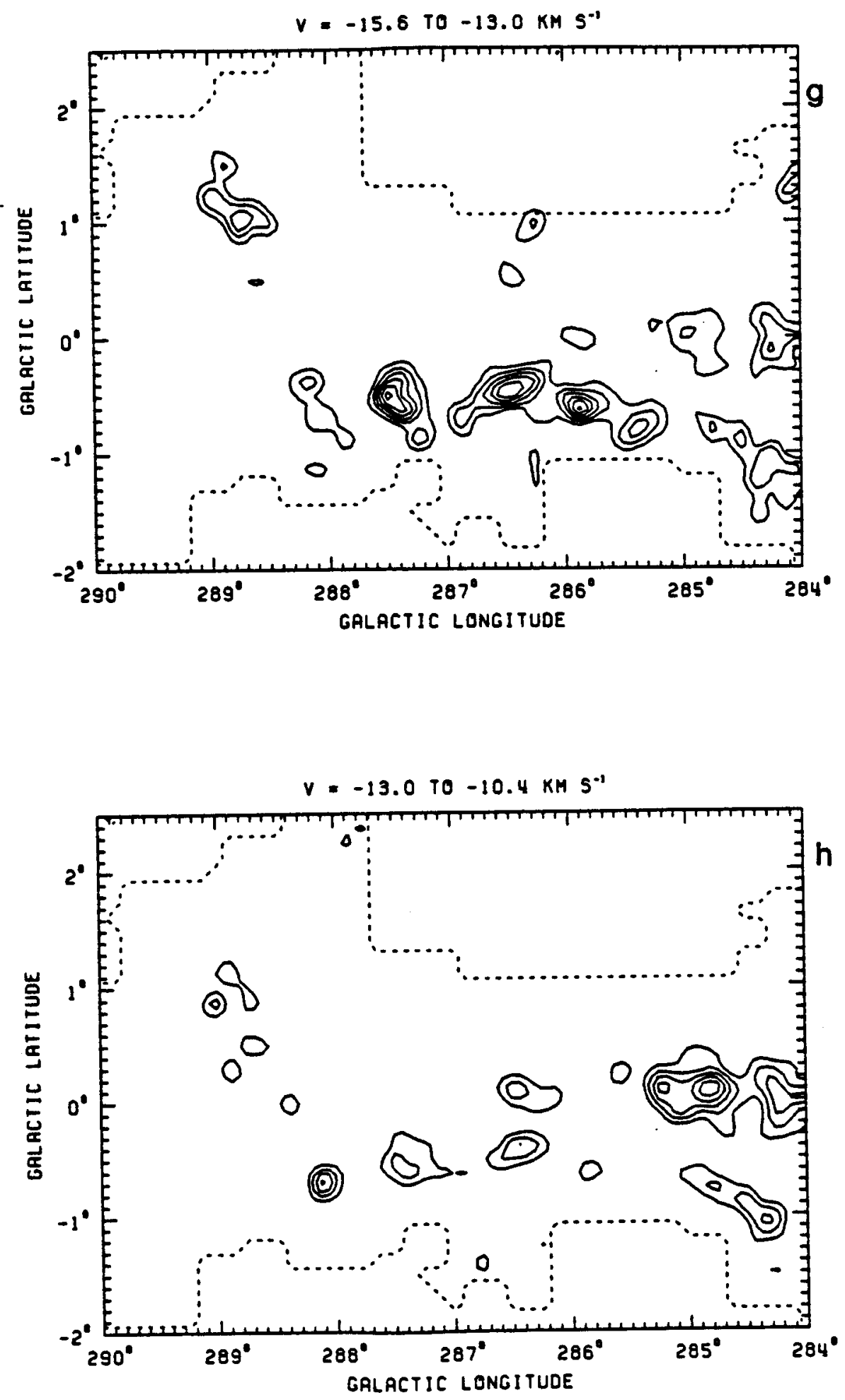

FIGURE V-4 (continued) 


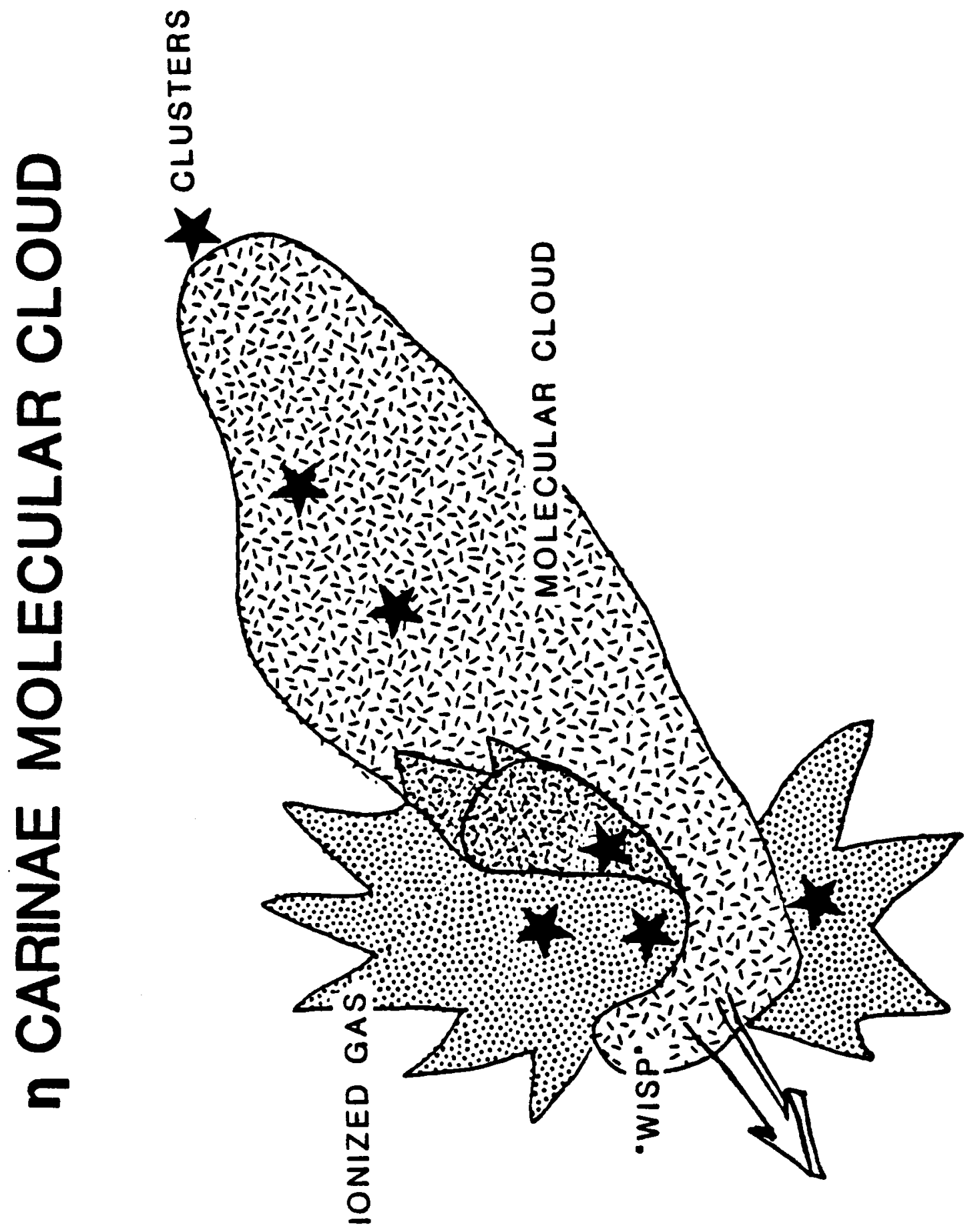

FIGURE V-5 


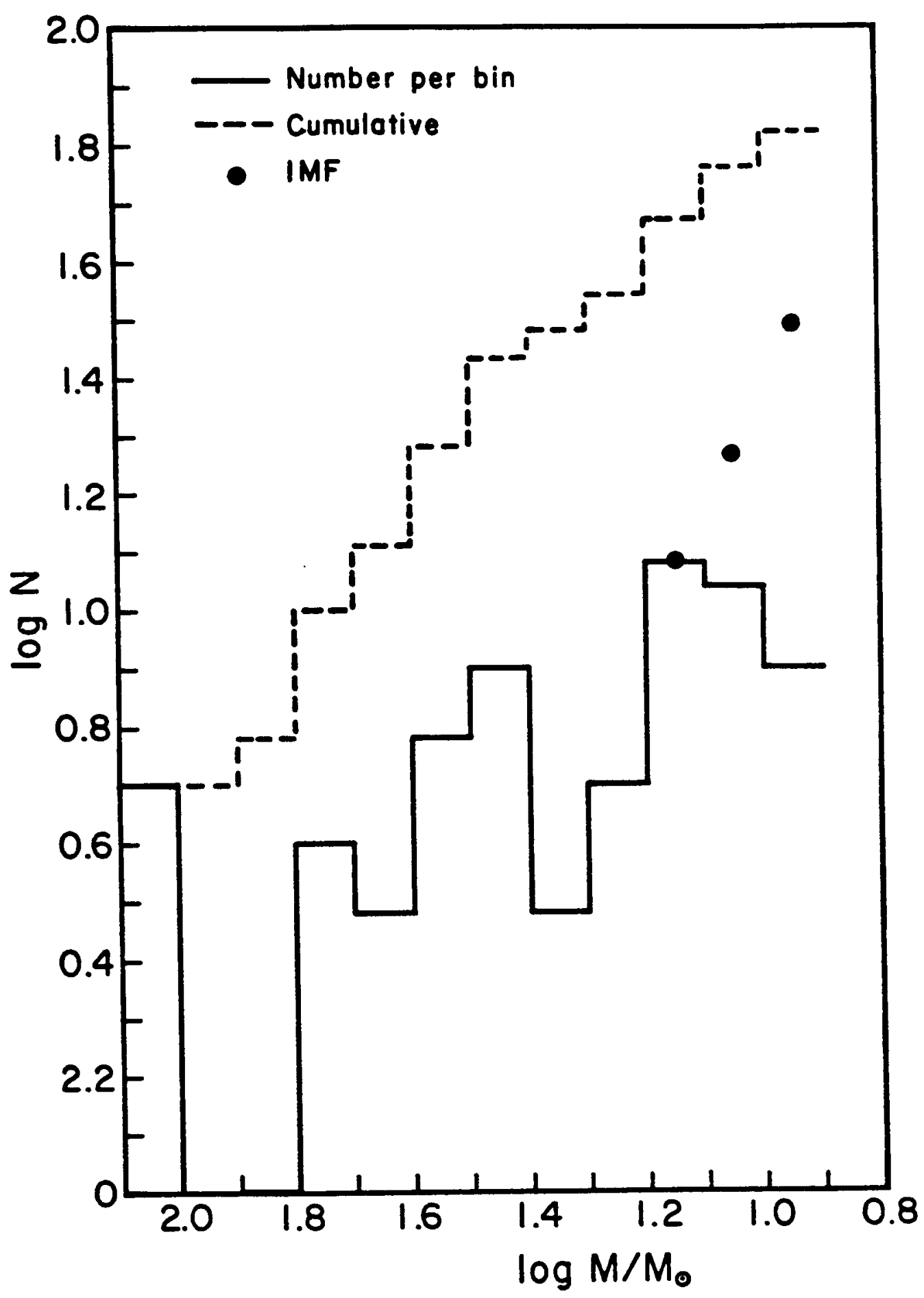

FIGURE V-6 

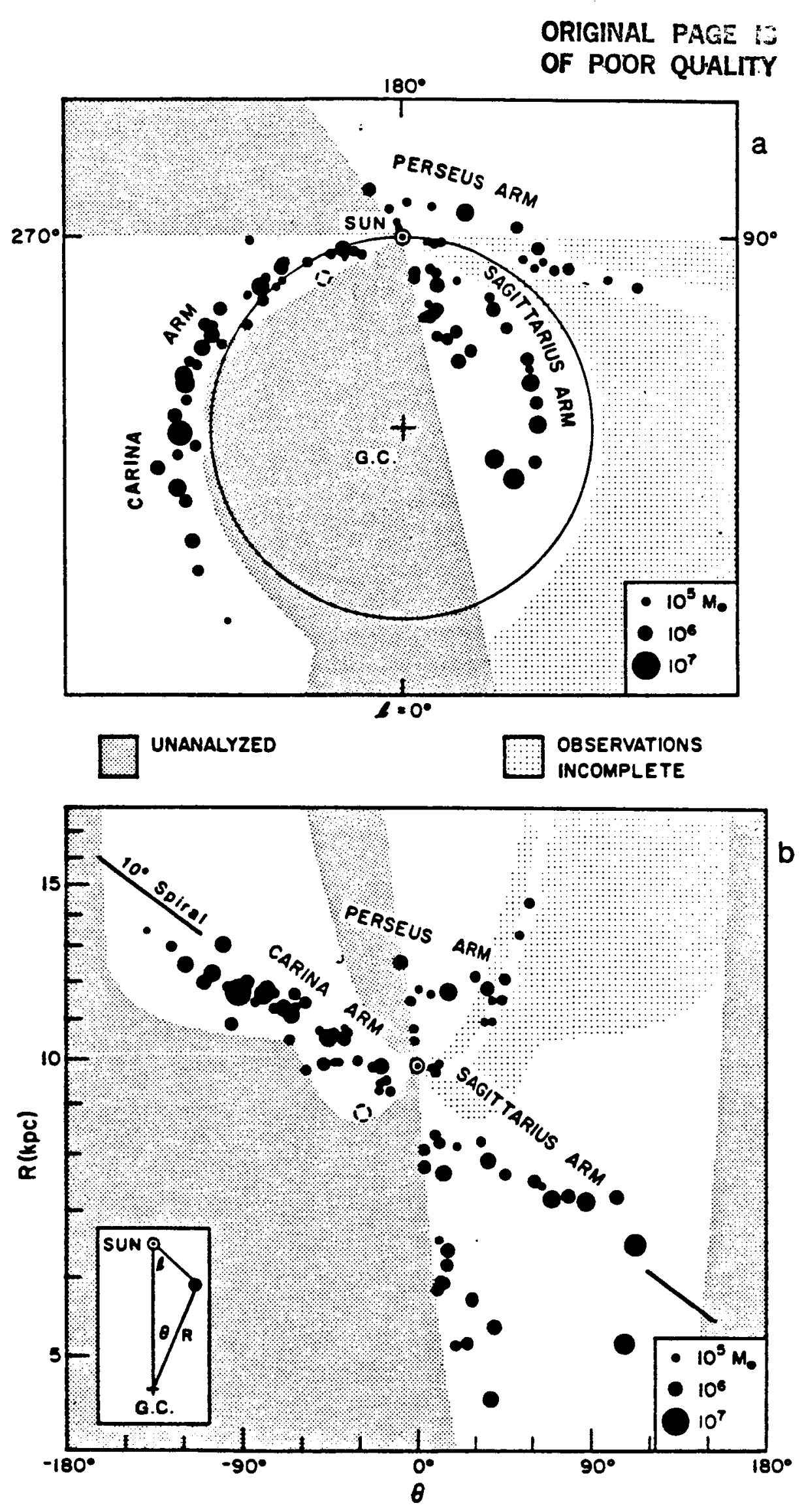

FIGURF $V-1$ 
ORIGINAL PAGE

OF POOR QUALTIY

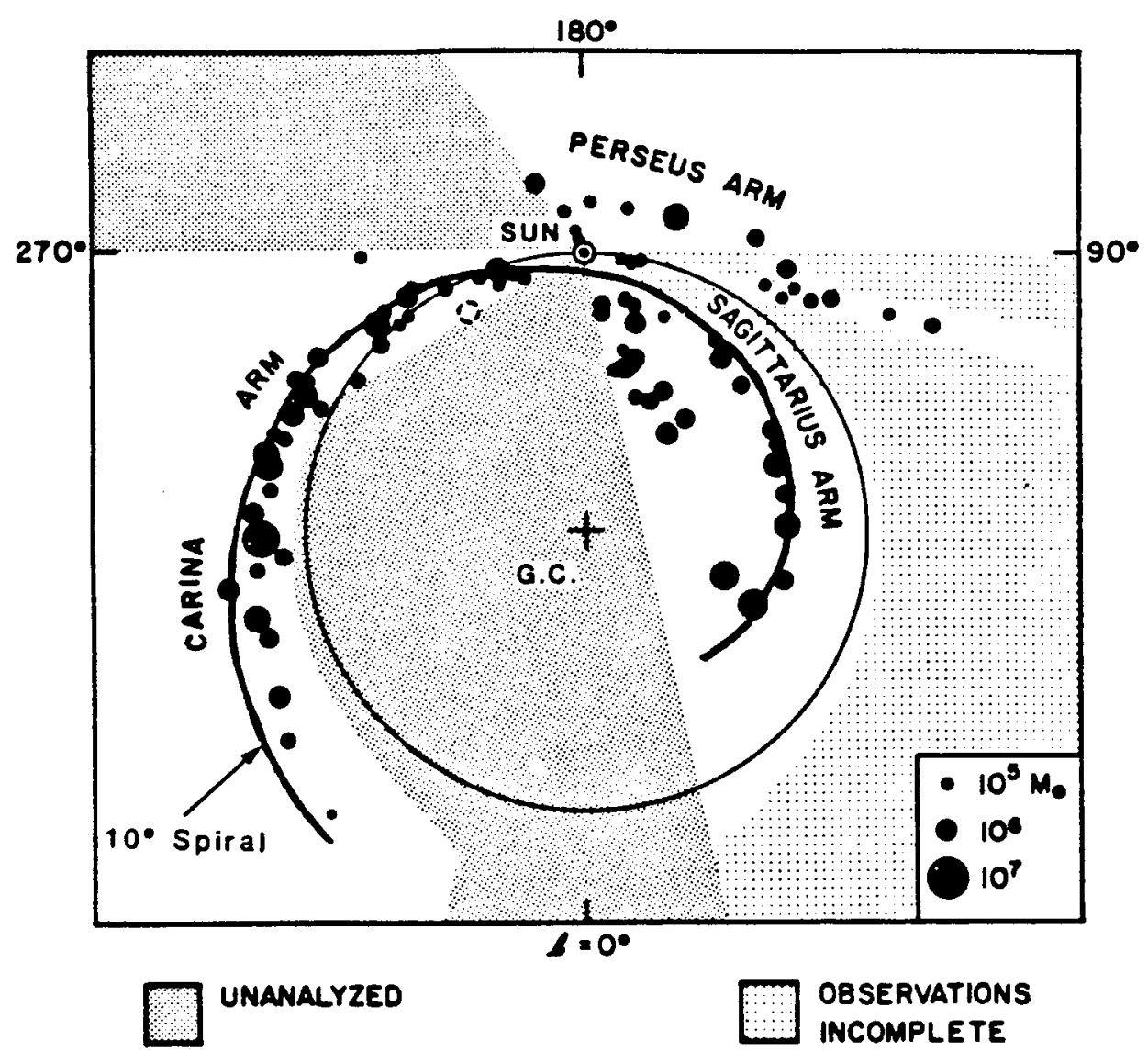

FIGURE V-8 


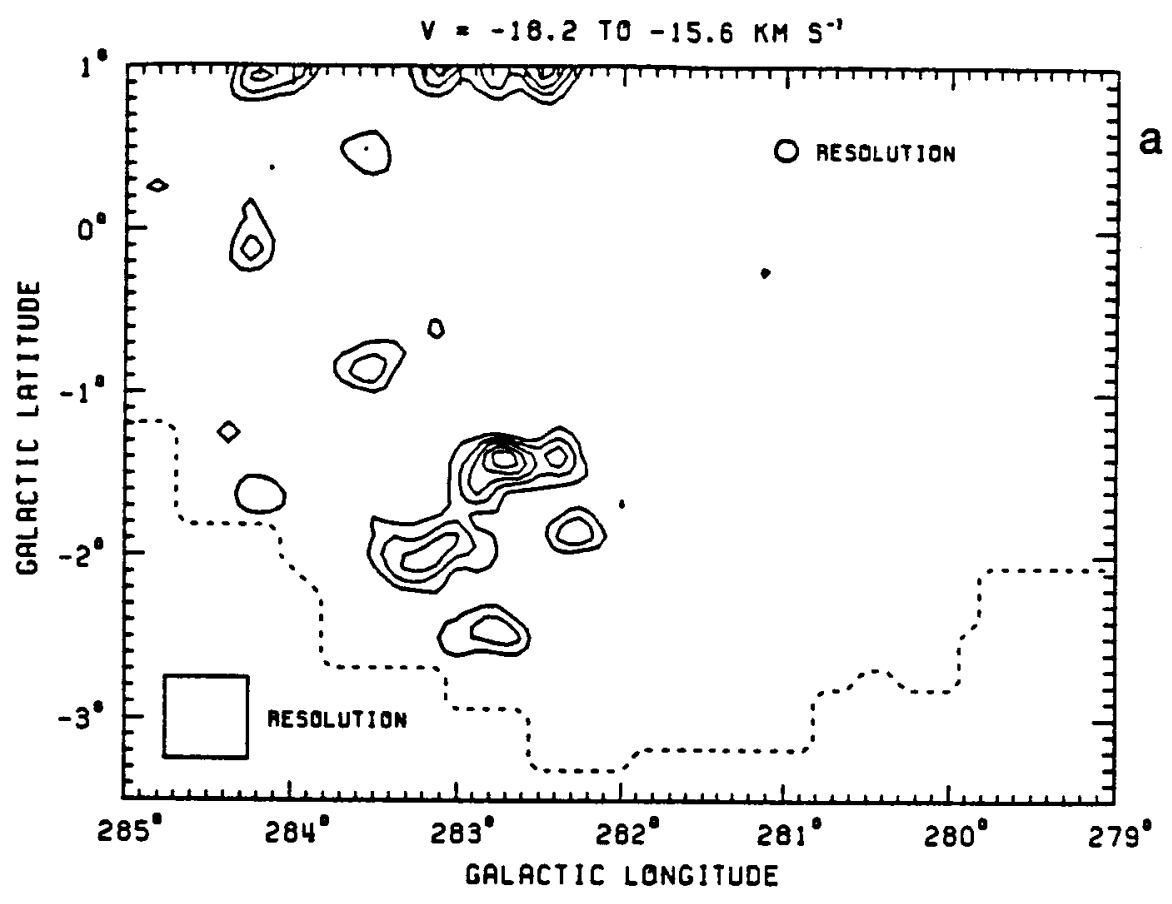

$V=-15.6 \mathrm{TO}-13.0 \mathrm{KM} \mathrm{\textrm {S } ^ { - 1 }}$

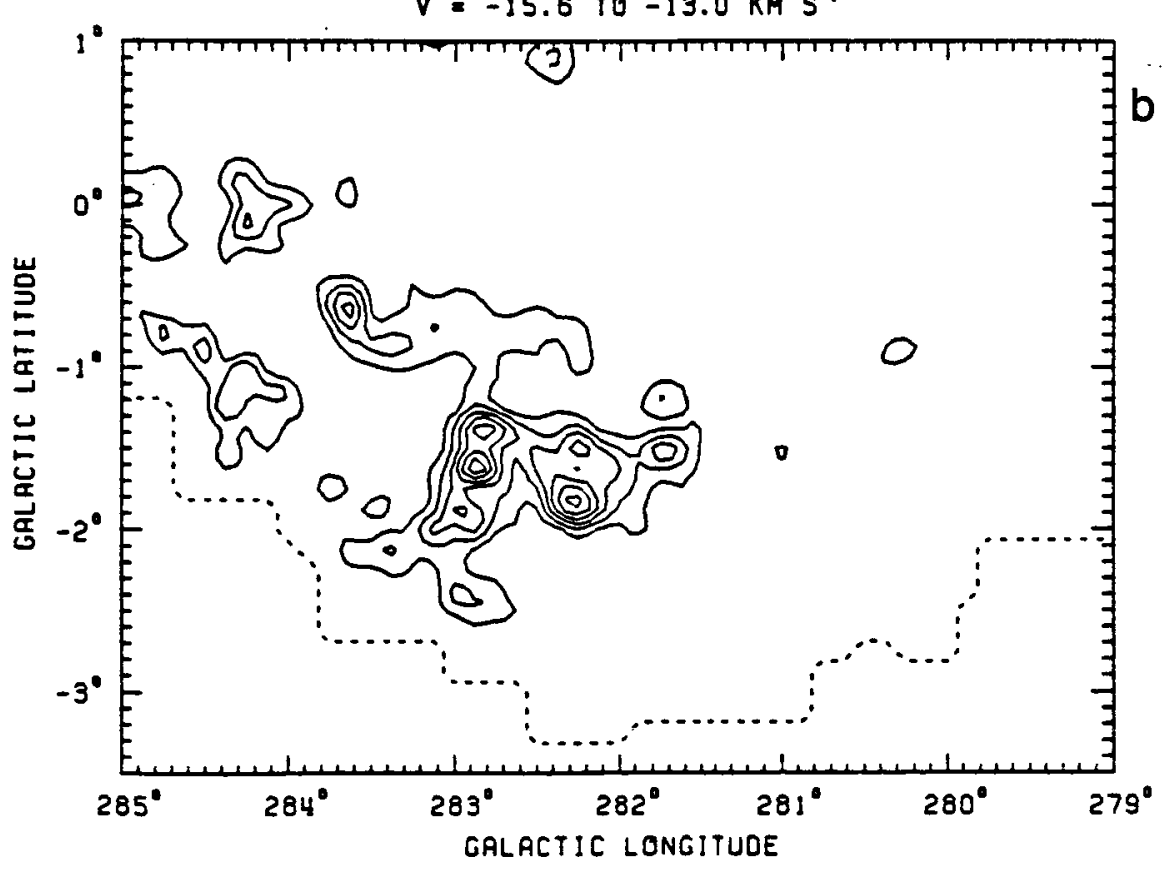

FIGURE V-9 

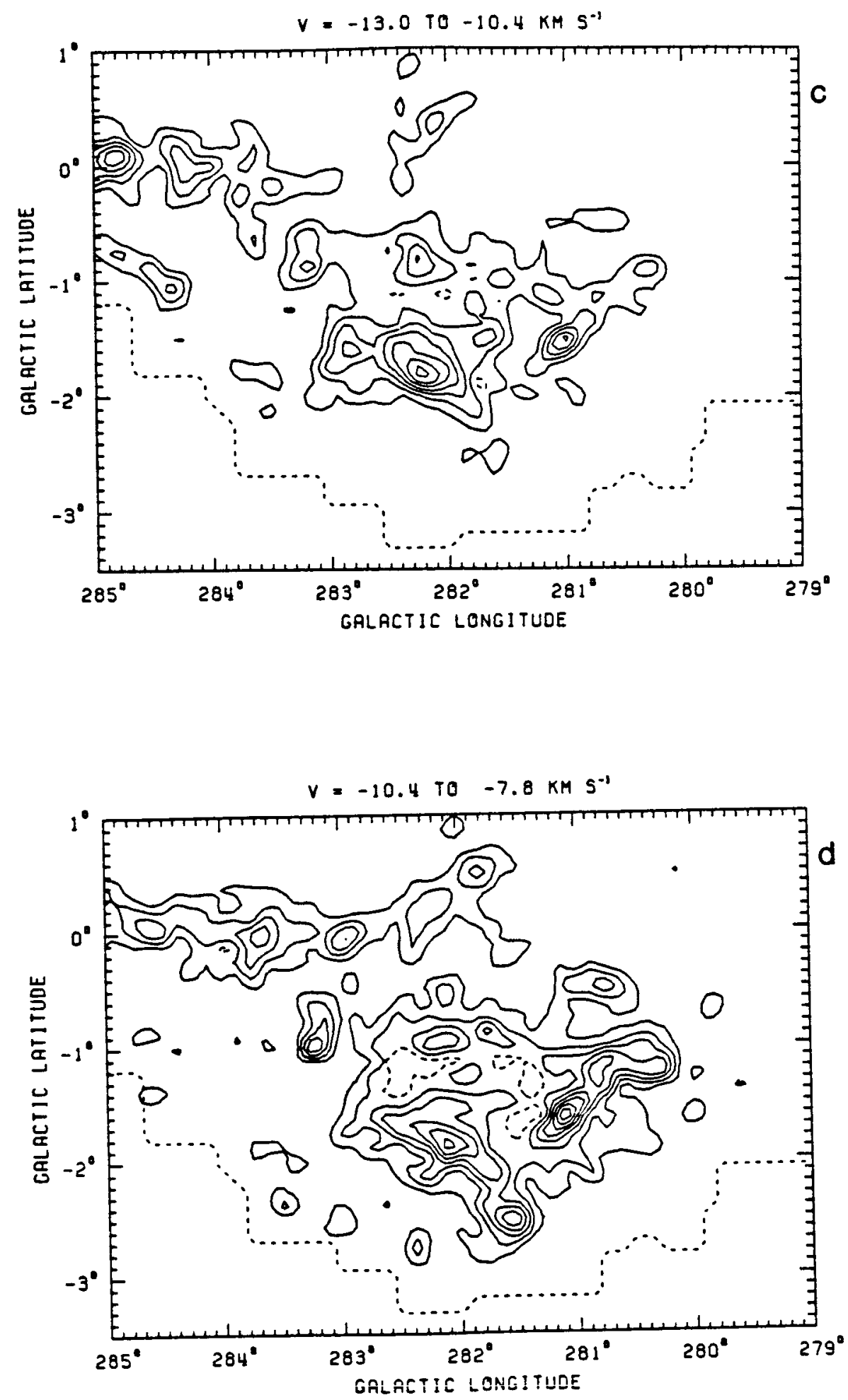

FIGURE V-9 (continued) 

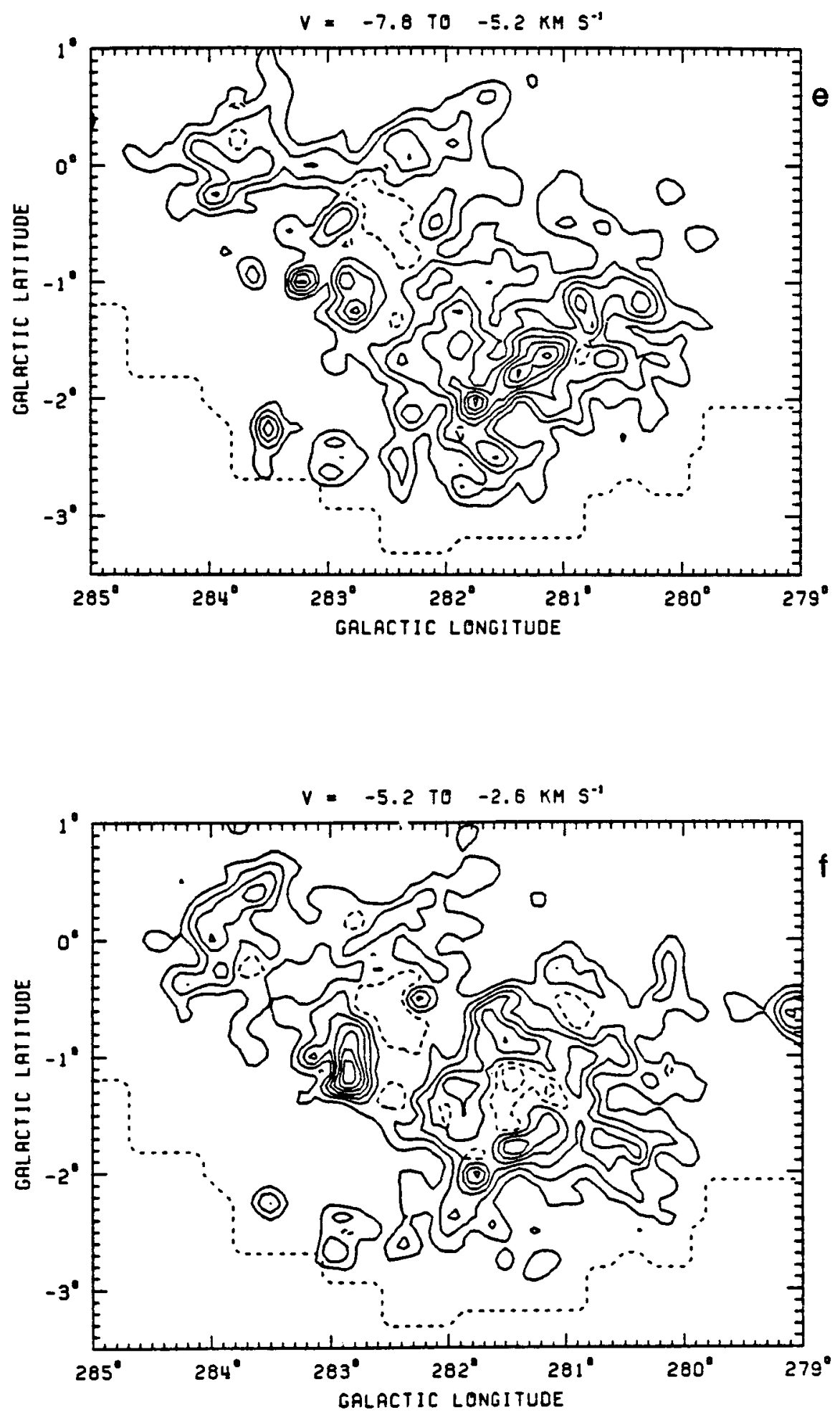

FIGURE V-9 (continued) 

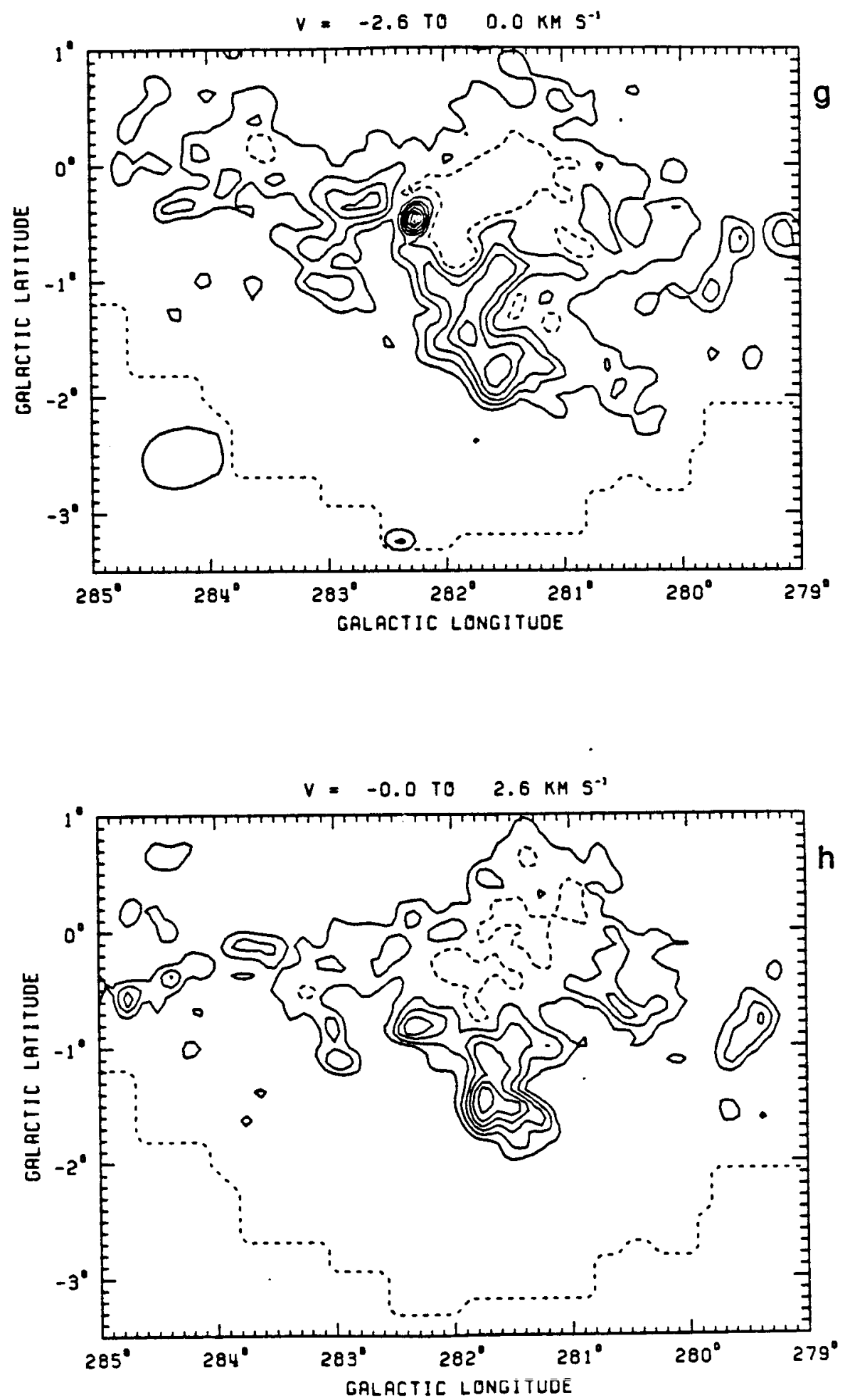

FIGURE V-9 (continued) 

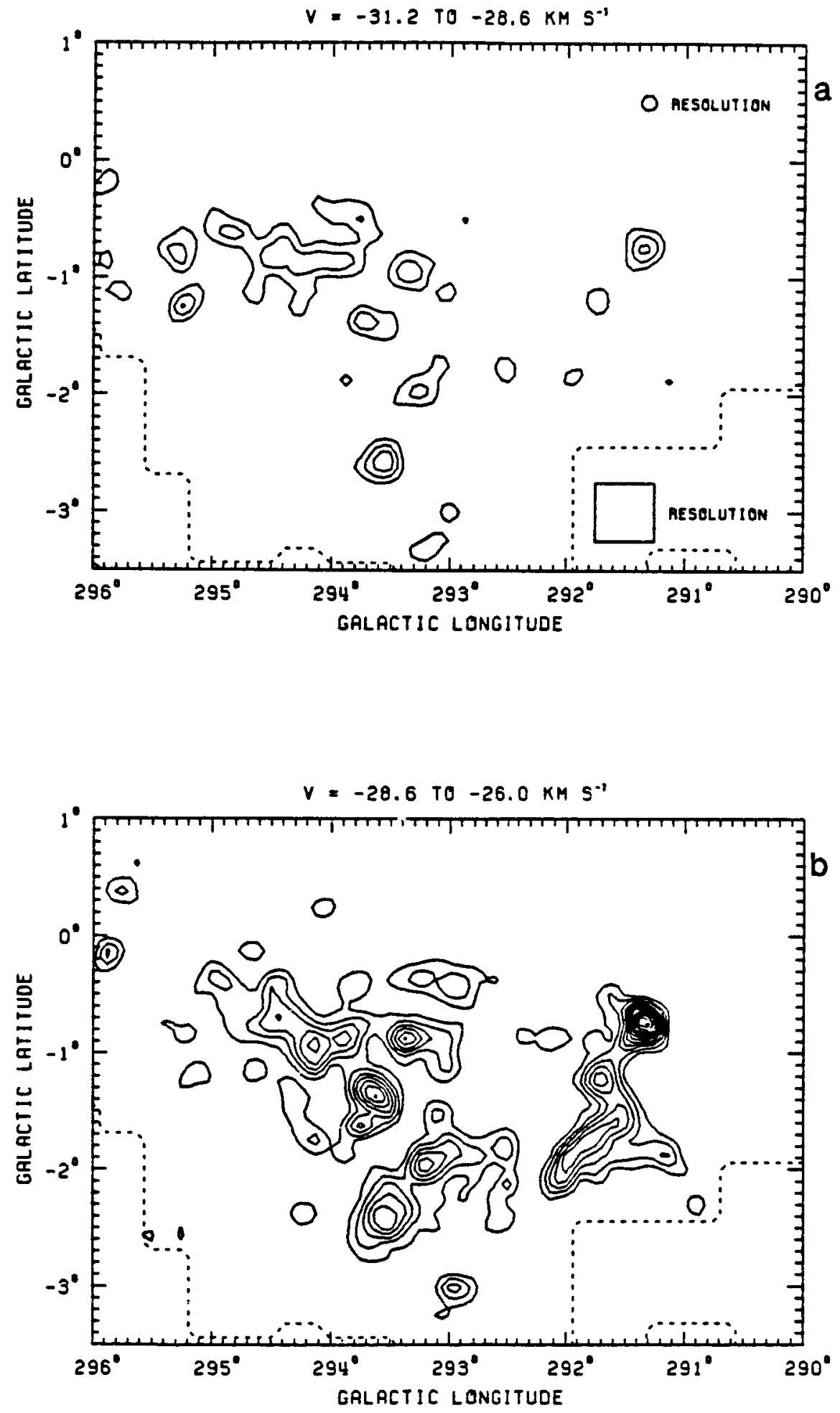

FIGURE V-10 

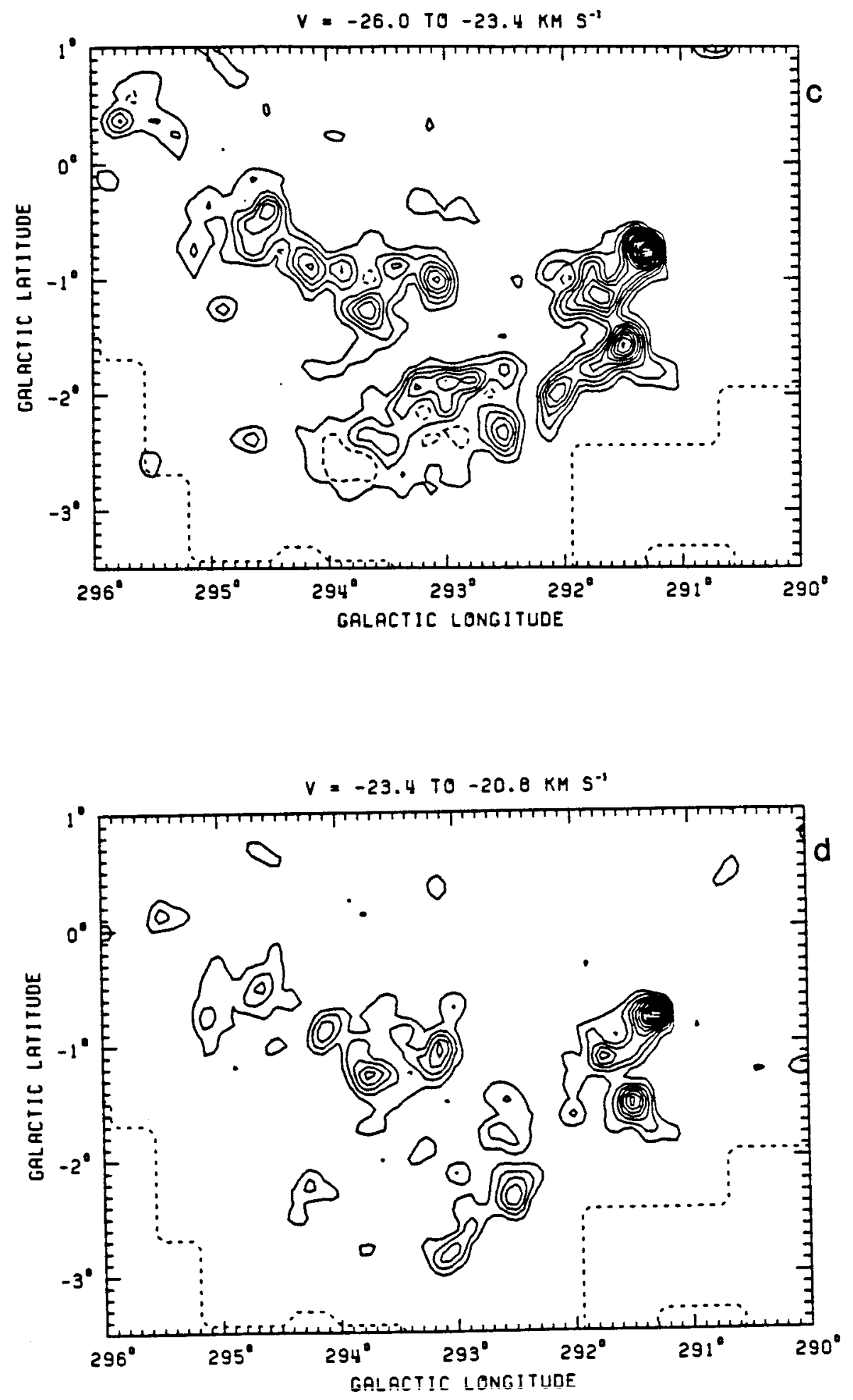

FIGURE $V-10$ (continued) 

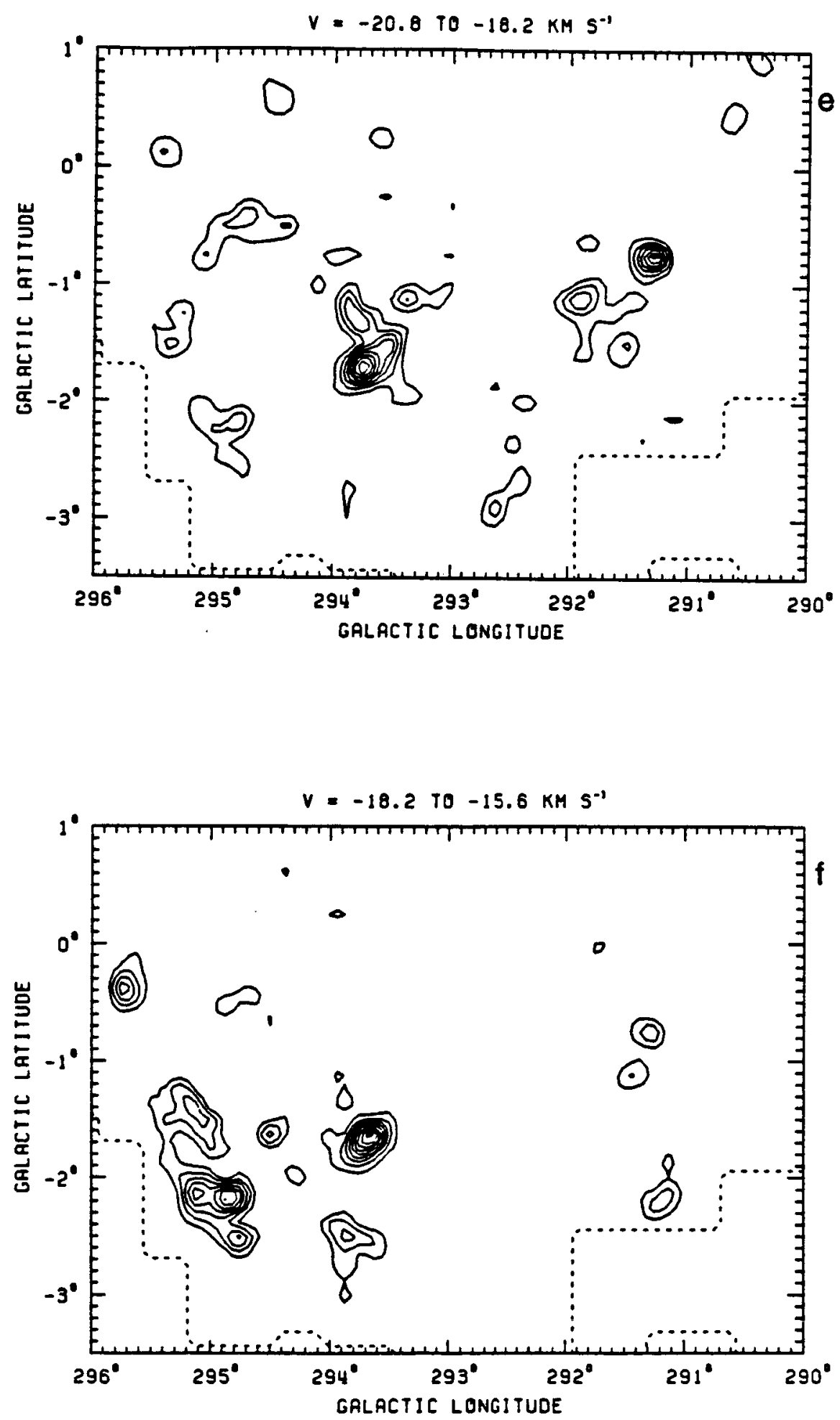

FIGURE V-10 (continued) 

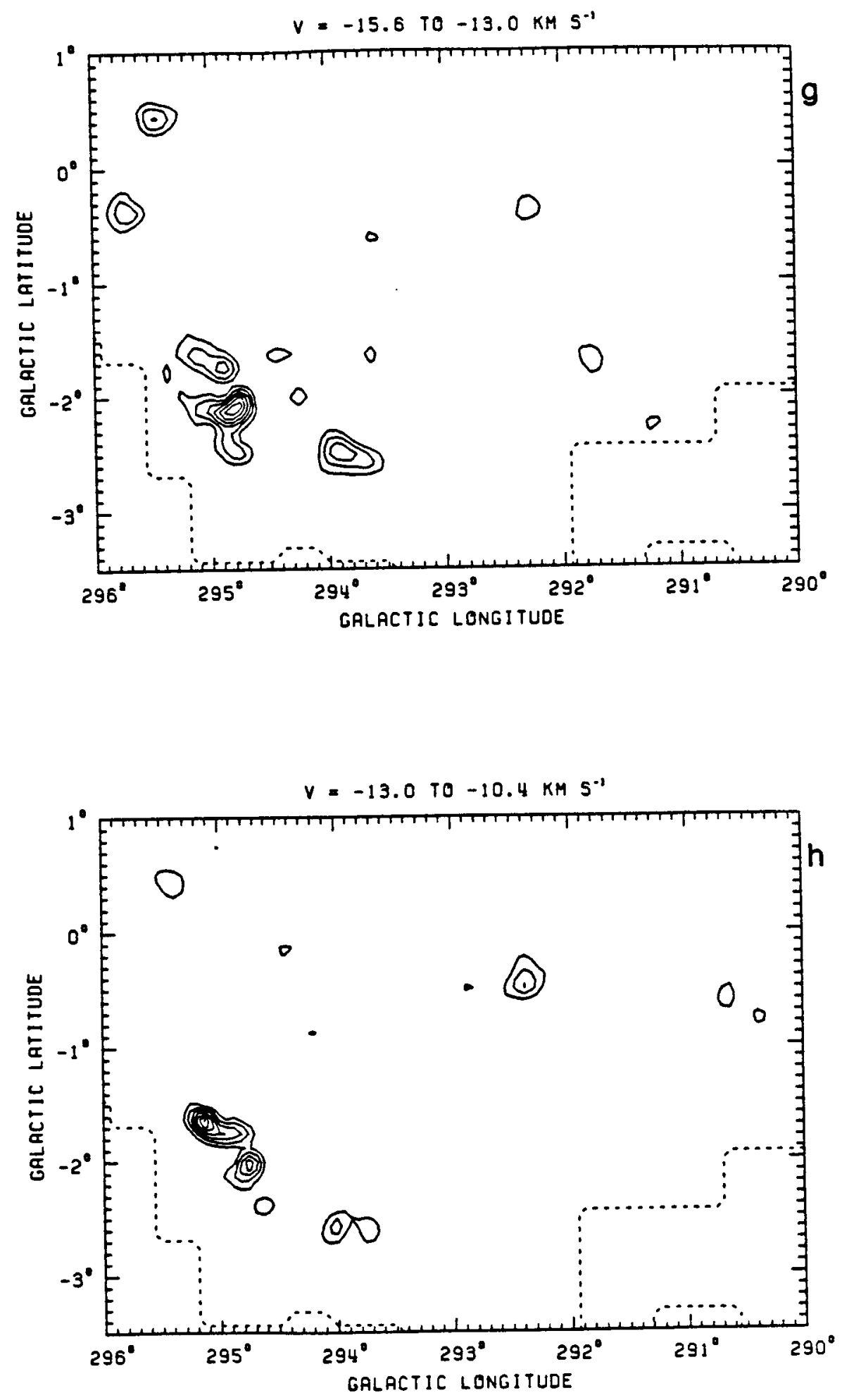

FIGURE V-10 (continued) 

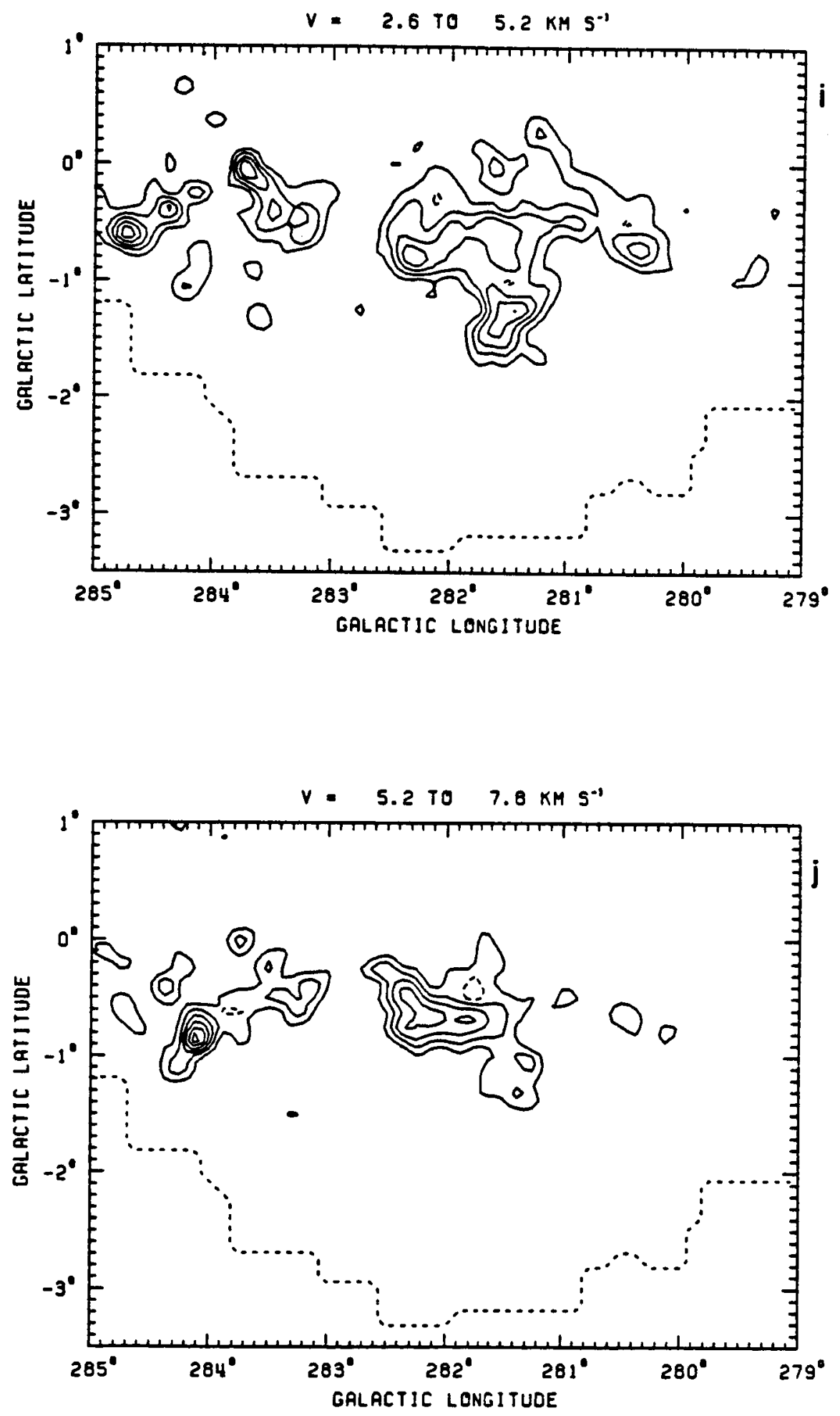

FIGURE $V-10$ (continued) 
SKY COVERAGE

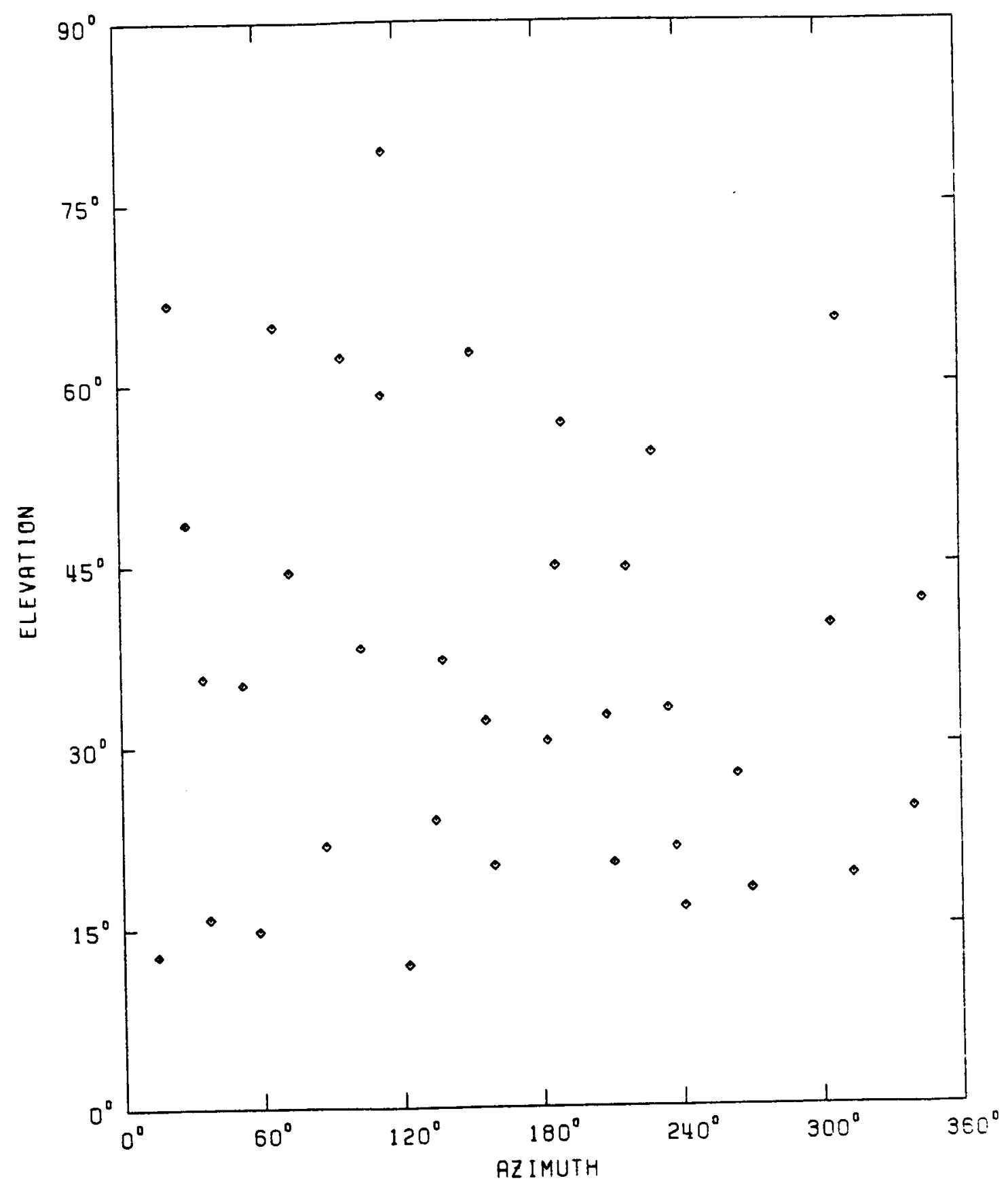

FIGURE $A-1$ 
ORIGINAL B:?

OF POOR QUALTT

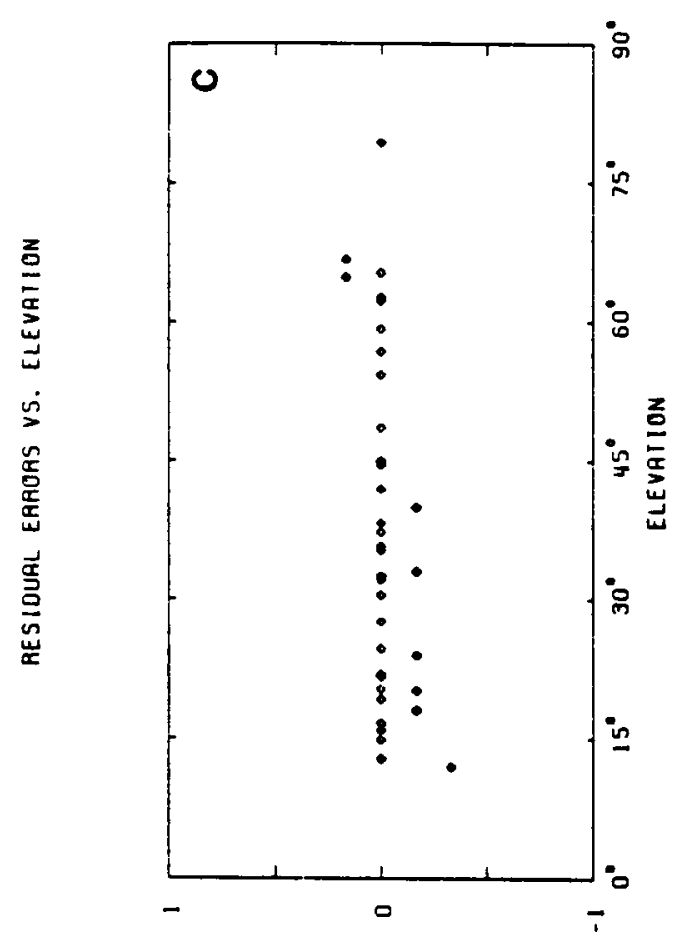

(NIW Jष甘) (73) 0

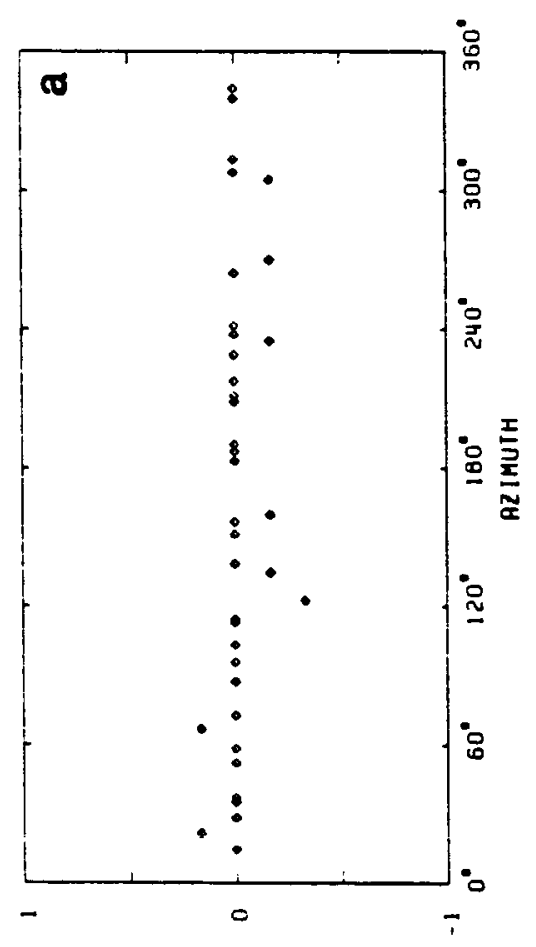

IN:H Zy甘I 17310
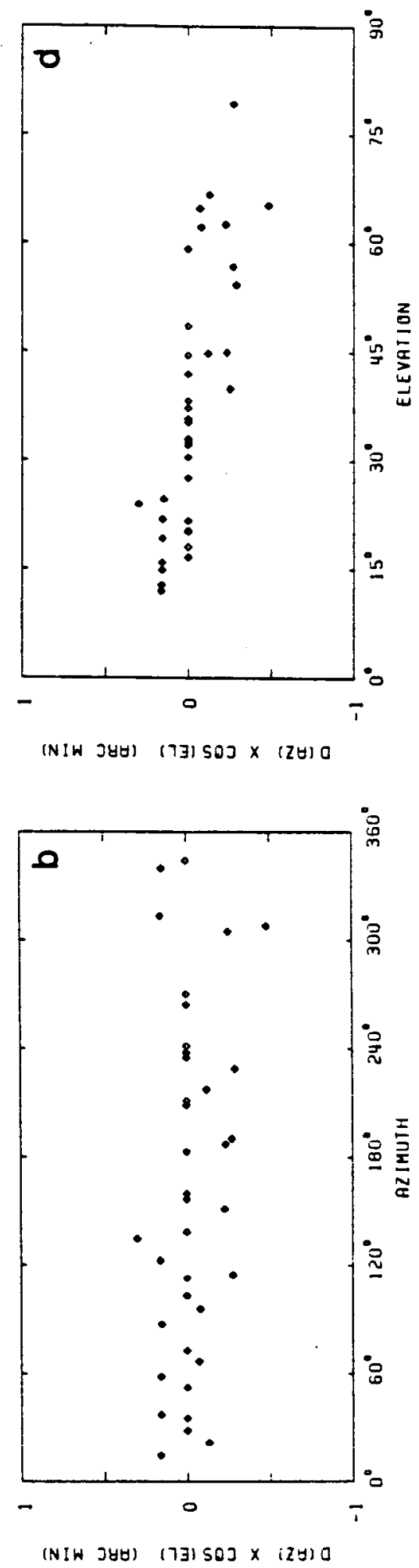

FIGURE A-2 
SUNPOINTING ERRORS VS. DAY Of 1983

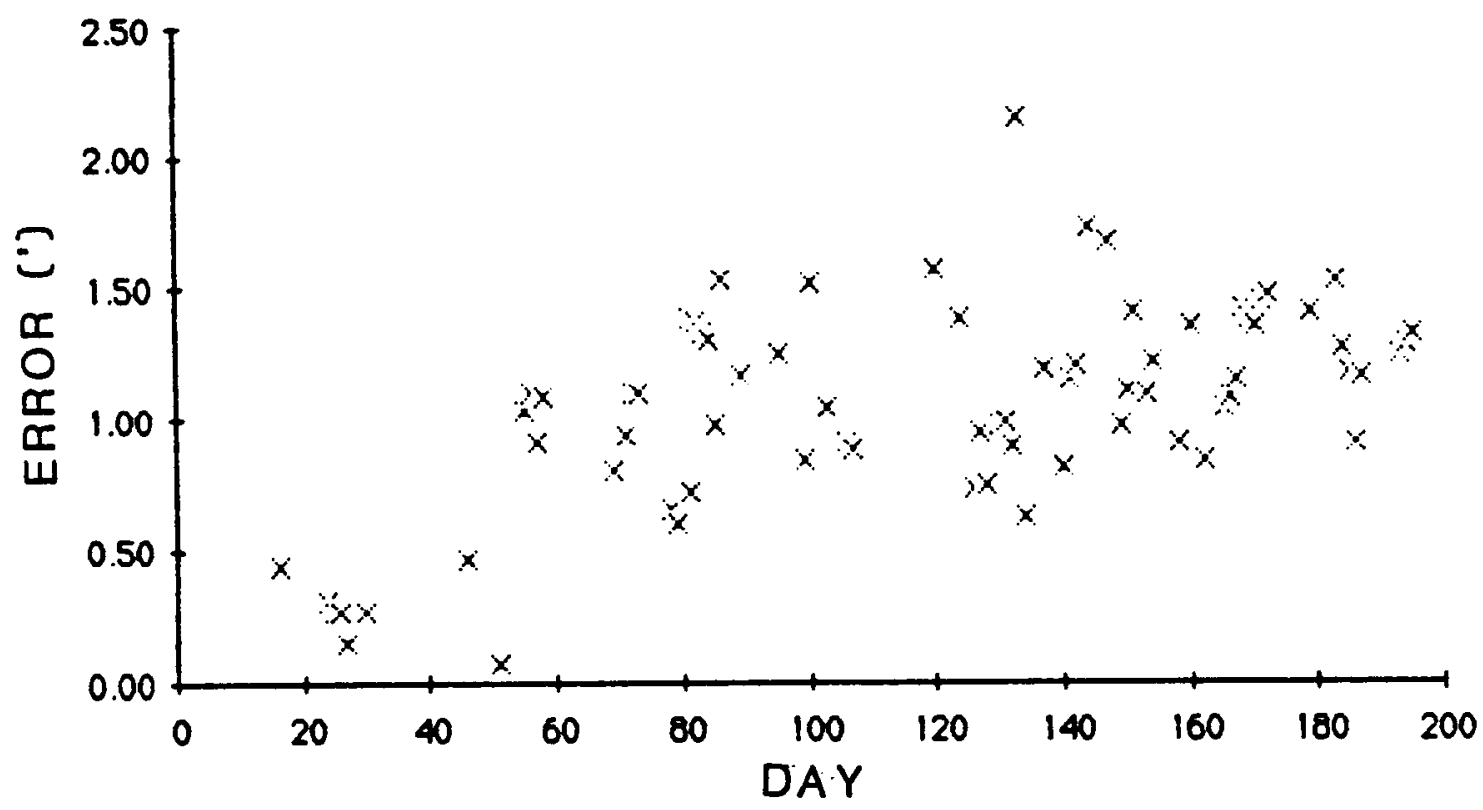

FIGURE $A-3$ 


\section{ORIGINAL FIAE IS}

OF POOR QUALITY

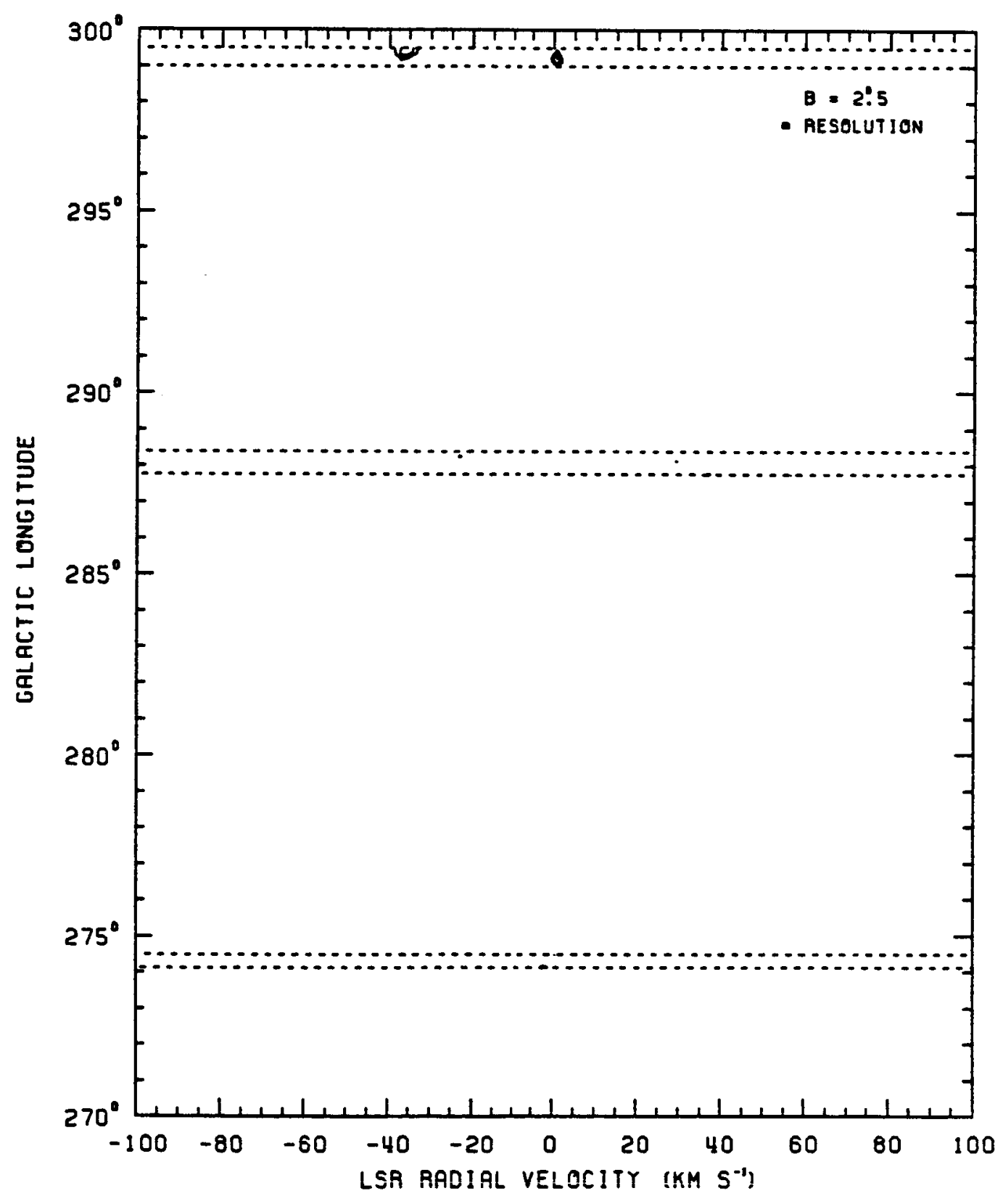

FIGURE B-1 


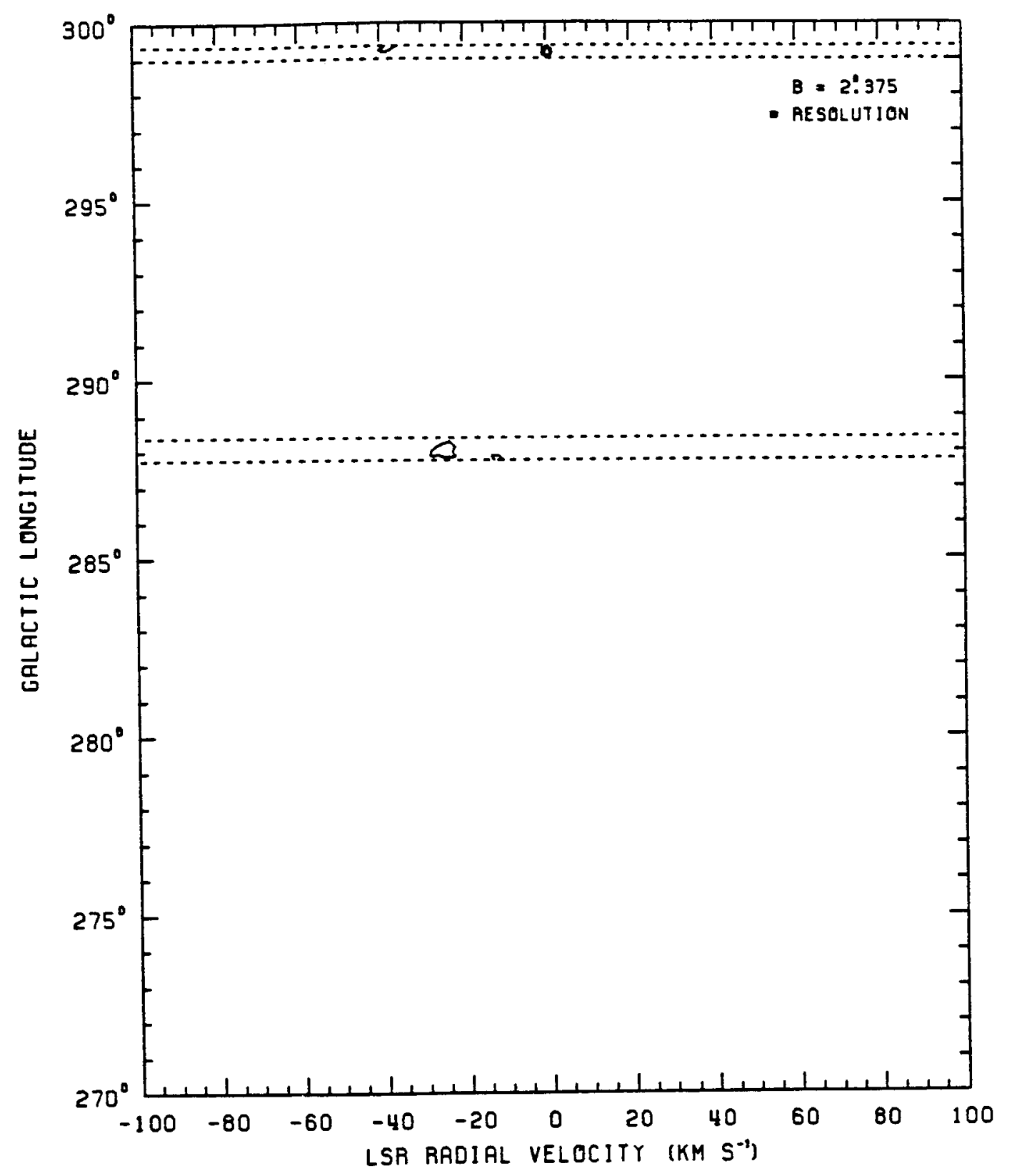

FIGURE B-1 (continued) 


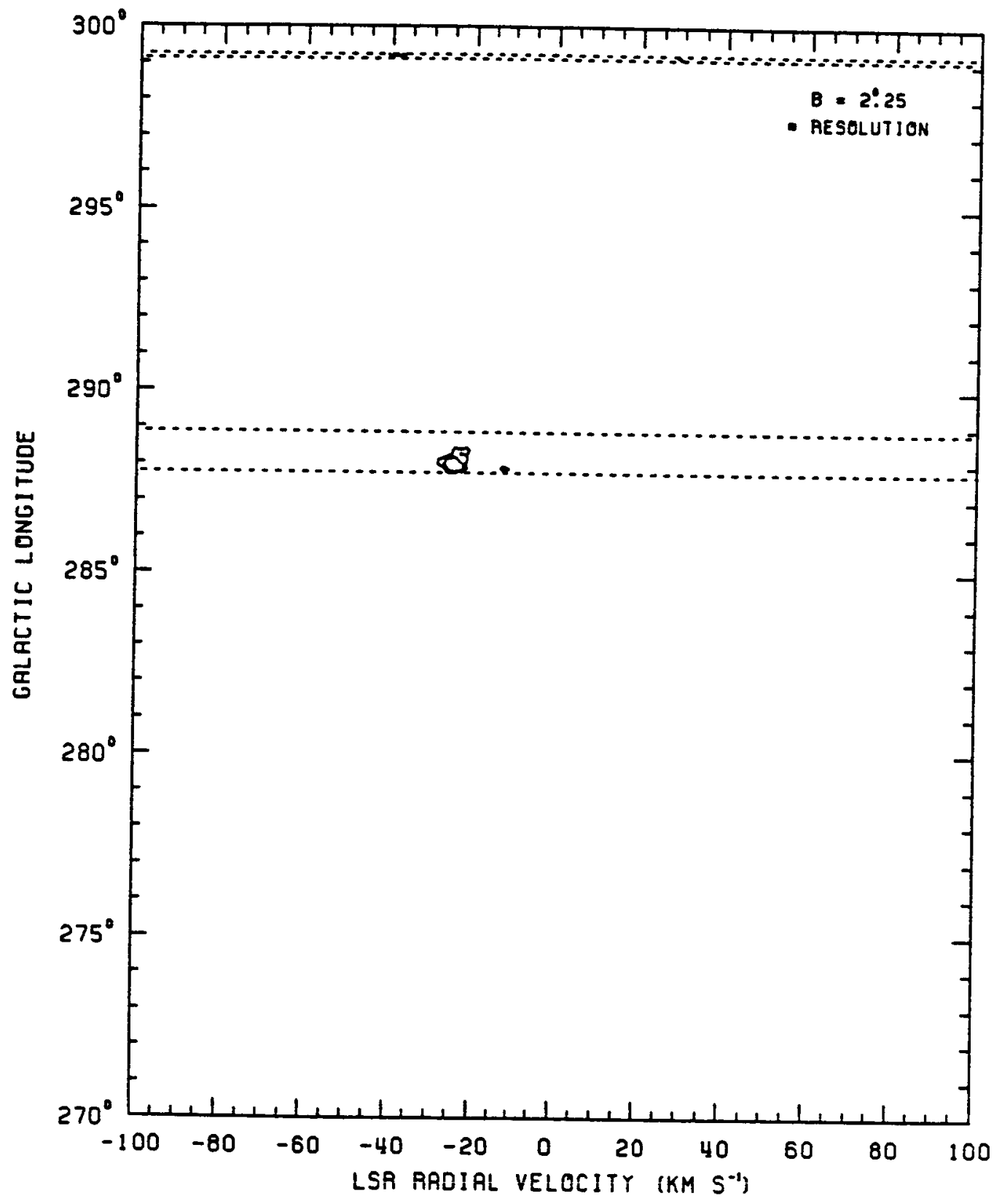

FIGURE B-1 (continued)

199 


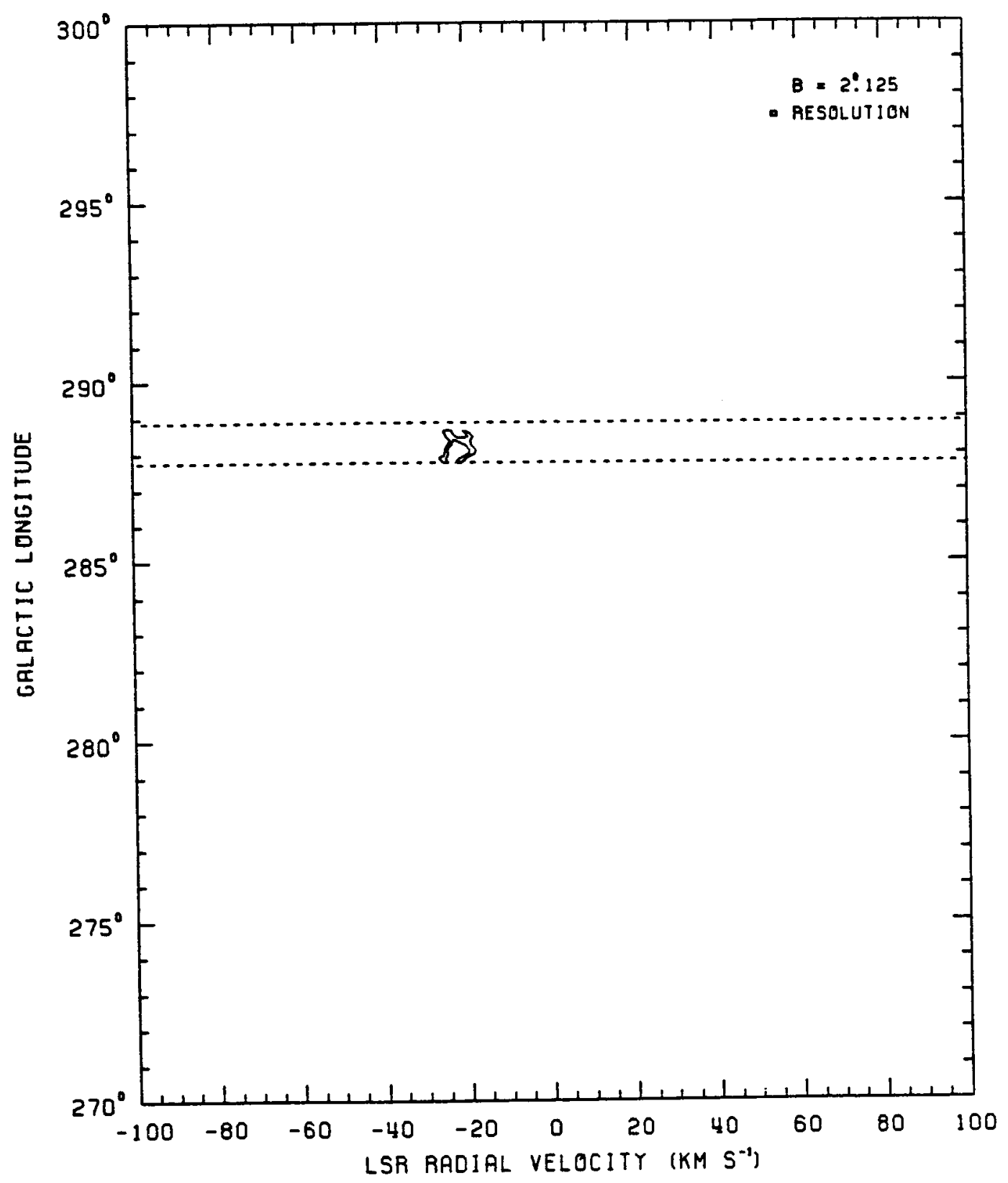

FIGURE B-1 (continued) 


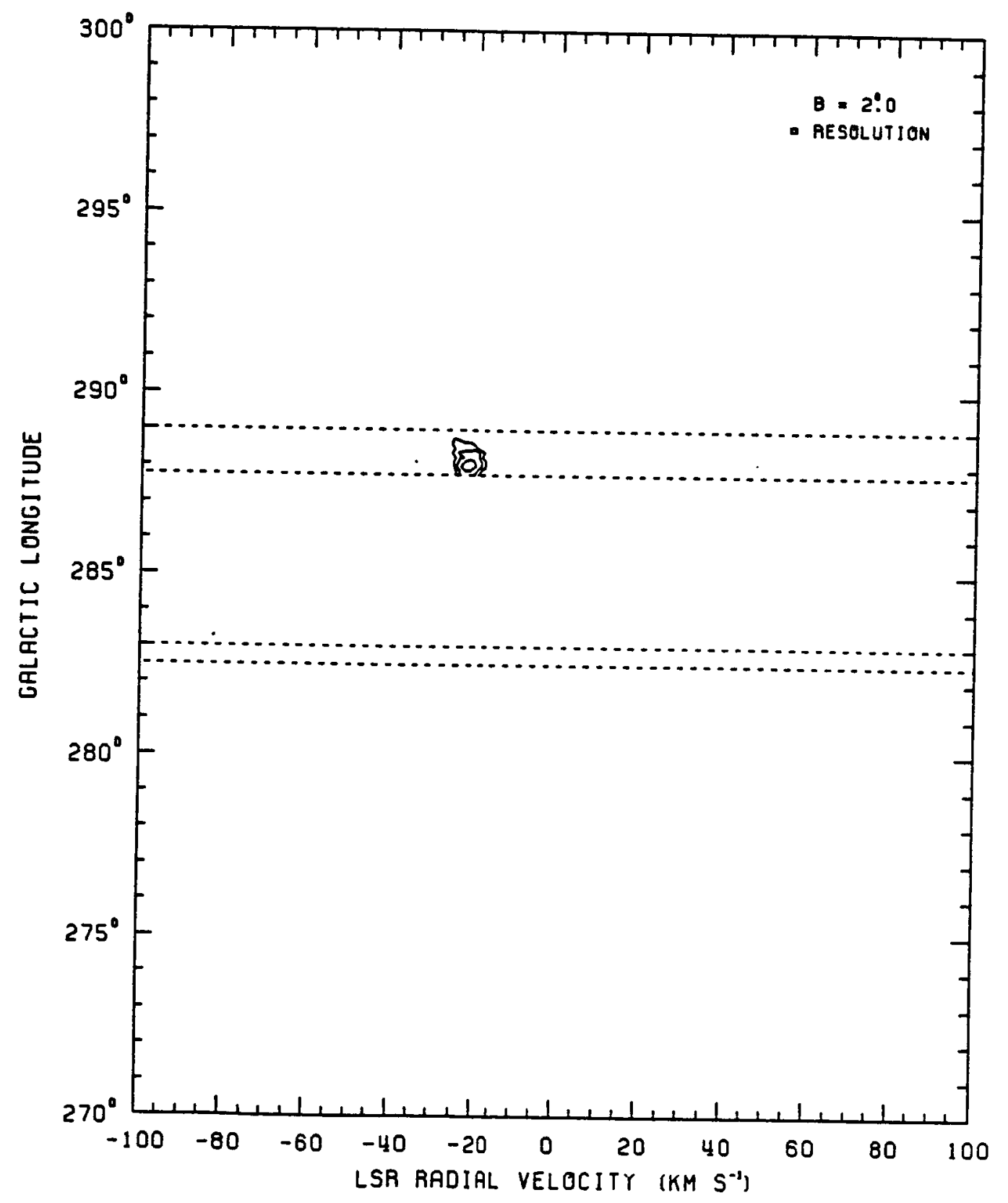

FIGURE B-1 (continued) 


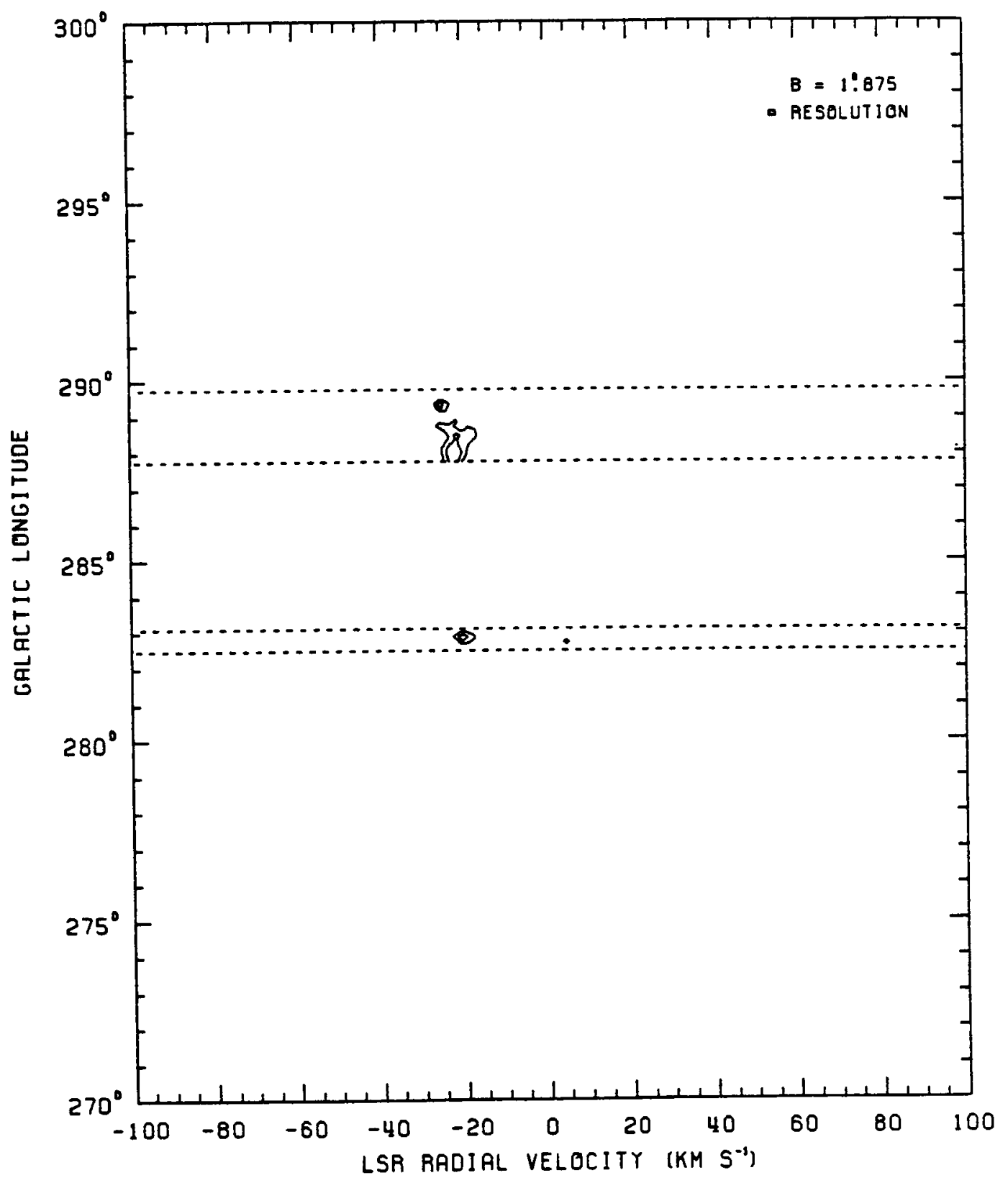

FIGURE B-I (continued) 


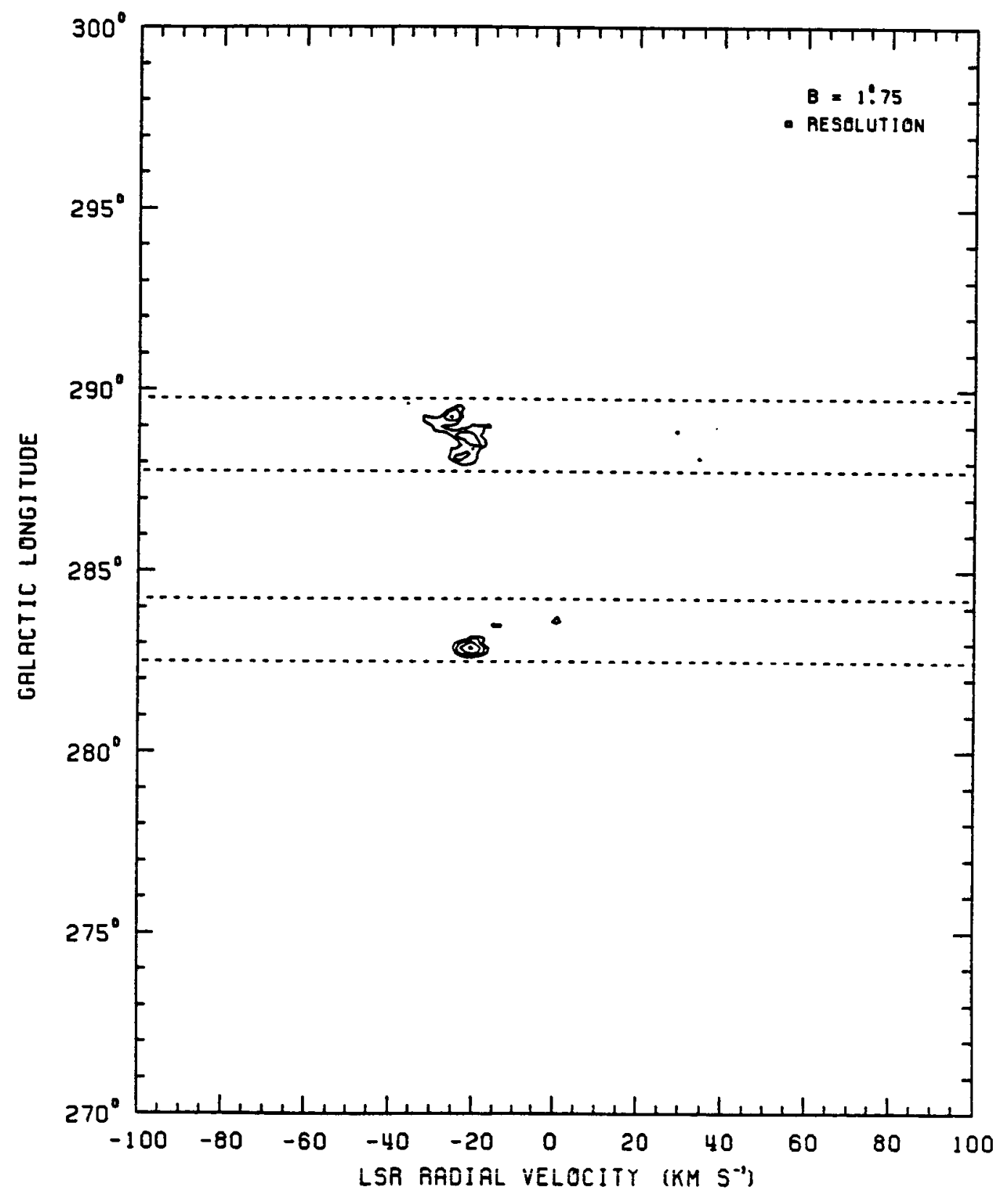

FIGURE B-1 (continued) 


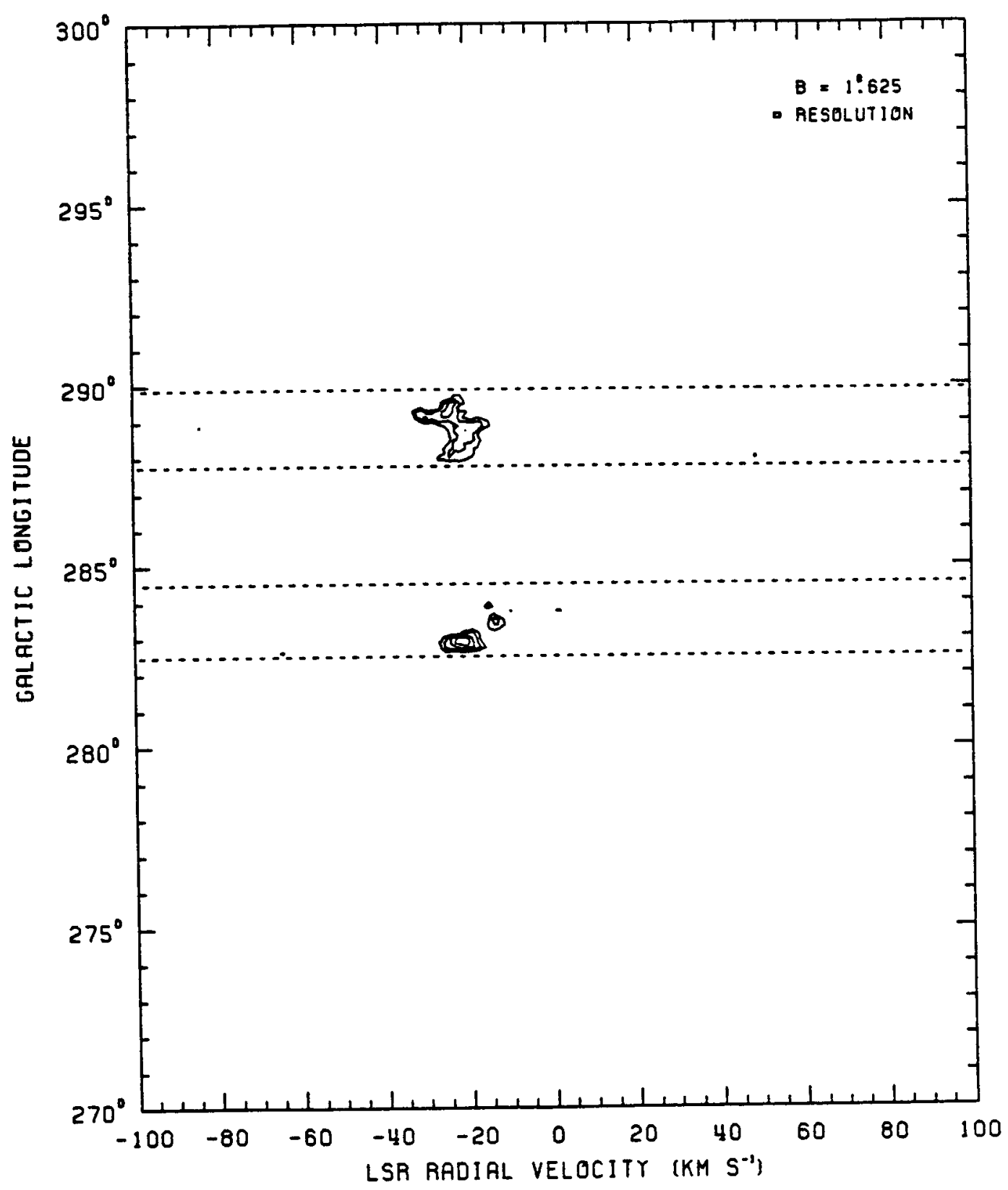

FIGURE B-1 (continued) 


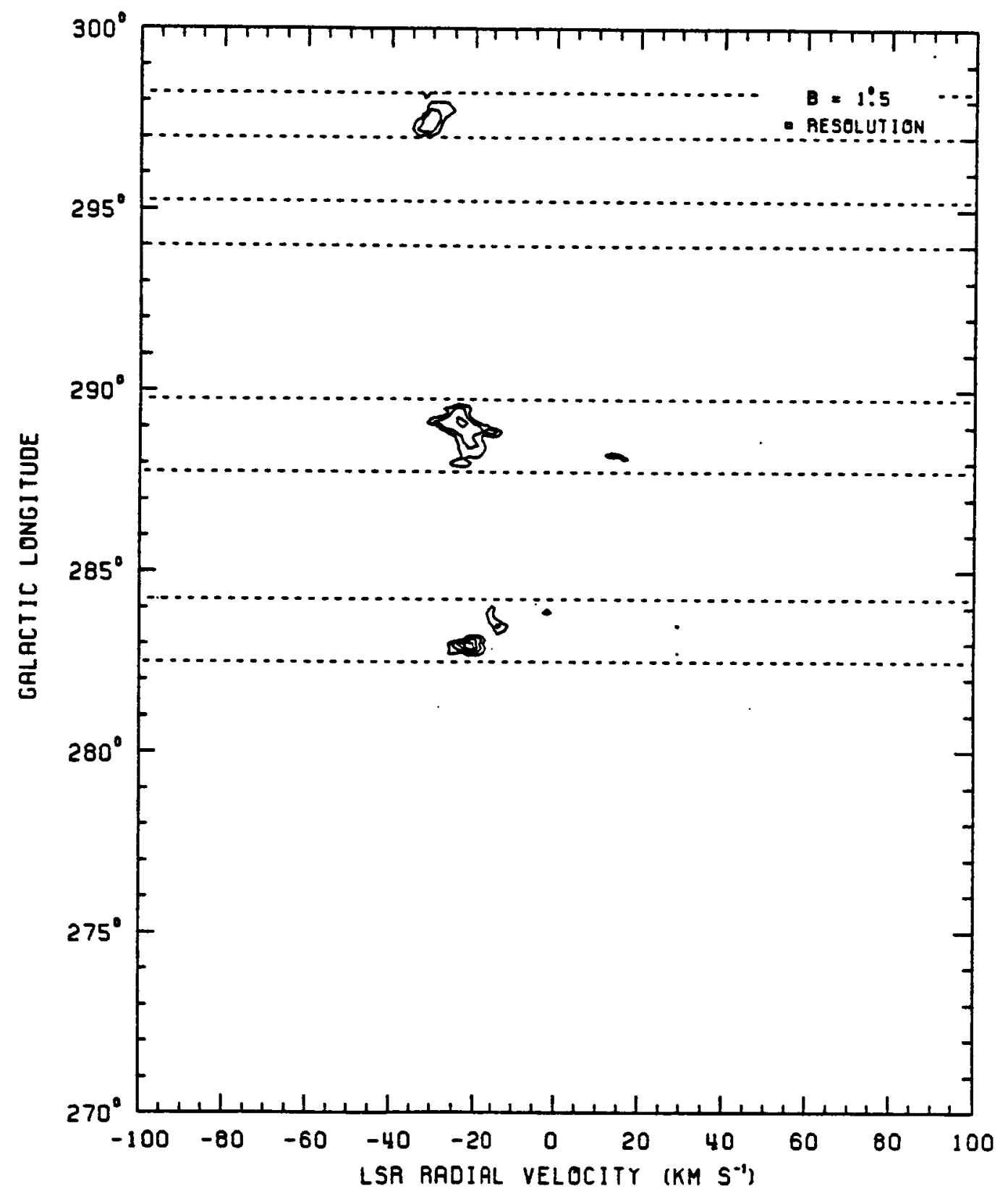

FIGURE B-1 (continued)

205 


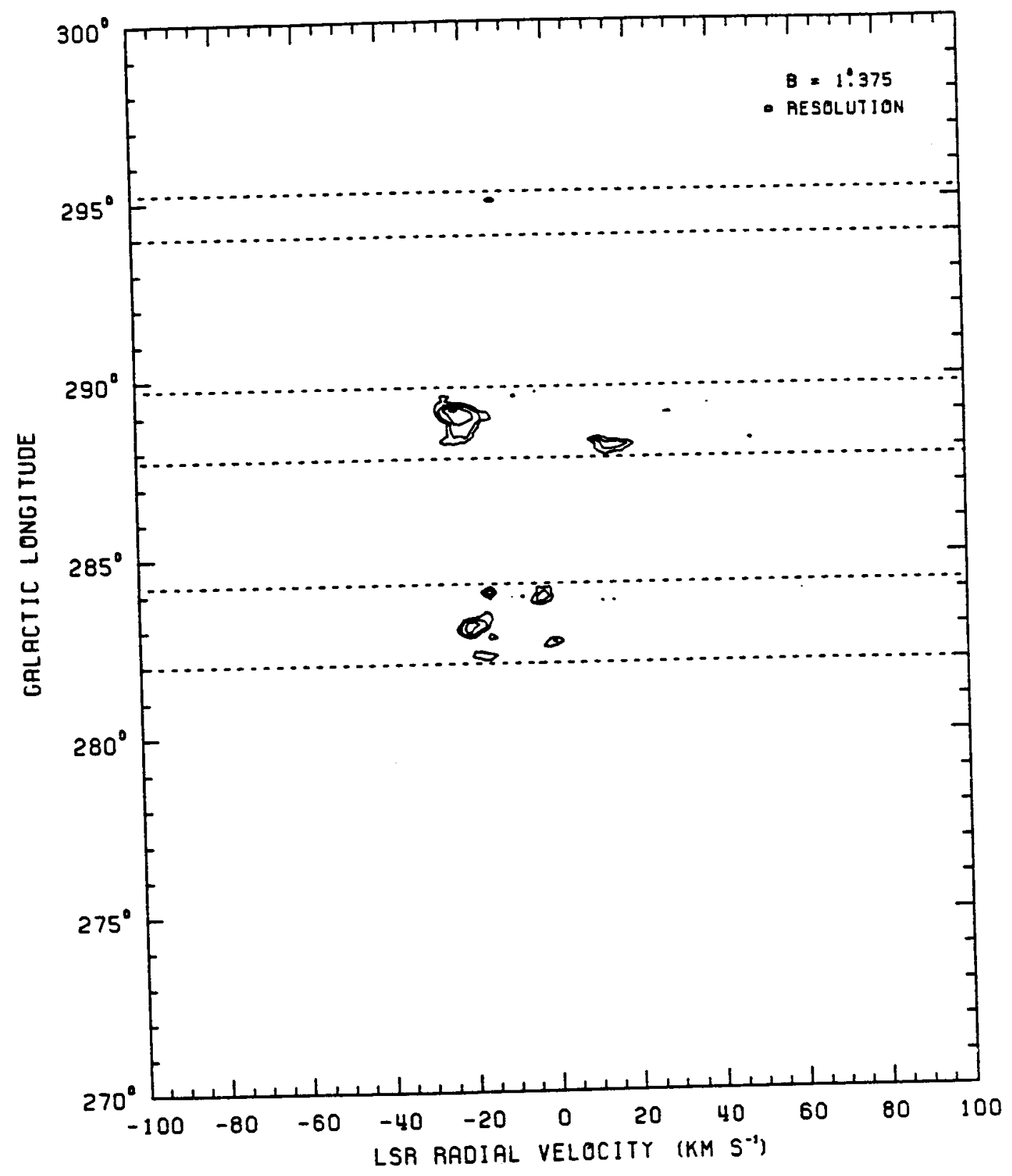

FIGURE B-1 (continued) 


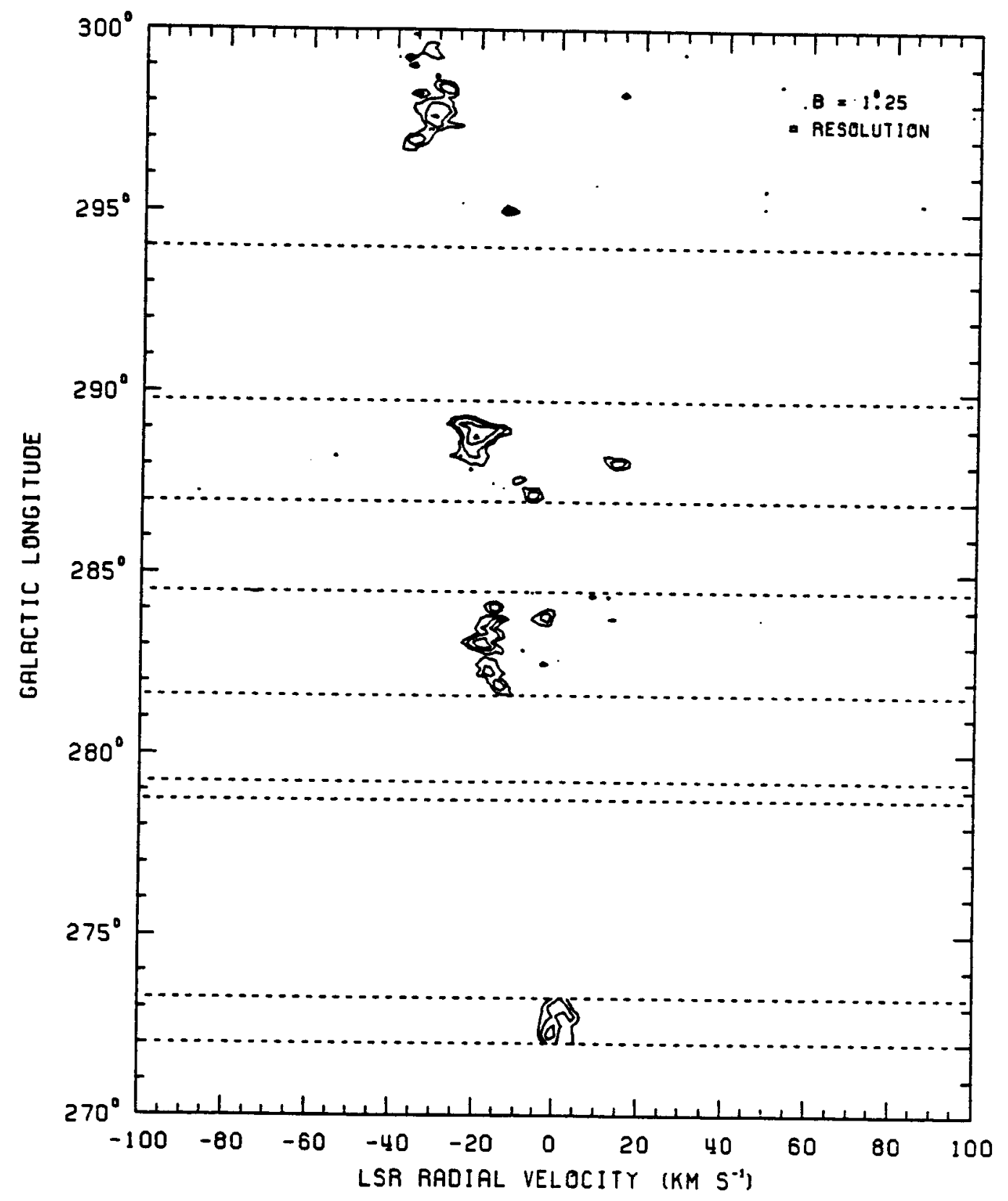

FIGURE B-1 (continued) 


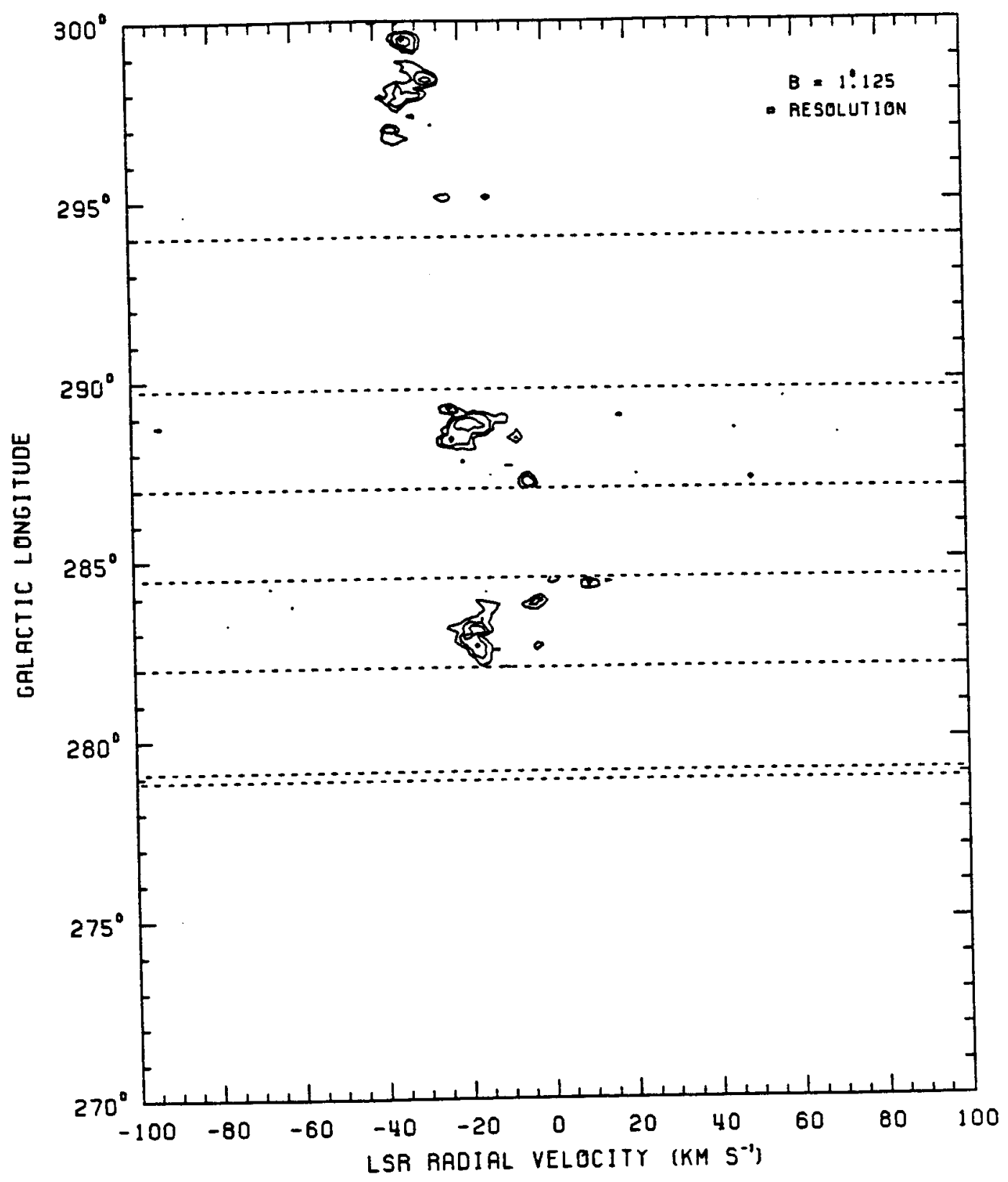

FIGURE B-1 (continued) 


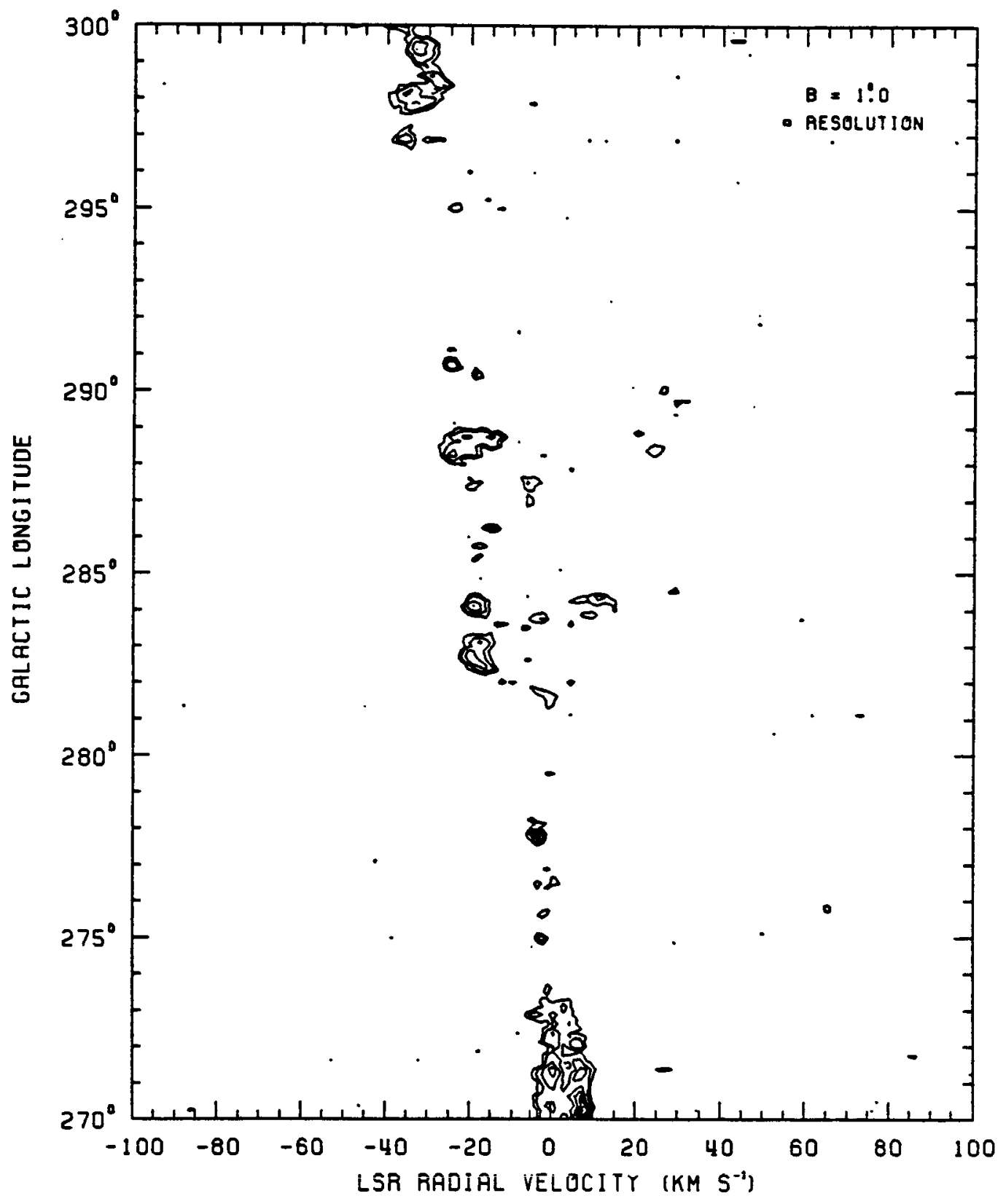

FIGURE B-1 (continued)

209 


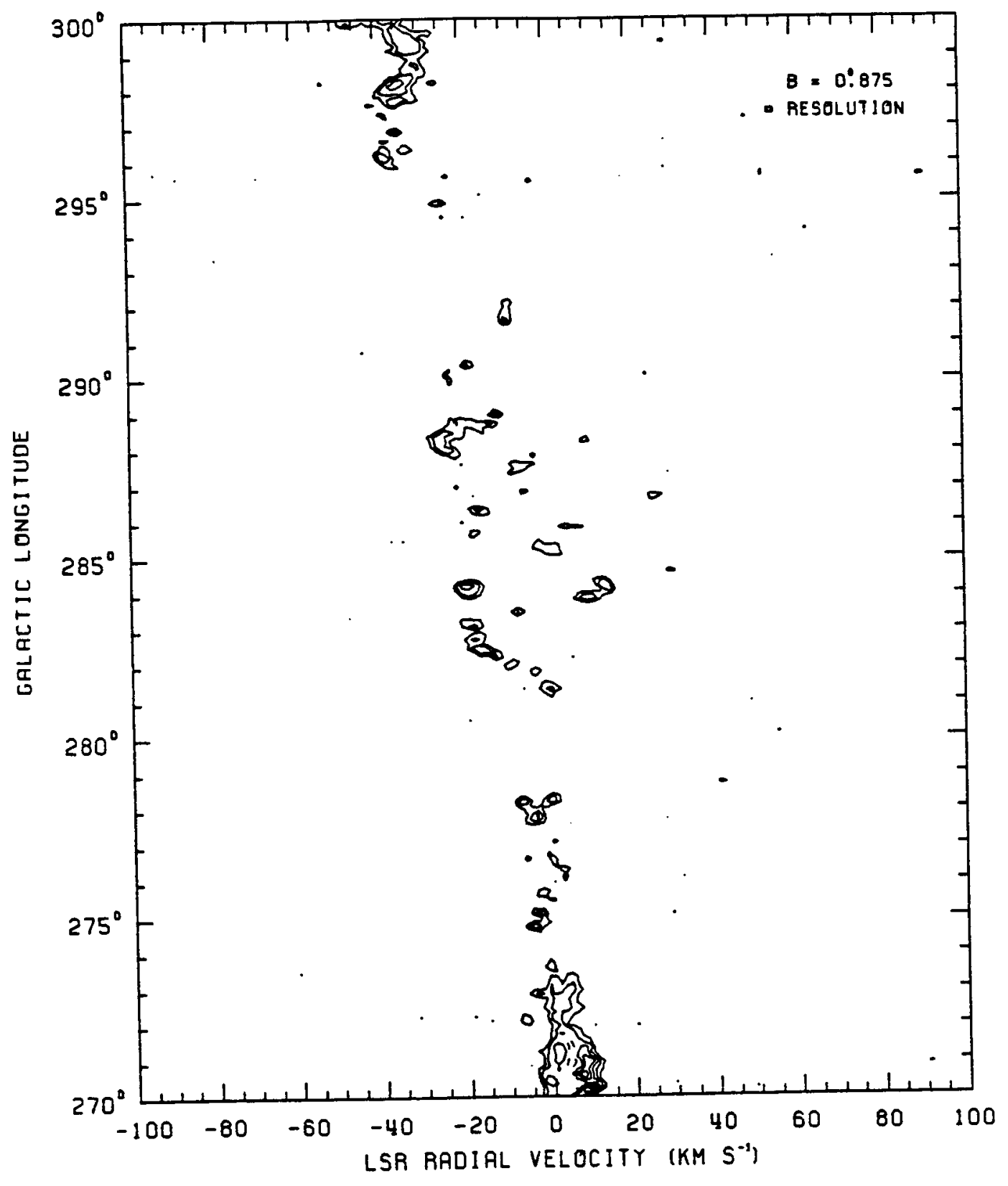

FIGURE B-1 (continued) 


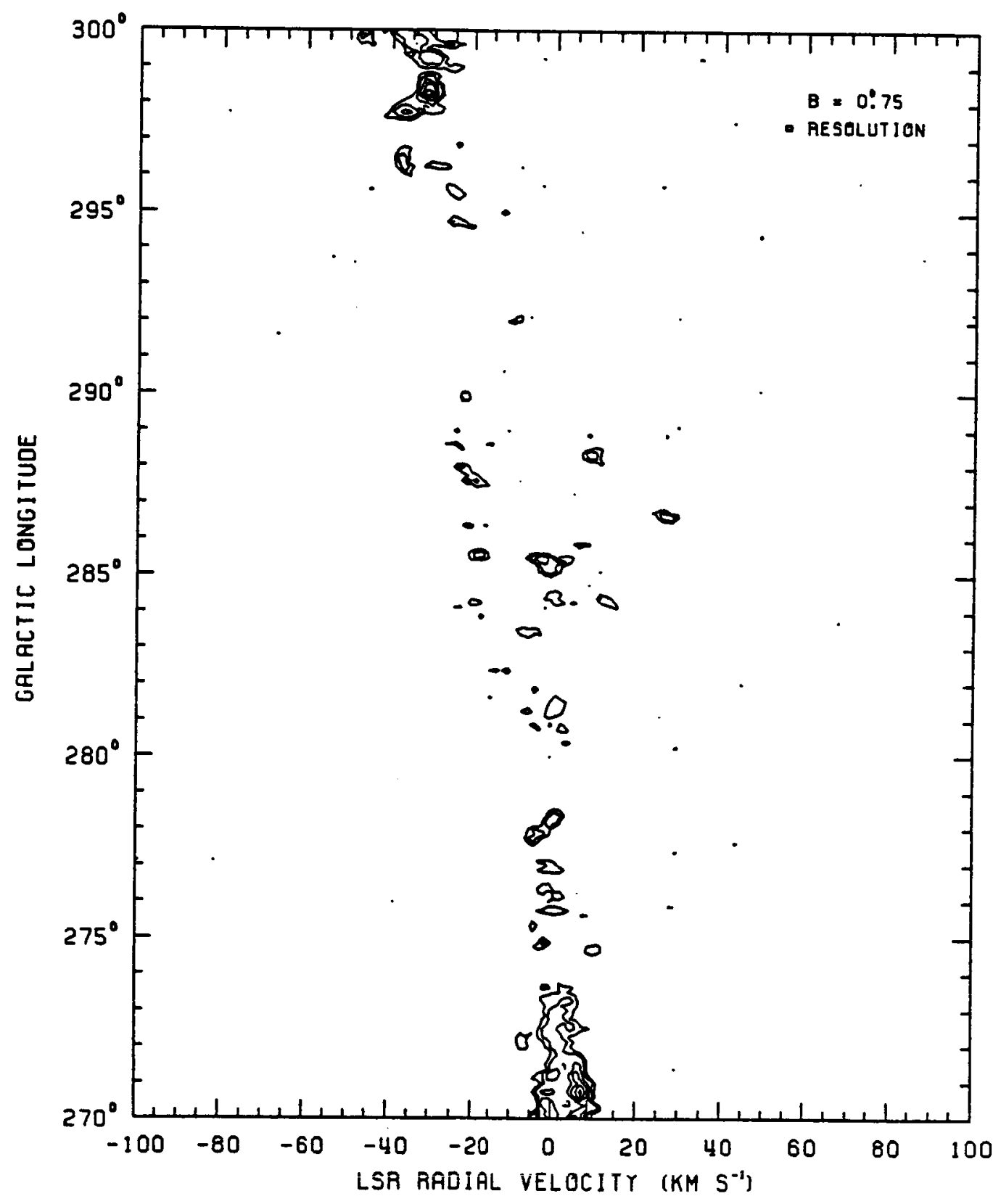

FIGURE B-1 (continued) 


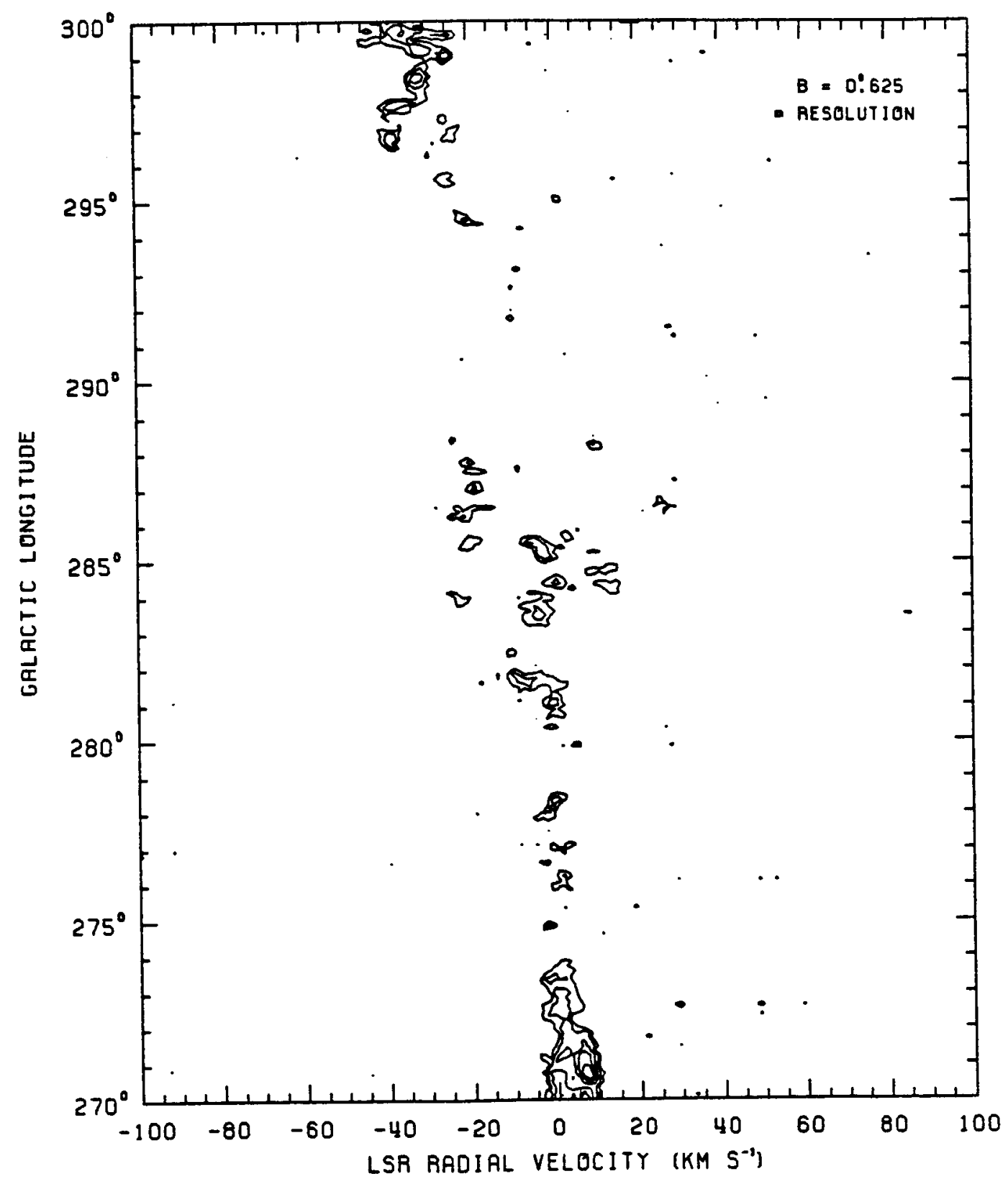

FIGURE B-1 (continued) 


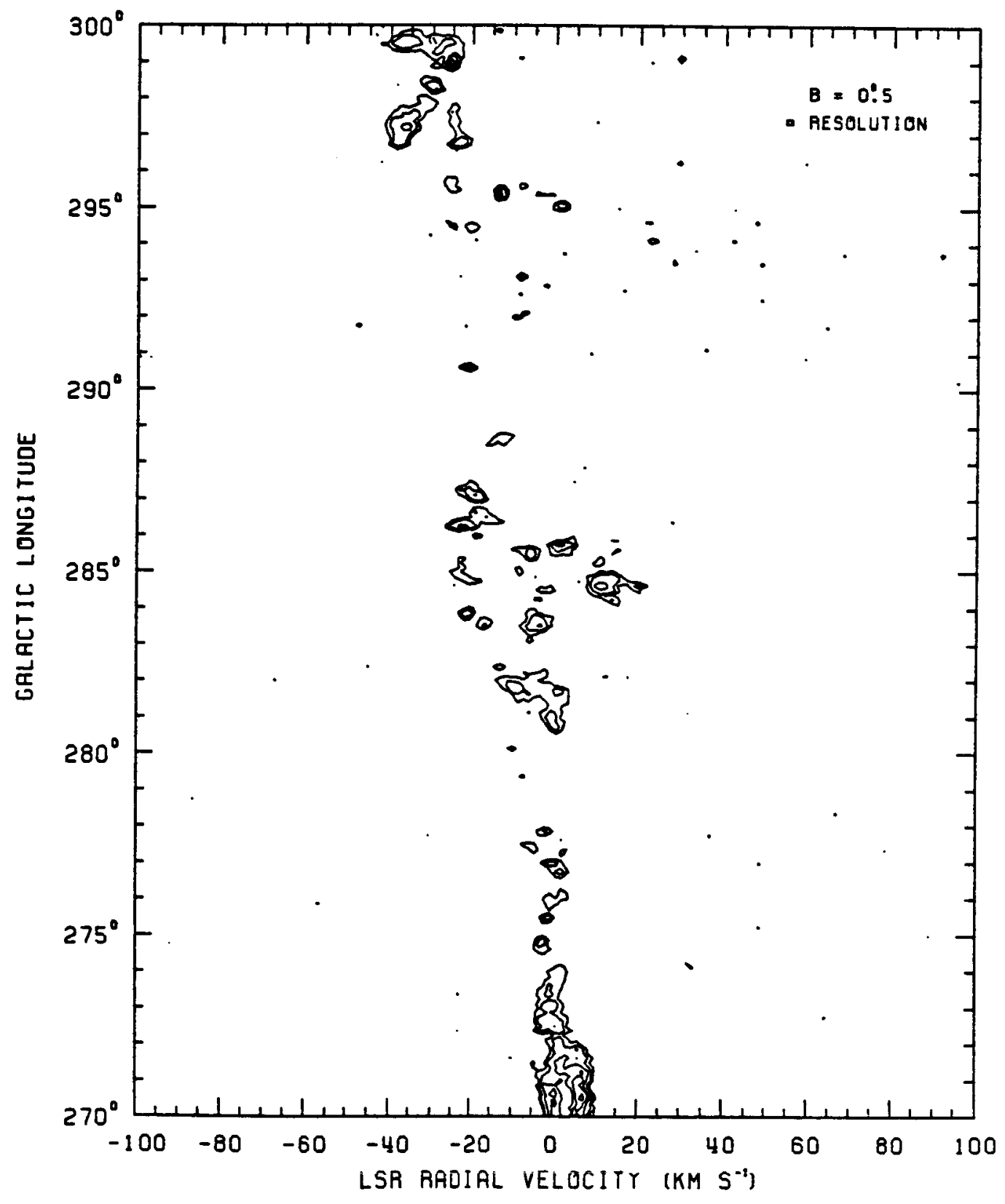

FIGURE B-I (continued)

213 


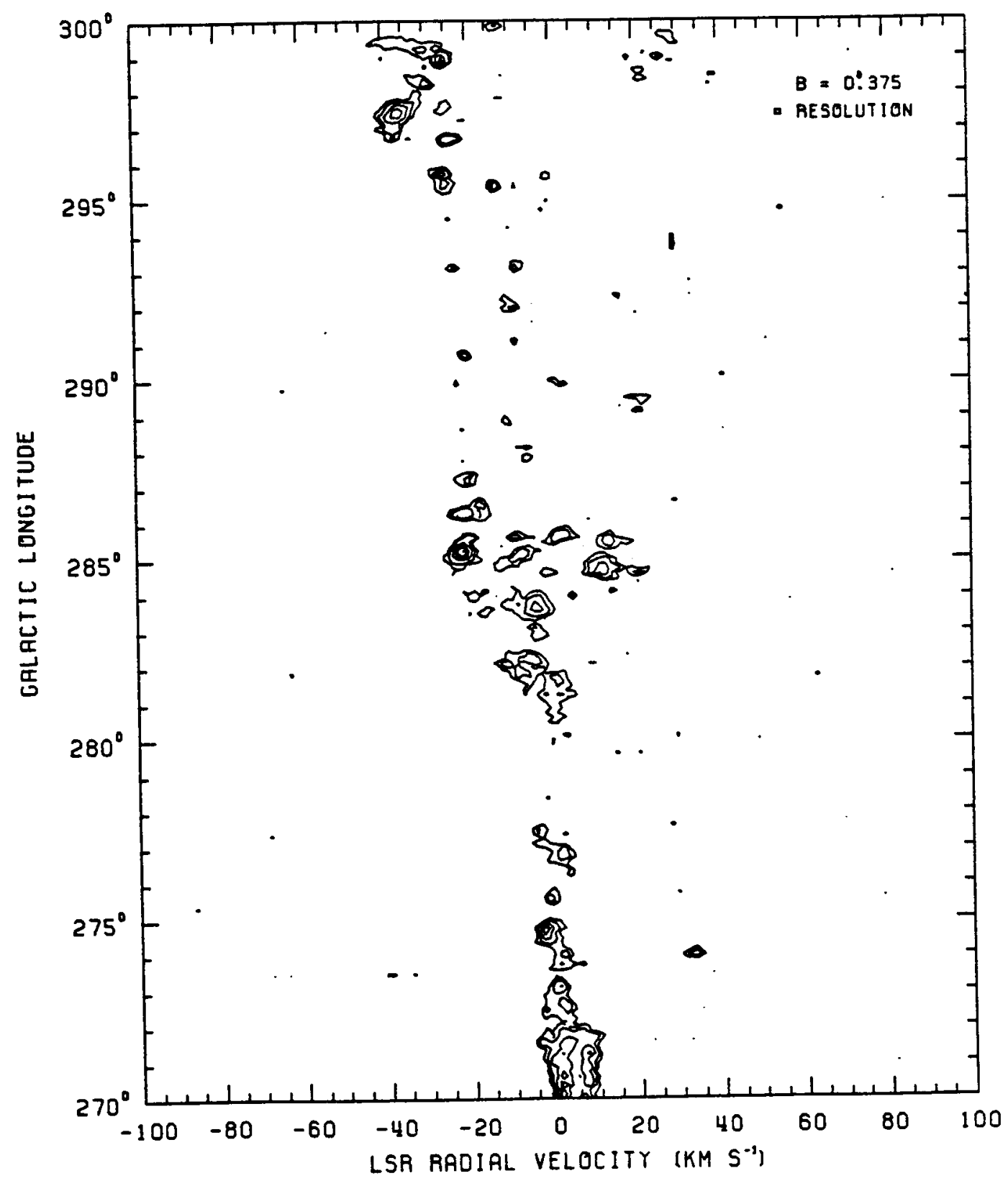

FIGURE B-1 (continued) 


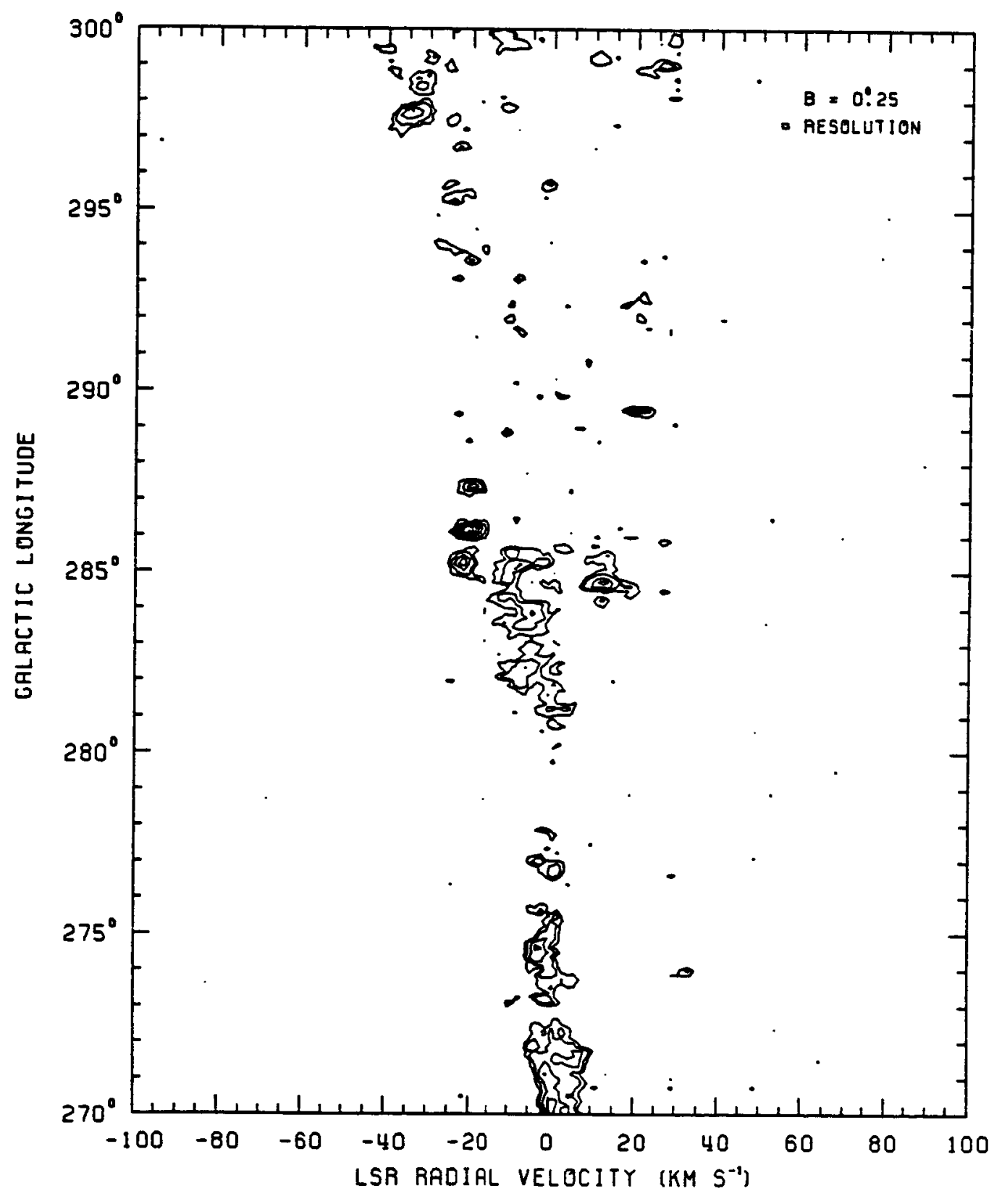

FIGURE B-1 (continued) 


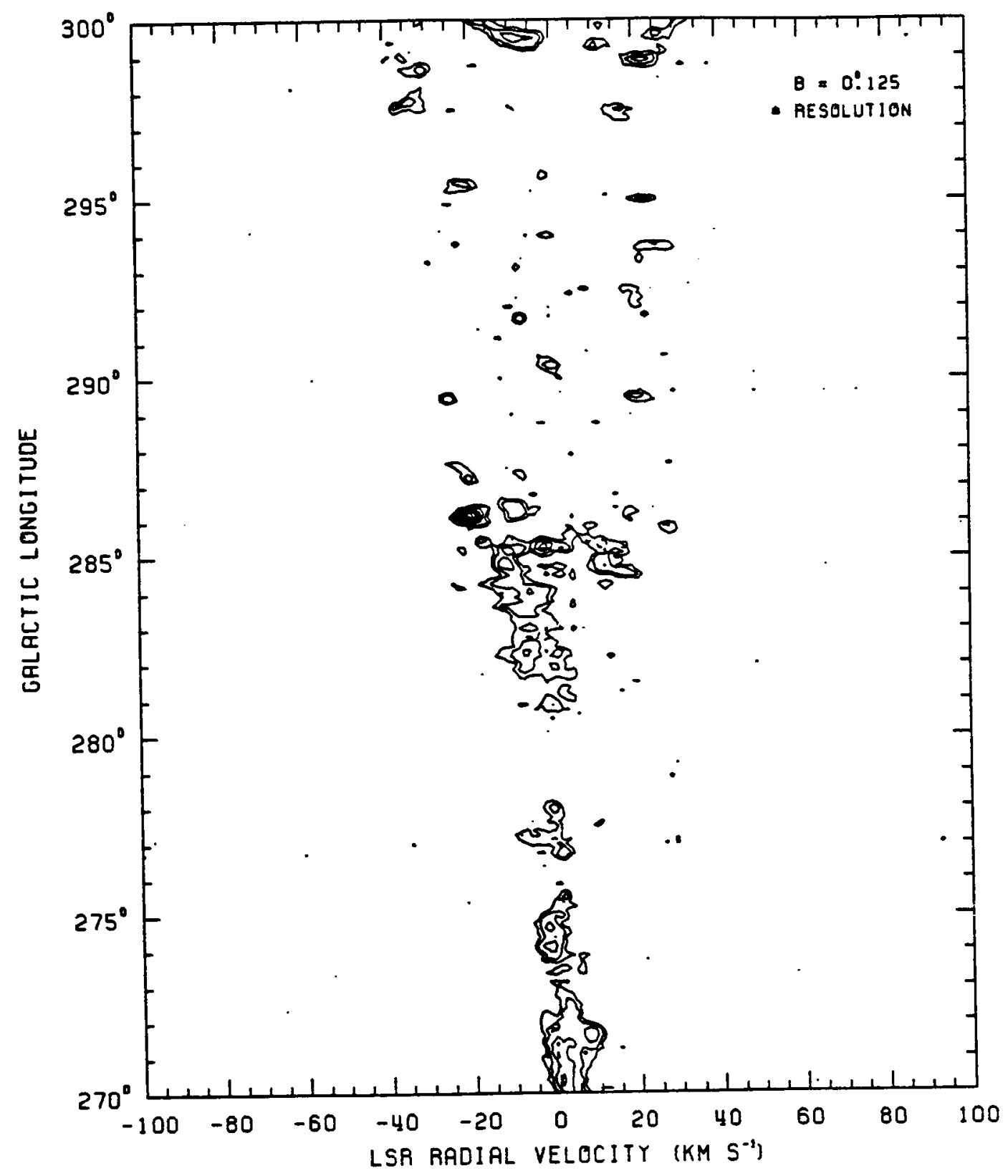

FIGURE B-1 (continued) 


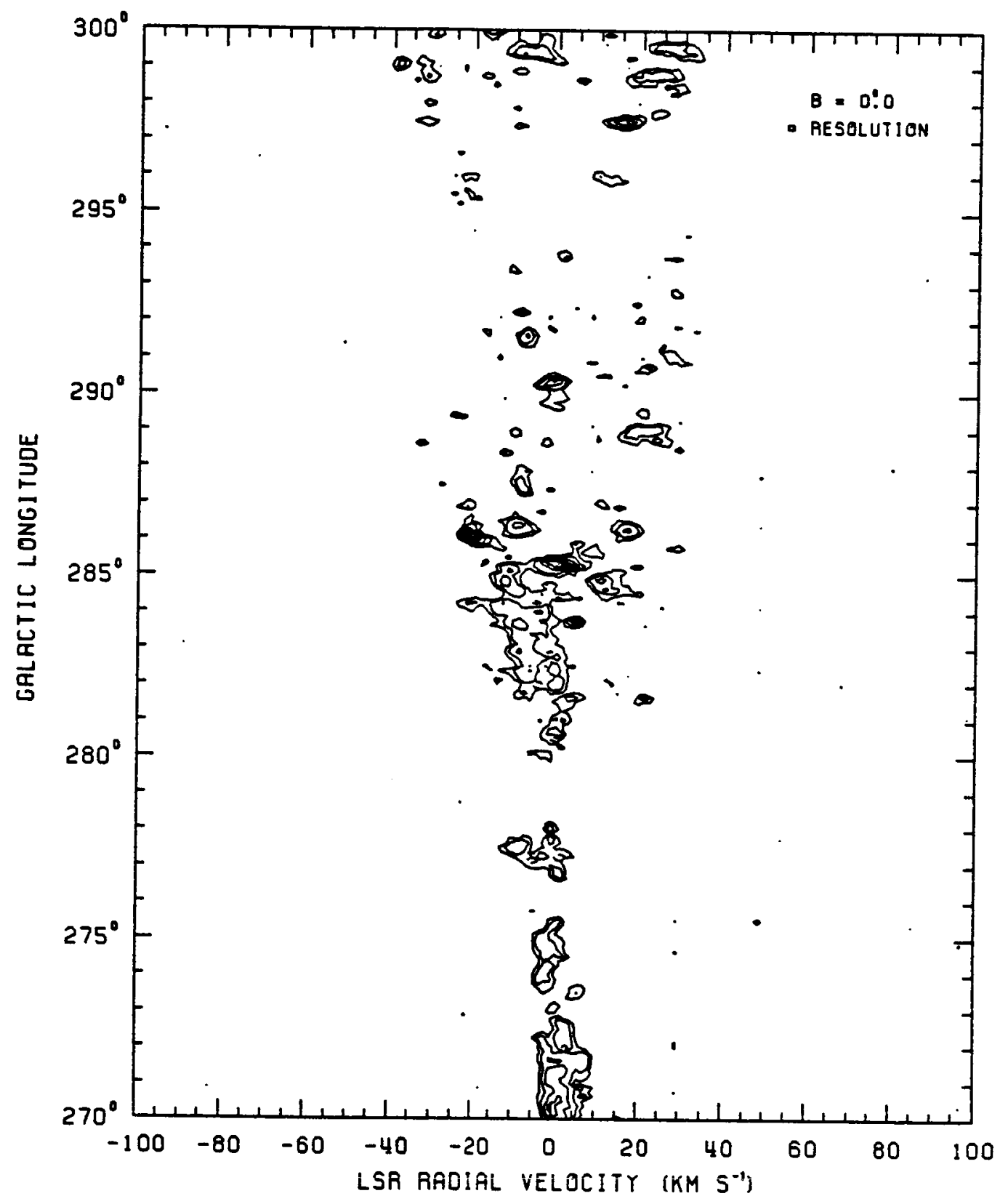

FIGURE B-1 (continued) 


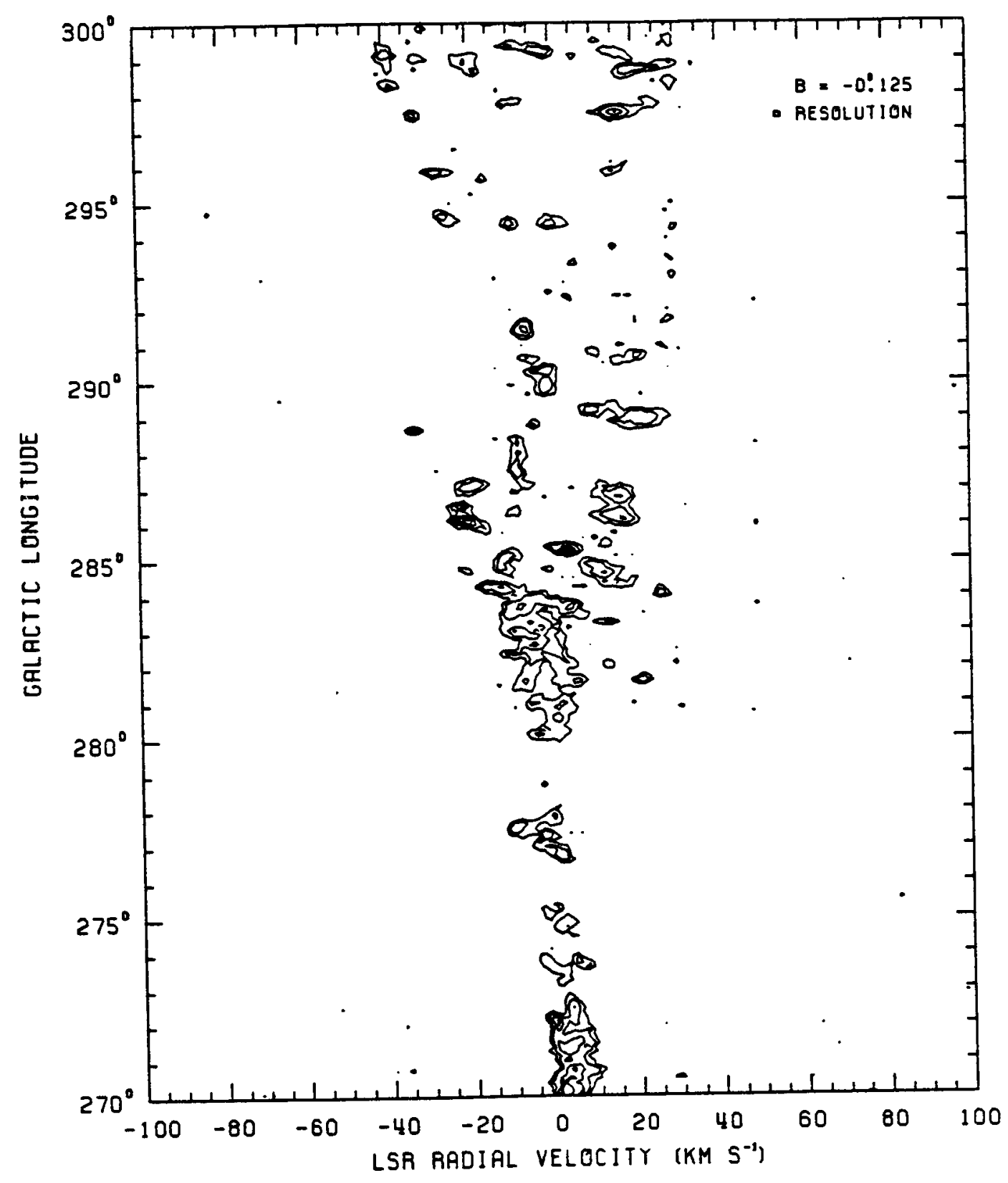

FIGURE B-1 (cont inued) 


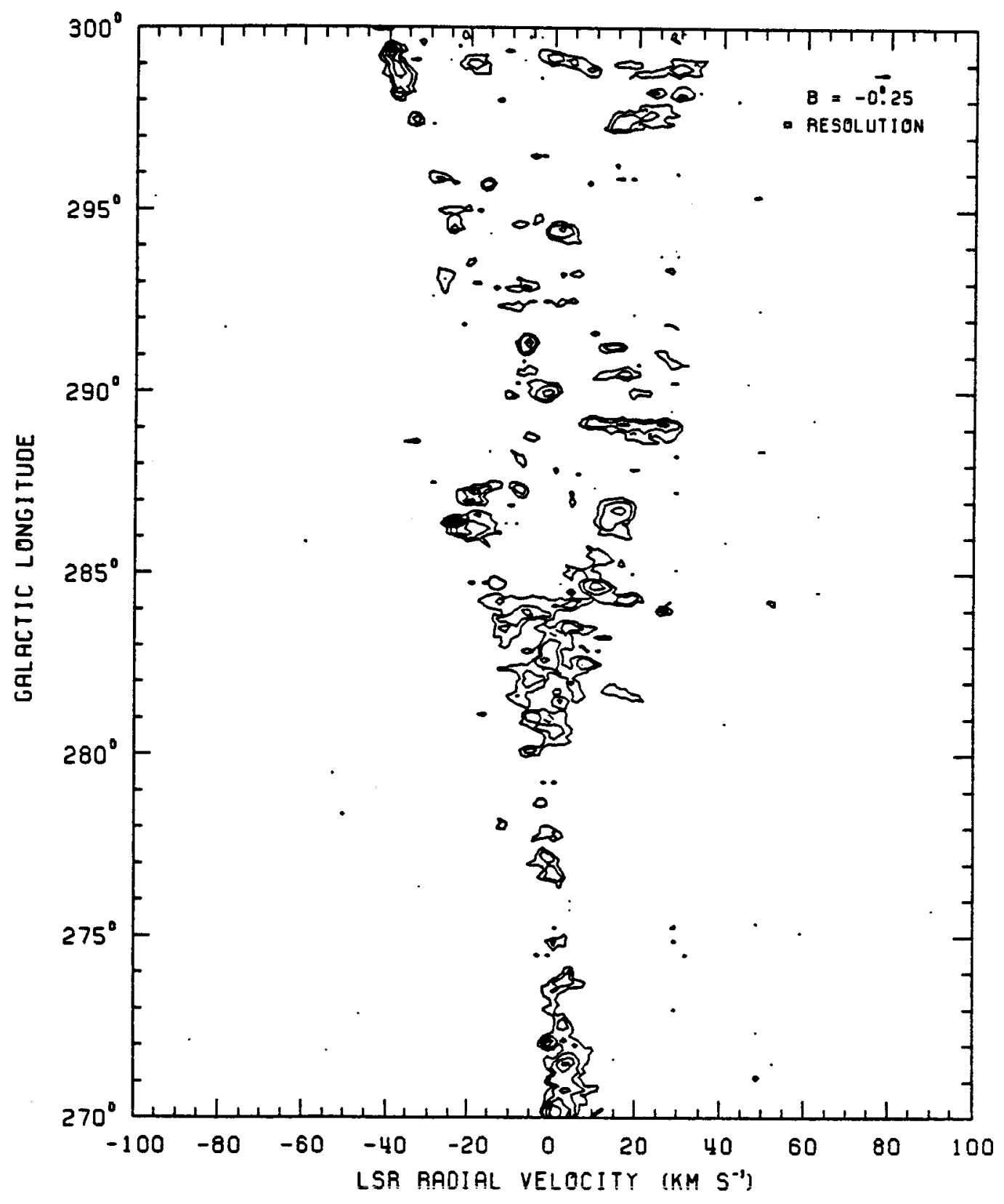

FIGURE B-1 (continued) 


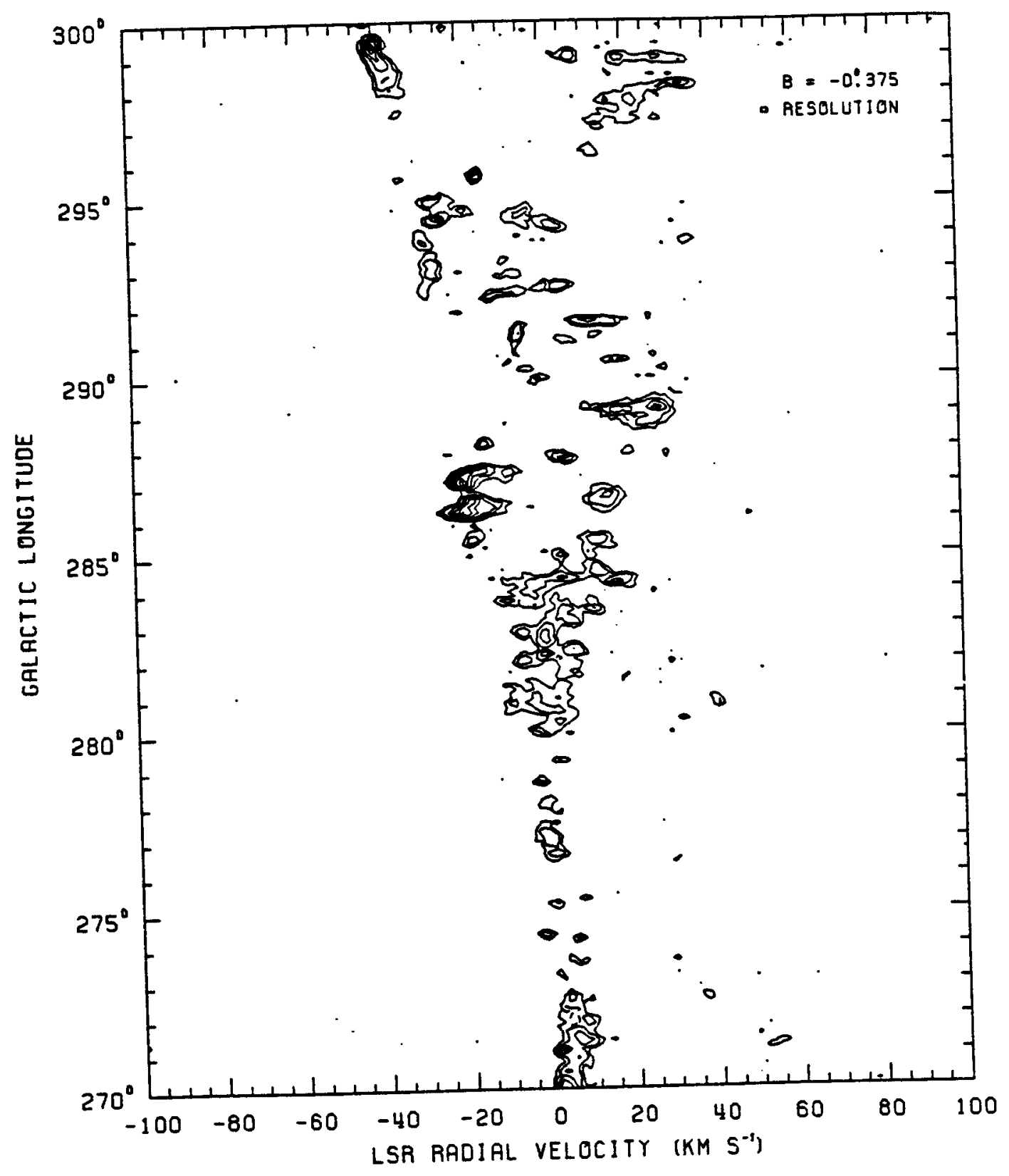

FIGURE B-1 (continued) 
ORIGINAL PAGE IS

OF POOR QUALITY

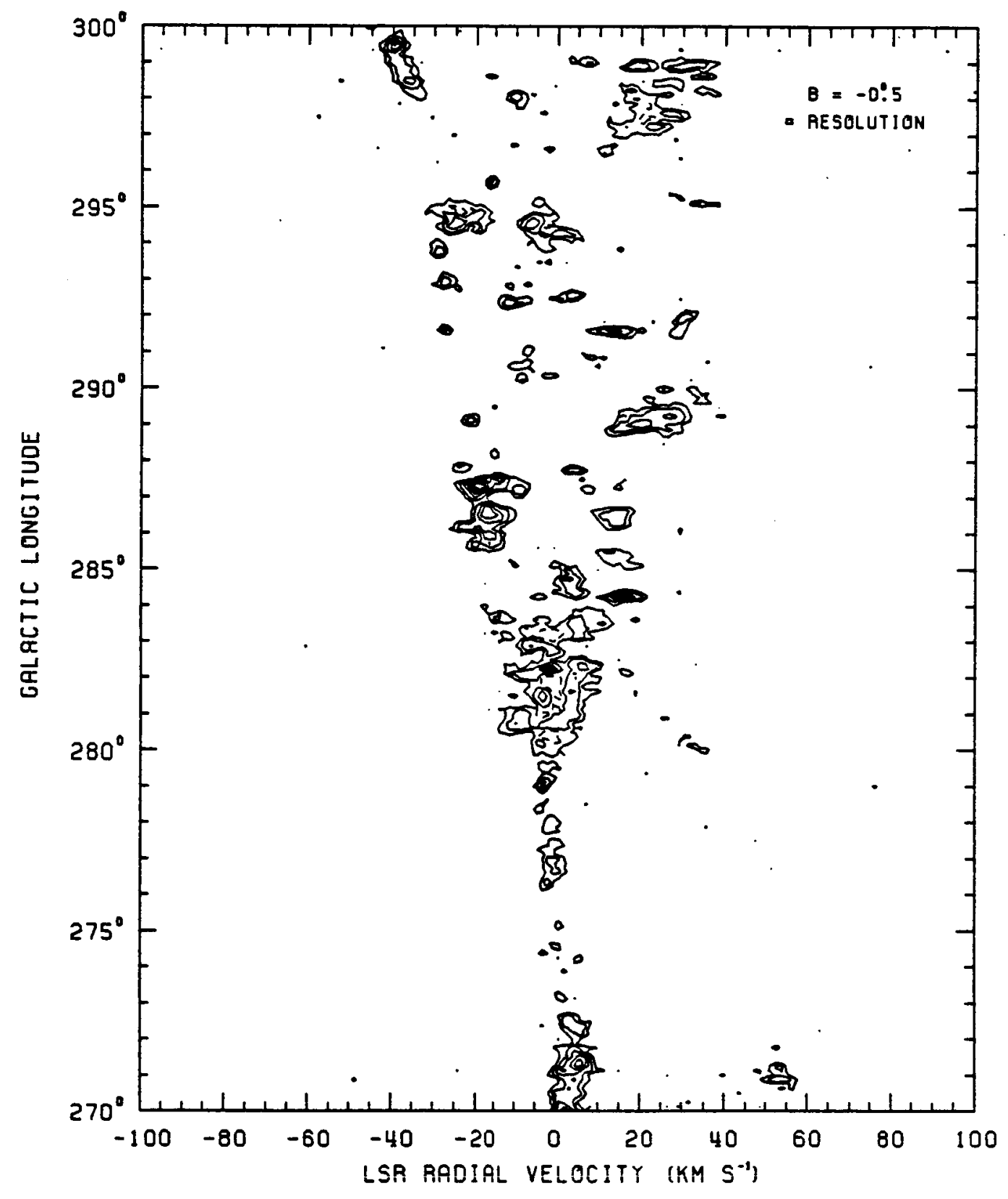

FIGURE B-1 (continued) 


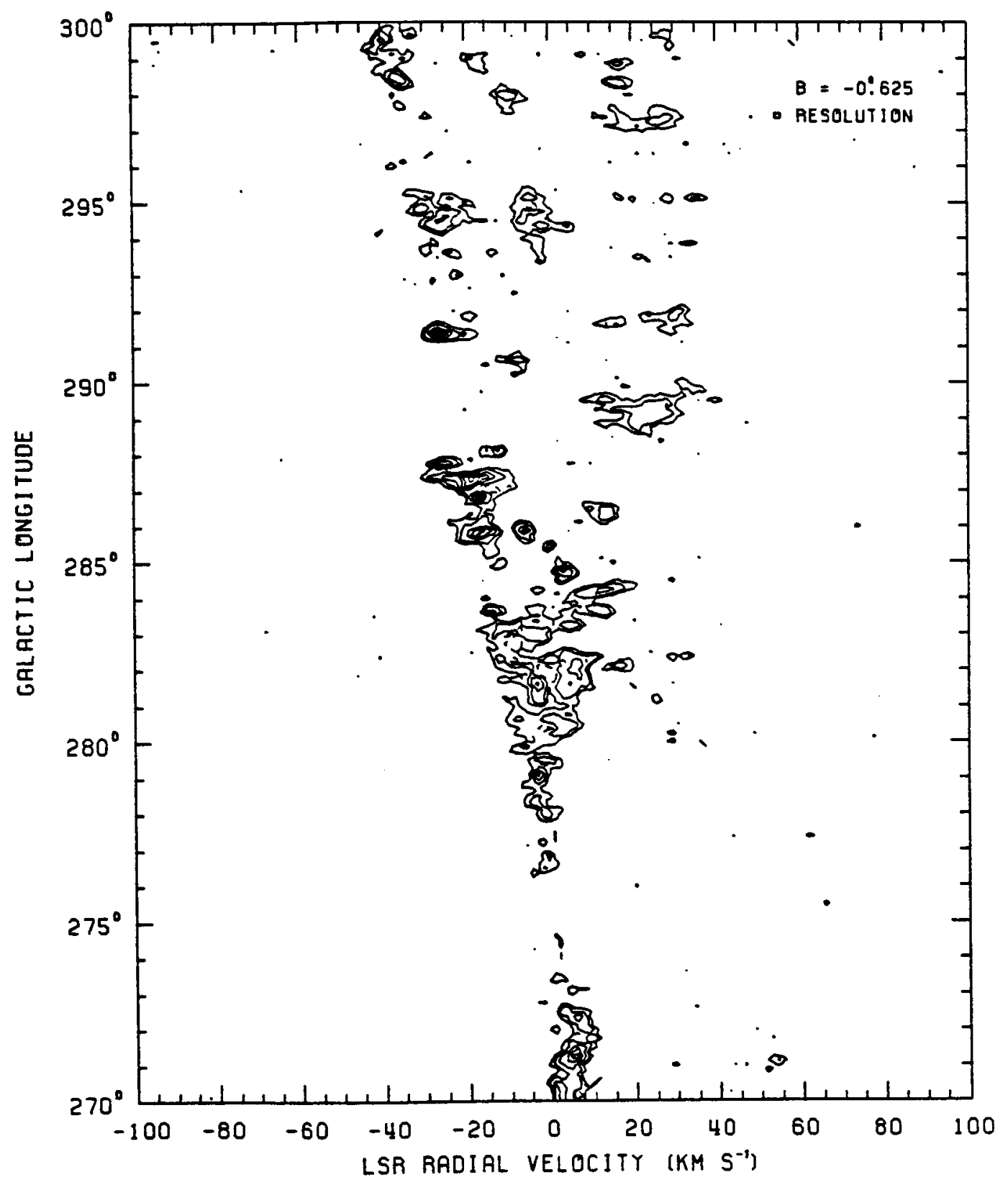

FIGURE B-1 (continued) 
ORIGINAL PAGE IS

OF POOR QUALITY

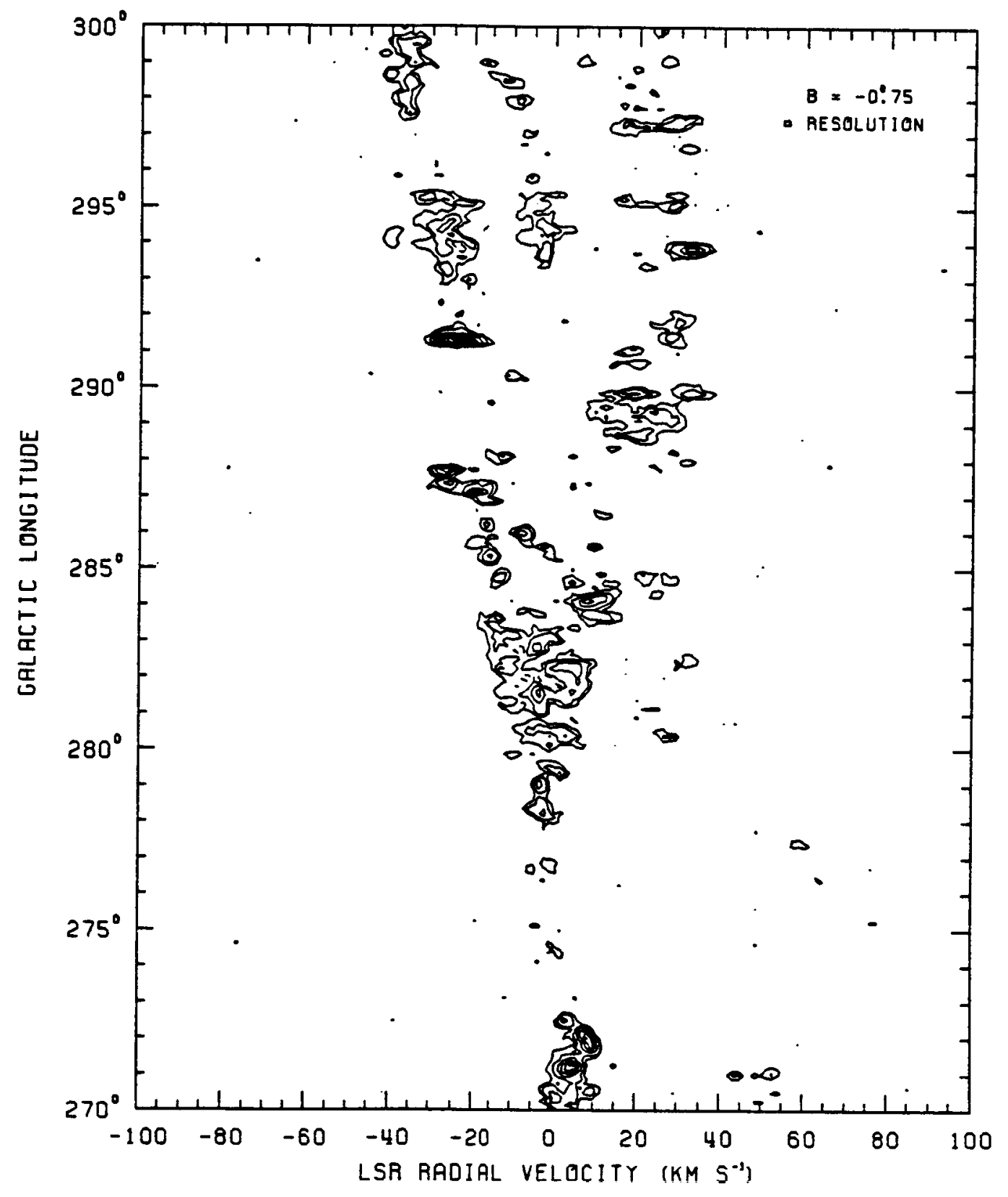

FIGURE B-1 (continued) 


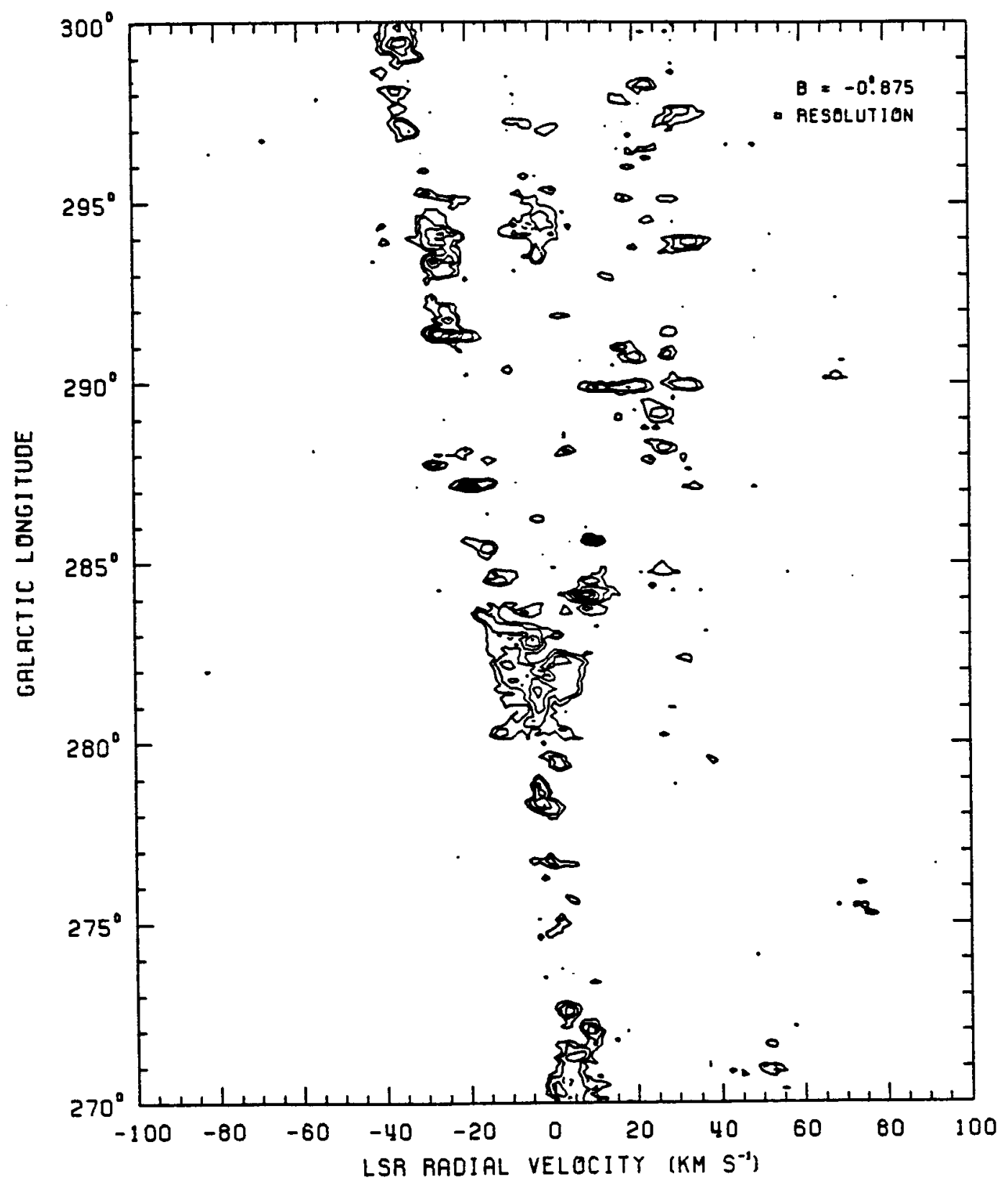

FIGURE B-1 (continued) 
ORIGINAL PAGE IS

OF POOR QUALITY

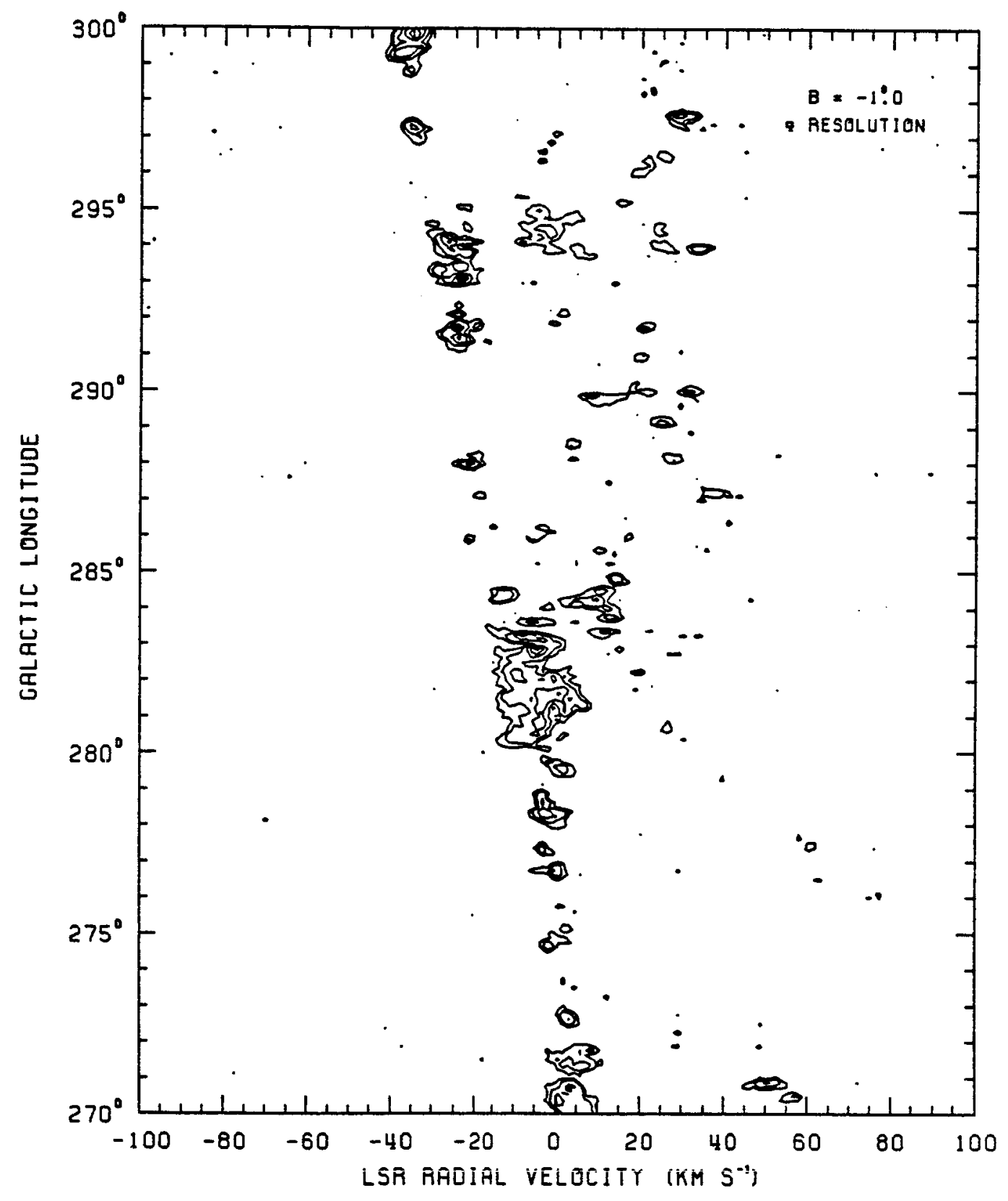

FIGURE B-1 (continued) 


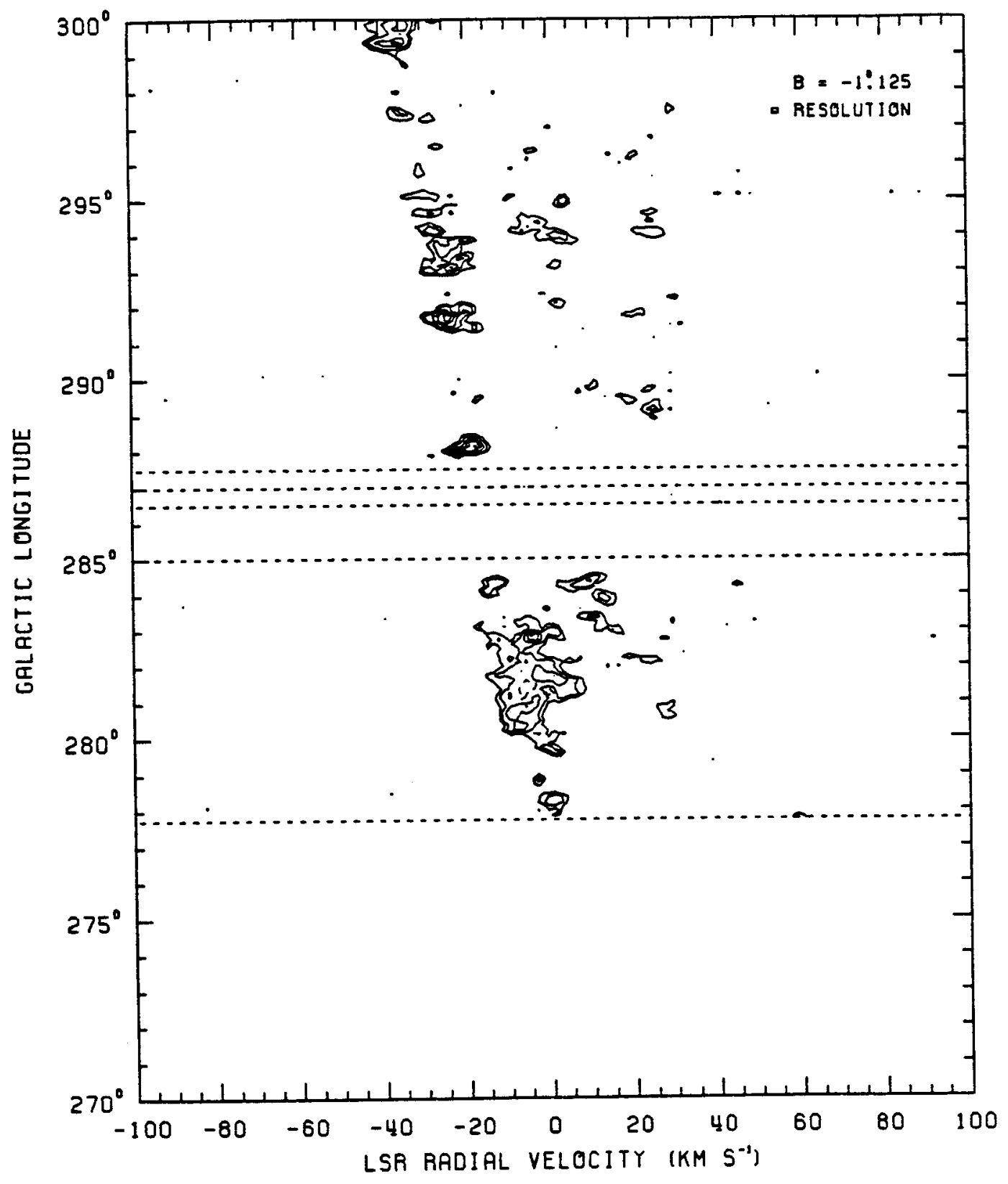

FIGURE B-1 (continued) 


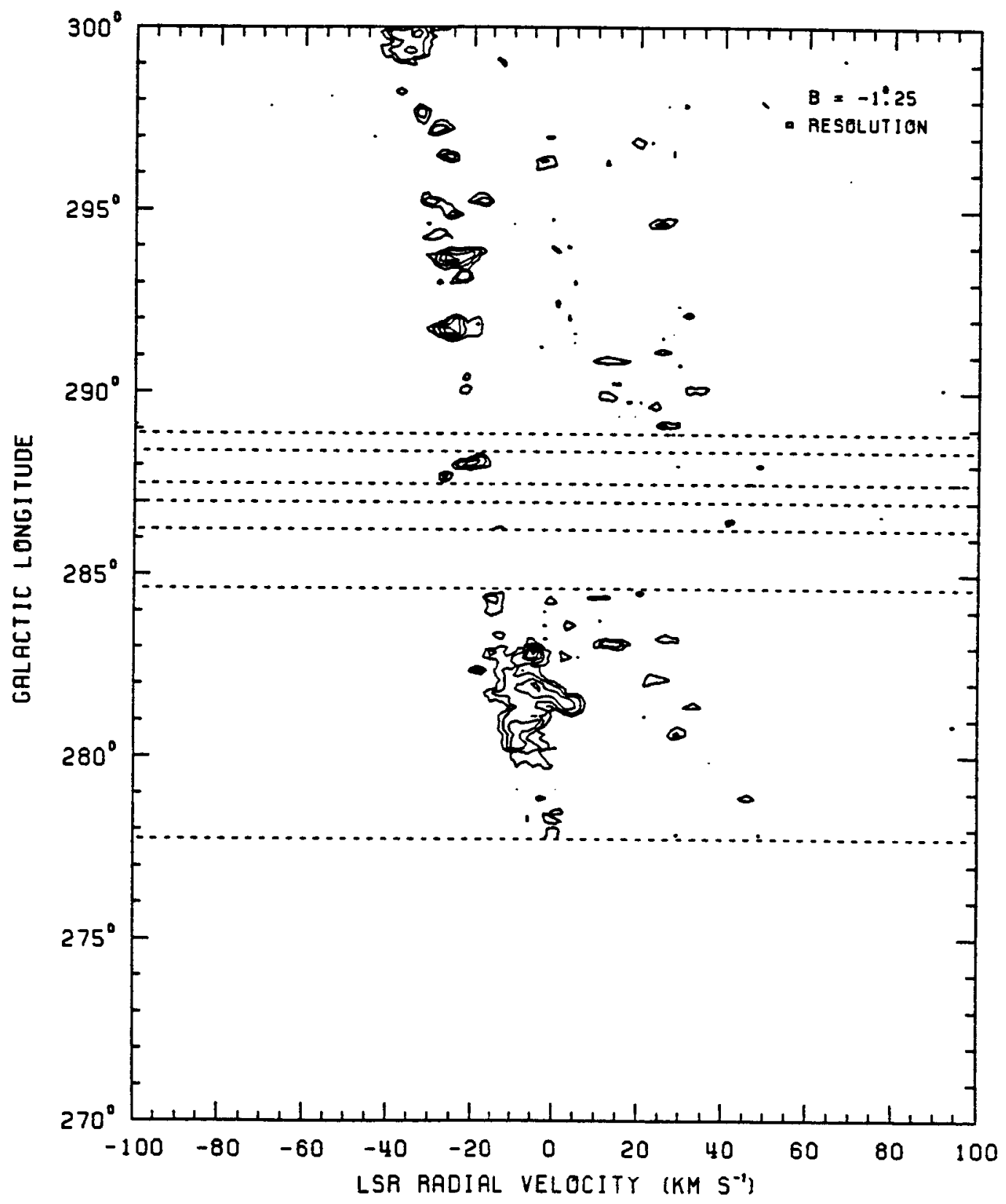

FIGURE B-1 (continued) 


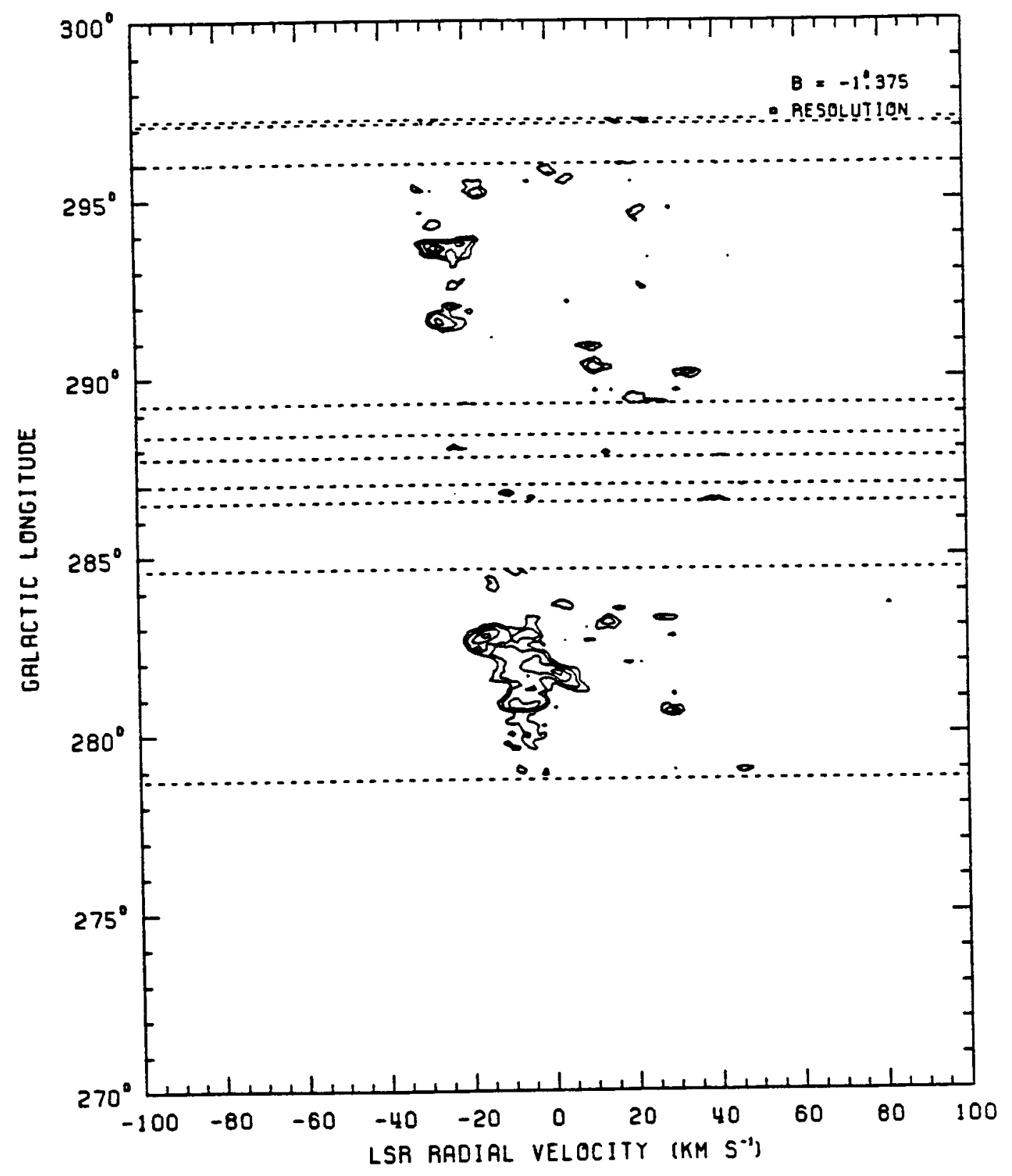

FIGURE B-1 (continued) 
ORIGINAL PAGE is

OF POOR QUALITY

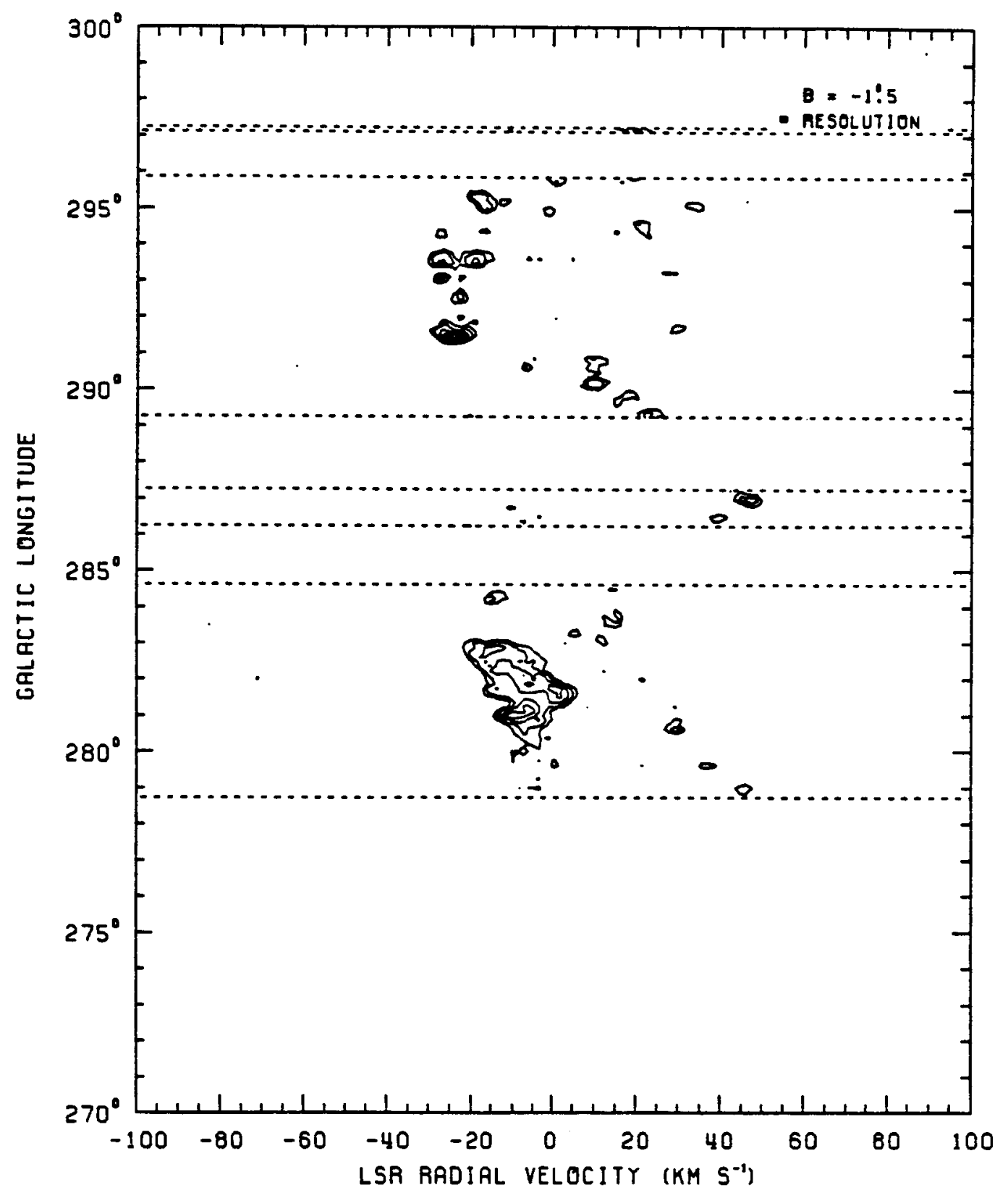

FIGURE B-1 (continued) 


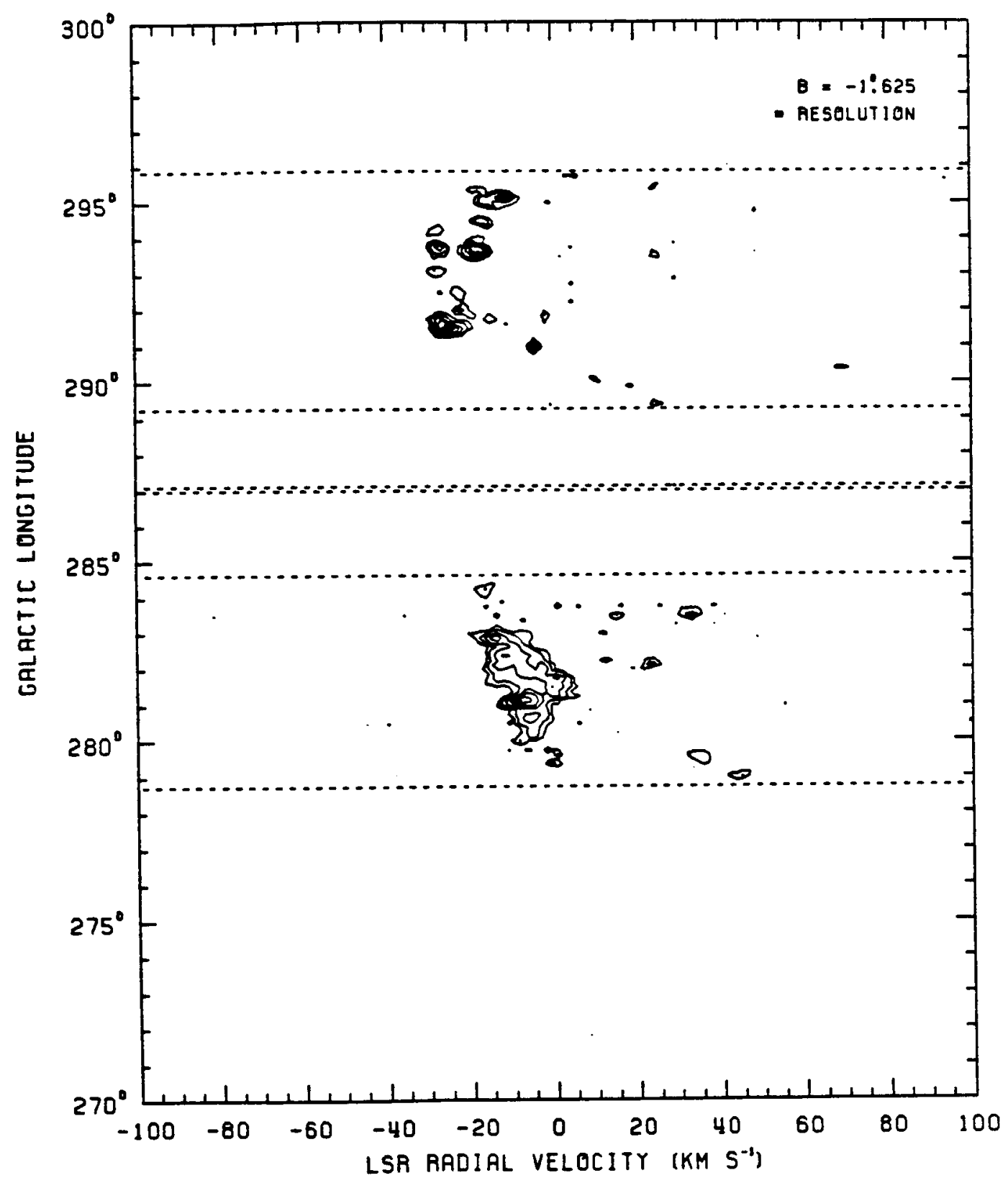

FIGURE B-1 (continued) 


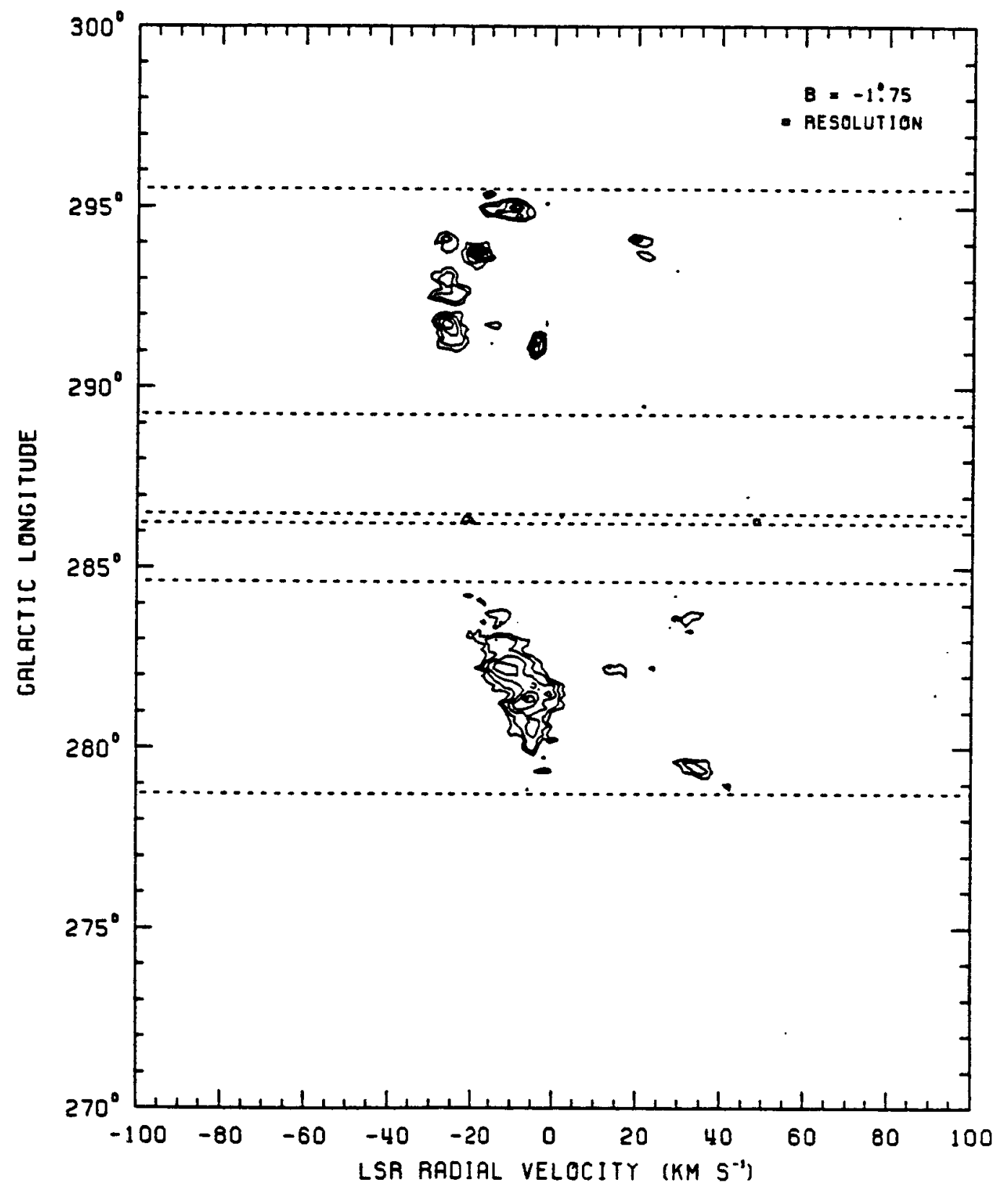

FIGURE B-1 (continued) 


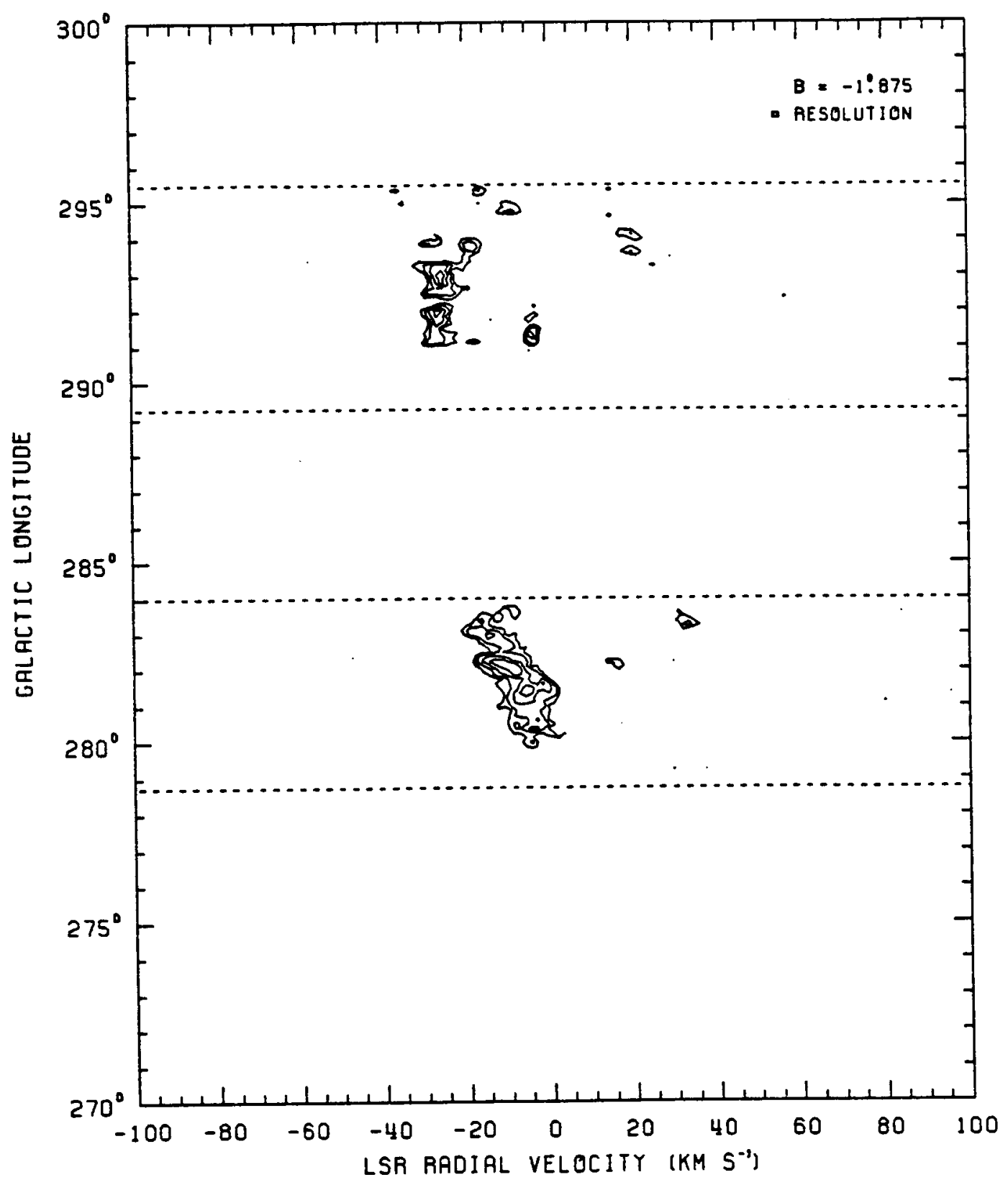

FIGURE B-1 (continued) 


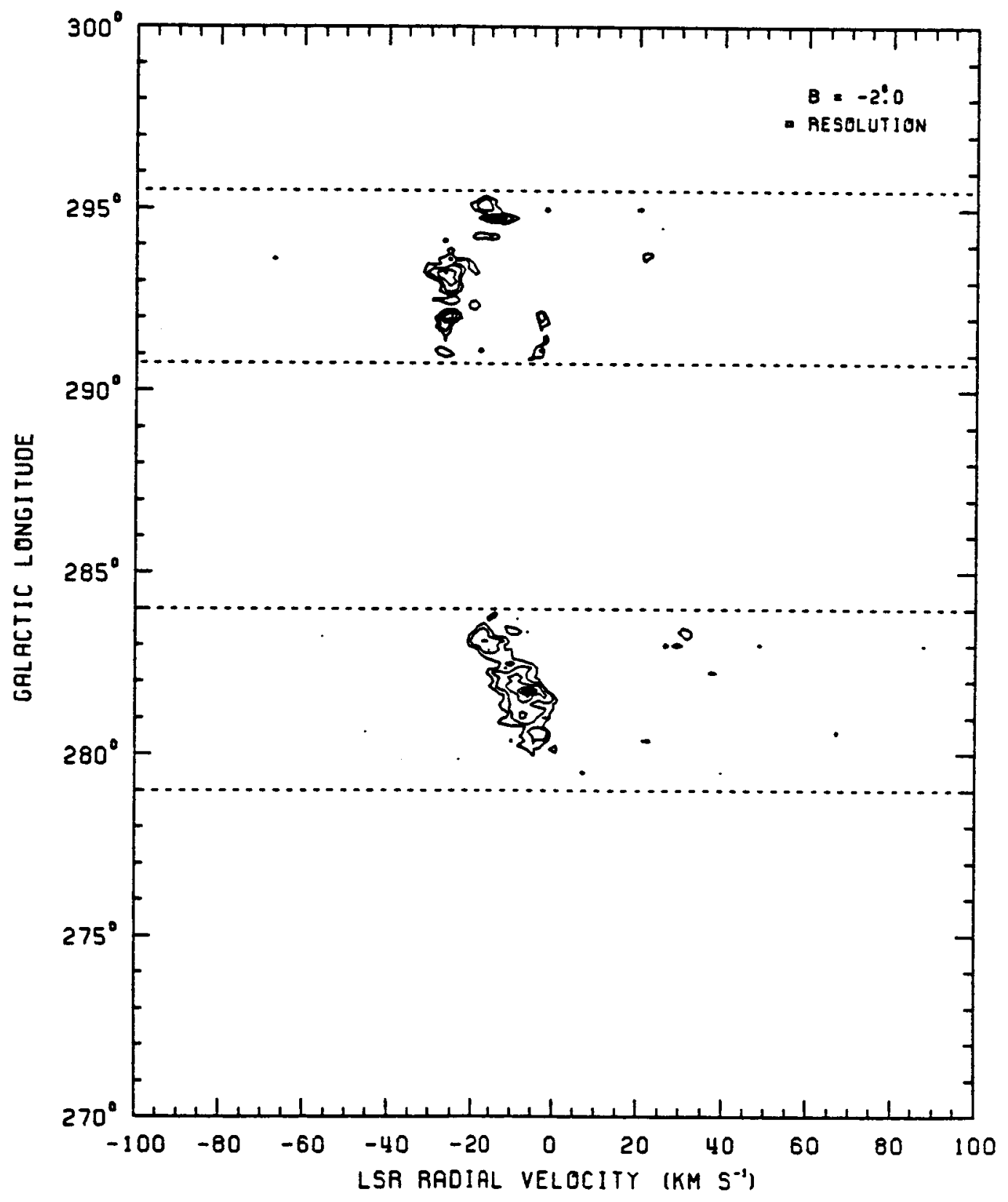

FIGURE B-1 (continued) 


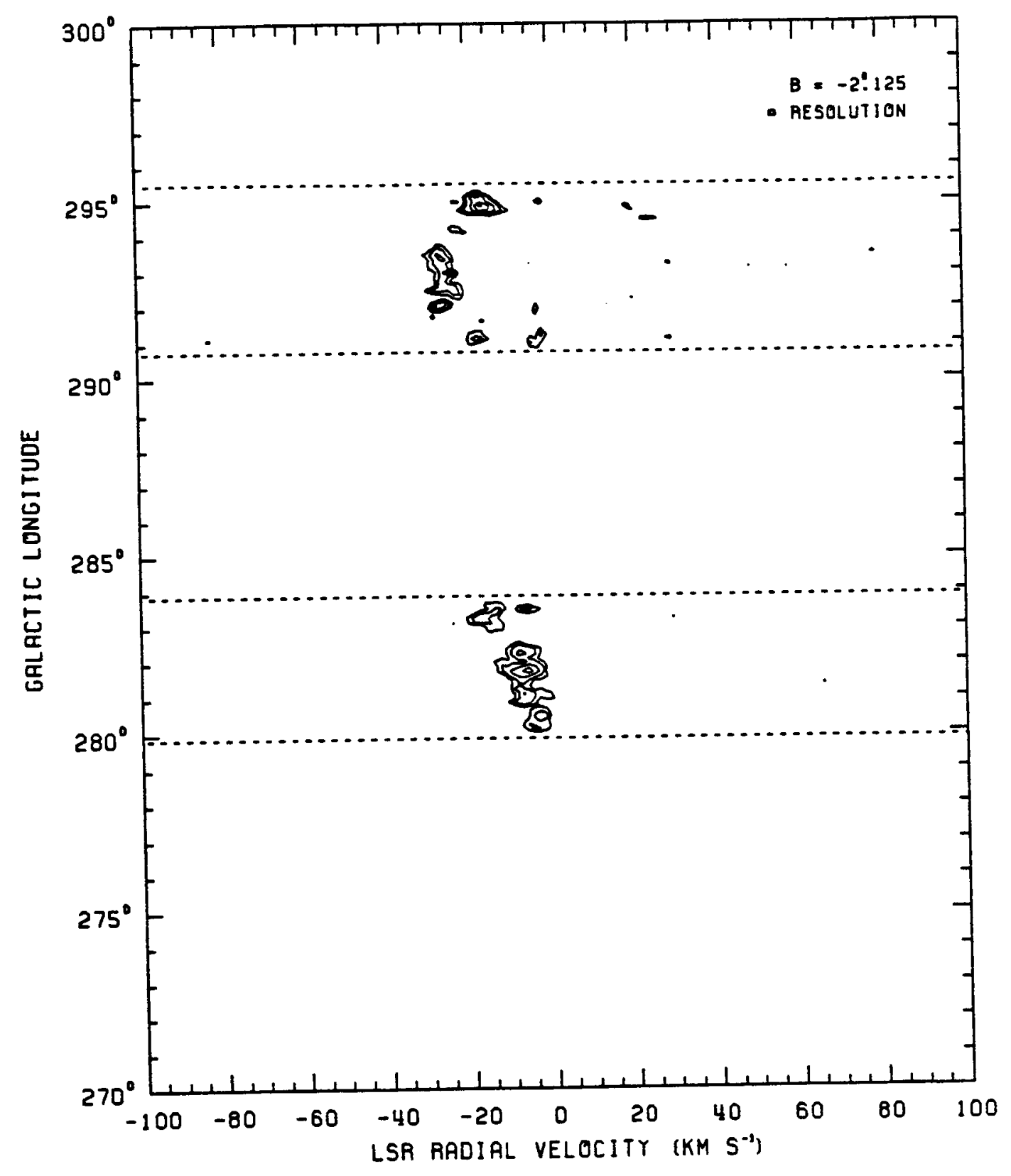

FIGURE B-1 (continued) 


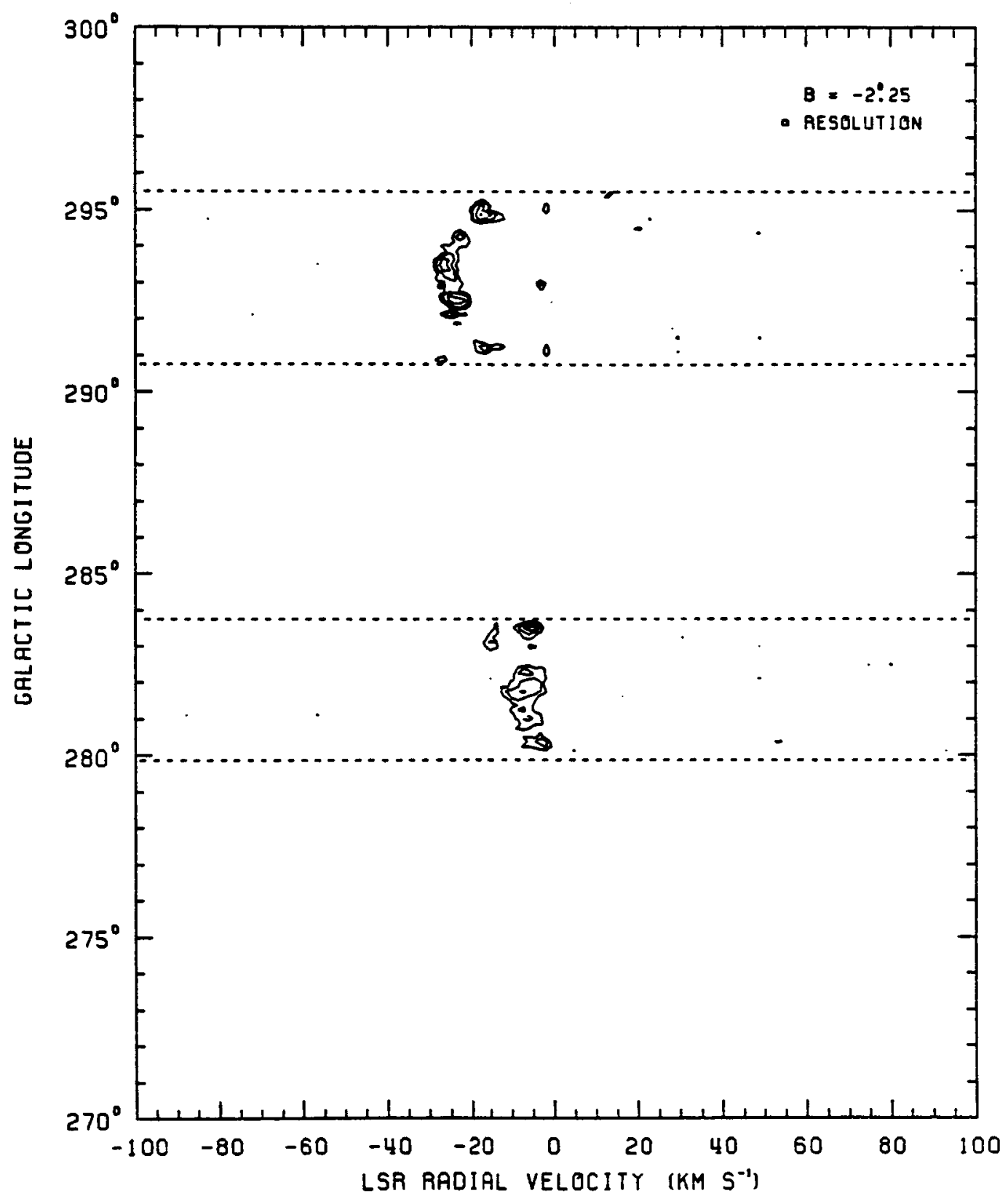

FIGURE B-1 (continued)

235 


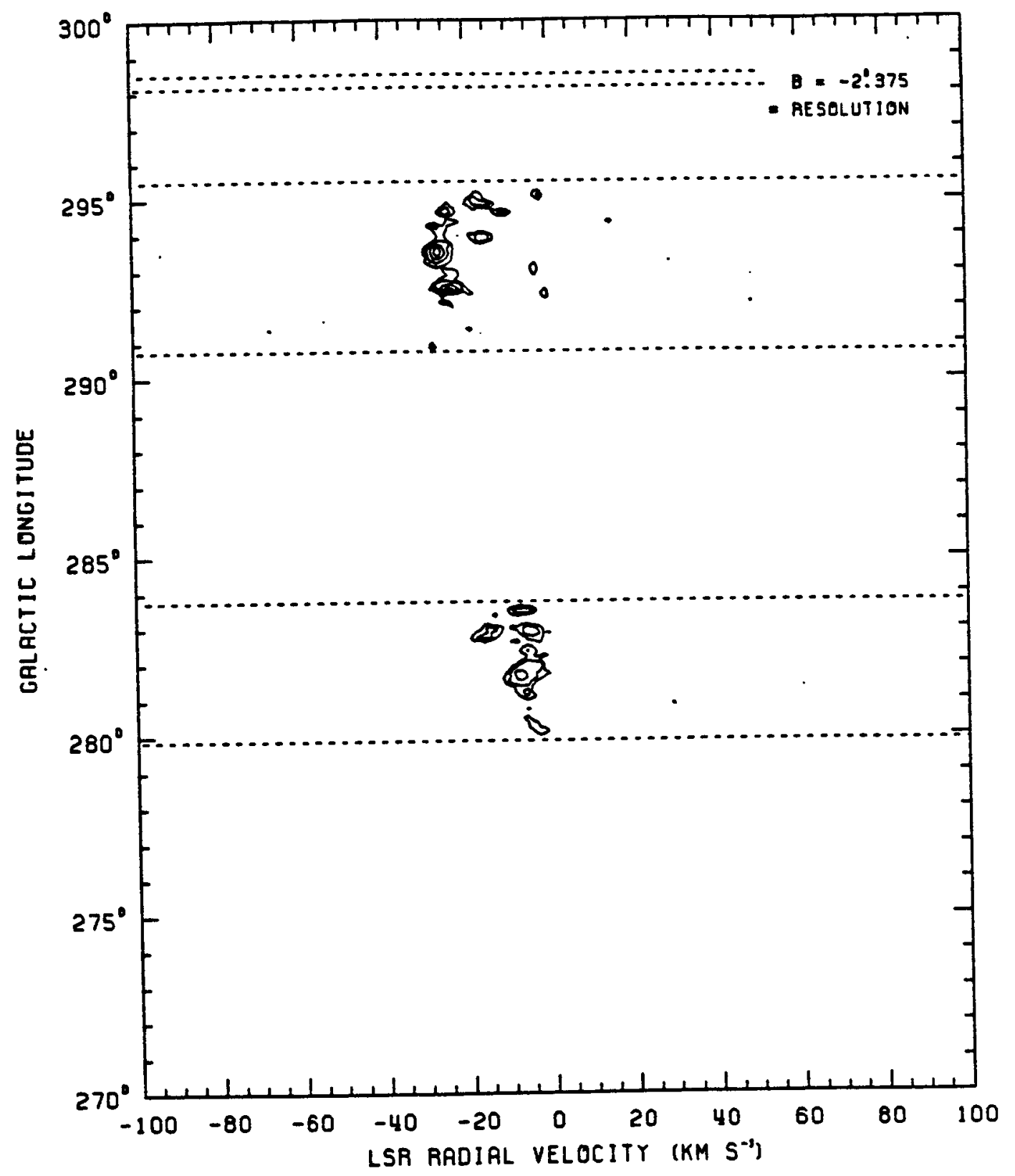

FIGURE B-1 (continued) 


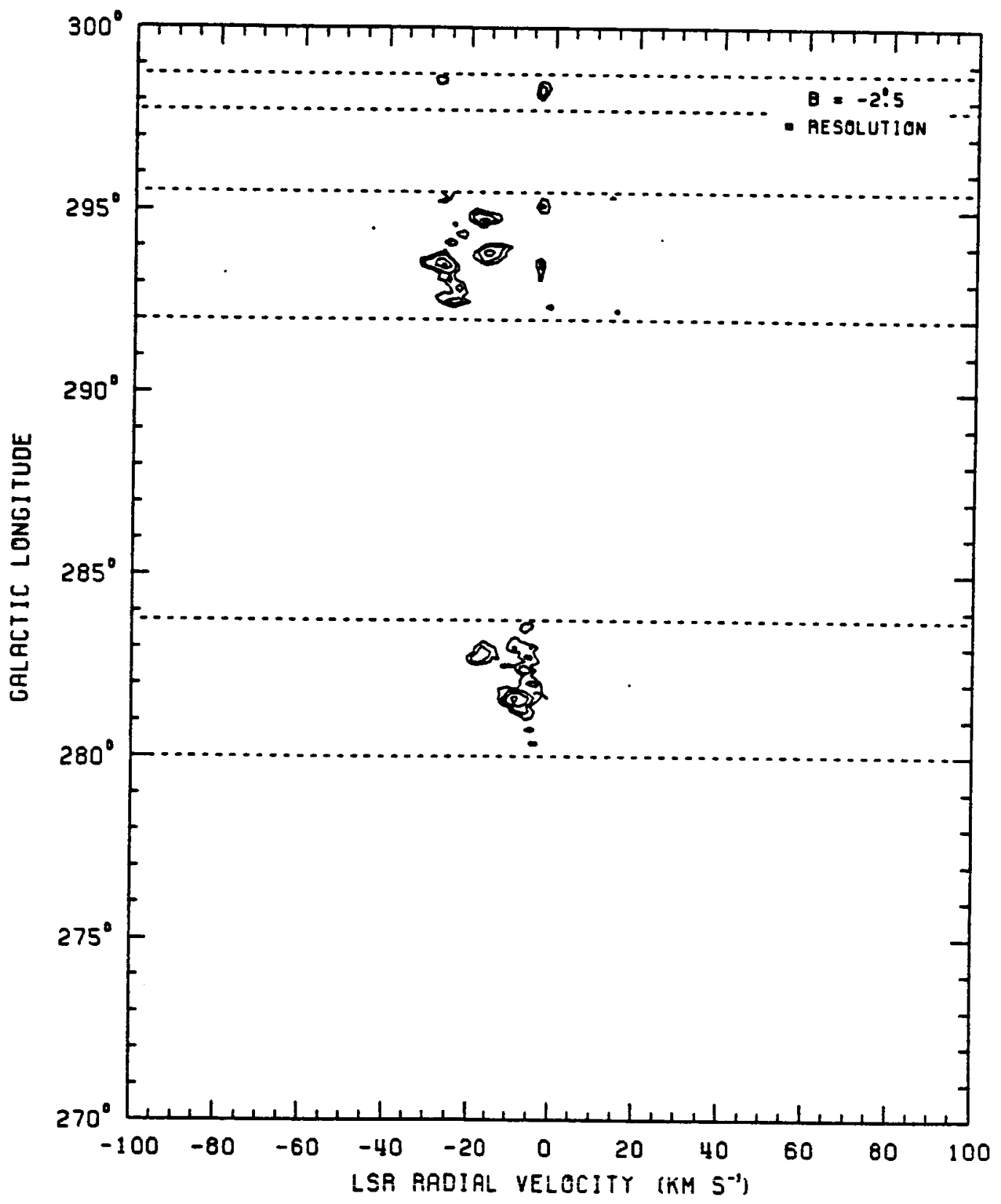

FIGURE B-1 (continued) 


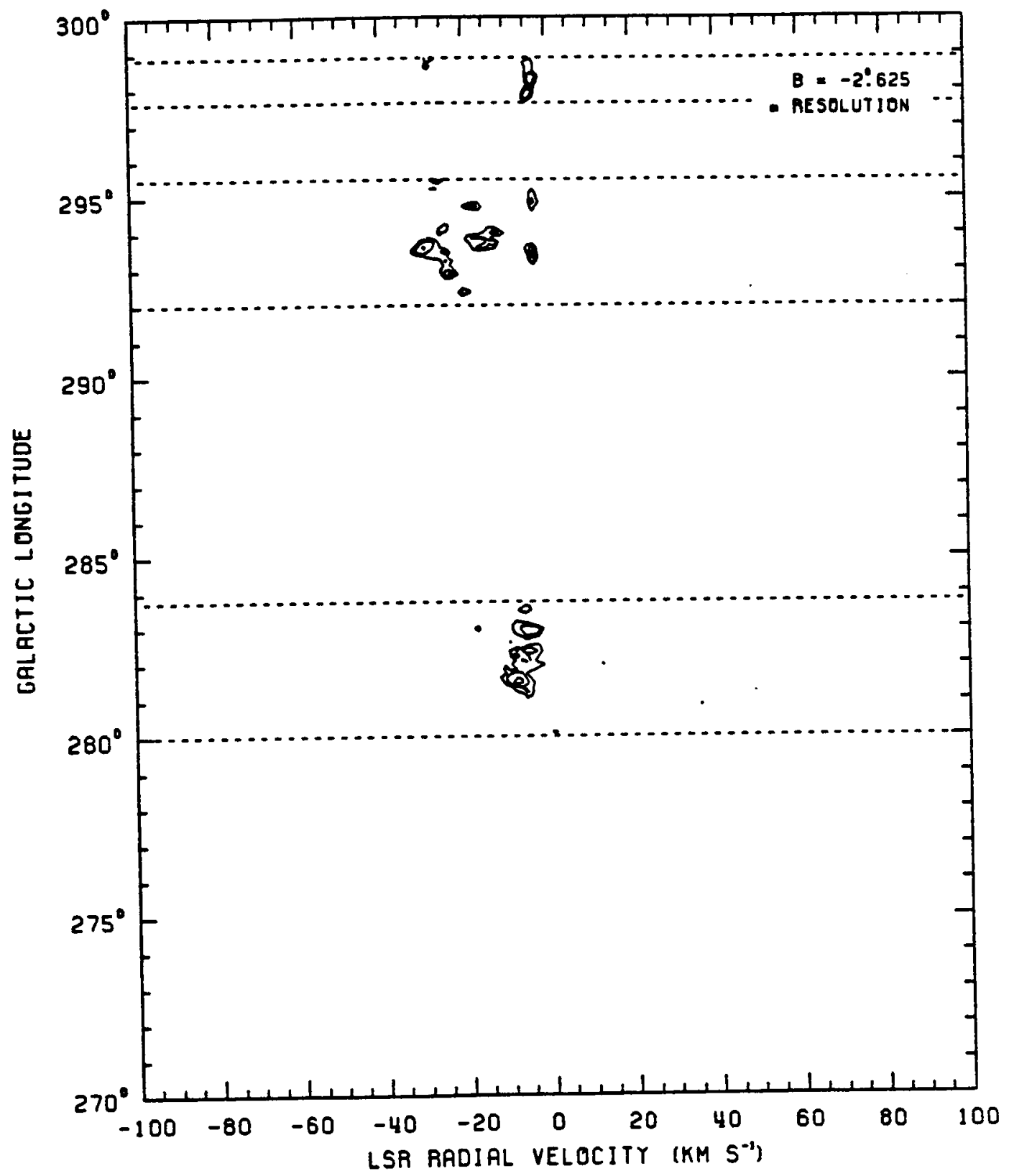

FIGURE B-I (continued) 


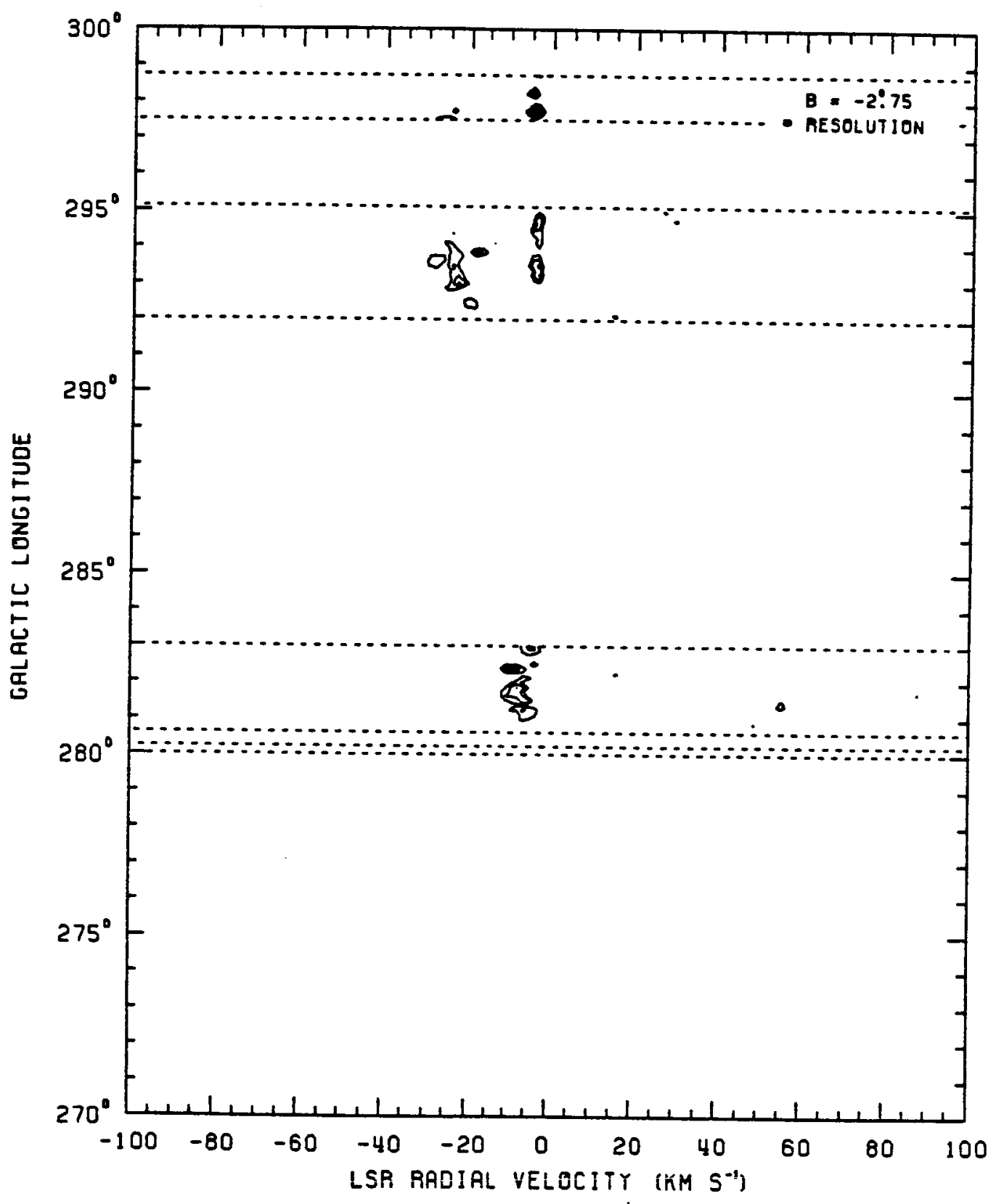

FIGURE B-1 (continued) 


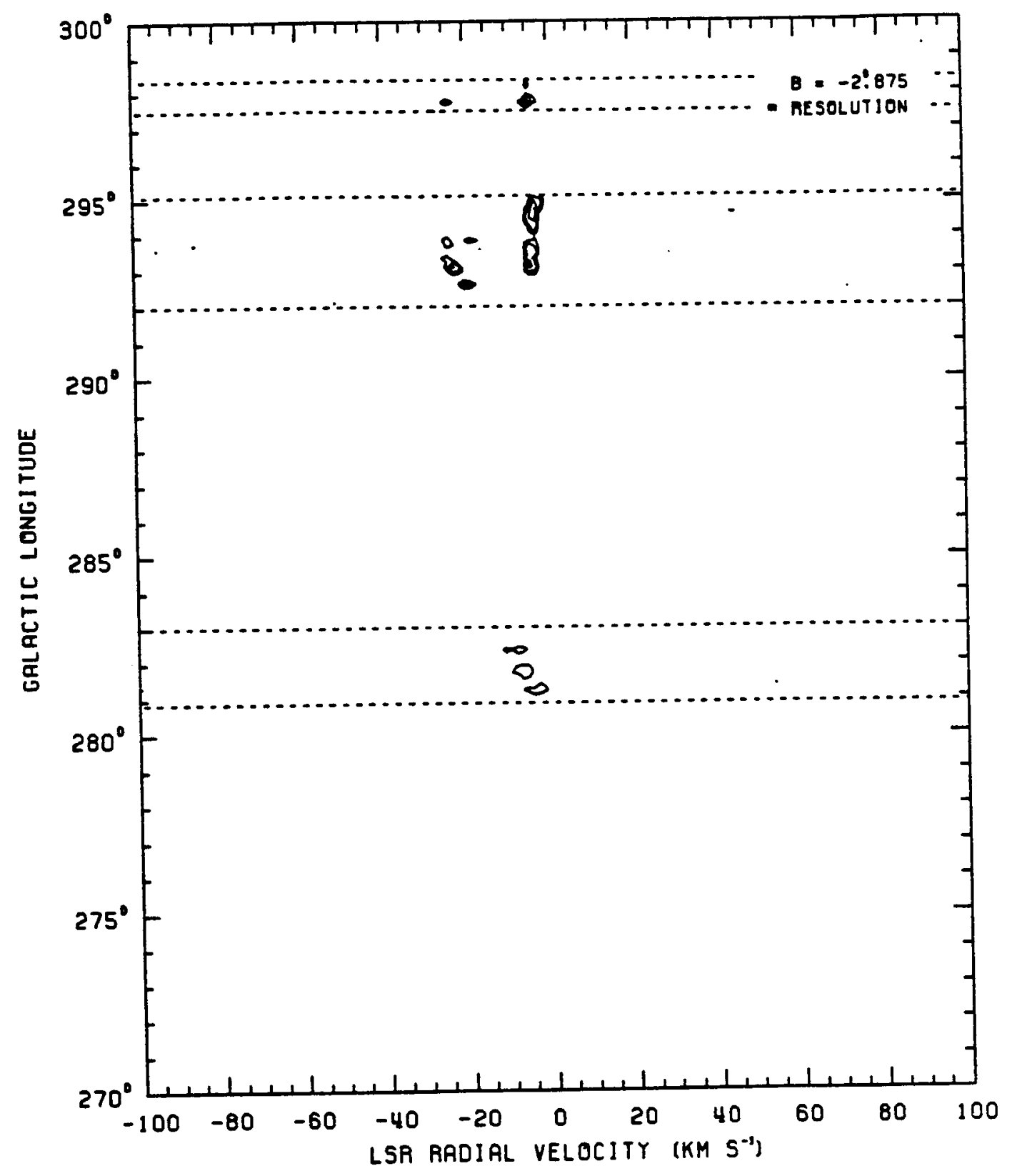

FIGURE B-1 (continued) 


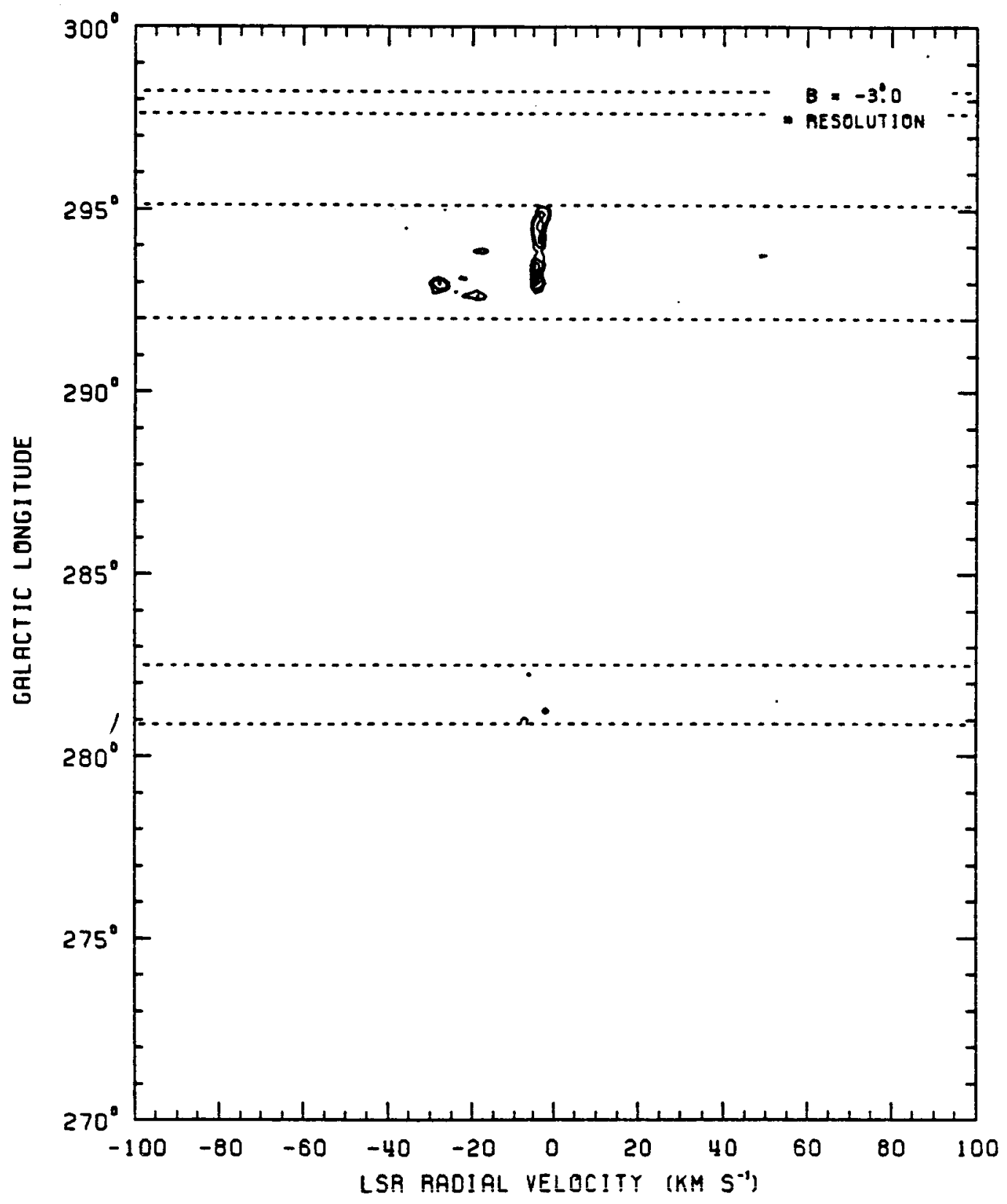

FIGURE B-1 (continued) 


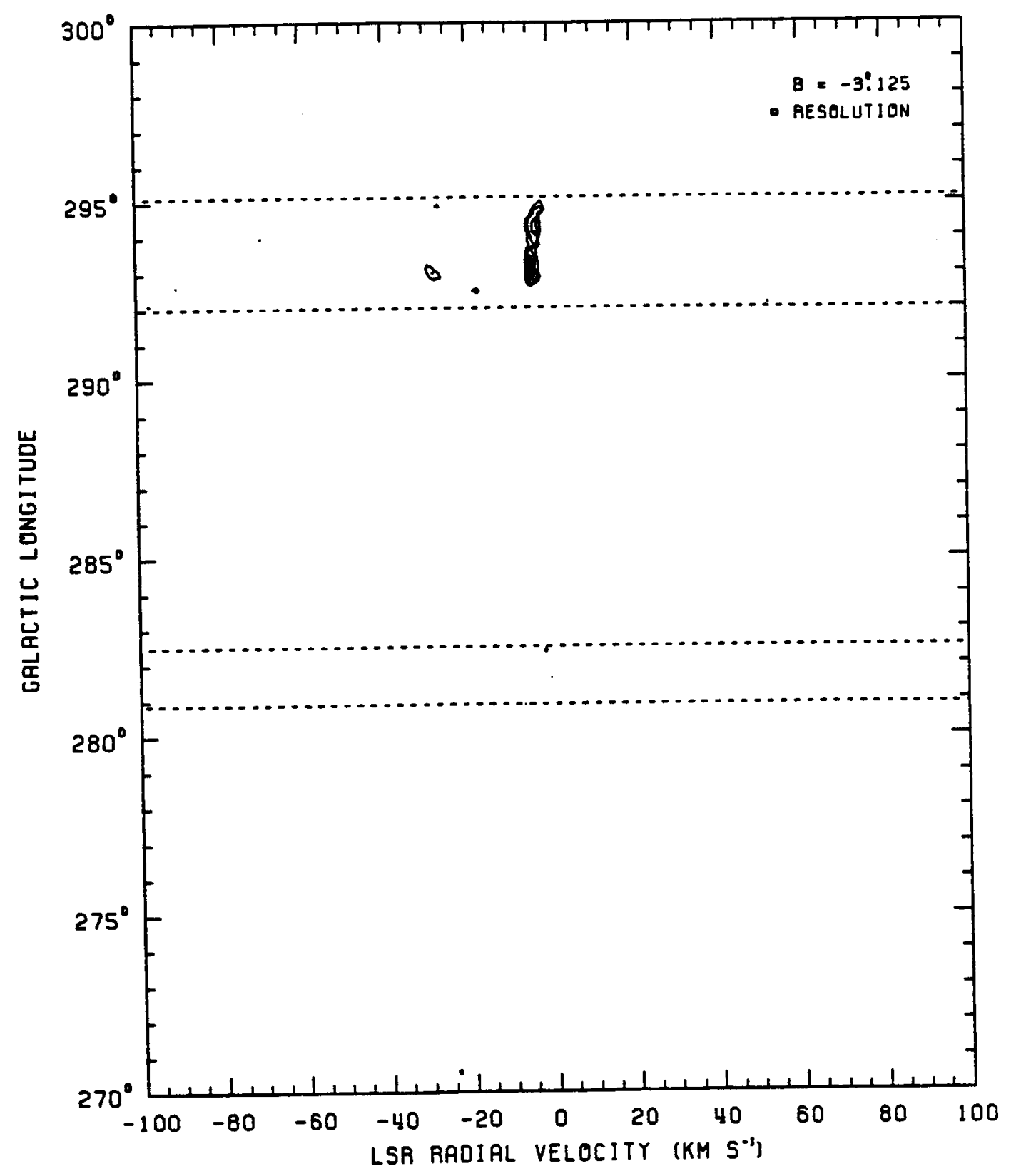

FIGURE B-1 (continued) 


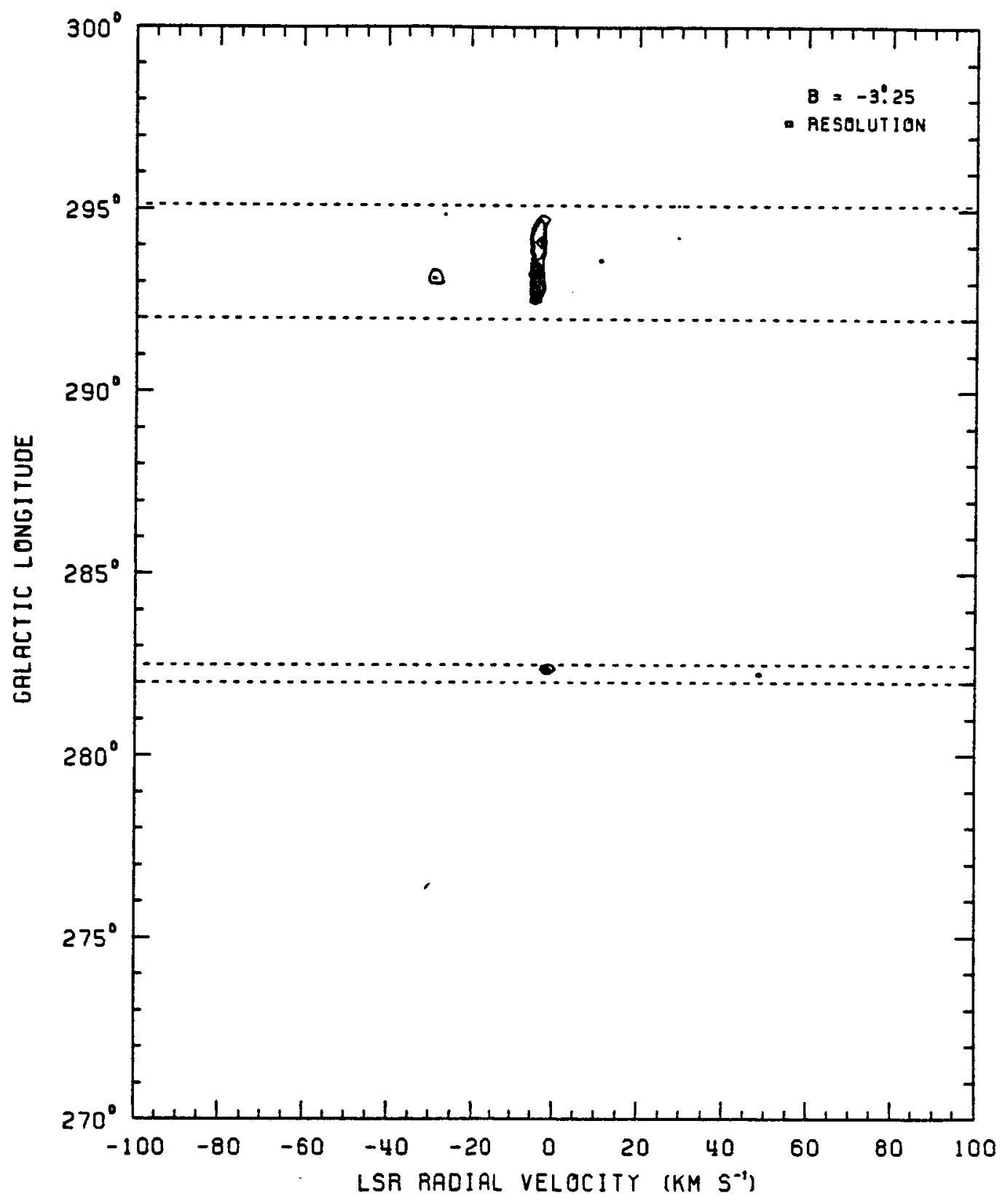

FIGURE B-1 (continued) 


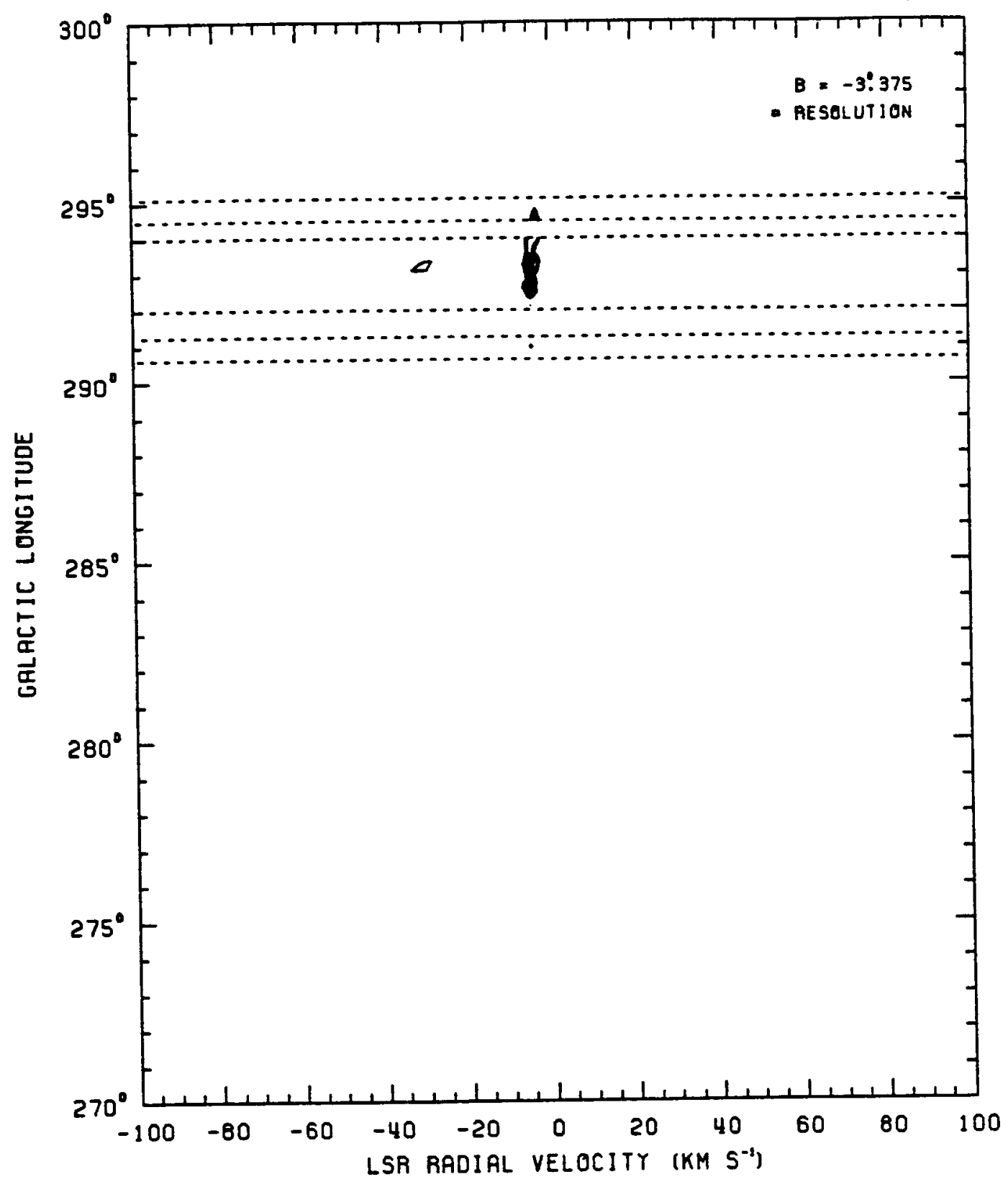

FIGURE B-1 (continued) 


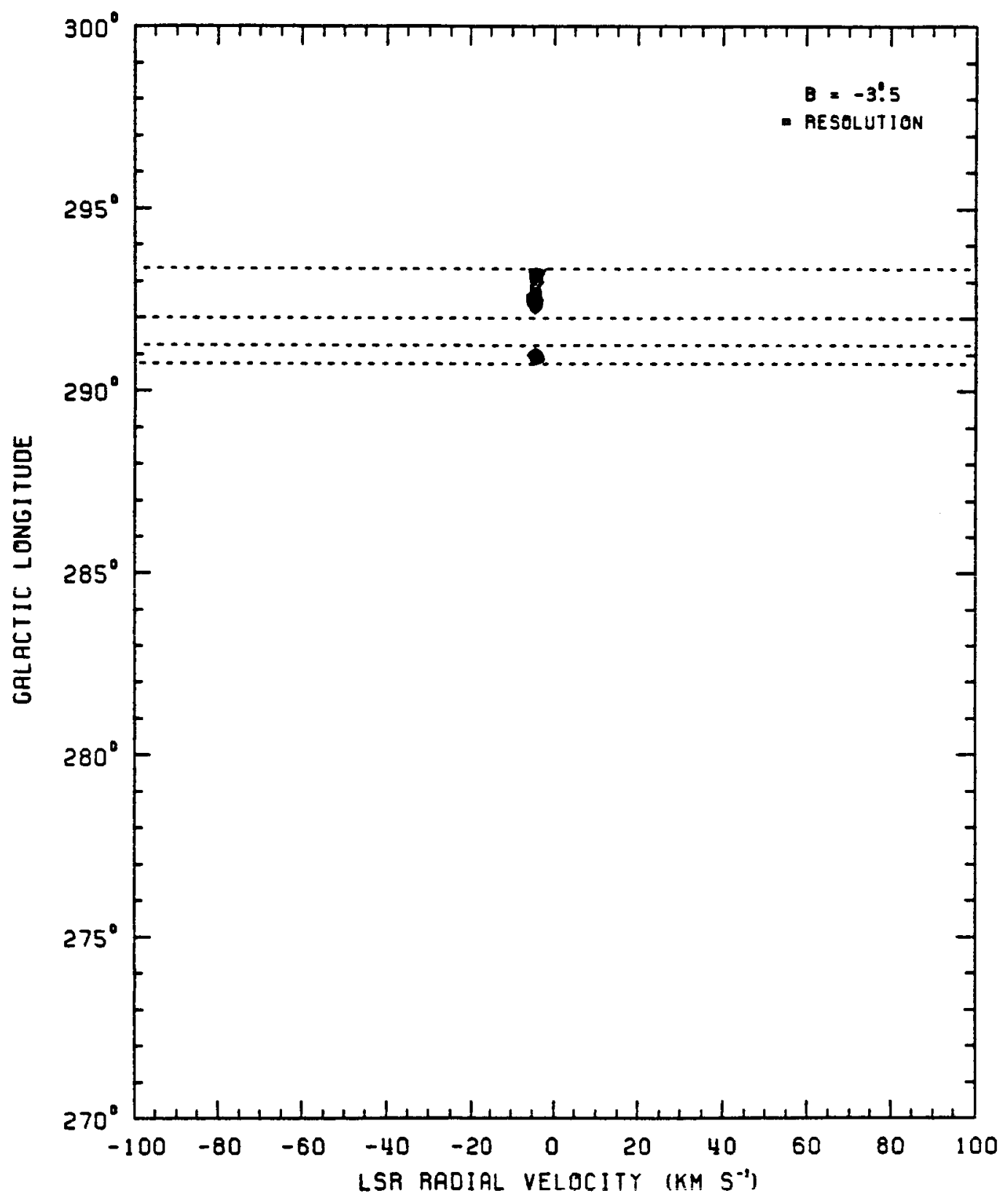

FIGURE B-1 (continued) 


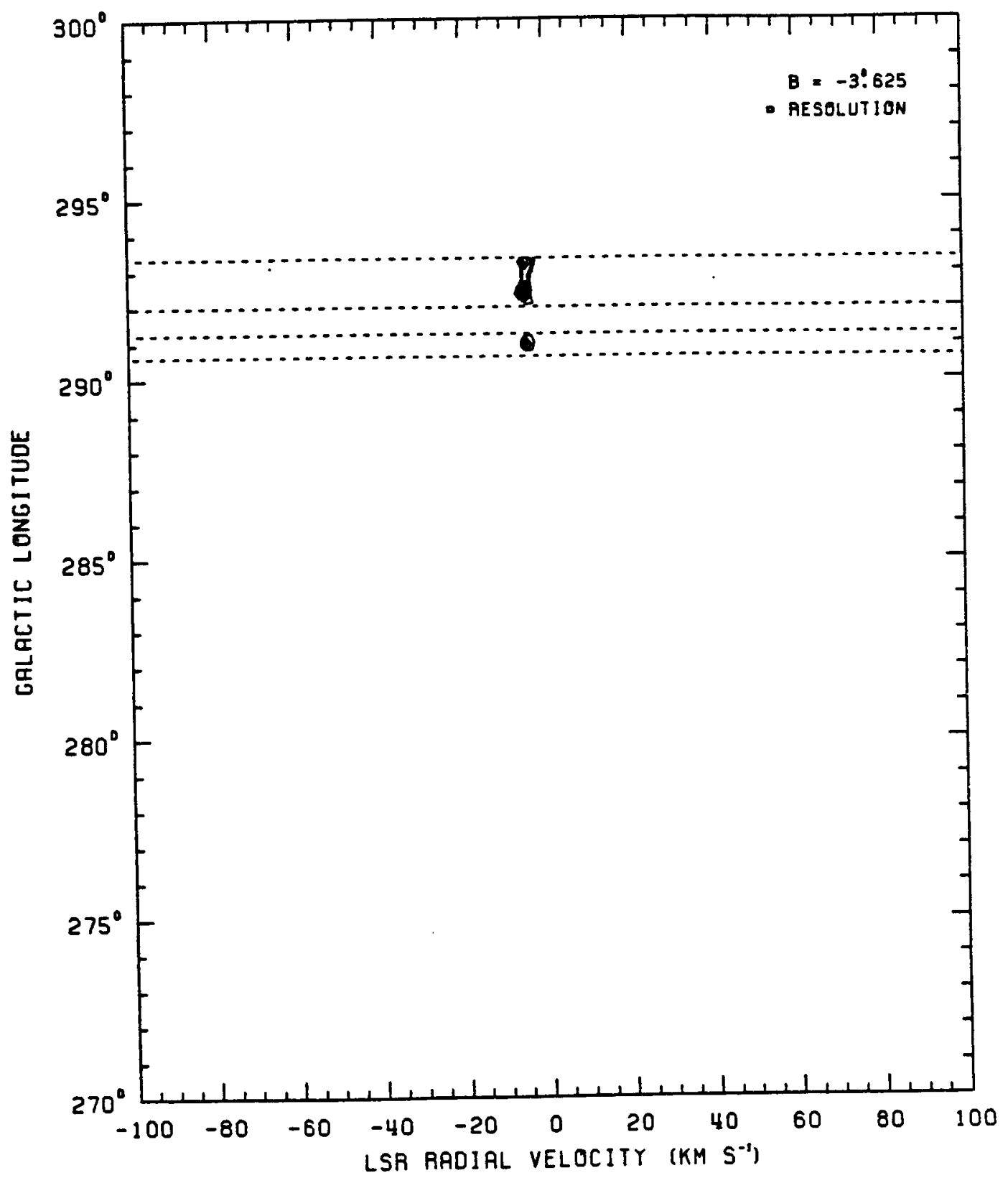

FIGURE B-l (continued) 


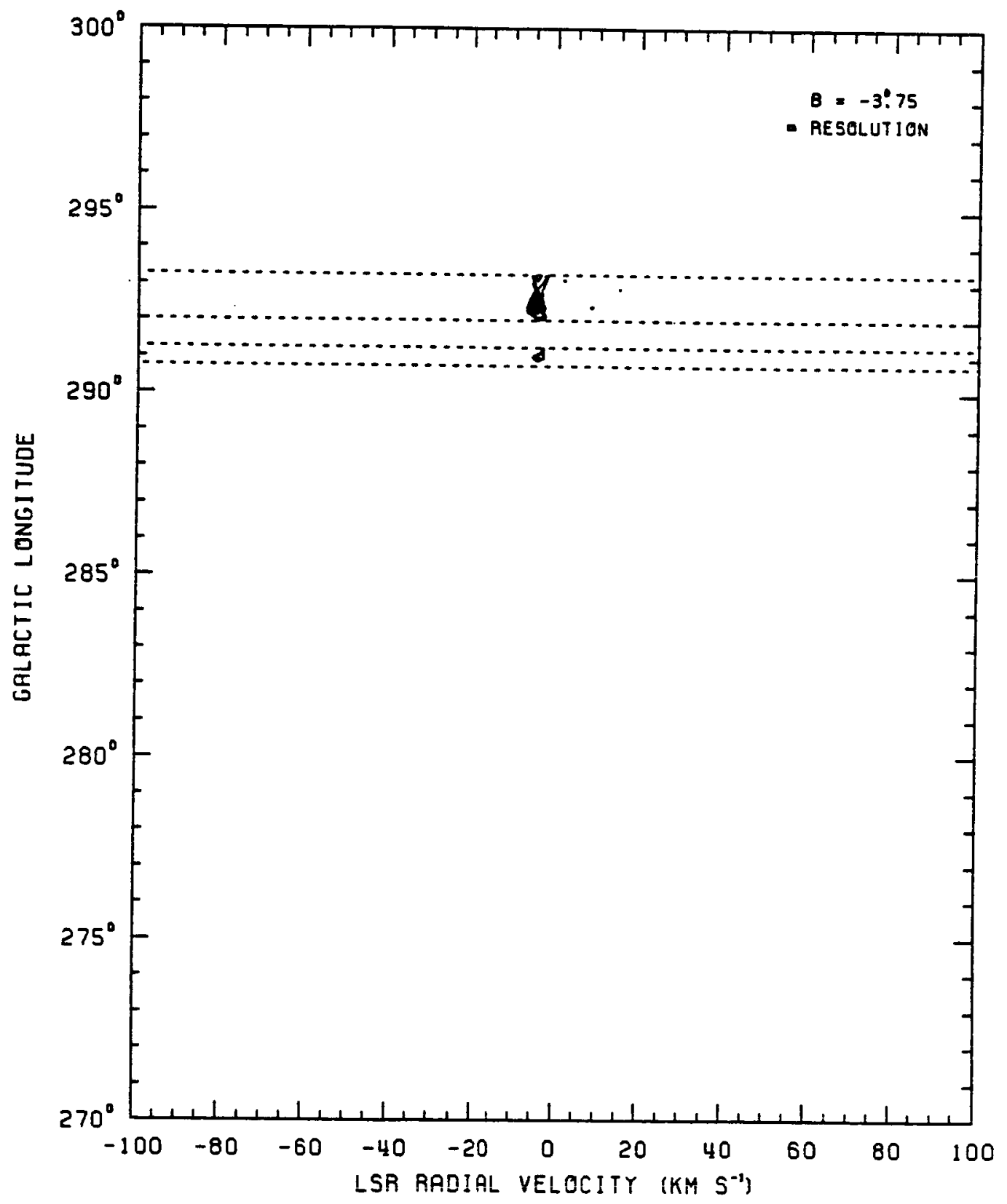

FIGURE B-1 (continued) 


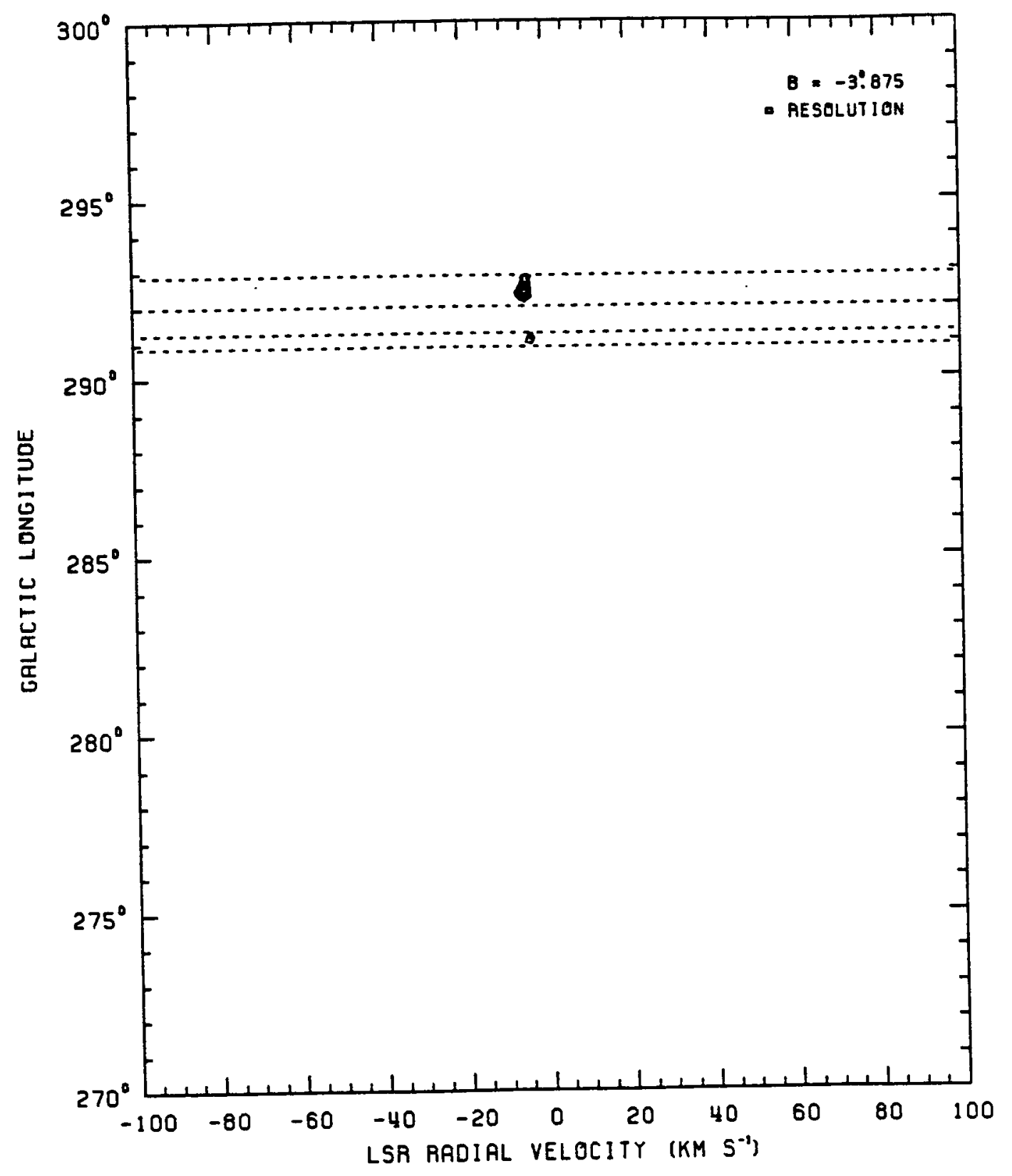

FIGURE B-1 (continued) 


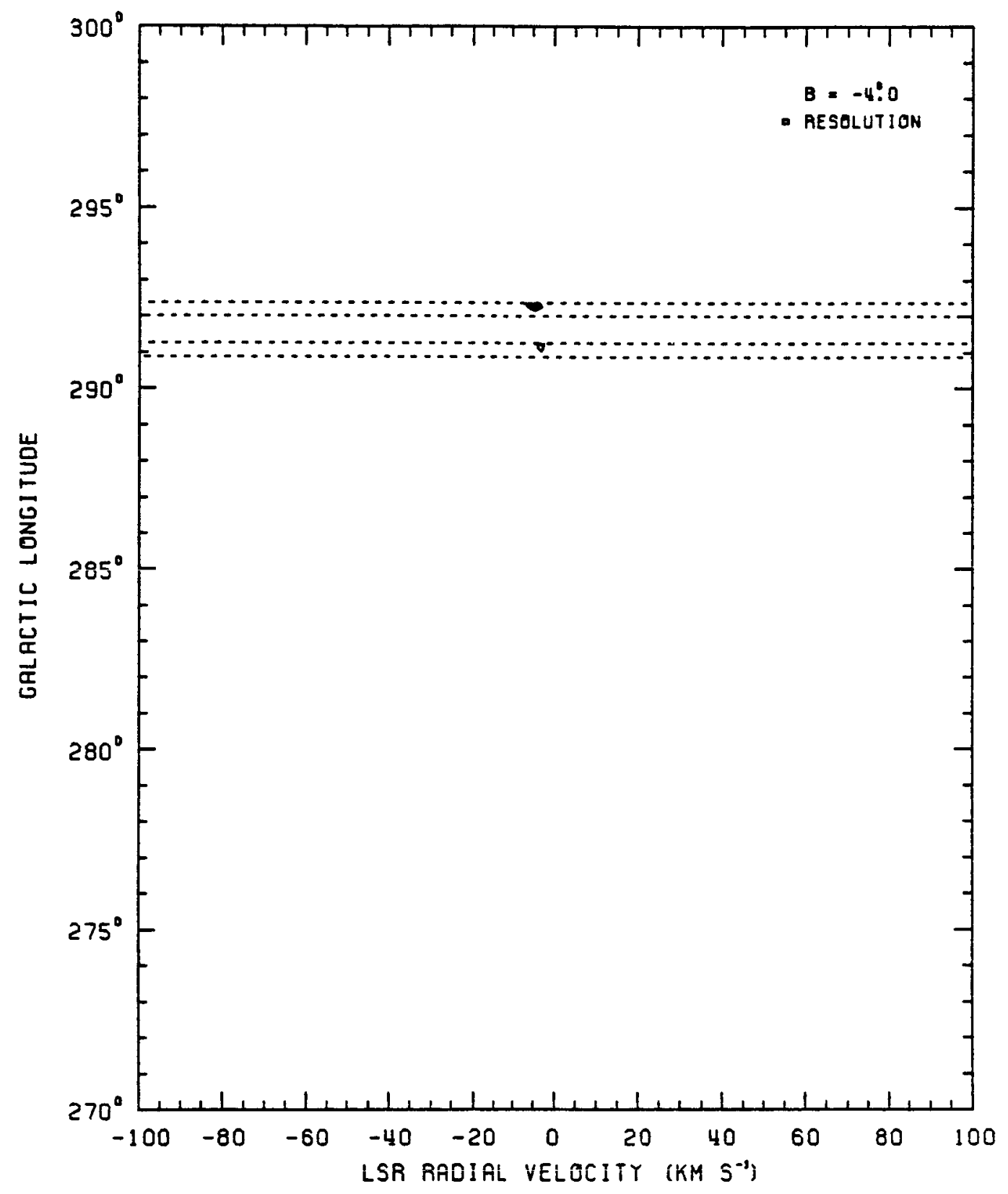

FIGURE B-1 (continued) 


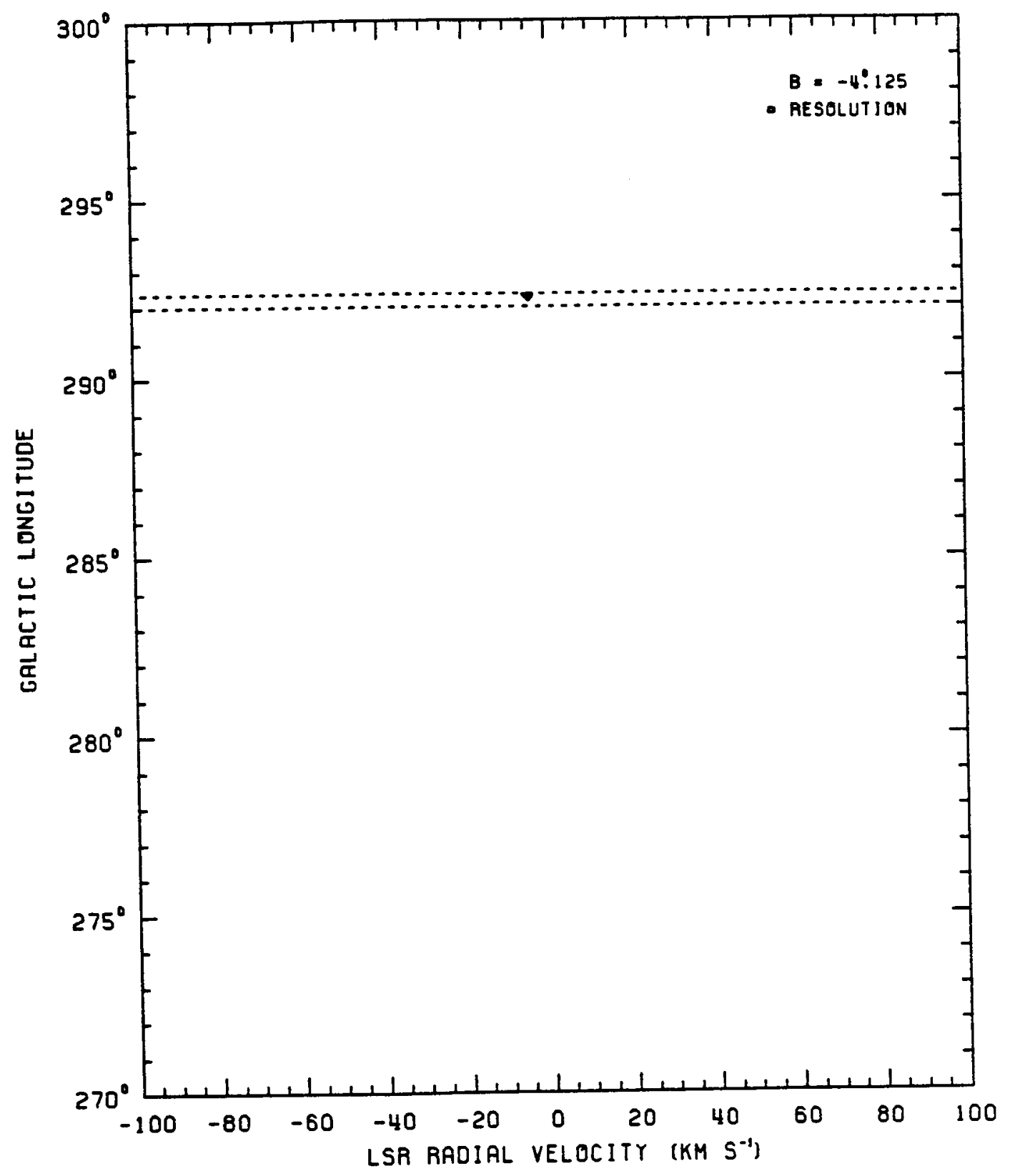

FIGURE B-1 (continued) 


\section{ORIGINAL PAGE IS
OF POOR QUALITY}
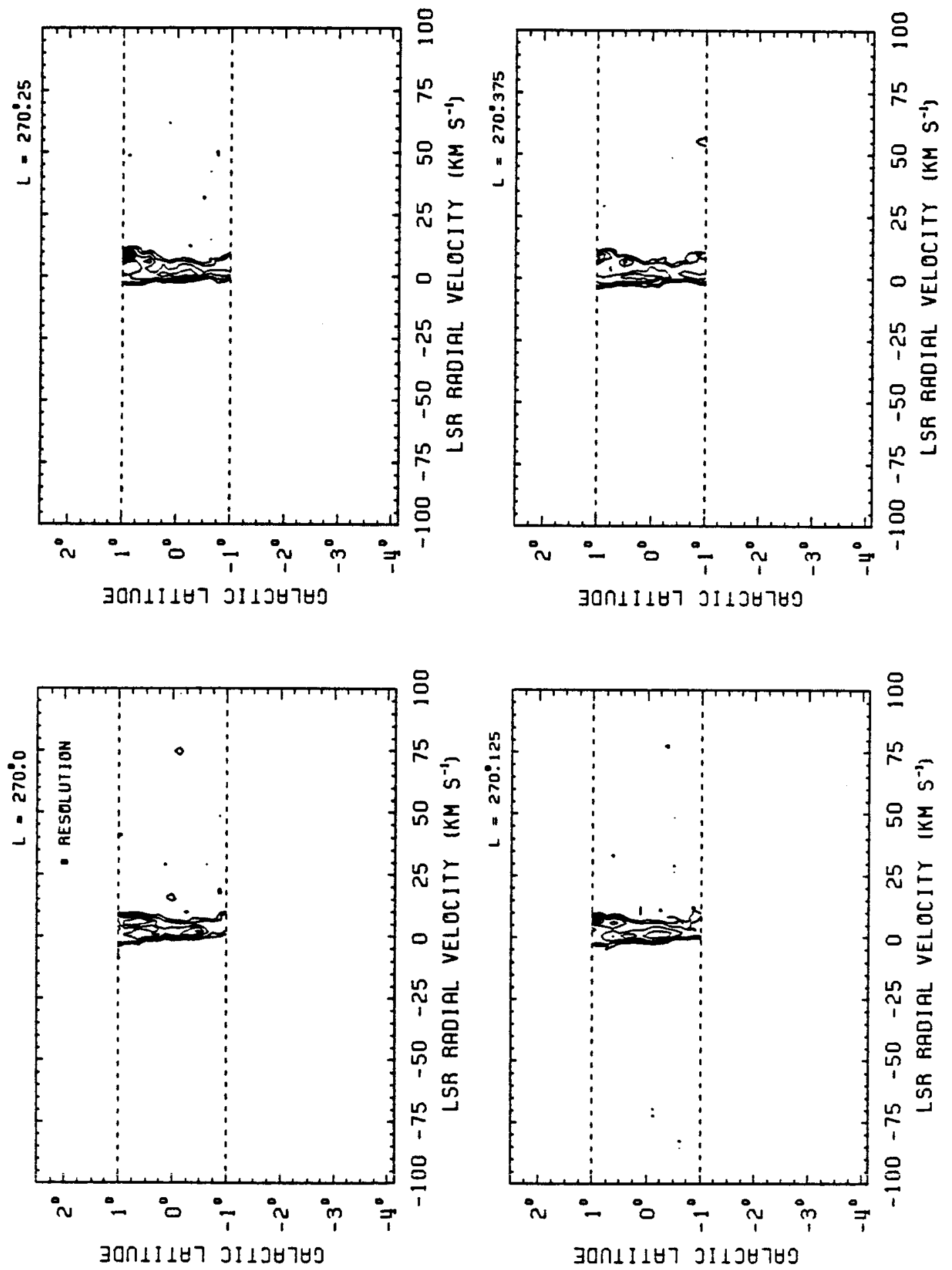

FIGURE B-2 

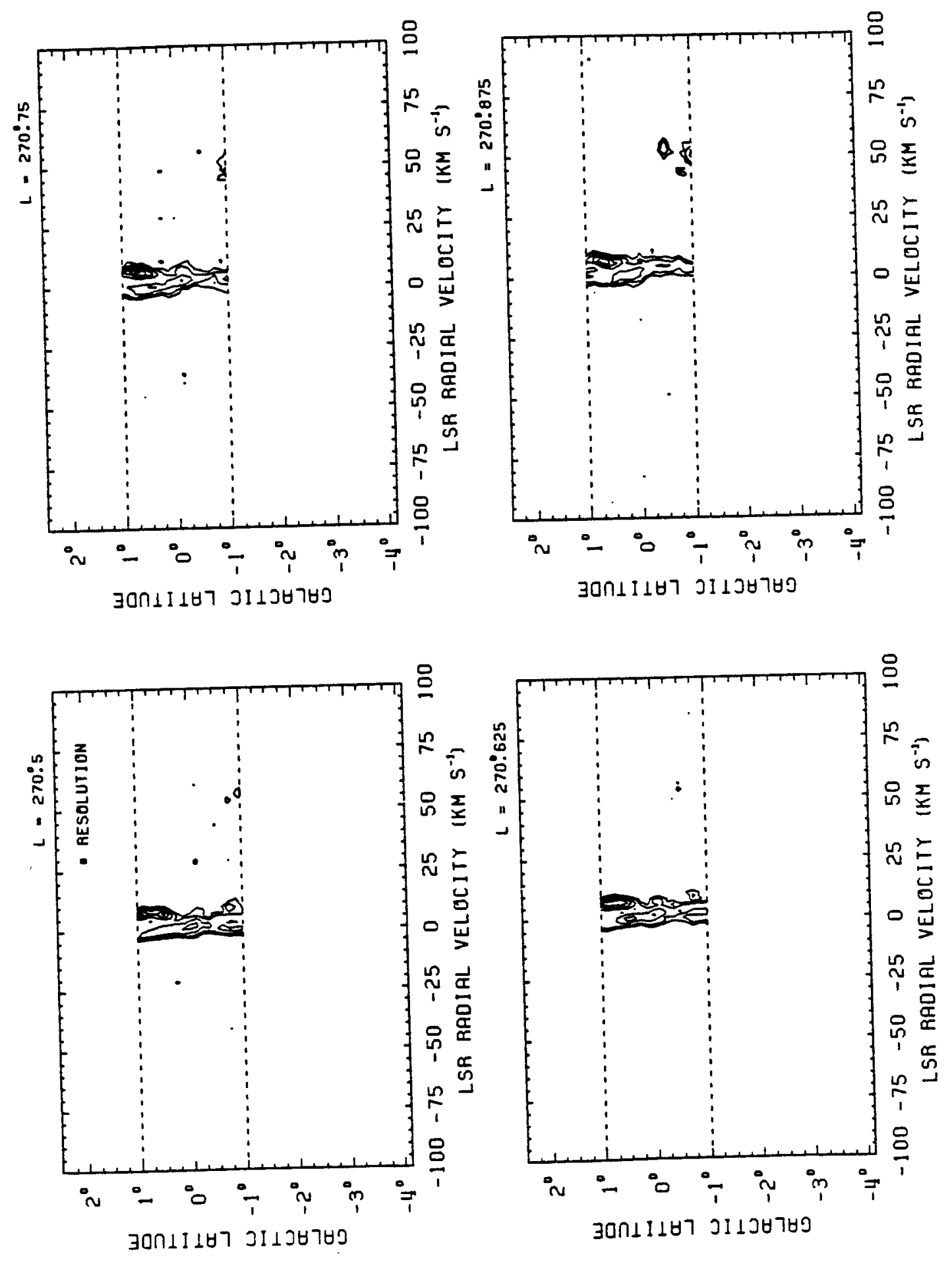

FIGURE B-2 (continued) 

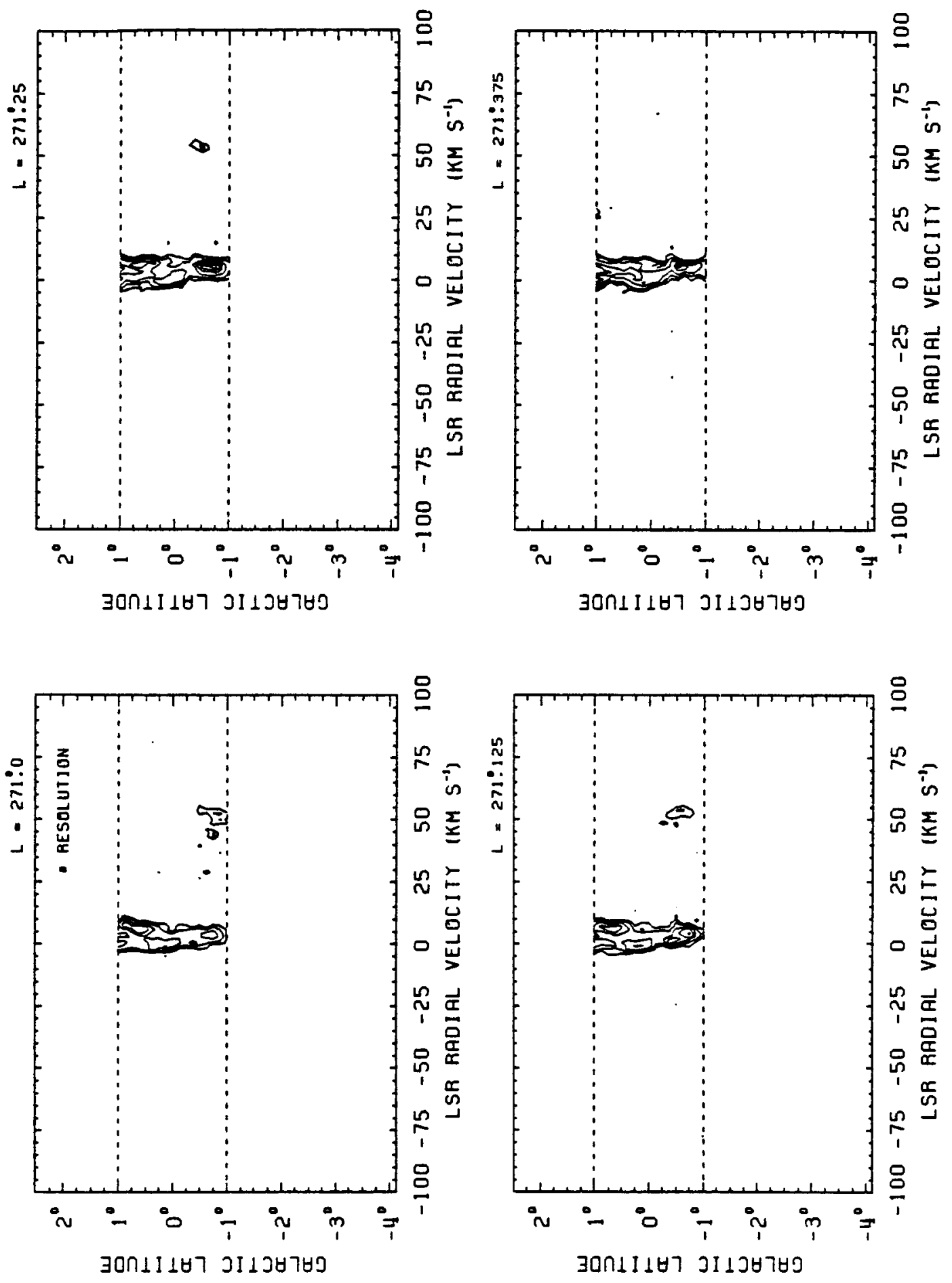

FIGURE B-2 (continued) 

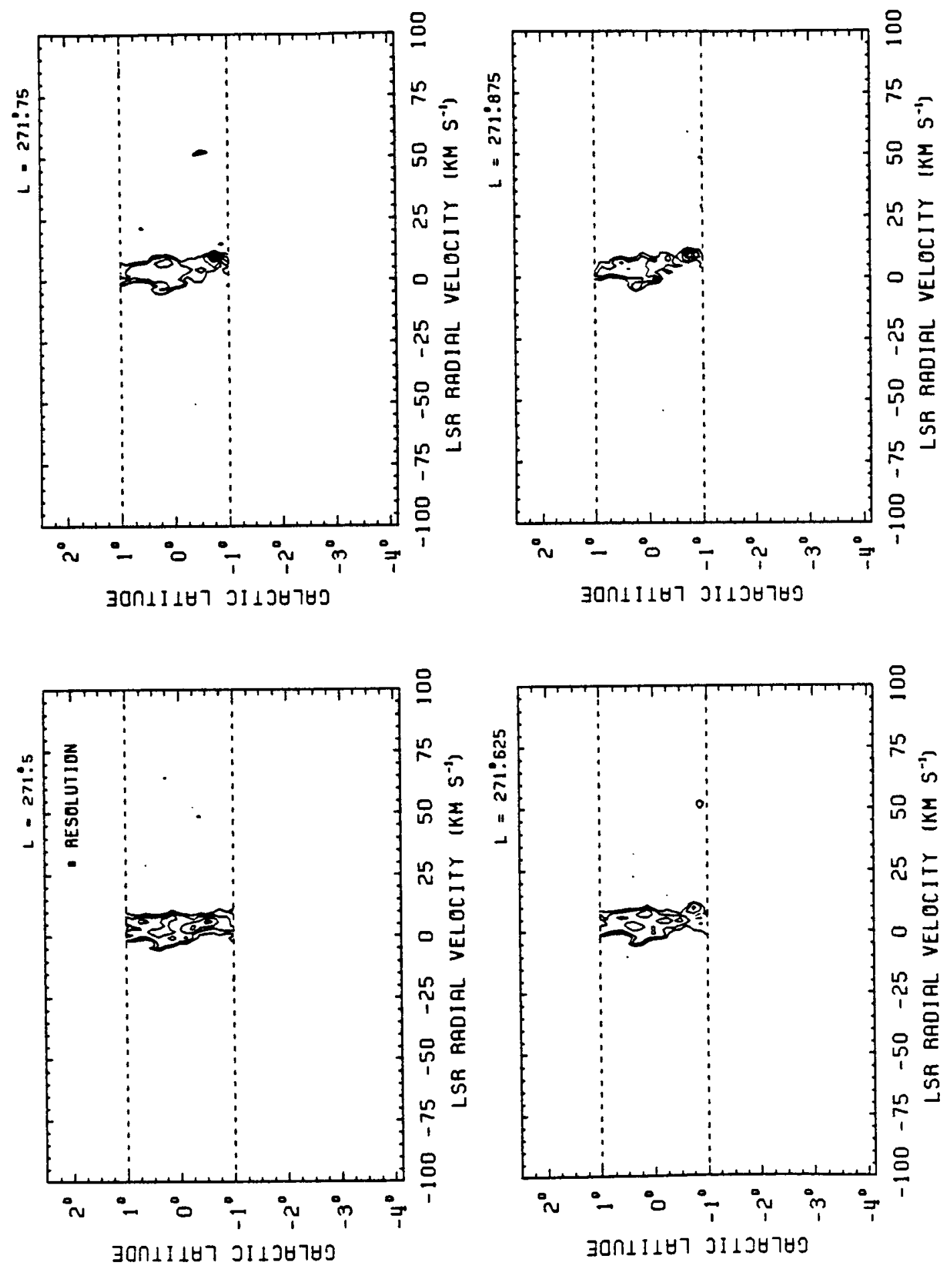

FIGURE B-2 (continued) 


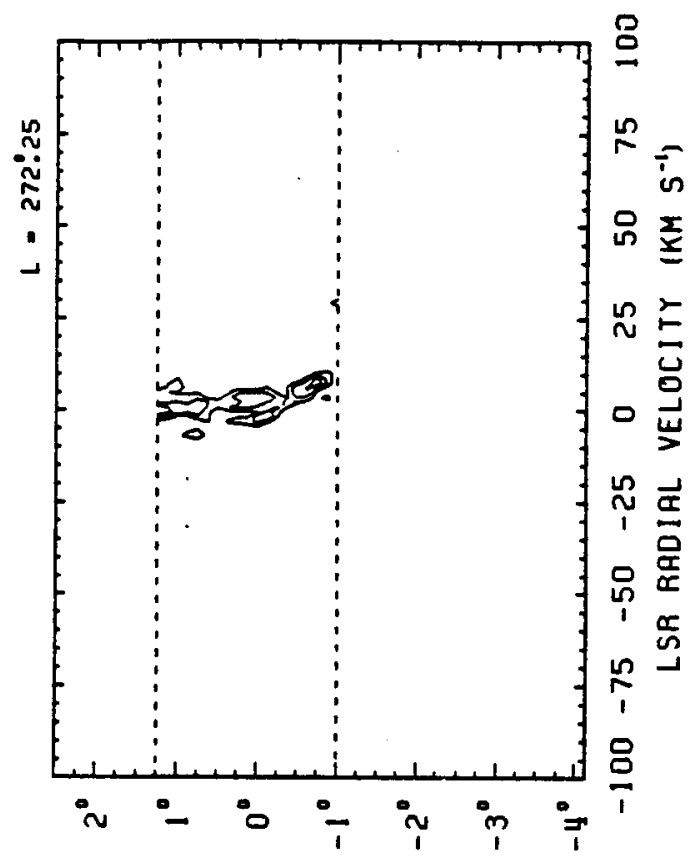

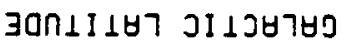
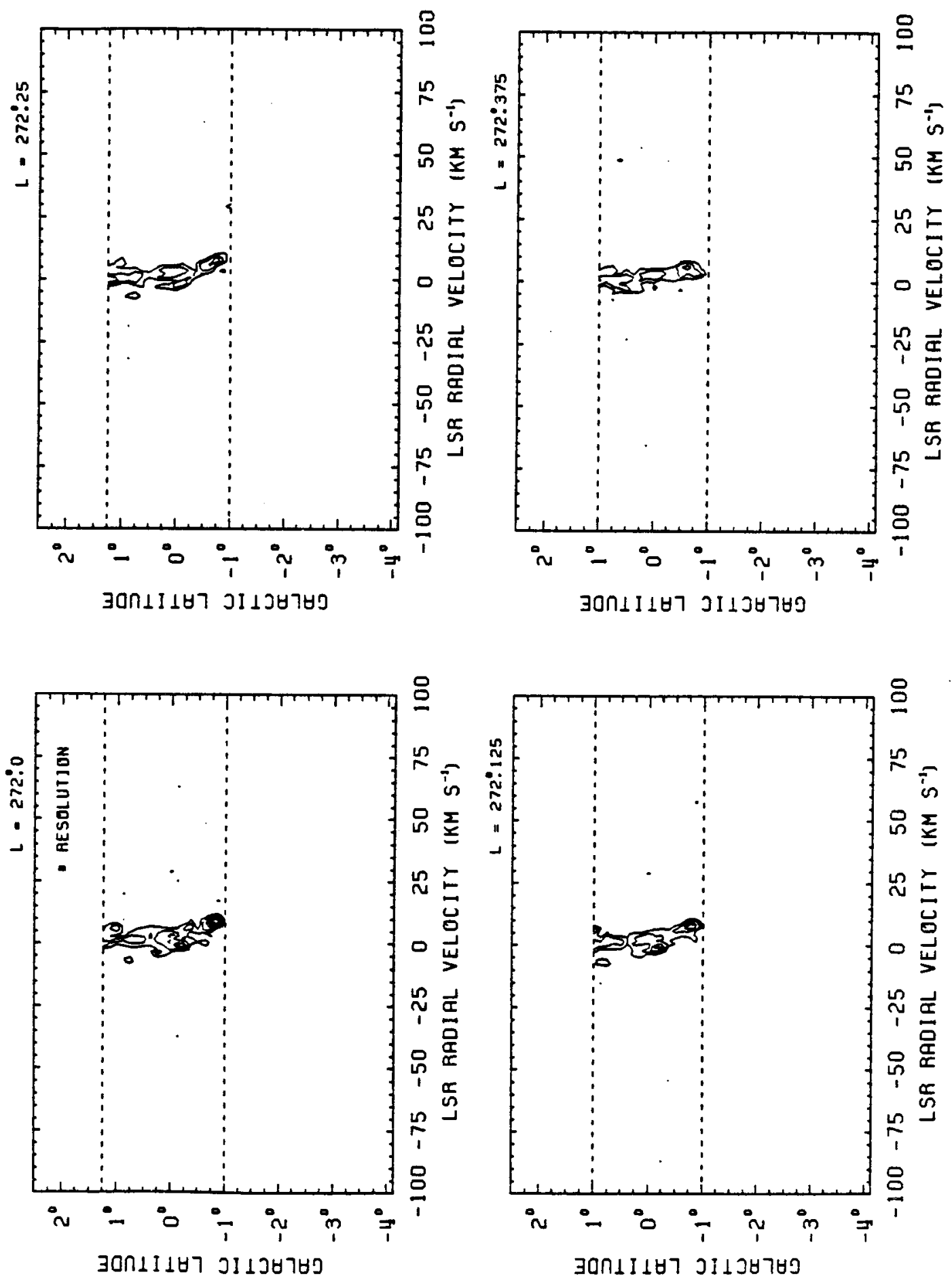

FIGURE B-2 (continued) 

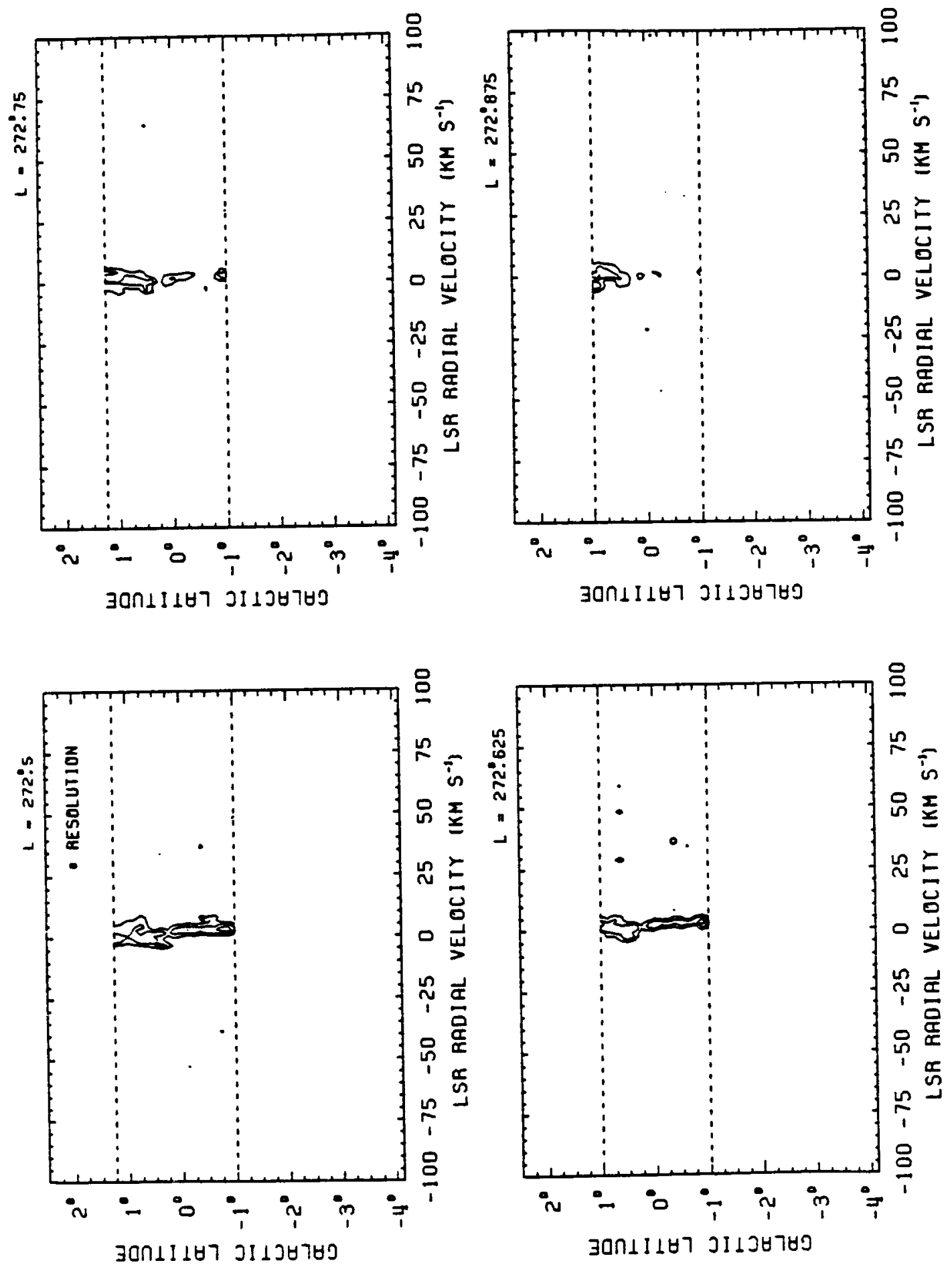

FIGURE B-2 (continued) 

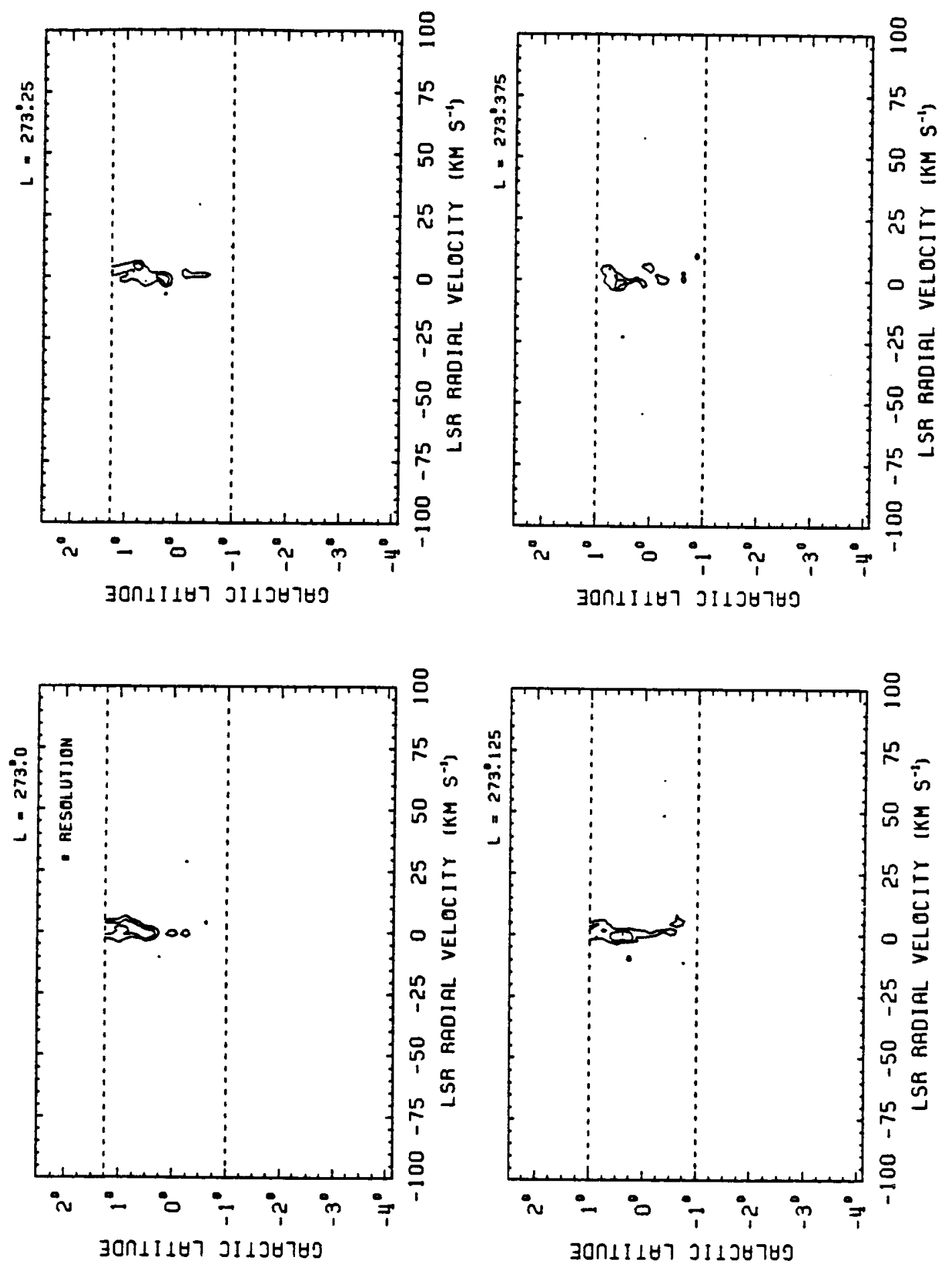

FIGURE B-2 (continued) 

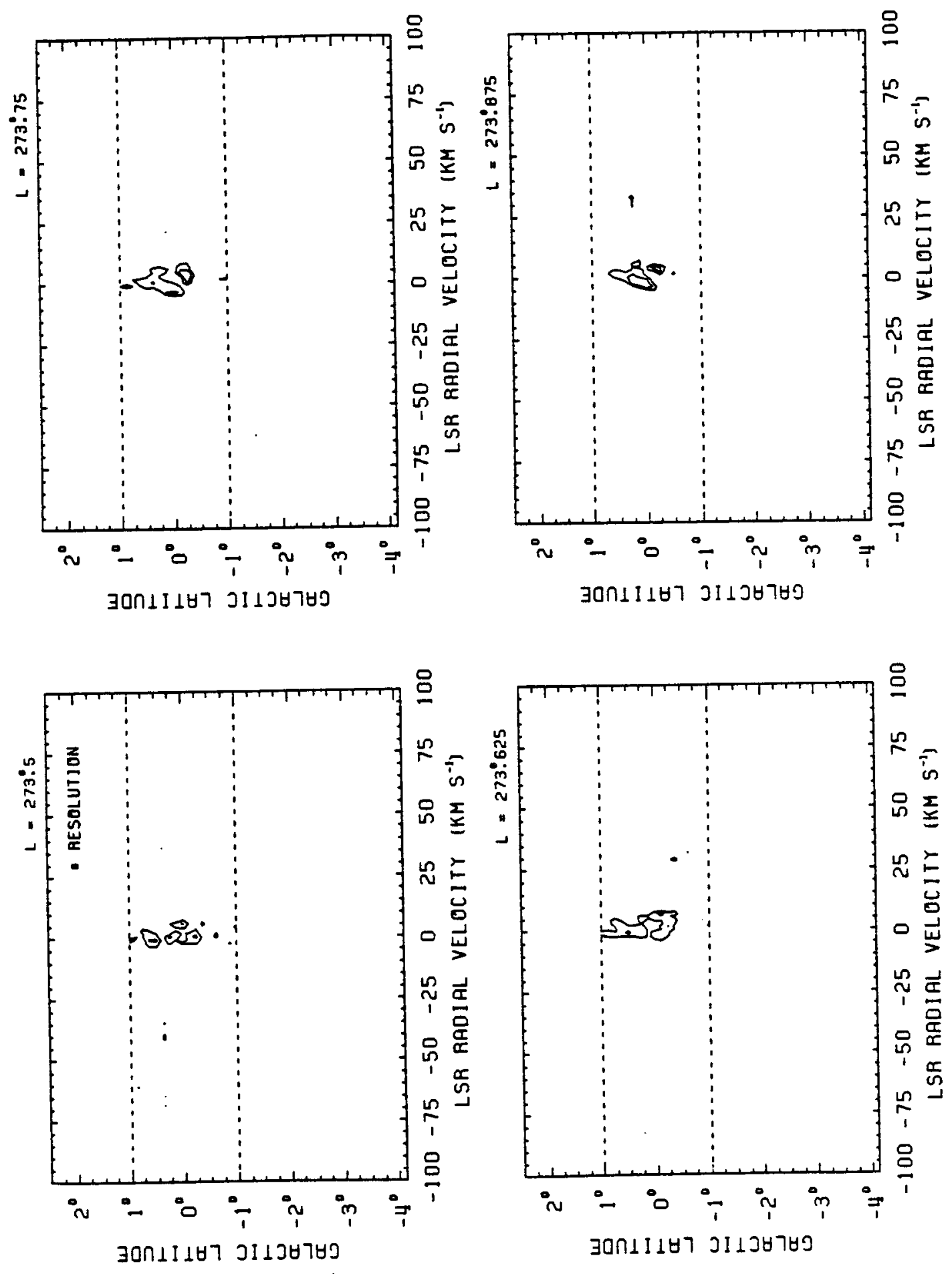

FIGURE B-2 (continued) 

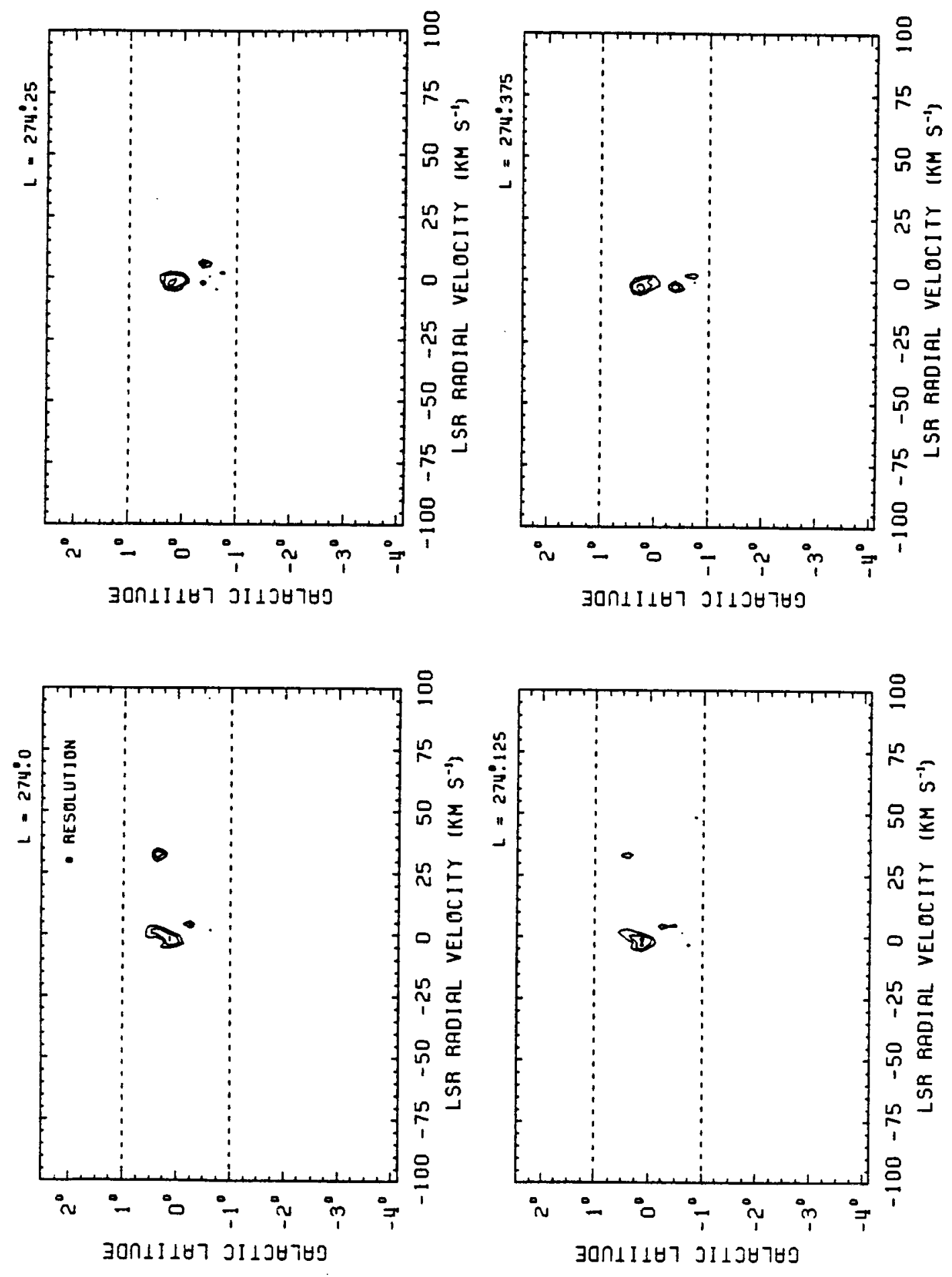

FIGURE B-2 (continued) 

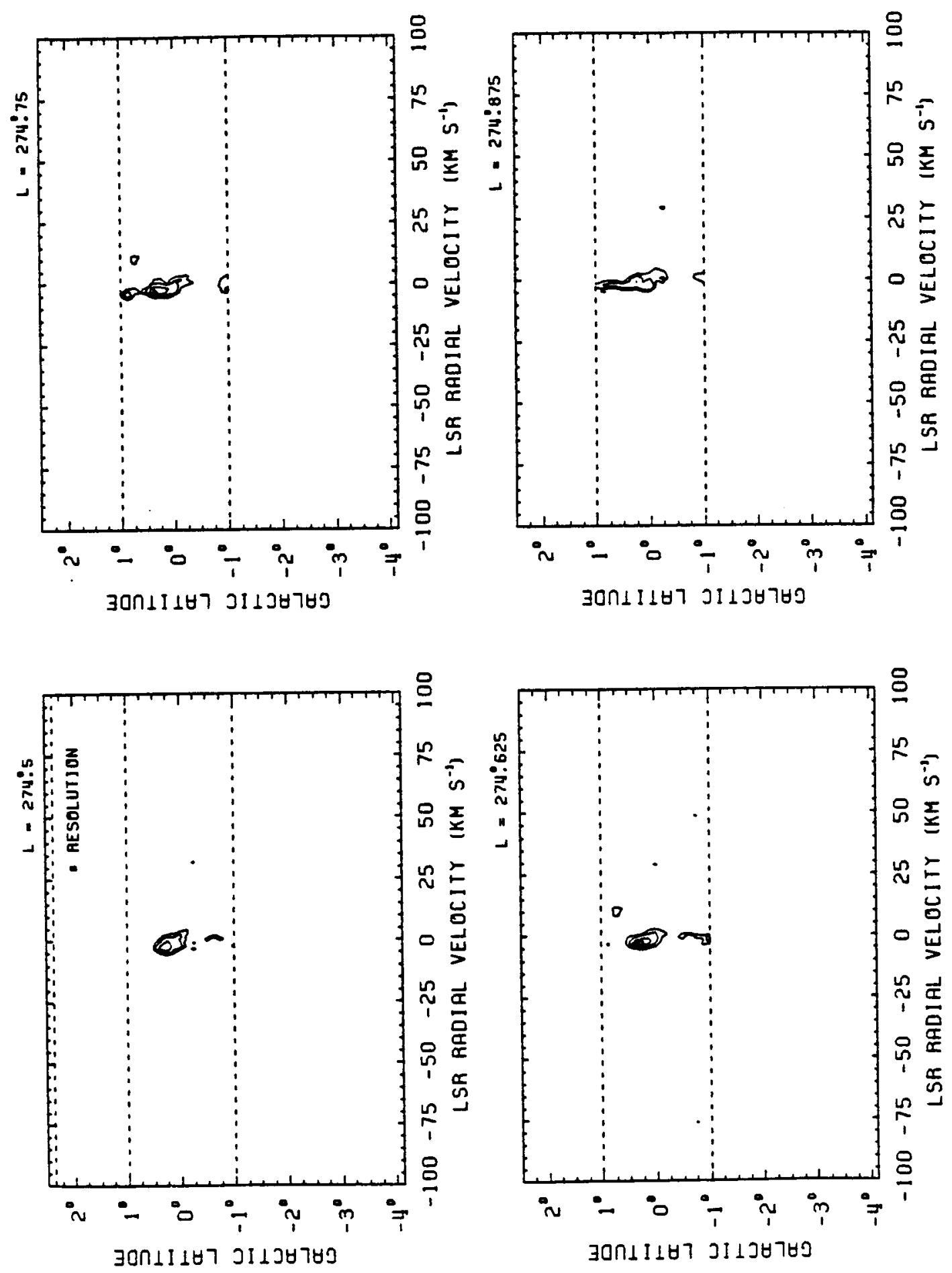

FIGURE B-2 (continued) 

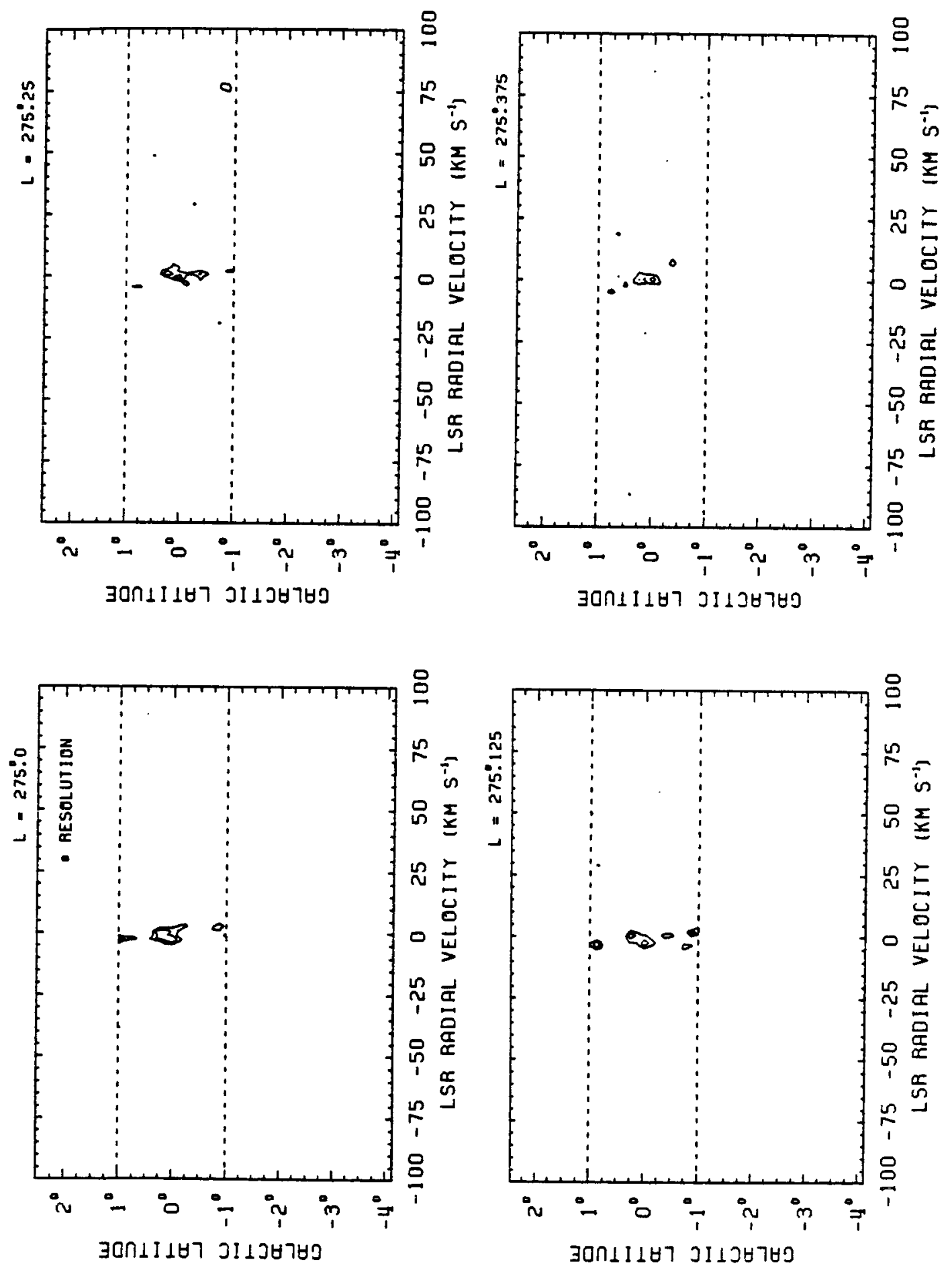

FIGURE B-2 (continued) 

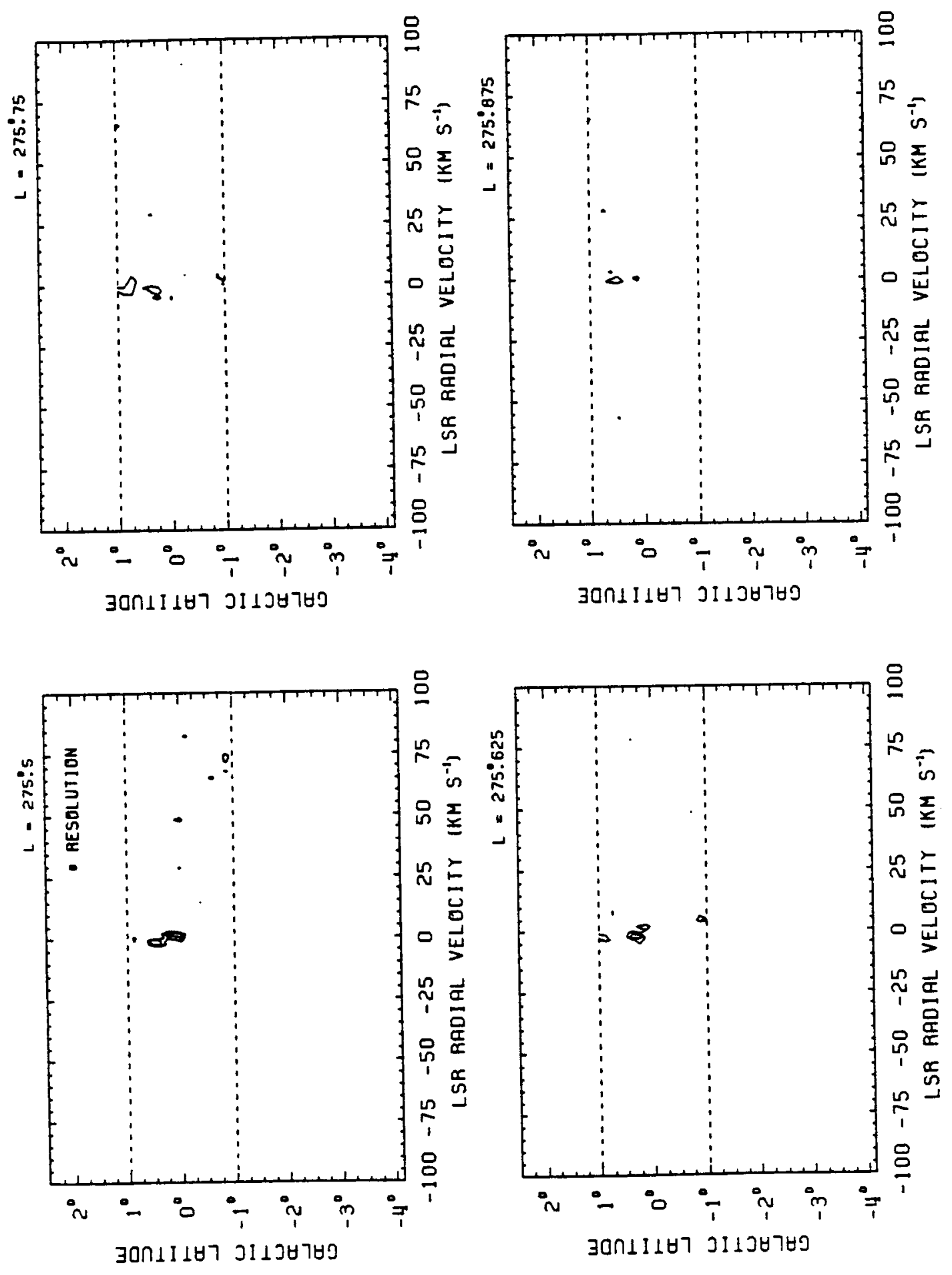

FIGURE B-2 (continued) 

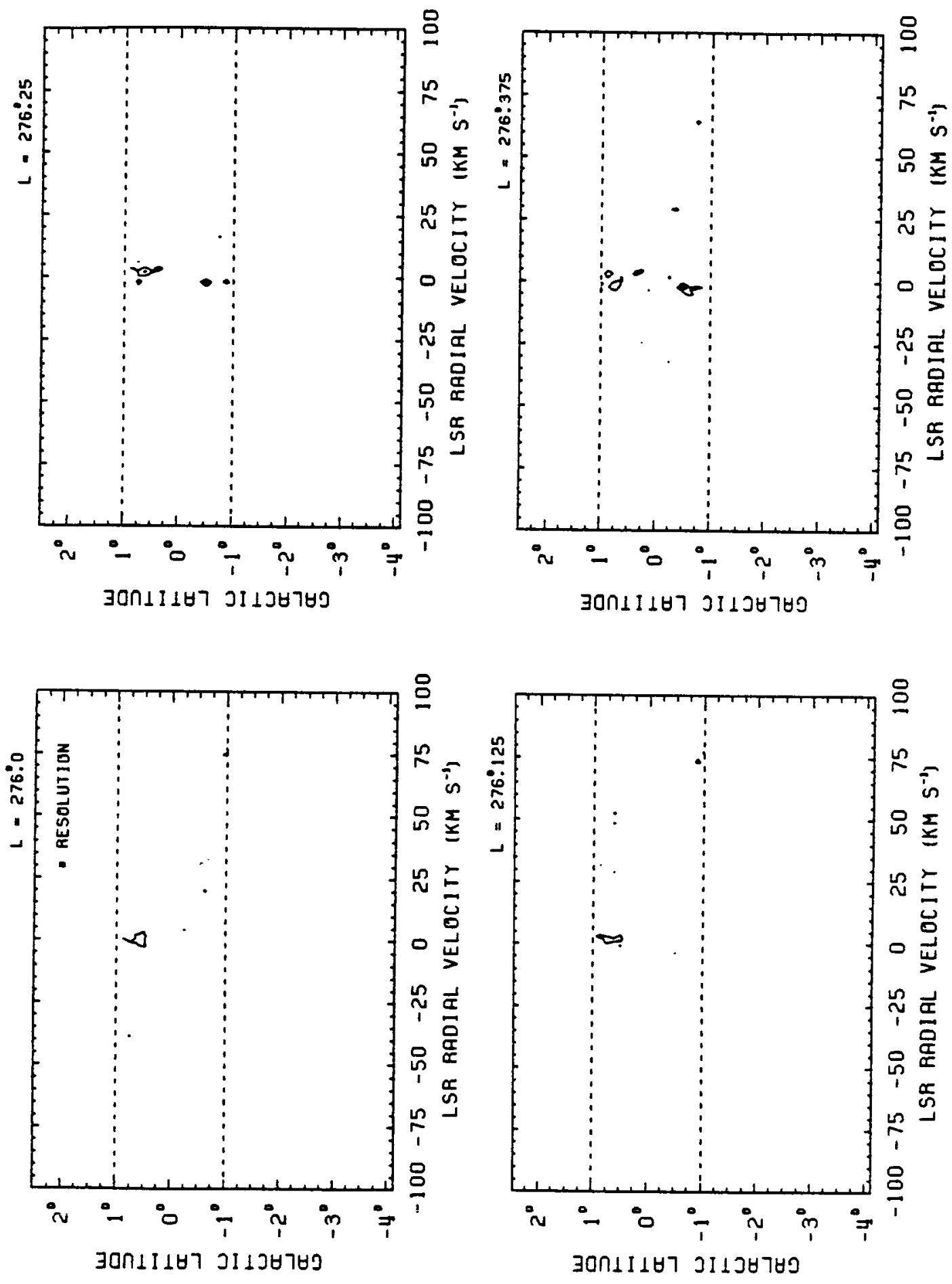

FIGURE B-2 (continued) 

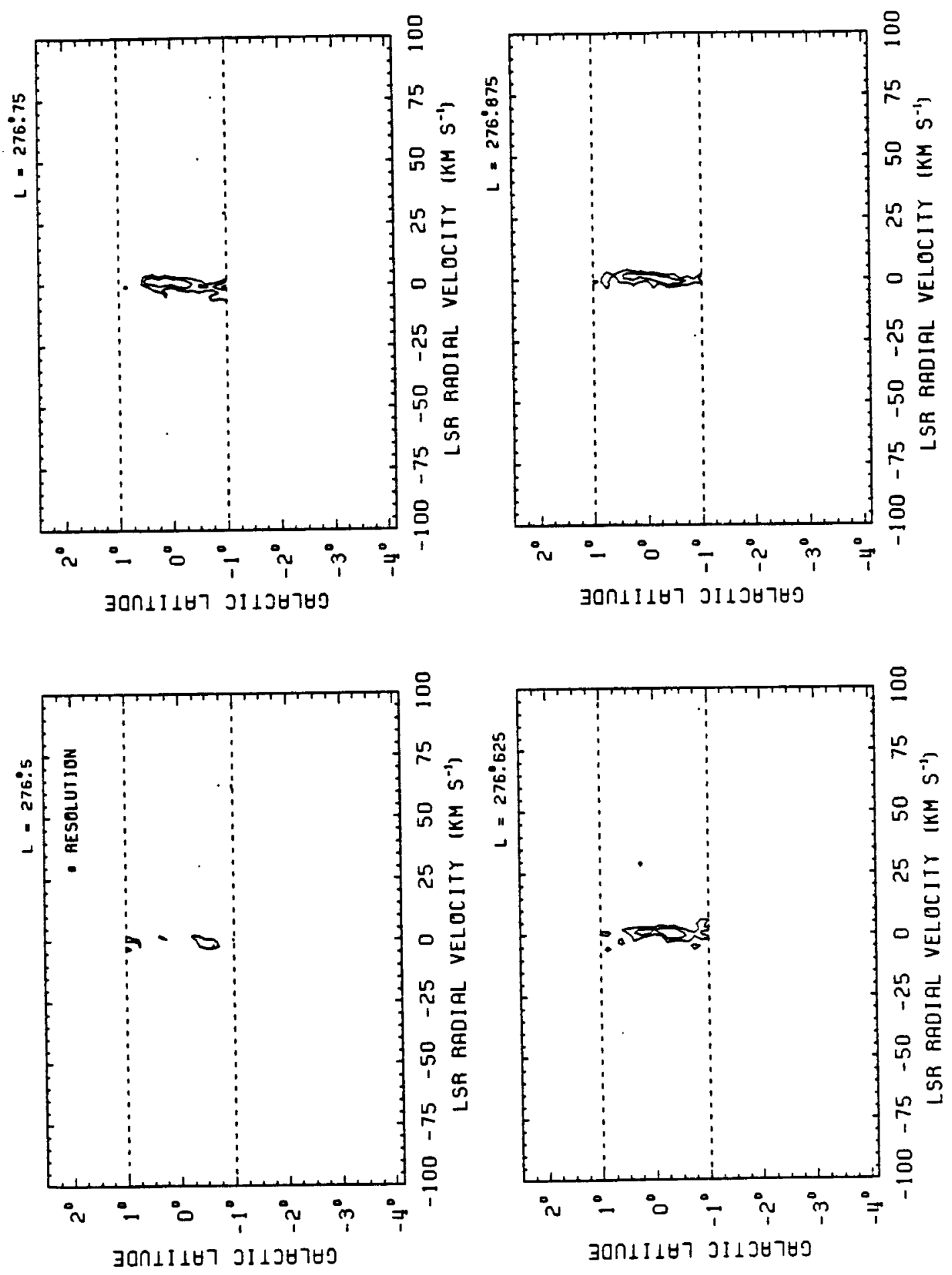

FIGURE B-2 (continued) 

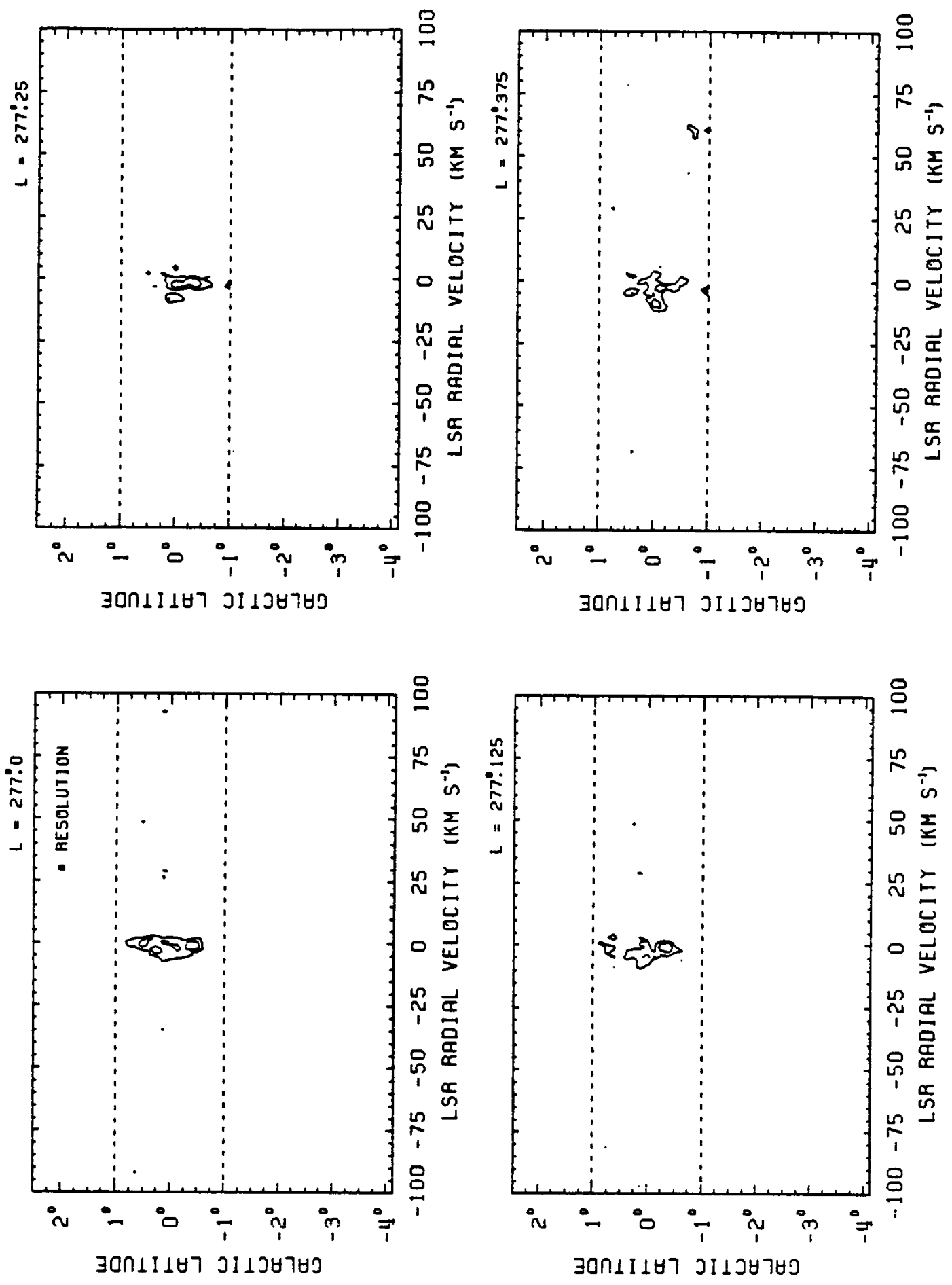

FIGURE B-2 (continued) 

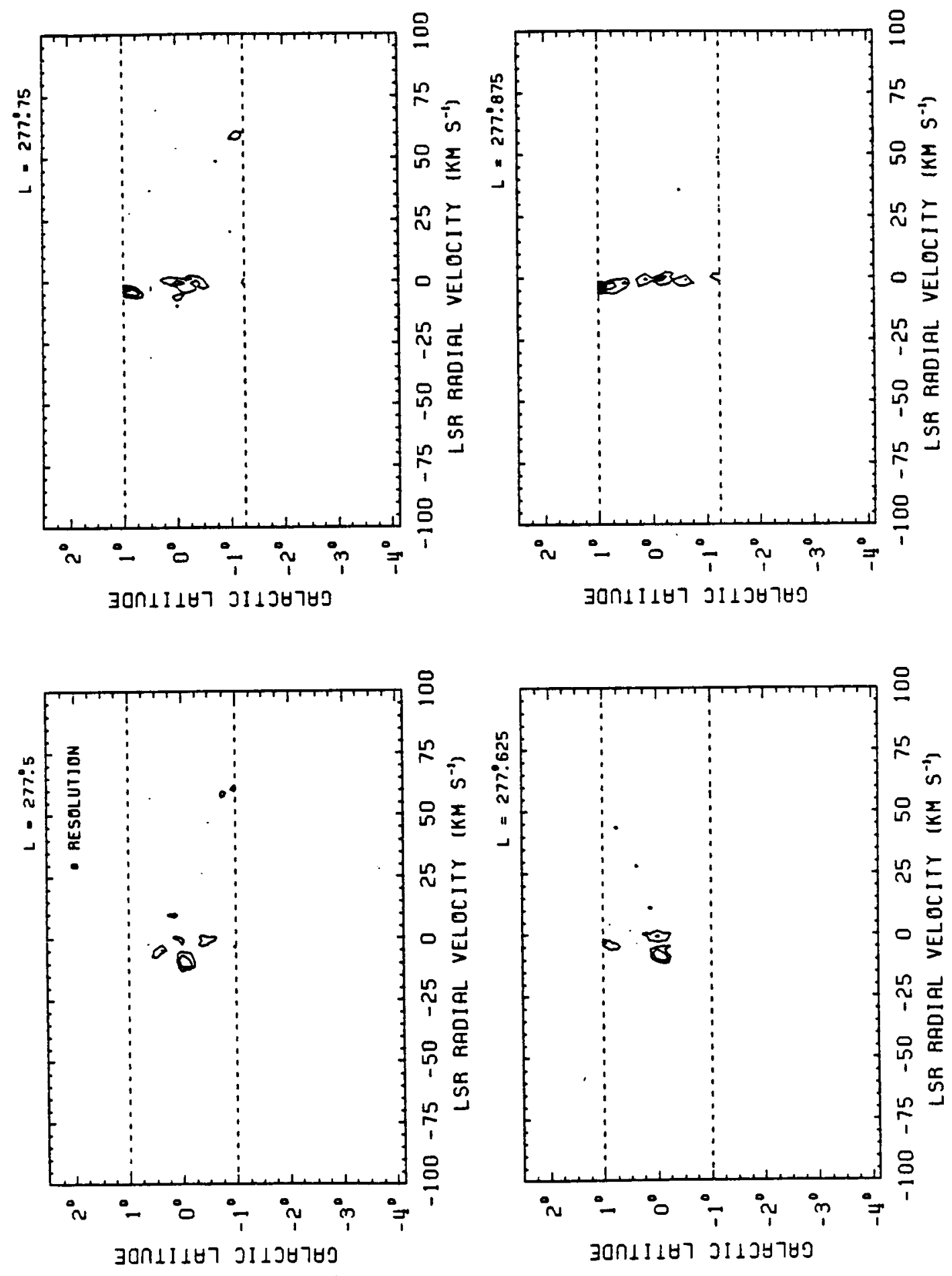

FIGURE B-2 (continued) 

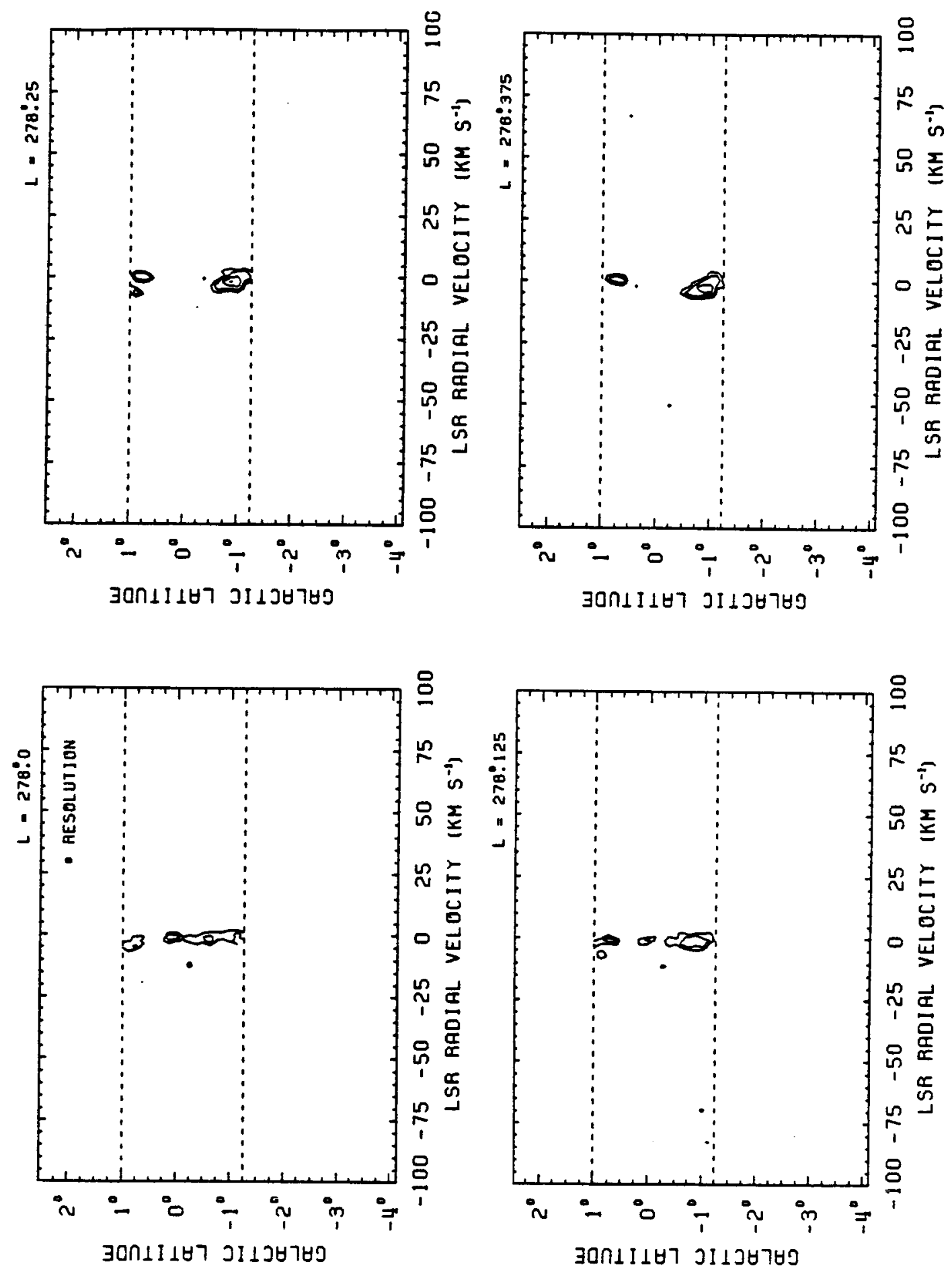

FIGURE B-2 (continued) 

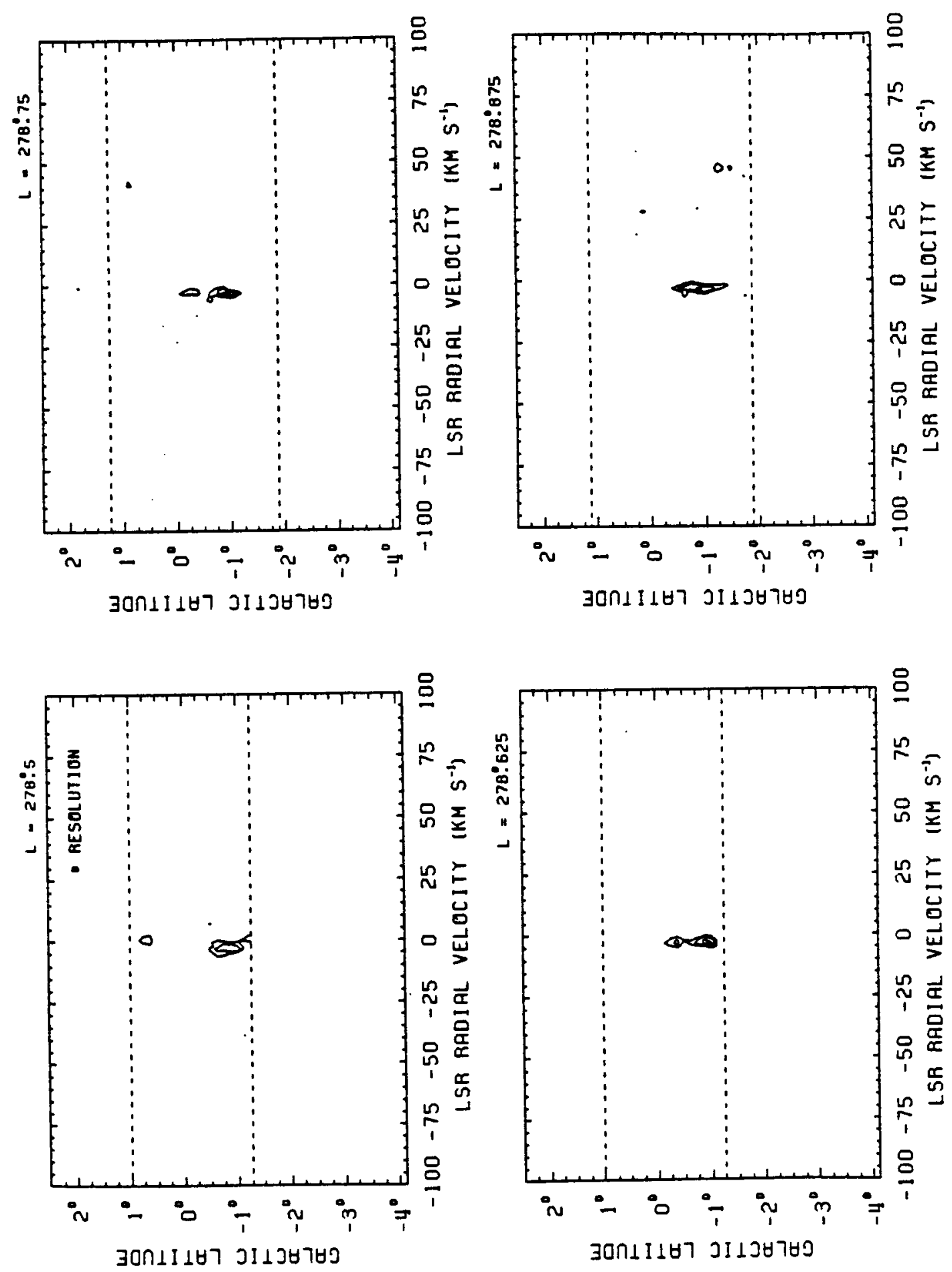

FIGURE B-2 (continued) 

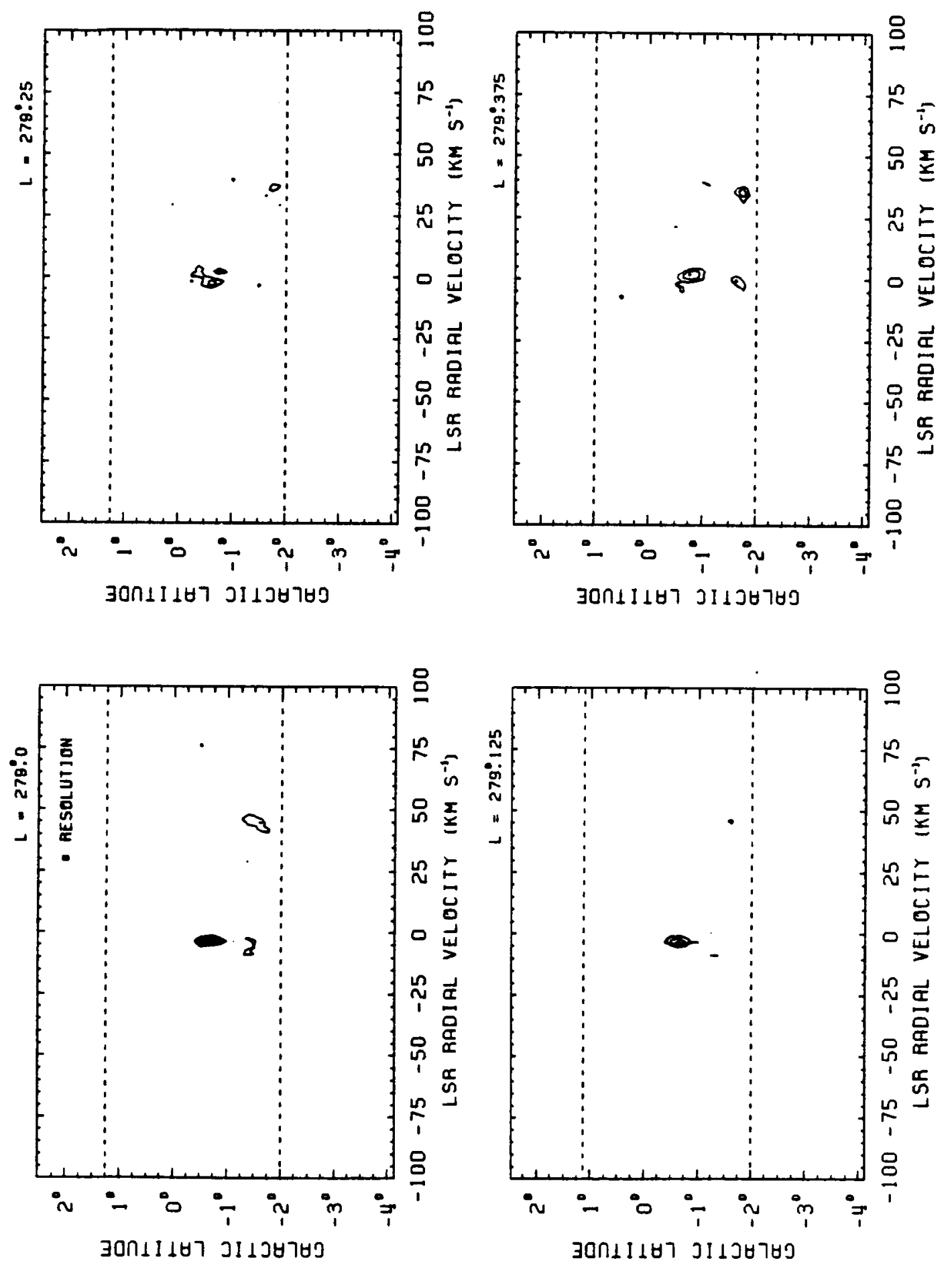

FIGURE B-2 (continued) 

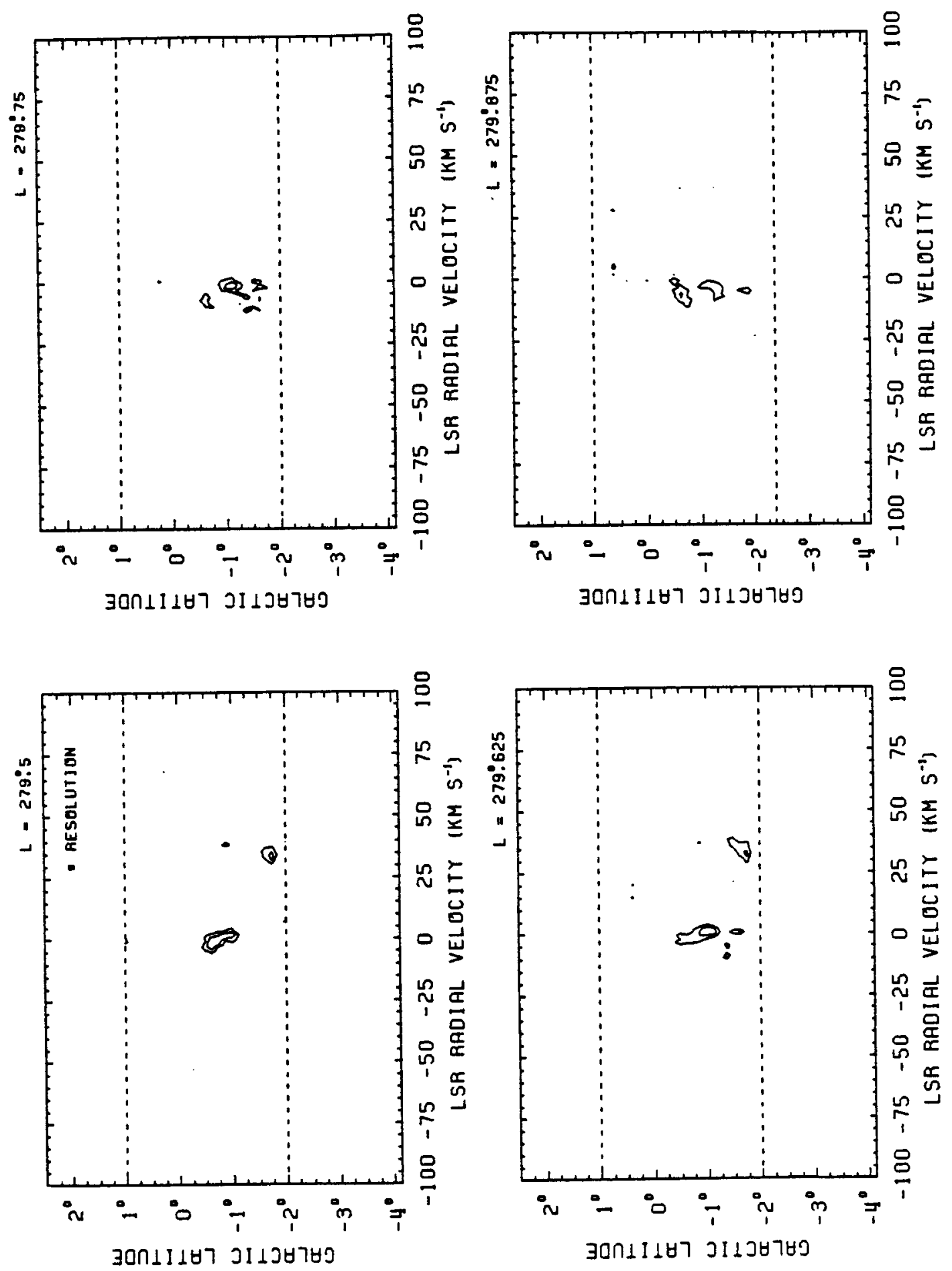

FIGURE B-2 (continued) 


\section{ORIGINAL PAGE IS \\ OF. RODR QUALITY}
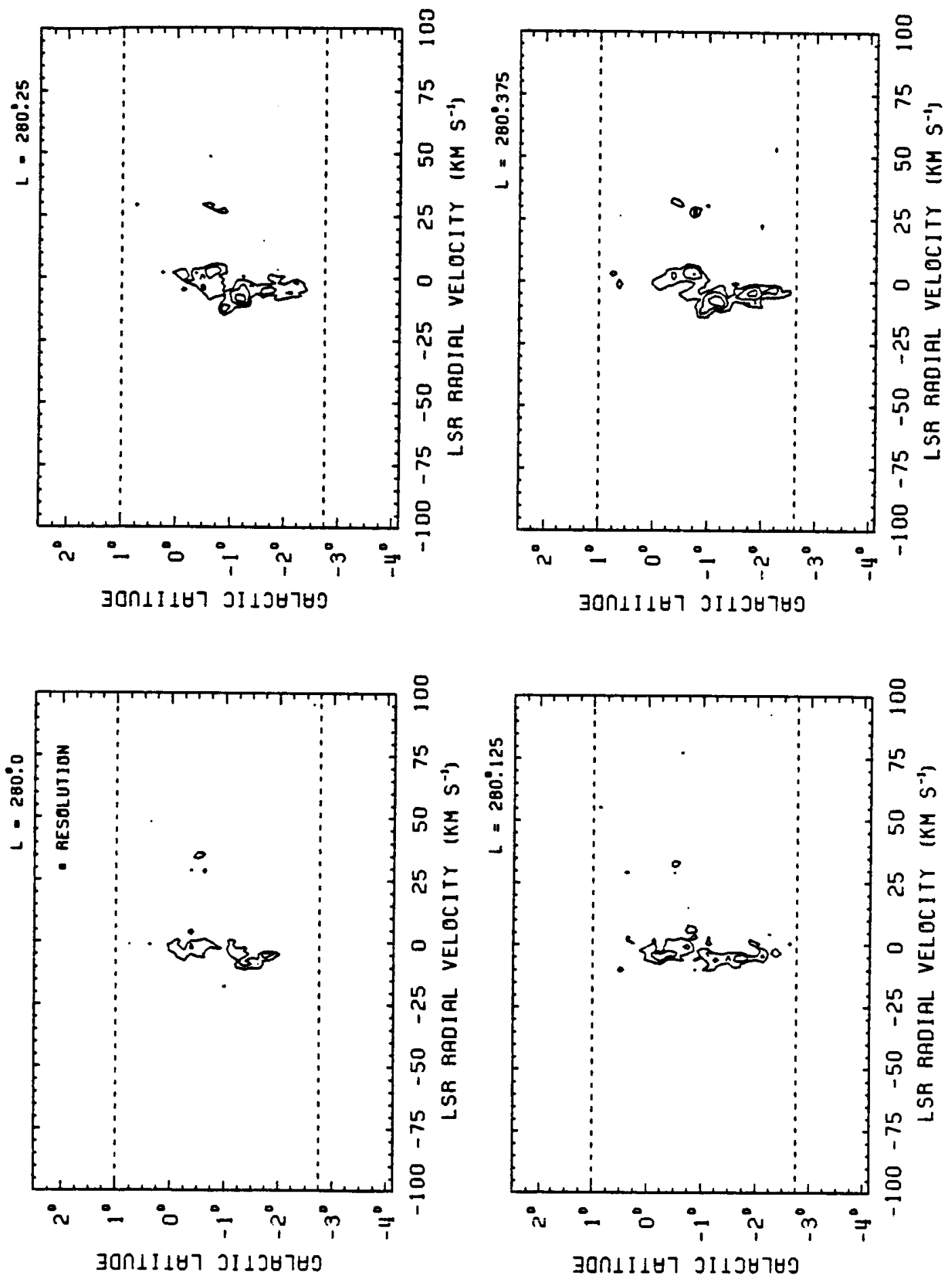

FIGURE B-2 (continued) 

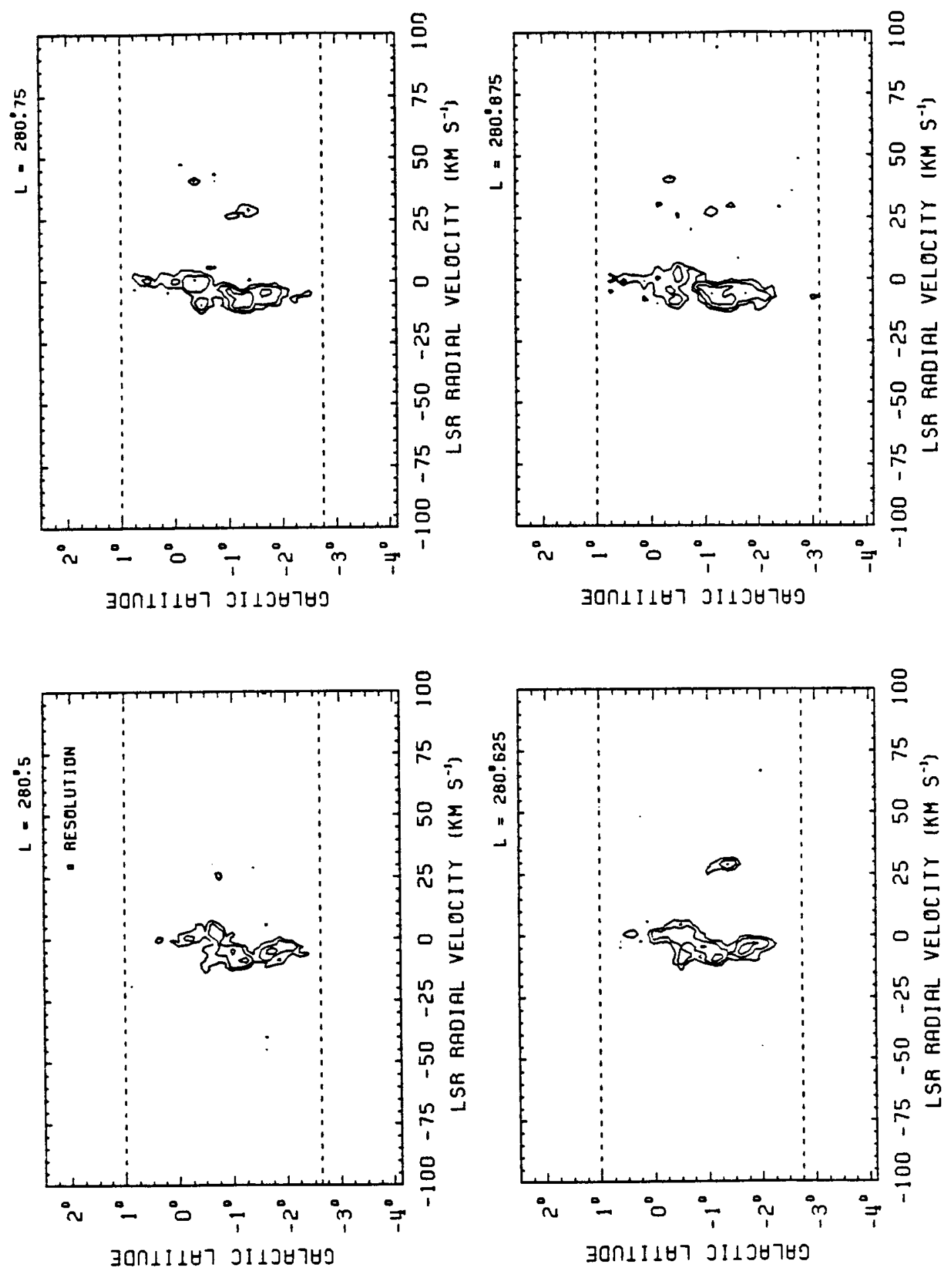

FIGURE B-2 (continued) 

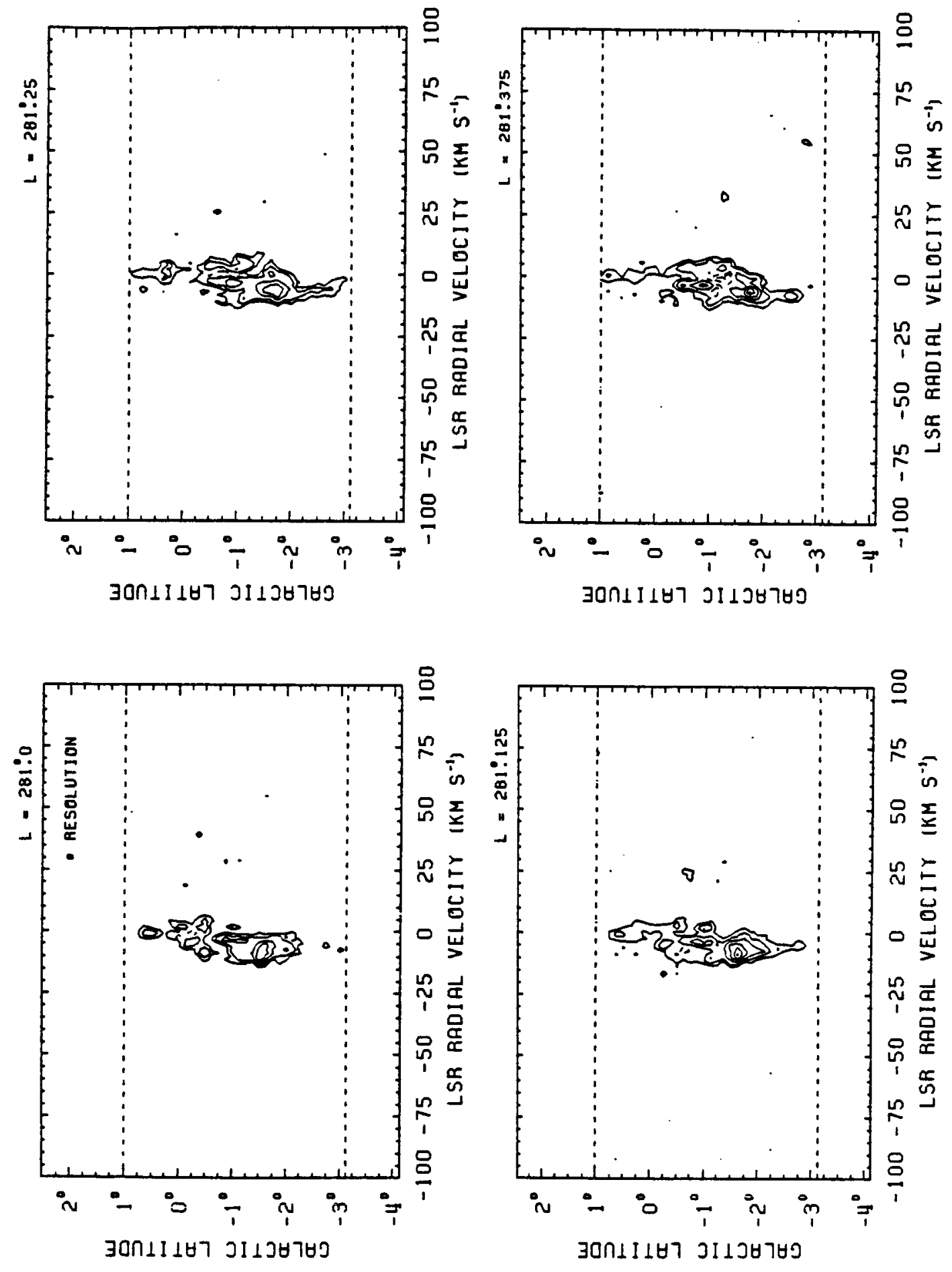

FIGURE B-2 (continued) 

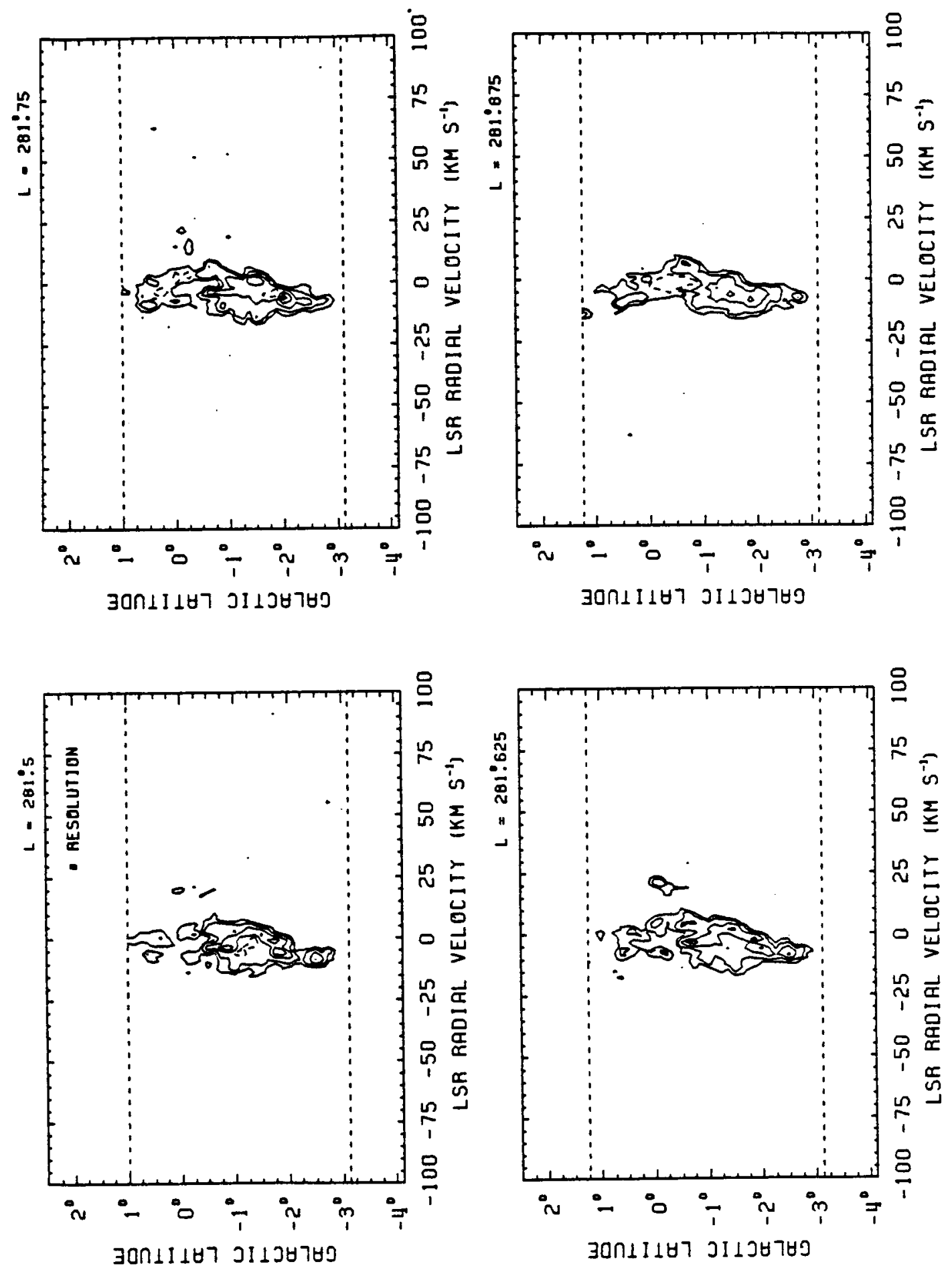

FIGURE B-2 (continued) 

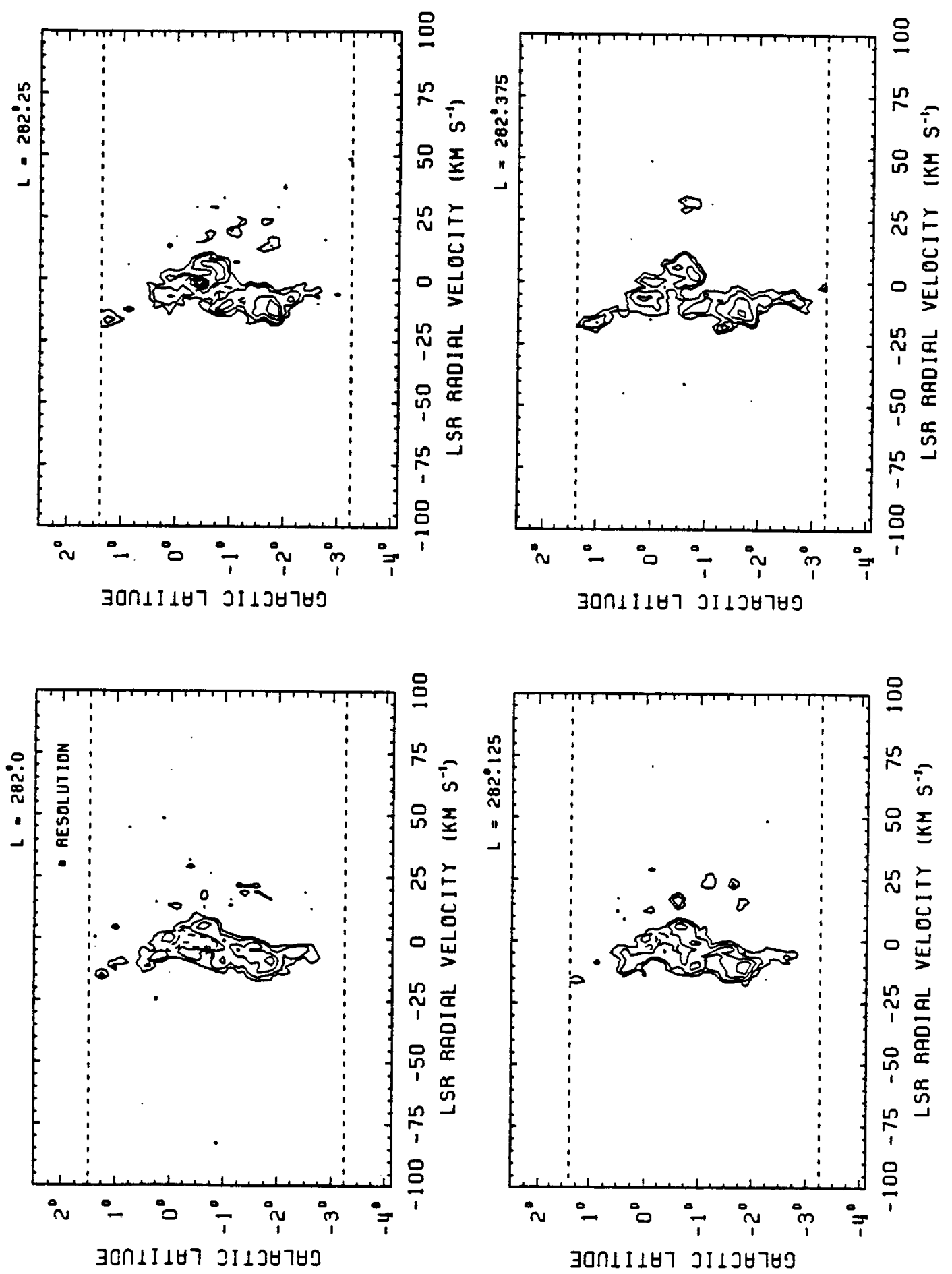

FIGURE B-2 (continued) 

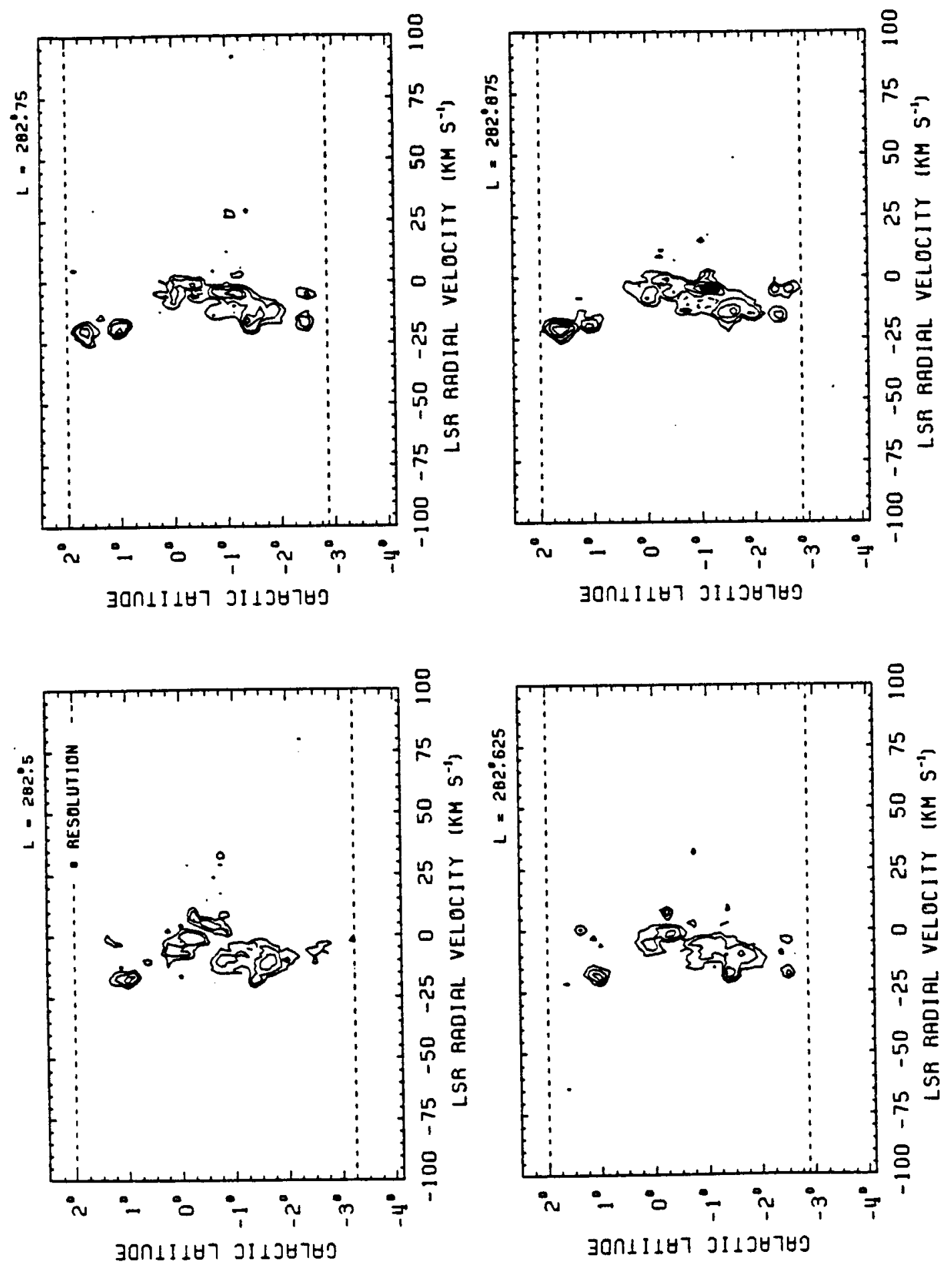

FIGURE B-2 (continued) 

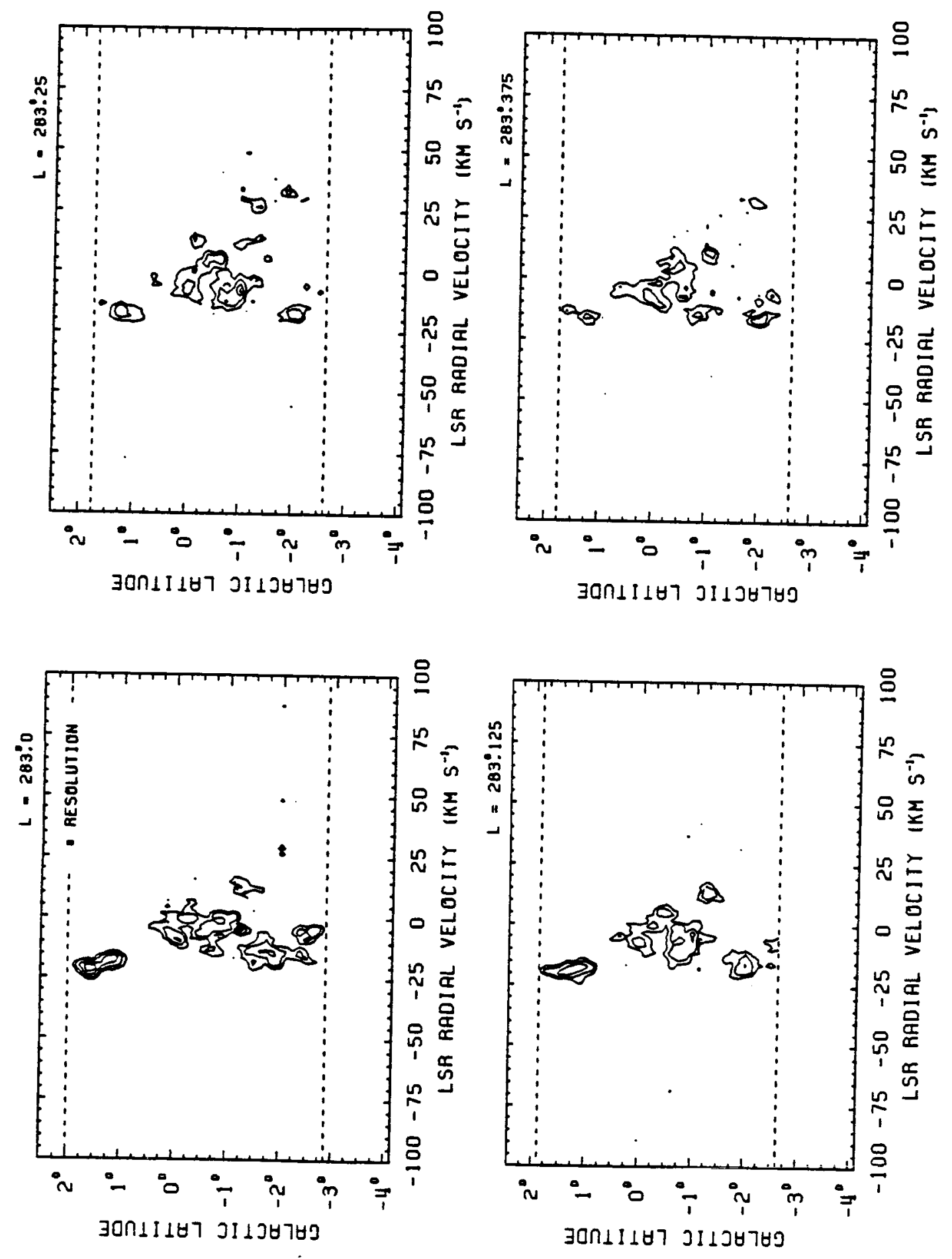

FIGURE B-2 (continued) 

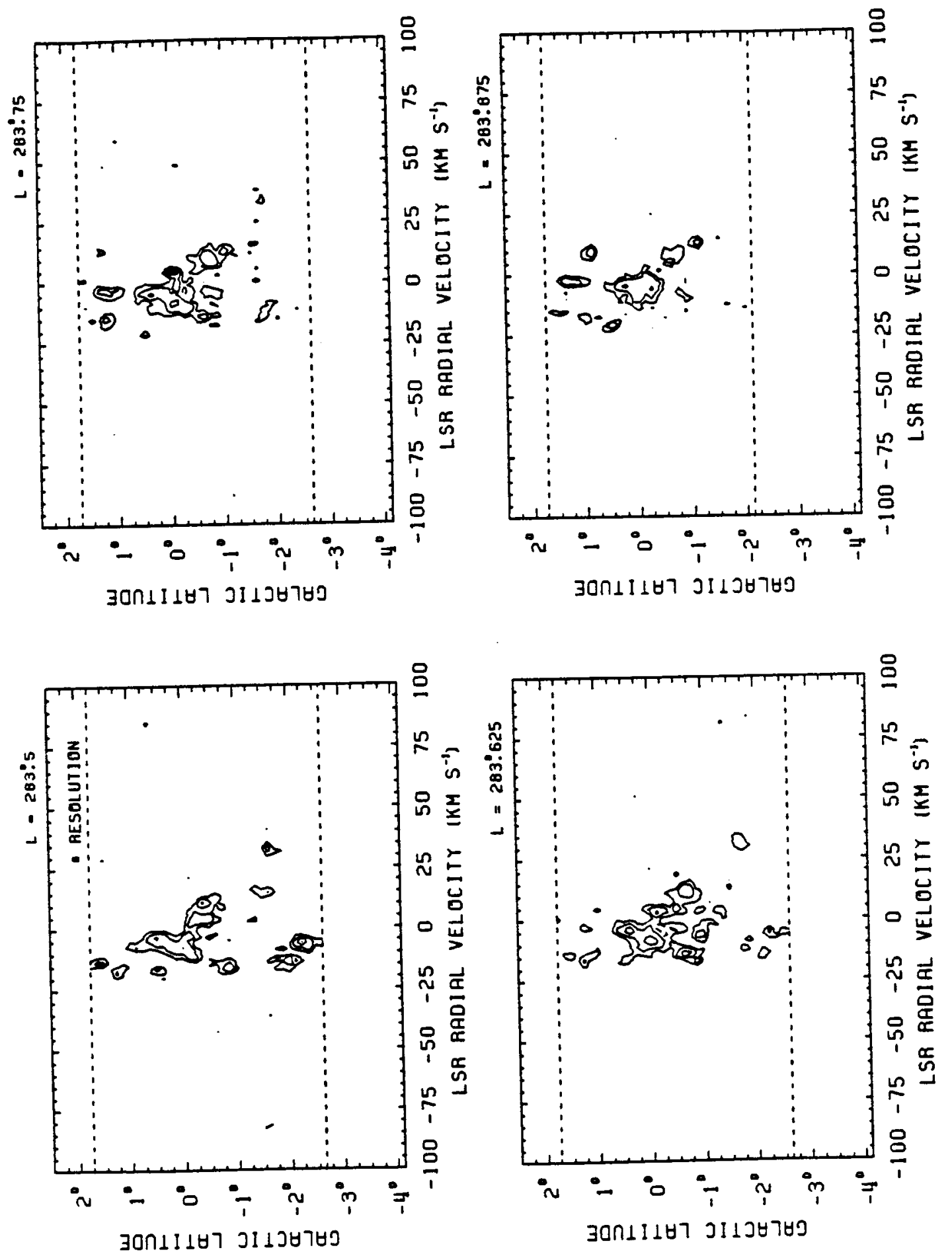

FIGURE B-2 (continued) 

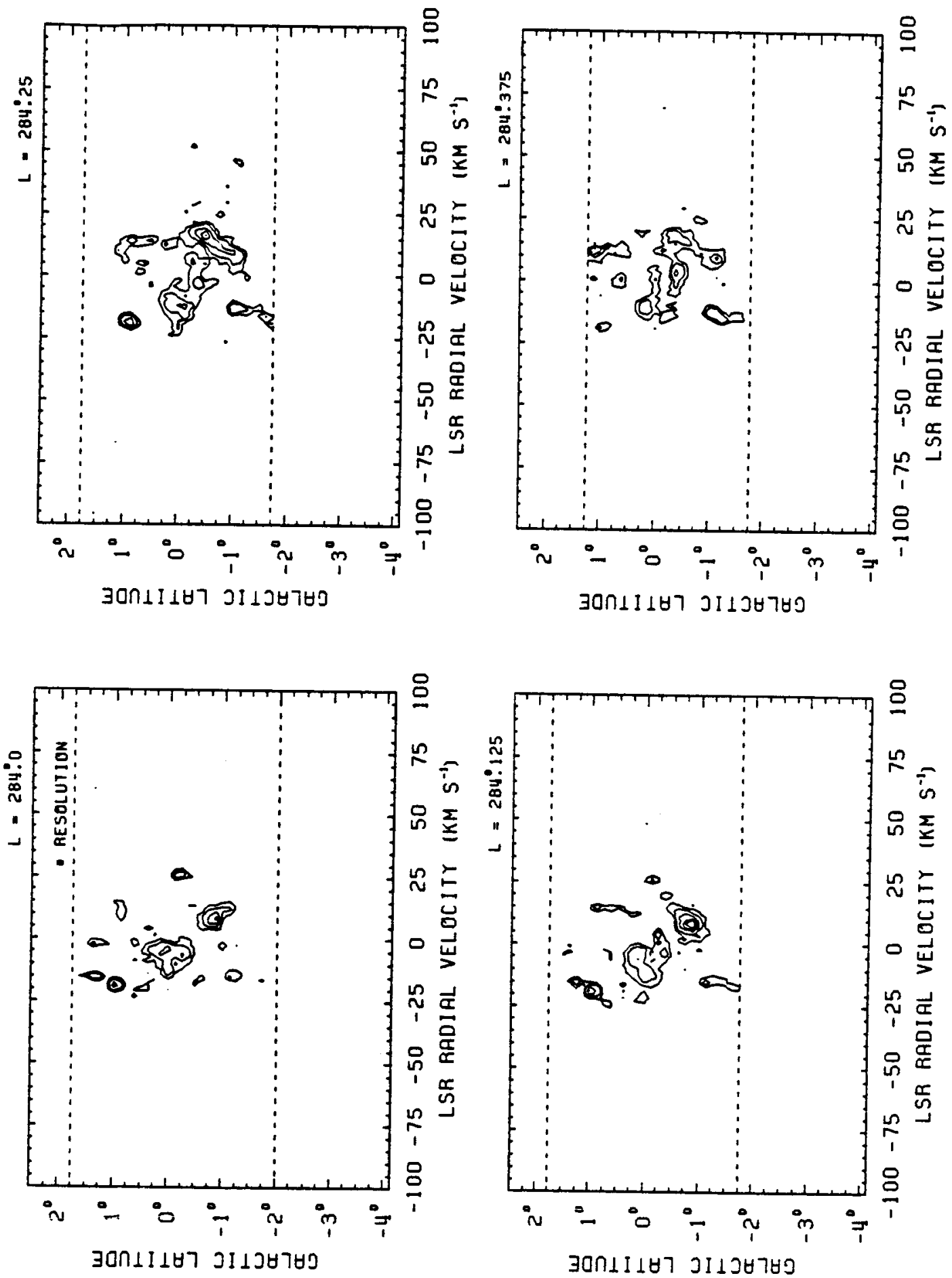

FIGURE B-2 (continued) 

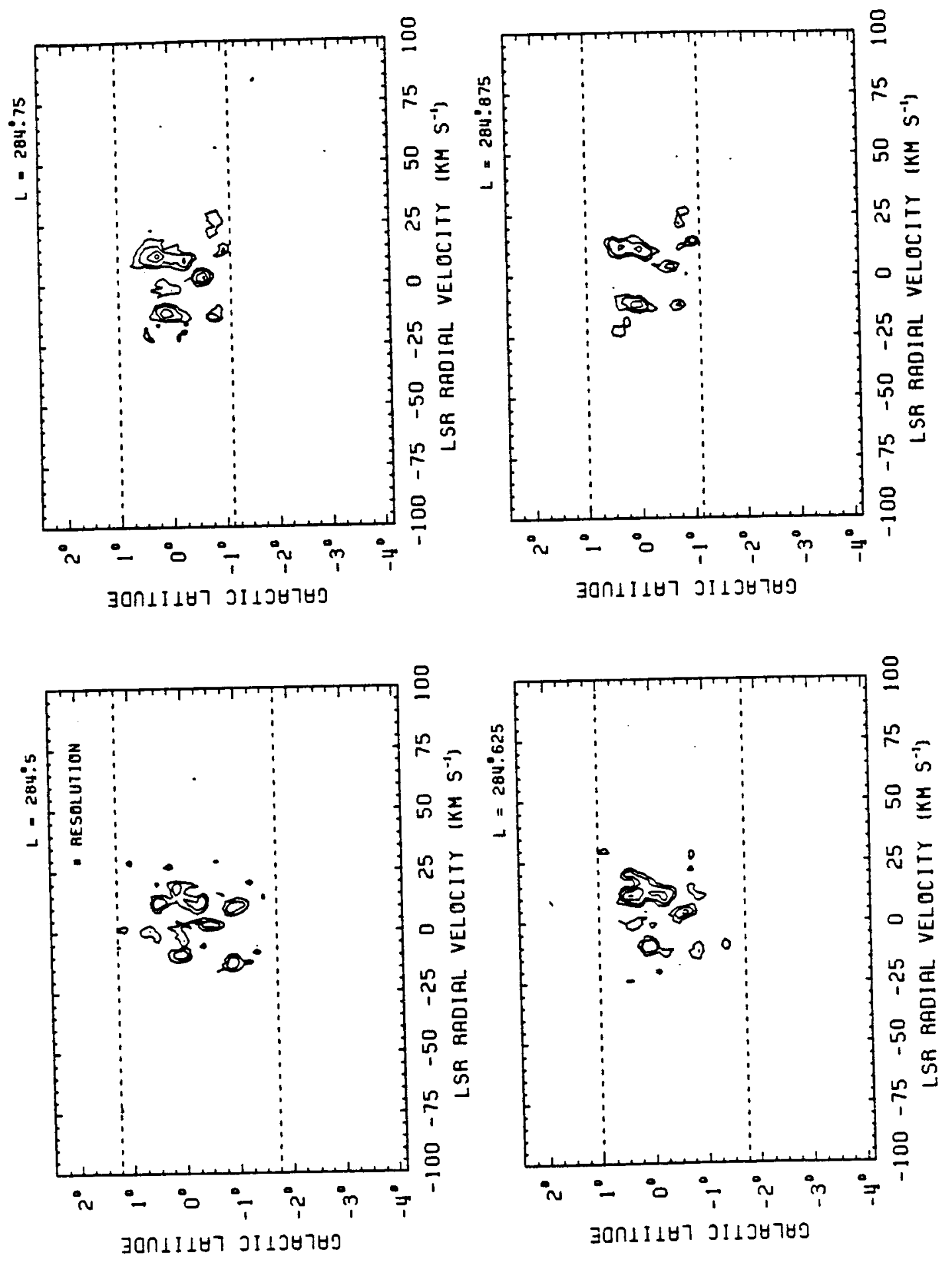

FIGURE B-2 (continued) 


\section{ORIGINAL PAGE IS \\ OF POOR QUALITY}
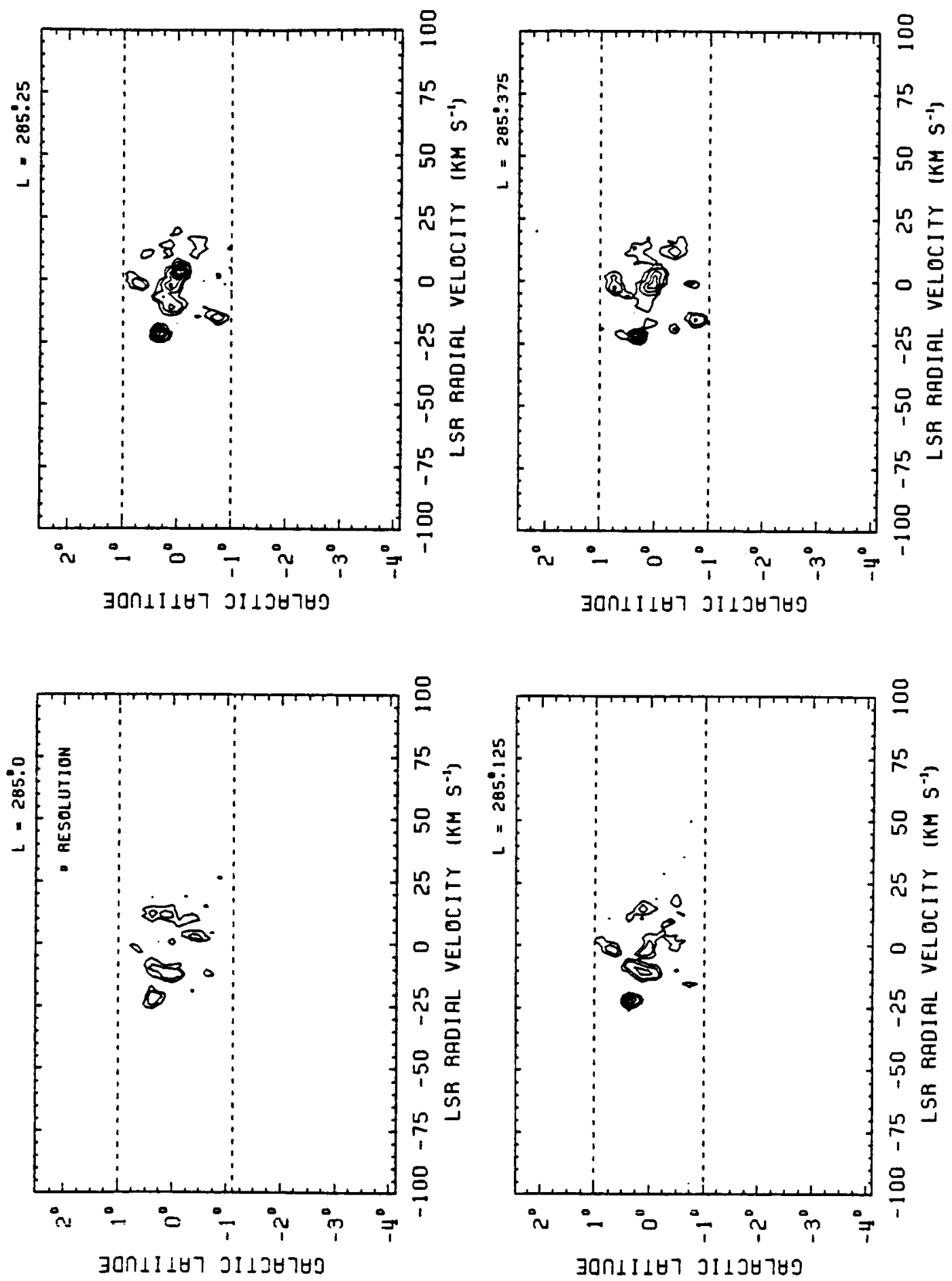

FIGURE B-2 (continued) 

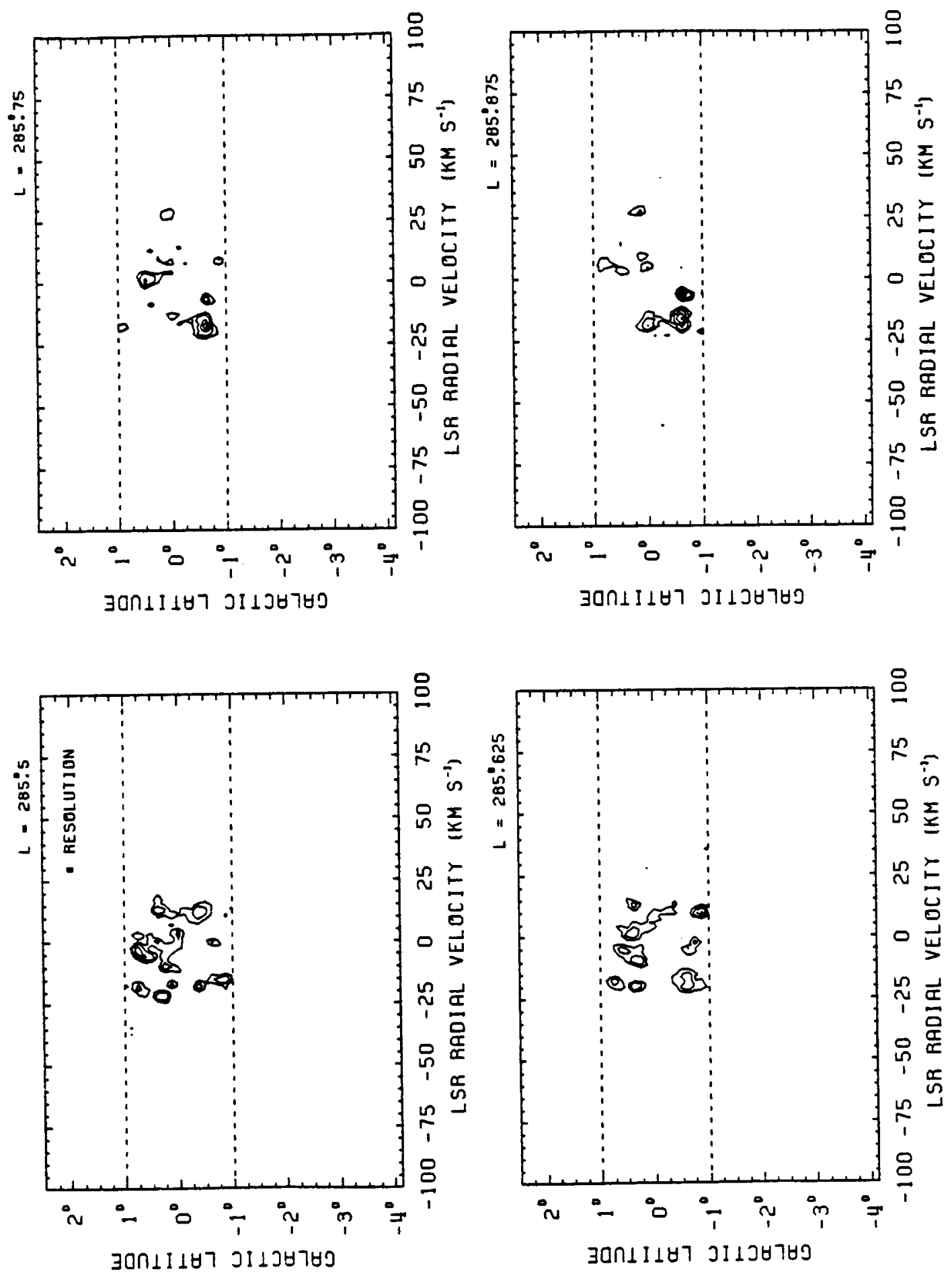

FIGURE B-2 (cont inued) 

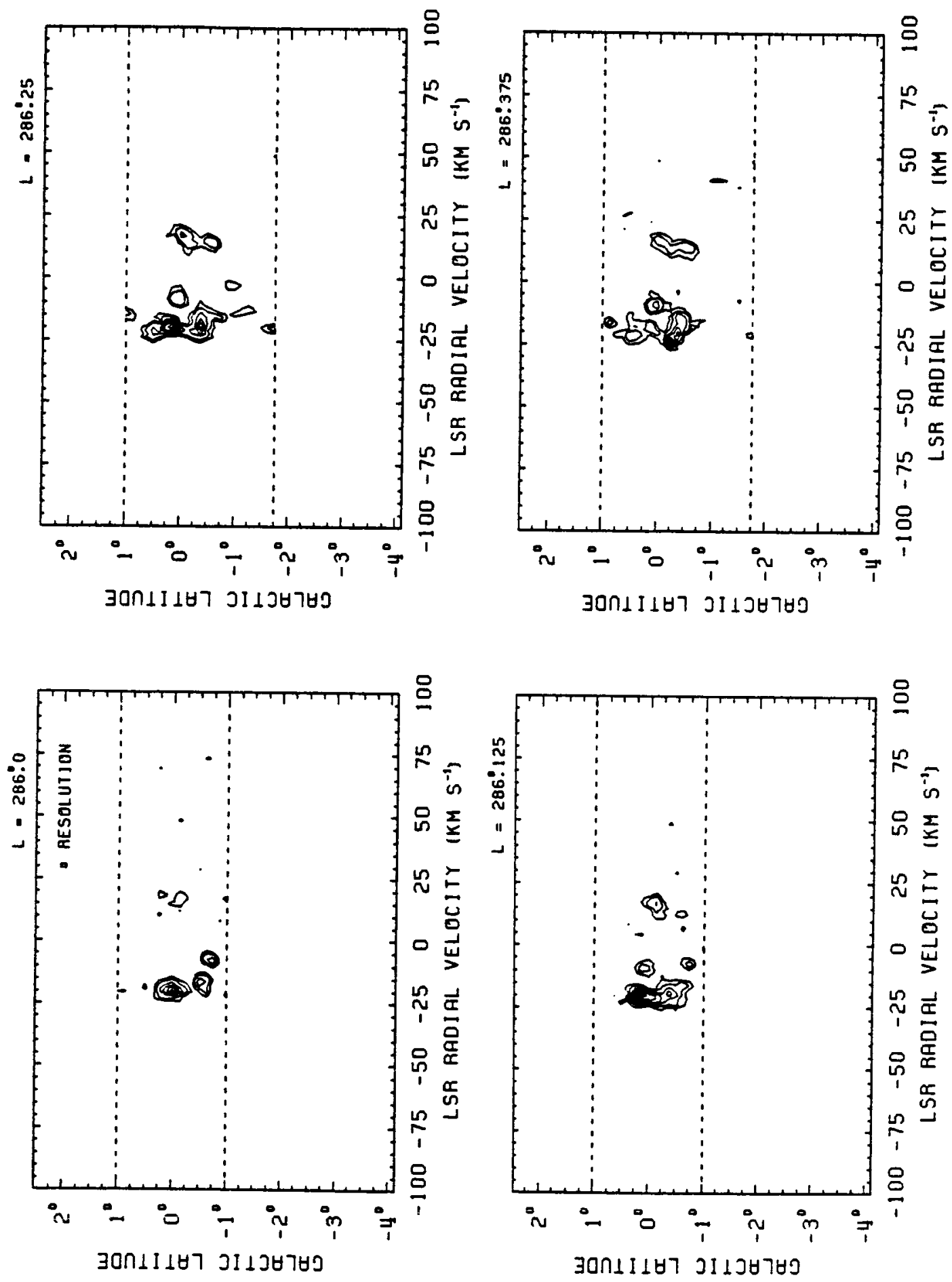

FIGURE B-2 (continued) 

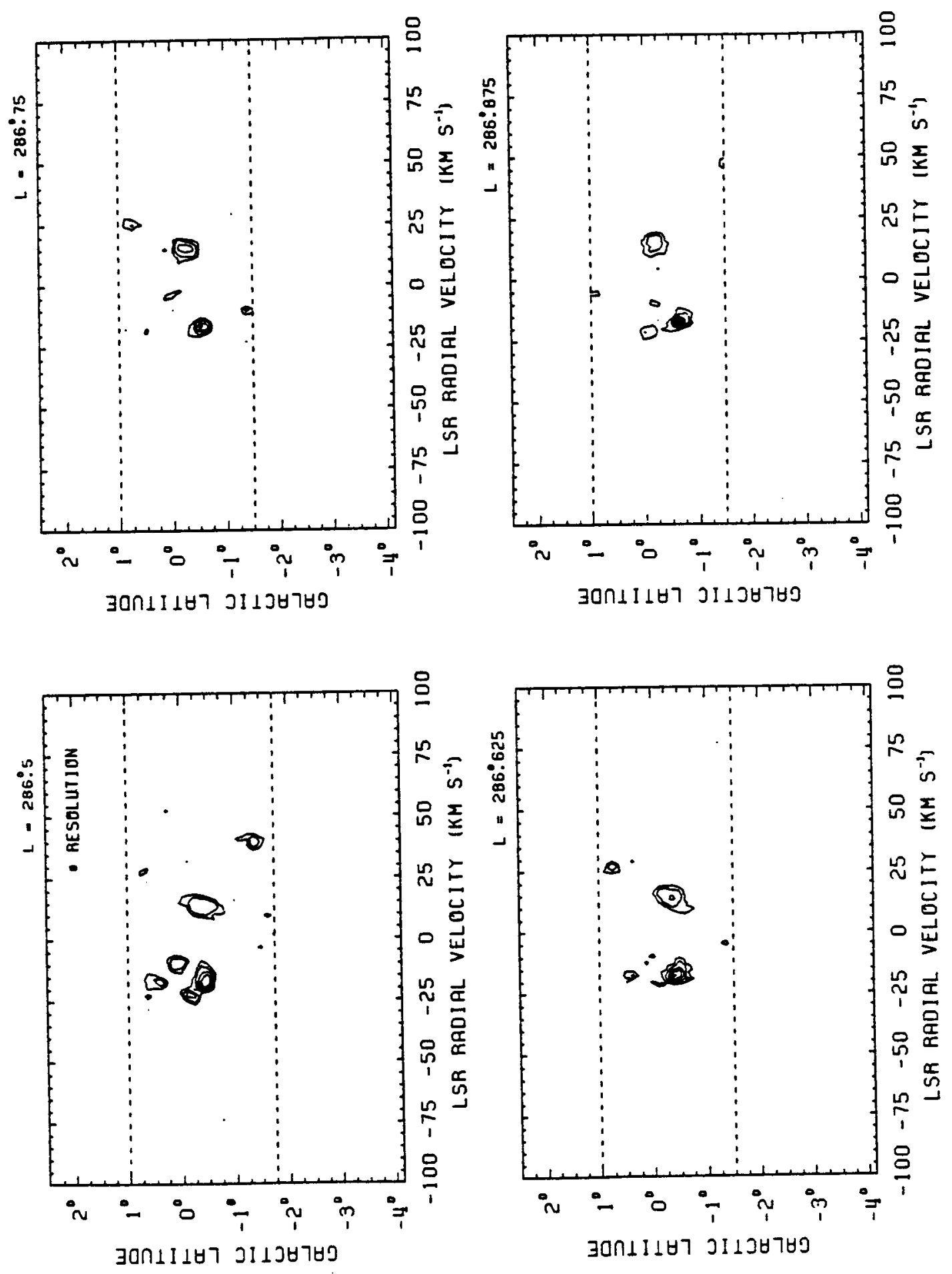

FIGURE B-2 (continued) 

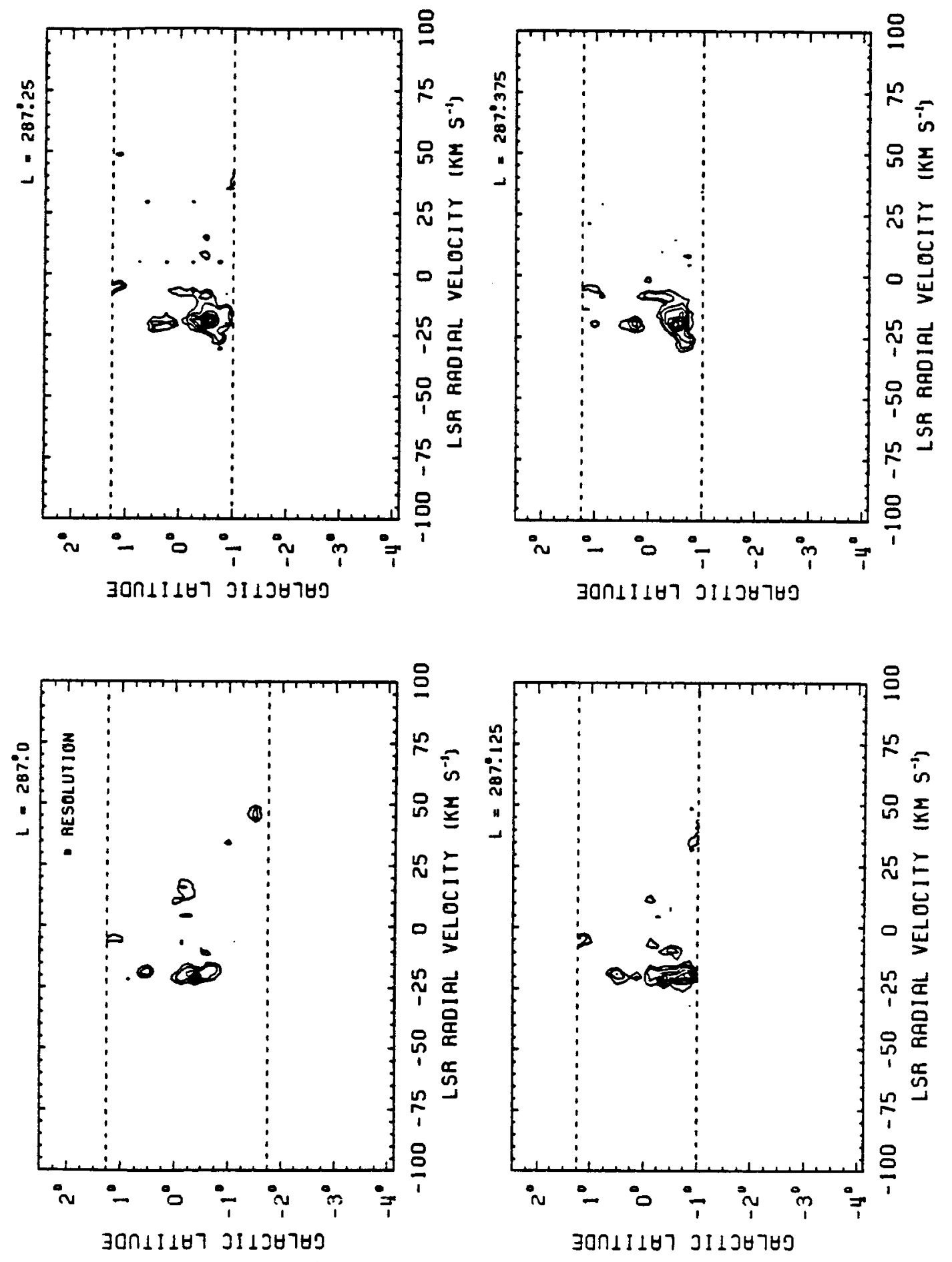

FIGURE B-2 (continued) 

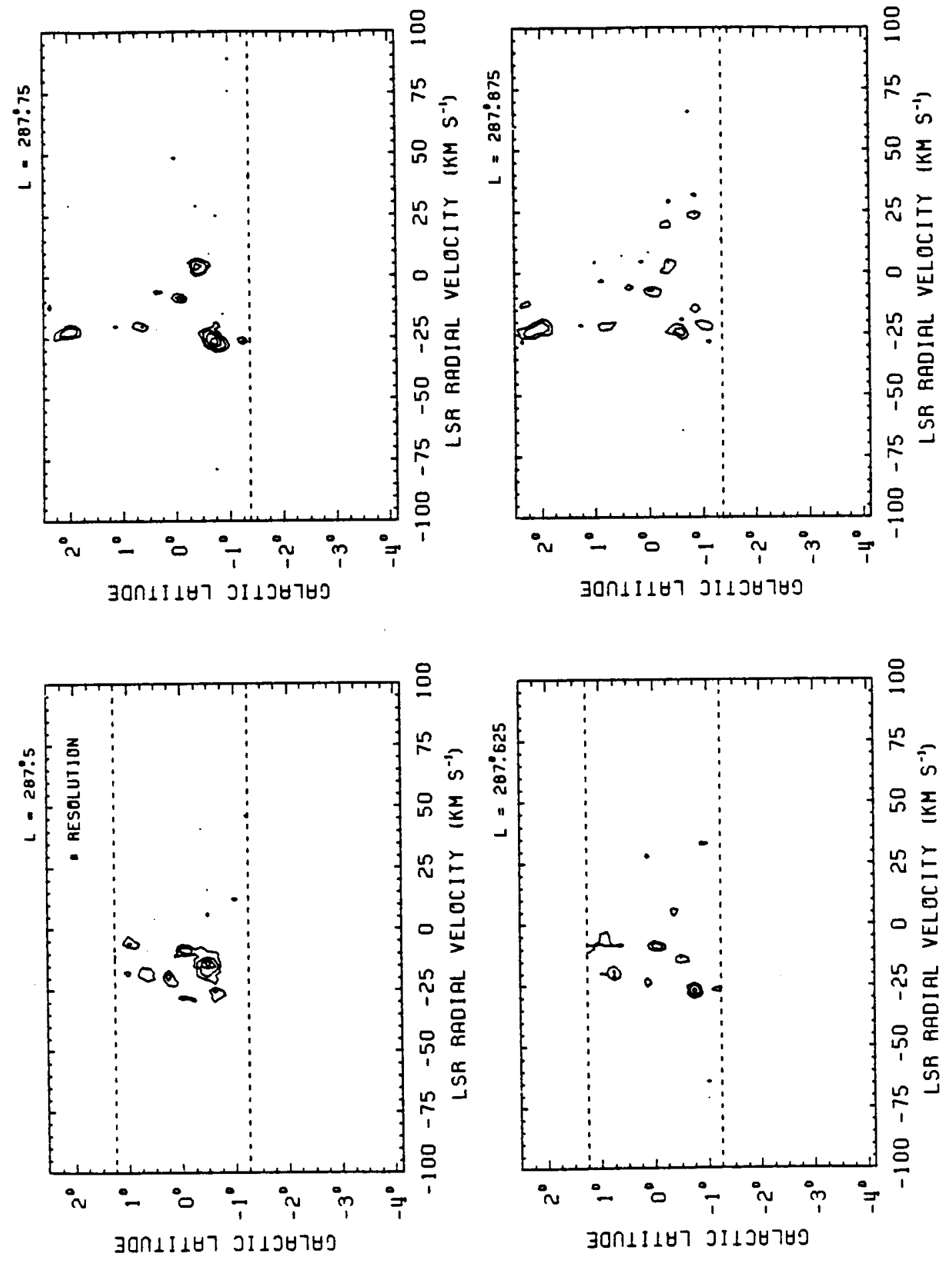

FIGURE B-2 (continued) 

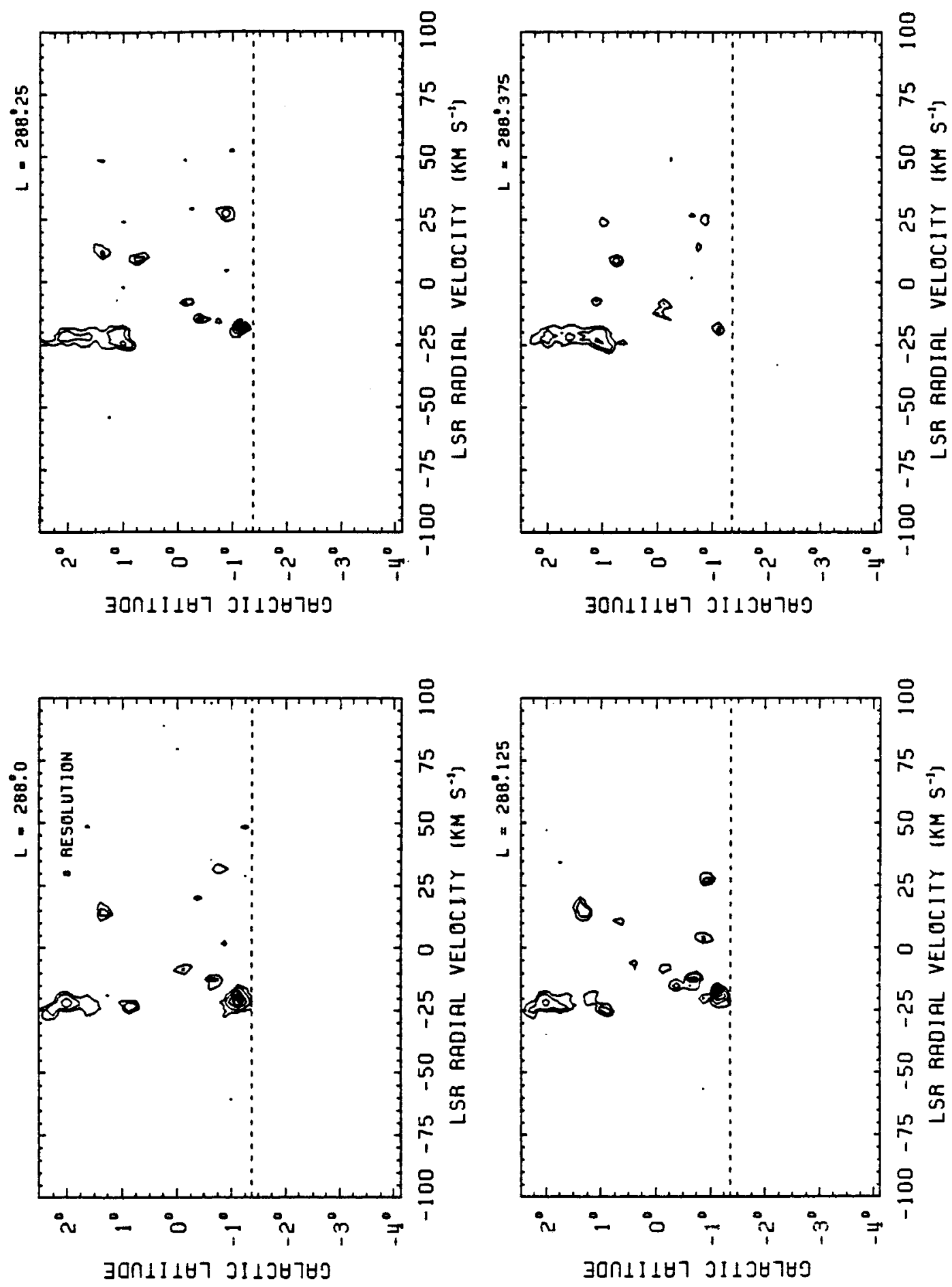

FIGURE B-2 (continued) 

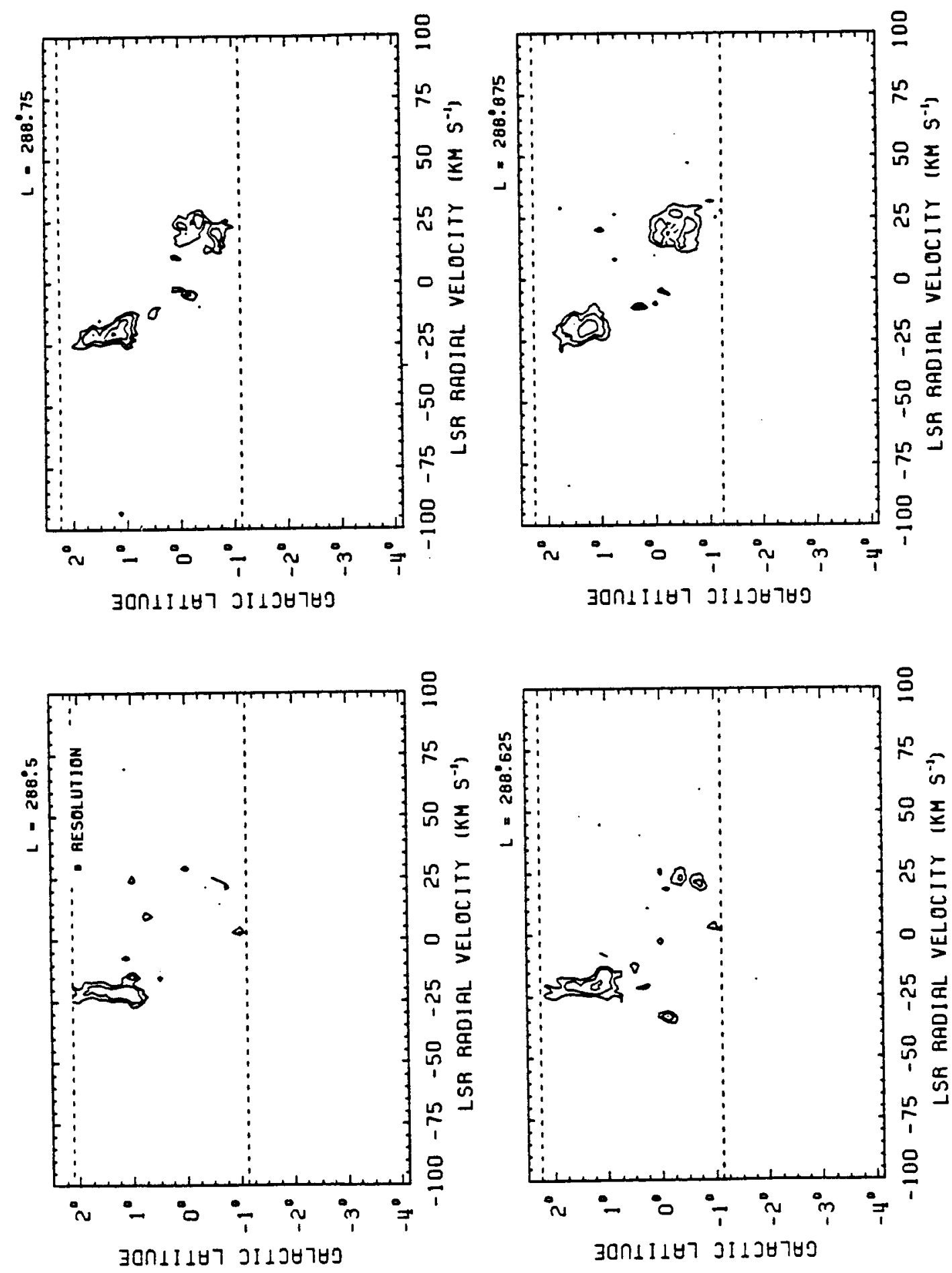

FIGURE B-2 (continued) 

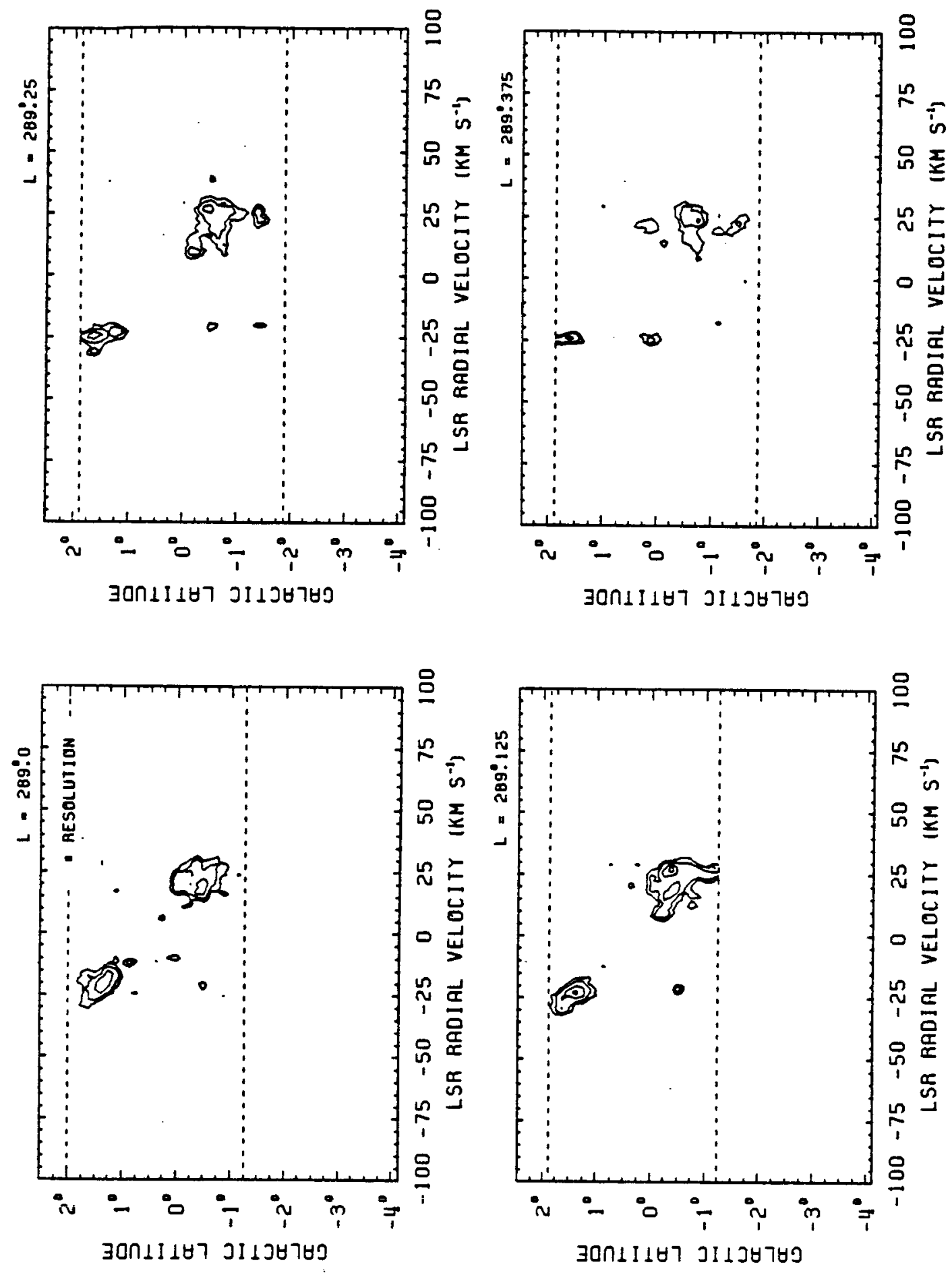

FIGURE B-2 (continued) 

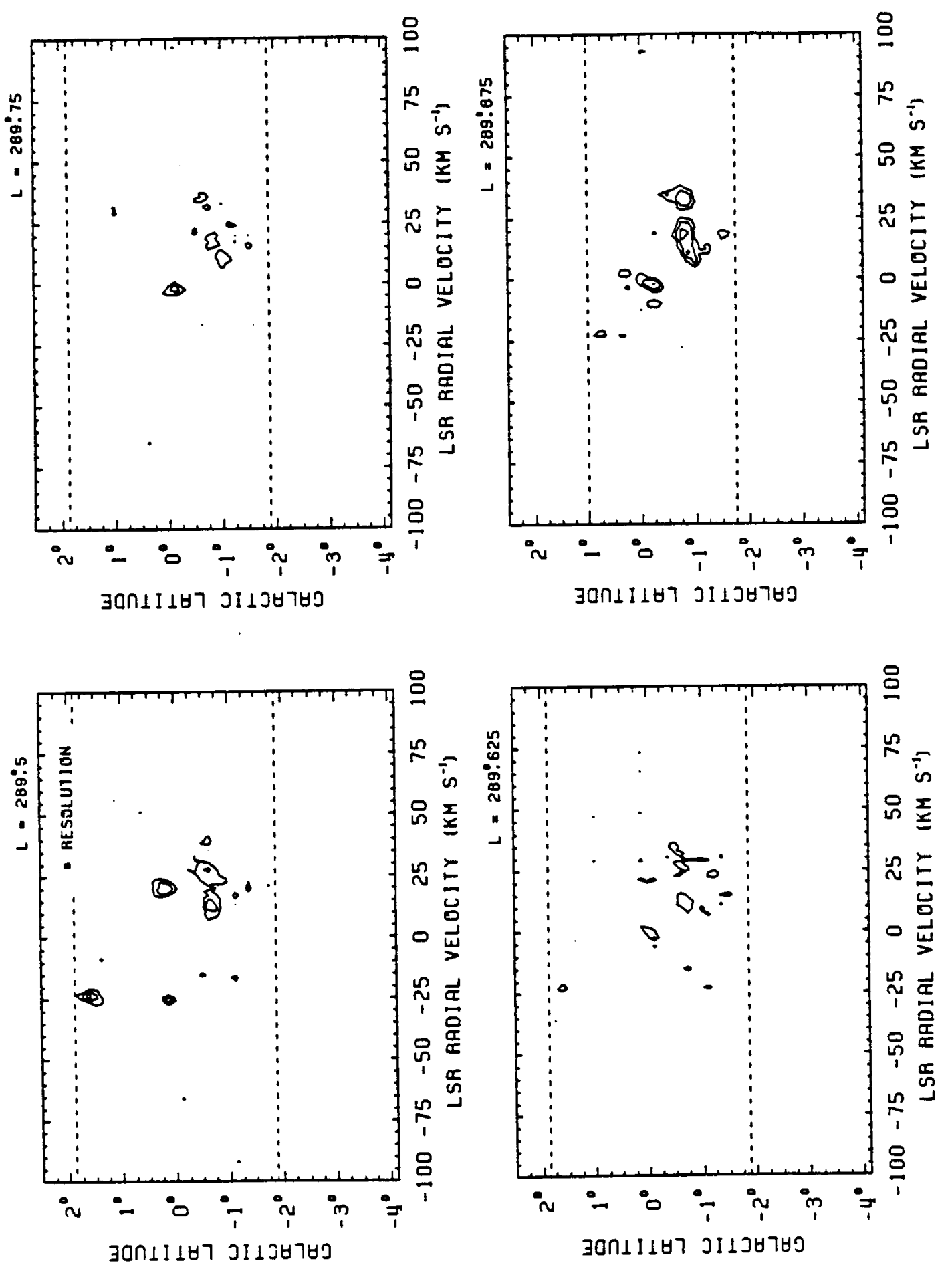

FIGURE B-2 (continued) 

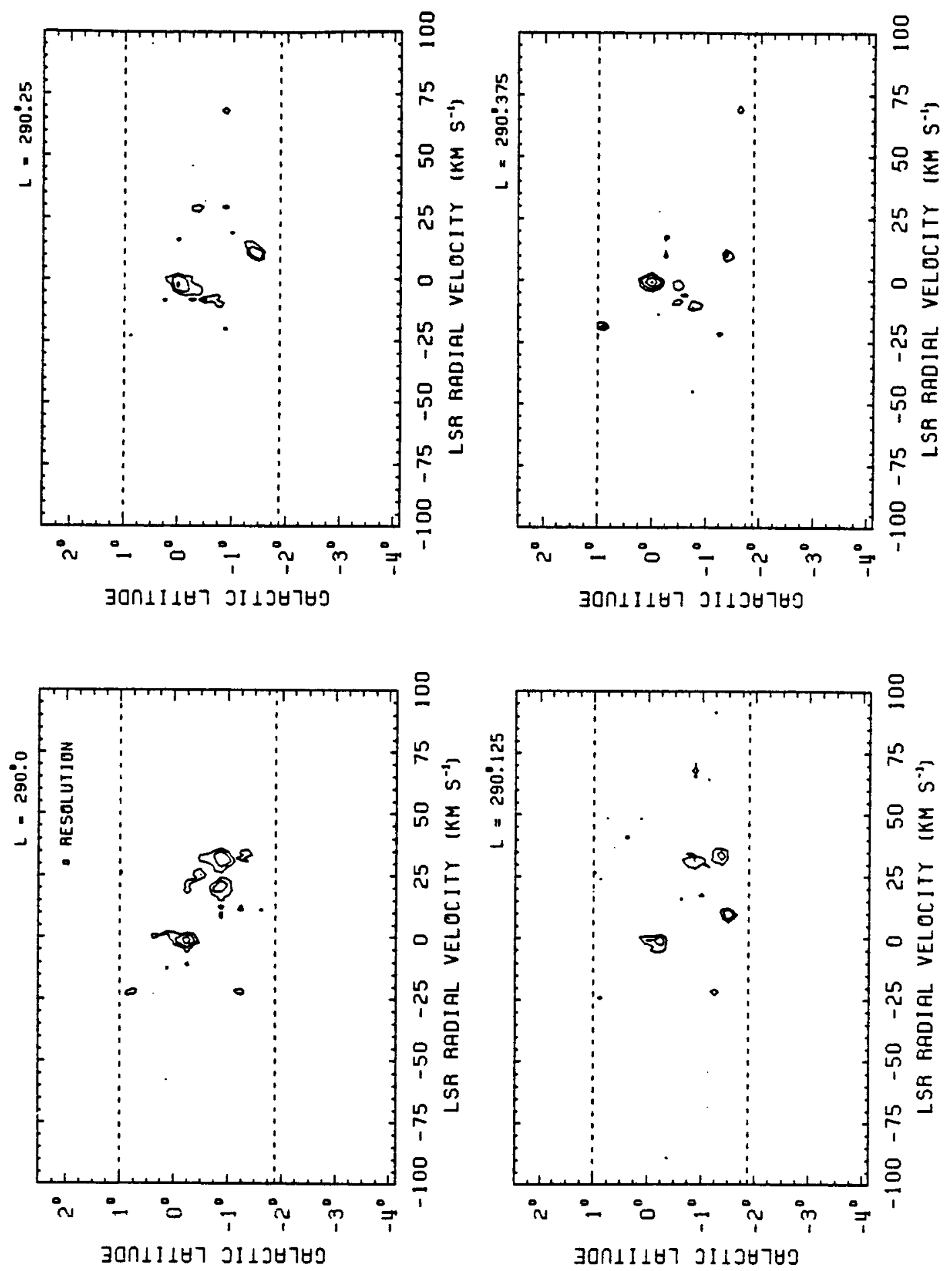

FIGURE B-2 (continued) 

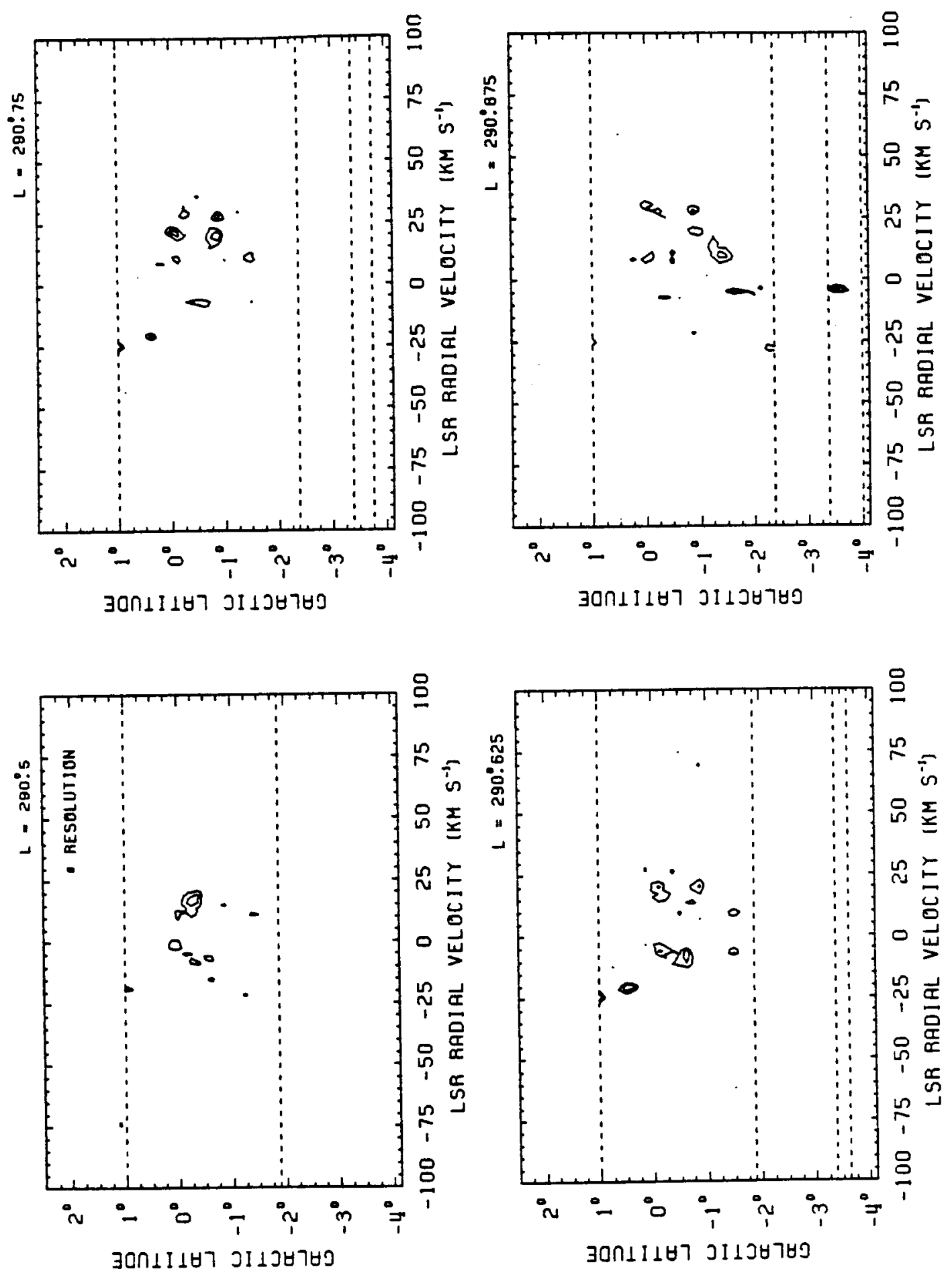

FIGURE B-2 (continued) 

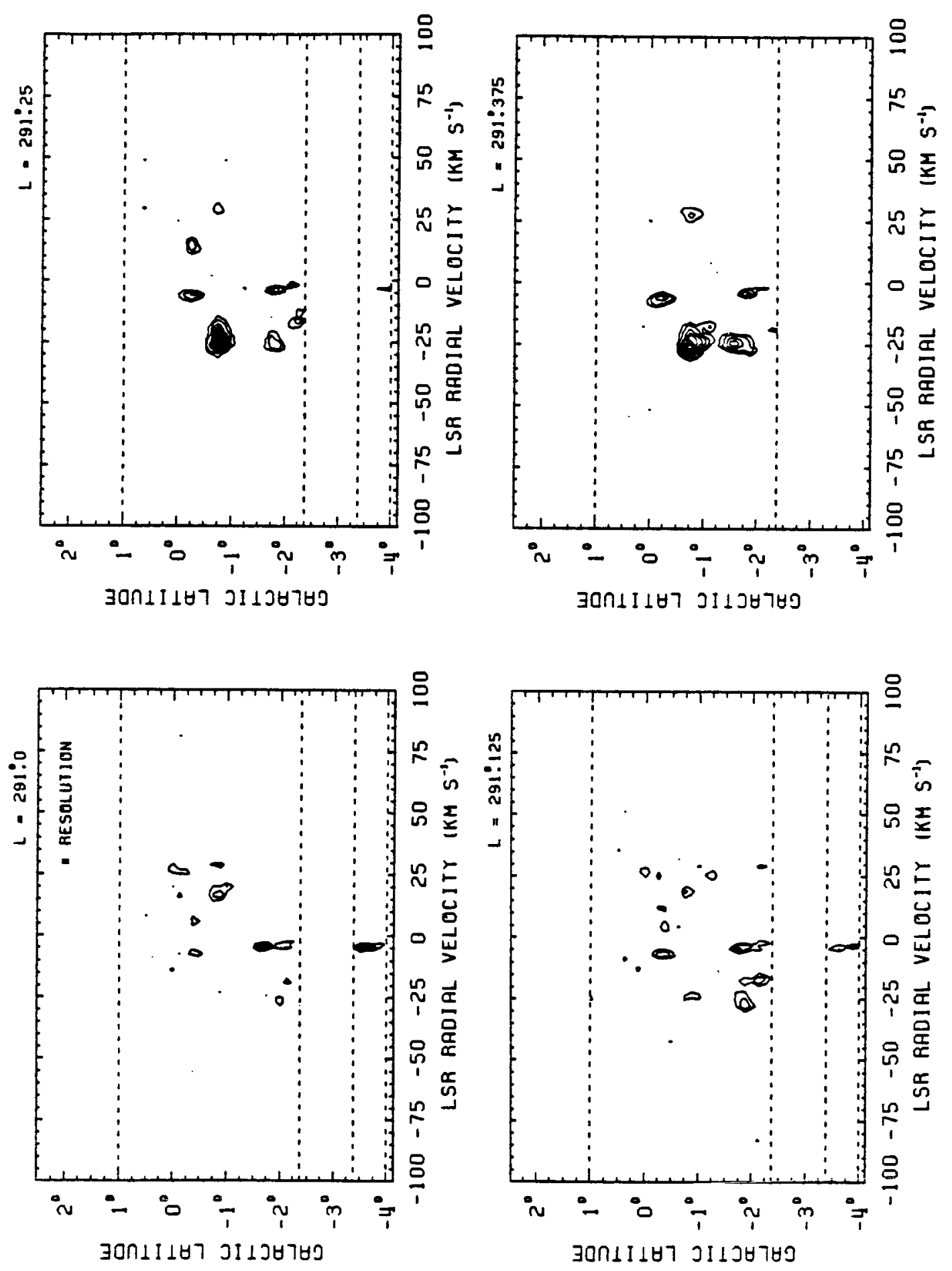

FIGURE B-2 (continued) 

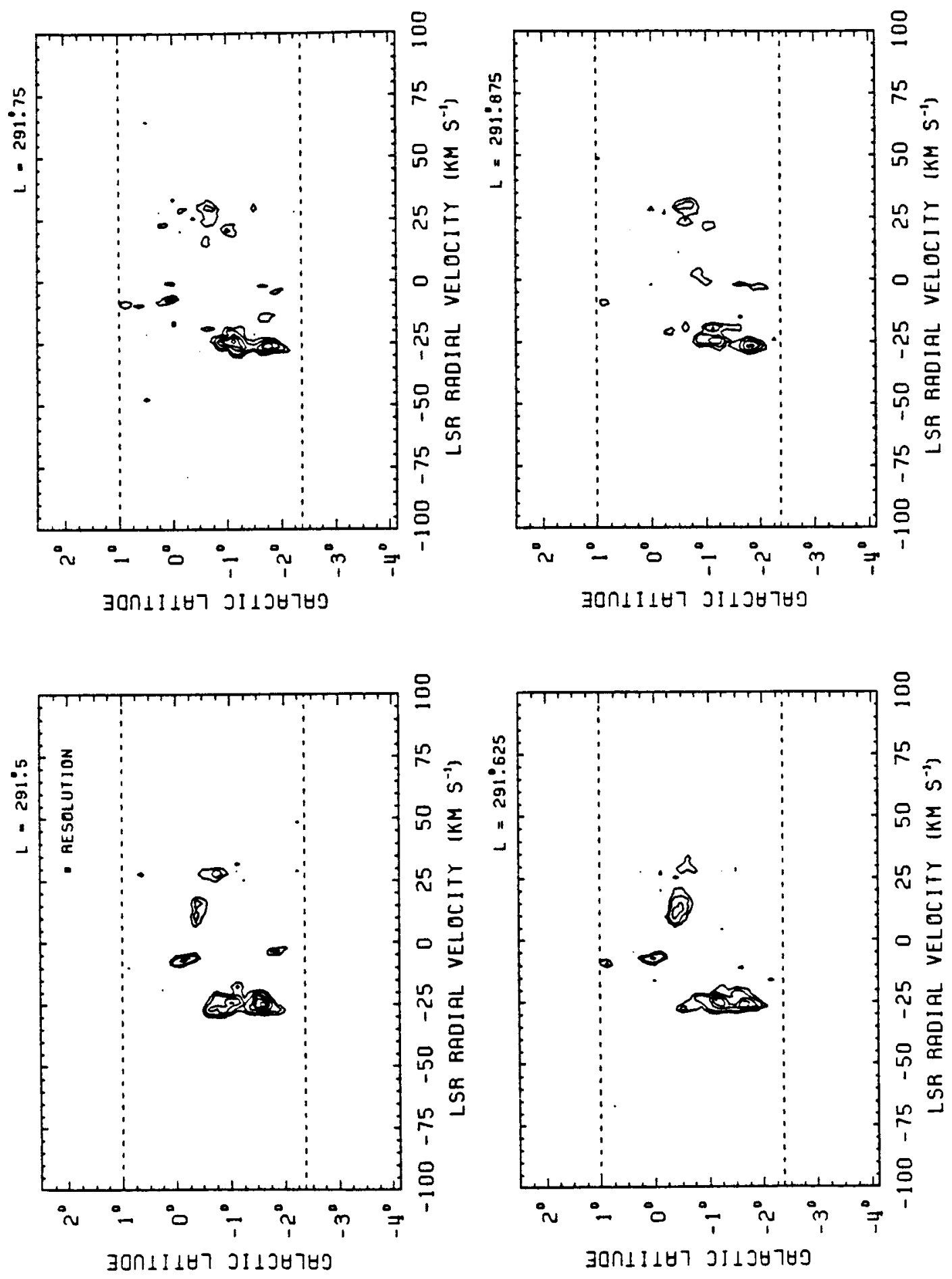

FIGURE B-2 (continued) 

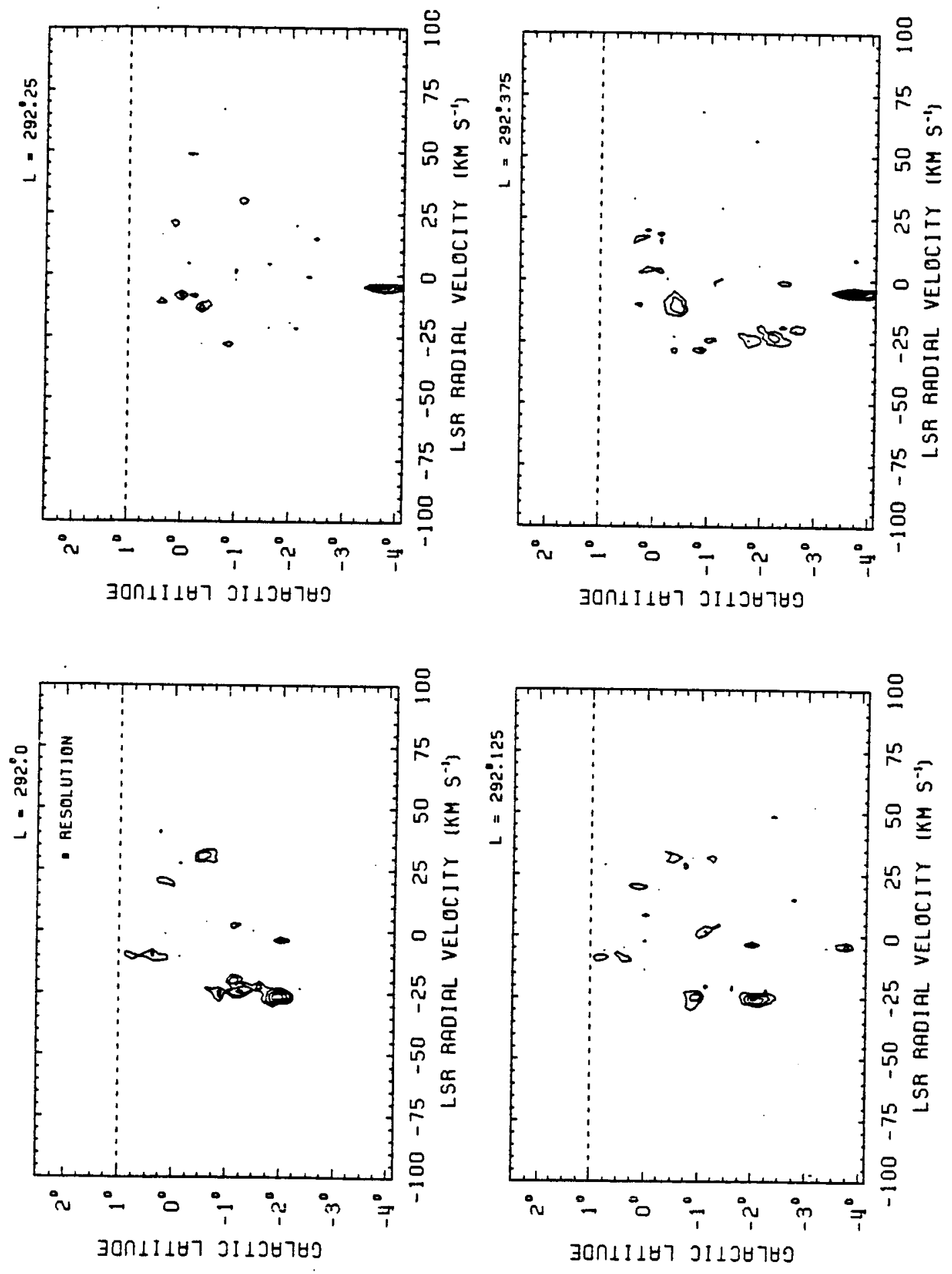

FIGURE B-2 (continued) 

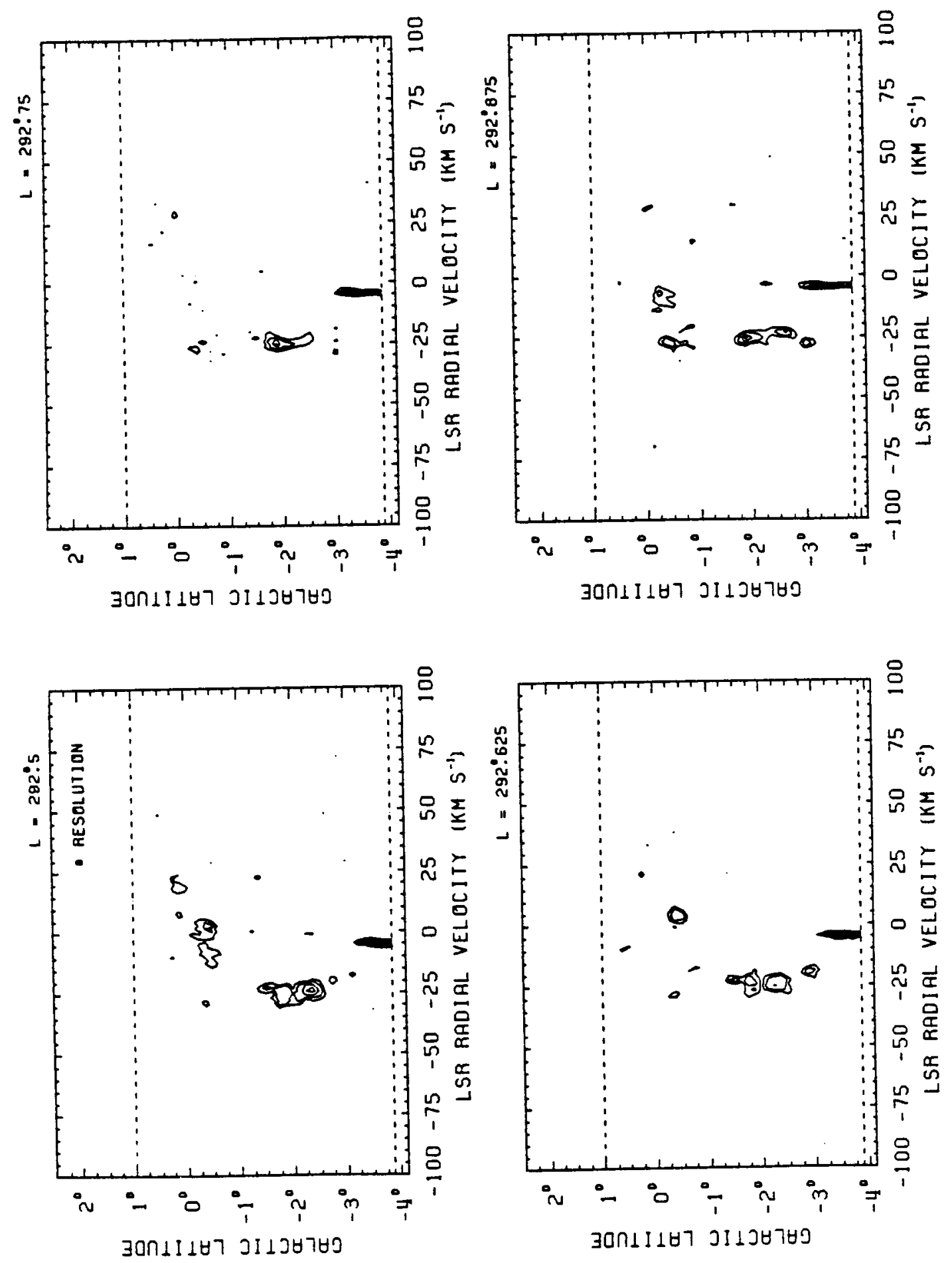

FIGURE B-2 (continued) 

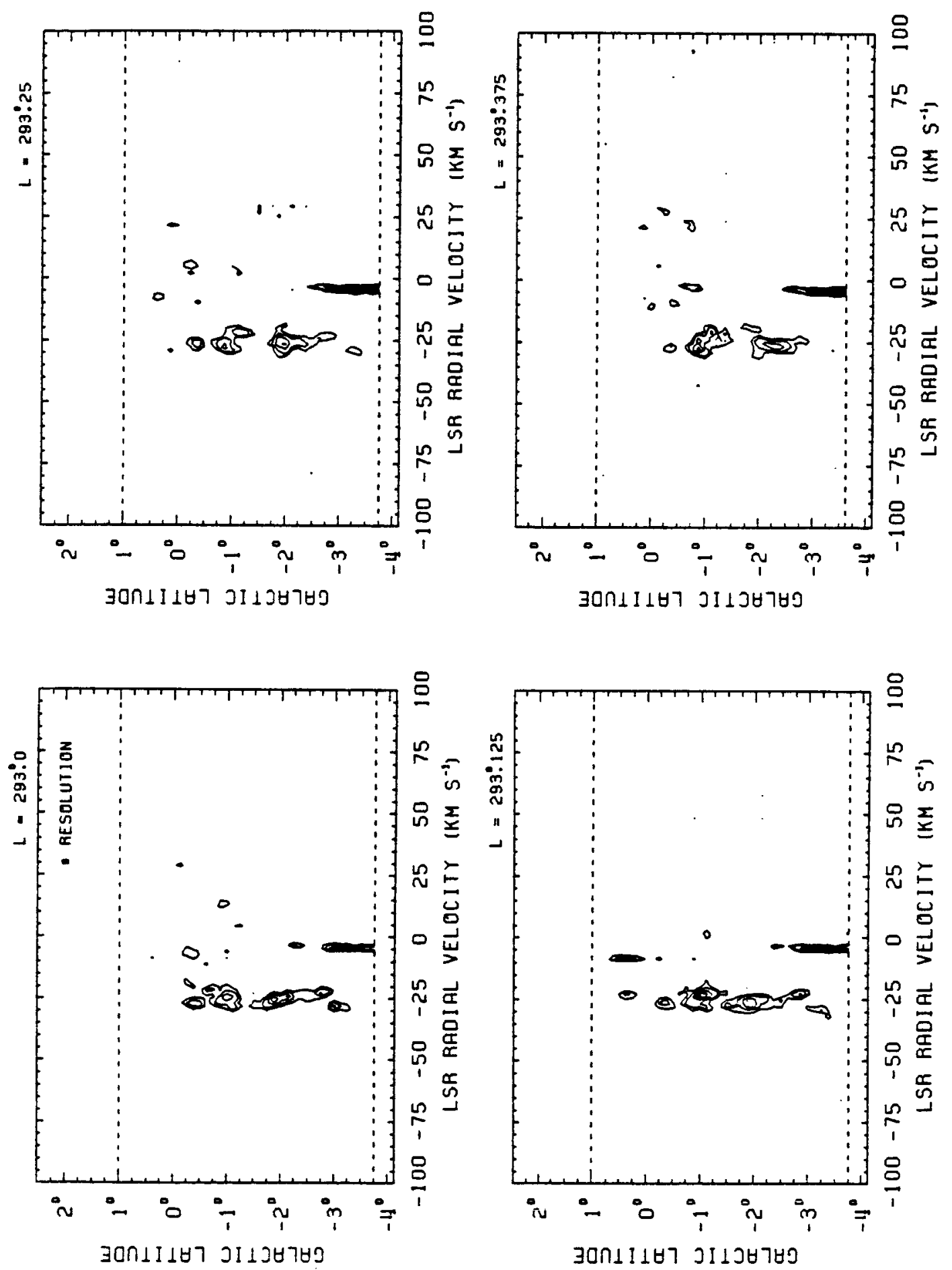

FIGURE B-2 (continued) 

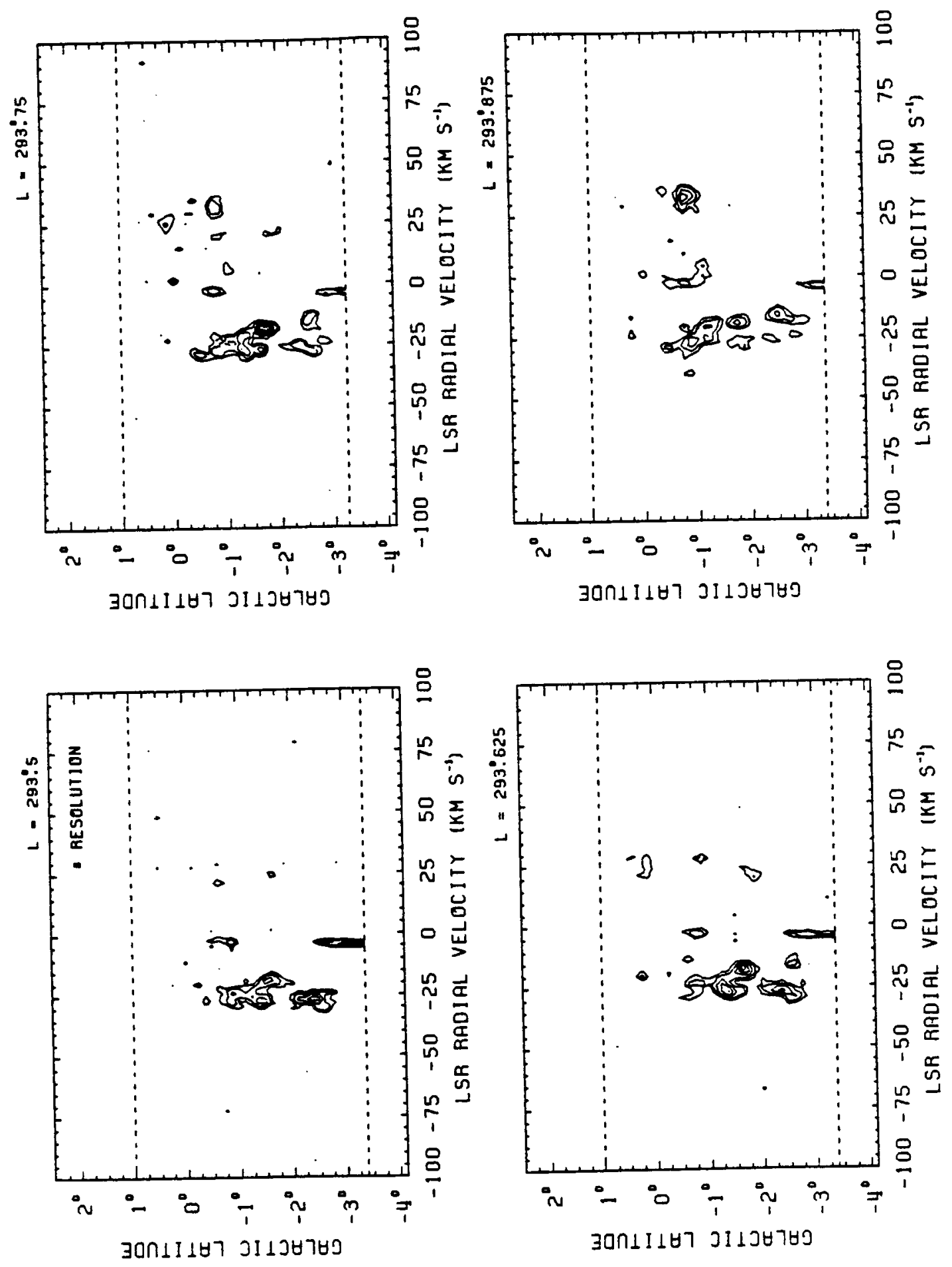

FIGURE B-2 (continued) 

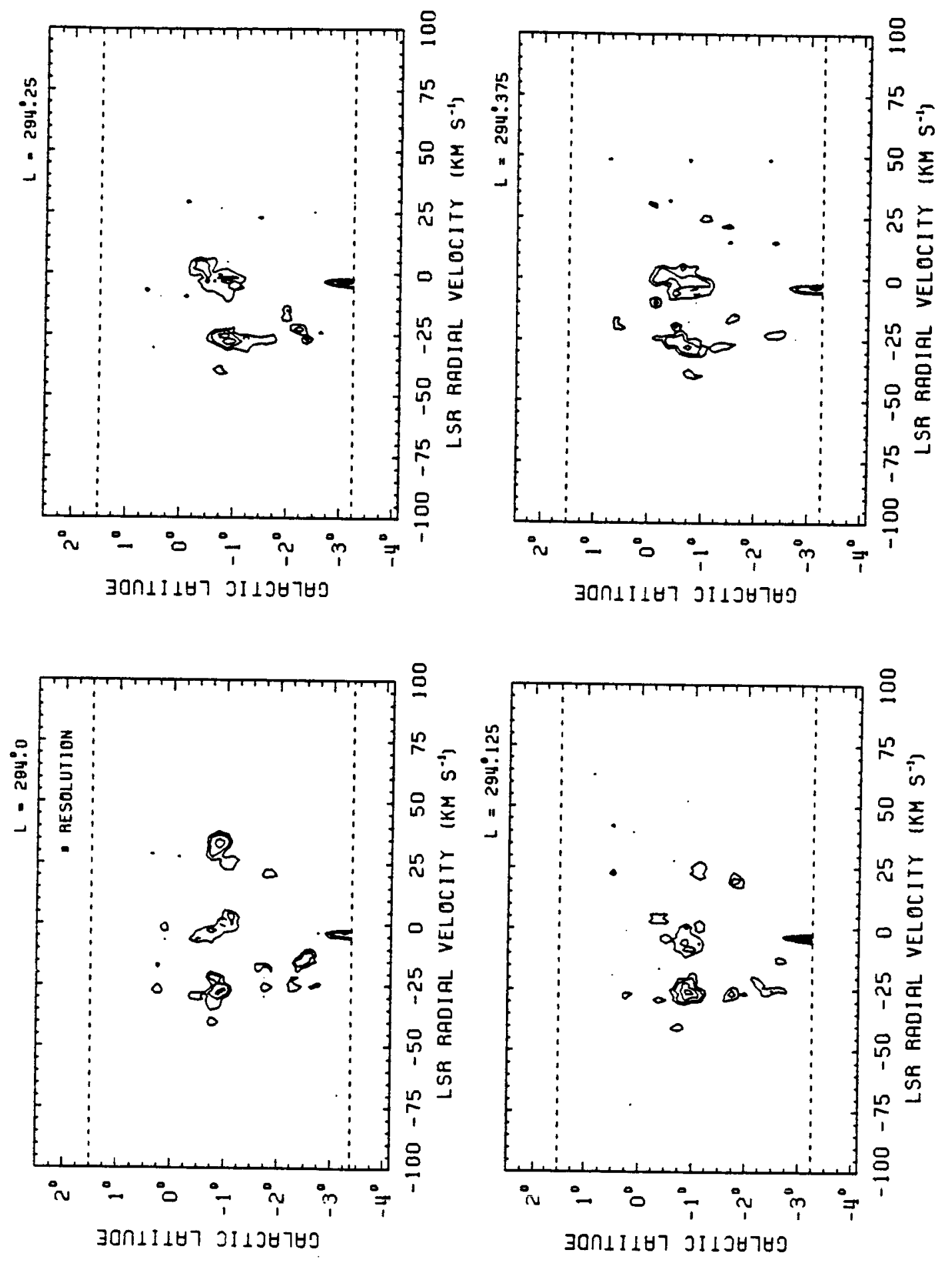

FIGURE B-2 (continued) 

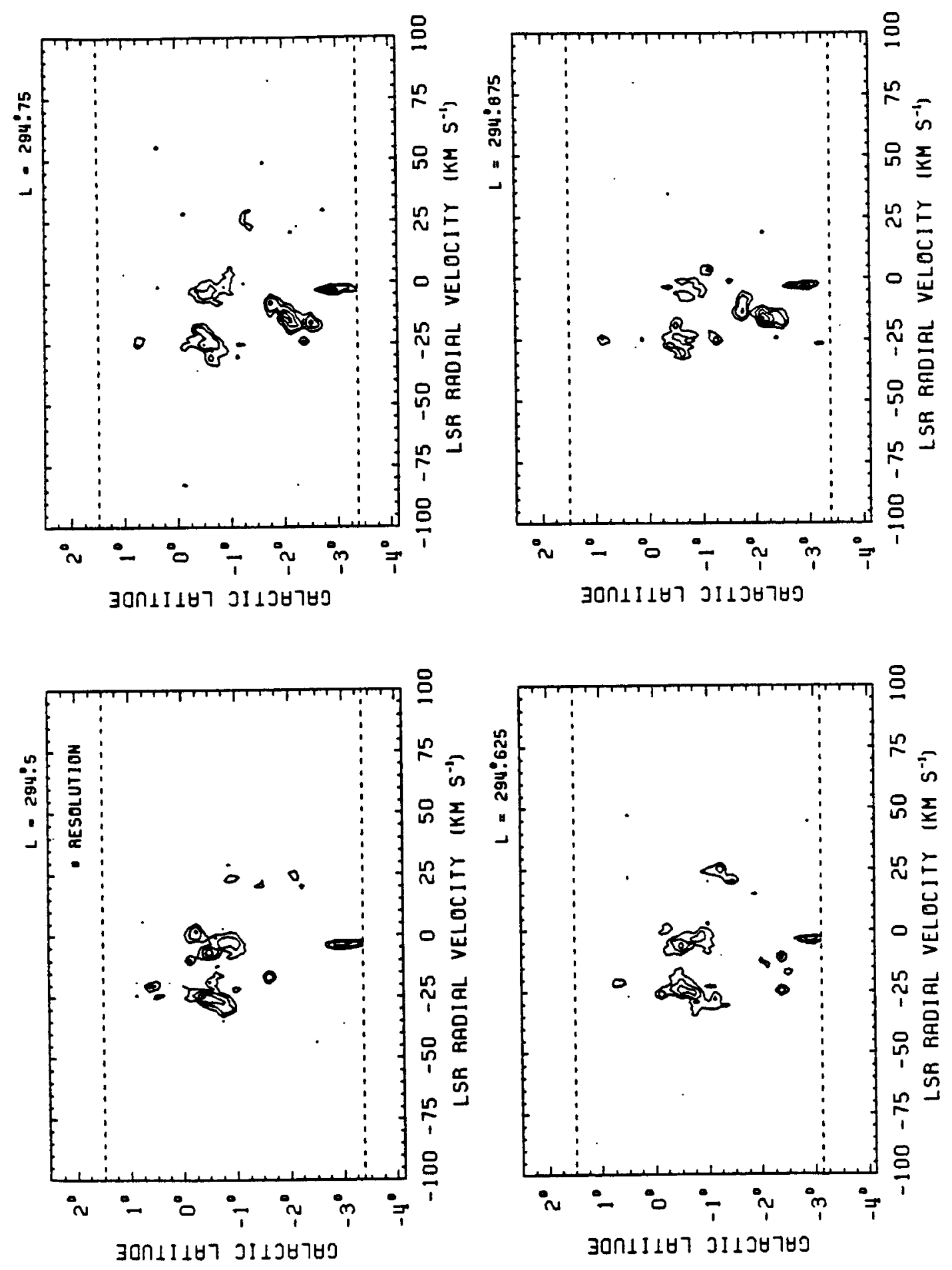

FIGURE B-2 (continued) 

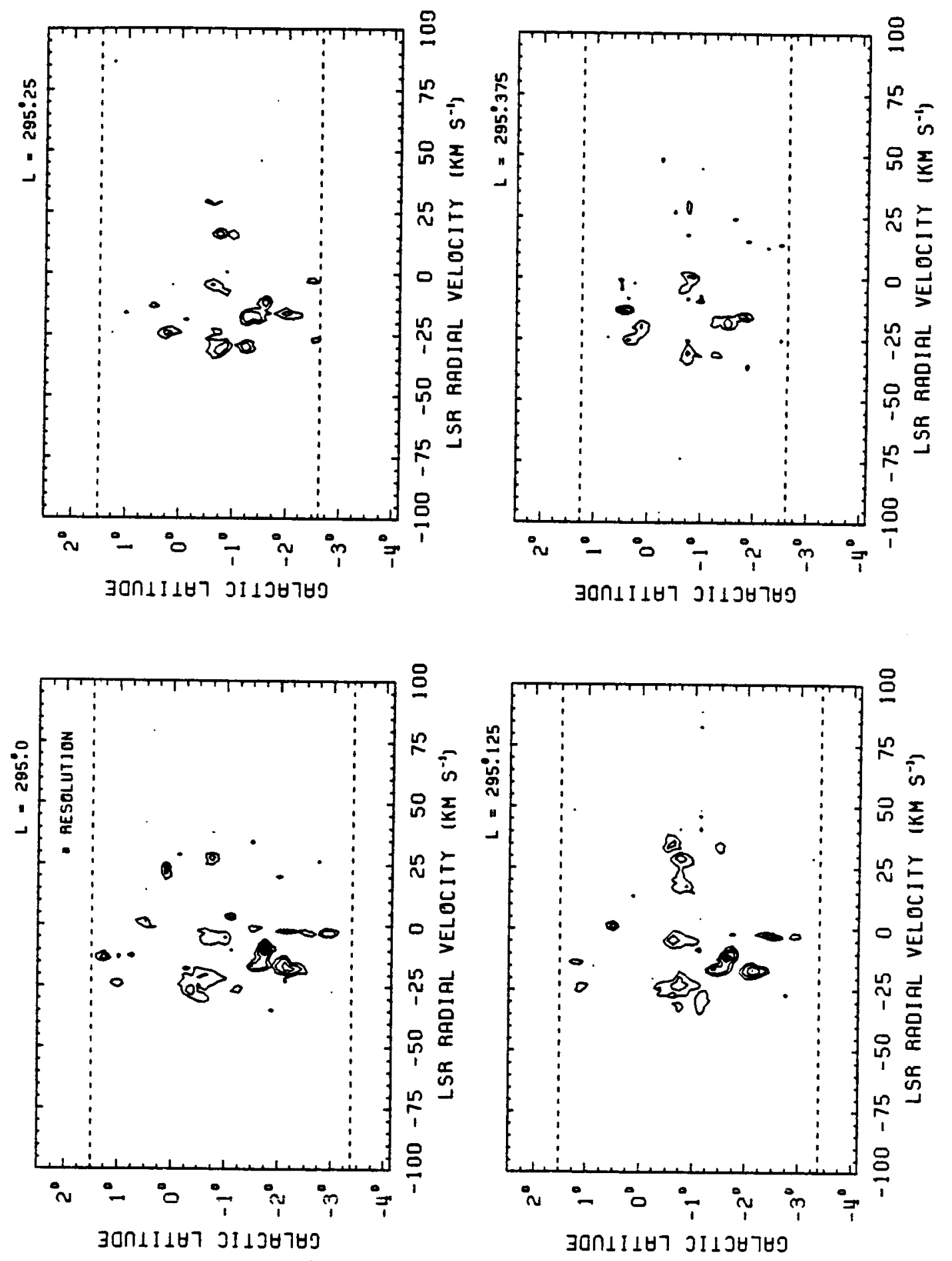

FIGURE B-2 (continued) 

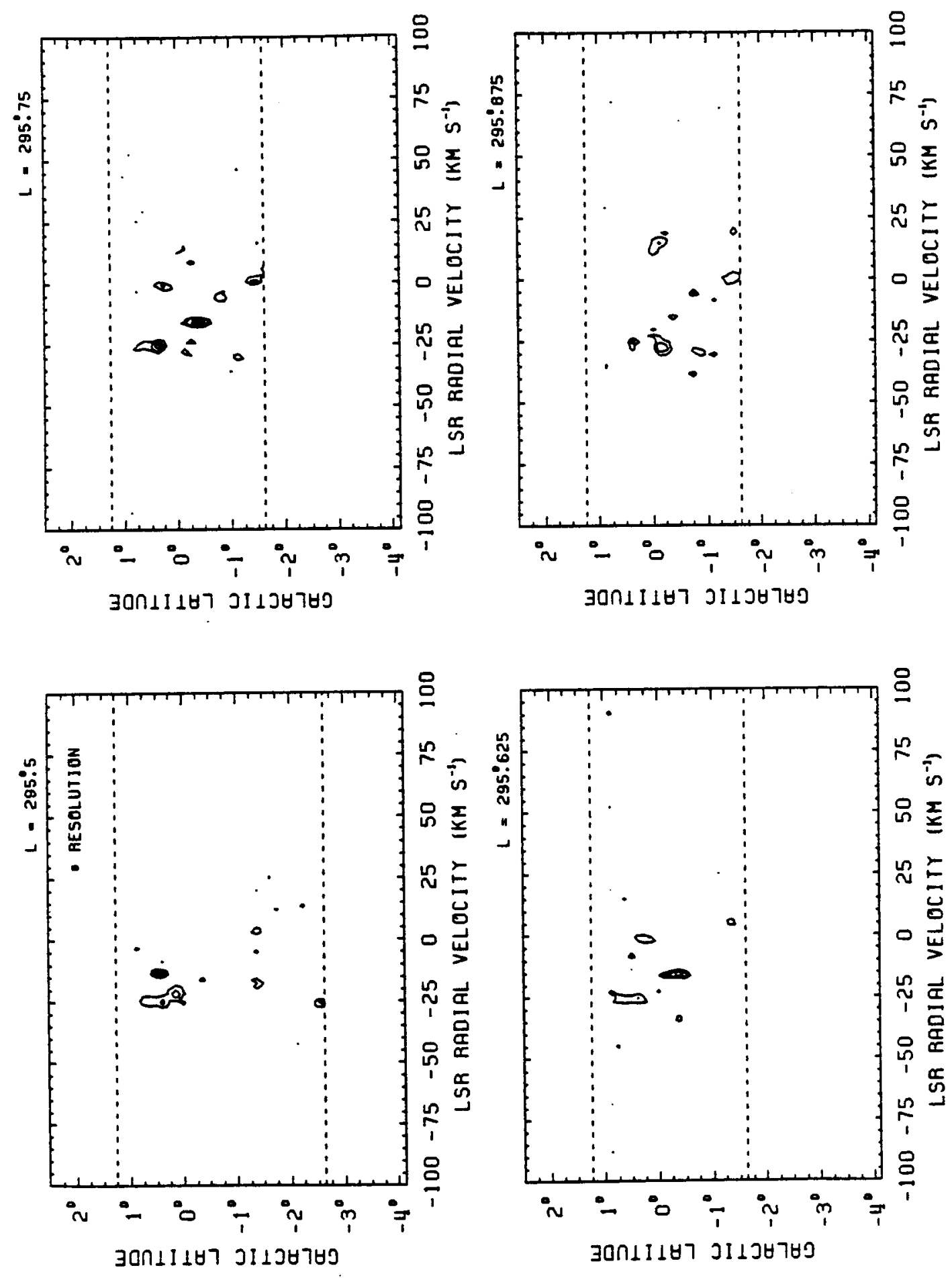

FIGURE B-2 (continued) 

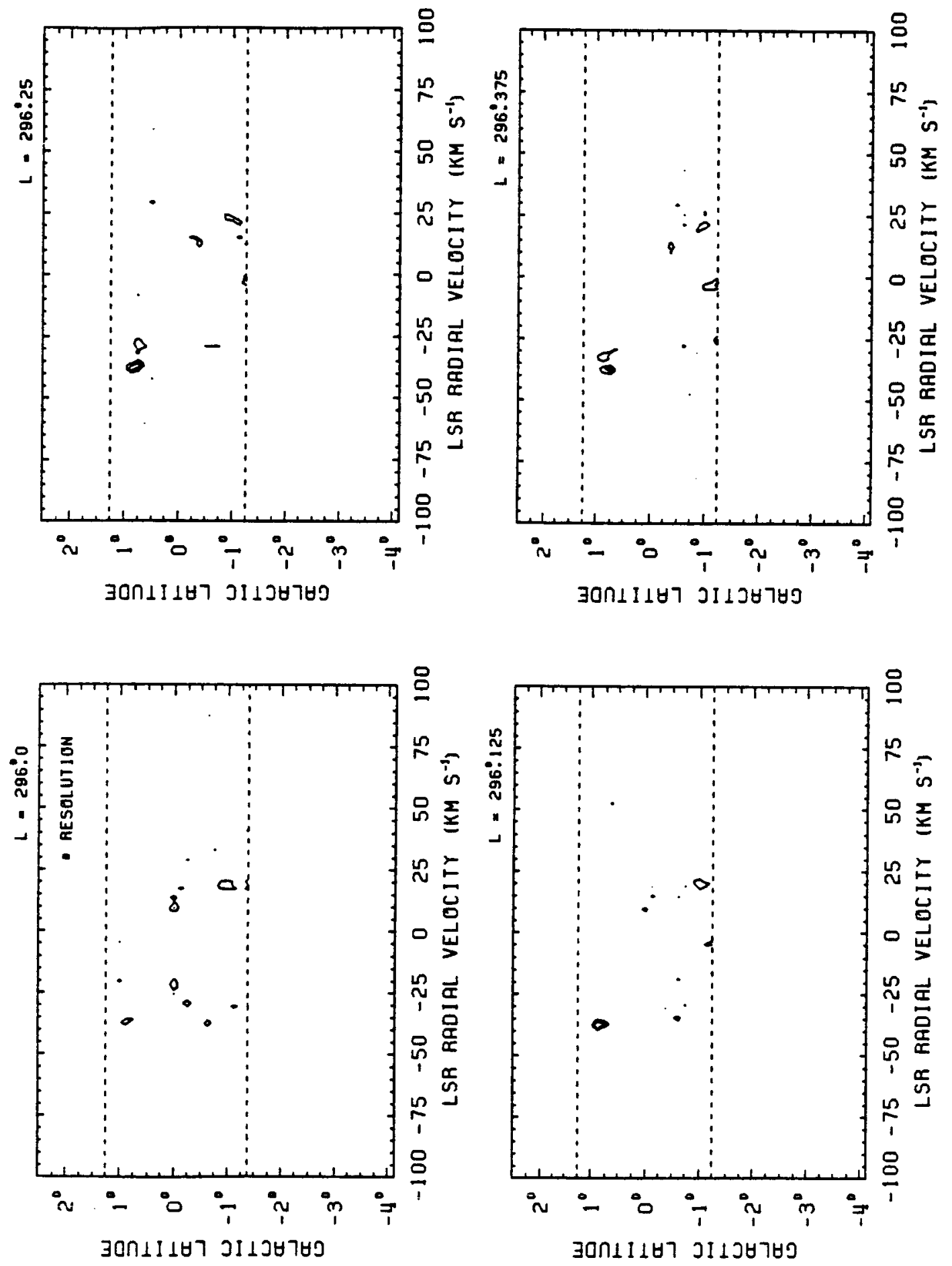

FIGURE B-2 (continued) 

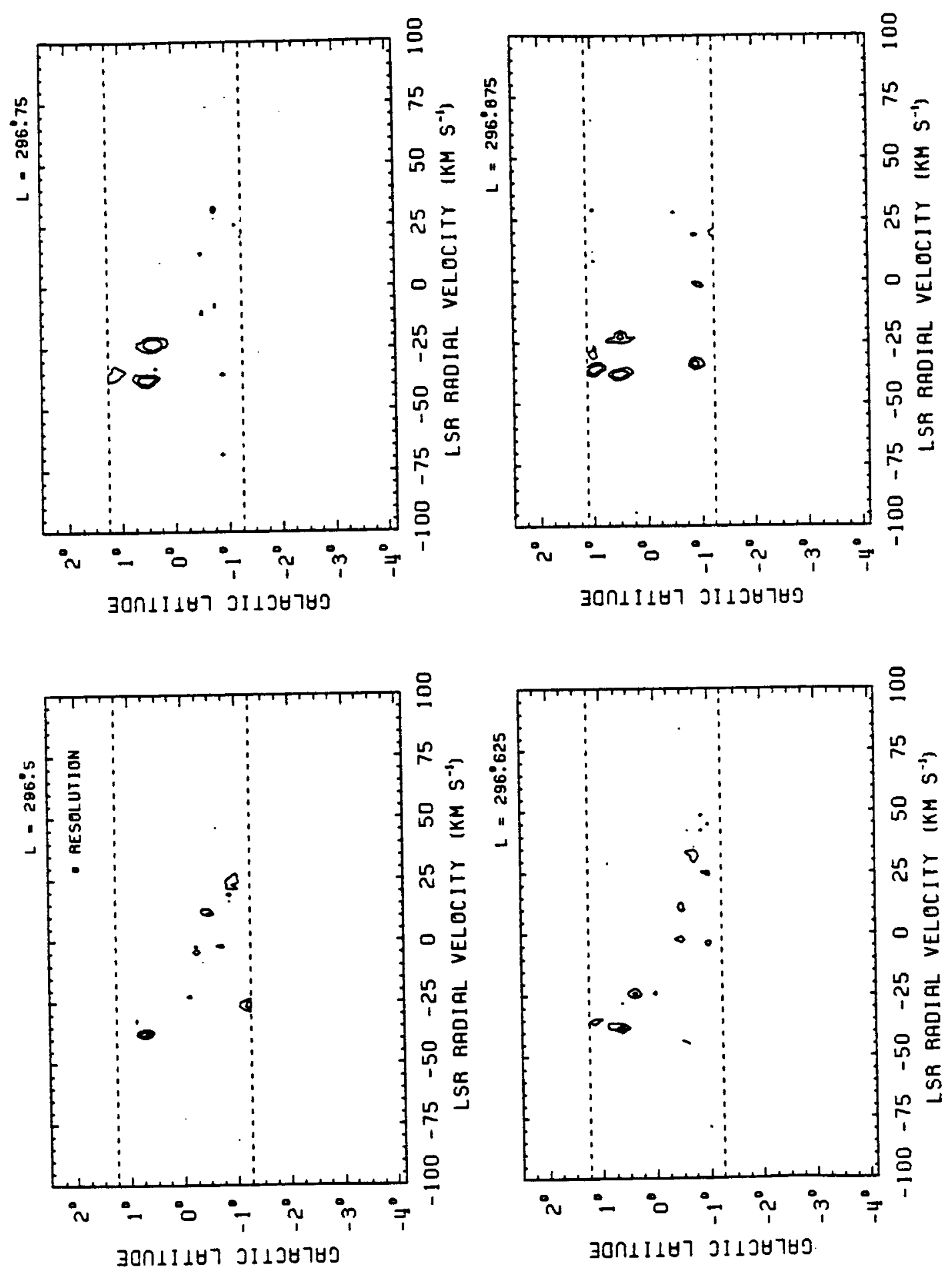

FIGURE B-2 (continued) 

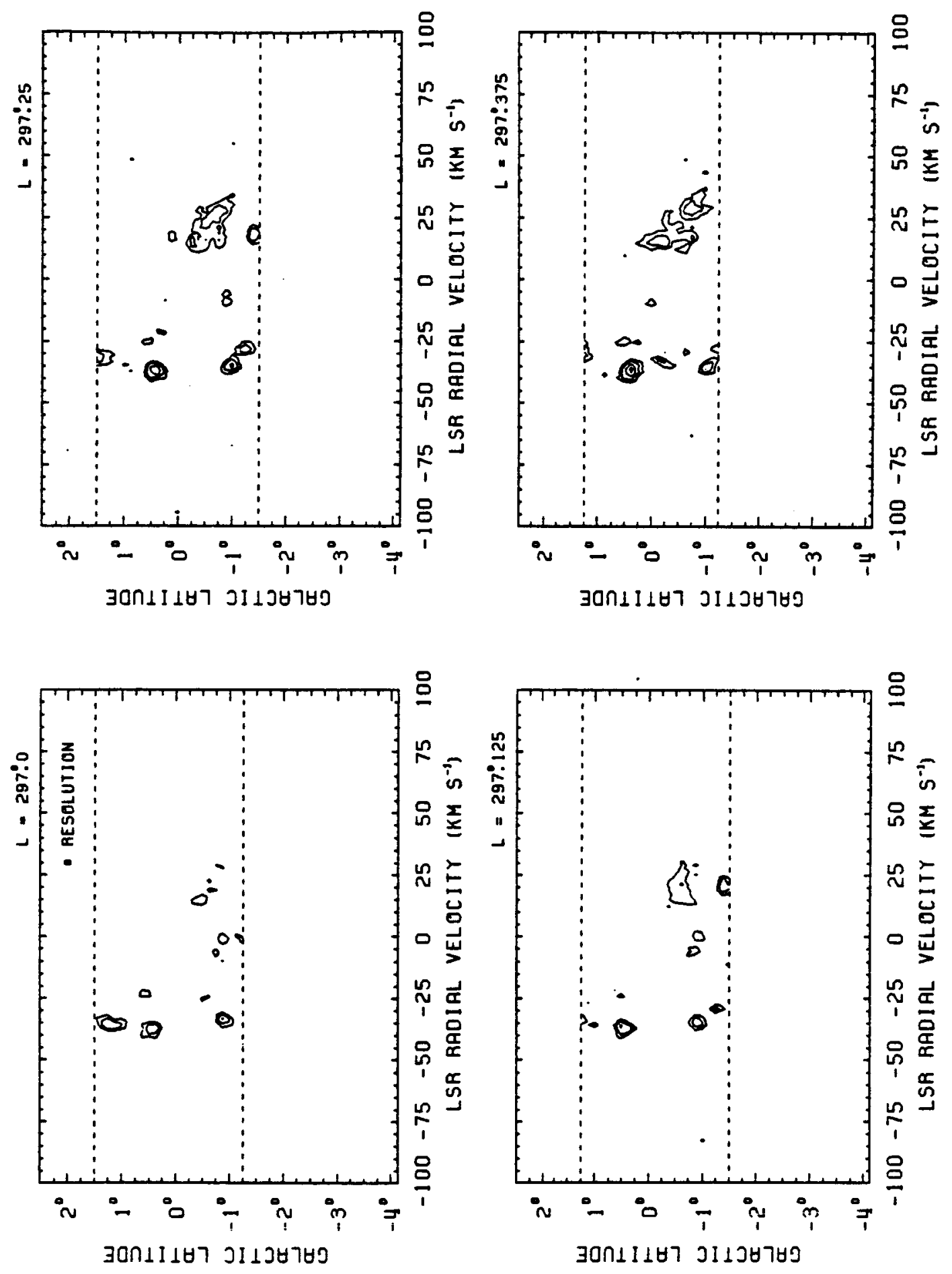

FIGURE B-2 (continued) 

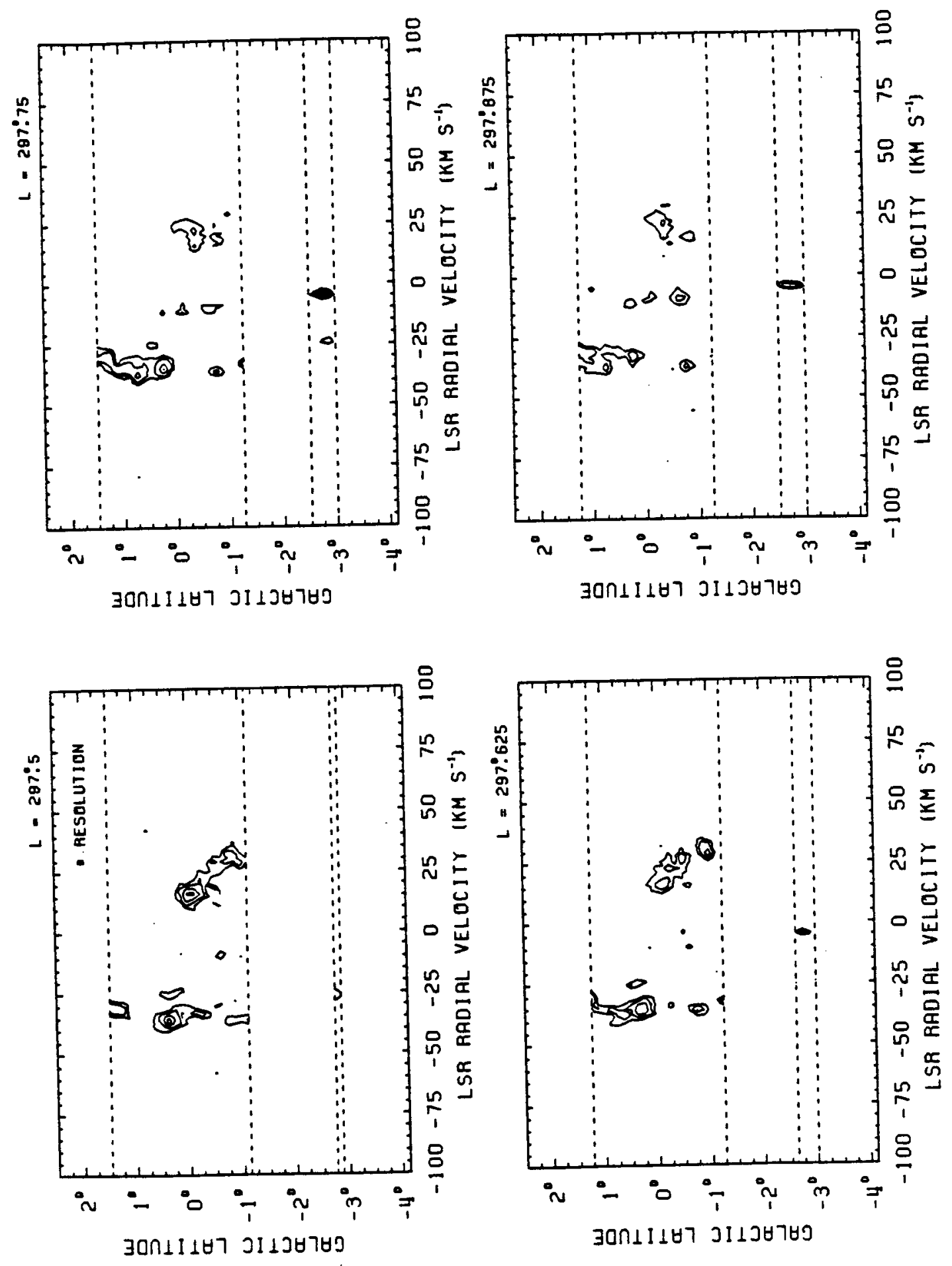

FIGURE B-2 (continued) 

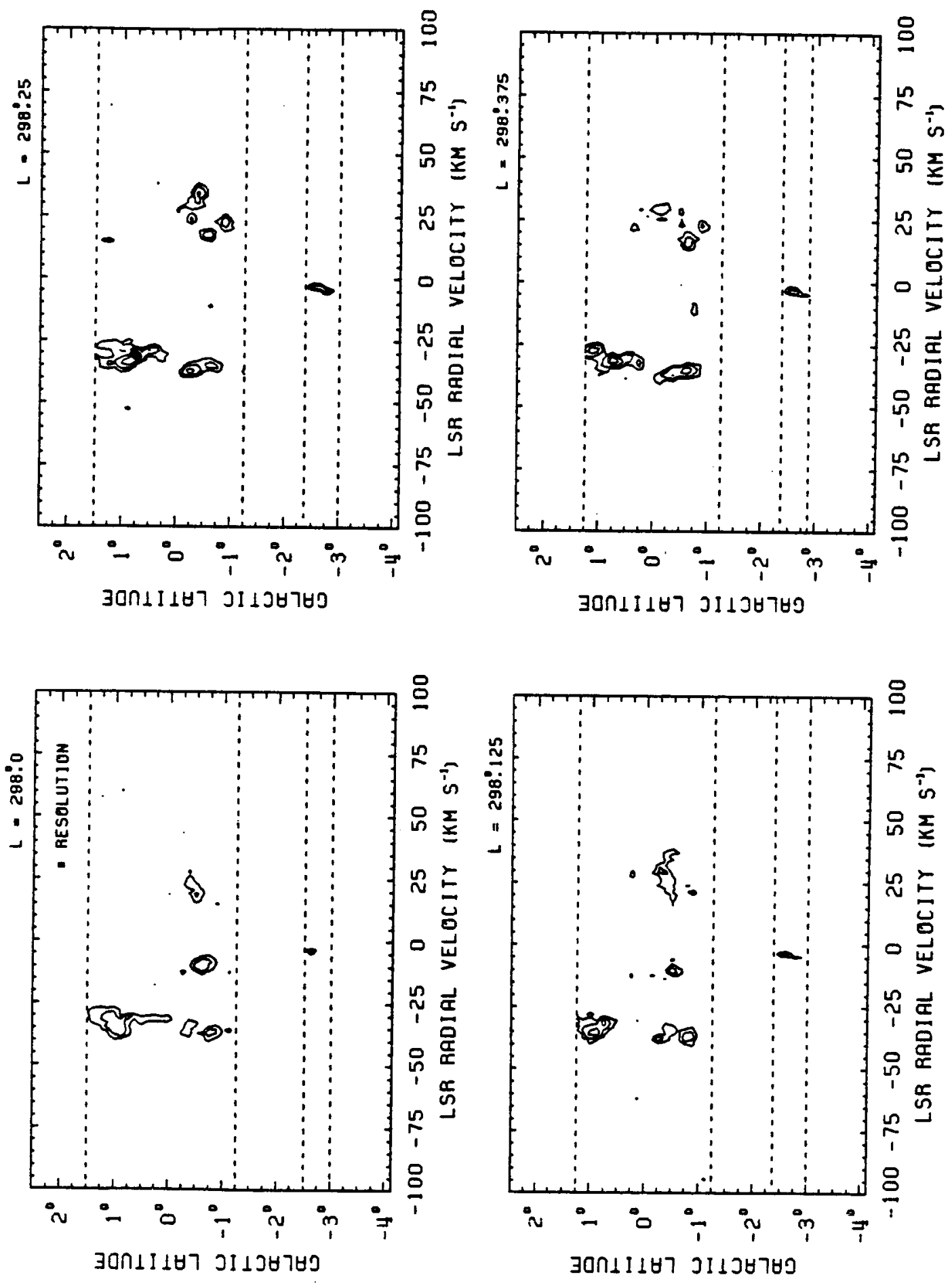

FIGURE B-2 (continued) 

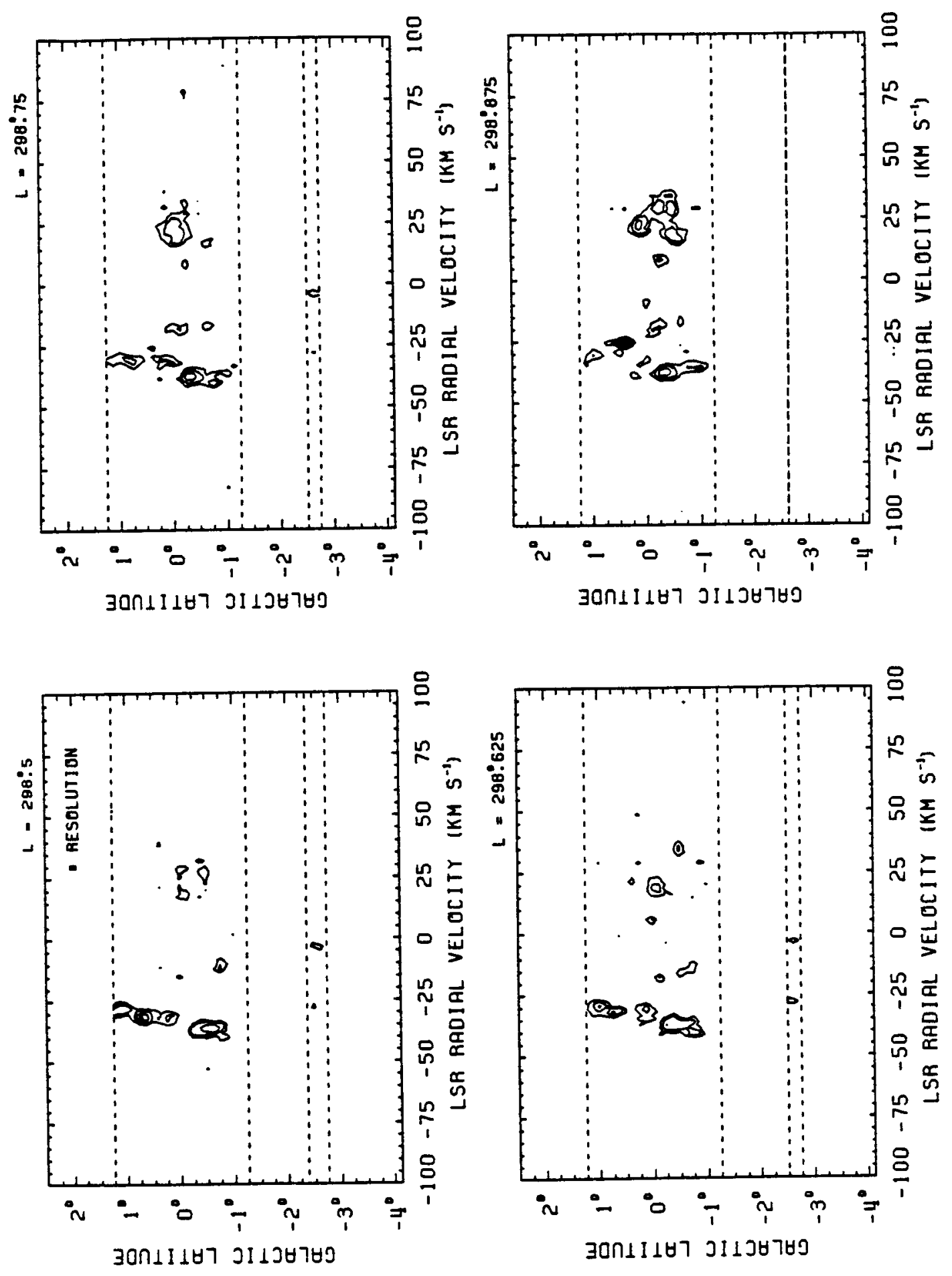

FIGURE B-2 (continued) 

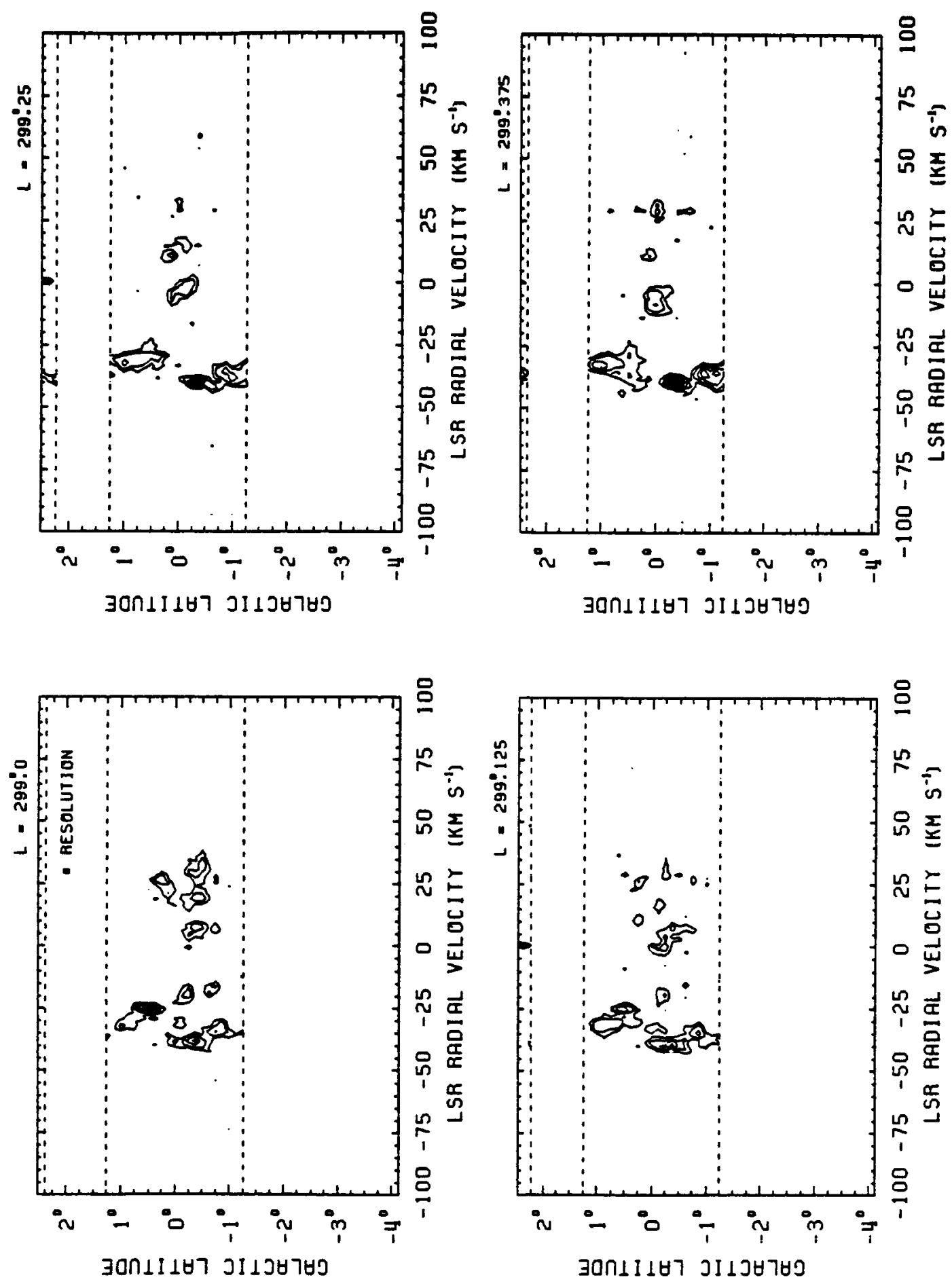

FIGURE B-2 (continued) 

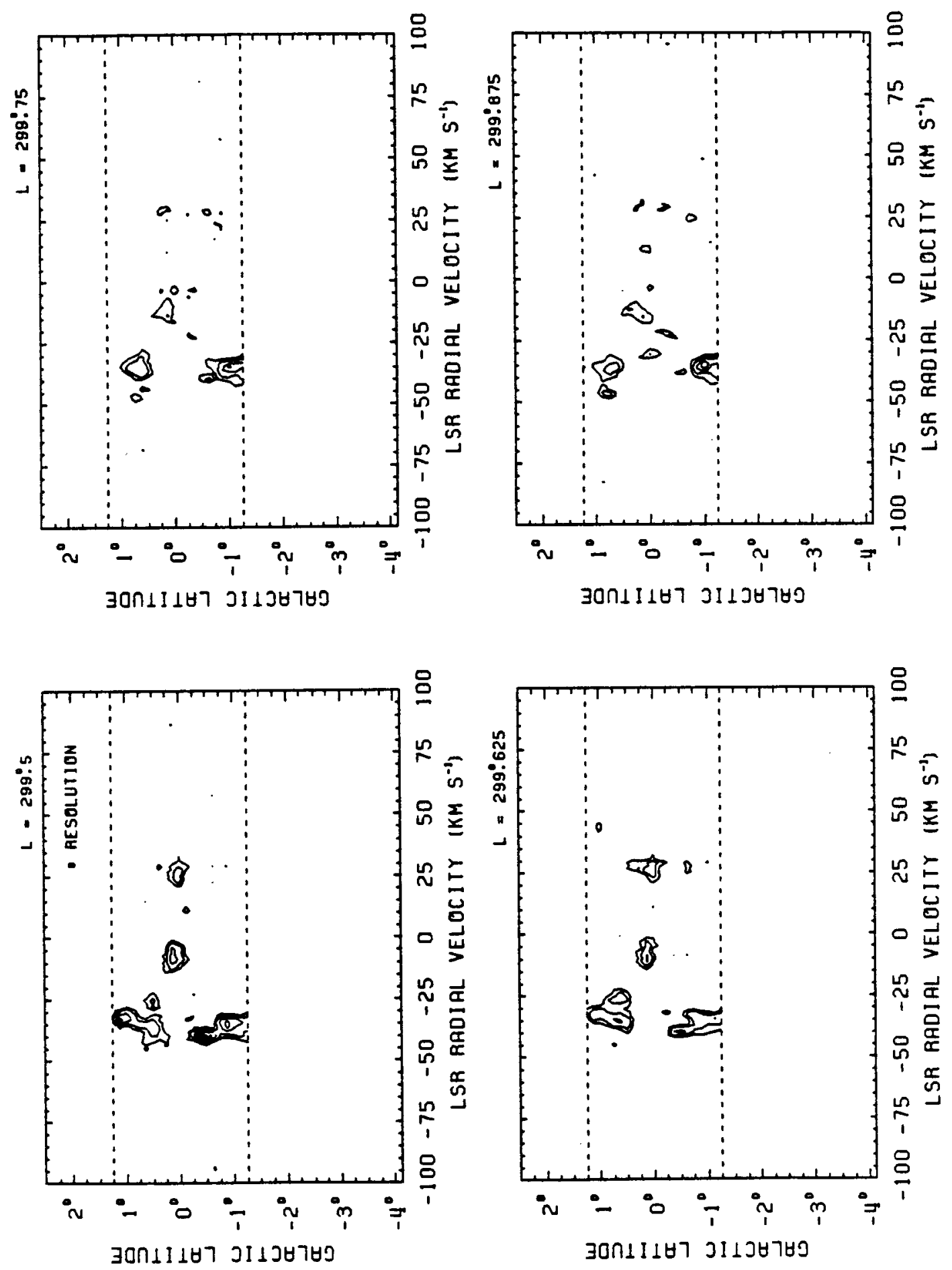

FIGURE B-2 (continued) 


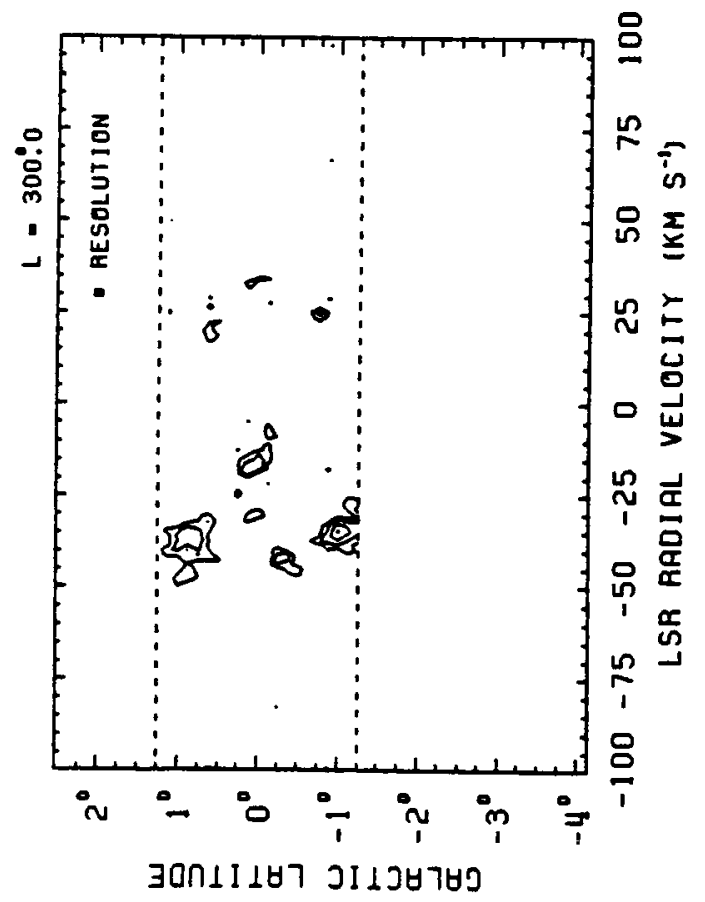

FIGURE B-2 (continued) 


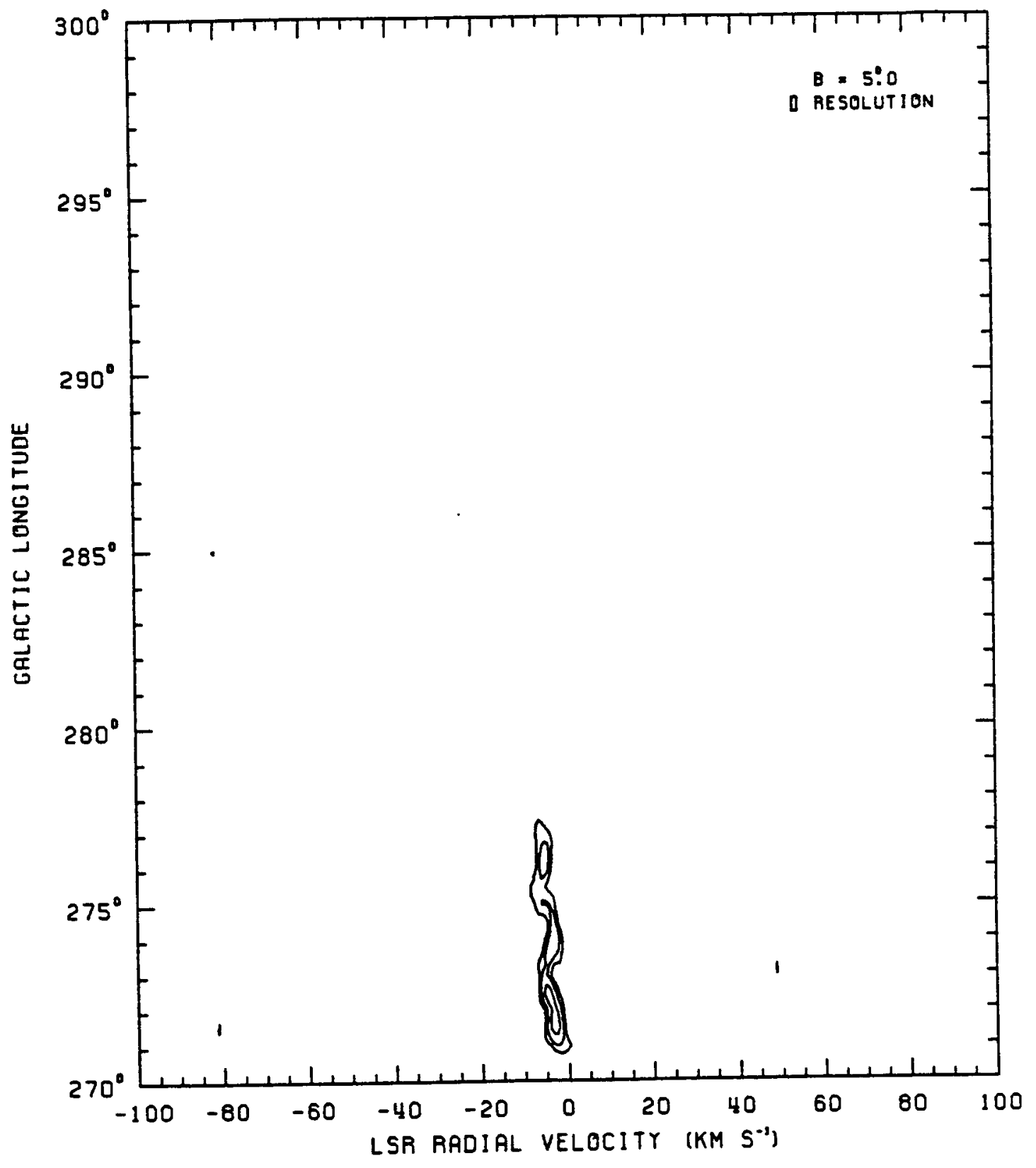

FIGURE B-3 


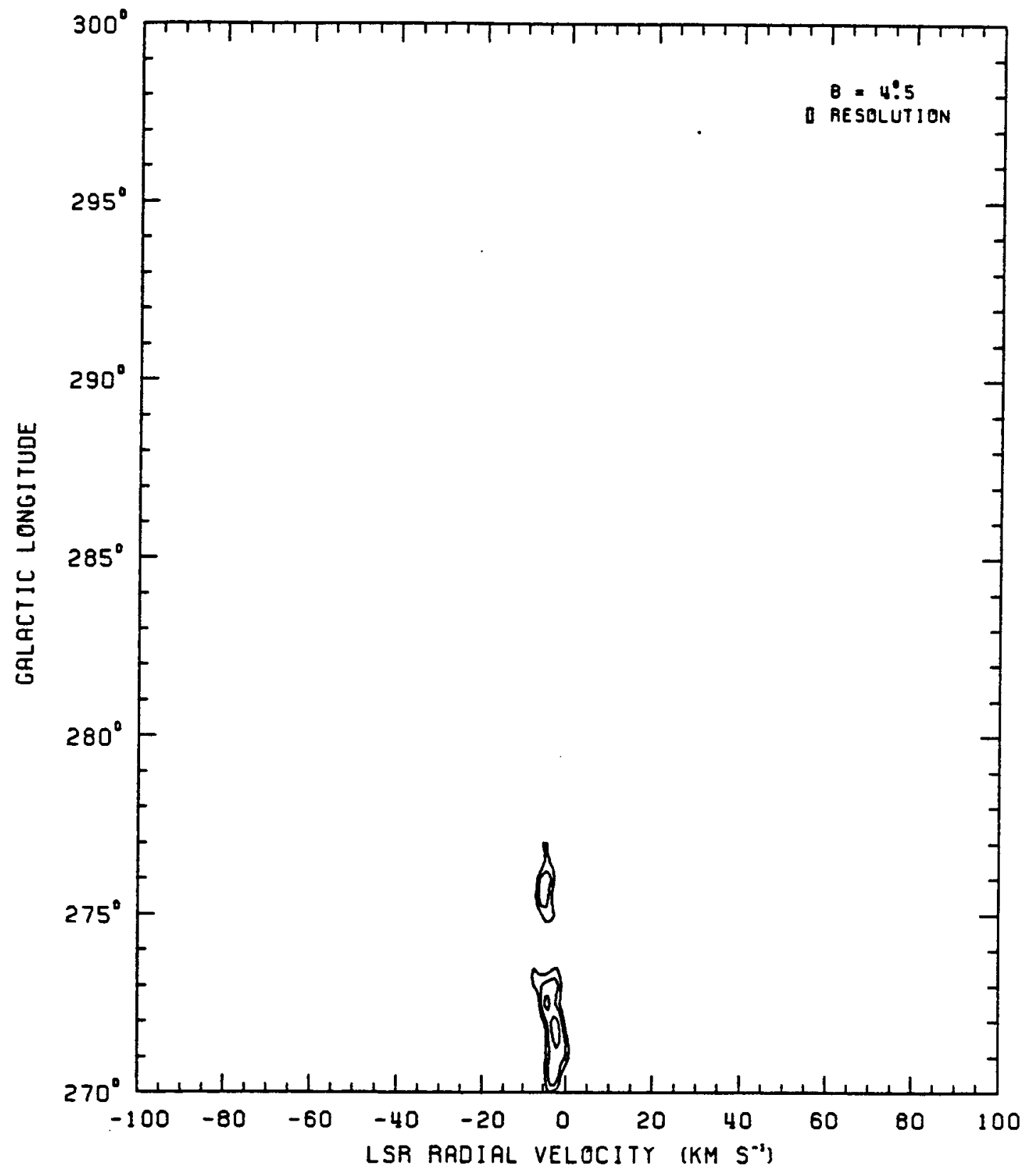

FIGURE B-3 (continued) 


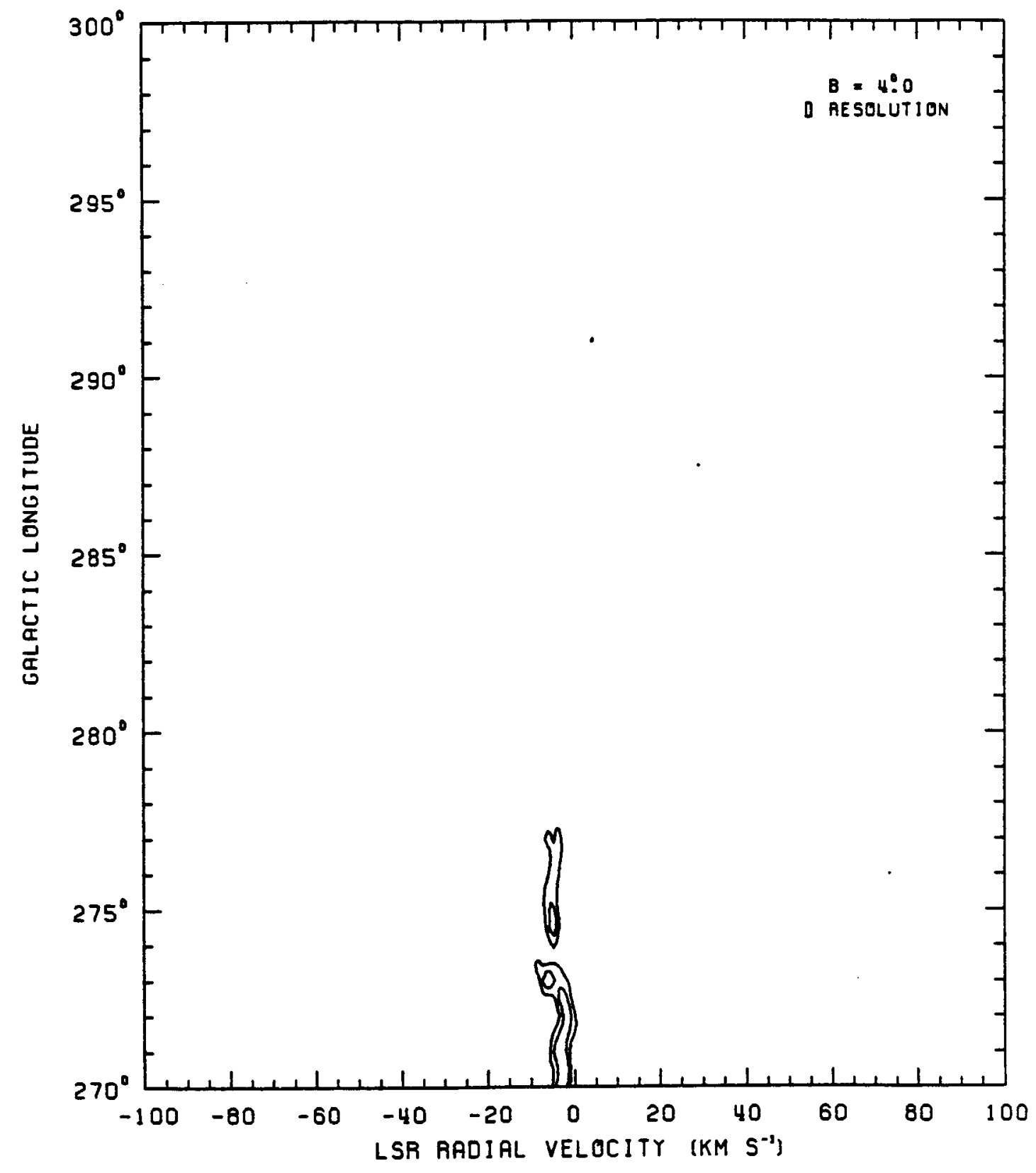

FIGURE B-3 (continued) 


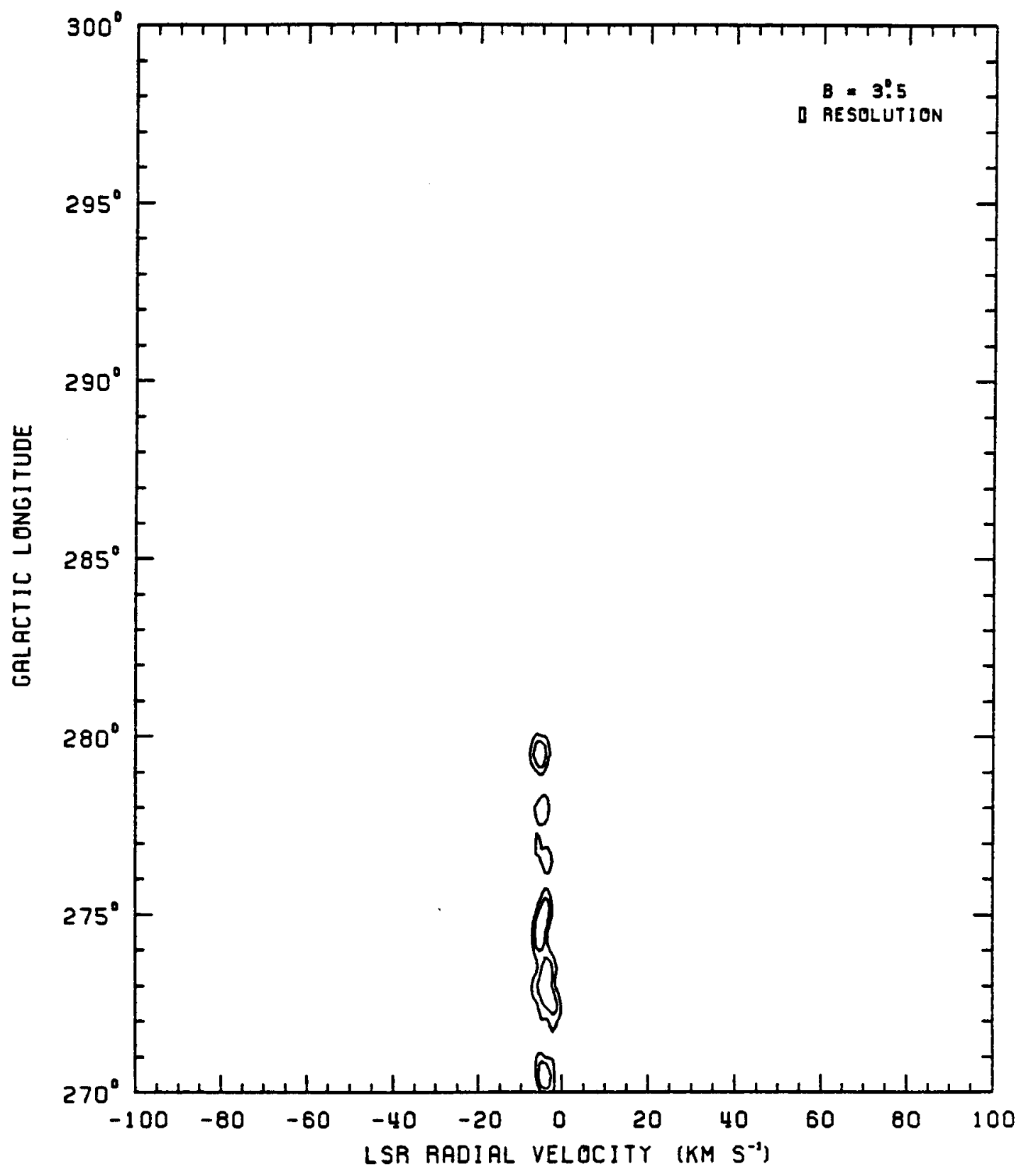

FIGURE B-3 (continued) 


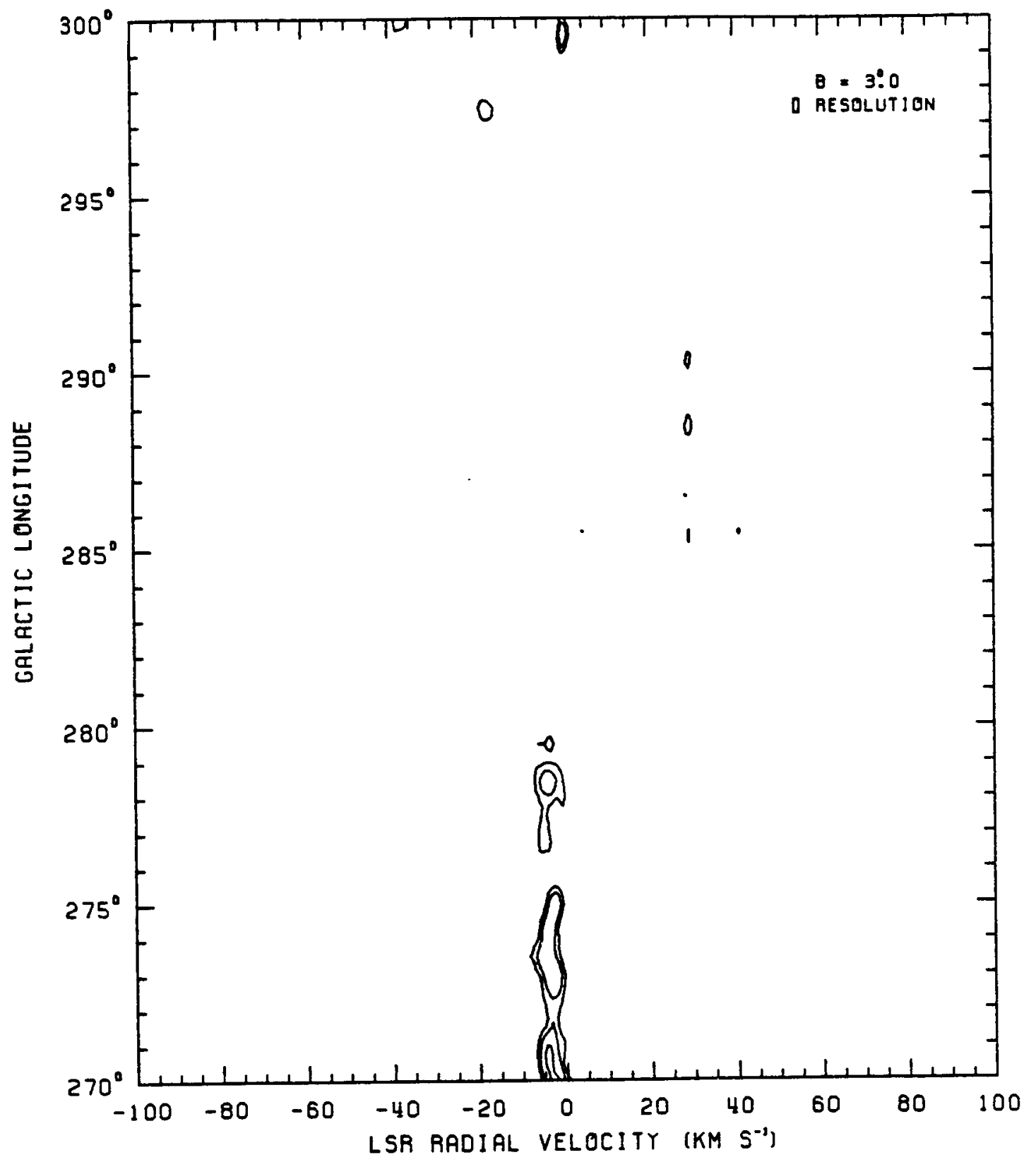

FIGURE B-3 (continued) 


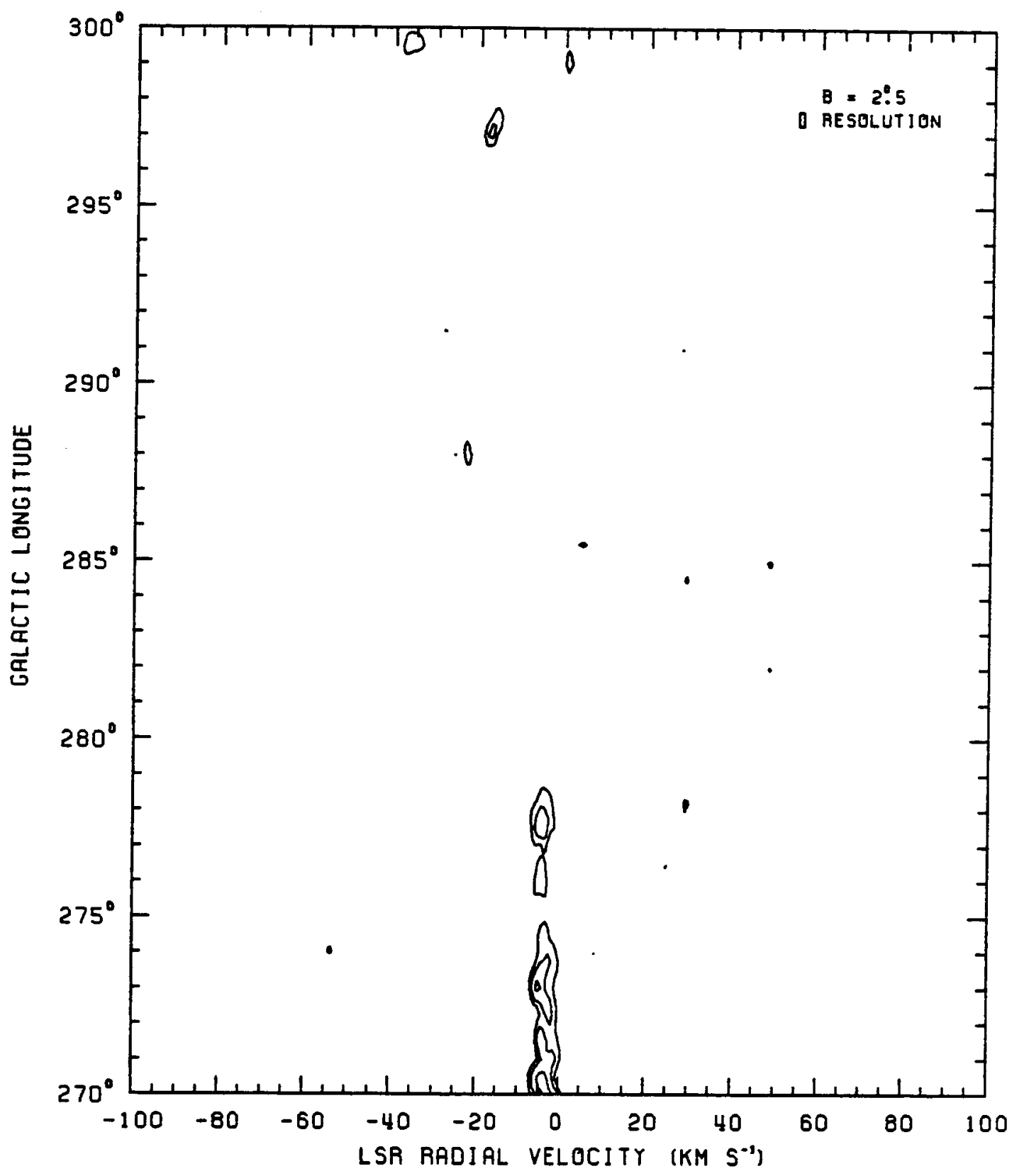

FIGURE B-3 (cont inued) 


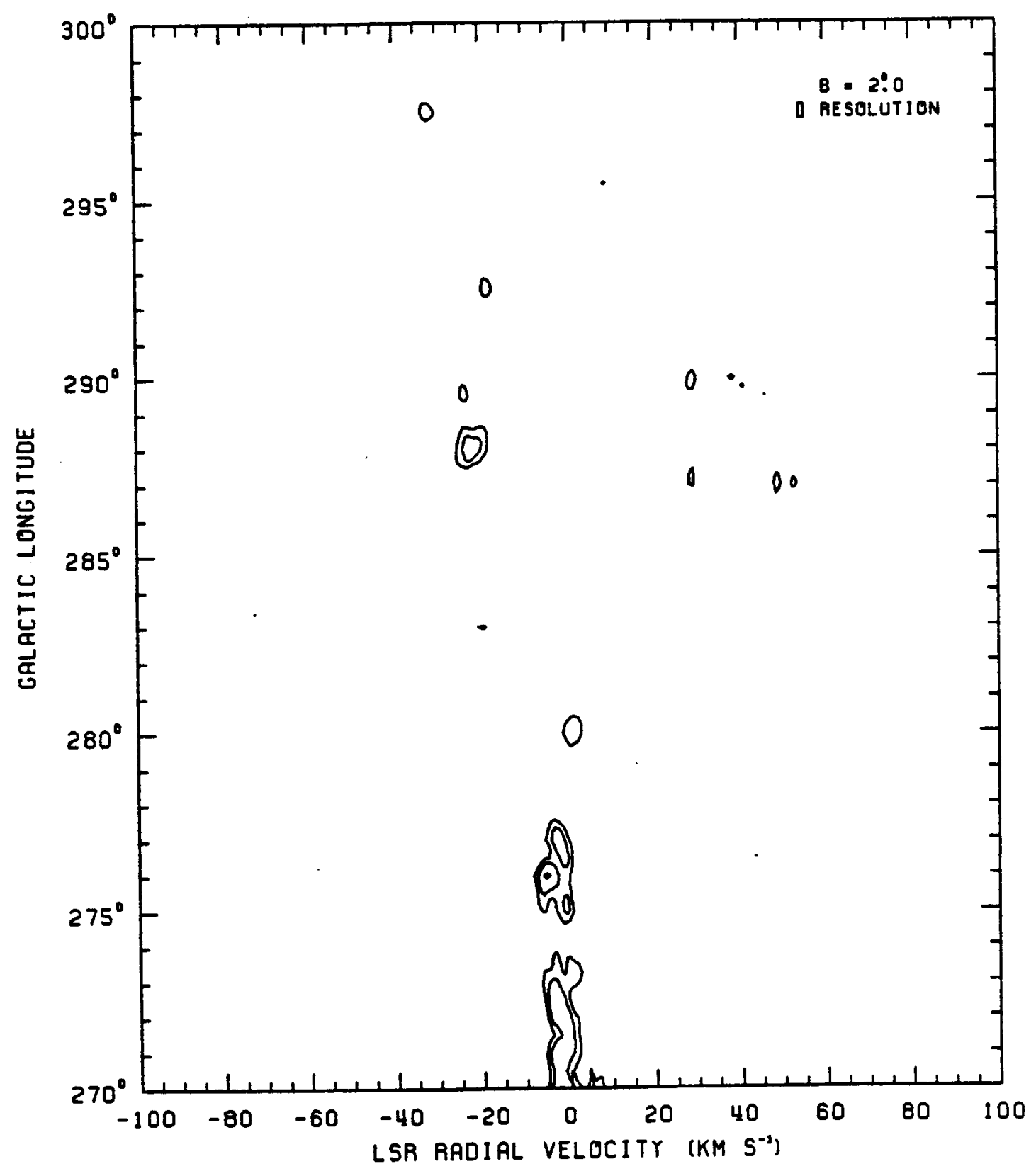

FIGURE B-3 (continued) 


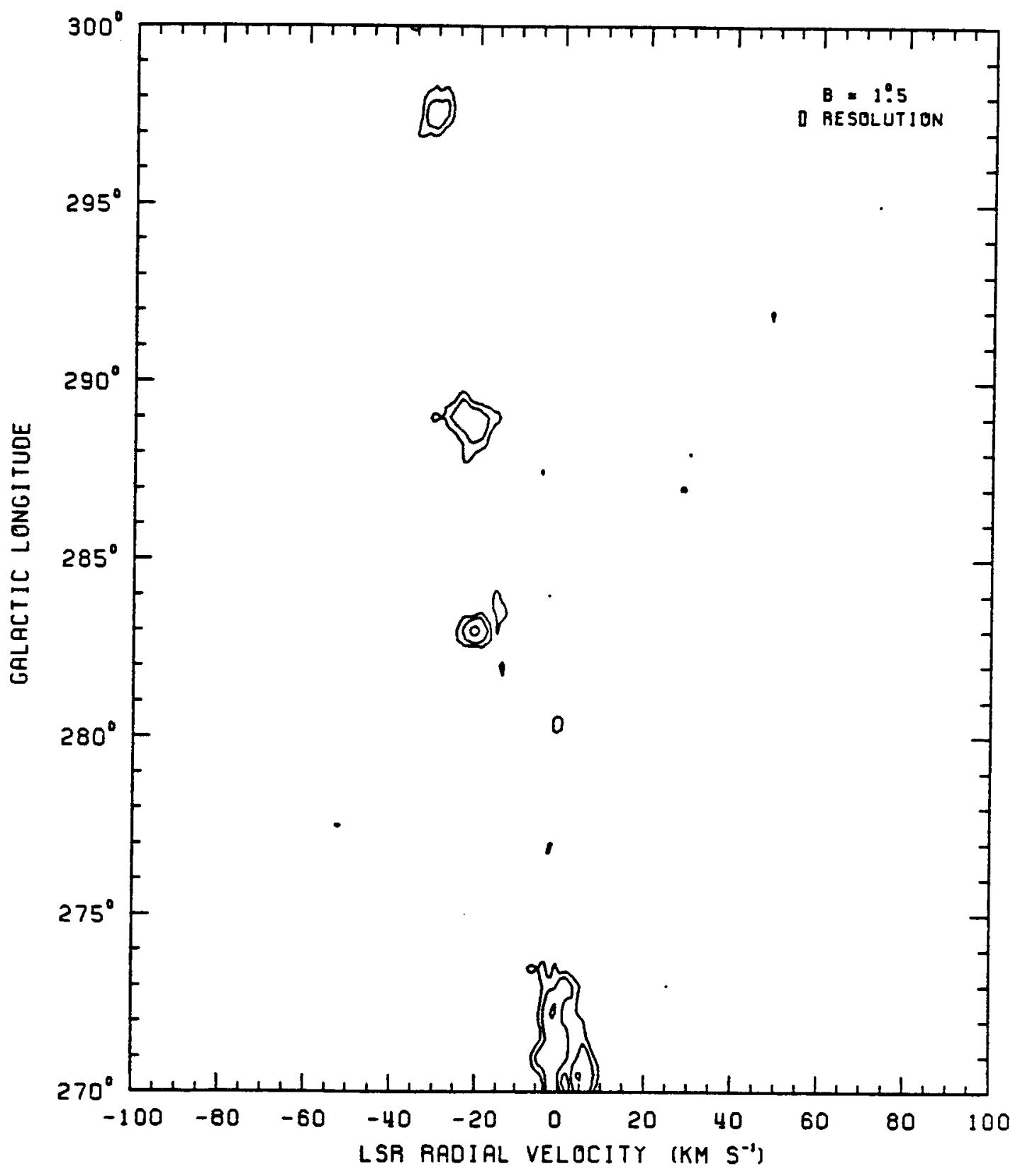

FIGURE B-3 (continued) 


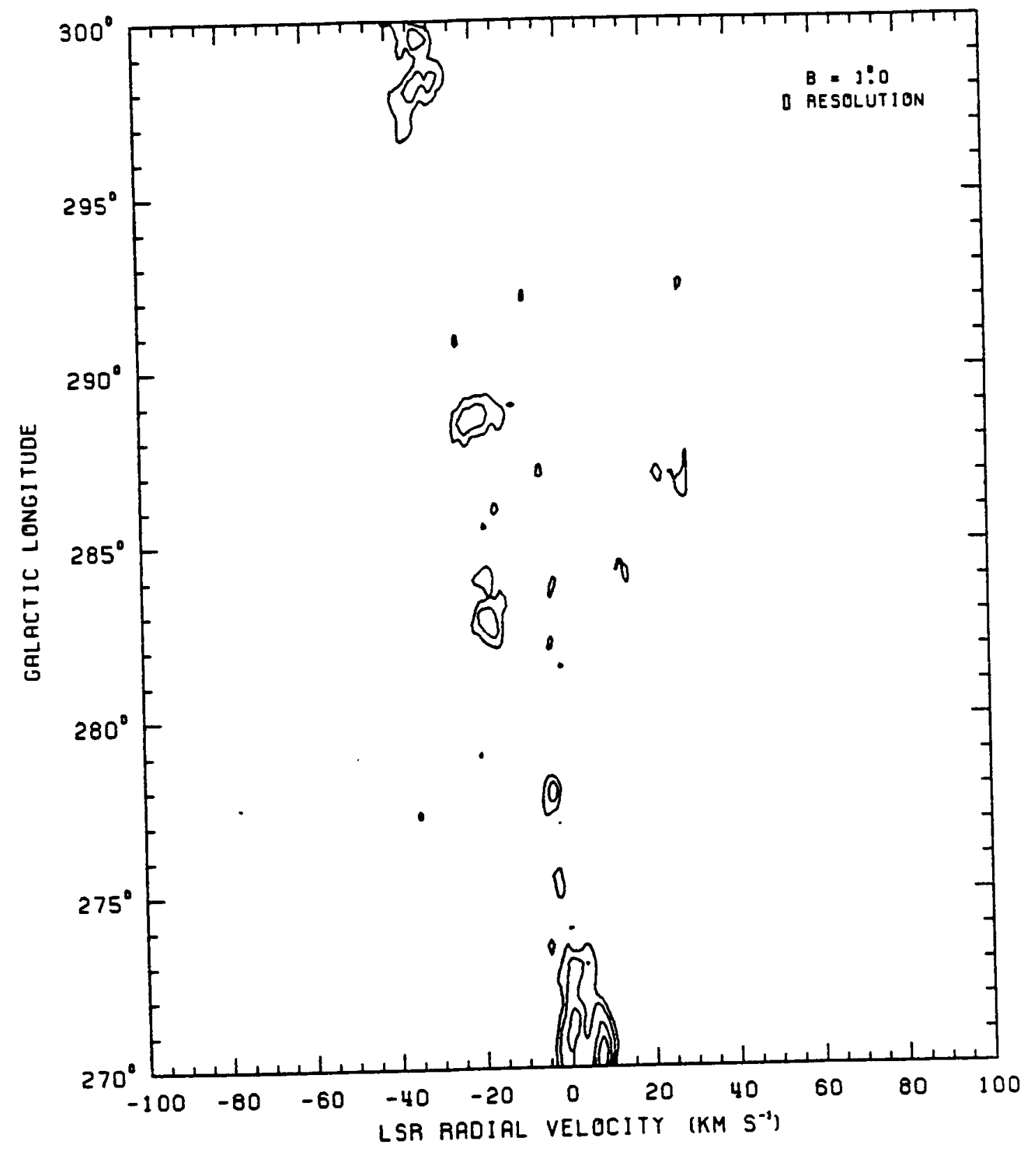

FIGURE B-3 (continued) 


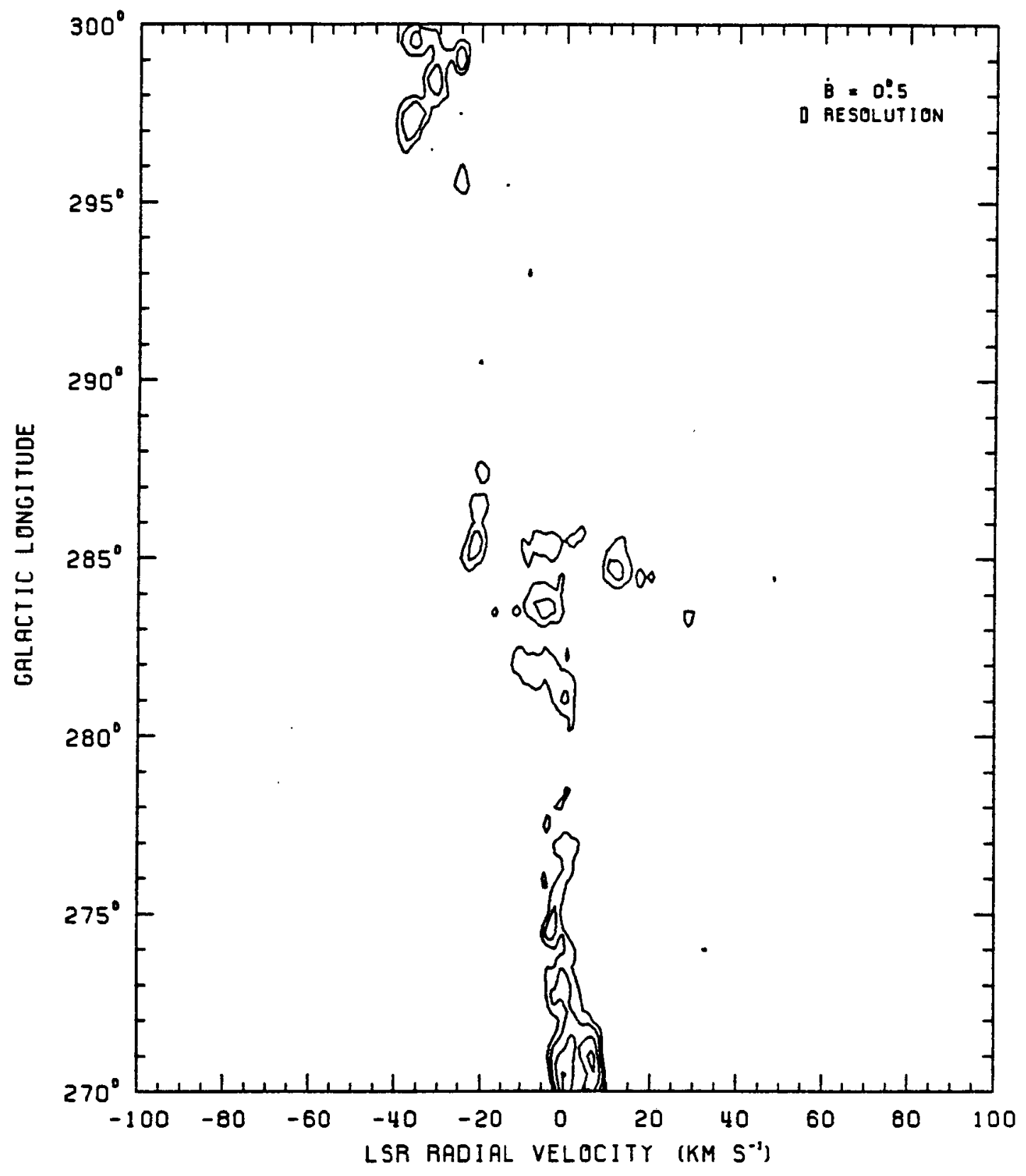

FIGURE B-3 (continued) 


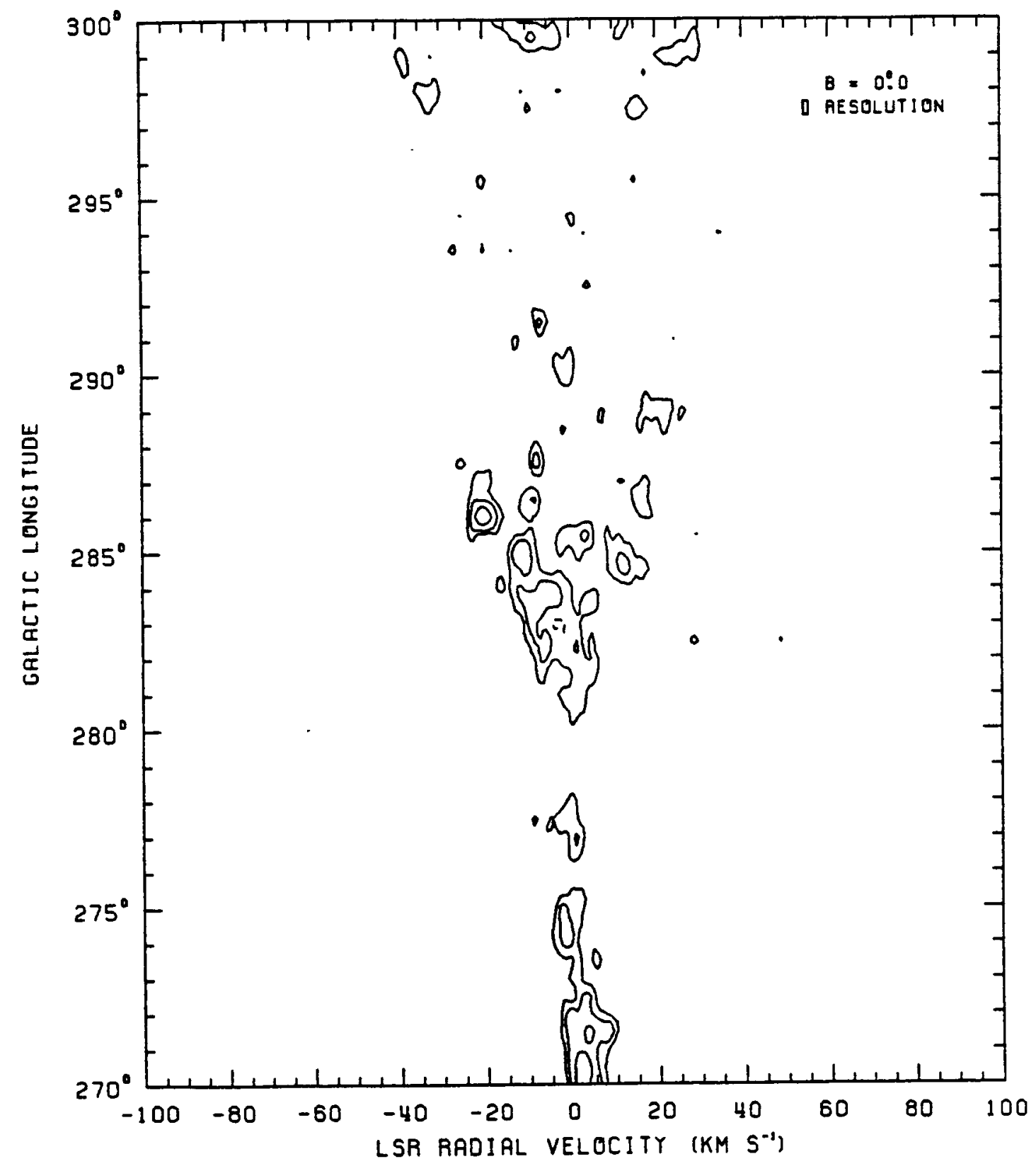

FIGURE B-3 (continued) 


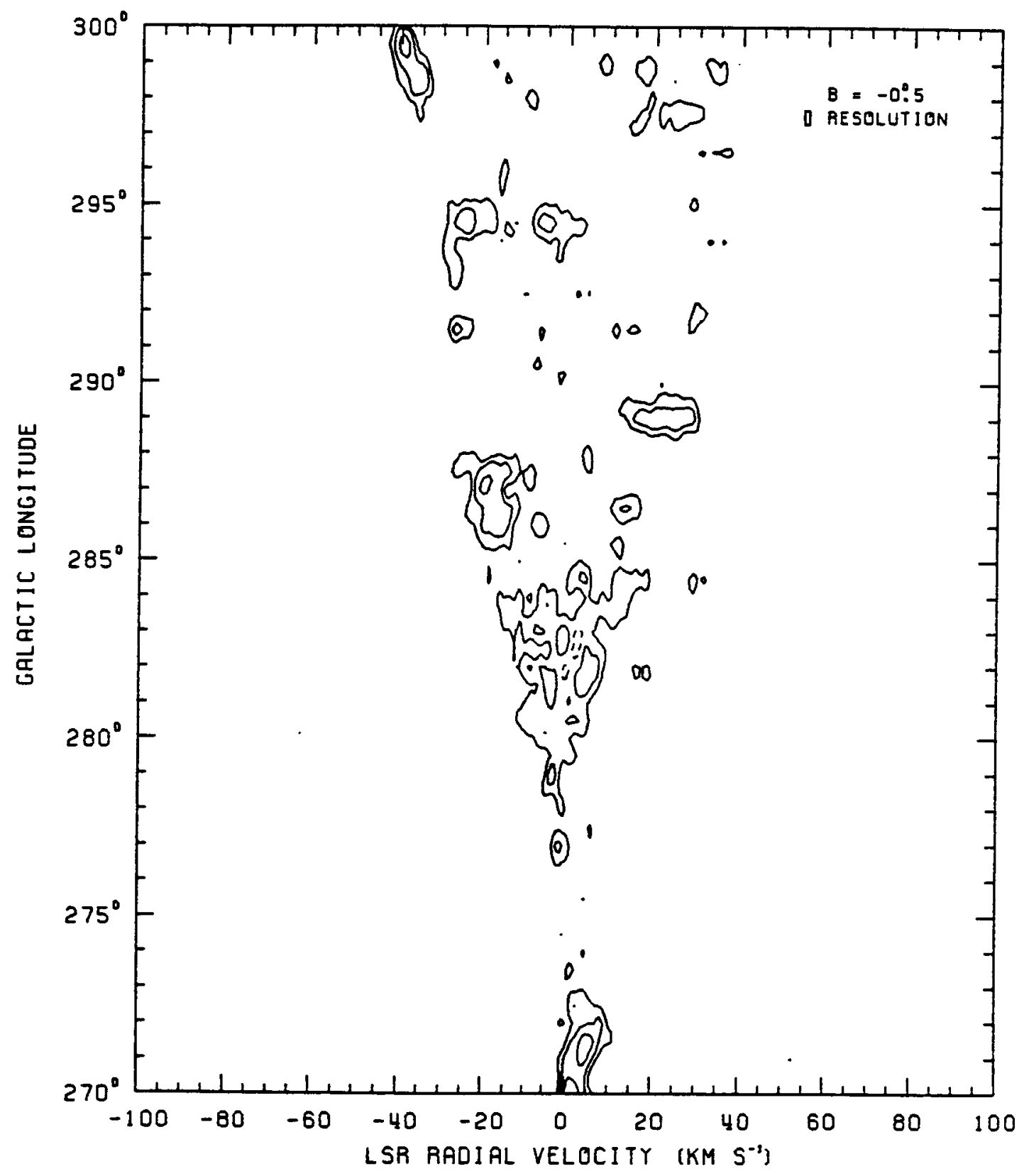

FIGURE B-3 (continued) 


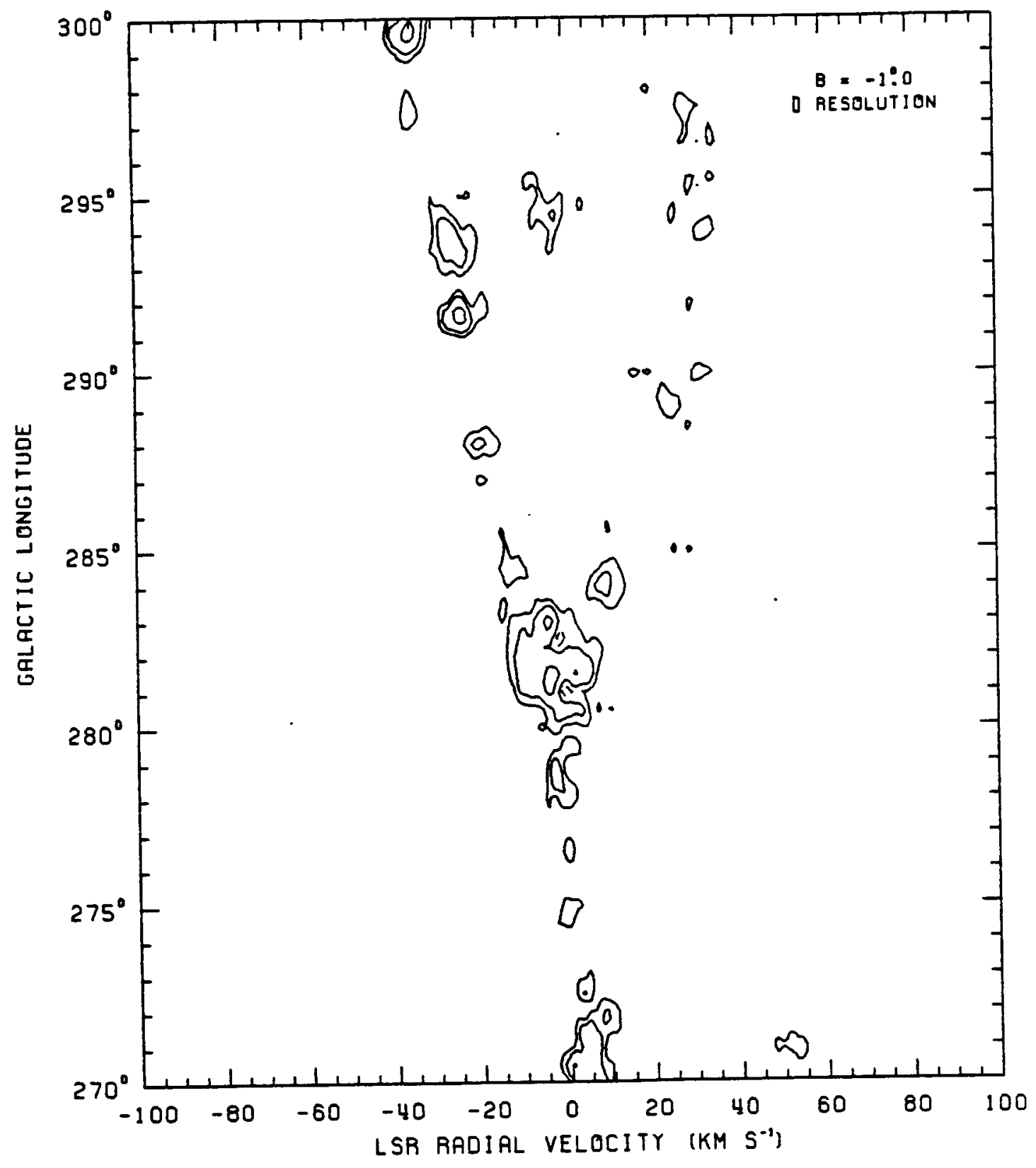

FIGURE B-3 (continued) 


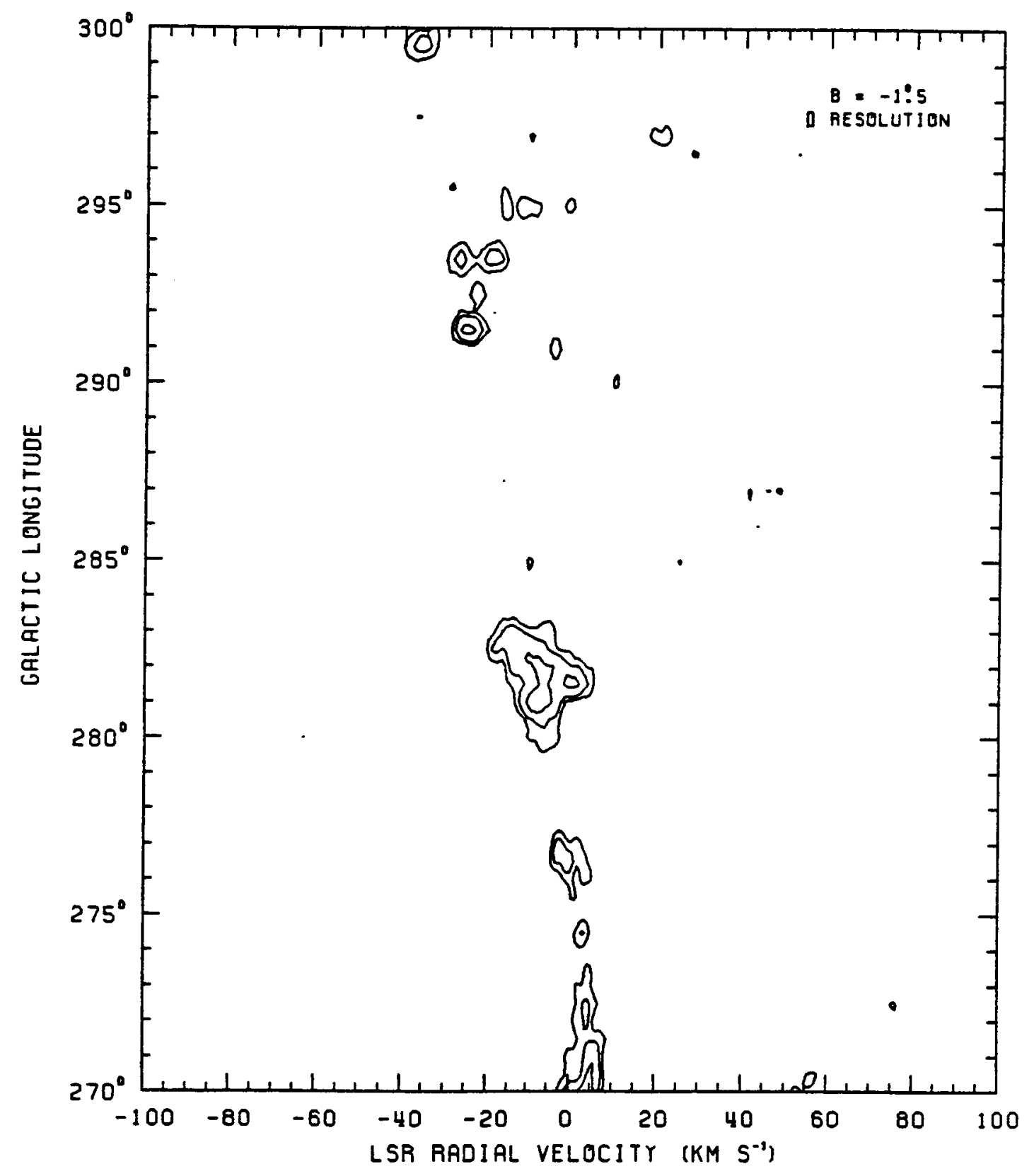

FIGURE B-3 (continued) 


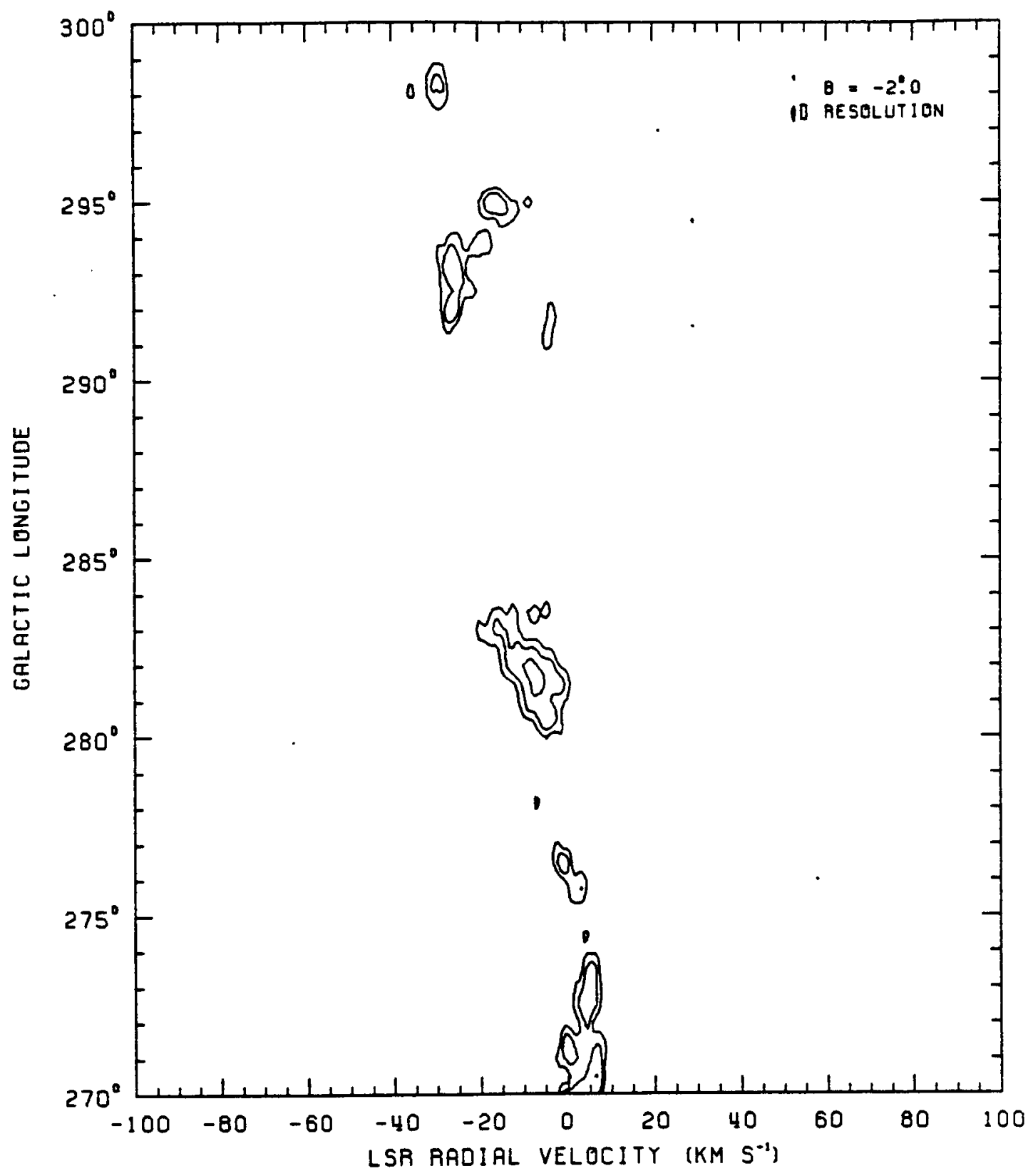

FIGURE B-3 (continued) 


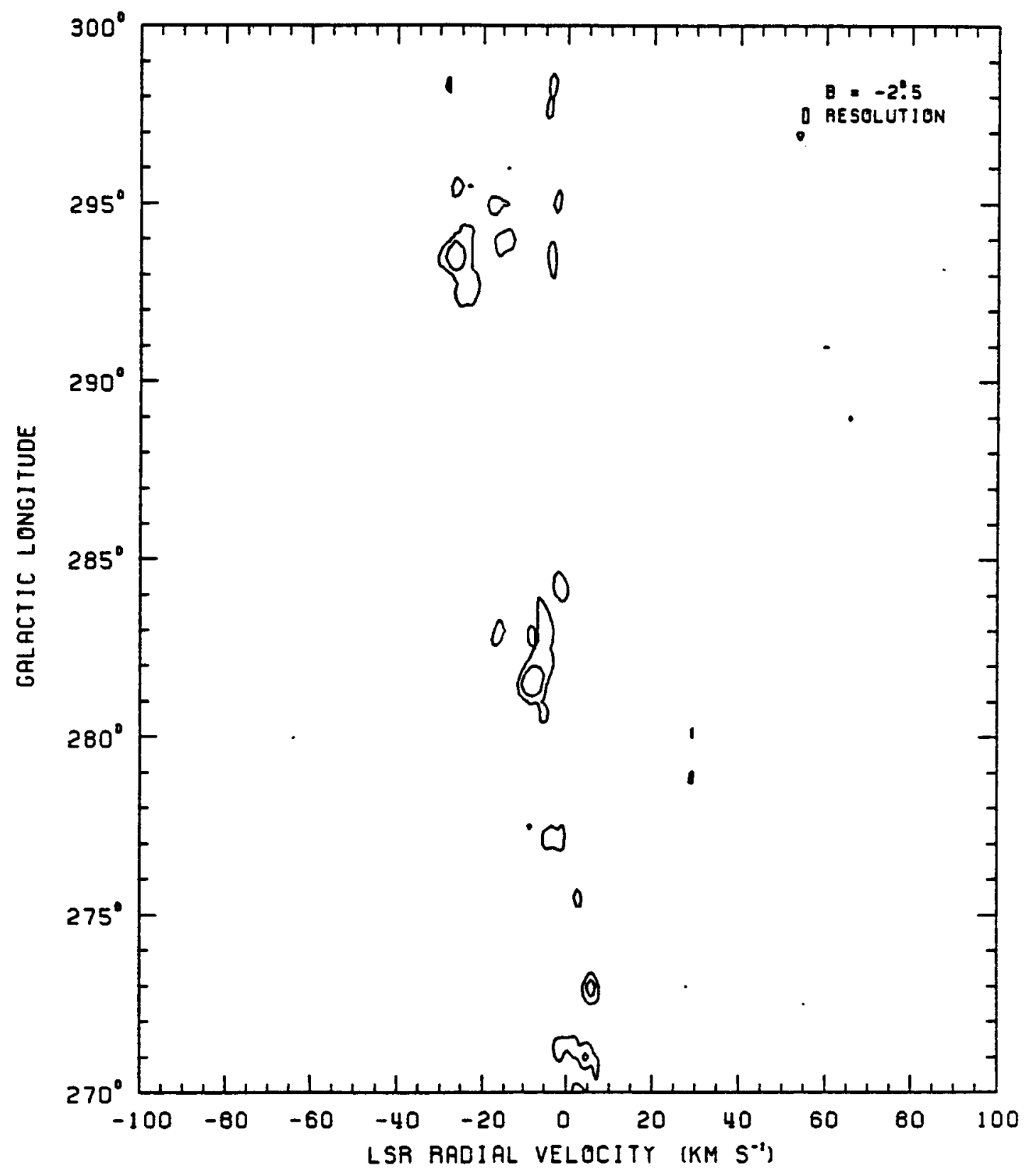

FIGURE B-3 (continued) 


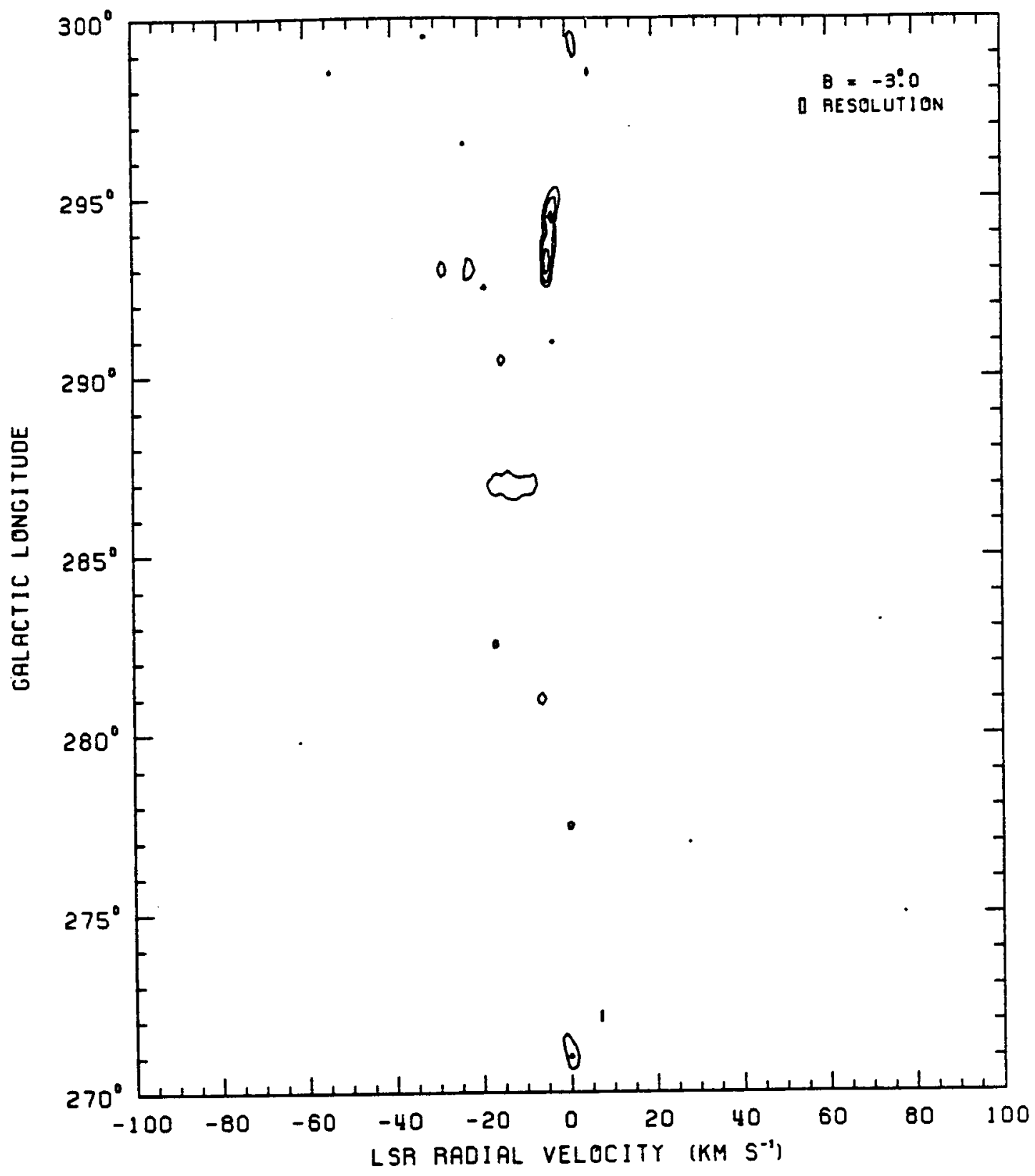

FIGURE B-3 (continued) 


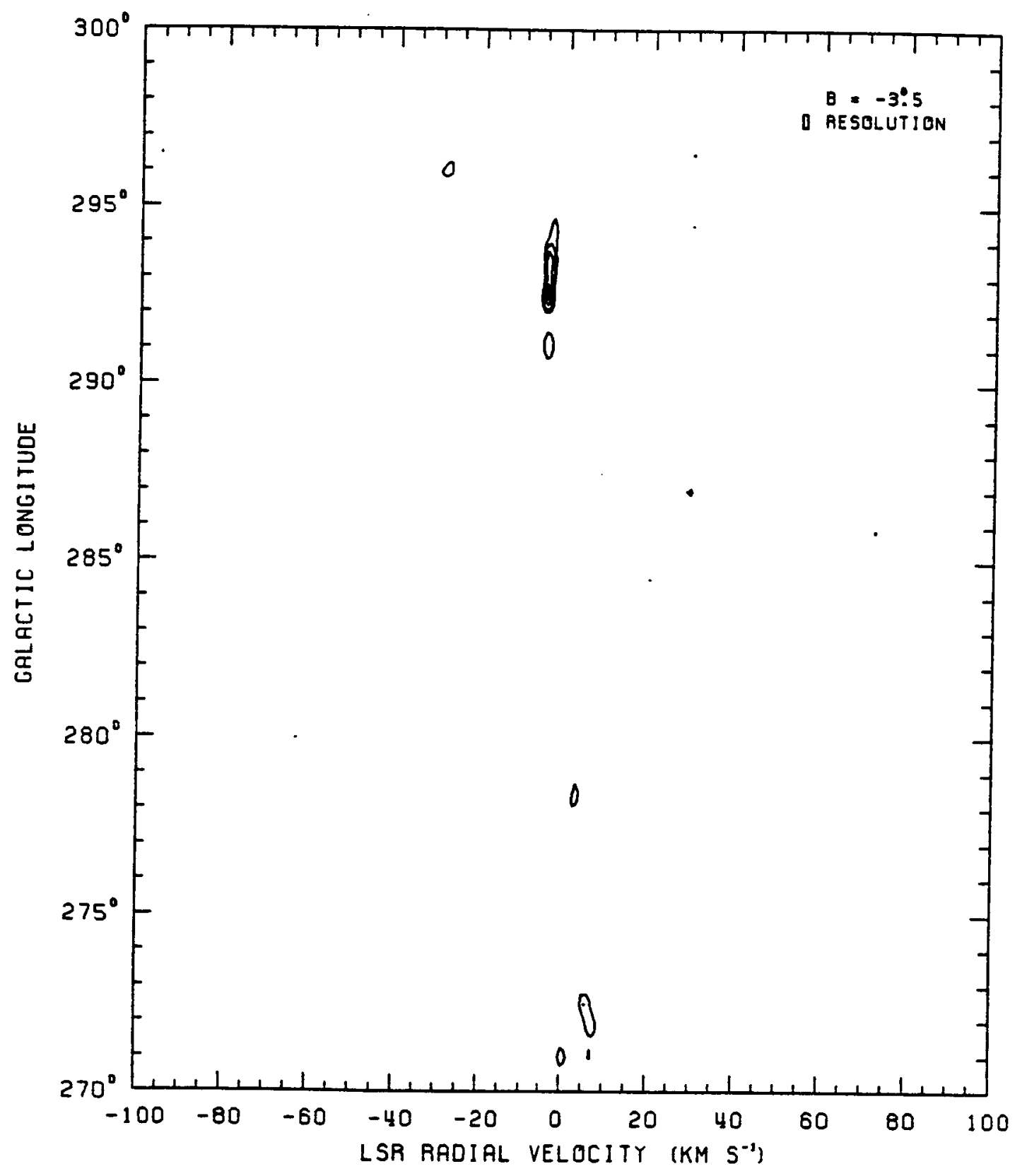

FIGURE B-3 (continued) 


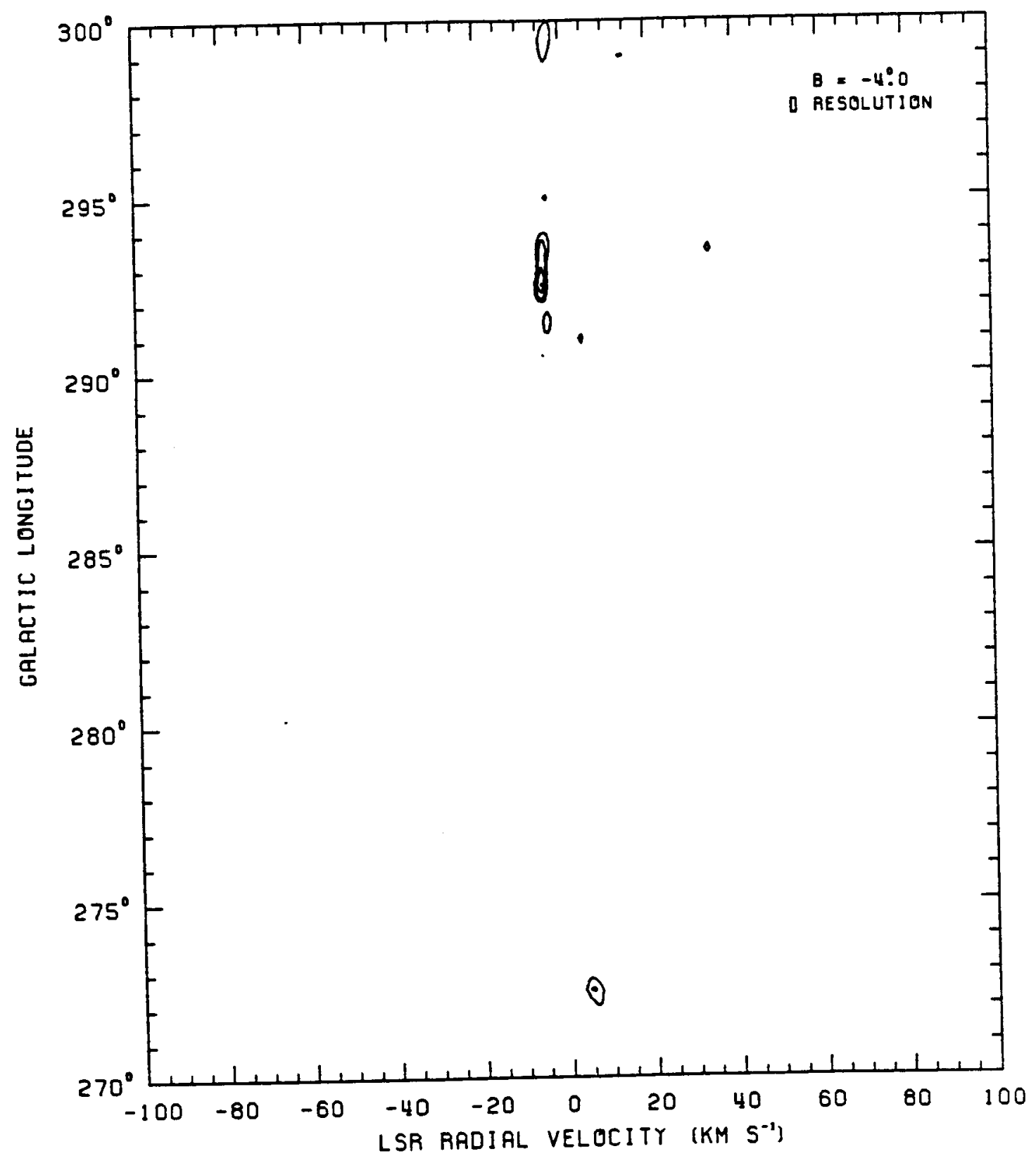

FIGURE B-3 (continued) 


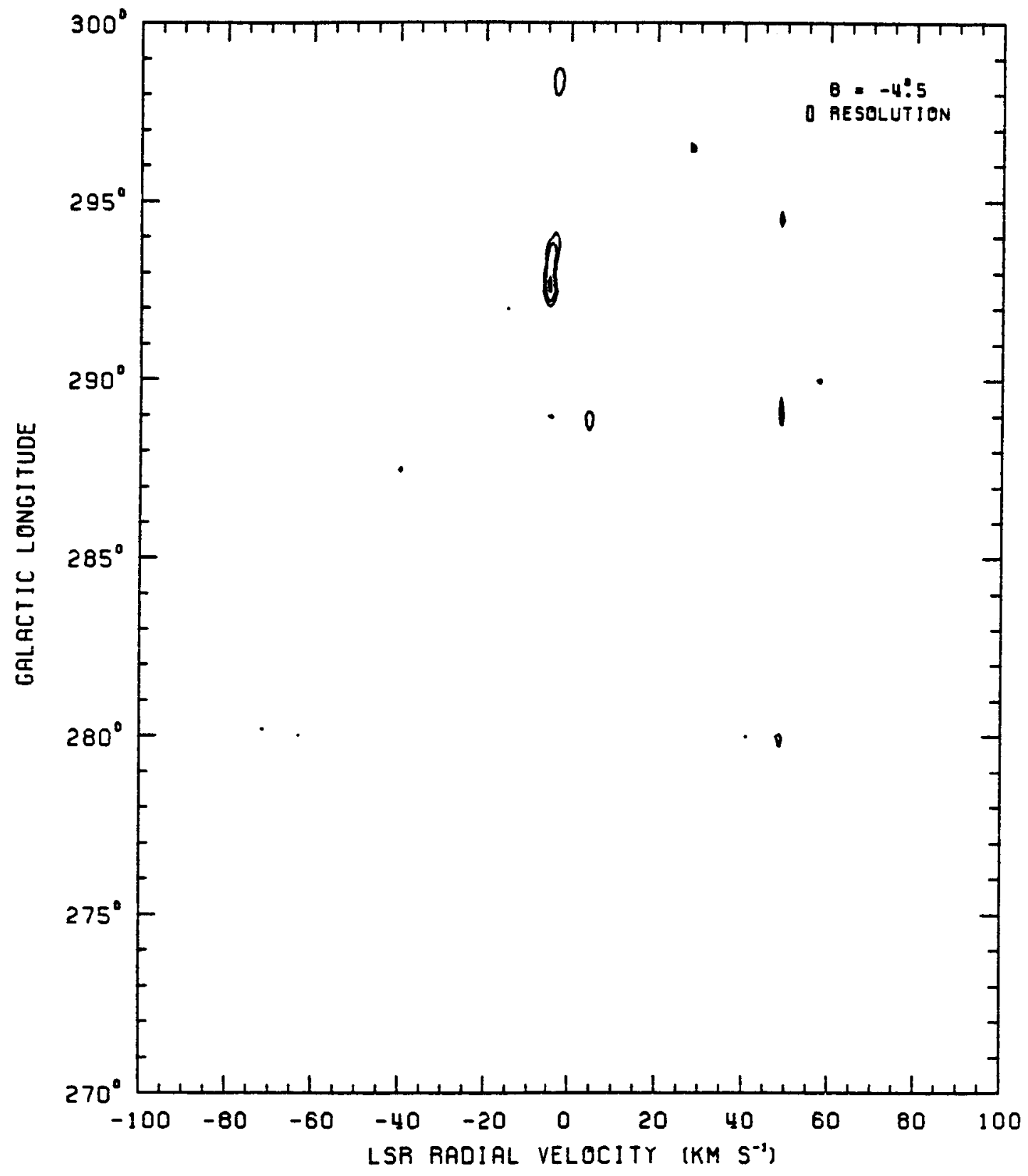

FIGURE B-3 (continued) 


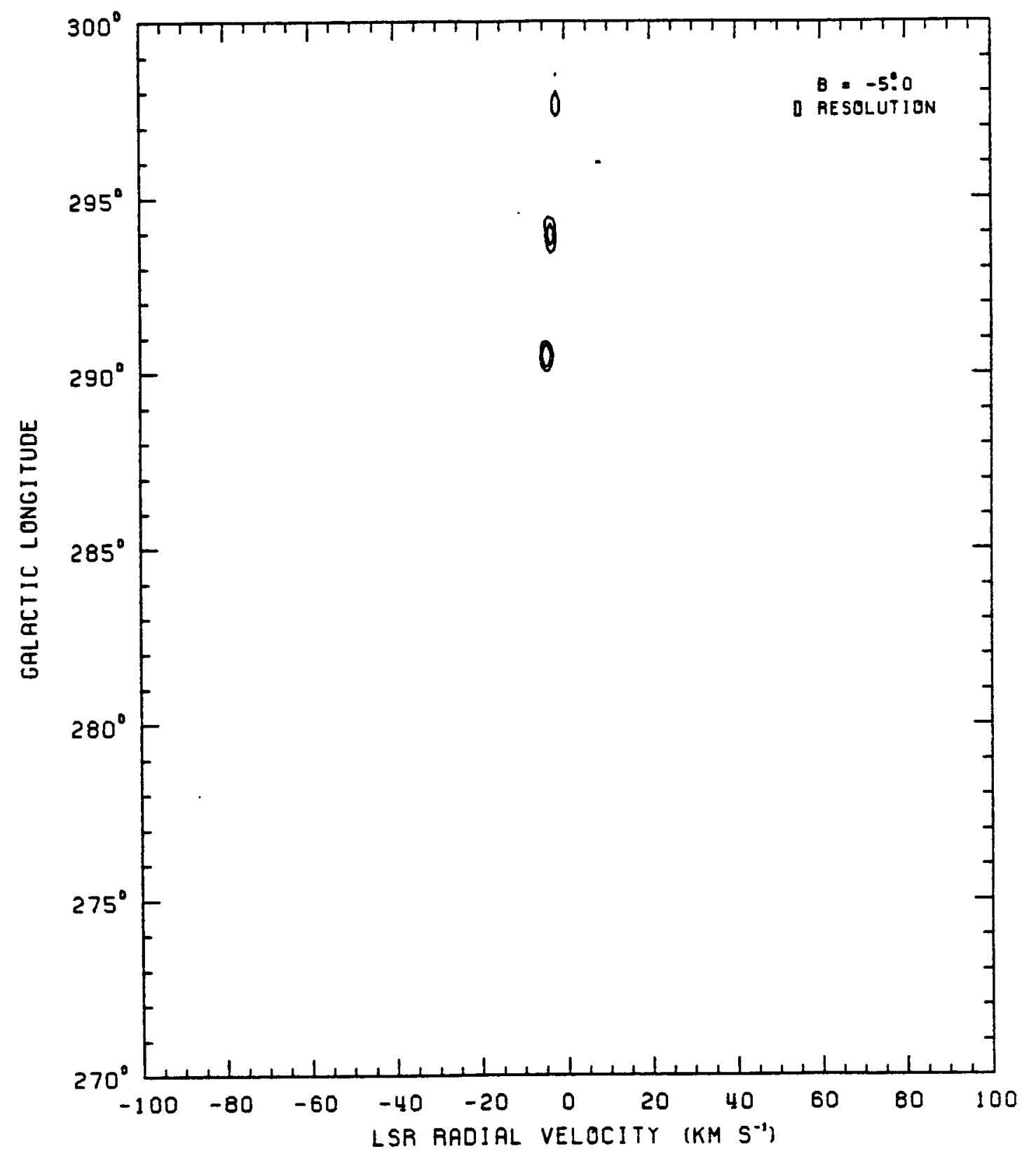

FIGURE B-3 (continued) 

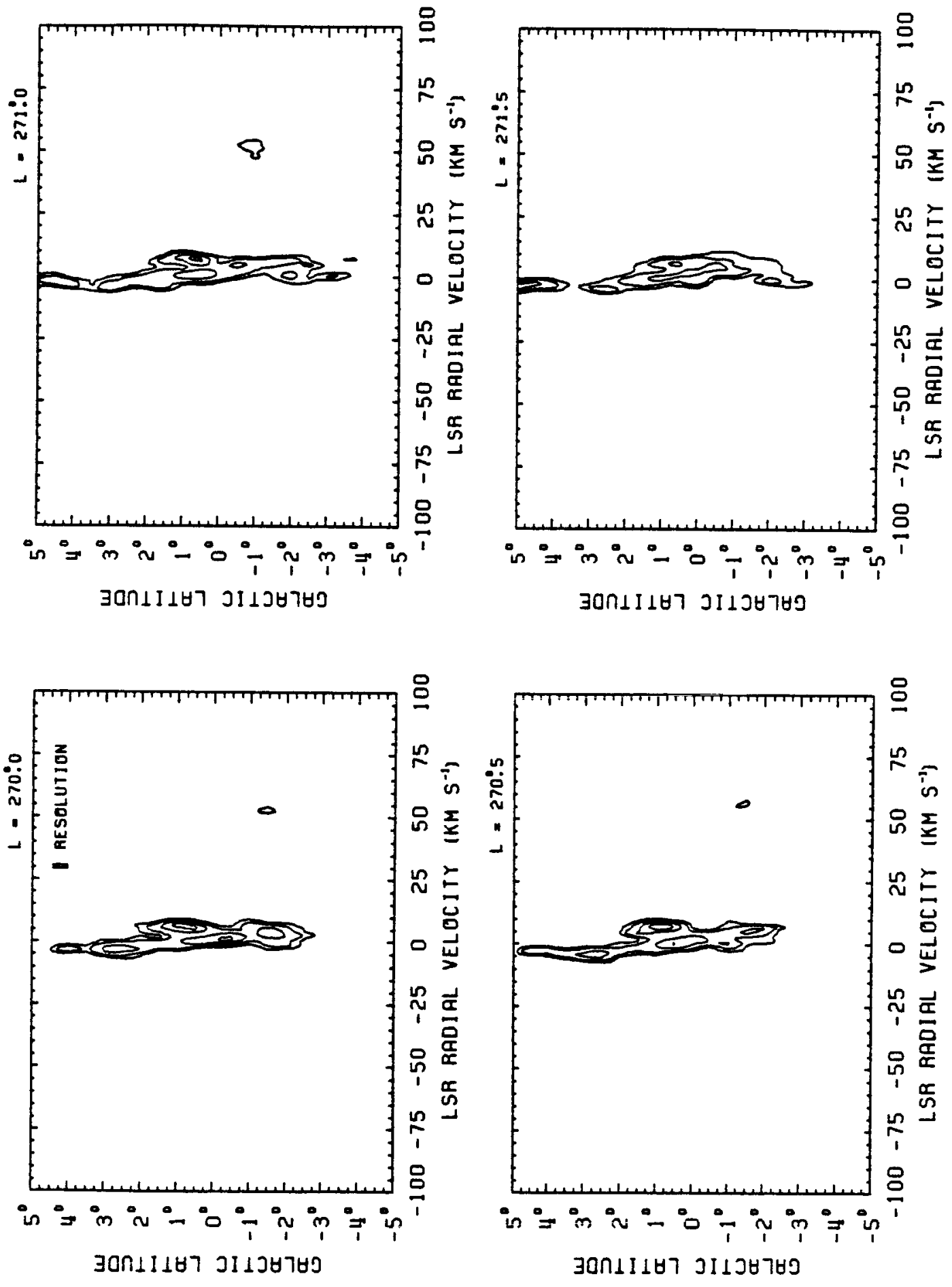

FIGURE B-4 

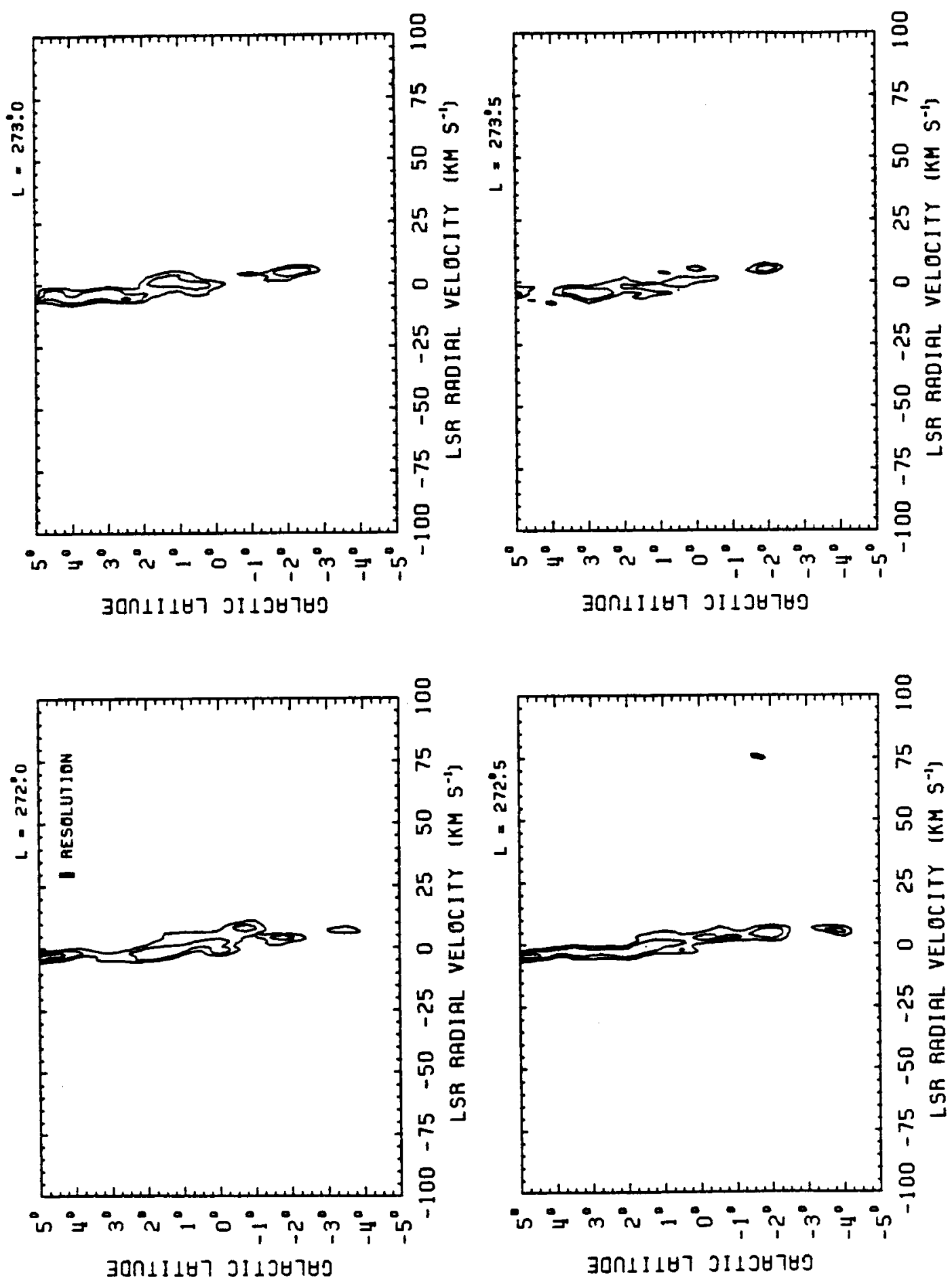

FIGURE B-4 (continued) 

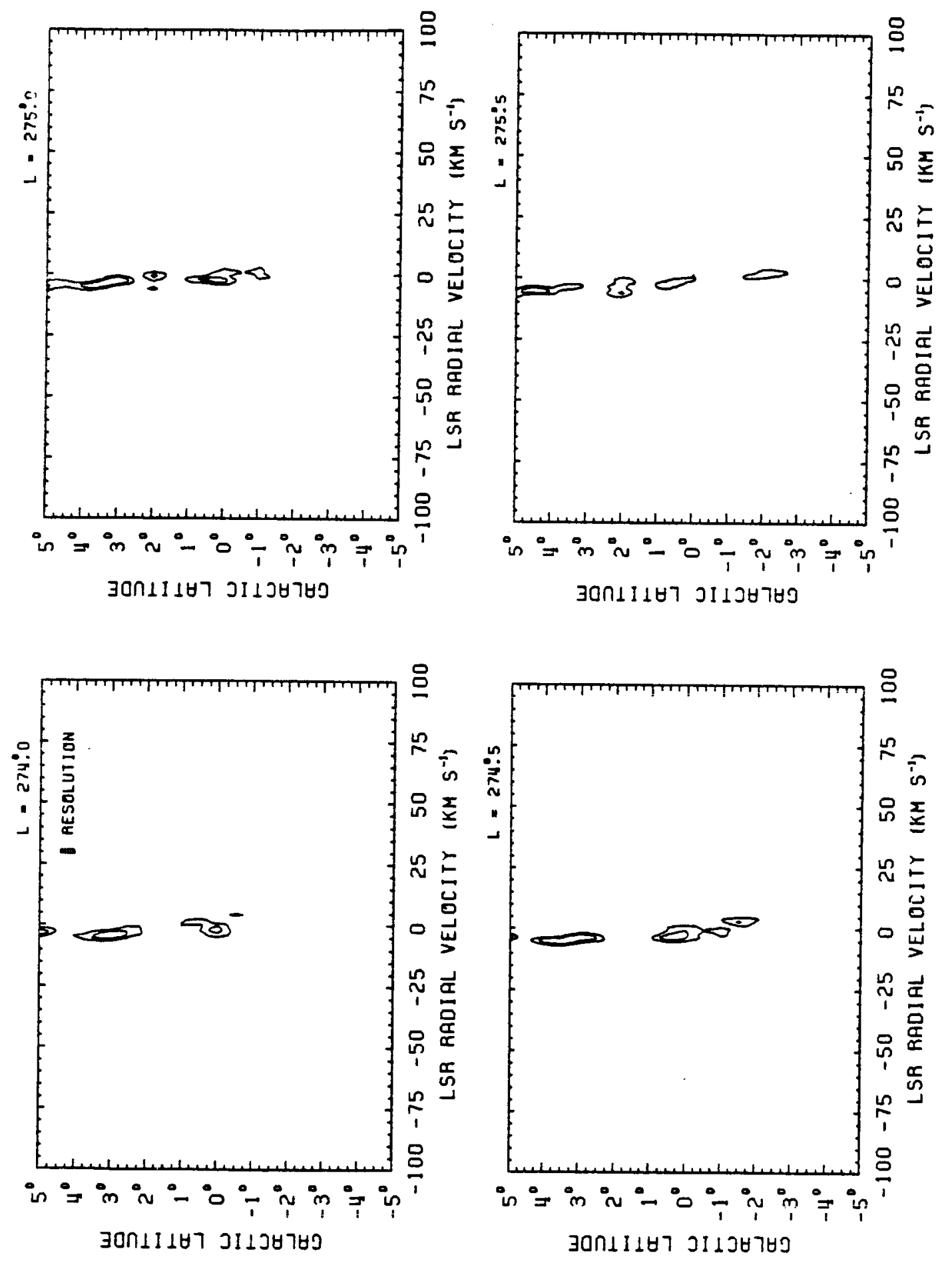

FIGURE B-4 (continued) 

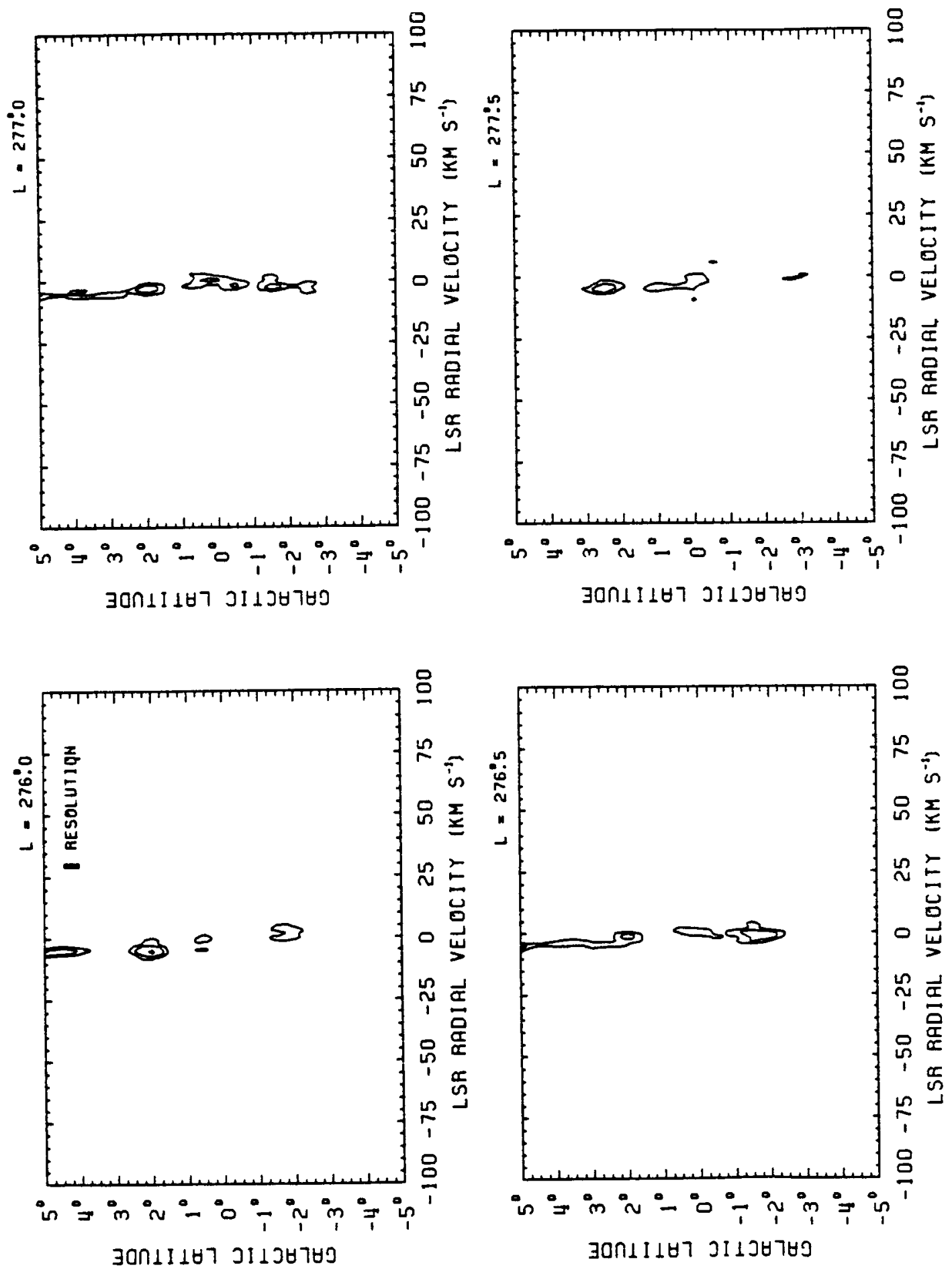

FIGURE B-4 (continued) 

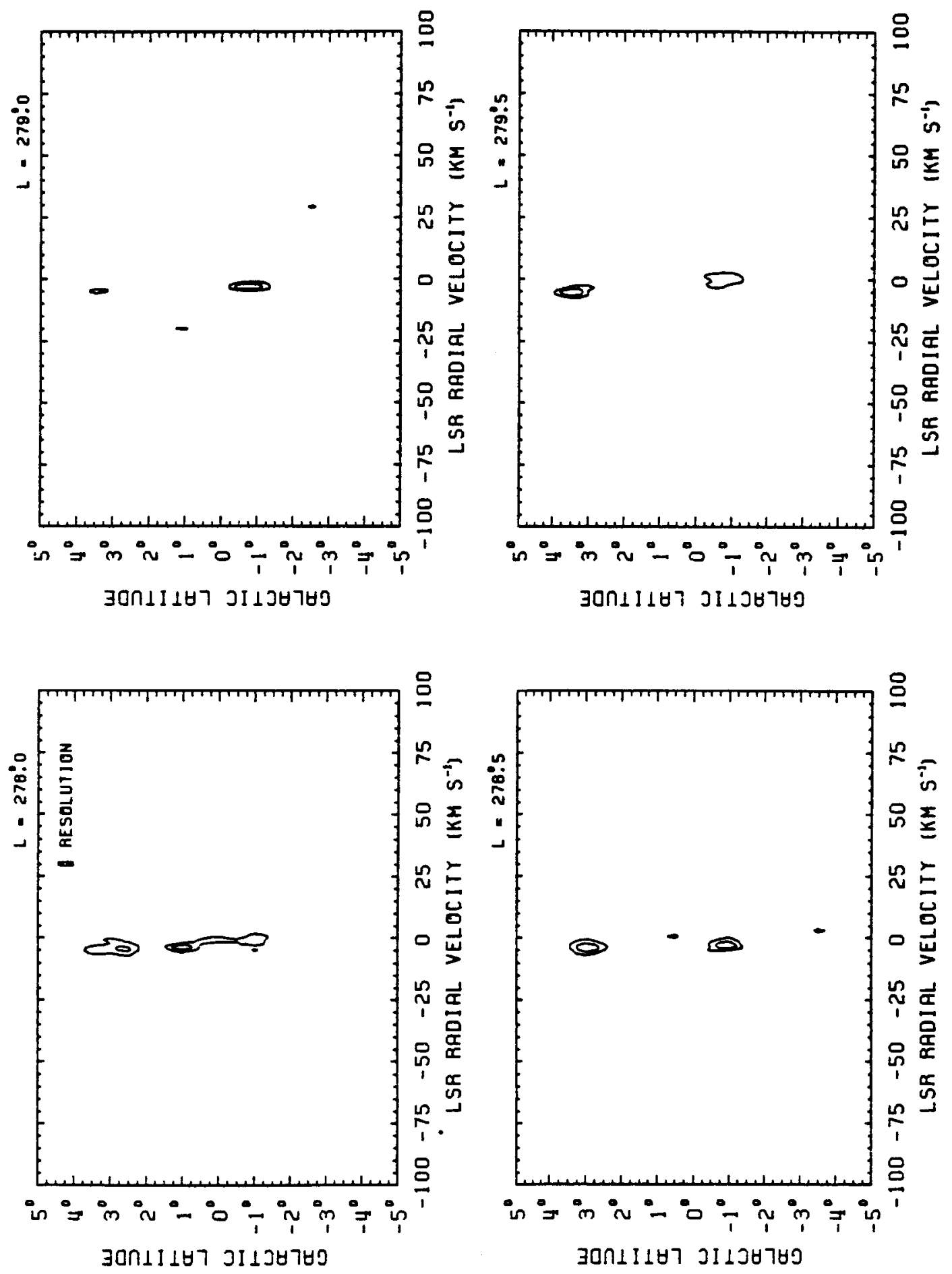

FIGURE B-4 (continued) 

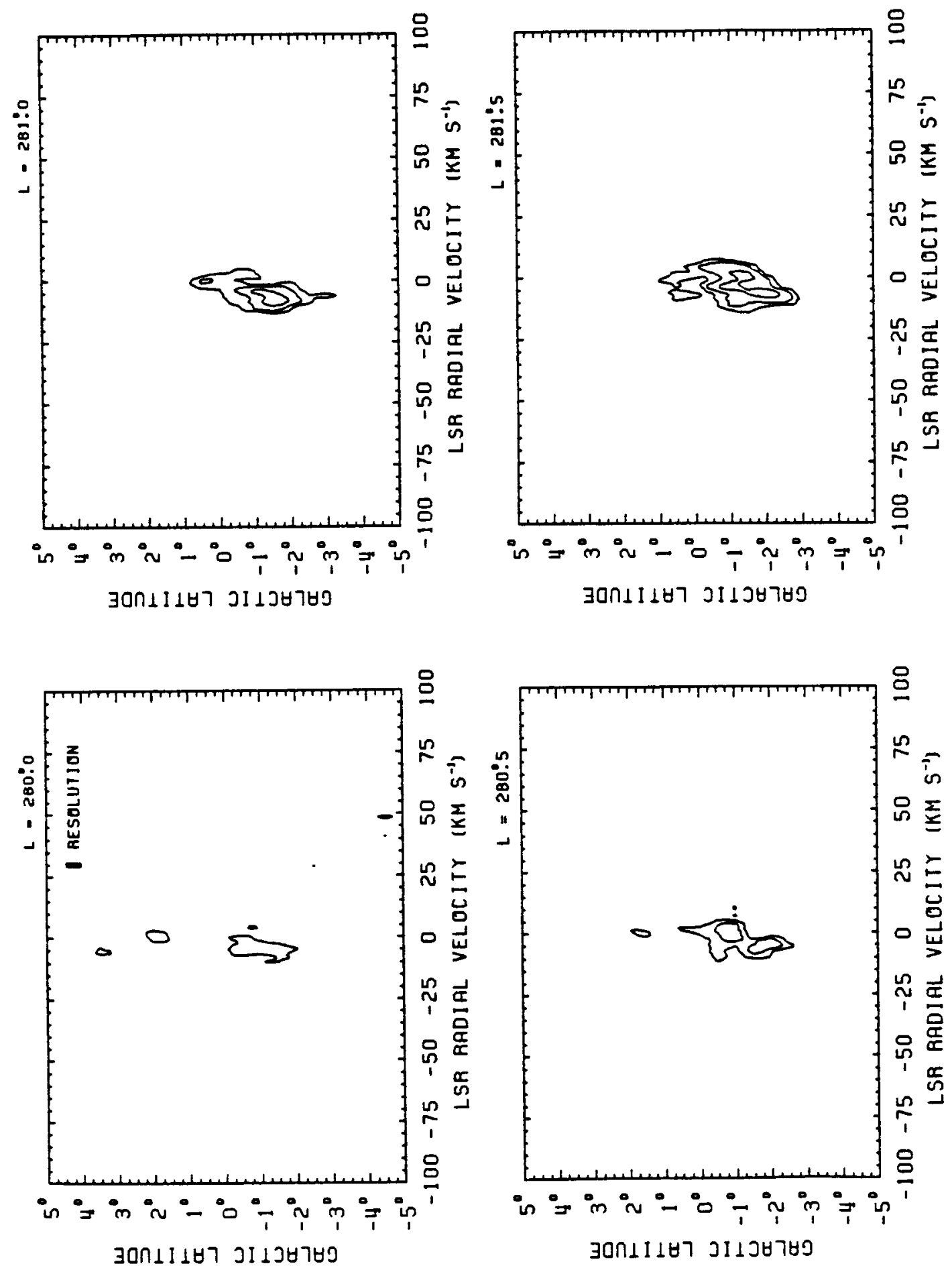

FIGURE B-4 (continued) 

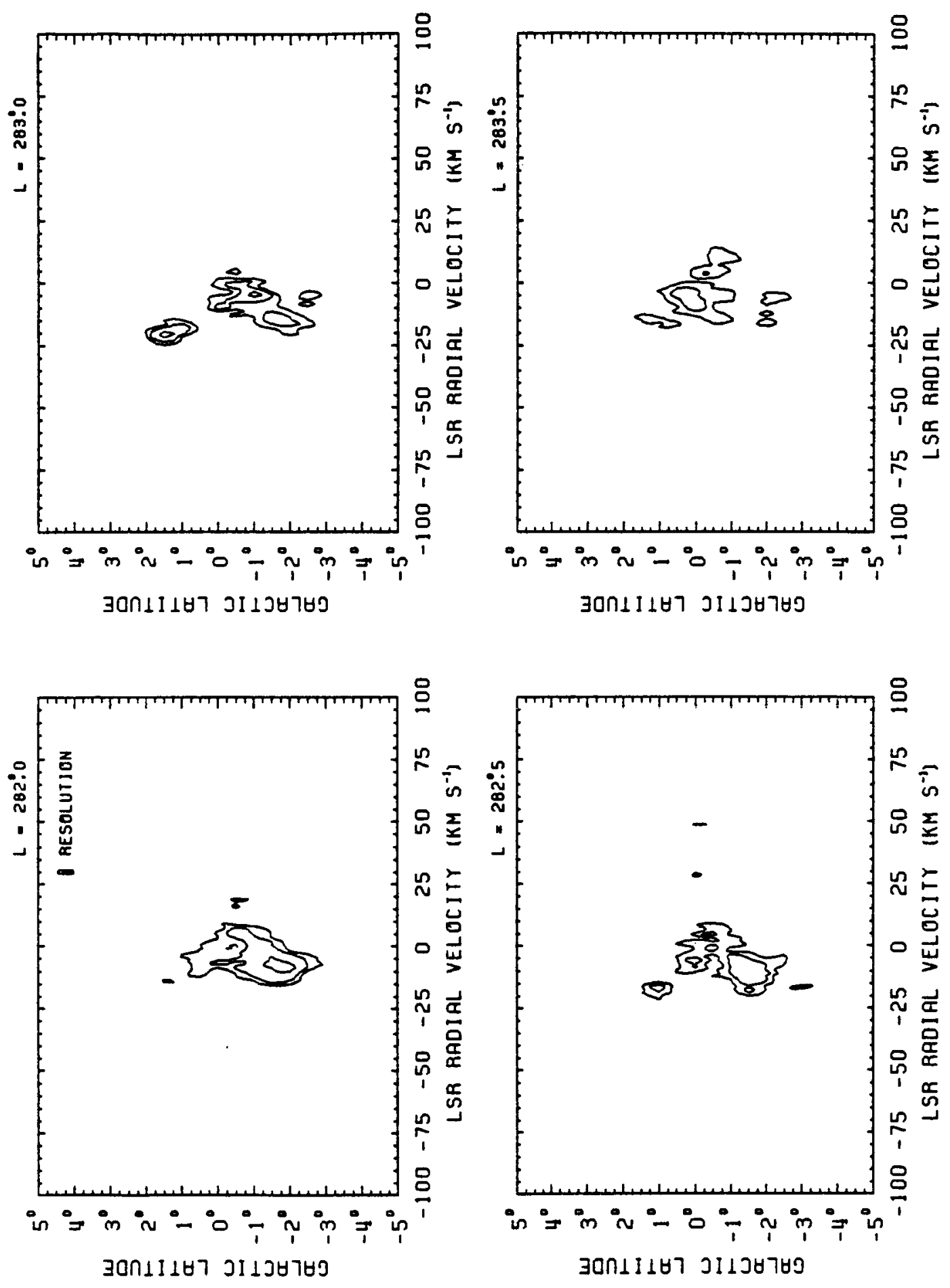

FIGURE B-4 (continued) 

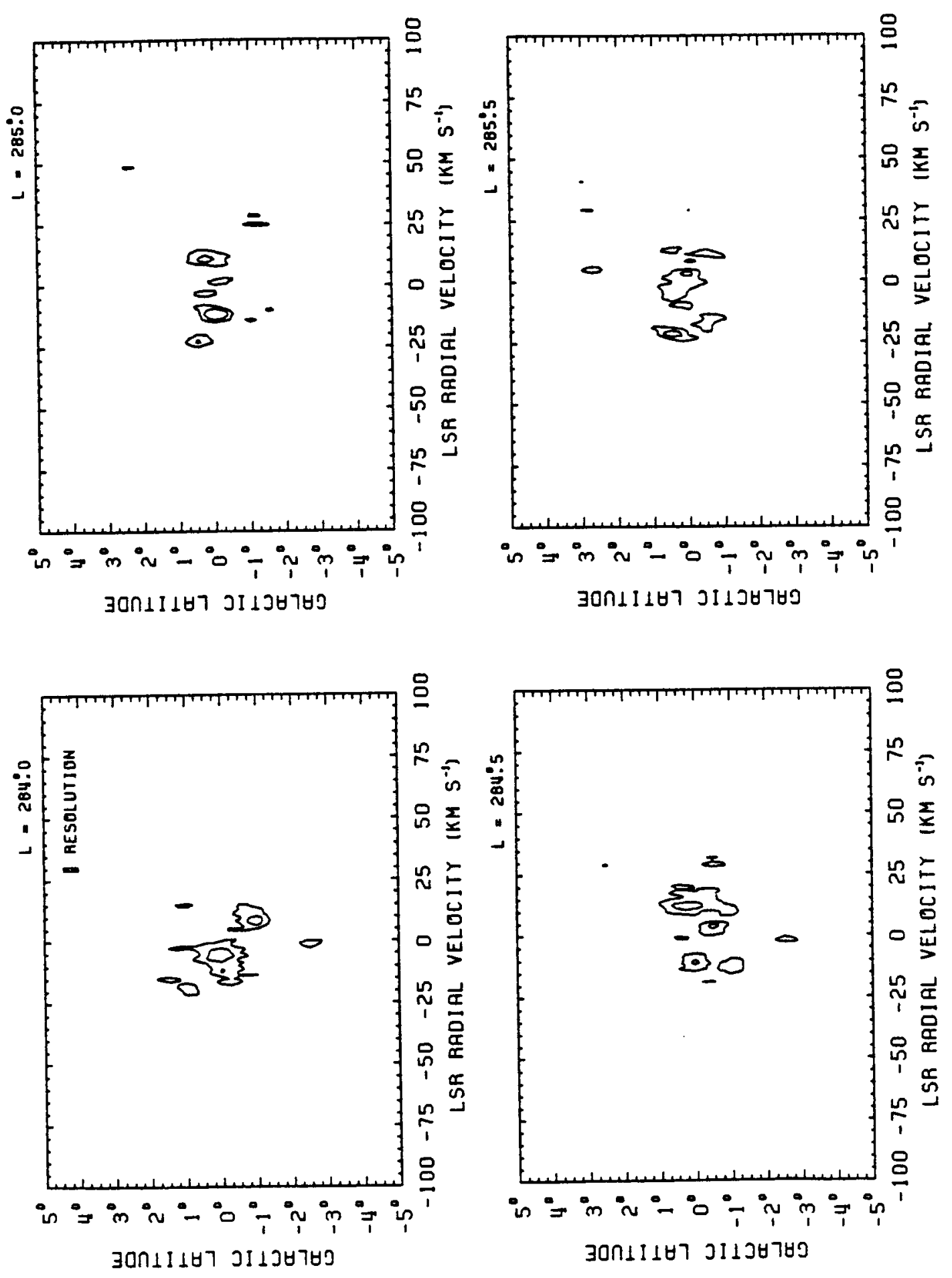

FIGURE B-4 (continued) 

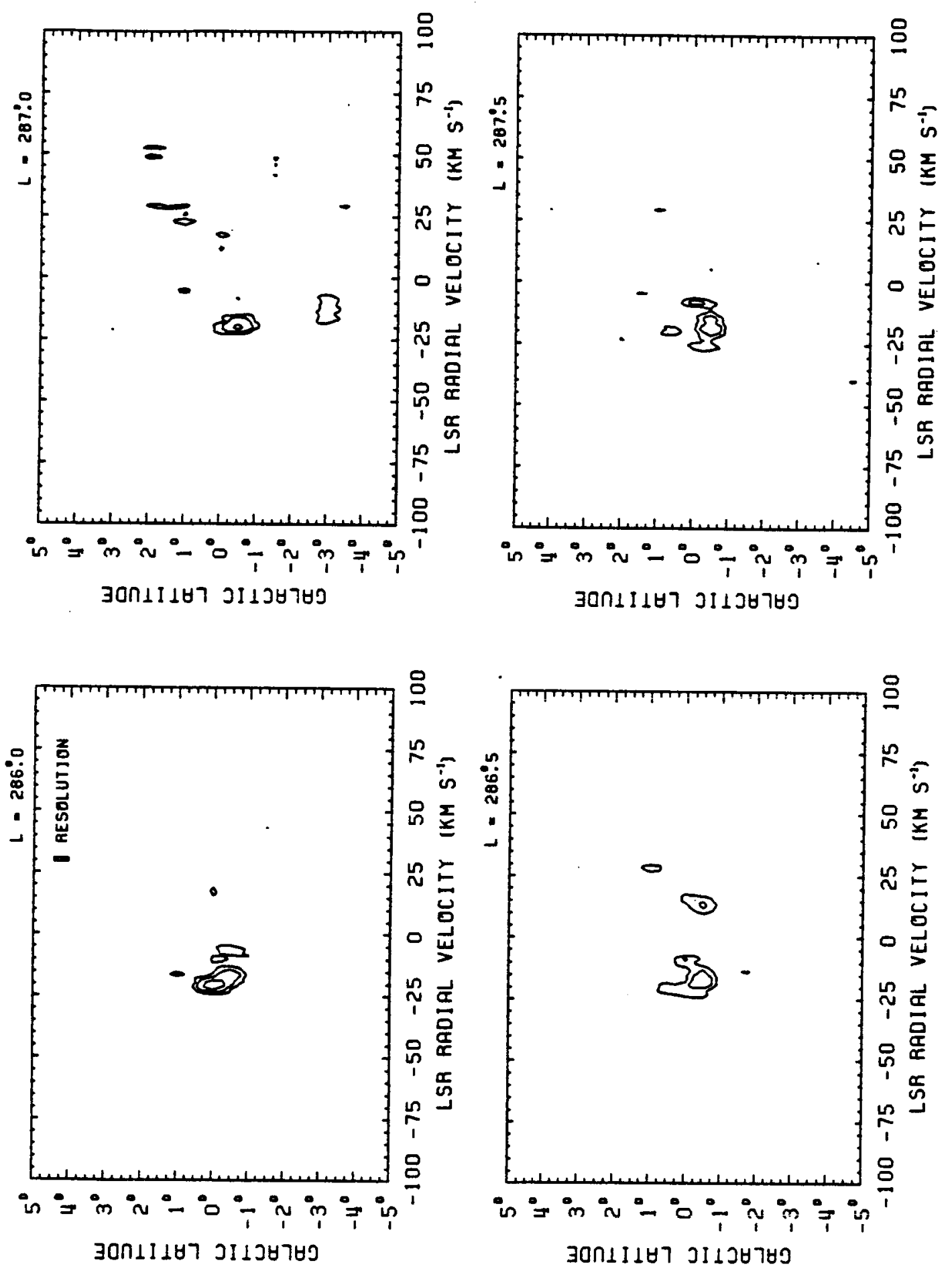

FIGURE B-4 (continued) 

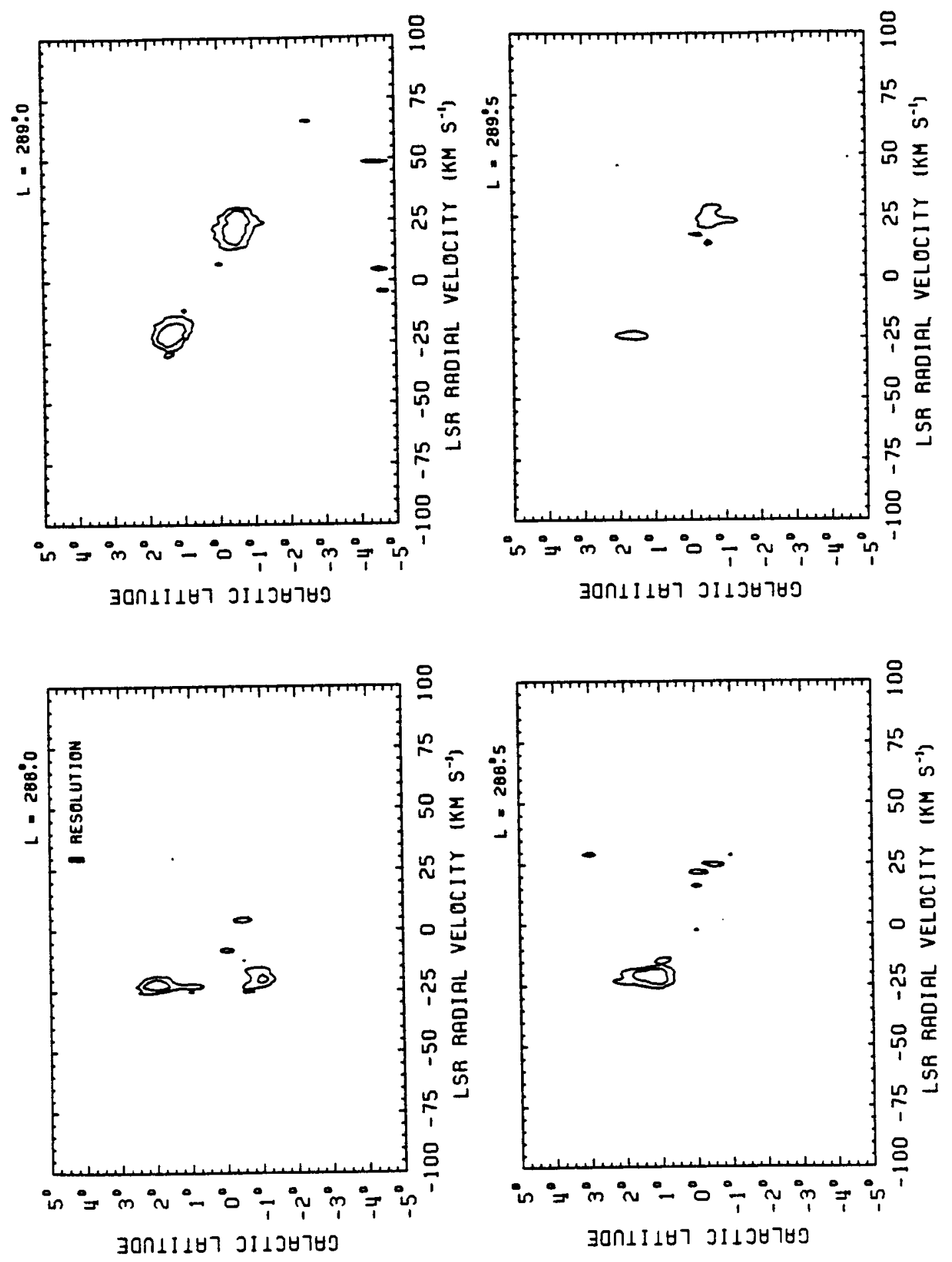

FIGURE B-4 (continued) 

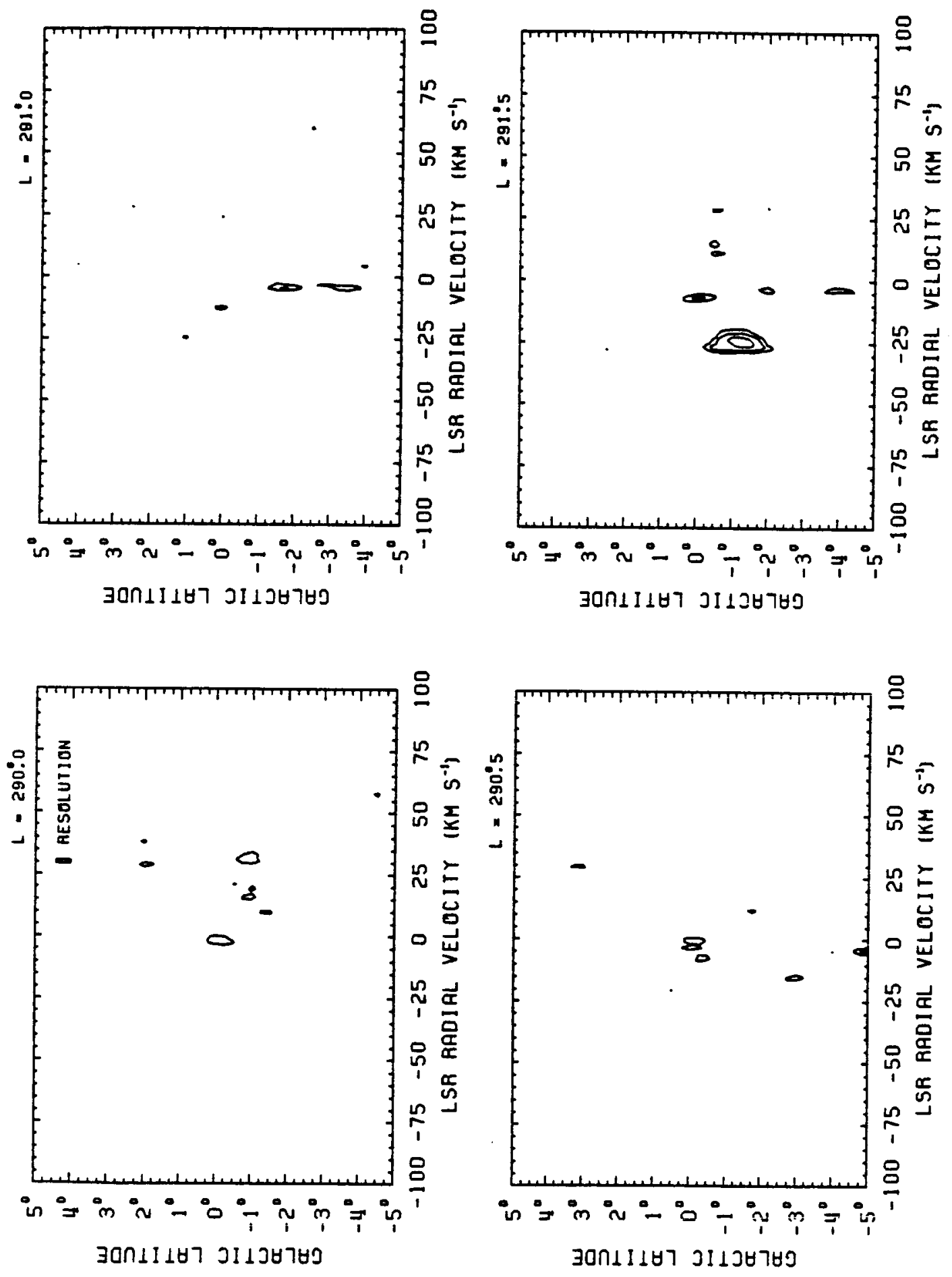

FIGURE B-4 (continued) 

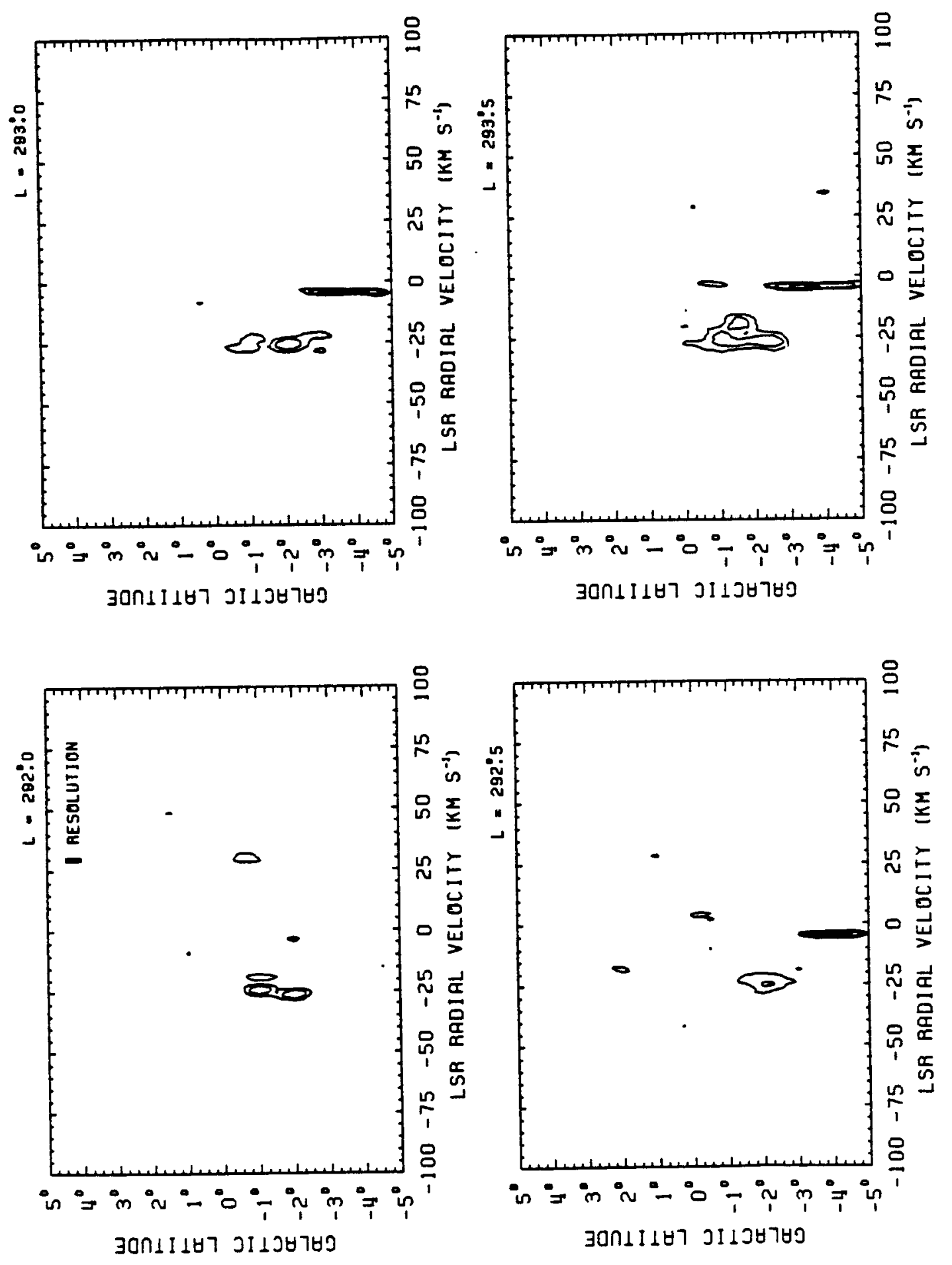

FIGURE B-4 (continued) 

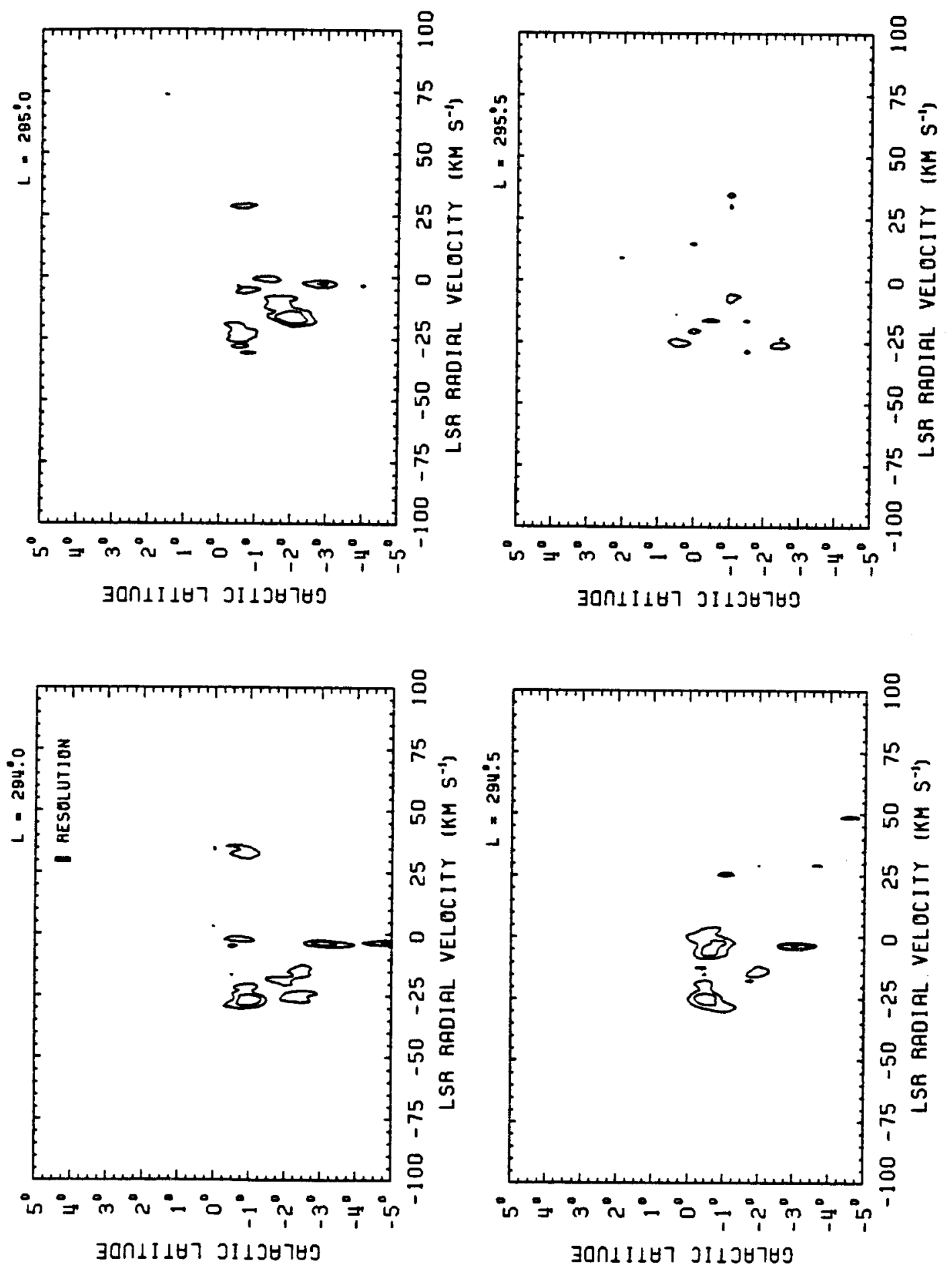

FIGURE B-4 (continued) 

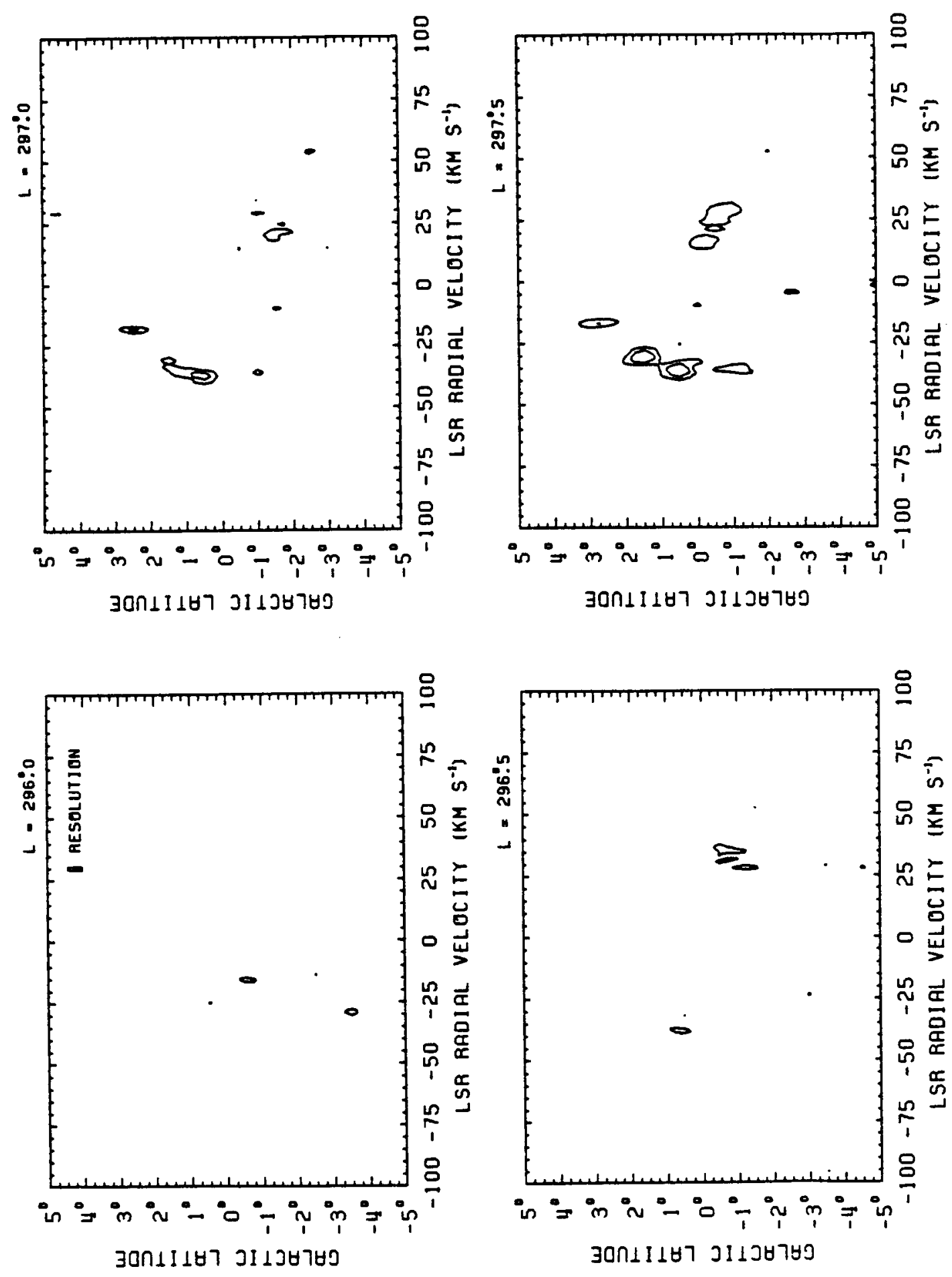

FIGURE B-4 (continued) 

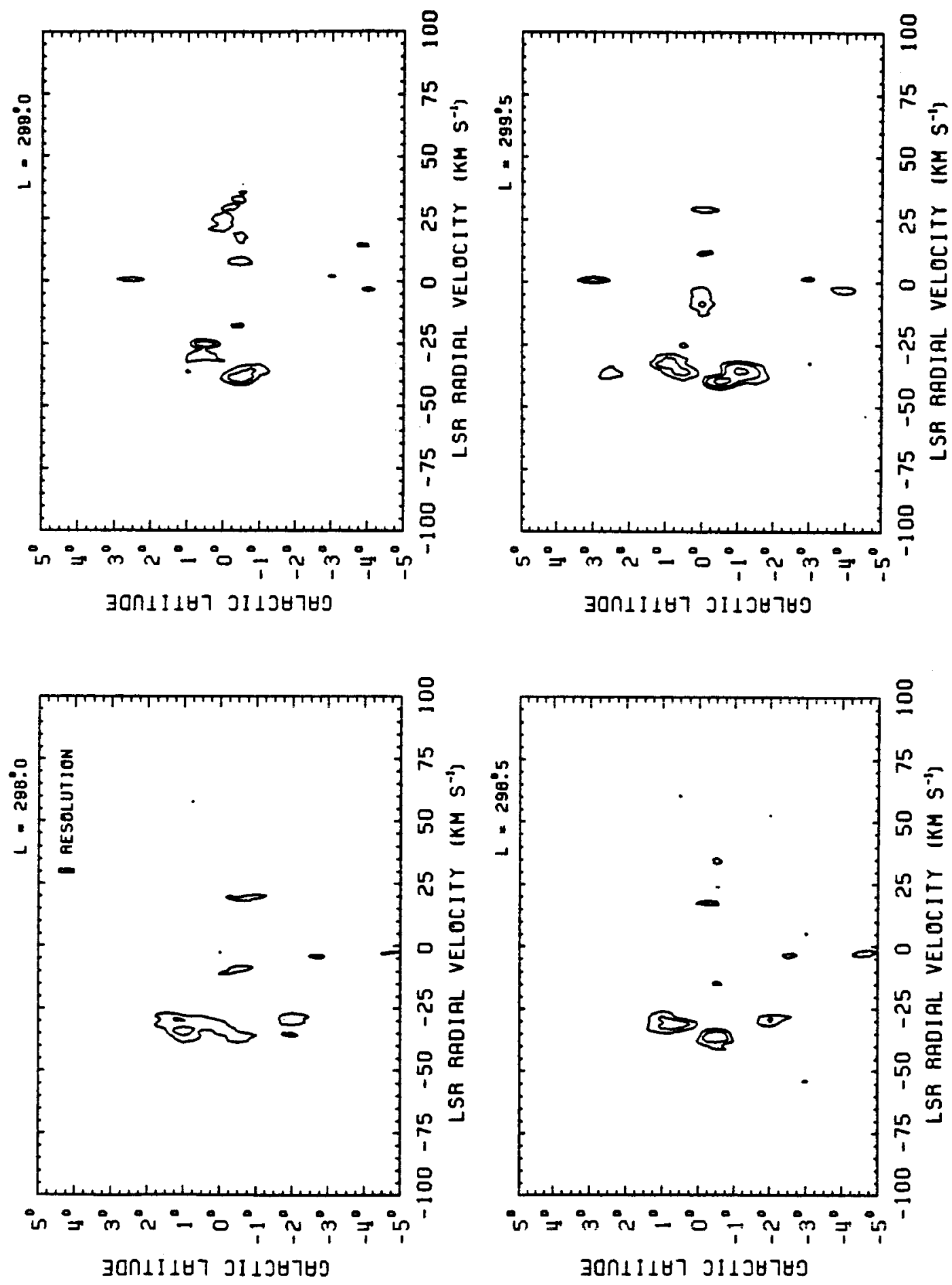

FIGURE B-4 (continued) 


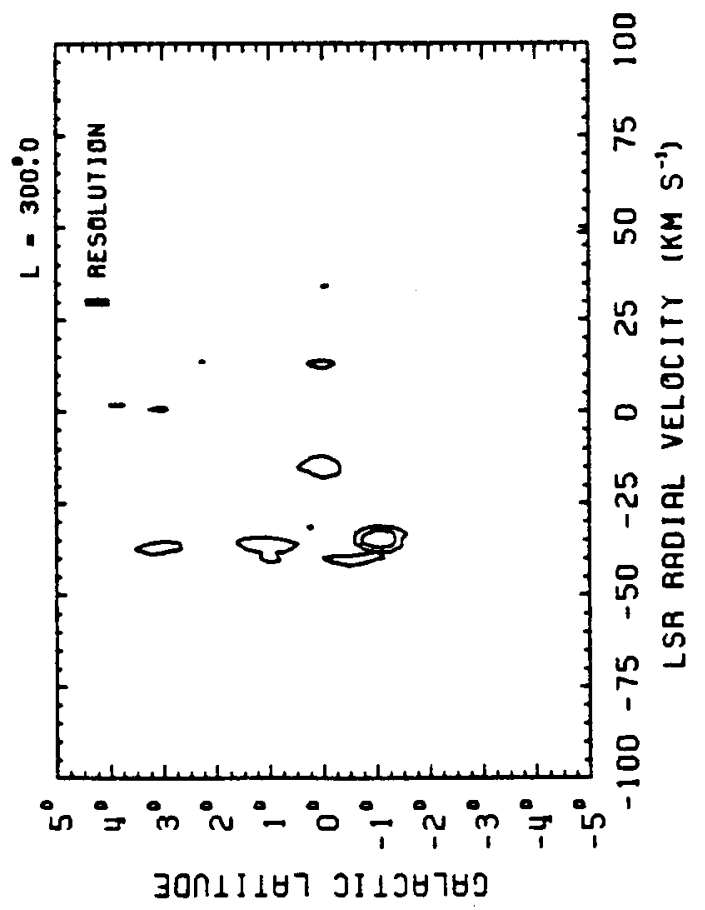

FIGURE B-4 (continued) 
BIBLIOGRAPHIC DATA SHEET

\begin{tabular}{|c|c|c|c|c|}
\hline $\begin{array}{l}\text { 1. Report No. } \\
\text { NASA TM-87798 }\end{array}$ & \multicolumn{2}{|c|}{ 2. Government Accession No. } & \multicolumn{2}{|c|}{ 3. Recipient's Catalog No. } \\
\hline \multicolumn{3}{|c|}{ 4. Title and Subtitle } & \multicolumn{2}{|c|}{$\begin{array}{l}\text { 5. Report Date } \\
\text { September } 1986\end{array}$} \\
\hline \multicolumn{3}{|c|}{ MOLECULAR CLOUDS IN THE CARINA ARM } & \multicolumn{2}{|c|}{ 6. Performing Organization Code } \\
\hline \multicolumn{3}{|c|}{ David Andrew Grabelsky* } & \multicolumn{2}{|c|}{ 8. Performing Organization Report No. } \\
\hline \multirow{3}{*}{\multicolumn{3}{|c|}{$\begin{array}{l}\text { 9. Performing Organization Name and Address } \\
\text { NASA/Goddard Institute for Space Studies } \\
\text { New York, NY } 10025\end{array}$}} & \multicolumn{2}{|l|}{ 10. Work Unit No. } \\
\hline & & & \multicolumn{2}{|c|}{ 11. Contract or Grant No. } \\
\hline & & & \multirow{2}{*}{\multicolumn{2}{|c|}{$\begin{array}{l}\text { 13. Type of Report and Period Covered } \\
\text { Technical Memorandum }\end{array}$}} \\
\hline \multirow{2}{*}{\multicolumn{3}{|c|}{$\begin{array}{l}\text { 12. Sponsoring Agency Name and Address } \\
\text { National Aeronautics \& Space Administration } \\
\text { Washington, DC } 20546\end{array}$}} & & \\
\hline & & & \multicolumn{2}{|c|}{ 14. Sponsoring Agency Code } \\
\hline \multicolumn{5}{|c|}{$\begin{array}{l}\text { 15. Supplementary Notes } \\
\qquad \begin{array}{l}\text { *David Andrew Grabelsky } \\
\text { Columbia University, New York, NY }\end{array}\end{array}$} \\
\hline \multicolumn{5}{|c|}{ 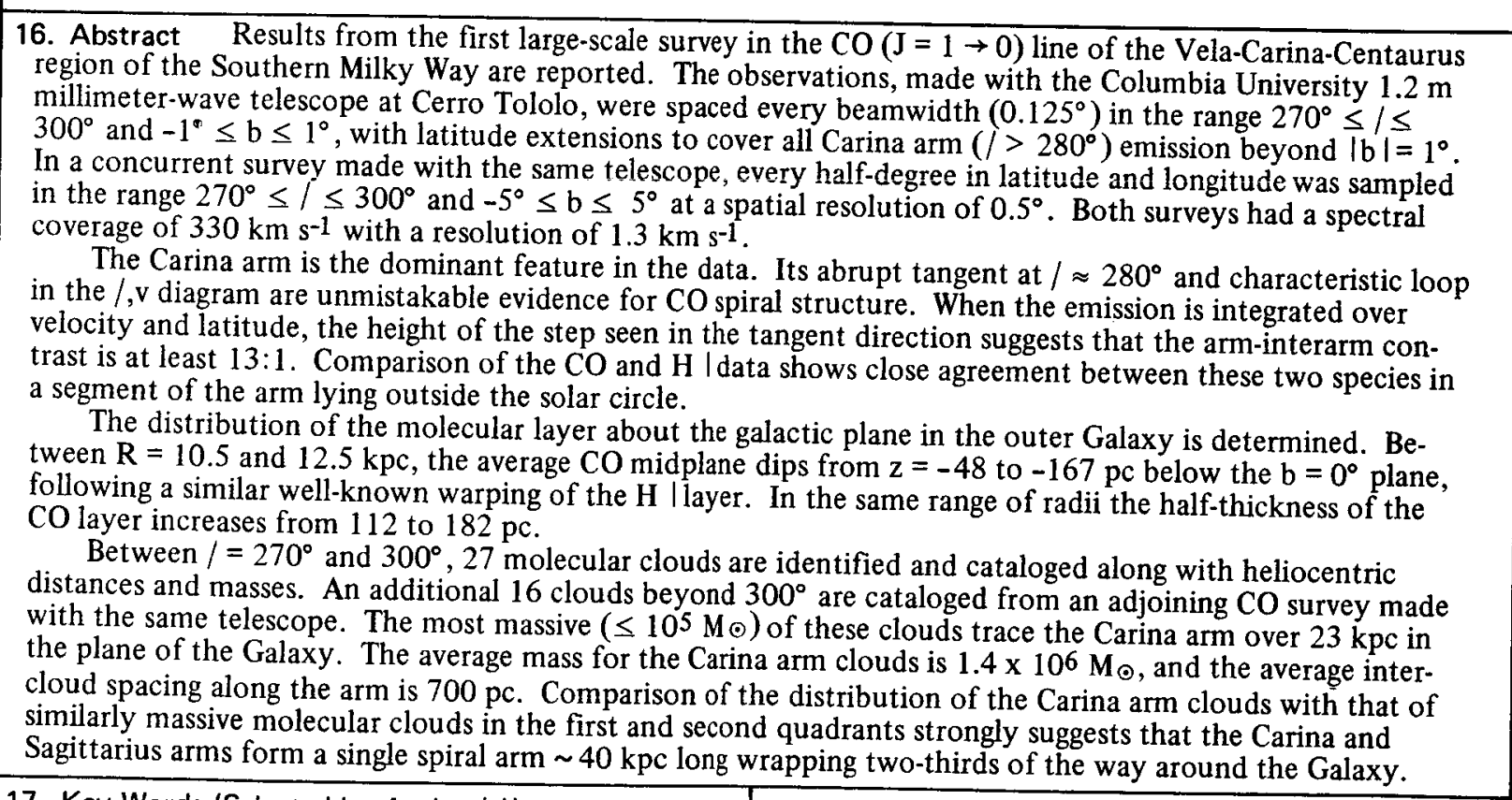 } \\
\hline \multirow{2}{*}{\multicolumn{2}{|c|}{$\begin{array}{l}\text { 17. Key Words (Selected by Author(s)) } \\
\text { CO, Molecular Clouds, Galactic Structure, } \\
\text { Star Formation }\end{array}$}} & \multicolumn{3}{|c|}{$\begin{array}{l}\text { 18. Distribution Statement } \\
\text { Unclassified - Unlimited }\end{array}$} \\
\hline & & & & ubject Category 89 \\
\hline $\begin{array}{l}\text { 19. Security Classif. (of this report) } \\
\text { Unclassified }\end{array}$ & $\begin{array}{l}\text { 20. Sect } \\
\text { Unc }\end{array}$ & sif. (of this page) & $\begin{array}{l}\text { 21. No. of Pages } \\
358\end{array}$ & $\begin{array}{l}\text { 22. } \text { Price }^{*} \\
\text { A16 }\end{array}$ \\
\hline
\end{tabular}

